\title{
VARIABILITY OF CURRENTS \\ IN GREAT SOUTH CHANNEL AND OVER GEORGES BANK: \\ OBSERVATION and MODELING
}

by

\section{Changsheng Chen}

M.S. in Marine Meteorology, Ocean University of Qingdao, 1983

M.S. in Physical Oceanography, MIT/WHOI Joint Program, 1989

Submitted in partial fulfillment of the

requirements for the degree of

DOCTOR OF PHILOSOPHY

at the

MASSACHUSETTS INSTITUTE OF TECHNOLOGY

and the

WOODS HOLE OCEANOGRAPHIC INSTITUTION

June 1992

(C) Changsheng Chen 1992

The author hereby grants to MIT and to WHOI permission to reproduce and to distribute copies of this thesis document in whole or in part.

Signature of Author

Joint Program in Physical Oceanography Massachusetts Institute of Technology

Woods Hole Oceanographic Institution

May 12, 1992

Certified by

Robert Beardsley

Senior Scientist

Thesis Supervisor

Accepted by

Lawrence J. Pratt

Chairman, Joint Committee for Physical Oceanography MASSACHUSETTS INSTITUTE A chusetts Institute of Technology

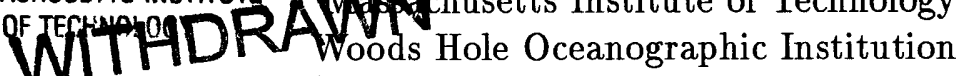
MAY 21 gPONA

N"NITEIBRAAts 


\title{
VARIABILITY OF CURRENTS \\ IN GREAT SOUTH CHANNEL AND OVER GEORGES BANK: OBSERVATION AND MODELING \\ by \\ Changsheng Chen
}

Submitted in partial fulfillment of the requirements for the degree of Doctor of Philosophy at the Massachusetts Institute of Technology and the Woods Hole Oceanographic Institution

May 11, 1992

\begin{abstract}
This thesis consists of two parts: (1) variability of currents and water properties in late spring in the northern Great South Channel and (2) numerical study of stratified tidal rectification over Georges Bank.

Part 1. Variability of Currents and Water Properties in Late Spring in the Northern Great South Channel

The South Channel Ocean Productivity Experiment (SCOPEX) was conducted to investigate springtime aggregations of right whales in the northern Great South Channel (GSC) region and the relationship between physical and biological variability. As part of SCOPEX, three regional CTD/ADCP surveys were made in the northern GSC during April 26-29, 1988, May 18-21, and June 6-11, 1989 to study water property distribution, structure of the low-salinity surface plume, tidal mixing boundaries, and the subtidal circulation. Satellite-tracked drifters drogued at 5 and $50 \mathrm{~m}$ were deployed during each survey. Several 24-hour CTD/ADCP anchor stations and separate CTD tow-yo transects were made at selected locations in 1989 in support of zooplankton vertical migration and spatial distribution studies.

The surface salinity patterns observed in late April, 1988 and June, 1989 differ significantly in the extent of the freshwater plume which occurs east of Cape Cod in spring. In April, 1988, the surface plume was just beginning to form along the outer coast of Cape Cod while six weeks later in 1989 , the minimum salinity was about $1.5^{\circ} \%$ less, and a large pool of water fresher than $31.6^{\circ} \%$ had pushed east over much of the northern GSC region. The difference in the amount of freshening observed between the two surveys is due primarily to the six-week difference in the seasonal cycle and increased river discharge in 1989. A significant difference is also found at mid-depth in the Maine Intermediate Water (MIW) for the two surveys. In April, 1988, the seasonal thermocline was just beginning to form, and the spatial structure of MIW was relatively uniform. In June 1989, a narrow core of temperature minimum water (with $T_{\min }$ in a range of $3.2^{\circ}$ to $4.4^{\circ} \mathrm{C}$ ) was found along
\end{abstract}


the western flank of the northern GSC between $40 \mathrm{~m}$ and $120 \mathrm{~m}$. This colder and fresher water spread to mix with the interior MIW as the core flowed southward into the central GSC. Hydrographic data plus satellite Sea Surface Temperature (SST) images showed a continuous tidal mixing boundary along the $80 \mathrm{~m}$ isobath in the GSC with a $10 \mathrm{~km}$ crossisobath variation during late spring 1988 and 1989, which agreed well with the contour of $\log _{10} \mathrm{~h} / \mathrm{D}=2.1$ based on energy arguments.

An empirical least-squares fit method has been used to separate the SCOPEX ADCP data into tidal and residual current components for each survey and the anchor stations. The resulting field of vertically averaged residual flow shows a cyclonic circulation pattern in the northern GSC, with strong currents in excess of $10 \mathrm{~cm} / \mathrm{s}$ flowing southward and southeastward along the western flank of the northern GSC, and turning northeastward to flow along the northern flank of Georges Bank. This cyclonic circulation was found at all levels throughout at least the upper $120 \mathrm{~m}$ (the maximum sampling depth of the ADCP), with maximum inflow and outflow occurring at the western and eastern ends of the crosschannel transects, respectively. There were two velocity maxima in the vertical; one was at the surface to drive the surface water, the other was at mid-depth to carry MIW into the northern GSC region. The residual ADCP current pattern is consistent with the vertical distributions of water masses and agrees well with trajectories of drifters deployed at 5 and $50 \mathrm{~m}$ in the GSC. A relatively strong, deep, jet-like flow was observed in June, 1989, corresponding to the narrow core of minimum temperature at a depth of $50 \mathrm{~m}$ along the western flank of the northern GSC. The vertical shears of geostrophic and ADCP residual flows were consistent within fitting errors in the deeper part of the northern GSC where the tidal current is weak, implying that buoyancy-driven flow was dominant in the central region of the GSC and tidal rectification over the shallower sides of the GSC in late spring.

\section{Part 2. Numerical Study of Stratified Tidal Rectification over Georges Bank}

Stratified tidal rectification over Georges Bank has been studied numerically using a two-dimensional primitive equation model with turbulent closure developed by Blumberg and Mellor (1987). A sequence of initial value experiments was first conducted for the homogeneous and stratified tidally driven flows over a symmetrical, finite-amplitude topographic bump, and then the model was applied to the real asymmetrical bottom topography over Georges Bank. In the unstratified case, the model predicts a topographically controlled clockwise residual circulation around Georges Bank, flowing northeastward as a strong jet with a maximum speed of about $16 \mathrm{~cm} / \mathrm{s}$ along the northern flank and southwestward as a relatively weak and broader flow with a maximum speed of about $5 \mathrm{~cm} / \mathrm{s}$ from the top of the Bank to the southern flank. As stratification is added, internal tidal rectification and tidal mixing intensify the along- and cross-isobath residual currents, and create tidal fronts which modify the vertical structure of the residual flow. During summer, the tidal front is located at the $40-\mathrm{m}$ isobath on the northern flank and at the $50-60-\mathrm{m}$ isobath on the southern flank, and the maximum of the along-bank current is increased up to about 
$32 \mathrm{~cm} / \mathrm{s}$ on the northern flank and to $8 \mathrm{~cm} / \mathrm{s}$ on the southern flank. During winter, the position of the tidal mixing front remains fixed on the northern flank, however, it moves to the shelf break on the southern flank. The winter maximum of clockwise along-bank residual flow is about $26 \mathrm{~cm} / \mathrm{s}$ on the northern flank and about $6 \mathrm{~cm} / \mathrm{s}$ at the shelf break on the southern flank. The model results are consistent with theories for stratified tidally driven flow, and in good agreement with observations on the northern flank. The predicted along-bank residual current is relatively weaker than observed on the southern flank, suggesting that buoyancy driving associated with the cross-bank density field and shelf break front is also important in generating residual flow on the southern flank.

Thesis Supervisor:

Dr. Robert Beardsley, Senior Scientist

Woods Hole Oceanographic Institution 


\section{Table of Contents}

$\begin{array}{ll}\text { Abstract } & 3\end{array}$

$\begin{array}{lr}\text { Acknowledgments } & 9\end{array}$

$\begin{array}{ll}\text { 1. Introduction } & 11\end{array}$

2. Variability of Currents and Water Properties in Late Spring in the Northern Great South Channel 22

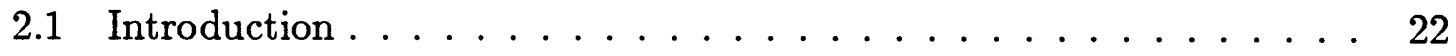

2.2 Variability of Water Properties in Late Spring in the Northern GSC from 1988 to 1989 . . . . . . . . . . . . . . . . . . . . 27

2.3 Separating the Low-Frequency and Tidal Currents from the ADCP Data . . . . . . . . . . . . . . . . . . 47

2.4 Structure of Tidal Currents in the Northern GSC . . . . . . . 65

2.5 Structure of Residual Flow in the Northern GSC . . . . . . . 78

2.6 Possible Relationship Between the High Concentration of Zooplankton and the Physical Environment in the Northern GSC . . . . . 109

2.7 Conclusion and Discussion . . . . . . . . . . . . . 113

3. Numerical Study of Stratified Tidal Rectification Over Georges Bank

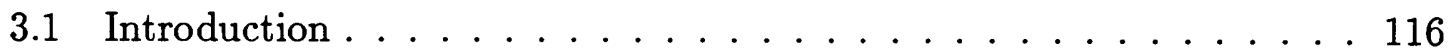

3.2 Review and Discussion of Previous Theoretical Works . . . . . . 120

3.3 The Numerical Model . . . . . . . . . . . . . . . . . . . . . . . . . . 129

3.4 Barotropic Tidal Rectification Over a Symmetrical Bank . . . . . . . 139

3.5 Stratified Tidal Rectification Over a Symmetrical Bank . . . . . . 168 
3.6 Tidal Rectification Over Georges Bank . . . . . . . . . . . . . 214

$\begin{array}{ll}\text { References } & 260\end{array}$

$\begin{array}{ll}\text { Appendix A } & 271\end{array}$

$\begin{array}{ll}\text { Appendix B } & 282\end{array}$ 


\section{Acknowledgments}

I would first of all like to thank my advisor Bob Beardsley for his patient guidance and encouragement, for many valuable discussions, and for his constructive criticisms of this work. Also, I would like to thank him for his efforts in editing my early, poorly written thesis drafts. His deep insight into a wide range of ocean sciences and his imagination have been a great help to me in both observational and numerical works.

I want to thank the members of my thesis committee, Dave Chapman, Glenn Flierl, Paola Rizzoli, and Glen Gawarkiewicz and the thesis defense chairman, Steve Lentz, for their helpful comments and suggestions. I would also like to thank Carl Wunsch, Ken Brink, John Loder, and Rocky Geyer for their interest in my thesis work and valuable comments. Dave gave me a great deal of help in preparing my thesis proposal in numerical modeling. His advice on the treatment of open boundary conditions saved me a lot of time in adding a radiation-plus-sponge open boundary condition in the Blumberg and Mellor model. Discussions with Glenn Flierl and Paola Rizzoli motivated me to study the driving mechanism of stratified tidal rectification using diagnostic analysis methods. Conversations with Glen Gawarkiewicz about the shelf break front were valuable in my interpretation of numerical results over Georges Bank.

Special thanks go to Alan Blumberg, who allowed me to use the new version of the Blumberg and Mellor model (ECOM3D-SI) for my numerical study of tidal rectification over Georges Bank. I would also like to thank Rich Signell for his help in teaching me the numerical code of ECON3D-SI and for providing the Fortran program for calculating the time series of each tidal constituent in the study area.

I would also like to thank Hsiao-ming Hsu for his help in learning SPEM model developed by Haidvogel et al. (1991). Although I did not use Haidvogel's model for my thesis work, working with Hsiao-ming enriched my experience in numerical modeling. 
I would like to thank Graham Giese, who gave me the tidal gauge data at Nauset and Chatham for comparison with the ADCP data; Jack Jossi, who permitted me to use the surface salinity data obtained on the NOAA Ship Opportunity Program (SOOP); and Howard Winn and Karen Wisher, who provided the sea surface temperature image, zooplankton, and whale sighting data. Richard Limeburner and Robert Beardsley were primarily in charge of the physical oceanographic field experiments of SCOPEX. Richard Limeburner processed the CTD and drifter data used in Chapter 2 of my thesis and also gave me a great deal of help in data analysis. In particular, I would like to thank him for his friendly and invaluable help during my five-year study in the MIT/WHOI Joint Program. I enjoyed sharing the information about the ADCP treatment with Julio Candela and thank him for nice comments on my work.

I also want to thank Steve Lentz for giving me his SUN 3/50 for my numerical work. I learned how to use the NCAR CRAY computer at WHOI from Dave Chapman, Hsiao-ming Hsu and Glen Gawarkiewicz. I cannot forget all kinds of help from Abbie Jackson in the Education Office in the last five years. Anne-Marie Michael taught me how to use $\mathrm{IAT}_{\mathrm{E}} \mathrm{X}$ and helped polish the final draft of this thesis. The fancy format and good organization of my thesis would have been impossible without her kind help. In addition, I wish to thank Barbara Gaffron for her editorial help on the final version of this thesis.

The friendship and support of my fellow Joint Program students have been invaluable. In particular, I would like to thank my classmates David Walsh, Kurt Polzin, Barry Klinger, and David Chester for their friendly help over the last five years.

Finally, I thank my darling wife Huichan Lin for her patient support during the last five years, and for the promise of continued love and happiness in the future.

This research was supported by the National Science Foundation under grants OCE 87-13988 and OCE 91-01034 and by the National Center for Atmospheric Research (NCAR) under computer time grants Nos. 35781029 and 35781035. 


\section{Chapter 1. Introduction}

The Gulf of Maine (GOM), located on the North American continental shelf between Cape Cod and Nova Scotia, is a semi-enclosed basin opening to the North Atlantic Ocean (Figure 1.1). The geometry of the GOM is mainly characterized by several deep basins and shallow submarine banks. On the seaward flank of the GOM is Georges Bank, which is separated from the Nantucket Shoals to the west by the Great South Channel (GSC) and from the Scotian Shelf to the east by the Northeast Channel. The bank is roughly elliptical in plane view with a length of about $200 \mathrm{~km}$ along the major axis and a width of about $150 \mathrm{~km}$ along the minor axis. The cross-bank bottom topography rises steeply with a slope of about 0.01 from $1000 \mathrm{~m}$ on the slope to $100 \mathrm{~m}$ at the shelf break, increases slowly with a slope of 0.0004 to $40 \mathrm{~m}$ over a distance of about $150 \mathrm{~km}$ on the top of the Bank, and then decreases rapidly with a slope of about 0.03 to a depth of $300 \mathrm{~m}$ in the deep basin just north of Georges Bank. GSC runs approximately north-south, with a sill depth of about $50 \mathrm{~m}$ located near $40^{\circ} 45^{\prime}$ to separate the mid and outer continental shelf to the south from the deeper GOM to the north with depths in excess of $150 \mathrm{~m}$. The deep connection between the GOM and the open ocean is mainly through Northeast Channel where the sill depth is about $230 \mathrm{~m}$.

The first systematic study of general circulation in the GOM can be traced back to Bigelow (1927). Based on a large number of surface drifter and hydrographic observations, he suggested that the summertime surface circulation in the GOM is dominated by two relatively large-scale gyres: a cyclonic circulation around Jordan Basin and an anticyclonic circulation around Georges Bank (Figure 1.2). Long-term direct Eulerian and Lagrangian current measurements in the western GOM and around Georges Bank summarized by Butman et al. (1982) demonstrate a permanent anticyclonic gyre around Georges Bank and a southward coastal flow along the southeast coast of Cape Cod that vary seasonally with a maximum in late spring and summer and a minimum in winter (Figure 1.3). This flow field 


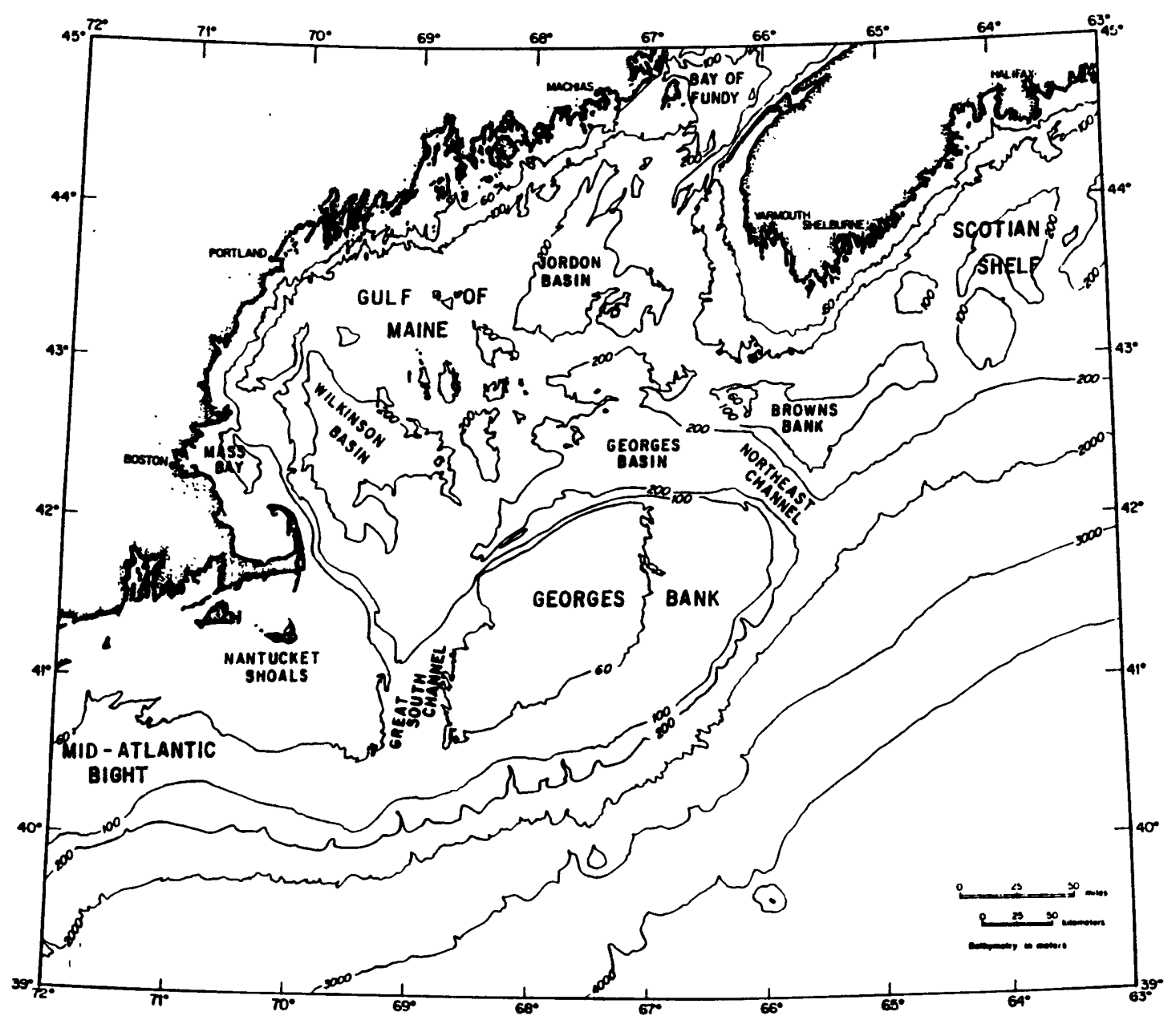

Figure 1.1: Bathymetry (in meters) of the southern New England continental margin (Uchupi and Austin, 1987). 


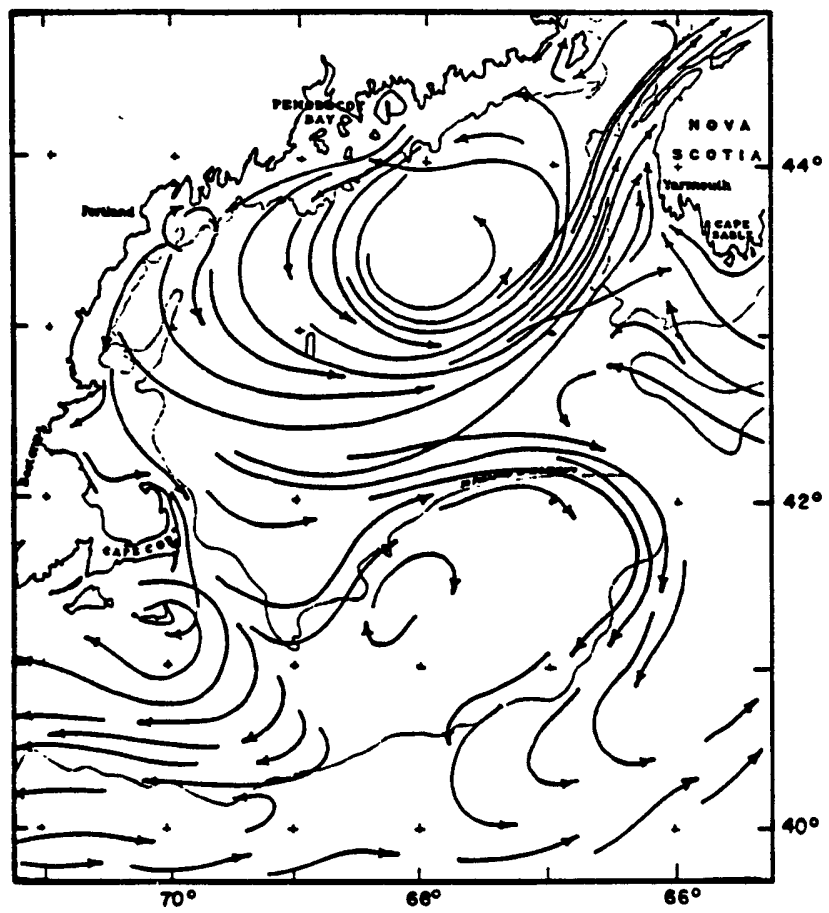

Figure 1.2: Summer near-surface, non-tidal circulation pattern in the Gulf of Maine suggested by Bigelow (1927).

is clearly illustrated by trajectories of the near-surface satellite-tracked drifters deployed by Limeburner and Beardsley during the 1988 and 1989 SCOPEX surveys, which show the closed nature of the around-bank circulation in summer caused by recirculation of water around Georges Bank and a southward coastal current flowing along the east coast of Cape Cod (Figure 1.5). The pattern of anticyclonic mean flow around Georges Bank has been attributed mainly to the rectification of strong semidiurnal tidal currents over the variable bottom topography (Loder, 1980, Loder and Wright, 1985 and Butman et al., 1982), which was also examined numerically by Greenberg (1983) using a vertically averaged model driven by the $M_{2}$ tide (Figure 1.4). The southward flow along the western flank of the GSC is believed to be due to both tidal rectification and river discharge (Butman et al., 1982 and Greenberg, 1983). There have been few direct current measurements in Jordan Basin to prove the closed nature of cyclonic circulation there (Brooks, 1986). 


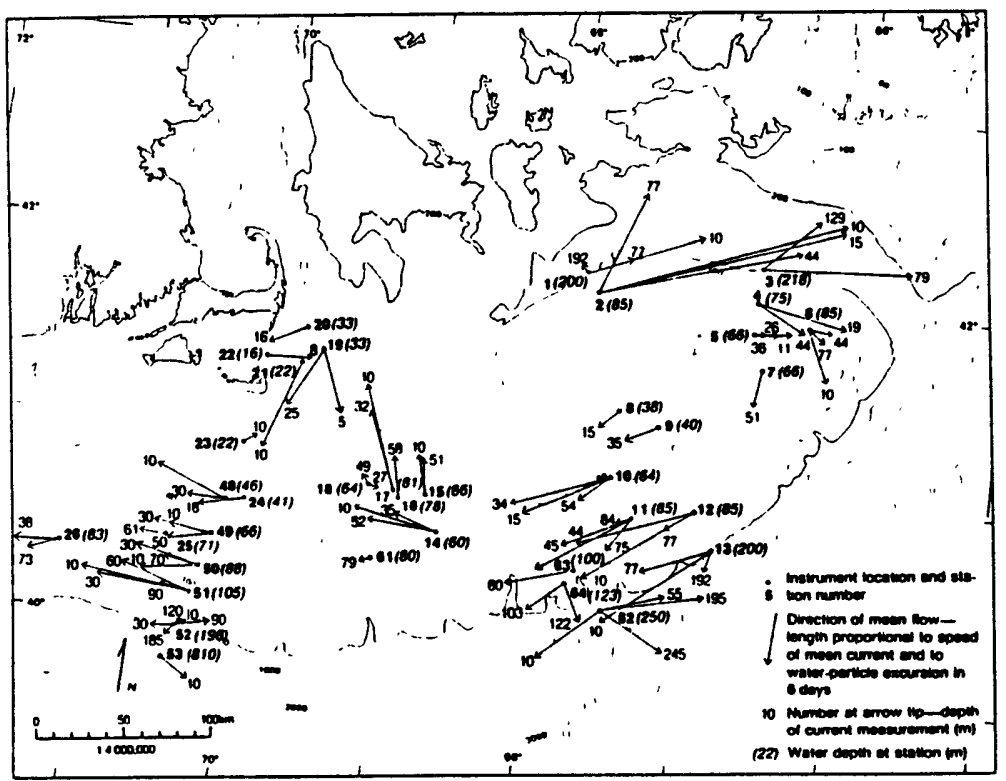

Figure 1.3: Mean Eulerian currents measured on Georges Bank and western GOM during 1975-1981 (Butman et al.,1982). The boldface numbers at origins of the current vectors are station identifiers. Italic numbers in parentheses indicate water depth $(\mathrm{m})$. Numbers at the tips of arrow indicate depth of measurement $(\mathrm{m})$. The speed scale is such that the length of current vector is equivalent to the mean displacement of a water particle during a six-day period at map scale.

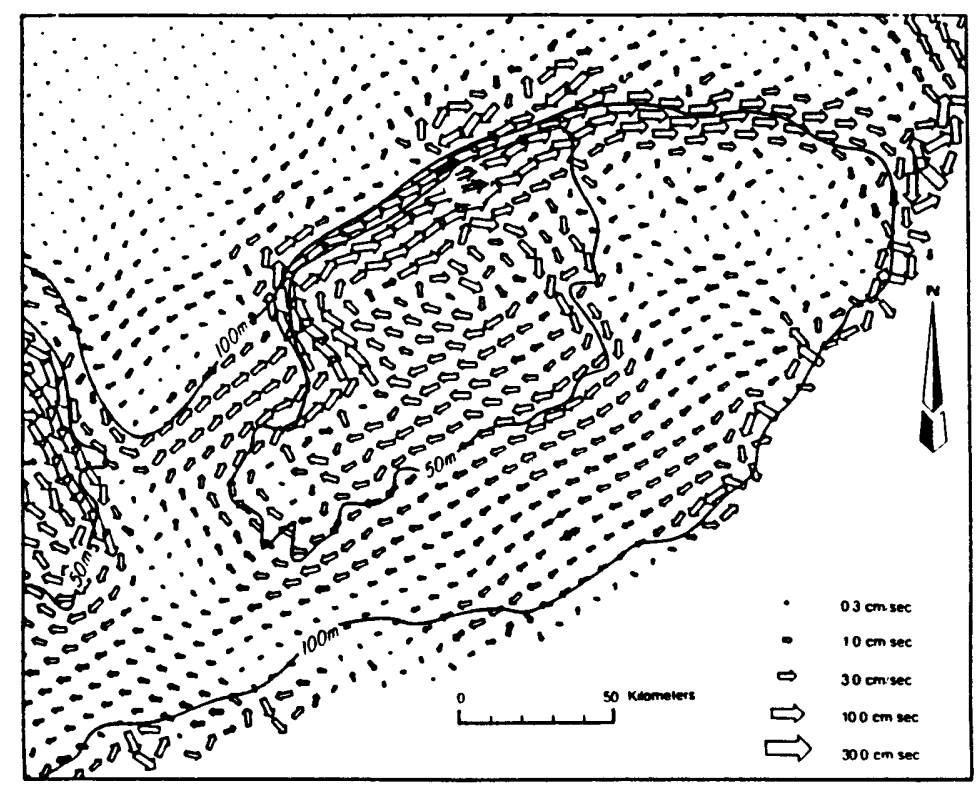

Figure 1.4: Residual currents in the Gulf of Maine induced by $M_{2}$ tidal forcing (Greenberg, 1983). A possible cyclonic recirculation cell is located north of $100-\mathrm{m}$ isobath in the GSC. 
Water properties in the GOM are mainly influenced by (1) exchange with the Atlantic Ocean through Northeast Channel, (2) freshwater input from both local and distant rivers, (3) atmospheric forcing, and (4) tidal mixing. Cold, low-salinity shelf water, entering the GOM from the Scotian Shelf at the surface (so defined as Scotia Shelf Water, SSW), and warm, high-salinity Slope Water (SW), flowing into the GOM directly through the Northeast Channel, provide the main sources of the Maine Surface and Bottom Waters (Smith, 1983, Mountain and Jessen, 1987 and Ramp et al., 1985). The winter overturning due to surface cooling and wind mixing leads to a vertical mixing between the cold, fresh surface water and warm, saline deep water within the GOM. During the following spring, surface warming re-stratifies the upper surface layer to cause a relatively prominent middepth temperature minimum water mass, which is characterized as Maine Intermediate Water (MIW, see Brown and Beardsley, 1978, and Hopkins and Garfield, 1979). Strong river discharge in late spring from Nova Scotia to Cape Cod causes an along-coast buoyancydriven, low-salinity plume, which is responsible for a nearshore band of low-salinity water near the surface in the western GOM in late spring and summer. Strong tidal currents mix the water over the shelf and the shallow bank, forming the vertically well-mixed water there (Garrett et al., 1978).

In spite of our knowledge of circulation and water mass properties in the western GOM, there are still many unanswered questions about the circulation pattern in the northern GSC and seasonal variability of mean circulation around Georges Bank. According to Bigelow's schematic description of nontidal circulation in the GOM, the surface circulation in the western GOM is a combination of a cyclonic flow in the northern part west of Jordan Basin, an anticyclonic recirculating flow in the southern part around Georges Bank, and a buoyancy-driven current along the western coast (Figure 1.2). However, the barotropic numerical model developed by Greenberg (1983) predicted a weaker tidally rectified cyclonic recirculation cell of order $30-50 \mathrm{~km}$ in scale and $1-3 \mathrm{~cm} / \mathrm{s}$ in strength in the northern GSC (Figure 1.4), which is different from Bigelow's classic schematic. What is the structure of nontidal flow in the northern GSC? Is there really a cyclonic eddy there as Greenberg 

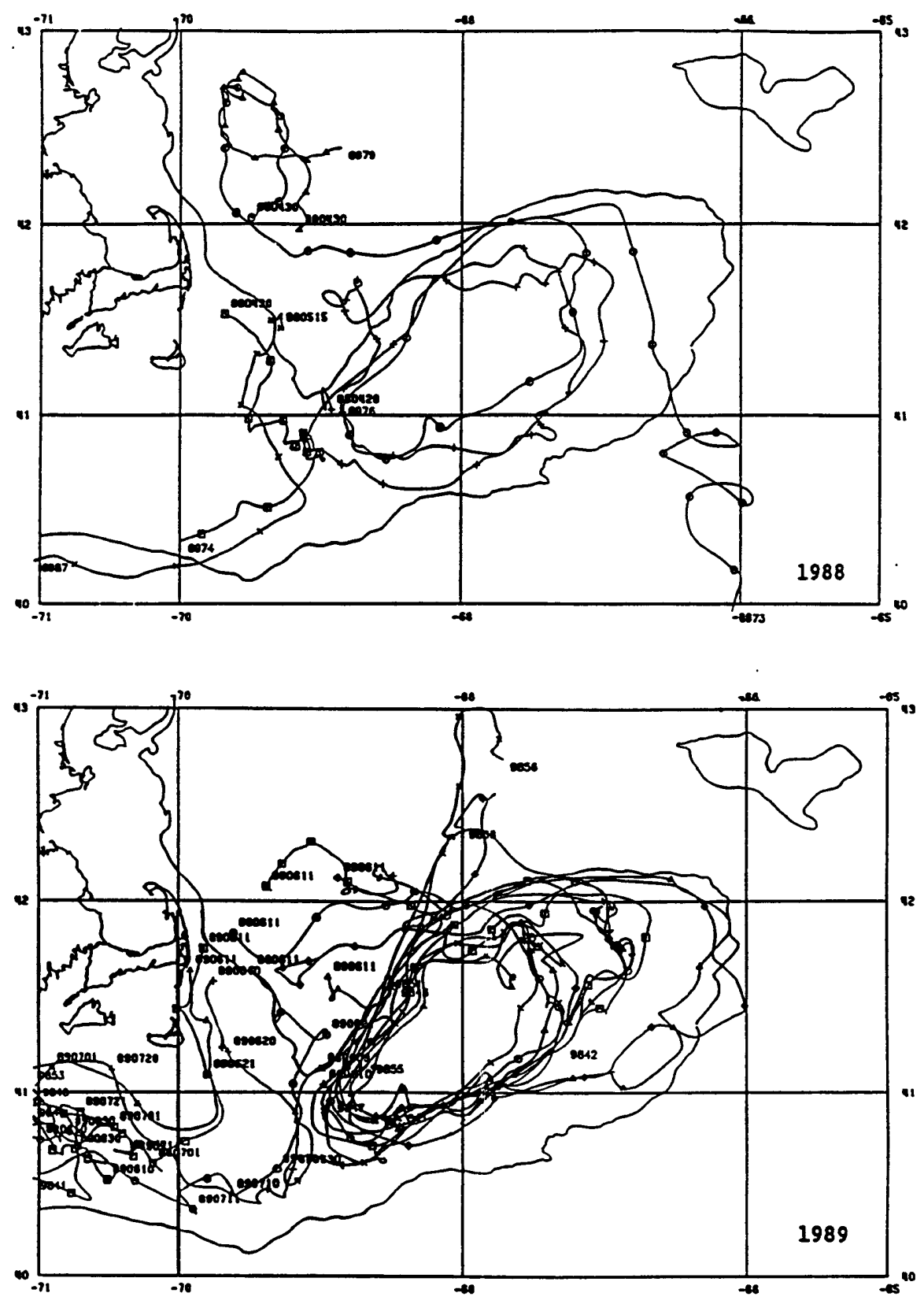

Figure 1.5: Trajectories of the SCOPEX 5-m satellite drifters deployed by Limeburner and Beardsley in late April 1988 (upper) and June 1989 (lower). The six-digit number is the measurement time, and four-digit number is the instrument label. 
predicted? Which physical process drives the residual flow in that area, tidal rectification or buoyancy forcing?

The exchange of water between the GOM and the New England shelf through the GSC is not yet clearly known. Hopkins and Garfield (1979) suggested a southward flow of MIW through the GSC to the New England shelf as a conservative mass based on the similar structure of the T-S envelope of MIW on both sides of the GSC. This suggestion may not be true because strong tidal mixing is usually observed in the southern GSC where the shallow sill of about $50 \mathrm{~m}$ is located. Where does the MIW go in the northern GSC? Does it recirculate like an eddy in Wilkinson Basin or flow along the local isobaths to join the anticyclonic gyre around Georges Bank? How much MIW can flow out to the New England shelf through the GSC? Two relatively complete regional hydrographic surveys, conducted in the western GOM during late spring, May 11-12, 1976 and May 24-June 7, 1979, show a surface low-salinity plume spreading along the western coast of the GOM and into the northern GSC region (Limeburner and Beardsley, 1982). This plume is believed to originate from spring river discharge into the GOM, primarily along the Maine and New Brunswick coasts, and the freshwater inflow from the Nova Scotia shelf. Does the low-salinity plume just flow along the western coast to the Nantucket Shoals like a buoyancy-driven, coastaltrapped current? How is the trajectory of this plume influenced by local and remote wind stress or deep circulation? The semidiurnal $M_{2}$ tide is dominant in the western GOM. Strong tidal mixing over the shelf results in the tidal mixing front over Nantucket Shoals, the GSC, and Georges Bank, separating the vertically well-mixed water over the shelf from the stratified water in the deeper northern GSC. Where is this front located in late spring? Is it continuous across the the GSC? All of these questions are closely associated with the aggregation and migration of zooplankton in the GSC, which will be described next.

Each spring, for at least the last ten years, almost the entire population of North Atlantic right whales migrates to the GSC region where unusually dense aggregations of the zooplankton Calanus finmarchicus, the primary food of the right whale, are found (Lime- 
burner and Beardsley, 1989). During the regional SCOPEX surveys in 1988 and 1989, the area of highest zooplankton concentration was observed in the western GSC in 1988 and in the eastern GSC in 1989 (Figure 1.6). Correspondingly, the observed right whales stayed in the western side of the GSC in 1988 but they moved to the eastern side in 1989 (Figure 1.7). In 1987, in the SCOPEX proposal, Beardsley and Limeburner suggested that such large aggregations and migration of biological productivity might be a direct result of the unique physical environment in the GSC. They proposed three possible physical mechanisms as follows: (1) the mechanical mixing of the water column by energetic tidal currents in the shallower region of the GSC; (2) the low-salinity plume; and (3) lateral mixing and convergence in the exchange region between the southward-flowing, low-salinity plume and the anticyclonic current around Georges Bank. The tidal mixing may result in a permanent front between the well-mixed water over the shelf and the temperature/salinity stratified water in the deeper areas of the GSC region so as to cause the richness of zooplankton there. The low-salinity plume may advect zooplankton and nutrients from the northwestern GOM into the GSC in spring. When the southward-flowing, low-salinity plume meets nutrient-rich, vertically well-mixed shelf water flowing northward through the GSC around Georges Bank, lateral mixing may lead to the increase of zooplankton in the GSC, and then, the convergence may help maintain a persistent high concentration of large zooplankton there. Moreover, the inter-annual variability of the low-salinity plume and local water motion in the northern GSC may be responsible for the inter-annual migration of the high zooplankton concentration in that area. Examination of these possible hypotheses requires more information about the flow field and water structure in the GSC.

Tidally rectified flow is a significant component in the GOM general circulation, especially over Georges Bank and Nantucket Shoals. Theoretical studies predict that an along-bank mean flow can be generated by a nonlinear transfer of vorticity and momentum from the tidal currents when a long barotropic tidal wave propagates from the deep ocean onto variable bottom topography (Huthnance, 1973 and 1981, Loder, 1980, and Zimmerman, 1978 and 1980). Friction, which creates a strong vertical shear in the tidal current near 


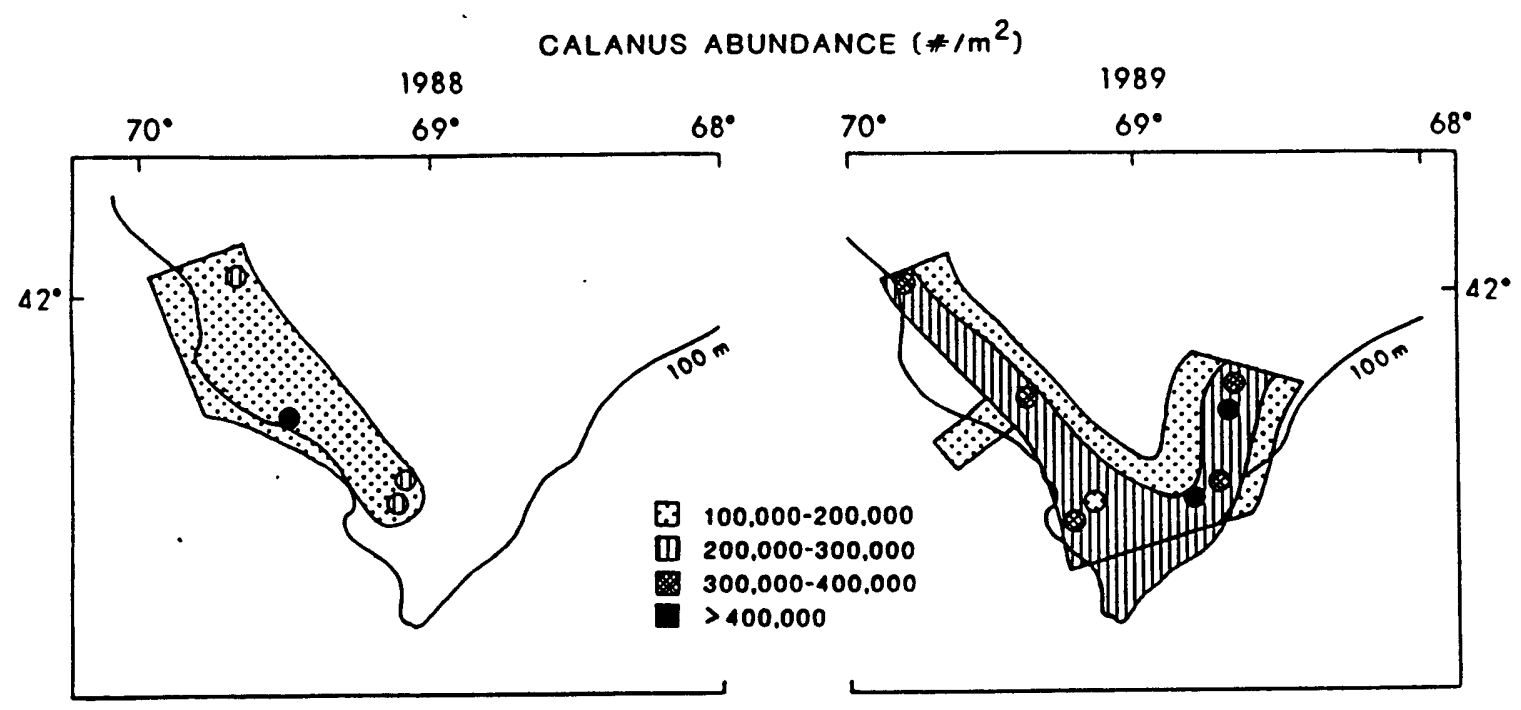

Figure 1.6: Calanus abundance $\left(\# / \mathrm{m}^{3}\right)$ in the late spring of 1988 (left) and 1989 (right). The solid line represents the $100-\mathrm{m}$ isobath and four different marked squares at middle of the figure show the ranges of Calanus numbers per square meter.
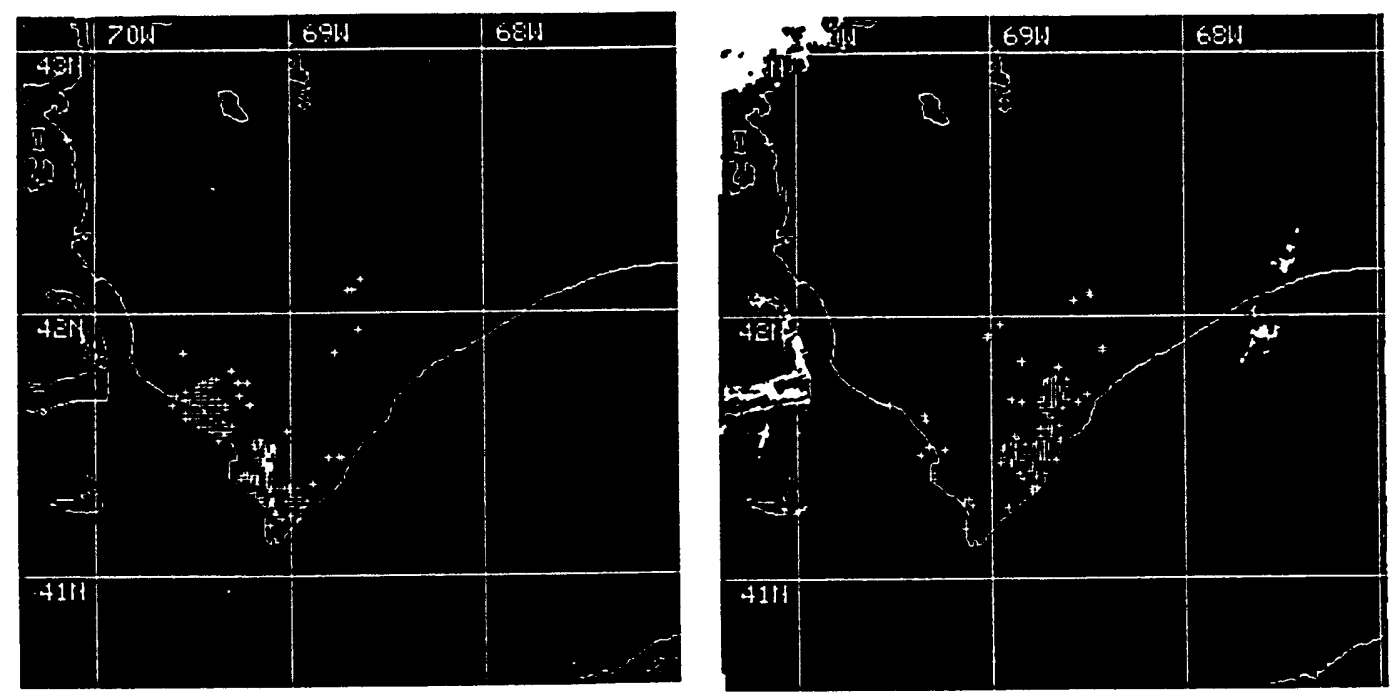

Figure 1.7: Right whale sightings in the late spring of 1988 (left) and 1989 (right). ' + ' is an individual whale sighting and the solid line represents the $100-\mathrm{m}$ isobath. 
the sea floor and shifts the tidal current phases, is important to balance the divergence in the tidal momentum flux across isobaths during the tidal rectification process (Wright and Loder, 1985). Whenever stratification is involved, strong tide-induced vertical mixing and internal tides can be generated over variable topography which may contribute to an alongbank mean flow through either a cross-bank density gradient (Loder and Wright, 1985) or baroclinic tidal rectification (Maas and Zimmerman, 1989a and $b$ ). The two-dimensional analytical or semi-analytical models (developed by Huthnance, 1973, Loder, 1980 and Loder and Wright, 1985, Zimmerman, 1978 and 1980, Maas and Zimmerman, 1989a and $b$ ) provide good insight into the dynamical mechanisms that generate residual flow over topography. However, they cannot quantitatively predict the detailed spatial structure and strength of residual currents because of the assumption of a small-amplitude bottom variation or harmonic truncation. How does the large and asymmetrical bottom slope influence the spatial distribution of residual current over Georges Bank? How important are the tidal mixing fronts and internal tidal rectification in the seasonal intensification of residual flow over finite-amplitude and asymmetrical bottom topography? Can the seasonal variability of the locations of tidal fronts be predicted with a numerical model?

This thesis consists of two parts: (I) a descriptive study of the mesoscale variability of water properties and currents in the northern GSC and (II) a numerical study of stratified tidal rectification over Georges Bank. In Chapter 2, the CTD, ADCP, satellitetracked drifter, SST, and surface wind data taken in late spring of 1988 and 1989 as part of the South Channel Ocean Productivity Experiment (SCOPEX) have been used (1) to describe the variability of water properties, location of tidal mixing fronts, and tidal and mean flows in the western GOM, with emphasis on the spring transition and spatial/temporal variability associated with the spring freshwater plume occurring off Cape Cod and deep Maine Intermediate Water (MIW) in the northern GSC, and (2) to investigate the relationship between variability in the zooplankton concentration and physical environment. In Chapter 3, a two-dimensional version of the Blumberg and Mellor numerical circulation model ENCOM3D-SI (Blumberg, 1991) with turbulence closure is developed using a peri- 
odic boundary condition in the along-bank direction and specifying a gravity wave radiation condition plus a sponge layer at the open boundary in the cross-bank direction. A sequence of initial experiments is conducted first over different finite-amplitude symmetrical topographic features or bumps to investigate the effects of topography, stratification, internal tidal rectification, and tidal mixing on the structure of stratified residual flows over variable bottom topographies, and then the model is applied to simulate stratified residual flow over the asymmetrical Georges Bank. 


\section{Chapter 2. Variability of Currents and Water Prop- erties in Late Spring in the Northern Great South Channel}

\section{$2.1 \quad$ Introduction}

As components of the SCOPEX field program, two regional CTD/ADCP surveys coupled with satellite-tracked drifter deployments were conducted in the northern GSC during April 26-29, 1988 and June 6-12, 1989 (Figure 2.1). ${ }^{1}$ The principal goals of these measurements were (a) to characterize water properties in regions of enhanced biological productivity, (b) to investigate the structure of the low-salinity surface plume observed east of Cape Cod in late spring, (c) to locate the front or boundary between the vertically well-mixed water over Nantucket Shoal, the GSC, Georges Bank, and the stratified water in the deeper northern GSC, and (d) to determine the three-dimensional structure of the flow field in the northern GSC and its relationship to the high zooplankton concentrations found there. A meso-scale regional CTD/ADCP survey was also made during May 1821,1989 on the western flank of the GSC east of Cape Cod to see if the surface freshwater plume was present and hence to determine the seasonal evolution of the plume during spring (Figure 2.2).

Mountain and Manning (1991) studied the annual and interannual variability of water properties in the GOM using the NOAA MARMAP ten-year hydrographic data set (from 1977 to 1987). They found that the variability of the surface low-salinity water in the western GOM is due primarily to the seasonal and interannual variability of local river discharge, with a maximum in late spring and a minimum in winter. Hydrographic survey data taken in the SCOPEX 1988 and 1989 CTD surveys provide snapshots of the distribu-

\footnotetext{
${ }^{1}$ All CTD/ADCP measurements were made by Limeburner and Beardsley. A detailed description of the CTD data correction can be found in the SCOPEX data report of CTD measurements which was published by Limeburner and Beardsley (1989).
} 

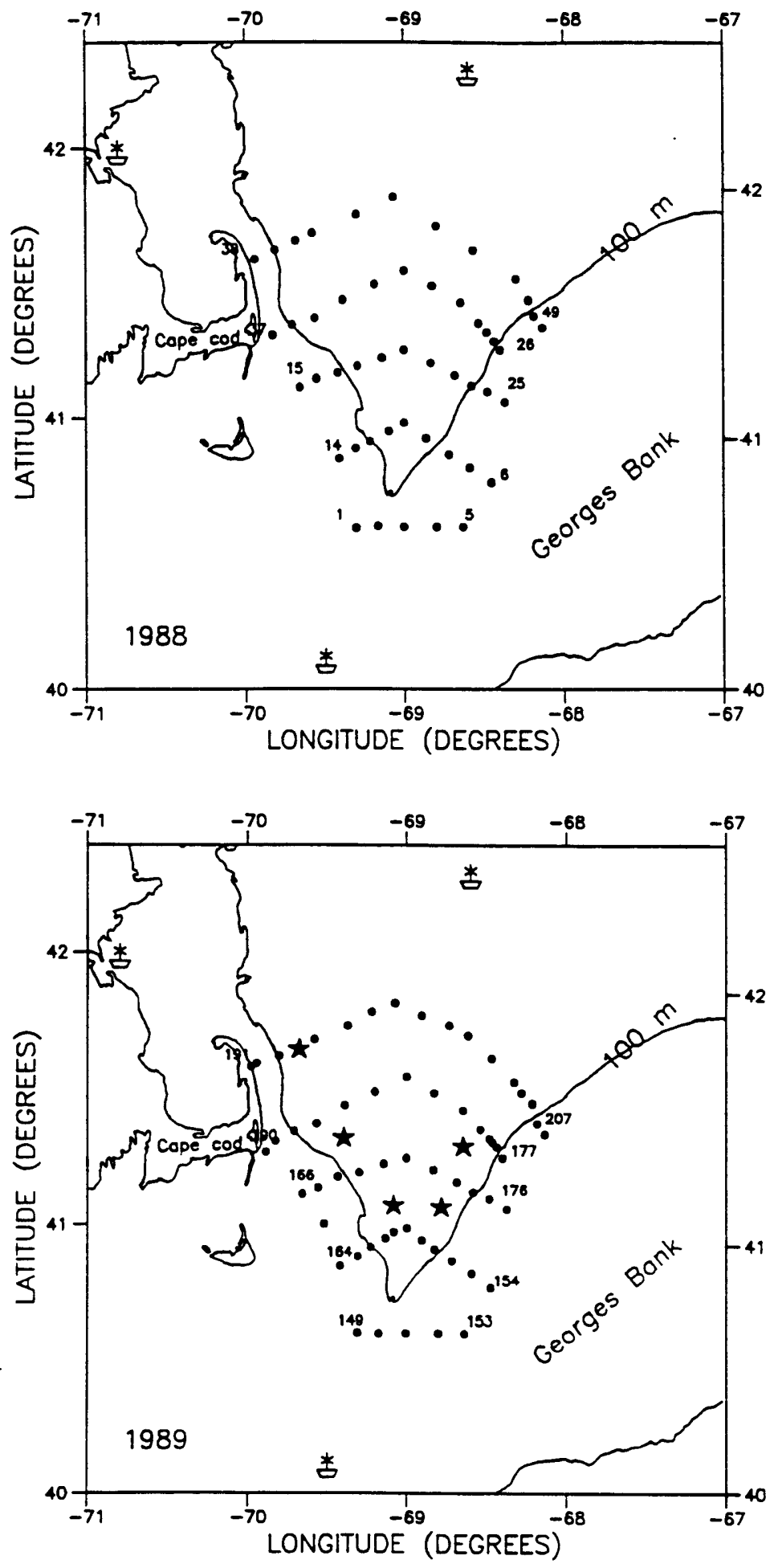

Figure 2.1: Locations of regional SCOPEX CTD survey stations (•) taken during April 26-29, 1988 (upper) and June 6-12, 1989 (lower). Integers in both the maps are the CTD station numbers and " $\star$ " represents the averaged spot of small-scale ADCP surveys conducted in late May and early June, 1989. " $\perp$ " indicates the position of NOAA meteorological buoys. Continuous ADCP records were taken along the path of the CTD measurements during both 1988 and 1989 surveys. 


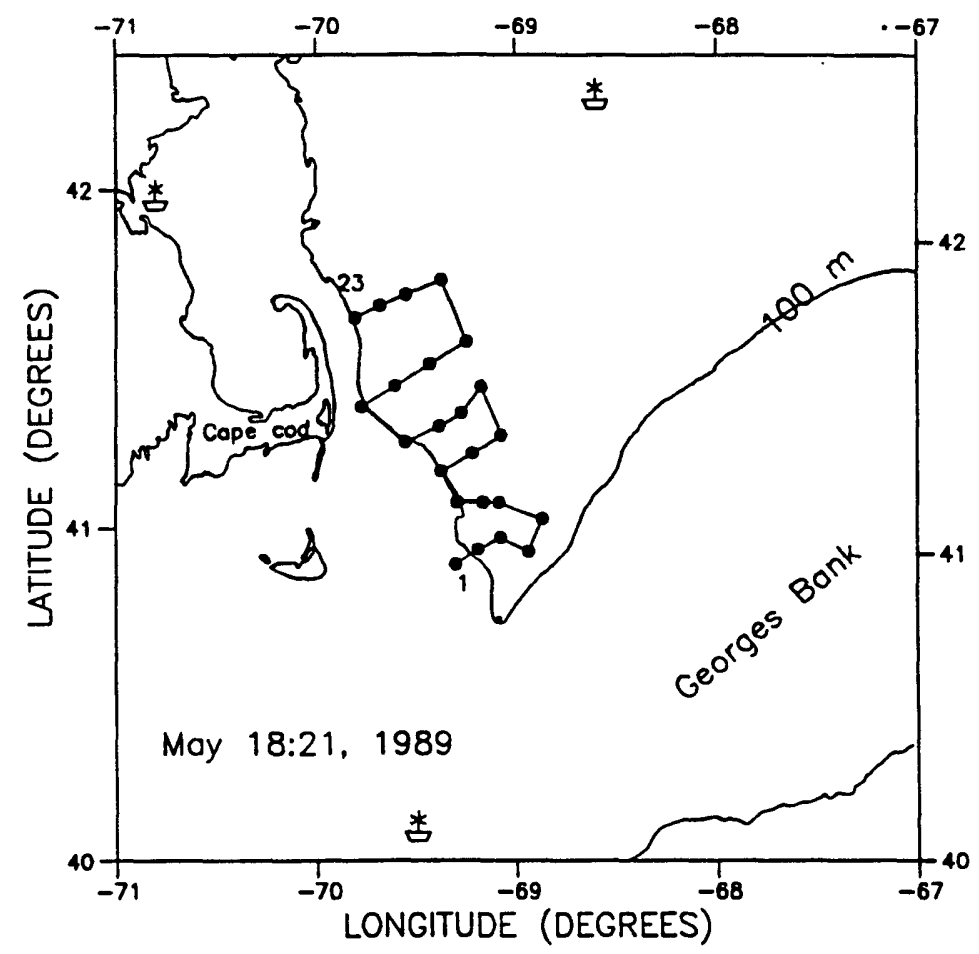

Figure 2.2: Locations of regional SCOPEX CTD/ADCP survey stations (•) taken during May 18-21, 1989. Integers are the CTD station numbers.

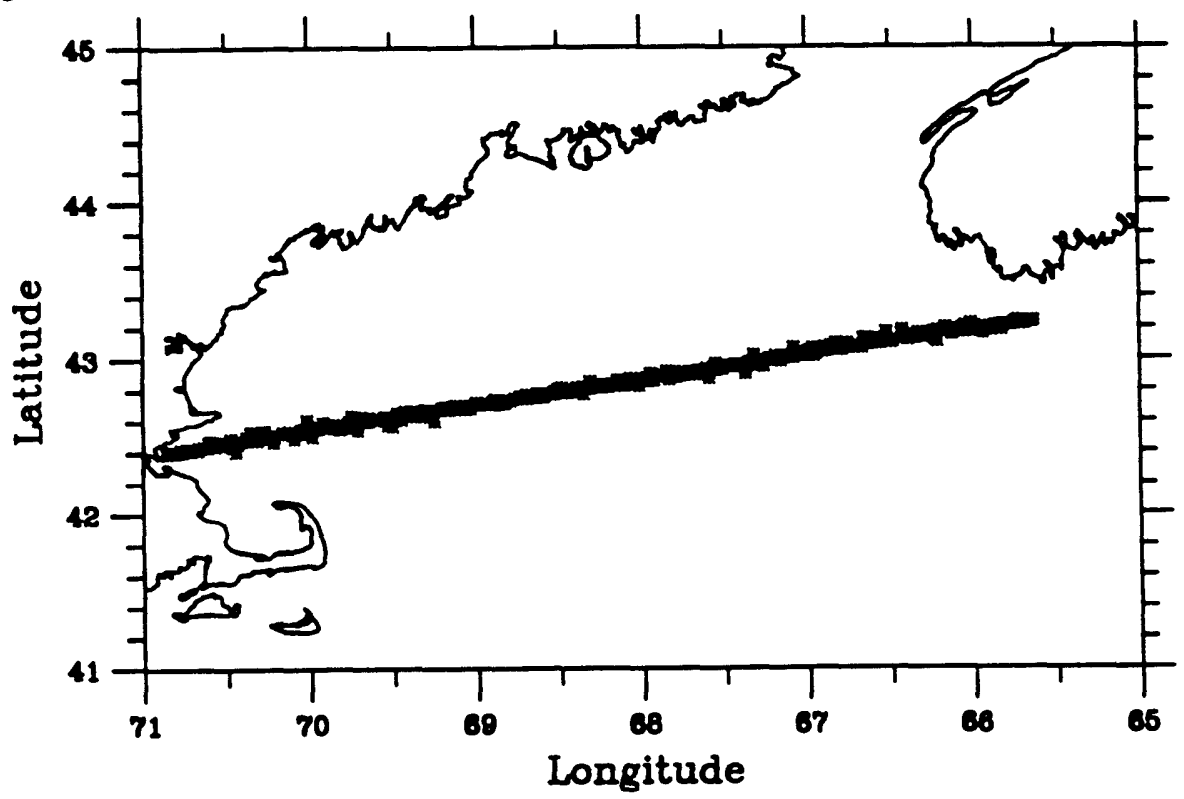

Figure 2.3: The SOOP transect across the GOM from Massachusetts Bay to Scotian Shelf from 1978 to 1989. 
tion of surface salinity in the northern GSC in late spring. To study the mechanisms which generate and move the low-salinity surface plume east of Cape Cod in late spring, we have also collected the surface wind data recorded continuously every hour on three NOAA environmental buoys around our study region during the 1988 and 1989 CTD/ADCP surveys, the 1988 and 1989 monthly freshwater river discharge data from Maine, New Hampshire, and Massachusetts, and monthly surface-salinity distribution data across the GOM on the NOAA Ship of Opportunity Program (SOOP) transect during the ten-year period 19781989 (see Figure 2.3). These data provide additional information about the sources of fresh water in the western GOM, and the effects of other processes on the evolution of the lowsalinity plume in late spring. Combined analysis of three hydrographic surveys, wind, and surface salinity data allow us to characterize directly the three-dimensional structure of water properties in the northern GSC in late spring. This analysis will be done within the context of the conceptual model of a seasonal springtime evolution of salinity field in the western GOM described by Mountain and Manning (1991), with the understanding that the SCOPEX hydrographic data considered in isolation cannot be used to prove that such a springtime cycle exists. The combined analysis will be discussed next in section 2.2 .

The relatively shallow depth of the SCOPEX survey region allowed good bottom tracking during most of the April, 1988 and the entire May-June, 1989 ADCP surveys in the northern GSC, so that high-quality absolute current profile data over the upper $140 \mathrm{~m}$ were obtained. However, the absolute currents obtained with the ADCP in the northern GSC include strong semidiurnal tidal signals which were almost one order of magnitude larger than the residual flow (Moody et al., 1984). In order to separate the ADCP current data into tidal and subtidal components, an empirical least squares fit method was used to treat the raw bottom-tracked ADCP data. Good agreement between fitted and observed tidal currents allows us to study the 3-D structure of the residual flow field and associated movements of water masses in the northern GSC. Details of the ADCP treatment and tidal and residual current structure will be discussed separately in sections $2.3,2.4$ and 2.5 . 
Combined analysis of hydrographic data and residual currents in the upper $30 \mathrm{~m}$ provides a detailed 3-D picture of the physical environment in regions of enhanced biological productivity. Study of the temperature/salinity front or tidal mixing front and associated convergence or divergence flow field allows us to test the three possible hypotheses proposed by Beardsley and Limeburner for the large aggregations of zooplankton found in the northern GSC. A simple analysis will be done in section 2.6 to study the possible relationship between the physical and biological processes.

Conclusions from the analysis of the two SCOPEX regional and one meso-scale CTD/ADCP survey data will be given in section 2.7 in which a conceptual model of the 3-D residual flow will be summarized based on ADCP and satellite-tracking drifters deployed at 5 and $50 \mathrm{~m}$ during the two SCOPEX surveys. Finally, some new questions arising from our analysis will be posed. 


\subsection{Variability of Water Properties in Late Spring in the Northern GSC from 1988 to 1989}

Five cross-channel hydrographic sections were conducted from west to east across the northern GSC in late spring during the April, 1988 and June, 1989 CTD/ADCP surveys, and six hydrographic sections were taken almost perpendicular to the $100-\mathrm{m}$ isobath east of Cape Cod from $41.0^{\circ} \mathrm{N}$ to $42.0^{\circ} \mathrm{N}$ in May, 1989. The June, 1989 hydrographic data are described in the data report published by Limeburner and Beardsley (1989).

\section{Surface Low-Salinity Plume East of Cape Cod in Late Spring}

There was a significant difference in the surface salinity patterns observed in late April, 1988 and May-June, 1989 in the extent of the low-salinity plume which occurs over the western flank of the northern GSC in spring (Figure 2.4). In April, 1988, the low-salinity plume was just beginning to form and was confined to the east coast of Cape Cod while in May-June, 1989, three to six weeks later, the plume was spreading offshore from Cape Cod and finally covered most of the northern GSC like a large pool from Wilkinson Basin to the southern sill of the GSC between Nantucket Shoals and Georges Bank. The minimum salinity of the plume was about $32.0^{\circ} \%$ in April, 1988 while it was $0.4-1.2 \%$ fresher in May-June, 1989. Such a big difference is also evident in the distribution of monthly surface salinity across the Gulf of Maine obtained on SOOP transects during 1988 and 1989 (Figure 2.5). Taking the $32.0 \%$ contour as a reference boundary of fresher water, we found in 1988 that the fresher water was beginning to appear in Massachusetts Bay in early April and extended to Wilkinson Basin after June, while in 1989, the fresher water was beginning to enter Wilkinson Basin in late April although it entered Massachusetts Bay in mid-April. The volume of low-salinity water found in Massachusetts Bay from May to August was considerably larger in 1989 than in 1988. The difference in the amount of freshening observed in the northern GSC between April, 1988 and June, 1989 is due to the increased local river discharge in 1989 and a three-to-six week time difference between the 


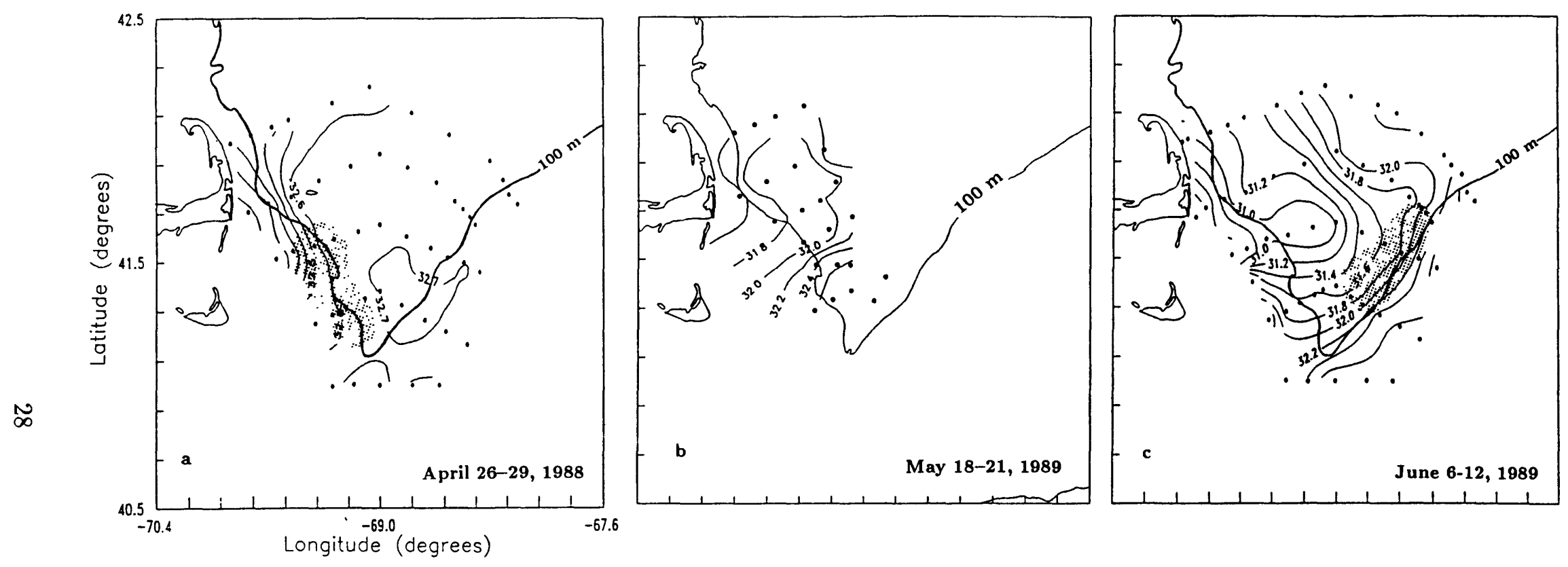

Figure 2.4: Surface (2 m) salinity distributions during April 26-29, 1988 (a), May 18-21 (b) and June 7-12, 1989 (c). The heavy solid line is the $100-\mathrm{m}$ isobath, the shaded areas represent the regions of the densest right whale sightings shown in Figure 1.7. The cross-channel sections running from the western flank of the northern GSC to its eastern flank are numbered A to E from south to north for the April, 1988 and June, 1989 surveys and $\mathrm{A}$ to $\mathrm{F}$ for the May, 1989 survey. 


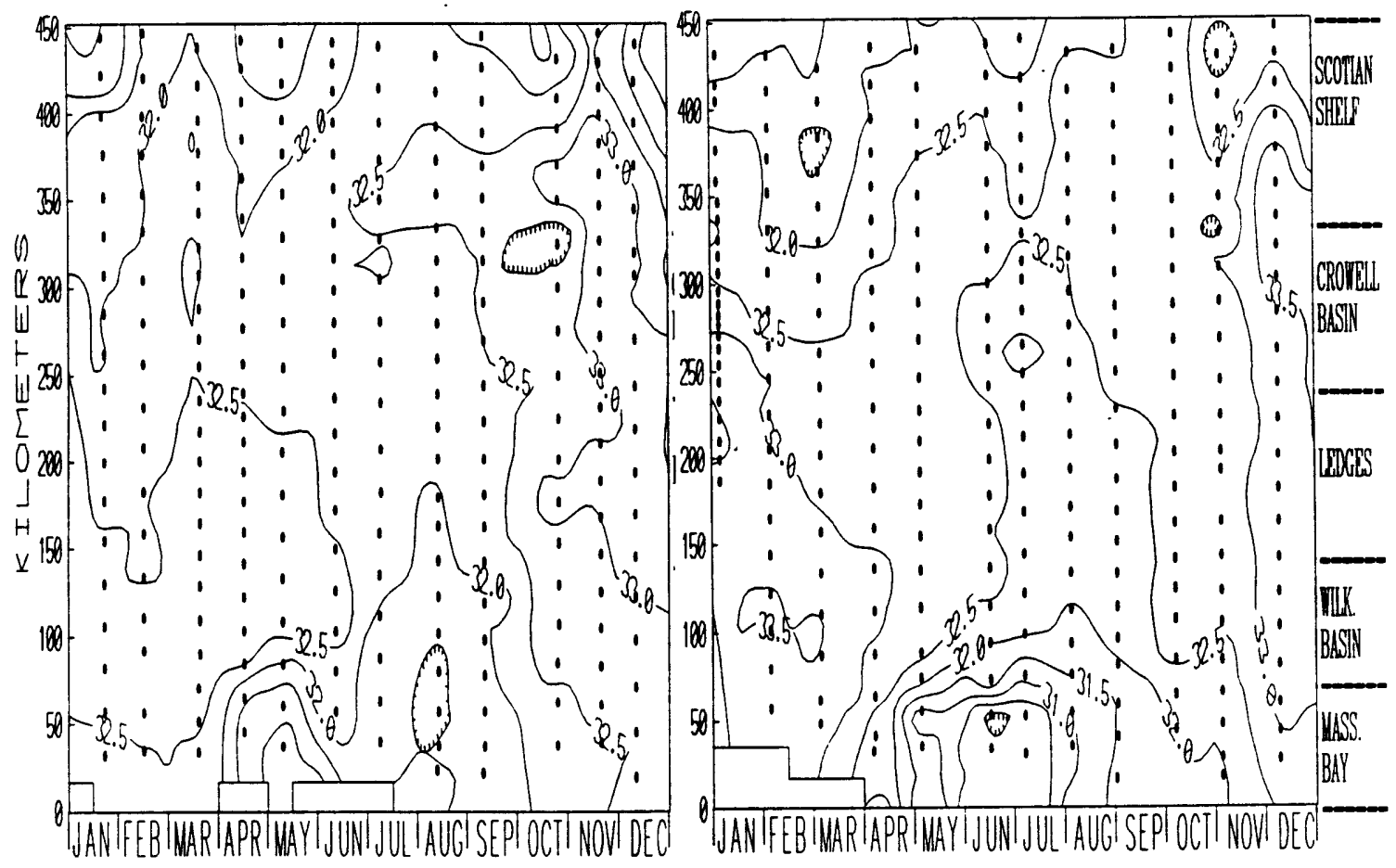

Figure 2.5: Surface salinity distributions across the GOM on the SOOP transect from Massachusetts Bay to Scotian shelf during 1988 (left) and 1989 (right). These maps were provided by J. W. Jossi of the Northeast Fisheries Center, Narragansett Laboratory.

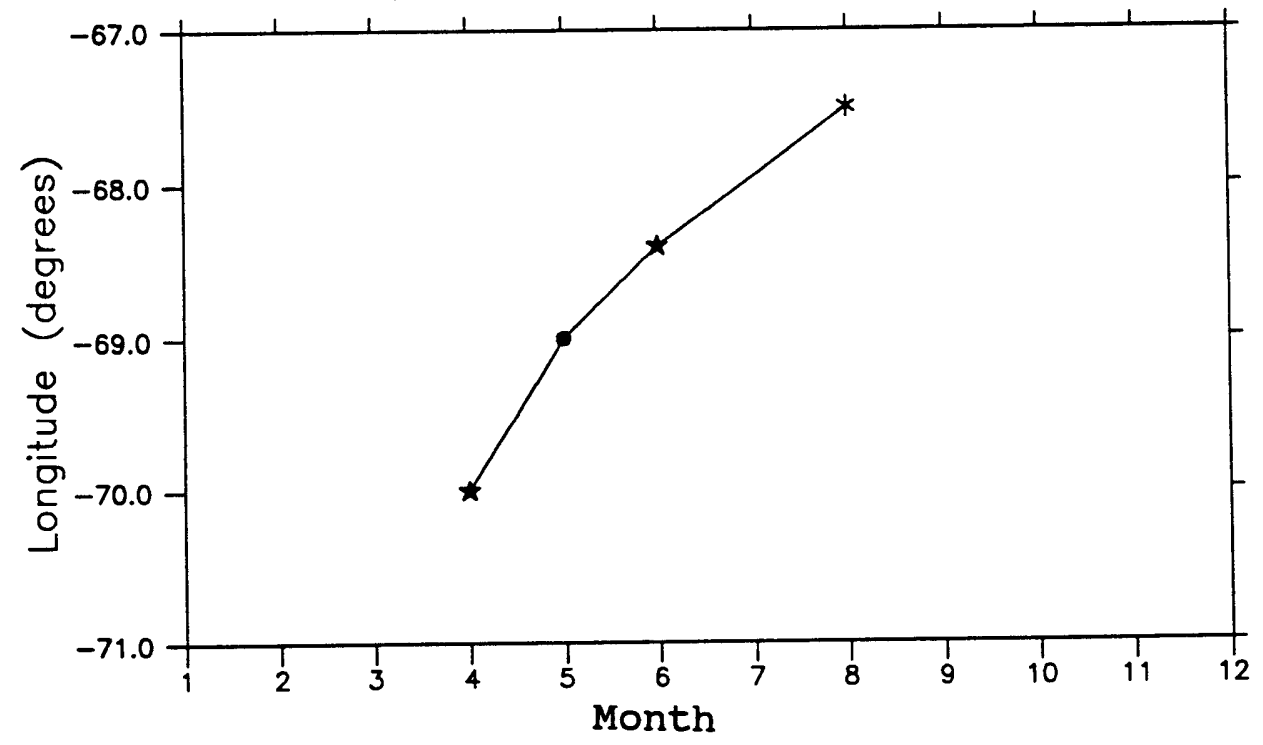

Figure 2.6: Monthly mean positions of the eastern boundary of surface ( $2 \mathrm{~m}$ ) low-salinity plume defined by the contour of $32.0 \%$. They are estimated from different hydrographic data sets taken during April 26-29, 1988, June 6-12, $1989(\star)$, May 11-21, 1976 (•, Limeburner and Beardsley, 1982) and July 28-August 5, 1987 (*, Garrison and Brown, 1989). 
two surveys. Distributions of monthly surface salinity during the last ten years from 1978 to 1989 clearly show two regions of fresher water, one in Massachusetts Bay and Wilkinson Basin and other on the Scotian Shelf (for example, see Figure 2.5). Larger river output was found from New Brunswick to Cape Cod in 1989 than in 1988. For an example, the Penobscot River maximum monthly averaged runoff was about $700 \mathrm{~m}^{3} / \mathrm{s}$ in April, 1988 while it increased to $1100 \mathrm{~m}^{3} / \mathrm{s}$ in May, 1989 (see Figure 2.7). Information on the structure of the low-salinity plume was also reported in previous hydrographic surveys taken in May, 1976 (Limeburner and Beardsley, 1982) and in July, 1987 (Garrison and Brown, 1989). Taking the $32.0 \%$ contour as a reference boundary of this low-salinity plume and ignoring its inter-annual variability, we found that the plume extended eastward with time in a seasonal cycle from just east of Cape Cod to the western flank of Georges Bank (see Figure 2.6). These data support our conceptual picture of a freshwater plume which spreads in the northern GSC and becomes fresher as time advances from April to June.

\section{Vertical Distributions of Water Properties in the Northern GSC}

Vertical sections of temperature and salinity are shown in Figures 2.8-2.10 for the April, 1988, May and June, 1989 surveys, respectively. The corresponding T/S diagrams for each section during the two year surveys are presented in Figure 2.11 for the April, 1988 and June, 1989 surveys and in Figure 2.12 for the May, 1989 survey. For the sake of comparison, section $\mathrm{E}$ for both April, 1988 and June, 1989 surveys and Section $\mathrm{F}$ for the May, 1989 survey, which cut from west to east across Wilkinson Basin, Franklin Swell and the southwestern end of Franklin Basin, are chosen as examples to study the seasonal variability of water properties in the northern GSC.

In April, 1988, the seasonal thermocline was just beginning to form and the freshwater plume can be clearly identified in the vertical salinity distribution over the western flank of the northern GSC where a strong salinity front was located. The maximum depth of the front was about $40 \mathrm{~m}$, and its cross-shelf scale was about $40 \mathrm{~km}$ extending east from Cape Cod. Farther offshore was located Maine Surface Water (MSW, Smith, 1983), which 

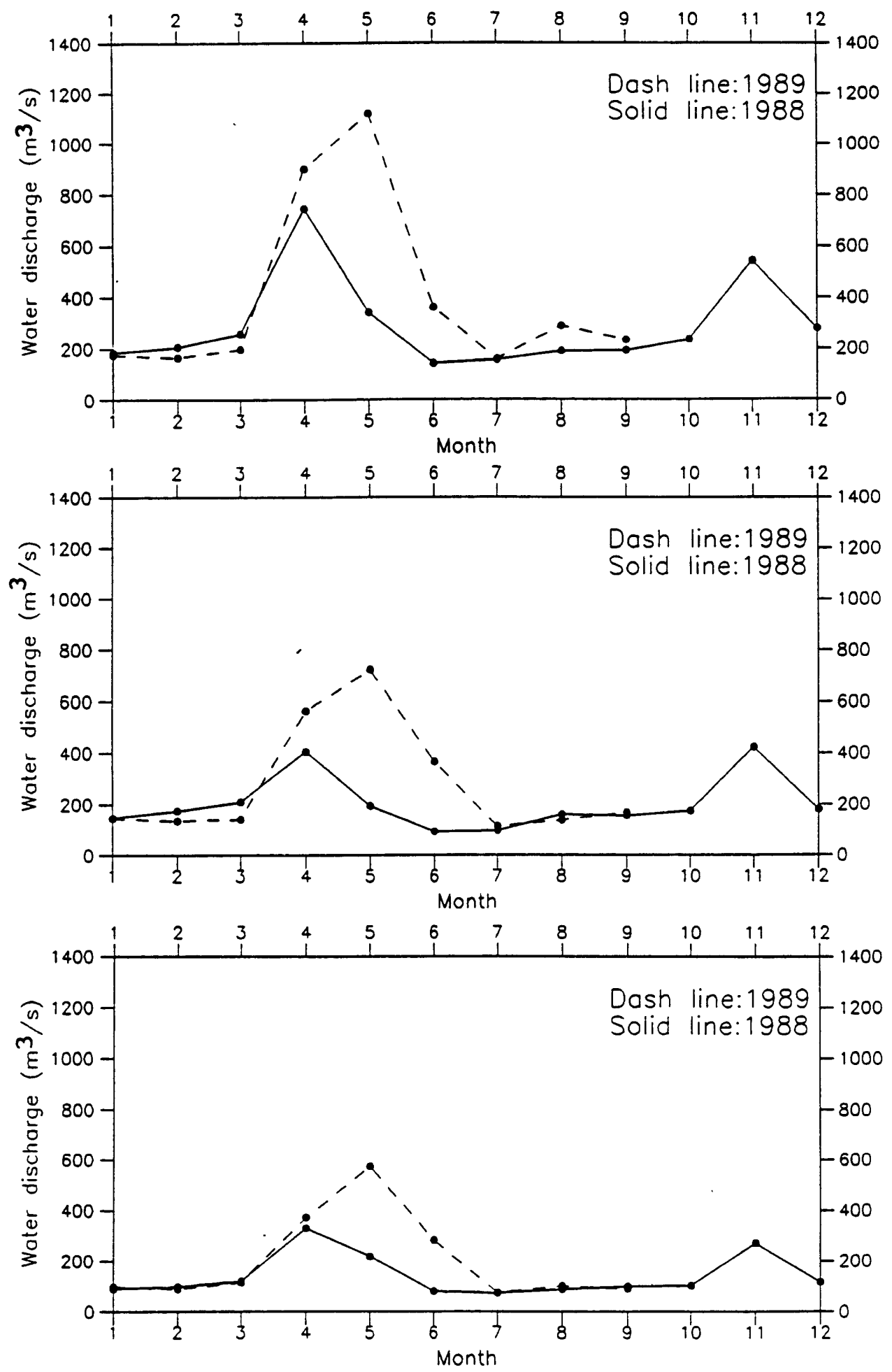

Figure 2.7: The 1988 and 1989 monthly averaged freshwater discharges from the Penobscot River at Eddington (upper), Kennebec River at North Sidney (middle) and Androscoggin River near Auburn (lower). 
was characterized by a range of temperature from $5.6^{\circ} \mathrm{C}$ at the surface to $4.8^{\circ} \mathrm{C}$ at $40 \mathrm{~m}$, and a relatively uniform salinity of less than $32.7 \%$. In May, 1989, a strong thermocline and halocline were observed in the upper $40 \mathrm{~m}$, while the thermocline and halocline were more concentrated in the upper $30 \mathrm{~m}$ in June, 1989, three weeks later in the seasonal cycle. At that time, the low-salinity plume had a surface salinity less than $32.0 \%$ ond extended far offshore east of Cape Cod into the central GSC region, although the freshest water was still found in a narrow surface band next to Cape Cod with a cross-shelf scale of $40 \mathrm{~km}$ and a vertical scale of $40 \mathrm{~m}$. Unlike April, 1988 and May, 1989, the low-salinity plume exhibited little vertical temperature and salinity structure in the upper $20 \mathrm{~m}$ in June, 1989, especially in the interior region away from the coastal tidal mixing front. Such a vertical relatively mixed structure may be the result of surface wind mixing. ${ }^{2}$

In all three surveys, Maine Intermediate Water (MIW) occupied the middle of the water column beneath the thermocline and halocline. In both 1988 and 1989, MIW was characterized by a temperature minimum less than $4.8^{\circ} \mathrm{C}$ centered between $40 \mathrm{~m}$ and $150 \mathrm{~m}$ and an intermediate salinity (see Figures 2.8-2.10). Tracing the core of temperature minimum by defining the $4.8^{\circ} \mathrm{C}$ contour as the boundary of the MIW for the 1988 and 1989 surveys, we found that the vertical thickness and horizontal extent of MIW tended to decrease eastward and southward, respectively, and finally disappeared over the sill of the GSC where the water was relatively well mixed in the vertical. This fact implies mixing between the MSW and MIW as the water flowed southward along the western flank of the northern GSC. Moreover, in April, 1988, the spatial structure of MIW was relatively uniform, while in May, 1989, a narrow core of temperature minimum less than $4.0^{\circ} \mathrm{C}$ was found along the western flank of the northern GSC between 40 and $150 \mathrm{~m}$. In June, 1989, three weeks later, this temperature minimum core had become much cooler (in a temperature range of $3.2^{\circ}-4.0^{\circ} \mathrm{C}$ ). This colder, fresher, and lighter water seemed to be isolated from the interior temperature minimum and spread offshore to mix with the interior MIW as the

\footnotetext{
${ }^{2}$ Note: the vertical gradient found in salinity but not in temperature in the upper $20 \mathrm{~m}$ in Figure 2.10 is due to the smaller contour plotting resolution used for salinity.
} 

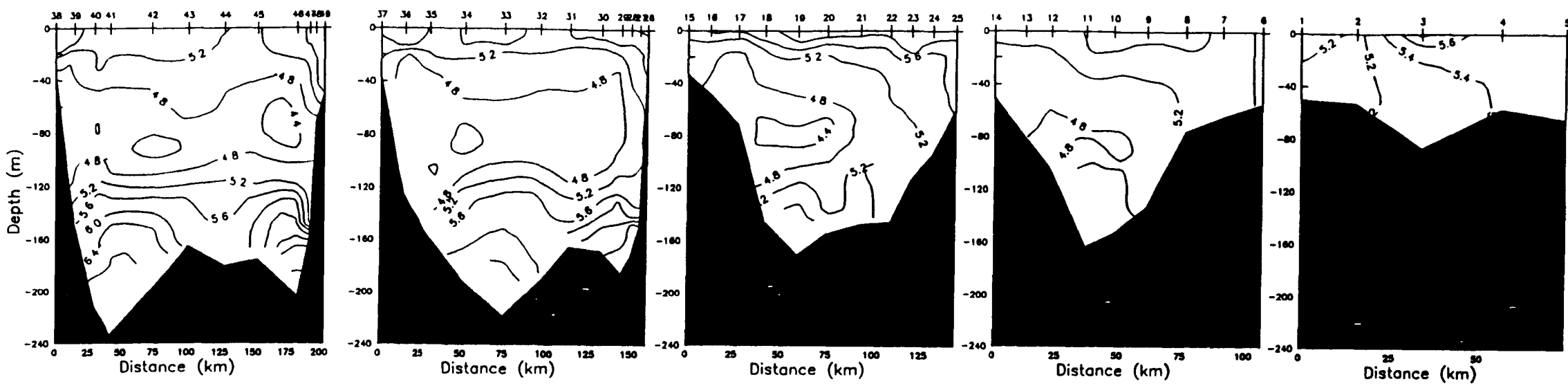

$\omega$
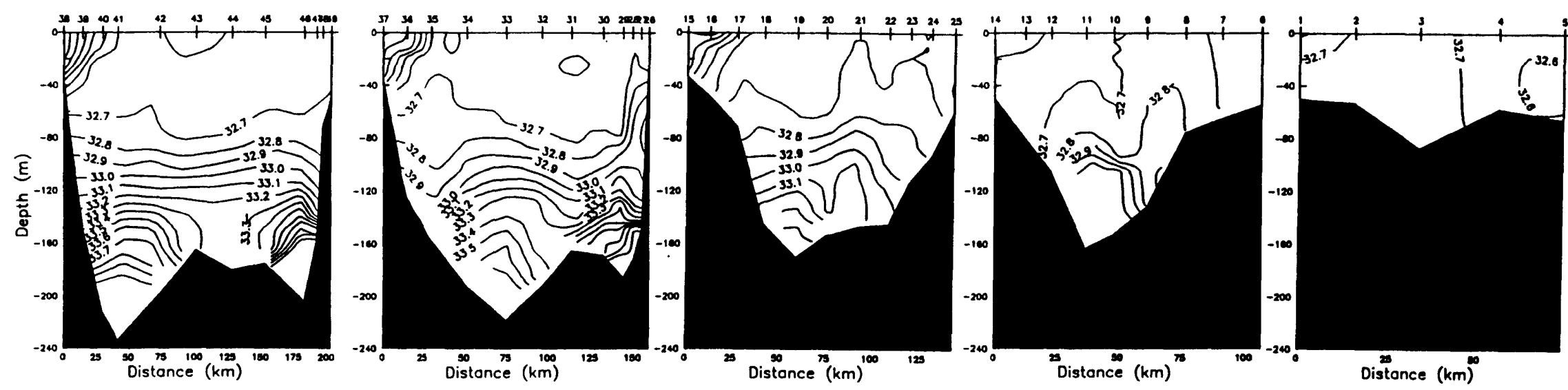

Figure 2.8: Vertical sections of temperature (upper) and salinity (lower) southward from section E (left) to section A (right) for the April 26-29 1988 CTD/ADCP surveys. 

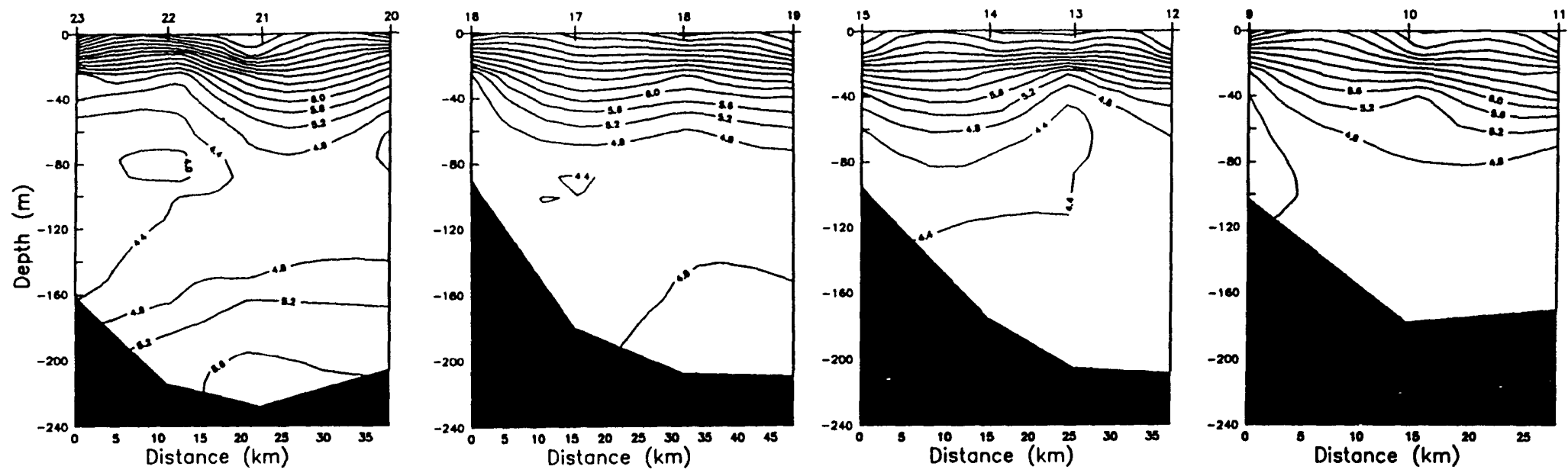

$\stackrel{\omega}{\Perp}$
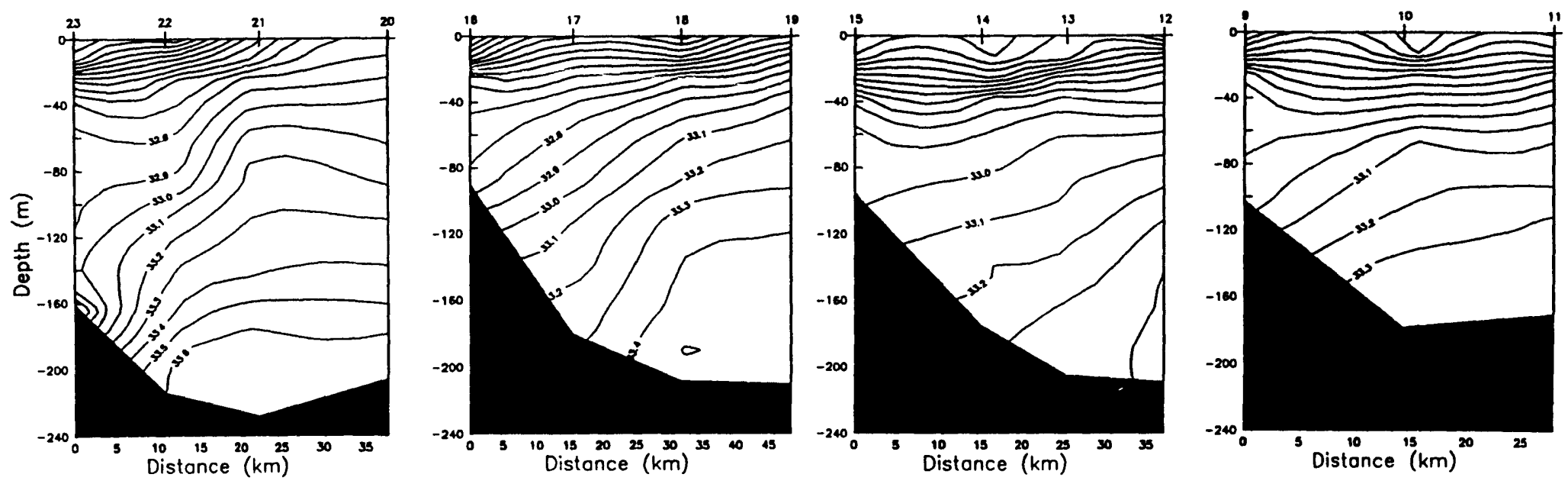

Figure 2.9: Vertical sections of temperature (upper) and salinity (lower) southward from section F (left) to section C (right) for the May 18-21 1989 CTD/ADCP survey 

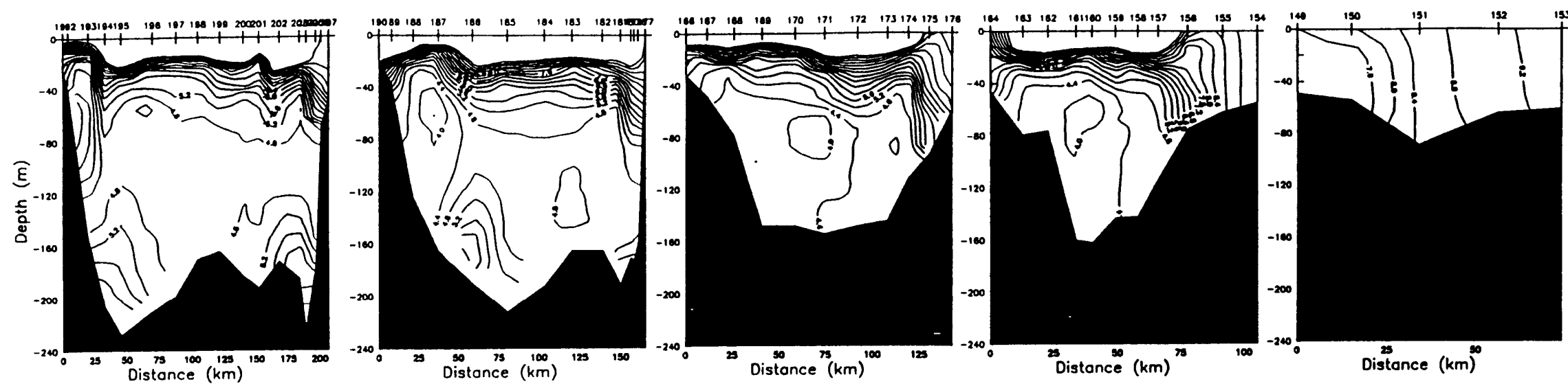

$\stackrel{\omega}{\sigma}$
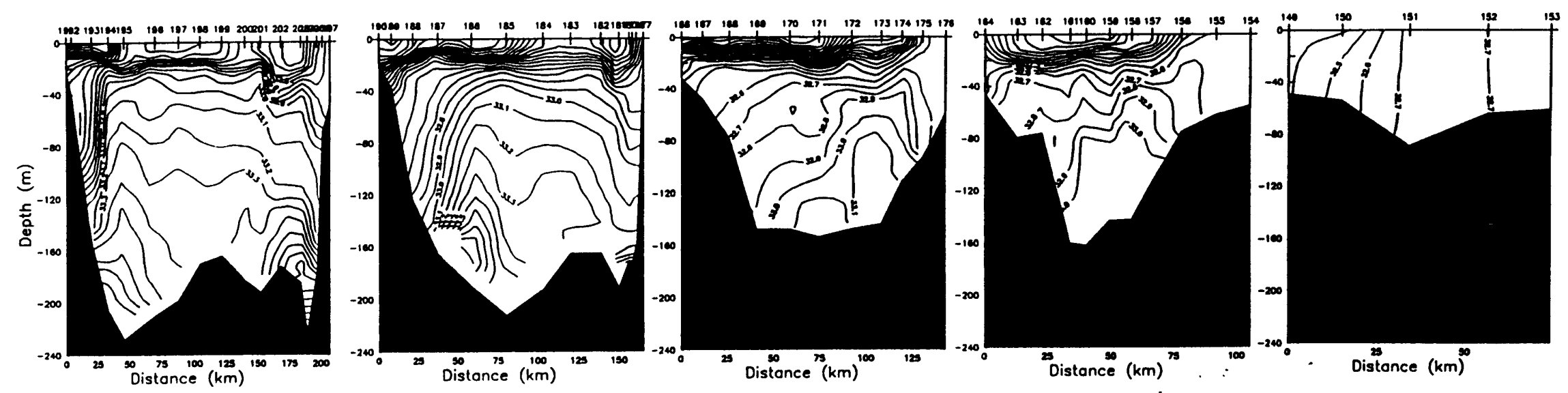

Figure 2.10: Vertical sections of temperature (upper) and salinity (lower) southward from section E (left) to section A (right) for the June 6-12 1989 CTD/ADCP survey . 
core flowed southward in the northern GSC (Figures 2.11-2.12). A similar structure was also reported in the May, 1979 hydrographic survey by Limeburner and Beardsley (1982), who suggested that upstream vertical mixing of the near-surface water may be one possible mechanism for the formation of such a low temperature minimum core water. It is difficult to prove this hypothesis based on our hydrographic survey data, even though we did find a very low surface salinity upstream in Massachusetts Bay during May-June, 1989.

A T/S analysis for the April, 1988 and June, 1989 surveys is made here to examine if MIW can pass south through the GSC onto the New England shelf. In April, 1988, the water was weakly stratified between stations 1 and 3 on the western side of section A. Nearly straight $\mathrm{T} / \mathrm{S}$ curves were observed at stations $1-3$, respectively, with a temperature and salinity of $5.2-6.1^{\circ} \mathrm{C}$ and $32.5-32.6^{\circ} \%$ at the surface and $5.1-5.3^{\circ} \mathrm{C}$ and $32.7-32.8^{\circ} \%$ at the bottom. These nearly linear T/S curves can be traced back to the northern section $B$ only in the upper $50 \mathrm{~m}$ where MSW was dominant, implying that only a small part of MIW shallower than $50 \mathrm{~m}$ may pass through the GSC as water mixed with MSW in April, 1988. A very different picture was found in June, 1989. The water was vertically well mixed at stations 149 and 151 and weakly stratified at station 150 on section A. Correspondingly, the $\mathrm{T} / \mathrm{S}$ diagram was characterized with a straight line at station 150 but with nearly single points at stations 149 and 151 . These $\mathrm{T} / \mathrm{S}$ relationships do not lie on the mixing line between MSW and MIW alone on the northern section B, implying (a) that little MIW was passing southward through the GSC in June, 1989 and (b) the existence of some other water source(s). A westward current was found at $51 \mathrm{~m}$ on section $\mathrm{A}$ in the ADCP data (see section 2.5), suggesting mixing between MSW, MIW, and Georges Bank water in the southern end of the GSC in June, 1989. In contrast with Hopkins and Garfield's (1979) suggestion, mixing plays an important role over the sill of the GSC so that no original MIW can pass south through the GSC without mixing with MSW during late spring.

The deepest water found in these surveys was Maine Bottom Water (MBW), caused by a westward penetration of warm and saline Slope Water from the Northeast 

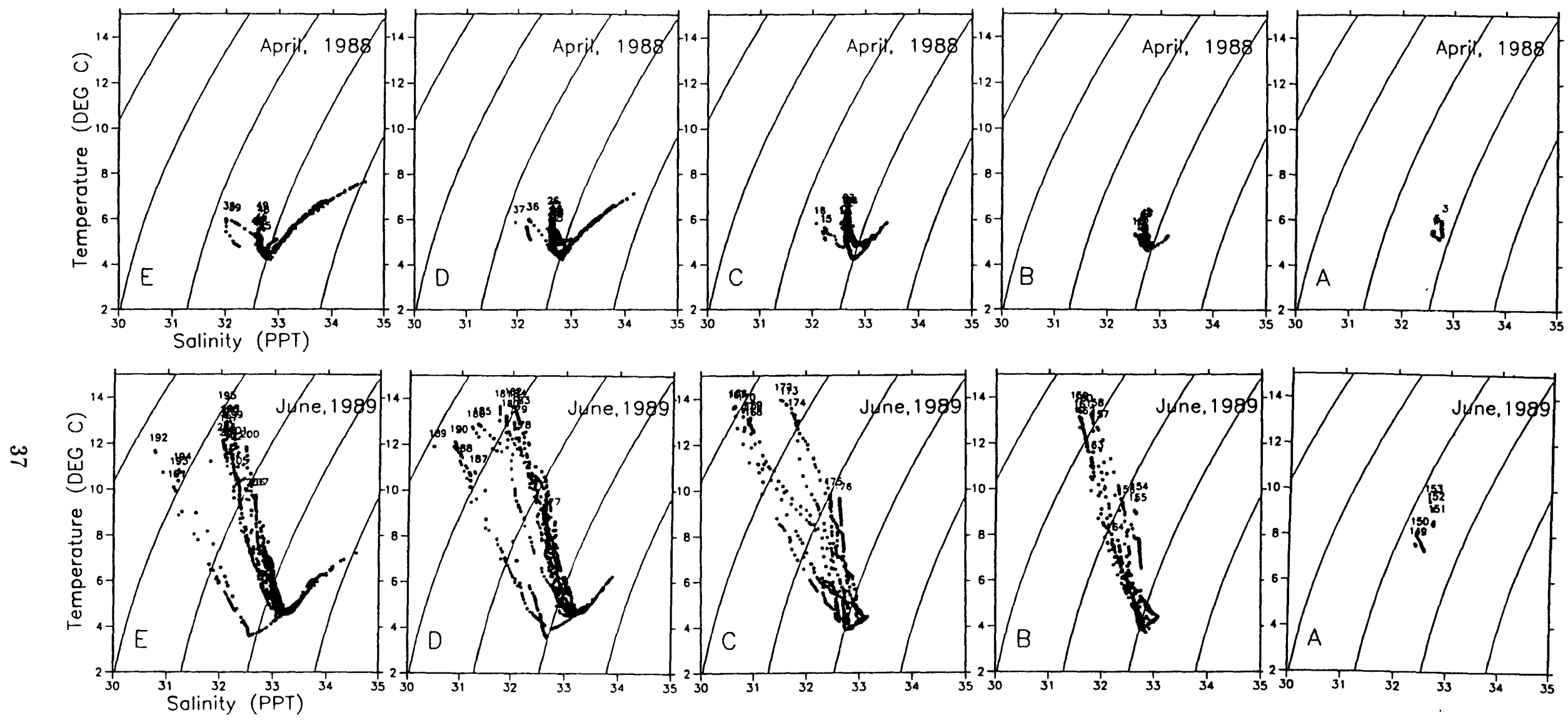

Figure 2.11: $T / S$ diagrams on each southward section from $E$ (left) to A (right) for the April 26-29, 1988 (upper) and June 6-12, 1989 CTD/ADCP surveys. The numbers in the maps are the CTD station identifiers, and the curves are the contours of $\sigma_{t}$ from 23.0 (left) to 27.0 (right). 

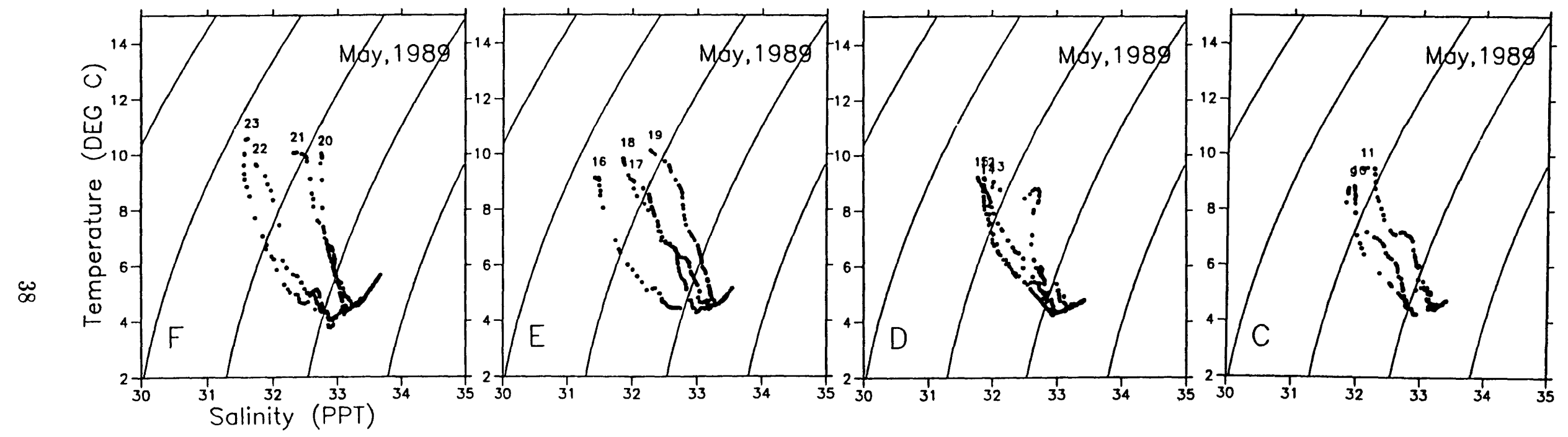

Figure 2.12: $\mathrm{T} / \mathrm{S}$ diagrams on each section southward from $\mathrm{F}$ (left) to $\mathrm{C}$ (right) for the May 18-21, $1989 \mathrm{CTD} / \mathrm{ADCP}$ survey 4 The numbers in the maps are the CTD station identifiers, and the curves are the contours of $\sigma_{t}$ from 23.0 (left) to 27.0 (right). 
Channel (Mountain and Jessen, 1987). The MBW is characterized here as that water lying beneath MIW between about $120 \mathrm{~m}$ and the bottom in which both temperature and salinity increase with depth (Figures 2.8-2.10). In addition, the MBW seemed to be more stratified in April, 1988 in the north central GSC than in June, 1989.

In summary, the three hydrographic surveys indicate that the low-salinity plume begins to form along the outer coast of Cape Cod in April and then spreads offshore into the northern GSC in May-June. The vertical stratification near the surface is mainly determined by surface heating, the low-salinity plume, and wind mixing. The strong thermocline was beginning to form in May, 1989 due to surface solar heating, and was concentrated at 20-30 $\mathrm{m}$ in June, 1989 due to the offshore extension of the low-salinity plume and wind mixing. The strong halocline was located in the upper $40 \mathrm{~m}$ on the western flank of the GSC in April, 1988, while it was concentrated at $20 \mathrm{~m}$ across the GSC in June, 1989 as the low-salinity plume extended offshore. The MIW occupied the middle of the water column between $40 \mathrm{~m}$ and $150 \mathrm{~m}$ in the 1988 and 1989 surveys. Most of the MIW was recirculated along local isobaths in Wilkinson Basin, and only a small part of it might have flowed southward along the western flank of the northern GSC as water mixed with the MSW. In addition, a colder and fresher core was found at mid-depth near the coast in June, 1989 and spread offshore to mix with the interior MIW as it flowed southward in the northern GSC.

\section{Mechanisms for the Offshore Spreading of the Low-Salinity Plume}

In general, the buoyancy-driven density current due to river discharge flows like a boundary jet along the coast in the direction of the propagation of coastal trapped waves. In the absence of sloping bottom topography over the shelf, the low-salinity plume may leave seaward from the coast because of a downstream accumulation of water caused by convergent currents along the coast (Stern et al., 1982; Chao, 1988). Whenever a sloping bottom is involved, the plume would be trapped near the coast so that there is no offshore spreading of the plume in the downstream direction (Chao, 1988). An increasing river discharge in late spring should only intensify the strength of a buoyancy-driven coastal current, and not 

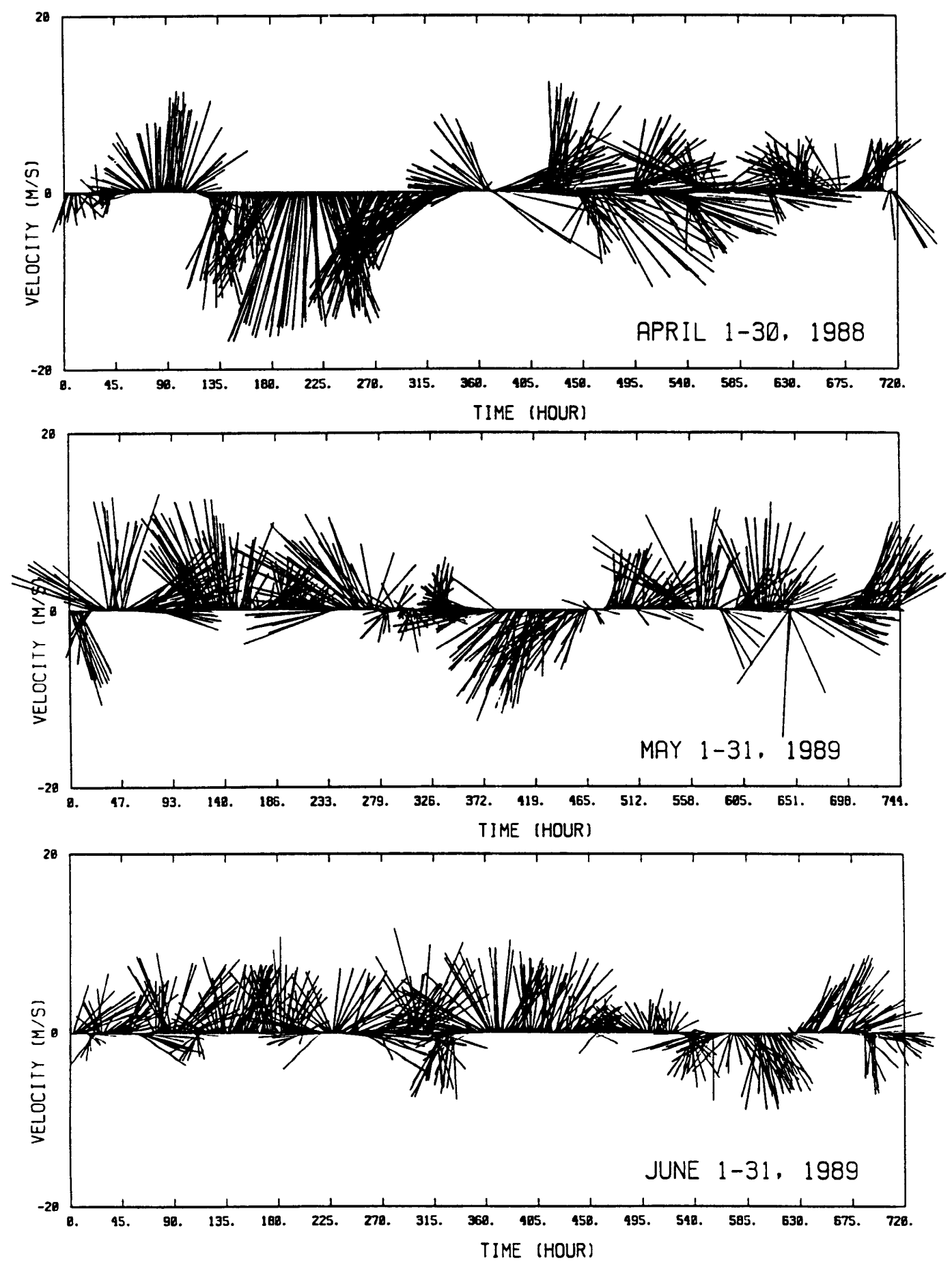

Figure 2.13: Hourly averaged wind measurements on buoy station No. 44008 at $69.5^{\circ} \mathrm{W}$ and $40.5^{\circ} \mathrm{N}$ from April 1 to 30, 1988 (upper) and May 1 to June 14, 1989 (middle and lower). The instrument is mounted at a height of $13.8 \mathrm{~m}$ above the sea surface. 
lead to offshore spreading. Thus, the observed spreading of the low-salinity plume east of Cape Cod in June, 1989 implies some additional dynamical mechanism. We will suggest two possible candidates next.

The shape of the low-salinity plume is sensitive to the local wind forcing through the surface Ekman drift. The time scale of the response of the GOM to both local and remote wind forcing is about 2-20 days (Brown and Irish, 1989). Upwelling-favorable winds could lead to a seaward spreading of the low-salinity plume by the offshore Ekman transport and cause a strong vertical stratification offshore through the resulting upwelling flow. In contrast, downwelling-favorable winds could confine the low-salinity plume to the coast by onshore Ekman transport and cause the plume to deepen in a narrow band near the coast by the downwelling flow. Wind data, were continuously recorded on three NOAA environmental buoys around the study area in late spring of 1988 and 1989 (see Figure 2.1). For an example, the hourly averaged wind velocities at the height of $13.8 \mathrm{~m}$ on buoy No. 44008 , south of the GSC, are presented in Figure 2.13. A strong downwelling-favorable wind higher than $15.0 \mathrm{~m} / \mathrm{s}$ was observed 10 days before the April, 1988, while a steady upwelling-favorable wind with an average speed of about $5 \mathrm{~m} / \mathrm{s}$ lasted for at least two months before the June, 1989 survey. The structure of the low-salinity plume observed in late spring of 1988 and 1989 is at least qualitatively consistent with this idea of local wind forcing.

A simple quantitative analysis for the offshore Ekman drift due to northward wind during late spring 1989 is done in the following. The wind stress is estimated from the observed wind data using a neutral, steady-state drag coefficient $C_{d n}$ at the height of $10 \mathrm{~m}$ and the $10-\mathrm{m}$ wind speed $U_{10}$ in a form as

$$
\tau=\rho_{a} C_{d n}\left(U_{10}\right) U_{10}^{2}
$$

where $\rho_{a}$ is the air density and is assumed to be constant at $1.22 \times 10^{-3} \mathrm{~g} / \mathrm{cm}^{3}$. Using the observed wind $U_{o}$ at the observation height $h_{o}$ (in meters), $C_{d n}$ and $U_{10}$ are calculated 
based on an iterative method introduced by Large and Pond (1981) in which

$$
C_{d n}=\left\{\begin{array}{lr}
1.2 \times 10^{-3} & U_{10}<11 \mathrm{~m} / \mathrm{s} \\
\left(0.49+0.065 U_{10}\right) \times 10^{-3} & 11 \mathrm{~m} / \mathrm{s}<U_{10}<25 \mathrm{~m} / \mathrm{s}
\end{array}\right.
$$

and

$$
U_{10}=\frac{U_{o}}{1+\frac{\sqrt{C_{d n}\left(U_{10}\right)}}{\kappa} \ln \frac{h_{o}}{10}} .
$$

where $\kappa$ is von Karman's constant and has a value of 0.4. The resulting surface wind stress for a value of $5 \mathrm{~m} / \mathrm{s}$ wind speed at $13.8 \mathrm{~m}$ is $0.34 \mathrm{dyn} / \mathrm{cm}^{2}$, and the Ekman transport for this wind stress is given by

$$
V_{E}=\frac{\tau}{\rho f}=0.35 \mathrm{~m}^{2} / \mathrm{s}
$$

where the Coriolis parameter $f$ is $9.57 \times 10^{-5}$ at $41^{\circ} \mathrm{N}$, and an average seawater density $\rho$ in the low-salinity plume is equal to $1.0235 \mathrm{~g} / \mathrm{cm}^{3}$. Taking the $32.0^{\circ} \%$ contour as the boundary of the low-salinity plume, the total volume of the plume was about $1.2 \times$ $10^{11} \mathrm{~m}^{3}$ with an area of $0.8^{\circ}$ latitude $\times 0.8^{\circ}$ longitude and a thickness of $20 \mathrm{~m}$. If the plume was pushed offshore only by Ekman transport with a value of $0.35 \mathrm{~m}^{2} / \mathrm{s}$, it would take about 46.3 days, or one and a half-months, to form the pool-like structure of near-surface, low-salinity water observed in the June, 1989 survey in the northern GSC. Observations on buoy station No. 44008 indeed showed at least two months of northward winds in the GSC which occurred before the June, 1989 survey, so that the offshore spreading of the low-salinity plume in late spring 1989 may have been caused by the surface wind stress.

The offshore spreading of the low-salinity plume might be also driven by deep circulation in the northern GSC. In section 2.5, we describe a cyclonic subtidal circulation in the intermediate depth water below $50 \mathrm{~m}$ in the northern GSC, flowing southeastward along the $100-\mathrm{m}$ isobath east of Cape Cod and then turning northeast to join the anti-cyclonic 
gyre around Georges Bank. This flow may produce a stress on the upper low-salinity plume so as to drive the upper layèr water offshore from Cape Cod. Therefore, we speculate here that the upwelling-favorable wind stress and the deep cyclonic current stress may both be responsible for the offshore spreading of the surface low-salinity plume in the northern GSC during late spring.

\section{Tidal Mixing Front in Late Spring the Northern GSC}

When wind mixing, horizontal diffusion and advection, and fresh water input are ignored, the vertical mixing in the coastal region is controlled primarily by a competition between the surface buoyancy flux due to surface solar heating and kinetic energy dissipation caused by oscillating tidal currents over the bottom. When tidal energy dissipation is stronger than the buoyancy input, the water will be vertically well mixed. In turn, the water will remain stratified when the buoyancy flux is dominant. The transition zone between well-mixed and stratified regions (called the tidal mixing or tidal front) should be located at a place where these two processes are balanced. Based on this argument, Simpson and Hunter (1974) suggested that the existence and position of the tidal mixing front can be predicted using a particular value of the ratio of potential energy required for complete vertical mixing and the rate of the dissipation of mechanical energy due to tidal currents (so called mixing efficiency). In an area of constant surface heat flux, thermal expansion coefficient, specific heat and bottom friction coefficient, this ratio is proportional to $h / U^{3}$ or $h / D$, where $h$ is the local water depth, $U$ is a characteristic tidal velocity, and $D$ is the rate of tidal energy dissipation.

Garrett et al. (1978) applied this criterion to the Gulf of Maine using available hydrographic data and vertically averaged tidal currents from the numerical tidal model developed by Greenberg (1979). They found that the regions of $\Delta \sigma_{t} \leq 0.5$ in the upper $40 \mathrm{~m}$ over Georges Bank and off southwestern Nova Scotia correspond reasonably well to the region of $\log _{10} h / D<2.0$, and predicted that a definite transition between stratified and well-mixed regions occurred at $\log _{10} h / D=1.9$. Loder and Greenberg (1986) examined 
the effects of wind mixing, oscillatory advection in a tidal cycle, and monthly and fortnightly variation in the strength of tidal mixing on the prediction of tidal front position. Adding the wind stress due to a $10-\mathrm{m}$ wind speed of 1 to $5 \mathrm{~m} / \mathrm{s}$ (a climatological mean wind speed in June and August in the GOM, Saunders, 1977) into the energetic argument, they found that the predicted frontal position for tidal plus wind mixing shifted to $\log _{10}(h /(D+0.59))=$ 1.65 (see Figure 2.14), and the predicted well-mixed area is slightly expanded from the case of tidal mixing alone (see the 1.65 dash-dot line in Figure 2.14). On the other hand, the oscillatory advection and monthly and fortnightly variation in the strength of tidal mixing can cause only a $10-\mathrm{km}$ deviation to the contour of $\log _{10} h / D=1.9$; then the transition zone between the well-mixed and stratified regions can shift to $\log _{10} h / D=2.1$ during the spring tide when the tidal dissipation is larger.

Figure 2.14 shows a combined map of $\Delta \sigma_{t}$ contours in the upper $40 \mathrm{~m}$ calculated from the June, 1989 CTD survey data (solid lines), a mean position of the thermal front averaged over satellite Sea Surface Temperature (SST) images from May 5 to June 12, 1989 (heavy solid line), frontal positions at both sides of the northern GSC found during the small-scale biological surveys on May 26 and June 3, 1989, and the contours of $\log _{10} h / D=$ 1.9 and 2.1 (dashed lines) and $\log _{10}(h /(D+0.59))=1.65$ digitized from Figures 3 and 5 in Loder and Greenberg's (1986) paper. ${ }^{3}$ Large temperature and $\sigma_{t}$ gradients were observed west of the GSC on May 26, 1989 between CTD stations 85 and 86 (see Limeburner and Beardsley, 1989). CTD station 85 showed a relatively well-mixed water column with a vertical difference of $0.4^{\circ} \mathrm{C}$ in temperature and 0.1 in $\sigma_{t}$ in the upper $40 \mathrm{~m}$, while CTD station 86 showed a strong thermocline from $10 \mathrm{~m}$ to $25 \mathrm{~m}$ near the surface. Using continuous SST data obtained between these two stations, we found that a thermal front with a sharp jump in temperature of $1.4^{\circ} \mathrm{C}$ over a distance of $0.4 \mathrm{~km}$ was located at $69^{\circ} 21.30^{\prime} \mathrm{W}$ and

\footnotetext{
${ }^{3}$ Note: since spatial averaging was done to smooth the water depth in Greenberg's (1983) tidal model, the positions of the $50-\mathrm{m}$ and $100-\mathrm{m}$ isobaths Loder and Greenberg refer to are significantly different from the real water depth, especially on the northern flank of Georges Bank where large gradients of bottom topography exist. To compare the frontal position obtained from the CTD and SST data with Loder and Greenberg's model results, we plot in Figure 2.14 the contour positions for $\log _{10} h / D=1.9$ and 2.1 and $\log _{10}(h / D+0.59)=1.65$ referred to the real $100-\mathrm{m}$ isobath rather than a direct copy of their absolute positions from Figures 3 and 5 in Loder and Greenberg's (1986) paper.
} 


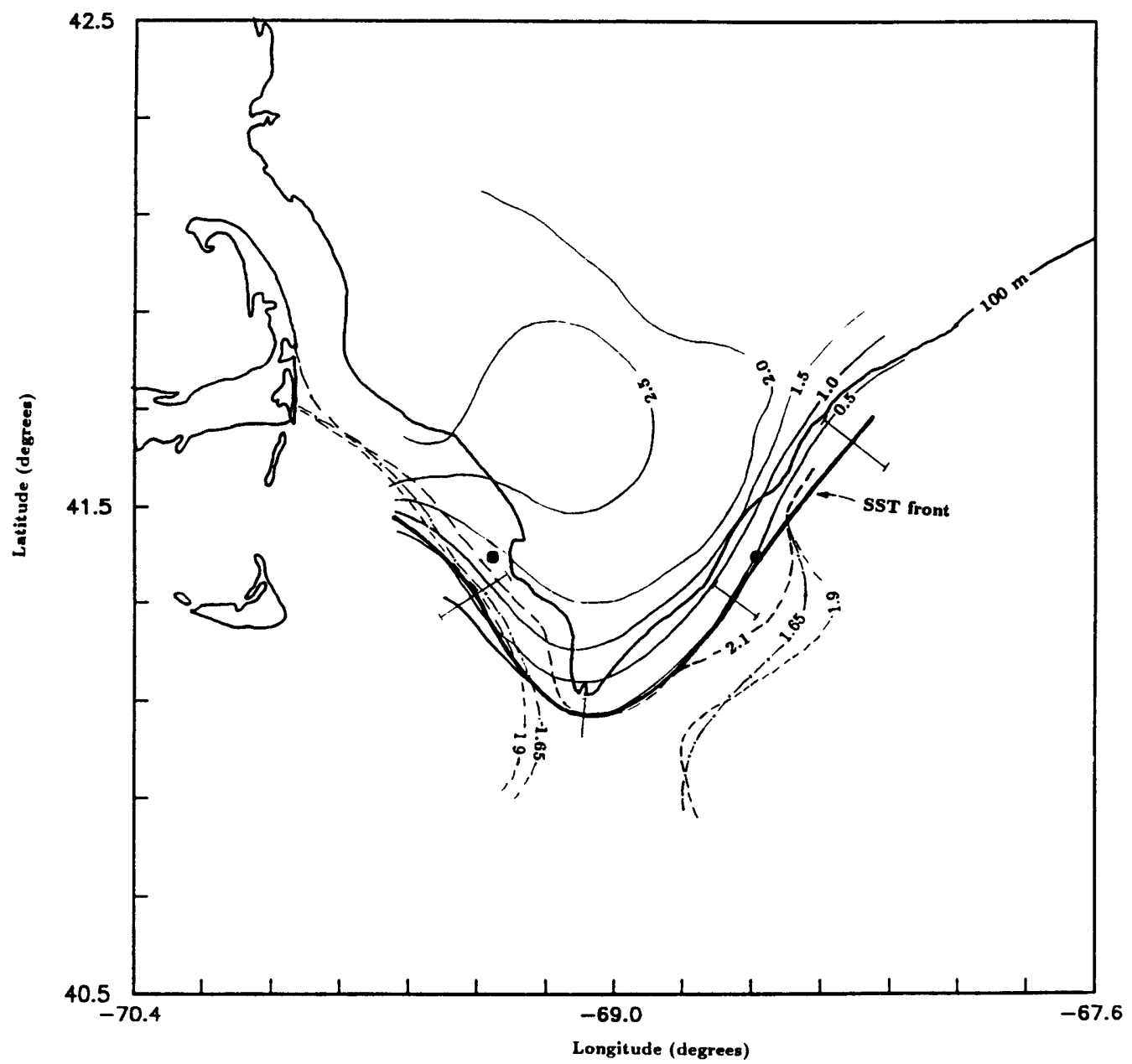

Figure 2.14: Comparison between observed and model tidal fronts over Nantucket Shoal, GSC and Georges Bank. Solid lines are $\Delta \sigma_{t}$ contours in the upper $40 \mathrm{~m}$ calculated from the June, 1989 CTD survey data. Heavy solid line is the monthly mean position of the thermal front averaged over the satellite SST images from May 5 to June 12, 1989. The deviation of the thermal front to the monthly mean position is given with the cross front solid line. "•" is the frontal position found during the small-scale biological survey on either May 26 or June 3, 1989. Dashed and dash-dot lines are the contours of $\log _{10} h / D=1.9$ and 2.1 for the case of tidal mixing alone and $\log _{10}(h /(D+0.59))=1.65$ for the case of tidal plus wind mixing, respectively. They are digitized from Figures 3 and 5 in Loder and Greenberg's (1986) paper. 
$41^{\circ} 23.75^{\prime} \mathrm{N}$, over Nantucket Shoals where the water depth is about $61 \mathrm{~m}$. A similar thermal front with a temperature jump of $2.0^{\circ} \mathrm{C}$ over $1.0 \mathrm{~km}$ at the surface was also observed on June 3,1989 at $68^{\circ} 35.15^{\prime} \mathrm{W}$ and $41^{\circ} 23.85^{\prime} \mathrm{N}$ near CTD station 136 , where the water depth is about $80 \mathrm{~m}$. The June, 1989 CTD survey showed a continuous contour of $\Delta \sigma_{t}=0.5$ [the boundary between the well-mixed and stratified regions defined by Garrett et al. (1978)] from Nantucket Shoals to the western flank of Georges Bank between the 80-m and 100$\mathrm{m}$ isobaths, in good agreement with the mean position of the SST front obtained from daily satellite SST images for the period May 5 to June 12, 1989. The April, 1988 CTD survey also showed a similar continuous contour of $\Delta \sigma_{t}=0.5$, implying that a relatively permanent vertical mixed region existed in the GSC near the $80-\mathrm{m}$ isobath in late spring in 1988 and 1989, and that the vertical mixed zones over Nantucket Shoals and Georges Bank were connected. This observed result is significantly different from those predicted by Garrett et al. (1978) for the case of tidal mixing alone and Loder and Greenberg (1986) for the case of tidal plus wind mixing but coincides well with the continuous contour of $\log _{10} h / D=2.1$ across the sill of the GSC estimated by Loder and Greenberg (1986) for the spring tide (see Figure 2.14). This fact suggests that the tidal mixing in the GSC during late spring is more intense than that predicted by the barotropic $M_{2}$ tidal dissipation and wind mixing alone. 


\subsection{Separating the Low-Frequency and Tidal Currents from the ADCP Data}

There are two main concerns whenever ship-mounted ADCP data are used to study residual flow in the coastal ocean: (1) contamination from the ship's velocity and (2) tidal currents. In water depths less than $200 \mathrm{~m}$, a 150-kHz ADCP can directly track the bottom along the ship's course, and thus the ship's velocity can be determined as the bottom tracking velocity. Since the bottom tracking velocity is recorded in the same coordinates as the raw ADCP current profile data, the largest measurement errors due to ship-mounted ADCP are automatically cancelled once the bottom tracking velocity is subtracted from the current profile data, leaving only misalignment and sensitivity errors (Joyce, 1989; Chen, 1989). On the other hand, tidal currents which are generally one order of magnitude or more larger than the subtidal flow may dominate the raw ADCP data so as to make them useless for the study of residual flow. Removing the tidal currents from the raw ADCP data requires detailed information about the spatial and temporal variability of tidal currents in the domain of study. Fortnightly or longer term variability of tidal currents due to a linear superposition of different tidal constituents may exist in the raw ADCP records, possibly making it difficult to resolve each tidal component from a short term ADCP measurement.

There has been no simple way available so far to filter tidal signals directly from the raw ADCP data recorded along a CTD survey track. As we know, the ship-mounted ADCP provides profiles of instantaneous current along the ship's track, which are a function of time and the ship's position in time. If the ship is stationary over at least one tidal cycle, the ADCP data can be treated as a time series and then tidal signals can be easily filtered from the raw ADCP data using harmonic analysis or least squares fit (Geyer and Signell, 1990; Lwisa and Bowers, 1990; Beardsley et al., 1990). If the ship is moving, however, a different approach that allows for spatial variability of tidal currents must be used to separate tidal and residual currents from the raw ADCP data. One possible method to deal with this problem is to use a numerical model to predict the spatial and temporal distribution of 
tidal current and then subtract them directly from the raw ADCP records (Freeland and Foreman, 1990). However, this approach is constrained from its general application in most coastal regions due to the lack of good numerical tidal models. Beardsley et al. (1990) treated the ADCP data (taken along a CTD tow-yo track within a small area about $5 \times 5 \mathrm{~km}$ in the GSC) as a time series and successfully used a least squares fit to separate the tidal and residual currents there. Candela et al. (1992) recently extended the least squares fit method to include a polynomial function to fit the spatial structure of tidal and subtidal currents observed in an ADCP survey. In the northern GSC, the cotidal and phase lines of the semidiurnal and diurnal tides almost parallel the local isobaths (Moody et al., 1984). This simple structure motivates us to apply the least squares fit method introduced by Candela et al. (1992) to treat the 1988 and 1989 SCOPEX regional ADCP data in the northern GSC. Since this approach is quite new and was being developed during the course of this research, some modifications of this method have been made here (1) to include the time variability of the fitted tidal currents due to the superposition of different tidal constituents during our surveys, and (2) to resolve the vertical structure of tidal and residual currents. A detailed discussion on separating tidal and residual flow from the SCOPEX ADCP data will be given next.

During the 1988 and 1989 SCOPEX regional hydrographic surveys, continuous acoustic Doppler current profile data were collected during April 26-29, 1988 (3 days), May 19-22, 1989 (4 days), and June 6-12, 1989 (6 days) using the R/V Endeavor 150$\mathrm{kHz}$ RDI ADCP. The relative shallow depth of the SCOPEX survey region allowed bottom tracking during the ADCP measurements so that high-quality absolute current profile data over the upper $140 \mathrm{~m}$ were obtained during the entire May-June, 1989 surveys and most of the April, 1988 survey. Due to unknown instrument problems, bottom tracking did not work well in the middle of the April, 1988 survey region. The GPS and Loran-C data recorded on board were not sufficient to allow accurate calculation of ship's velocity. For this reason, we only analyzed the ADCP data with bottom tracking for the April, 1988 survey. The vertical resolution of the ADCP data is four meters and the time interval of 
measurement is five minutes. The instantaneous raw ADCP records were dominated by semidiurnal tidal currents, especially during the May-June, 1989 surveys.

Without considering the nonlinear interaction between the tidal and subtidal currents, the total current field can be simply represented by

$$
\vec{u}=\vec{u}_{r}+\sum_{n=1}^{K}\left(\vec{a}_{n} \cos \omega_{n} t+\vec{b}_{n} \sin \omega_{n} t\right)
$$

where the index $n$ denotes the semidiurnal and diurnal tidal components used in our fitting, $\vec{u}_{r}$ is the residual current vector, and $\left(\vec{a}_{n}, \vec{b}_{n}\right)$ are the amplitudes of the tidal components. Candela et al. (1992) succeeded in using first and second order polynomials or splines as the empirical spatial functions of $\vec{u}_{r}$ and $\left(\vec{a}_{n}, \vec{b}_{n}\right)$, respectively, to fit the vertical integral transports of residual flow and semidiurnal $\left(M_{2}\right)$ tidal currents in the northern East China Sea. In the northern GSC, however, the superposition of different tidal constituents (three semidiurnal tides $M_{2}, N_{2}$, and $S_{2}$ and two diurnal tides $K_{1}$ and $O_{1}$ ) makes a significant contribution to the long term variability of the tidal currents, even though the ratio of either $N_{2}$ and $S_{2}$ to $M_{2}$ for the equilibrium tide is only about 0.2 and the ratio of $K_{1}$ or $O_{1}$ to $M_{2}$ is less than 0.1 (Moody et al., 1984). Six tidal elevation stations near our study area were chosen to investigate the fortnightly modulation due to superposition of semidiurnal and diurnal tides during these surveys (see Figure 2.15). In June, 1989, the amplitudes of $S_{2}$ and $N_{2}$ were of the same order, while their phases were about $180^{\circ}$ different at the beginning and then decreased with time during the survey. The combined contribution of these two tidal constituents resulted in an approximately linear decrease of the amplitude of the total semidiurnal tide (a sum of $M_{2}, S_{2}$ and $N_{2}$ ) during the survey but did not cause a frequency shift from $M_{2}$ (see Figure 2.16). In May, 1989, three weeks earlier, the amplitudes of $S_{2}$ and $N_{2}$ cancelled each other during most of the survey so that the total semidiurnal tidal component was equal to $M_{2}$ except for a little phase shift (Figure 2.17). In contrast, a small phase difference was found between $N_{2}$ and $S_{2}$ at the beginning of the April, 1988 survey; later it became larger and larger, leading to an 


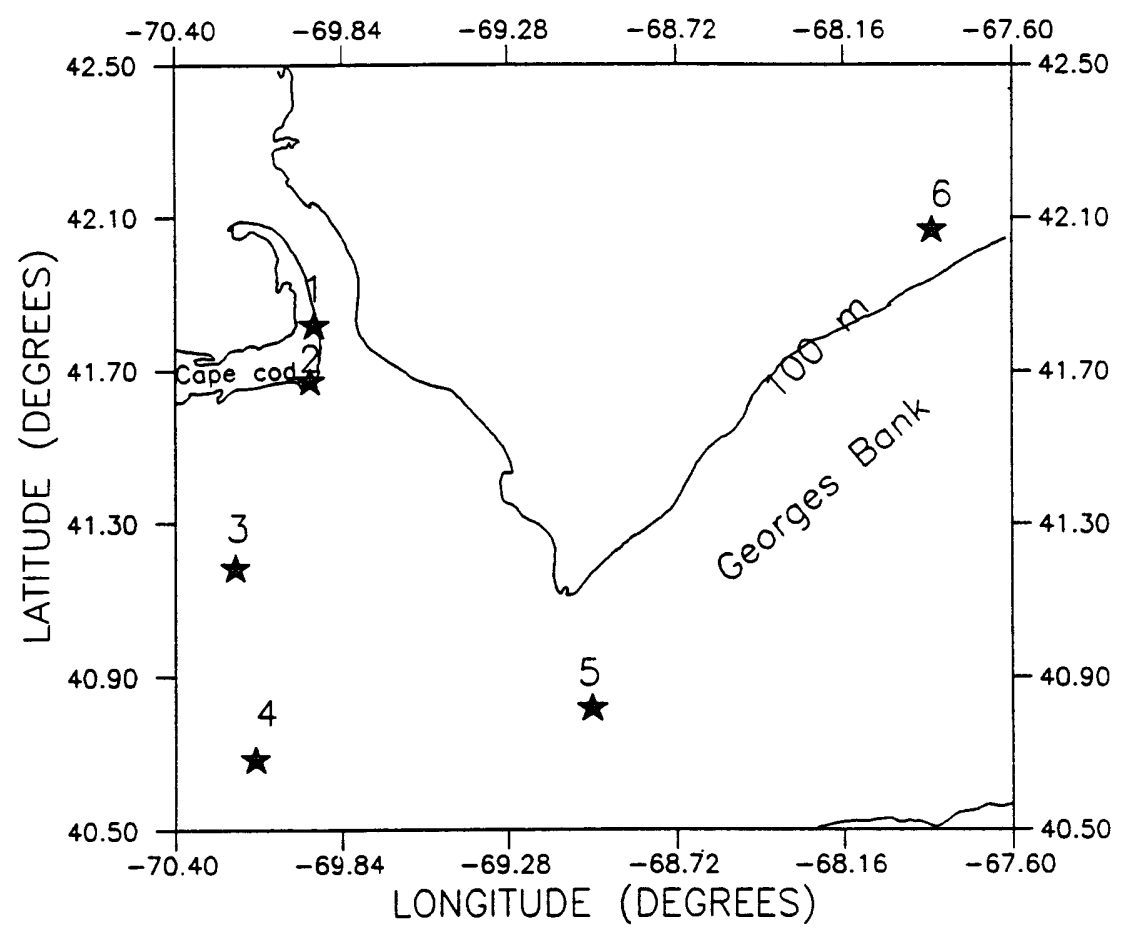

Figure 2.15: Stations of surface tidal elevation. Stars represent the positions of observational stations which are numbered 1 to 6 from Nauset to Georges Bank.

approximately linear increase of total semidiurnal tide with time during that survey. During all three surveys, the period of the total diurnal tide was equal to an average period of $K_{1}$ and $O_{1}$ tides, and its amplitude was almost two times as large as either of these two tidal constituents (see Figures 2.18 and 2.19 for examples). Based on these results, we decided to choose the $M_{2}$ frequency $\left(\omega_{1}=2 \pi / 12.4206 \mathrm{hrs}\right)$ and the mean frequency of $K_{1}$ and $O_{1}\left(\omega_{2}=2 \pi / 24.8769 \mathrm{hrs}\right)$ as the frequencies of the total semidiurnal and diurnal tidal components in our tidal fit for the SCOPEX ADCP data.

The spatial variability of the combined effect of $N_{2}$ and $S_{2}$ on the total semidiurnal tide has been examined next at the six stations by calculating the ratio of total semidiurnal tidal elevation $\left(\zeta_{M_{2}+N_{2}+S_{2}}\right)$ to its fitted value $\left(\widehat{\zeta}_{M_{2}}\right)$ using the $M_{2}$ frequency alone. The values of $\zeta_{M_{2}}+N_{2}+S_{2} / \widehat{\zeta}_{M_{2}}$ were the same for a given time at all of the six stations and then linearly increased or decreased with time at the same rate during the June, 1989 or April, 1988 survey (see Figure 2.20), implying that no spatial variability of total 

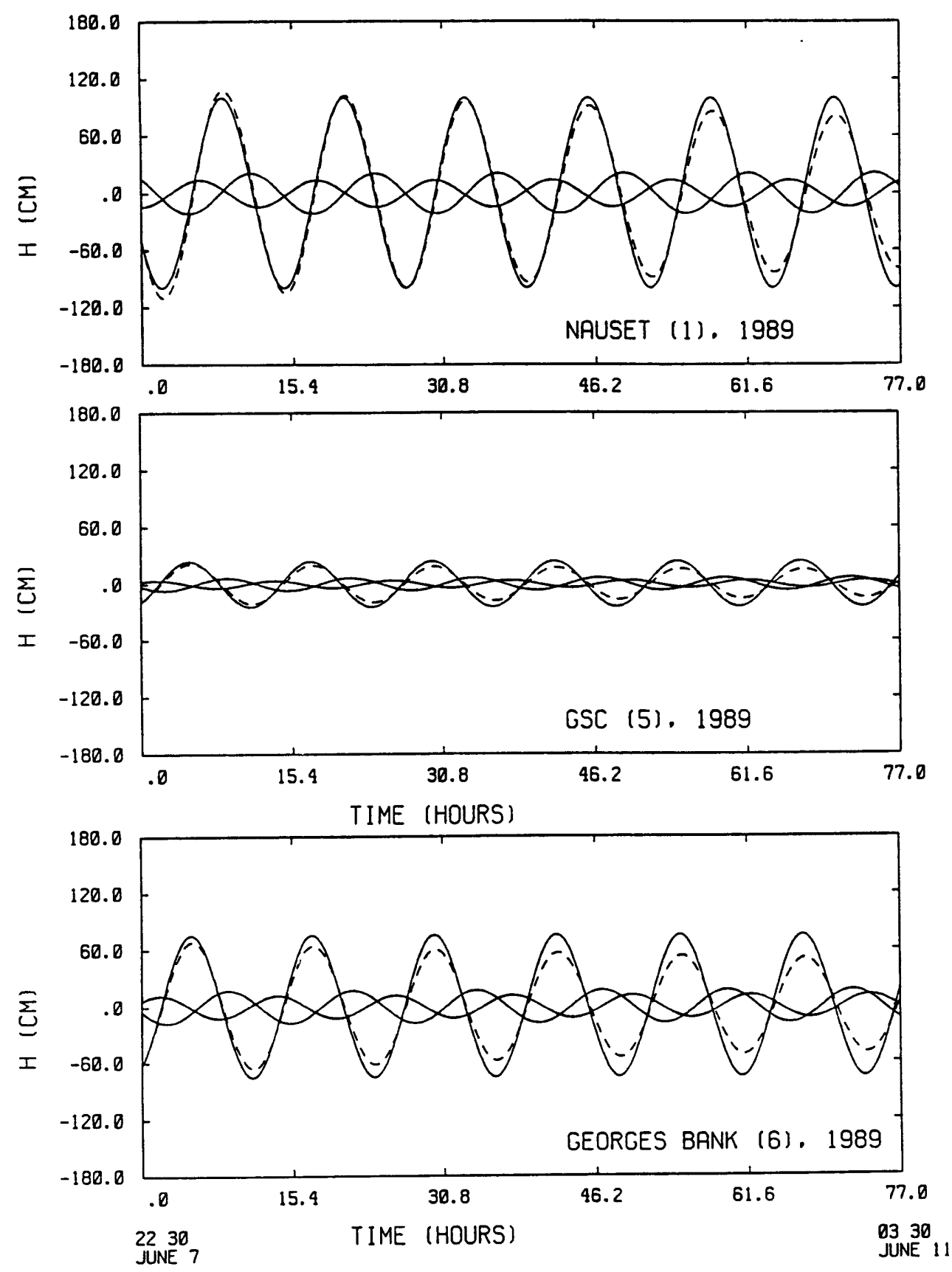

Figure 2.16: Time series of semidiurnal tidal elevations at Nauset, Great South Channel (GSC) and Georges Bank during the June, 1989 CTD/ADCP survey. The solid lines refer to $M_{2}$ (Lunar semidiurnal), $S_{2}$ (Solar semidiurnal) and $N_{2}$ (Large lunar elliptic), respectively. The dashed line represents a sum of $M_{2}+S_{2}+N_{2}$. 


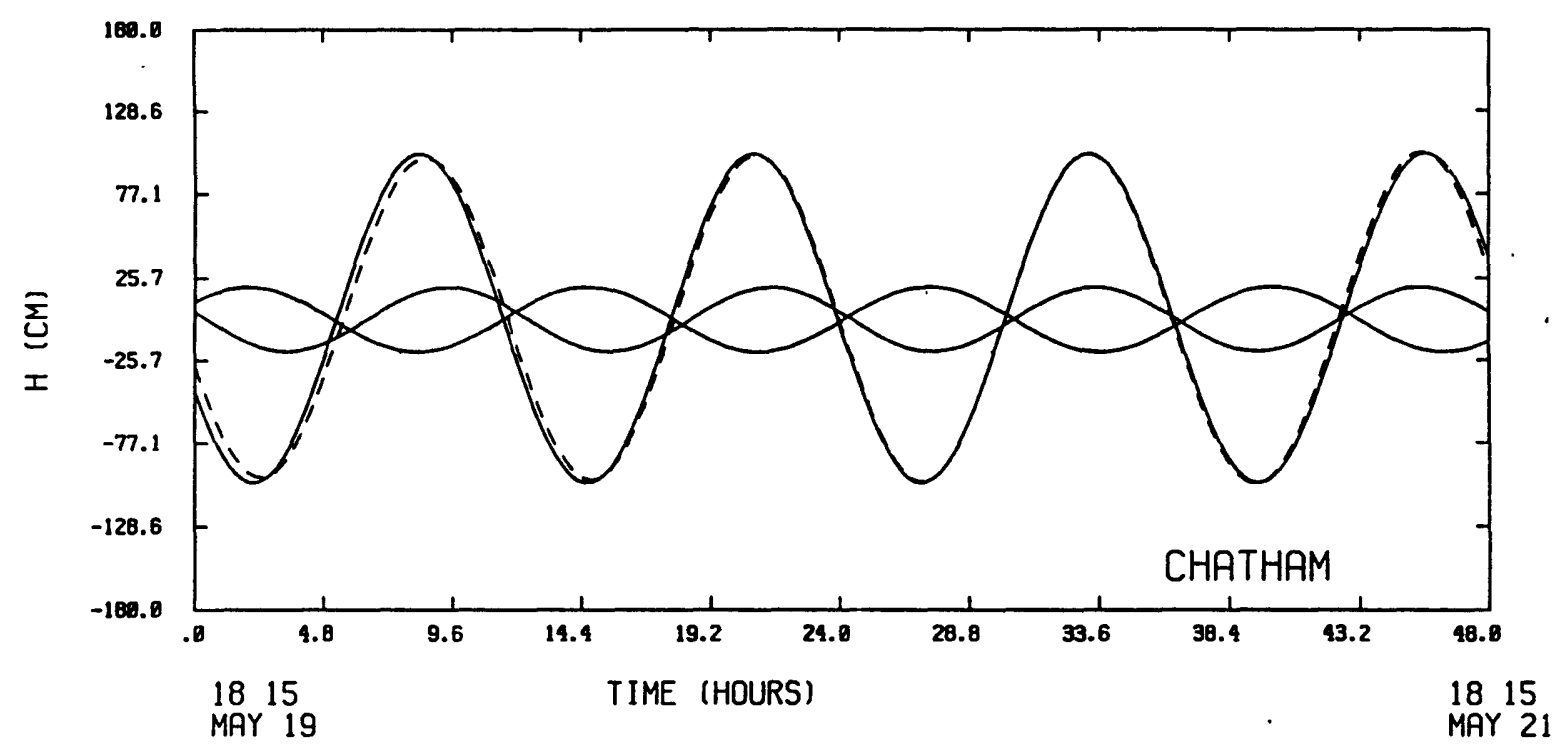

Figure 2.17: Time series of semidiurnal tidal elevations at Chatham during the May, 1989 CTD/ADCP survey. The solid lines refer to $M_{2}$ (Lunar semidiurnal), $S_{2}$ (Solar semidiurnal) and $N_{2}$ (Large lunar elliptic), respectively. The dashed line represents a sum of $M_{2}+S_{2}+N_{2}$.

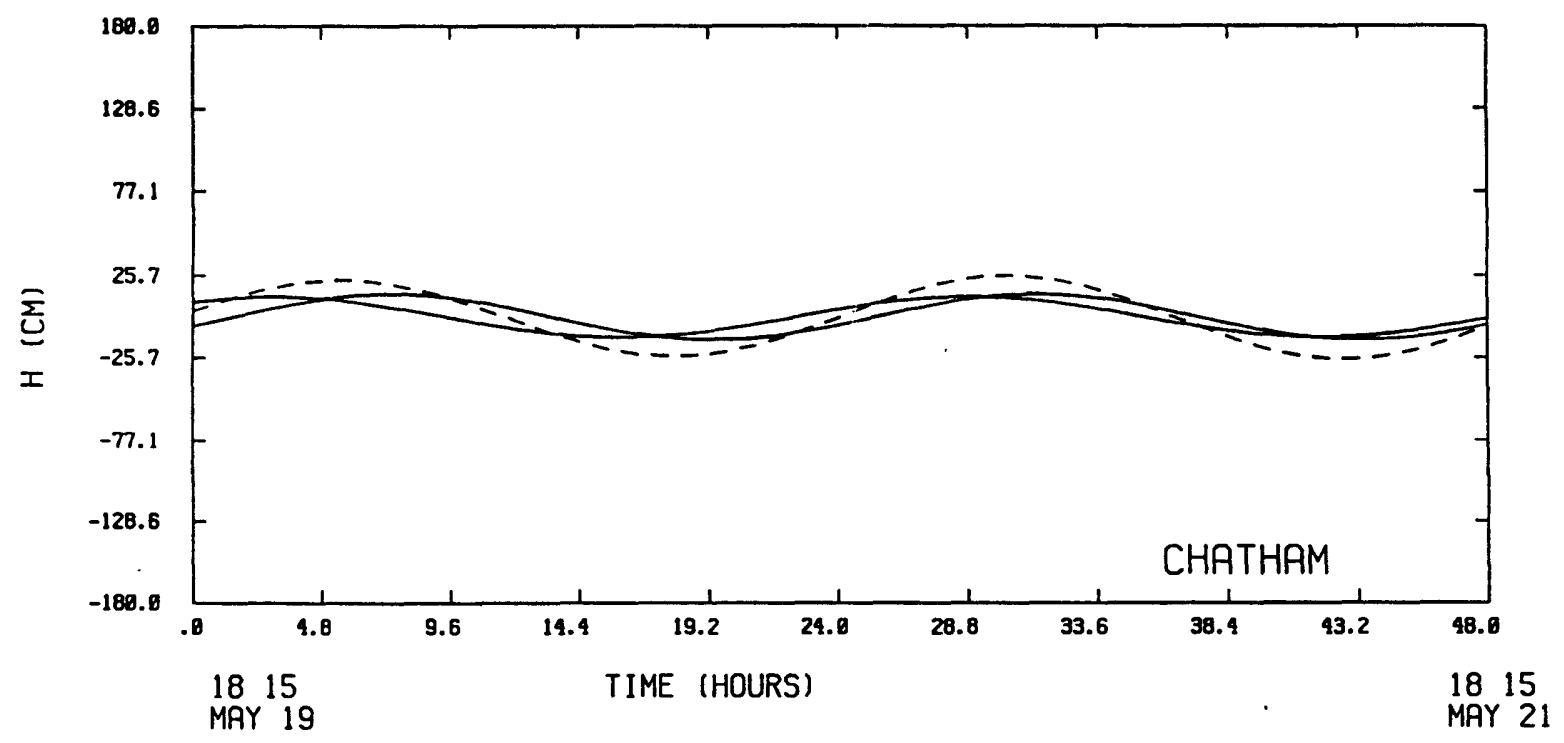

Figure 2.18: Time series of diurnal tidal elevation at Chatham during the May, 1989 CTD/ADCP survey. The solid lines refer to $K_{1}$ (Luni-solar diurnal) and $O_{1}$ (Lunar diurnal). The dashed line represents a sum of $K_{1}+O_{1}$. 

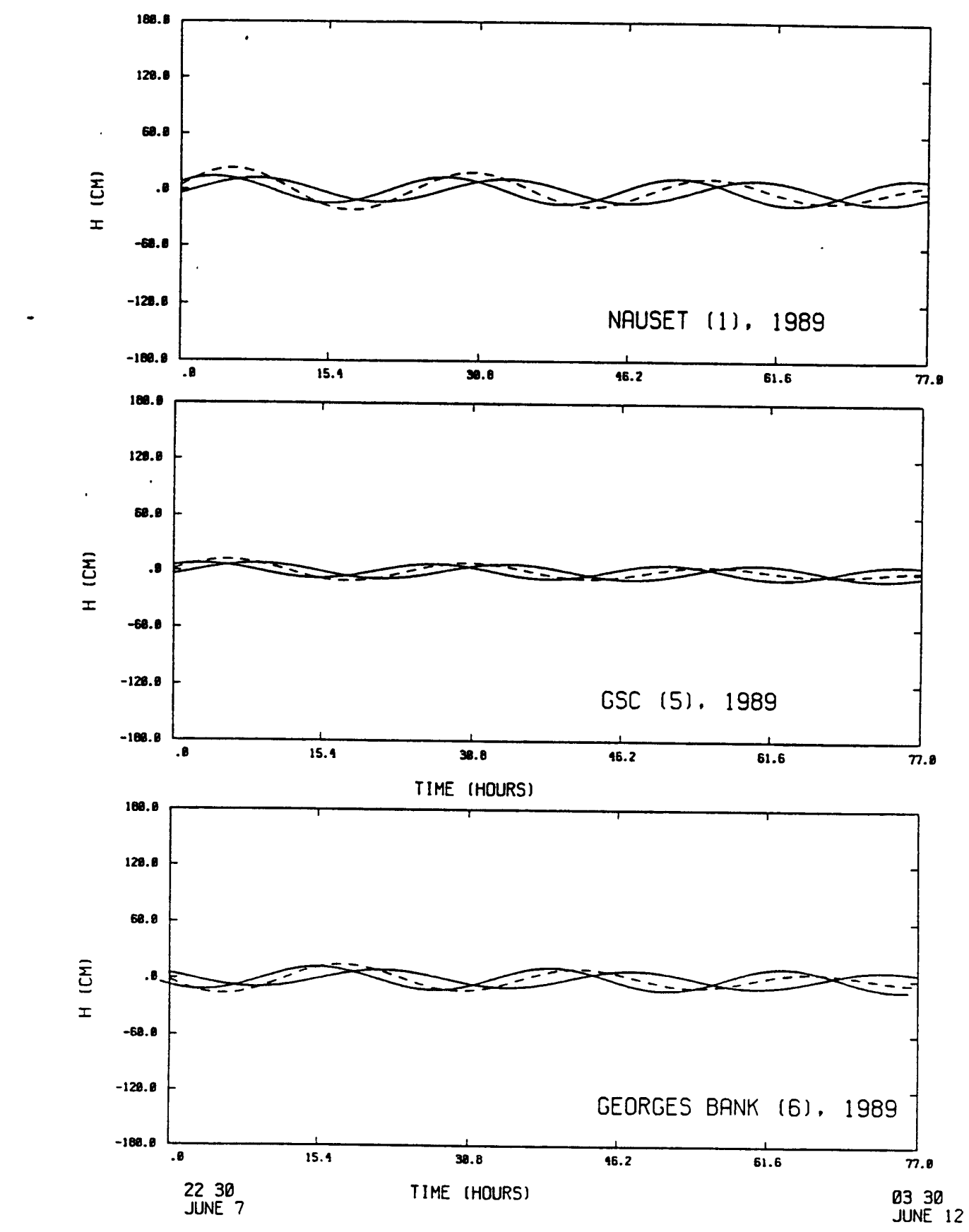

Figure 2.19: Time series of diurnal tidal elevation at Nauset, Great South Channel (GSC), and Georges Bank during the June, $1989 \mathrm{CTD} / \mathrm{ADCP}$ survey. The solid lines refer to $K_{1}$ (Luni-solar diurnal) and $O_{1}$ (Lunar diurnal). The dashed line represents a sum of $K_{1}+O_{1}$. 

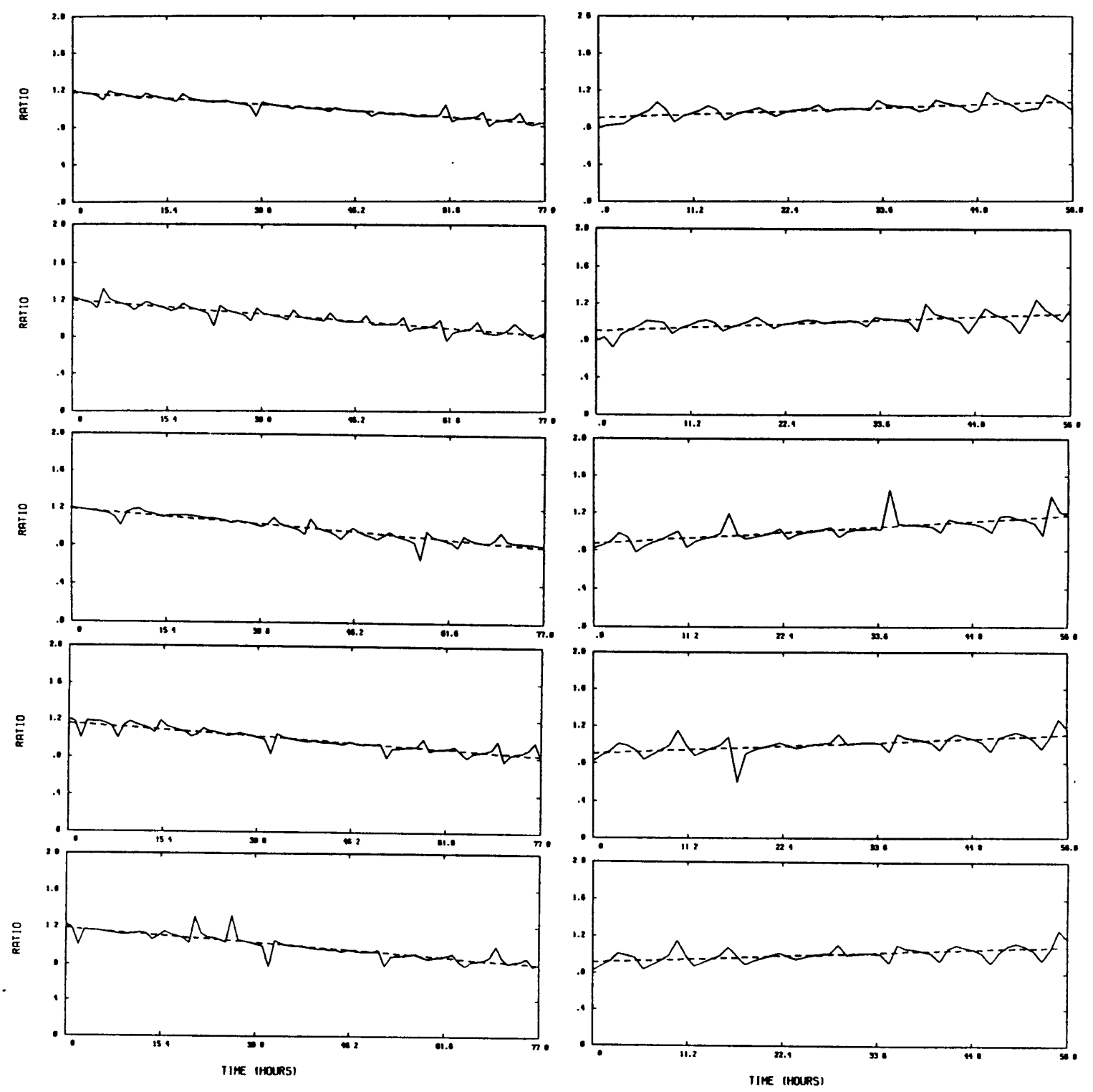

Figure 2.20: Ratio of total semidiurnal tidal elevation $\zeta_{M_{2}}+N_{2}+S_{2}$ to its fitted value $\widehat{\zeta}_{M_{2}}$ at a frequency of $M_{2}$ (solid line) at Nauset, Chatham, Nantucket Shoal, Great South Channel and Georges Bank for the June, 1989 (left) and April, 1988 (right) CTD/ADCP surveys. Dashed lines are least squares fit for the ratio of $\zeta_{M_{2}}+N_{2}+S_{2}$ to $\widehat{\zeta}_{M_{2}}$. 
semidiurnal tidal elevation was caused by the phase difference of $N_{2}$ and $S_{2}$ during the April, 1988 and June, 1989 surveys. Therefore, the combined contribution of $N_{2}$ and $S_{2}$ to total semidiurnal tidal elevation during these two surveys can be simply included by multiplying the amplitude of fitted semidiurnal tidal elevation with a linearly time-dependent and spatially uniform correction term. Since the tidal currents are proportional to the spatial gradient of tidal elevation, we expect here that the same spatially uniform correction can be also used for the semidiurnal tidal current field in the northern GSC during the April, 1988 and June, 1989 surveys. Good agreement was found between $\zeta_{M_{2}}+N_{2}+S_{2}$ and $\widehat{\zeta}_{M_{2}}$ in the May, 1989 survey. The ratio of $\zeta_{M_{2}}+N_{2}+S_{2}$ to $\widehat{\zeta}_{M_{2}}$ was about 1.0 during the whole survey so that the combined contribution of $N_{2}$ and $S_{2}$ does not need to be included for the total semidiurnal fitting during the May, 1989 survey. A similar analysis has been made for the diurnal tide for the April, 1988, May and June, 1989 surveys. Since the correction of the amplitude due to the phase difference of $K_{1}$ and $O_{1}$ was very small compared with that of the semidiurnal tides, we neglect it here in the diurnal tidal fitting.

Taking the time-dependent correction for the semidiurnal tidal currents into account, the low frequency residual flow and the amplitudes of tidal currents can be then separately expressed by polynomial functions of the form

$$
\begin{aligned}
\vec{u}_{r} & =\sum_{k=0}^{L} \sum_{i=0}^{k} \vec{u}_{r l_{k+\imath}} x^{k-i} y^{i} ; \\
\left(\begin{array}{l}
\vec{a}_{1} \\
\vec{b}_{1}
\end{array}\right) & =\varepsilon \sum_{k=0}^{m_{1}} \sum_{i=1}^{k}\left(\begin{array}{ll}
\vec{a}_{1} l_{k+\imath} \\
\vec{b}_{1} l_{k+\imath}
\end{array}\right) x^{k-i} y^{i} ; \\
\left(\begin{array}{l}
\vec{a}_{2} \\
\vec{b}_{2}
\end{array}\right) & =\sum_{k=0}^{m_{2}} \sum_{i=1}^{k}\left(\begin{array}{ll}
\vec{a}_{2} l_{k+\imath} \\
\vec{b}_{2} l_{k+\imath}
\end{array}\right) x^{k-i} y^{i},
\end{aligned}
$$

where $\mathrm{L}, m_{1}$ and $m_{2}$ are degrees of the polynomial for the low frequency, semidiurnal and diurnal tidal currents, respectively. $l_{k+i}=\sum_{i}^{k} i$ is an integer which is equal to the total number of previous terms. $\varepsilon$ is the linearly time-dependent correction factor for the 
semidiurnal tidal currents, given by

$$
\varepsilon= \begin{cases}1.2-\frac{0.40}{t_{N}-t_{o}}\left(t-t_{o}\right) & \text { for the June, 1989 survey } \\ 1.0 & \text { for the May, 1989 survey } \\ 0.9+\frac{0.18}{t_{N}-t_{o}}\left(t-t_{o}\right) & \text { for the April, 1988 survey }\end{cases}
$$

where $t_{o}$ and $t_{N}$ are the initial and final time of the survey.

It may be useful to note here that instead of finding a correction factor for the semidiurnal tidal fit, the effect of $N_{2}$ and $S_{2}$ tidal constituents on the total semidiurnal tidal currents for the 1988 and 1989 surveys can be also taken into account by adding a linearly time-dependent correction term into the amplitude of the fitted semidiurnal tidal currents expressed by a polynomial function since the contribution of mixture terms of time and space to the tidal amplitude was very small. Such an approach, however, must be done at the expense of reducing the degrees of freedom for the fitting function, possibly increasing the fitting uncertainty within a given confidence level. For this reason, we prefer here to make a correction for $N_{2}$ and $S_{2}$ to the total semidiurnal tidal currents by multiplying the tidal amplitude with a fixed linearly time-dependent factor obtained from the ratio of $\zeta_{M_{2}}+N_{2}+S_{2} / \widehat{\zeta}_{M_{2}}$, respectively, for the 1988 and 1989 surveys.

The multiple least squares regression method to fit the raw ADCP data is briefly described next (Brownlee, 1965; Draper and Smith, 1966; Fofonoff and Bryden, 1975). Given data $Y_{n}$ for $u_{n}$ or $v_{n} ; \mathrm{n}=1,2,3, \ldots, \mathrm{N}$, the regression formula (2.1) of $\mathbf{r}$ degrees of the polynomials for total low-frequency and tidal currents can be simply rewritten as

$$
\widehat{Y}_{n}=\sum_{i=1}^{\mathbf{r}} a_{\imath} X_{n i}
$$

where $\widehat{Y}_{n}$ is the predicted value for $Y_{n} . X_{n i}$ is an independent variable consisting of the different terms of the polynomials or products of the polynomials with the periodic function 
given by tidal frequencies. The least squares estimates for $a_{i}$ can be obtained subject to minimization of the squares residuals

$$
G_{N r}=\sum_{n=1}^{N}\left(Y_{n}-\widehat{Y}_{n}\right)^{2}
$$

Minimization of $G_{N}$ with respect to $a_{i}$ yields the normal equations in the matrix form

$$
\left(\mathbf{X}^{\prime} \mathbf{X}\right) \mathbf{A}=\mathbf{Y}
$$

where $\mathbf{X}=\left\{X_{n i}\right\}, \mathbf{A}=\left\{a_{i}\right\}$ and $\mathbf{Y}=\left\{\sum_{n=1}^{N} X_{n i} Y_{n}\right\}$. The prime' denotes the transpose of the matrix. Let $\mathbf{C}$ be the inverse of matrix $\left(\mathbf{X}^{\prime} \mathbf{X}\right)$, then

$$
\mathbf{A}=\mathbf{C} \mathbf{Y}
$$

If $Y_{n}$ is assumed to be a random variable normally distributed about a true value with variance $\sigma^{2}$, the coefficient $a_{i}$ will also be random variables with variance given by

$$
\sigma_{a_{i}}^{2}=C_{i i} \sigma^{2}
$$

and the unbiased expected value for $\sigma^{2}$ is

$$
\sigma^{2}=\frac{G_{N}}{(N-r)}
$$

where $(N-r)$ is the degrees of freedom for the least squares regression.

Two statistical tests using the student's $t$-distribution and the $F$-distribution have been made to test whether each $a_{i}$ is significantly different from zero at the $95 \%$ confidence level and if adding higher order polynomials into the regression gives a significantly better fit. For a normal distribution, the ratio $a_{i} / \sigma \sqrt{C_{i i}}$ satisfies a student's $t$-distribution with 
$N-r$ degrees of freedom. Therefore, $a_{i}$ become significantly different from zero at a $95 \%$ confidence level only if

$$
\left|\frac{a_{i}}{\sigma \sqrt{C_{i i}}}\right|>t_{0.05}(N-r)
$$

A higher order of polynomial is important only if it significantly reduces the square residual for fitting. For a normal distribution, the ratio $G_{N q}-G_{N r} /(q-r) \sigma_{q}$ has an $F$-distribution with $q-r$ degrees of freedom for the first fit and $N-1-r$ degrees of freedom for the second fit. At a $95 \%$ confidence level, additional terms $(q-r)$ are significant only if

$$
\frac{G_{N r}-G_{N q}}{(q-r) \sigma_{q}}>F_{0.05}(q-r, N-1-r)
$$

The uncertainty in the predicted value $\hat{Y}_{n}$ from the true value $Y_{n}$ is given by the standard deviation of $\widehat{Y}_{n}$, which is equal to

$$
\sigma_{\widehat{Y}_{n}}=\sigma \sqrt{\mathbf{X}_{n}^{\prime} \mathbf{C ~ \mathbf { X } _ { n }}}
$$

At a $95 \%$ confidence limit $(\alpha=0.05)$ on the true mean value of $Y_{n}$ at $\mathrm{X}_{n}$, the fitting error of least squares regression at position $\mathbf{X}_{n}$ is given by

$$
\Delta Y_{n}= \pm t\left\{(N-r-1), 1-\frac{1}{2} \alpha\right\} \sigma \sqrt{\mathbf{X}_{n}^{\prime} \mathbf{C ~ \mathbf { X } _ { n }}}
$$

which is a function of position $(x, y)$ and degrees of freedom $(N-r-1)$.

The critical factor in application of the least squares method to separate the tidal and subtidal currents from the ADCP data is the number of independent data records $N$. For a given time series, in general, $N$ can be determined by an analysis of the auto-correlation function of the time series. That is,

$$
N=\frac{M}{T},
$$


where $M$ is the total time length of data records and $T$ is the correlation time scale of the time series which is defined as

$$
T=\int_{0}^{\infty} R(\tau) d \tau
$$

where $R(\tau)$ is the auto-correlation coefficient of the time series. However, narrow band tidal signals at frequencies $\omega_{1}$ (semidiurnal) and $\omega_{2}$ (diurnal), when superimposed on a random residual signal in the raw SCOPEX ADCP data, caused a periodic auto-correlation coefficient for $u$ or $v$ (Figure 2.21), thus the time scale for independent data records can not be directly determined using the auto-correlation coefficient. In fact, a pure harmonic time series contains only two degrees of freedom (amplitude and phase). Therefore, the existence of narrow band tidal variability in the ADCP data must reduce the statistical significance for the residual flow by reducing the number of independent realizations in the sample record. For this reason, we first subtracted the tidal currents from the total water velocity by a least squares fit for the raw 5-min ADCP data and then calculated the auto-correlation coefficient for residual velocity $u_{r}$ or $v_{r}$ (Figure 2.22). The resulting auto-correlation coefficient showed a correlation time scale of about one-hour for each of $u_{r}$ and $v_{r}$ during both the 1988 and 1989 surveys, thus the raw ADCP data for all surveys were averaged into one-hour, non-overlapping values along the ship's track to construct an independent data set for the residual flow analysis.

Direct fit for the one-hour averaged ADCP currents using the empirical formulae (2.1)-(2.4) showed high correlations of 0.95 for $u$ and 0.99 for $v$ between the predicted and raw ADCP currents (see Figures 2.23 and 2.24 as examples for the 1989 surveys). As discussed before, such high correlations may not be statistically meaningful because of the existence of narrow band tidal signals at semidiurnal and diurnal frequencies (see Chelton, 1982). In other words, the high correlation found from the least squares fit of total ADCP data may reflect the dominance of the tidal components. On the other hand, it is also 

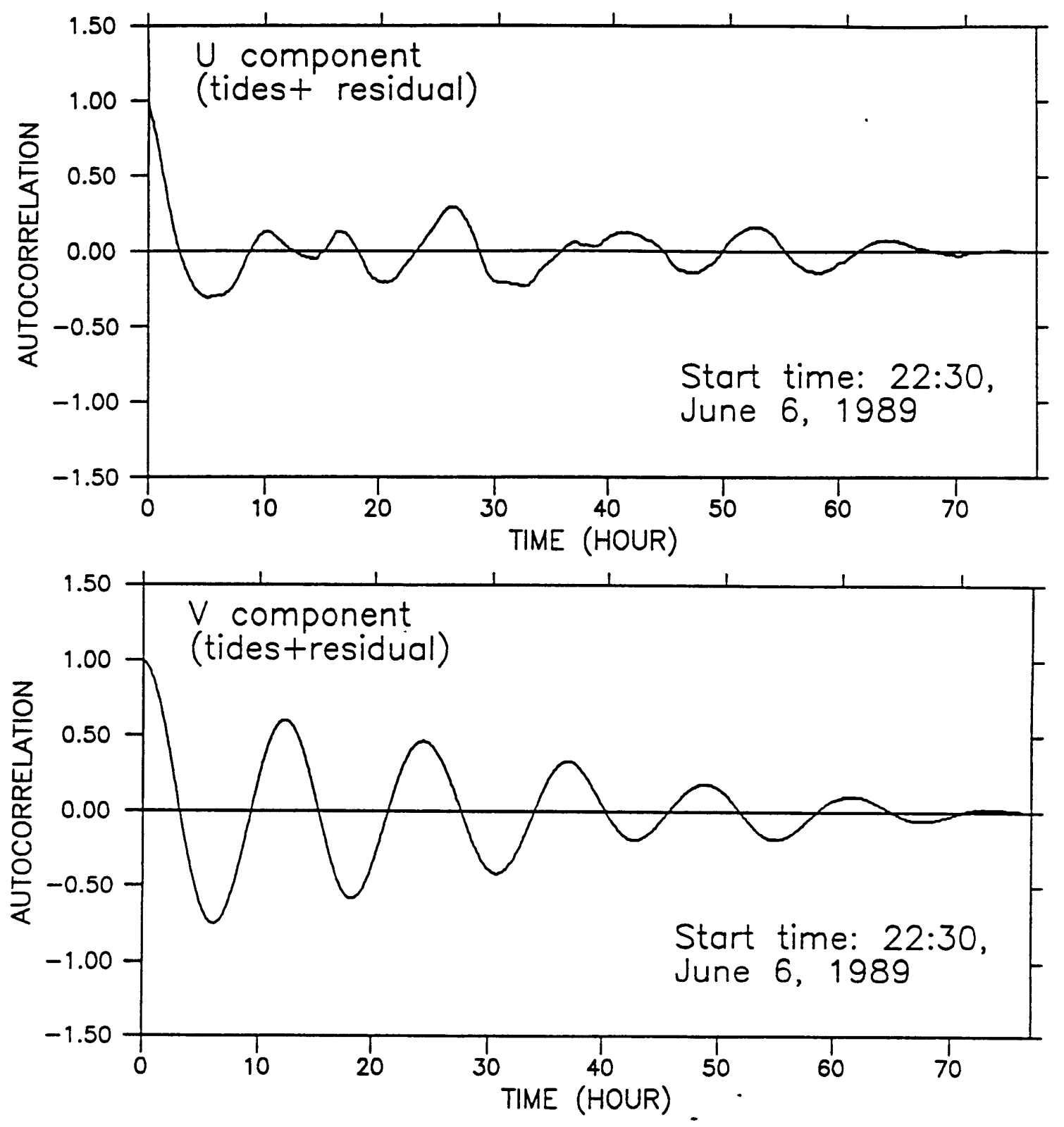

Figure 2.21: Auto-correlation coefficient of the raw ADCP velocity $u$ (upper) and $v$ (lower) for the June, 1989 CTD/ADCP survey. The resolution of the raw ADCP data is five minutes during the whole survey. 

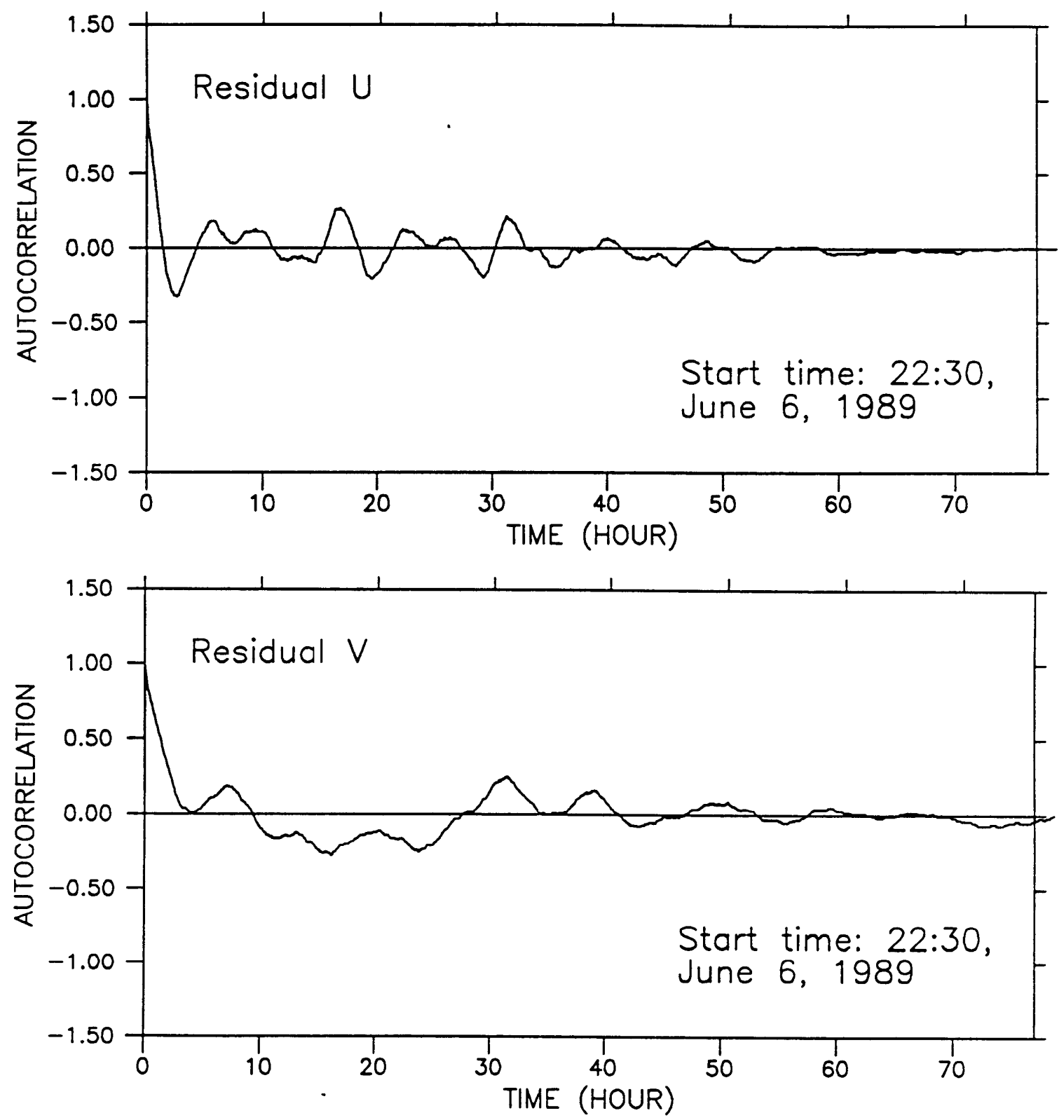

Figure 2.22: Auto-correlation coefficient of residual flow $u_{r}$ (upper) and $v_{r}$ (lower) for the June, 1989 CTD/ADCP survey. The resolution of the raw ADCP data is five minutes during the whole survey. 
difficult to find out the statistical reliability for our tidal fit since there are only two degrees of freedom for each tidal component. For these reasons, two steps were taken to fit the tidal and residual flow in the SCOPEX one-hour averaged ADCP data. The first step was to find the best fit for the tidal currents using the empirical formulae (2.1)-(2.4) with a low order polynomial for the residual flow. The second step was to fit a "raw" residual current data series that was obtained by subtracting the model tidal currents found in step one from the original one-hour averaged ADCP currents. The uncertainty of model tidal currents is determined from the standard deviation of predicted tidal elevation obtained using the least squares fit from the total observed elevation of semidiurnal and diurnal tides at the six tidal stations shown in Figure $2.15^{4}$, while the errors in the model residual flow are calculated based on the measurement error and fitting uncertainty estimated from the formula (2.14) where the number of independent data samples is equal to the number of one-hour averaged data records. Good agreement between the predicted tidal currents and previous observations demonstrates the efficiency of this least squares fit approach for the ADCP data, while the consistency between the model residual flow field and water distributions and drifter trajectories in the 1988 and 1989 surveys give further encouragement that the analysis method yields an accurate picture of the nontidal flow. These results will be discussed in the next two sections on tidal and residual flows.

\footnotetext{
${ }^{4}$ Note: since the tidal elevation is associated with the integral of tidal currents, the uncertainty may be greater in the model tidal currents than in the tidal evelation.
} 

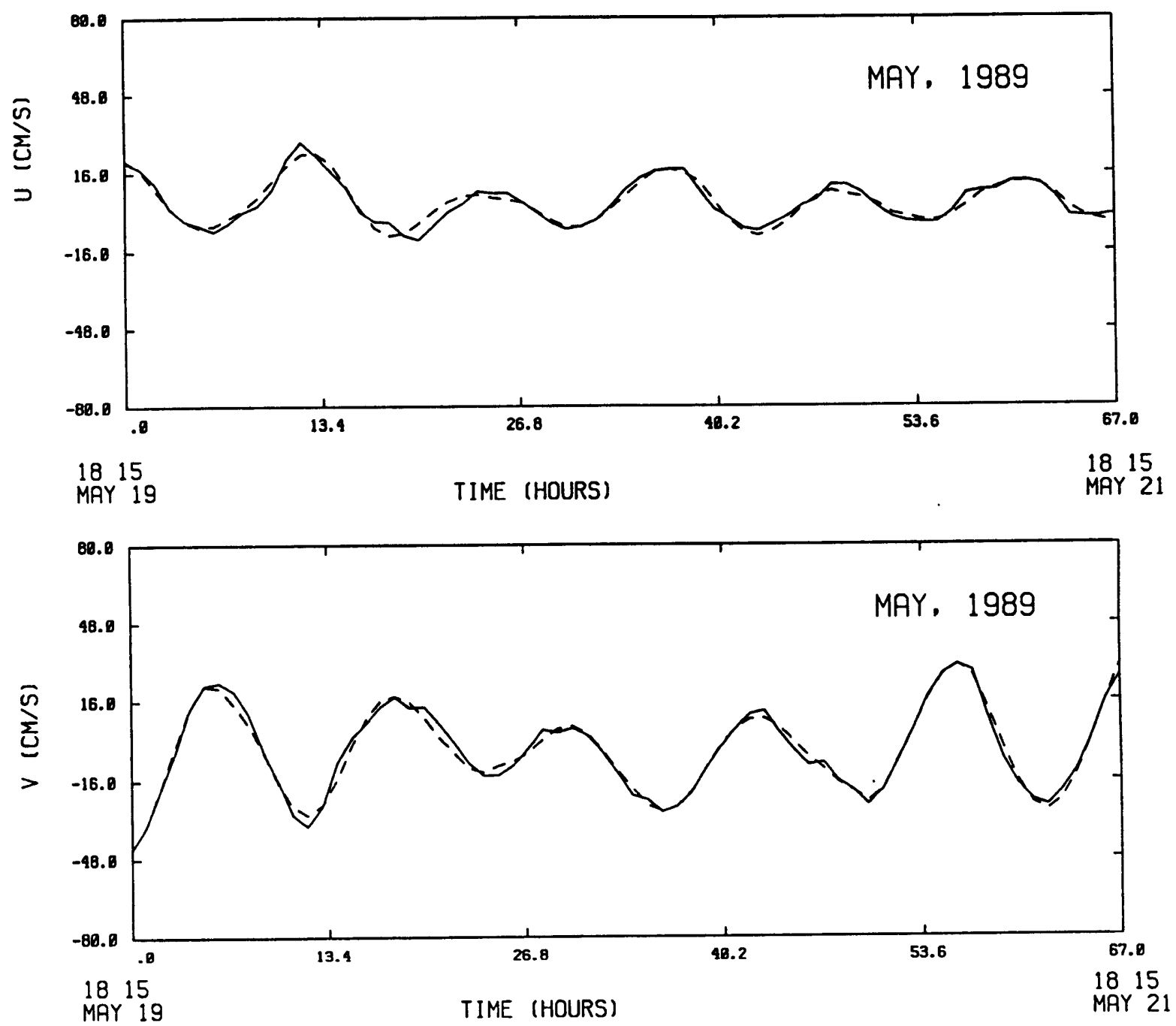

Figure 2.23: Comparison of observed and predicted vertically-averaged ADCP velocity $u$ (upper) and $v$ (lower) for the May, $1989 \mathrm{CTD} / \mathrm{ADCP}$ survey. The solid line represents the one-hour averaged ADCP velocity while the dashed line represents the predicted value using the least squares fit. 

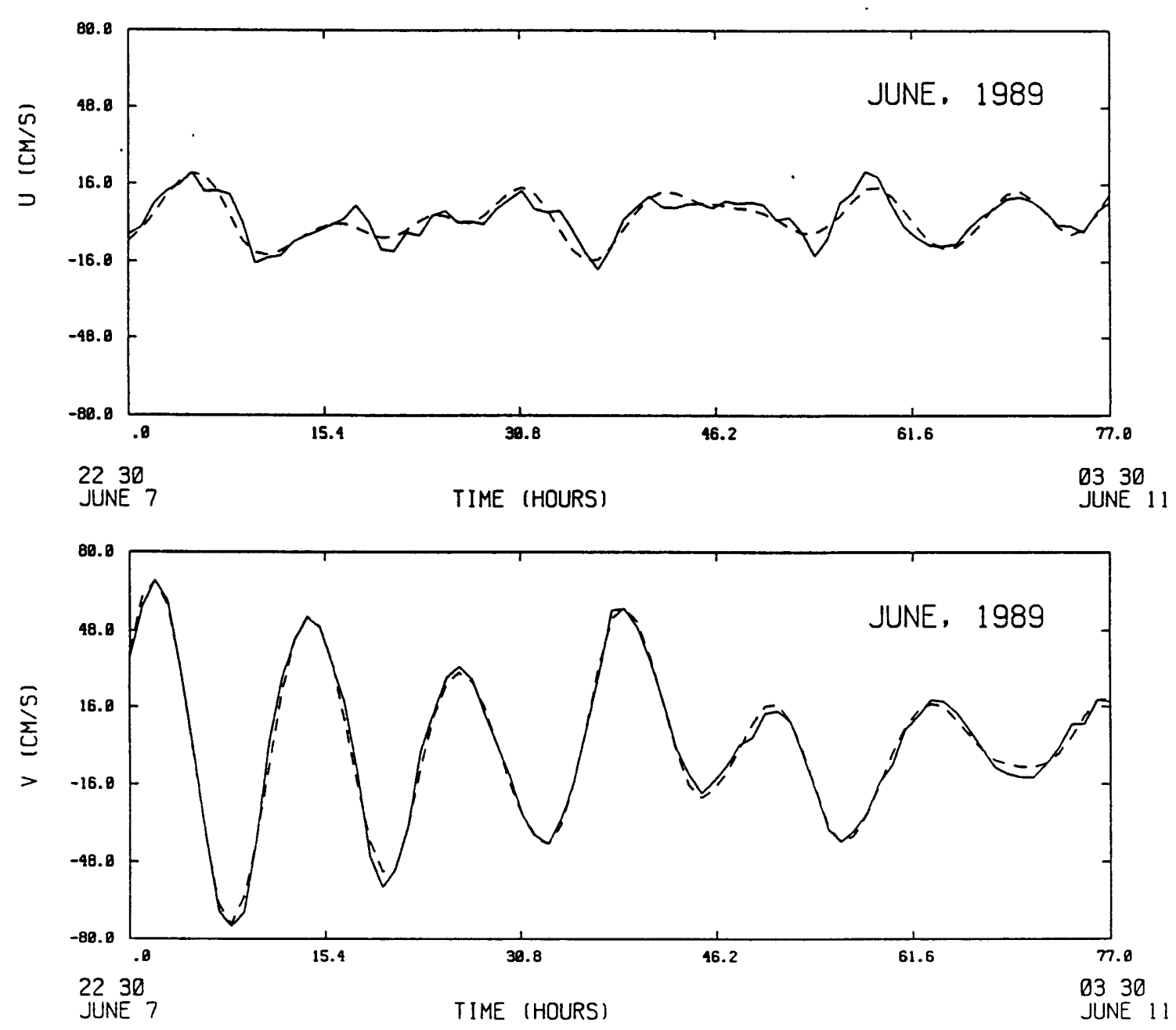

Figure 2.24: Comparison of observed and predicted vertically-averaged ADCP velocity $u$ (upper) and $v$ (lower) for the June, $1989 \mathrm{CTD} / \mathrm{ADCP}$ survey. The solid line represents the one-hour averaged ADCP velocity while the dashed line represents the predicted value using the least squares fit. 


\subsection{Structure of Tidal Currents in the Northern GSC}

As discussed before, whether the least squares fit for the ship-mounted ADCP data is effective or not critically depends on how accurate the tidal fitting is. To check the tidal fitting, however, one needs to find out the tidal constants (amplitude and phase) for a single tidal component. Otherwise, it is impossible to compare the tidal fitting results with observations because of temporal variability caused by the superposition of different tidal constituents. In the case of the 1988 and 1989 SCOPEX CTD/ADCP surveys, the semidiurnal tidal current was fitted based on the $M_{2}$ frequency plus a linearly time-dependent correction due to the combined effect of $N_{2}$ and $S_{2}$, so that it is possible to make an estimate for the $M_{2}$ tide on the assumption that the ratio of $\zeta_{M_{2}}$ to $\hat{\zeta}_{M_{2}}$ is uniform in space. However, it is impossible to separate either $K_{1}$ or $O_{1}$ tidal currents from the model diurnal tidal fit since it was fitted using the average of $K_{1}$ and $O_{1}$ frequencies. For this reason, we will focus our discussion in this section only on the structure of the $M_{2}$ tide.

\section{Cotidal Chart and Tidal Ellipse of $M_{2}$ Tide}

A simple way to find the cotidal chart of the $M_{2}$ tide is to fit the volume transport of the $\mathrm{ADCP}$ velocity profile directly since the tidal elevation is proportional to the convergence or divergence of the tidal current transport. However, the regional April, 1988 and June, 1989 SCOPEX ADCP surveys were conducted along a series of crosschannel legs, which produced a periodic-like distribution of water depth along the ship's track. A direct fit for the total transport mistook such a depth variation as part of the tidal variation so as to create a meaningless tidal and residual current fit. To avoid this problem, we first fitted the vertically averaged tidal and subtidal velocities at each point along the ship's track, and then constructed a single tidal velocity for the $M_{2}$ tide by removing the linear time variation for amplitude and phase from the total semidiurnal tidal fit. Finally, substituting the vertically averaged $M_{2}$ tidal velocity and local water depth 
into the continuity equation, we directly calculated the amplitude and phase of the $M_{2}$ tidal elevation and then constructed a cotidal chart over the survey region.
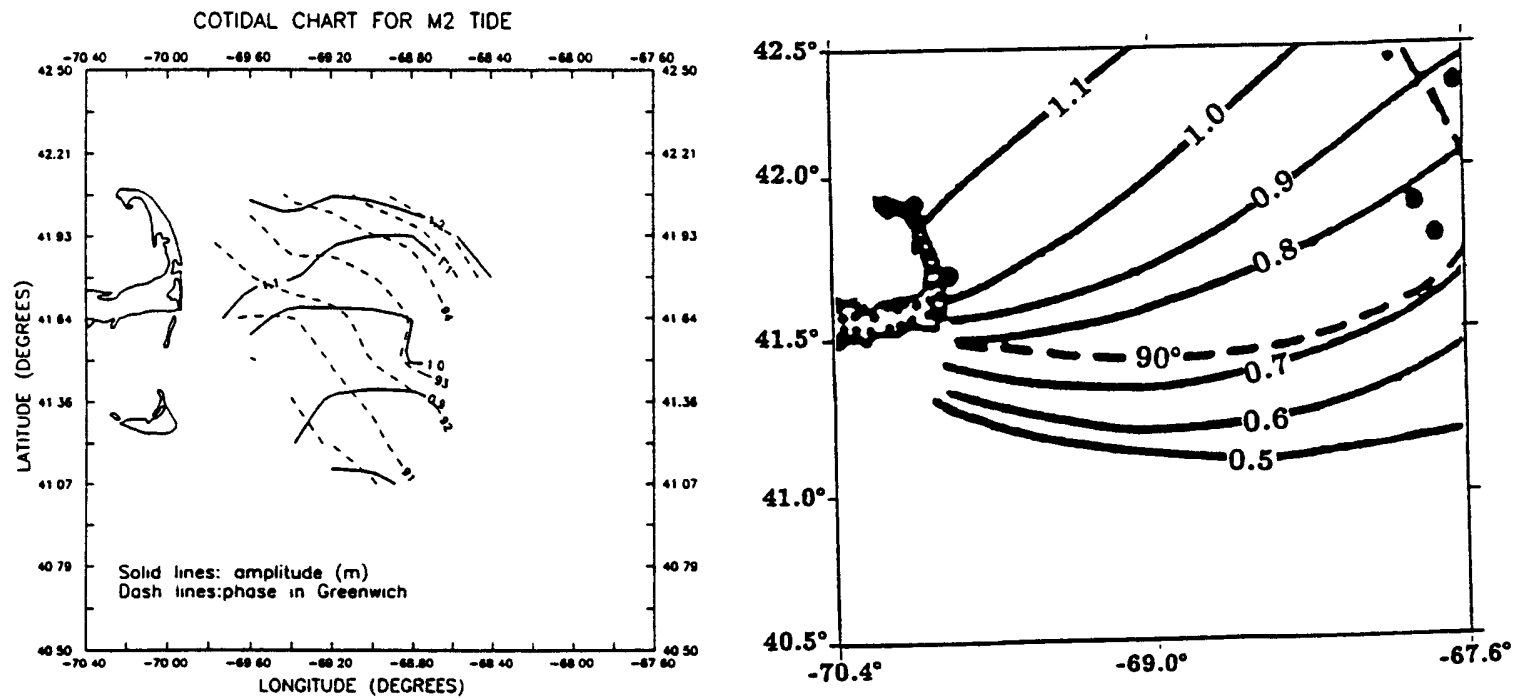

Figure 2.25: Cotidal charts for the $M_{2}$ tidal elevation predicted from the June, 1989 ADCP data (left) and a harmonic analysis (right) done by Moody et al. (1984). The solid and dashed lines represent the contours of amplitude in meters and phase in a degree of Greenwich, respectively, while the black dots in right map indicate the positions of tidal stations used for the harmonic analysis in Moody et al.'s work.

As an example, the cotidal chart for the $M_{2}$ tide obtained from the June, 1989 SCOPEX CTD/ADCP survey is shown in Figure 2.25. The fitting error of the $M_{2}$ tide was estimated according to the uncertainty caused by the inversion of the total semidiurnal tidal elevation to the $M_{2}$ tidal elevation at the six tidal observation stations shown in Figure 2.15. The uncertainty in the cotidal chart of the $M_{2}$ tide constructed from the ADCP data is about $0.2 \mathrm{~m}$ in amplitude and about $4^{\circ}$ in phase. Within the range of these errors, the amplitude and phase of the $M_{2}$ tide increase from $0.8 \mathrm{~m}$ and $90^{\circ}$ near the sill in the southern GSC to about $1.0 \mathrm{~m}$ and $95^{\circ}$ close to Wilkinson Basin. A sharp jump in the 1.0-m contour for the $M_{2}$ tidal amplitude was found on the western side of the survey region in Figure 2.25, and may be caused by a local error in water depth gradient since smoothing of the depth data was carried out only along the ship's track in our fitting. For comparison 
with previous observations, we duplicated the part of Moody et al.'s cotidal chart for the $M_{2}$ tidal elevation in the northern GSC (Moody et al., 1984) that was contoured based on harmonic analysis of bottom pressure at several stations near Cape Cod, Nantucket Shoals and Georges Bank. Although there is low resolution in Moody et al.'s map, it still seems to agree well with our result to the extent of uncertainty of the estimated $M_{2}$ tidal transport.

Table 2.1: Comparison of $M_{2}$ Tidal Elevations

\begin{tabular}{|c|c|c|c|c|}
\hline \multirow{2}{*}{ Stations } & \multicolumn{2}{|c|}{ Bottom Pressure } & \multicolumn{2}{c|}{ ADCP Fitting } \\
\cline { 2 - 5 } & Amp (m) & Phase $\left(^{\circ}\right)$ & Amp (m) & Phase ( $\left.{ }^{\circ}\right)$ \\
\hline Nauset & 1.03 & 102.0 & $1.15 \pm 0.14$ & $91.5 \pm 3.7$ \\
\hline Chatham & 1.05 & 85.0 & $1.11 \pm 0.13$ & $90.0 \pm 3.6$ \\
\hline
\end{tabular}

Table 2.1 shows the comparison of tidal constants between the bottom pressure and the ADCP analyses at the Nauset and Chatham stations, which are located on the east coast of Cape Cod, about $10 \mathrm{~km}$ away from our survey region. Good agreement is found in amplitude and phase within a range of tidal transport errors between these two different measurements except for a phase difference of $10^{\circ}$ at Nauset. The relatively large phase difference at Nauset was probably due to the spatial separation between tidal and ADCP stations and unknown errors in the bottom pressure calculation. Since the contribution of local stratification and surface pressure to the surface tidal elevation, which may be significant in late spring, was not taken into account in the bottom pressure analysis, the uncertainty in the estimate of the $M_{2}$ tidal elevation may be relatively large.

More comparisons with the results of harmonic analysis are made in Table 2.2 to check the quality of the ADCP tidal fitting. There are three current meter stations covered by the regional SCOPEX CTD/ADCP surveys; they are located respectively at A: $68^{\circ} 49^{\prime} \mathrm{W}$, $40^{\circ} 51^{\prime} \mathrm{N}, \mathrm{B}: 69^{\circ} 01^{\prime} \mathrm{W}, 40^{\circ} 51^{\prime} \mathrm{N}$, and $\mathrm{C}: 69^{\circ} 07^{\prime} \mathrm{W}, 42^{\circ} 21^{\prime} \mathrm{N}$. The maximum differences between ellipse parameters for harmonic method and ADCP treatment are about $10 \mathrm{~cm} / \mathrm{s}$ in major 
axis at station $A, 14 \mathrm{~cm} / \mathrm{s}$ in minor axis and $7^{\circ}$ at station $\mathrm{B}$, and $11^{\circ}$ in orientation at station C. If we consider all tidal parameters in Table 2.2, the mean difference between these two analyses is about $9 \mathrm{~cm} / \mathrm{s}$ in major and minor axes, $3.5^{\circ}$ in phase, and $6^{\circ}$ in orientation. Such differences in tidal parameters are most likely due to variability in the $M_{2}$ tidal currents caused by internal tides in late spring since no attempts were made to estimate or filter the internal tides from the calculation of tidal currents in both harmonic method and ADCP treatment. In this sense, we conclude here that the $M_{2}$ tidal fitting from the ADCP data at each depth of data records coincide well with previous current meter measurements within the range of estimated errors due to internal tidal variability and inversion of $M_{2}$ tide from the total fitted semidiurnal tide.

\section{Vertical Structure of the $M_{2}$ Tidal Current}

Small-scale biological surveys were made in late May to early June, 1989 following feeding whales in the western GOM. These surveys provided at least 20 hour-long ADCP data sets with a vertical resolution of $4 \mathrm{~m}$ at five stations along the $100-\mathrm{m}$ isobath in the northern GSC (Figure 2.26). The least squares method described in section 2.3 was applied to fit the ADCP data profile at each station assuming spatial uniformity along the ship's track there. The correlation coefficient of the fitted current to the raw ADCP data is 0.99 for both $U$ and $V$ components except at station 4 where a strongly resonant-like signal was found in the middle of the survey time (see Figure 2.27). Based on the fitted tidal currents, we first calculated the profile of the $M_{2}$ tidal ellipse at each time series stations, and then compared these results with those obtained from the June, 1989 ADCP survey to look for temporal variability of vertical structure of the $M_{2}$ currents in the late spring, 1989.

The three-dimensional, depth-rotation and magnitude of the $M_{2}$ tidal current at a specific time at the five stations are shown in Figure 2.28. Tidal currents not only changed the direction of rotation one or two times in the vertical at each station but also behaved very differently from one to another. As a result, tidal currents tended to increase 
Table 2.2: Comparison of $M_{2}$ tidal current parameters between the harmonic method and ADCP treatment

\begin{tabular}{|c|c|c|c|c|c|c|c|c|c|c|c|c|}
\hline \multirow[b]{2}{*}{ Location } & \multicolumn{6}{|c|}{ Harmonic method } & \multicolumn{6}{|c|}{ ADCP treatment } \\
\hline & $\begin{array}{l}\text { Instr } \\
\text { depth } \\
\text { (m) }\end{array}$ & $\begin{array}{l}\text { water } \\
\text { depth } \\
(\mathrm{m})\end{array}$ & $\begin{array}{l}\text { umajor } \\
(\mathrm{cm} / \mathrm{s})\end{array}$ & $\begin{array}{l}\text { uminor } \\
(\mathrm{cm} / \mathrm{s})\end{array}$ & $\begin{array}{l}\text { phase } \\
\text { (Deg-G) }\end{array}$ & $\begin{array}{l}\text { orien } \\
\text { (Deg) }\end{array}$ & $\begin{array}{c}\text { instr } \\
\text { depth } \\
\text { (m) }\end{array}$ & $\begin{array}{c}\text { water } \\
\text { depth } \\
(\mathrm{m})\end{array}$ & $\begin{array}{l}\text { umajor } \\
(\mathrm{cm} / \mathrm{s})\end{array}$ & $\begin{array}{l}\text { uminor } \\
(\mathrm{cm} / \mathrm{s})\end{array}$ & $\begin{array}{l}\text { phase } \\
\text { (Deg-G) }\end{array}$ & $\begin{array}{l}\text { orien } \\
\text { (Deg) }\end{array}$ \\
\hline \multirow{2}{*}{$\begin{array}{l}40^{\circ} 51^{\prime} \mathrm{N} \\
68^{\circ} 49^{\prime} \mathrm{W}\end{array}$} & 10.0 & 66.0 & $73.2 \pm 3.1$ & $-28.9 \pm 1.8$ & $36.7 \pm 2.0$ & $-86.7 \pm 2.0$ & 11.0 & 66.0 & $75.2 \pm 6.2$ & $-15.7 \pm 1.5$ & $34.9 \pm 0.8$ & $-79.6 \pm 4.4$ \\
\hline & 51.0 & 66.0 & $59.6 \pm 2.0$ & $-21.8 \pm 1.7$ & $29.4 \pm 1.0$ & $-88.3 \pm 1.0$ & 51.0 & 66.0 & $69.9 \pm 5.6$ & $-27.9 \pm 2.7$ & $29.8 \pm 0.7$ & $-85.1 \pm 4.7$ \\
\hline \multirow{3}{*}{$\begin{array}{l}40^{\circ} 51^{\prime} \mathrm{N} \\
69^{\circ} 01^{\prime} \mathrm{W}\end{array}$} & 10.0 & 83.0 & 71.5 & -26.6 & 43.8 & -79.8 & 11.0 & 83.0 & $73.2 \pm 5.9$ & $-12.7 \pm 1.2$ & $37.0 \pm 0.9$ & $-81.8 \pm 4.5$ \\
\hline & 32.0 & 83.0 & 70.1 & -25.9 & 40.4 & -80.9 & 31.0 & 83.0 & $79.3 \pm 6.3$ & $-16.4 \pm 1.6$ & $33.5 \pm 0.8$ & $-83.6 \pm 4.6$ \\
\hline & 68.0 & 83.0 & $57.1 \pm 0.9$ & $-19.3 \pm 0.6$ & $34.8 \pm 1.0$ & $-79.0 \pm 1.0$ & 67.0 & 83.0 & $52.6 \pm 4.2$ & $-27.7 \pm 2.6$ & $29.6 \pm 2.6$ & $-78.7 \pm 1.8$ \\
\hline \multirow{2}{*}{$\begin{array}{l}40^{\circ} 52^{\prime} \mathrm{N} \\
69^{\circ} 07^{\prime} \mathrm{W}\end{array}$} & 27.0 & 64.0 & 62.9 & -20.5 & 43.4 & -70.4 & 27.0 & 64.0 & $69.3 \pm 5.5$ & $-17.1 \pm 1.6$ & $40.2 \pm 0.9$ & $-81.2 \pm 4.5$ \\
\hline & 49.0 & 64.0 & 53.0 & -17.4 & 41.7 & -65.1 & 51.0 & 64.0 & $57.7 \pm 4.6$ & $-24.8 \pm 2.4$ & $36.5 \pm 0.8$ & $-76.3 \pm 0.9$ \\
\hline
\end{tabular}

Note: Umajor-amplitude of the major axis; Uminor-amplitude of the minor axis; Phase-time of maximum current (Deg-G: Greenwich phase in degrees); Orien-orientation of the major axis. The orientation is measured counterclockwise from east. The negative sign in Uminor indicates a clockwise rotation of current vector. Otherwise, current vector rotates counterclockwise. 


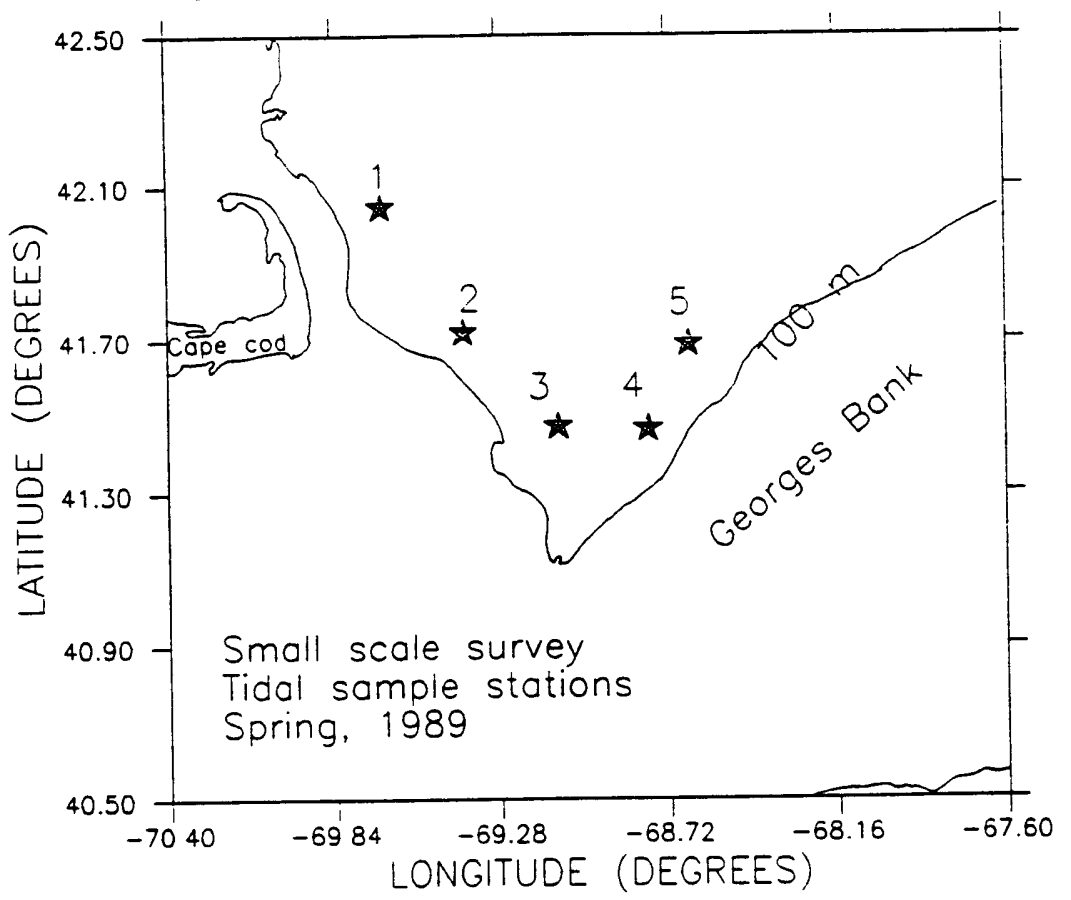

Figure 2.26: Positions of the ADCP stations for the local biological survey taken from May 22 to June 5, 1989. The stars represent the stations of the small-scale ADCP measurements which are numbered 1 to 5 from west to east.

in magnitude with depth and reached the maximum at a depth of about $100 \mathrm{~m}$ near the bottom. Maas and Zimmerman (1989a and $b$ ) analytically studied tidally driven residual flow over variable bottom topography and found that stratification can cause an internal tide, which is generated through the propagation of a barotropic tidal wave over a variable bottom, to be trapped at the bottom. The small-scale SCOPEX biological survey was made in May-June when the stratification became stronger due to increasing surface solar heating: the observed bottom-trapped structure of $M_{2}$ tidal currents at the five stations is most likely due to the combined effects of stratification and topography.

The vertical structure of the $M_{2}$ tidal ellipse for the May-June, small-scale, biological ADCP survey is shown in Figure 2.29 (solid lines) to compare with that (dashed lines) for the June, 1989 regional CTD/ADCP survey. The tidal ellipse parameters of the $M_{2}$ tidal current, such as major axis, minor axis, phase and orientation, exhibited clearly the vertical modes of the tidal current at each station for these two surveys, implying the 

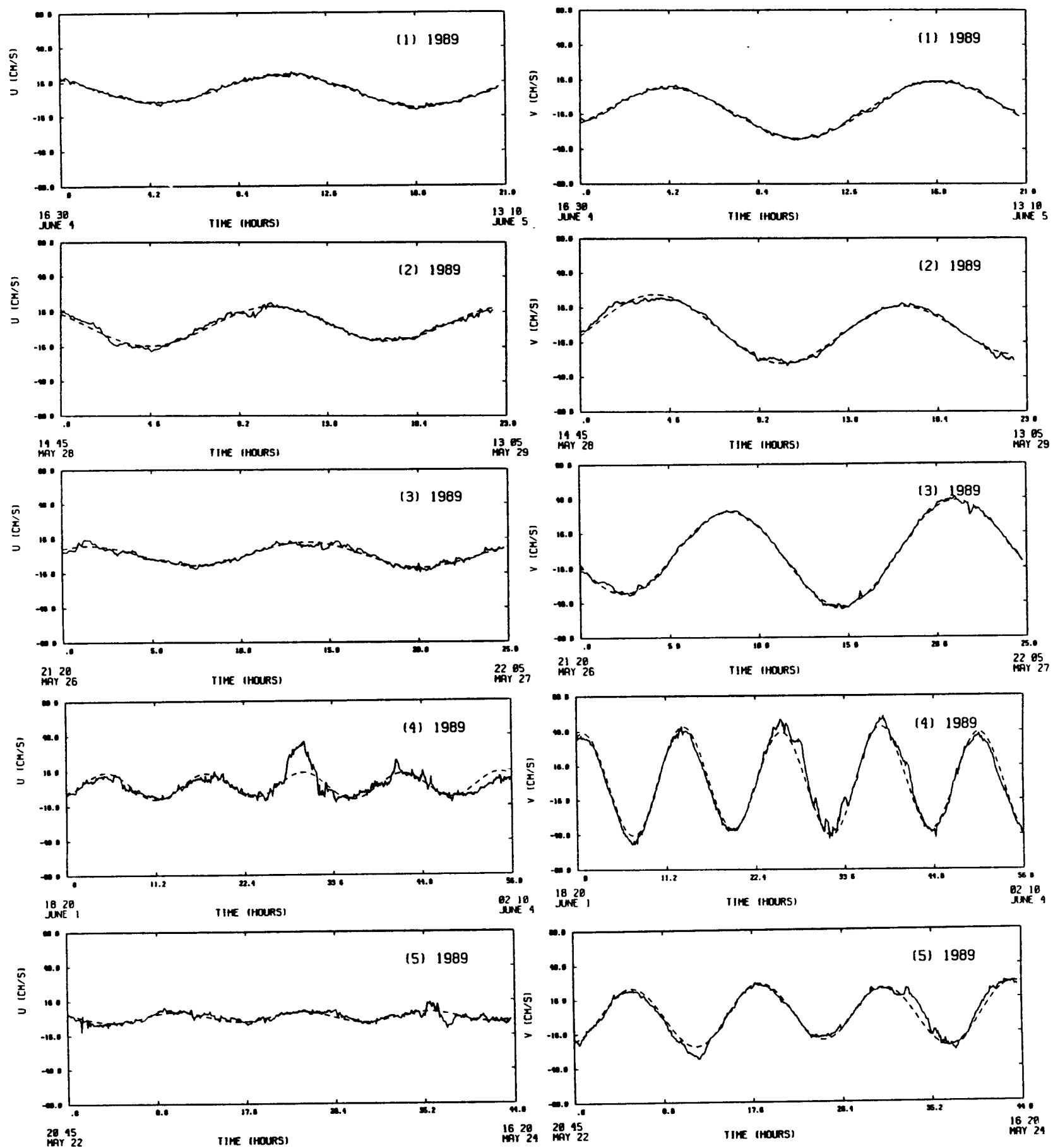

Figure 2.27: Comparisons between observed (solid line) and predicted (dashed line) velocities ( $u$ and $v$ ). A least squares method with a linearly time-dependent correction for the effect of $N_{2}$ and $S_{2}$ was used to fit the raw 5-min ADCP data. Stations 1 to 5 are arranged from top to bottom, while the comparison for $u$ velocity components is put at left and comparison for $v$ velocity component is at right. 


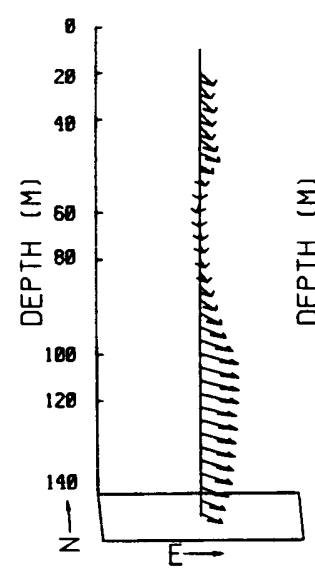

(1)

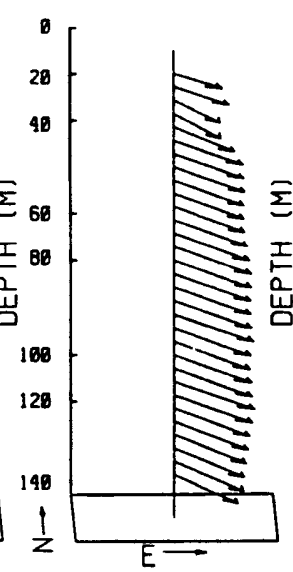

(2)

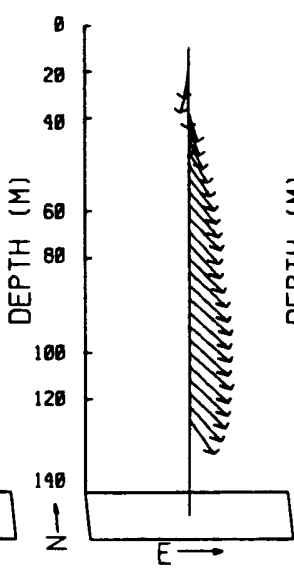

(3)

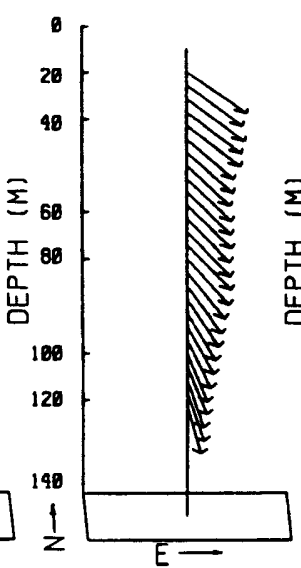

(4)

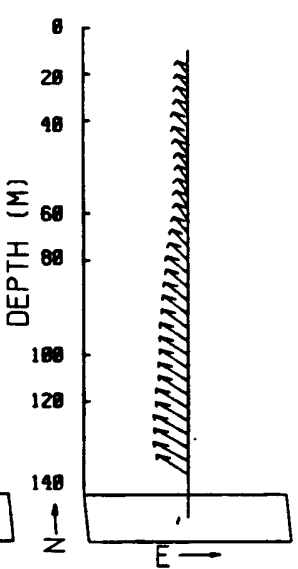

(5)
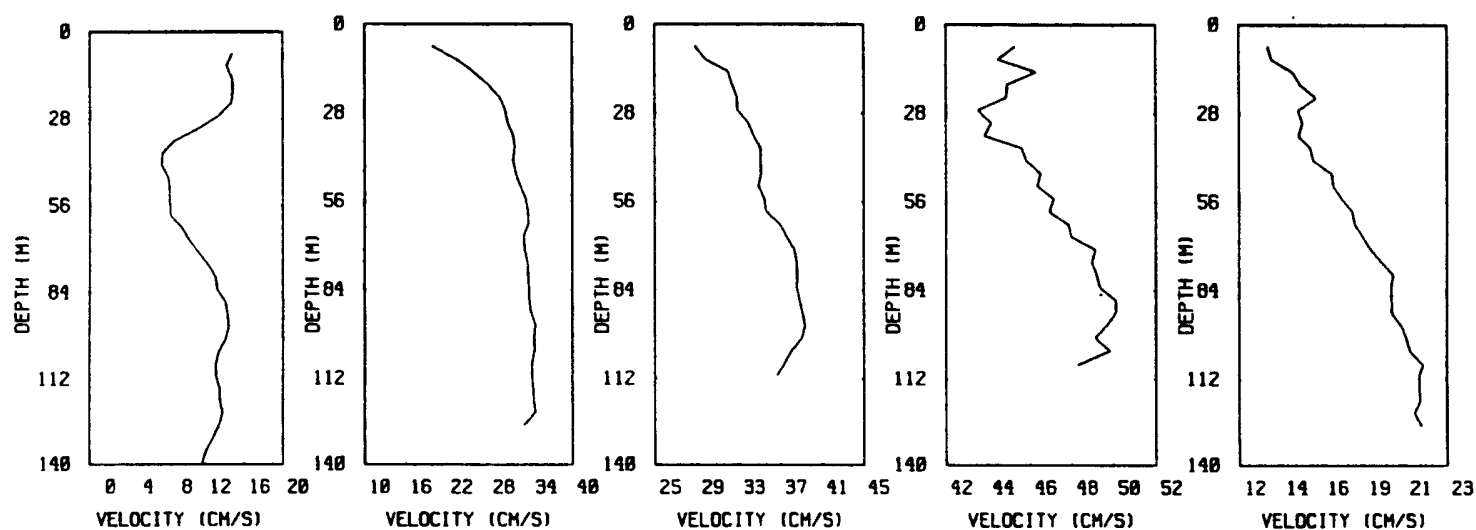

Figure 2.28: Three-dimensional depth-rotation (upper) and magnitude (lower) of the $M_{2}$ tidal currents for a specified time $T=0$. The station numbers are represented under rotation picture of velocity vector from west to east. 

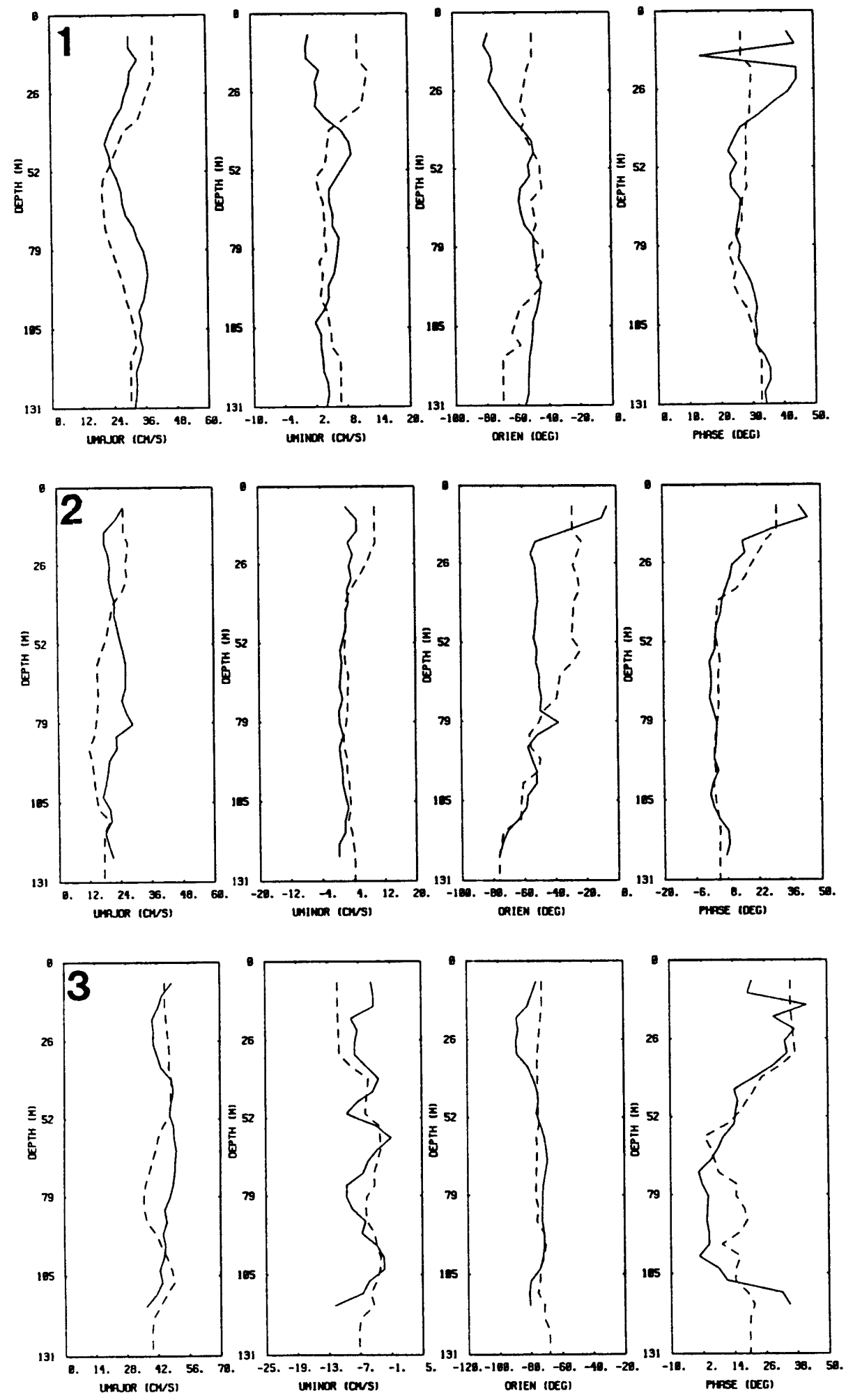

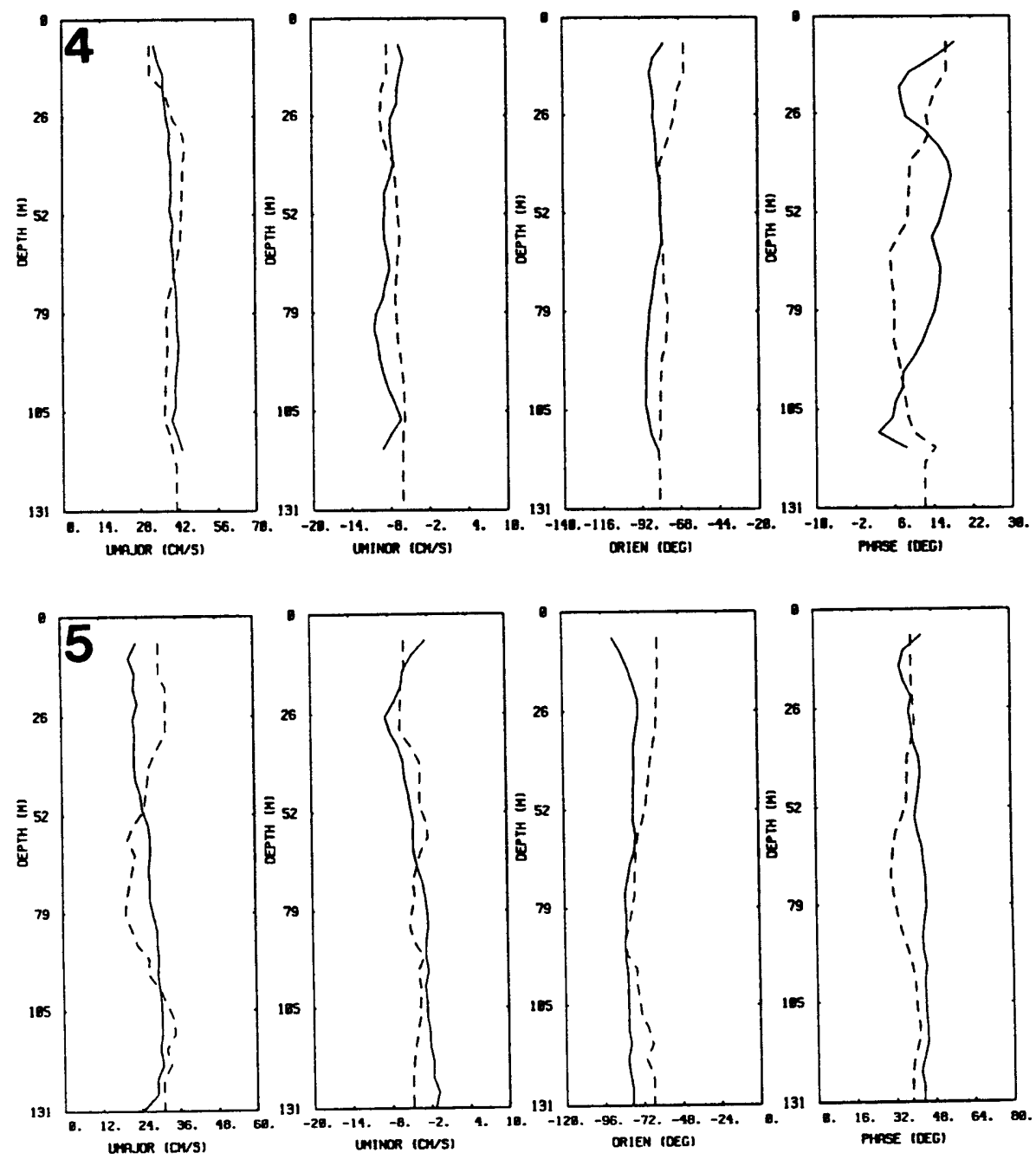

Figure 2.29: Comparisons between the tidal ellipse parameters obtained from the May-June small-scale biological (solid lines) and June regional (dashed lines) ADCP surveys. Stations 1-5 are numbered at the upper left corner in each picture. Note: a $10 \mathrm{~m}$ smoothing was made on the tidal parameters obtained from the June, 1989 regional ADCP in fit to filter the small-scale "noise" in the vertical distribution of fitted tidal current. 
existence of an internal tide at the $M_{2}$ frequency in the results of the $M_{2}$ tidal fitting. Therefore, the comparison of tidal parameters from the two surveys may be complicated by any temporal variability of the internal tides. In spite of this, we can still see that the tendencies of tidal parameters were very similar to each other at the five stations and that the temporal variation of the tidal ellipse from late May (start time for the biological survey) to mid-June (end time for the regional survey) was only about $10 \%$ of the total value except at station 1 where a large difference was found in the upper $40 \mathrm{~m}$ in minor axis, orientation, and phase. If these variations are all attributed to the temporal variability of internal tides, then the fitting uncertainty for the barotropic $M_{2}$ tidal current from the ADCP data is about $10 \%$. This means that the barotropic component of the $M_{2}$ tidal current is still dominant in the northern GSC in late spring regardless of the existence of strong stratification at that time.

An effort was also made to filter the internal tide from the fitted $M_{2}$ tidal current by vertically averaging over the whole water column for both small-scale and regional ADCP surveys. Good agreement was found in the vertically averaged ellipse parameters between these two surveys in which the difference in tidal ellipses was greatly reduced to $1-3 \%$ of the total vertically averaged value (see Table 2.3 ). On the one hand, this result implies a weak nonlinear interaction between internal and barotropic tidal currents during these two SCOPEX surveys, and on the other hand, it demonstrates again the effectiveness of the least squares fit of empirical formula (2.1) for the separation of tidal and residual flow in ship-mounted ADCP data in the GSC region.

The tidal ellipses of the vertically averaged $M_{2}$ tide obtained from the May-June, 1989 small-scale biological survey are plotted in Figure 2.30. On the western side of the northern GSC between $41.7^{\circ} \mathrm{N}$ and $42.1^{\circ} \mathrm{N}$, the orientation of the $M_{2}$ tidal ellipse was approximately parallel to the local isobath and the current rotated counterclockwise in a tidal cycle. On the eastern side of the northern GSC, however, the $M_{2}$ tidal current tended to cross the isobaths and rotate clockwise in a tidal cycle. As long as these five 
Table 2.3: Comparison of $M_{2}$ tidal current parameters between the small and large scale ADCP surveys

\begin{tabular}{|c|c|c|c|c|c|c|c|c|}
\hline \multirow[b]{2}{*}{ Location } & \multicolumn{4}{|c|}{ Small scale survey } & \multicolumn{4}{|c|}{ Large scale survey } \\
\hline & $\begin{array}{l}\text { Umajor } \\
(\mathrm{cm} / \mathrm{s})\end{array}$ & $\begin{array}{l}\text { Uminor } \\
(\mathrm{cm} / \mathrm{s})\end{array}$ & $\begin{array}{l}\text { Phase } \\
\text { (Deg-G) }\end{array}$ & $\begin{array}{l}\text { Orien } \\
\text { (Deg) }\end{array}$ & $\begin{array}{l}\text { Umajor } \\
(\mathrm{cm} / \mathrm{s})\end{array}$ & $\begin{array}{l}\text { Uminor } \\
(\mathrm{cm} / \mathrm{s})\end{array}$ & $\begin{array}{l}\text { Phase } \\
\text { (Deg-G) }\end{array}$ & $\begin{array}{l}\text { Orien } \\
\text { (Deg) }\end{array}$ \\
\hline $42^{\circ} 03^{\prime} \mathrm{N}, 69^{\circ} 41^{\prime} \mathrm{W}$ & $30.48 \pm 1.07$ & $4.36 \pm 0.72$ & $30.79 \pm 0.62$ & $-57.24 \pm 0.79$ & $28.97 \pm 1.65$ & $5.75 \pm 0.55$ & $29.90 \pm 0.69$ & $-53.27 \pm 2.93$ \\
\hline $41^{\circ} 28^{\prime} \mathrm{N}, 69^{\circ} 05^{\prime} \mathrm{W}$ & $45.36 \pm 1.59$ & $-5.95 \pm 0.98$ & $16.51 \pm 0.33$ & $-76.55 \pm 0.92$ & $42.85 \pm 2.44$ & $-5.92 \pm 0.57$ & $19.41 \pm 0.45$ & $-73.24 \pm 4.03$ \\
\hline $41^{\circ} 28^{\prime} \mathrm{N}, 68^{\circ} 47^{\prime} \mathrm{W}$ & $39.43 \pm 1.38$ & $-8.21 \pm 1.35$ & $12.17 \pm 0.24$ & $-84.27 \pm 1.01$ & $38.98 \pm 2.22$ & $-7.22 \pm 0.69$ & $10.56 \pm 0.24$ & $-76.28 \pm 4.20$ \\
\hline $41^{\circ} 41^{\prime} \mathrm{N} 68^{\circ} 39^{\prime} \mathrm{W}$ & $26.46 \pm 0.93$ & $-3.86 \pm 0.64$ & $42.20 \pm 0.84$ & $-79.44 \pm 0.95$ & $27.57 \pm 1.57$ & $-4.36 \pm 0.42$ & $37.34 \pm 0.86$ & $-70.45 \pm 3.87$ \\
\hline
\end{tabular}

Note: Umajor-amplitude of the major axis; Uminor-amplitude of the minor axis; Phase-time of maximum current (Deg-G: Greenwich phase in degrees); Orien-orientation of the major axis. The orientation is measured counterclockwise from east. The negative sign in Uminor indicates a clockwise rotation of current vector. Otherwise, current vector rotates counterclockwise. 


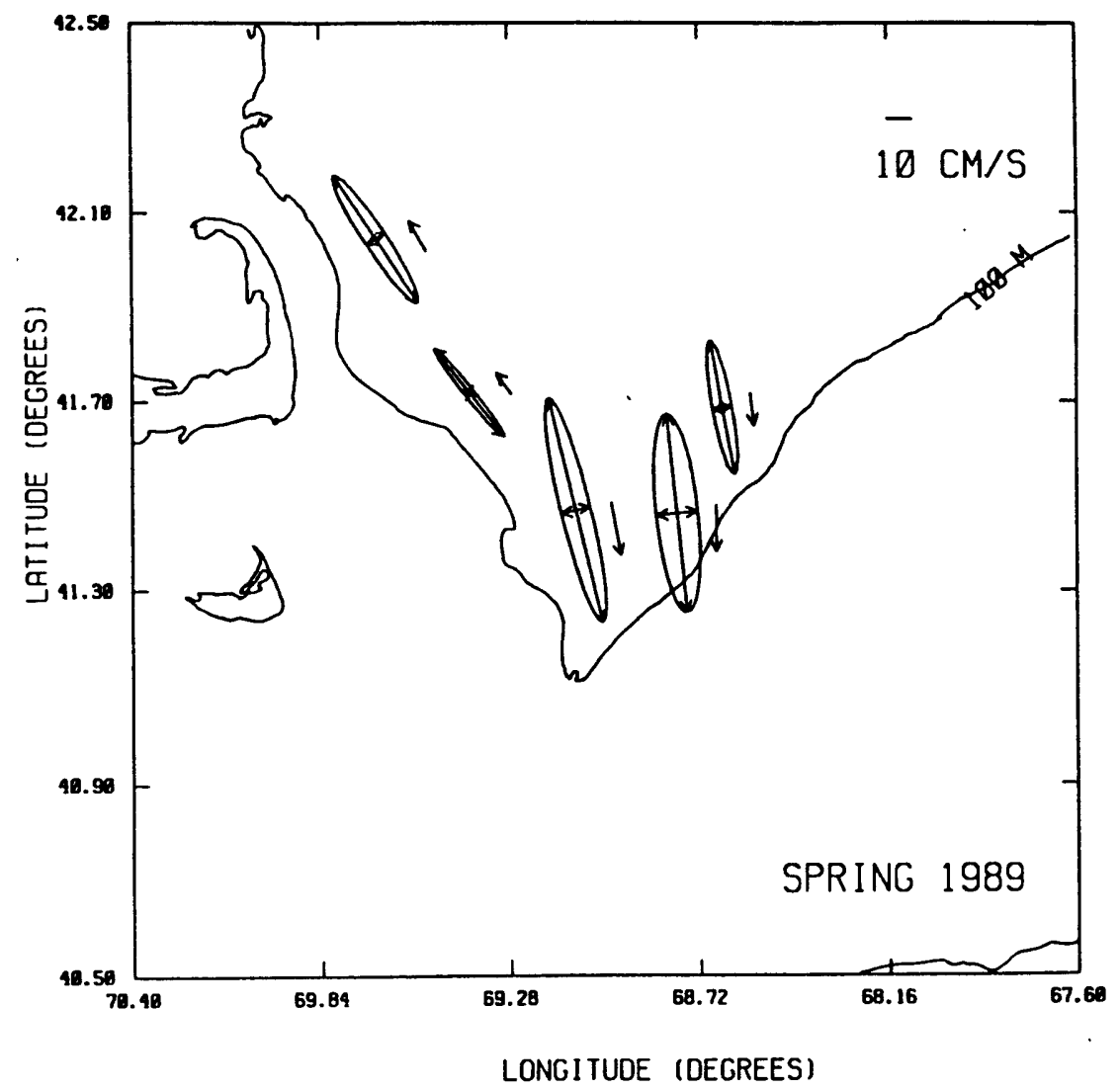

Figure 2.30: Tidal ellipse of the $M_{2}$ current at each station from number 1 (west) to 5 (east). The solid curve in the map is the $100-\mathrm{m}$ isobath, and velocity scale is shown at the upper right corner of this map.

stations were concerned, the maximum tidal current was located in the middle of the GSC at station 3, where the major axis of the $M_{2}$ tidal ellipse was about $45 \mathrm{~cm} / \mathrm{s}$ and oriented about $13^{\circ}$ counterclockwise from North. These pictures are consistent with the results of harmonic analysis done by Moody et al. (1984). 


\subsection{Structure of Residual Flow in the Northern GSC}

In principle, the residual flow can be directly obtained by subtracting the fitted tidal currents from the ADCP currents since good tidal fitting implies a weak nonlinear interaction between the tidal and subtidal currents. However, the residual flow so obtained may not provide a good snapshot of the Eulerian subtidal current field since the "raw" residual flow contains both the temporal and spatial subtidal current variability and measurement noise. For this reason, a spatial polynomial has been applied in this section to refit the subtidal current again to resolve the steady structure of residual flow with a $95 \%$ confidence level in a statistical sense. Comparison has been made between the 1988 and 1989 residual flows to study the inter-annual variability of mean flow in the northern GSC in late spring. Also the fitted residual flow has been compared with the satellite-tracked drifter data at 5 and $50 \mathrm{~m}$ to investigate the mean circulation patterns at surface and mid-depth in this region. Results of these comparisons will be presented in this section.

\section{Vertically Averaged Structure of Residual Flow in the Northern GSC}

The lower panel of Figure 2.31 shows the vertically averaged subtidal residual current vectors obtained directly by subtracting the fitted semidiurnal and diurnal tidal currents from the June, 1989 ADCP data. The time resolution of the residual velocity was chosen as one-hour, which was determined to be the independent time scale of the residual current time series. Although this picture appears to be a little messy, it basically shows a cyclonic circulation pattern in the northern GSC where a strong coastal current flowed southeastward along the local topography east of Cape Cod with a speed of order $10 \mathrm{~cm} / \mathrm{s}$ or more and then turned northeastward on the western flank of Georges Bank (see the trajectories of drifters drogued at the 5 and $50 \mathrm{~m}$ in Figures 2.42-2.45). Relatively weak currents of order $3-4 \mathrm{~cm} / \mathrm{s}$ were observed north of $41.5^{\circ} \mathrm{N}$ in the middle of the northern GSC where the water depth is deeper than $100 \mathrm{~m}$. Recirculation around Georges Bank is 

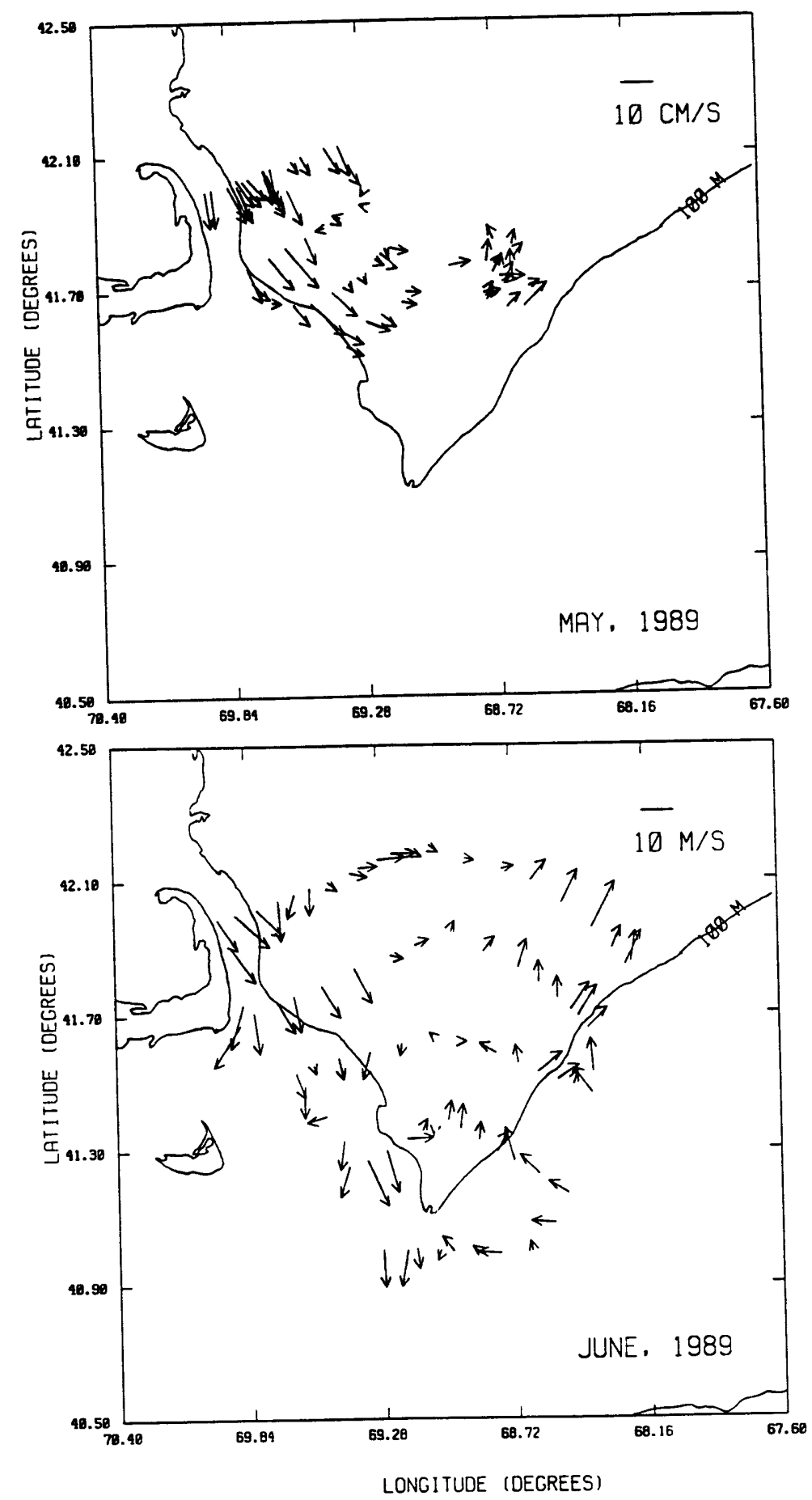

Figure 2.31: Vertically averaged residual current vectors obtained directly by subtracting the fitted semidiurnal and diurnal tidal currents from the May (upper), and June (lower), 1989 ADCP data. Current vectors are in centimeters per second and the velocity scale is shown near the top-right corner. 
also seen at the southeast corner of the survey area where continuous currents were found to flow into the GSC from the southwest flank of Georges Bank.

In order to resolve the mean circulation pattern better during this survey, we have taken these residual currents as a "raw" data set and then used the spatial polynomial to refit it with the least squares method. The $F$-test showed a best fit for the "raw" residual flow with a second order polynomial, in which the correlation between the "raw" and fitted residual flow was found to be 0.65 for $u$ and 0.91 for $v$,and the standard deviation of this fitting with the degrees of freedom $(N-r)$ was equal as $3.7 \mathrm{~cm} / \mathrm{s}$ for $u$ and $3.3 \mathrm{~cm} / \mathrm{s}$ for $v$. Direct comparison was made between the "raw" and fitted residual velocities in Figure 2.32 which shows a good fit in both $u$ and $v$ along the ship's track except for the unfitted relatively high frequency variation. The field of fitted mean current vectors is shown in Figure 2.34 (upper), and the uncertainty in the fitted residual flow at a $95 \%$ confidence level is given in Figure 2.34 (lower) according to the formula (2.14) given in section 2.3. The fitting errors were distributed as a function of space along the ship's track during the June, 1989 survey; they were equal to $\pm 3.0--3.5 \mathrm{~cm} / \mathrm{s}$ in $u$ and $\pm 2.5--3.0 \mathrm{~cm} / \mathrm{s}$ in $v$ in the interior region of the ADCP survey, but about $4.0 \mathrm{~cm} / \mathrm{s}$ for both $u$ and $v$ just east of Cape Cod, in the southern GSC less than $70 \mathrm{~m}$, and at the northeast corner of the survey area where the residual flow was in general much stronger. The field of mean residual flow clearly shows a cyclonic circulation pattern consistent with the satellite-tracked drifter trajectories in the northern GSC within uncertainties of fitting errors. The vertically averaged water exchange between the GOM and the outer shelf was confined to the shallow region where GOM water was found to flow out along the western flank of the GSC at a speed of over $10 \pm 4.0 \mathrm{~cm} / \mathrm{s}$ and Georges Bank water flowed over the eastern flank of the GSC at a speed of about $6.0 \pm 3.5 \mathrm{~cm} / \mathrm{s}$.

The magnitude of fitting errors defined as $\sqrt{\Delta u^{2}+\Delta v^{2}}(\Delta u$ and $\Delta v$ are the fitting errors for $u$ and $v$, respectively) is plotted in Figure 2.35 along the ship's track to compare with the measurement errors found from vertically averaged ADCP data. The 

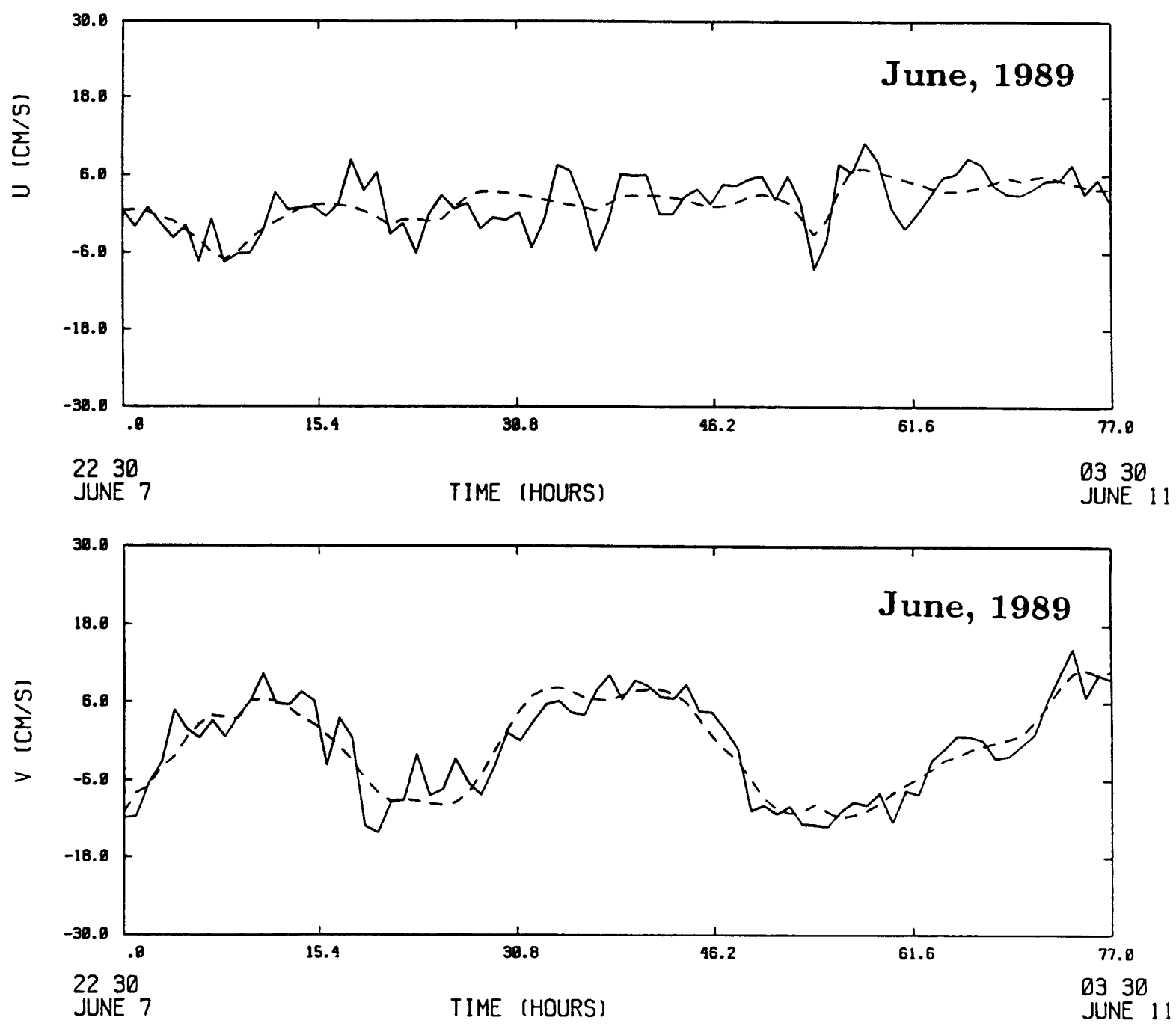

Figure 2.32: Comparison between the "raw" (solid line) and fitted (dashed line) vertically averaged residual current components $u$ (upper) and $v$ (lower) along the ship's track during June 7-11, 1989 CTD/ADCP surveys. The time resolution is one-hour and velocities are in centimeters per second. 
measurement errors of the ADCP data were reduced to $\pm 2--3 \mathrm{~cm} / \mathrm{s}$ after vertical and one-hour averaging, which were almost the same order as the least squares fitting errors. Spectral analysis for the fitting errors $\Delta u$ and $\Delta v$ was done to investigate the energy distribution of unfitted noises over the frequency domain, and hence to find the dominant frequency of temporal variability of the residual currents. However, this analysis did not provide useful information about temporal variability because the spectral density for the unfitted noise was almost uniformly distributed over the whole frequency domain. This is because the temporal variability of the residual flow during the June, 1989 survey was so small and mixed with random measurement noise that we could not resolve it.

ADCP data were also collected along with CTD measurements in the south-central and western flank of the GSC three weeks before the June, 1989 survey. This survey started at 17:45, May 19, at CTD station 9 on CTD section 3 (numbered from south to north) and ended at 15:05, May 22 on the western flank of Georges Bank. The vertically averaged "raw" subtidal current vectors, obtained directly by subtracting the fitted semidiurnal and diurnal tidal currents from the one-hour averaged raw ADCP data, are shown in Figure 2.31 (upper), which also indicates a cyclonic circulation pattern in the northern GSC with a strong southward coastal jet-like flow east of Cape Cod. The best fit for these "raw" subtidal flow data was found using an $F$-test with a third order polynomial, resulting in a correlation of 0.64 for $u$ and 0.93 for $v$ and a standard deviation of $2.2 \mathrm{~cm} / \mathrm{s}$ for $u$ and $2.3 \mathrm{~cm} / \mathrm{s}$ for $v$. The comparison between the "raw" and fitted residual velocities along the ship's track agreed well in magnitude during the survey except for some unfitted peaks near the $100-\mathrm{m}$ isobath on CTD section 4 on the western flank of the GSC (Figure 2.36). The fitted mean current vectors are plotted in Figure 2.33 (upper). The fitting uncertainty for the residual flow at a $95 \%$ confidence level ranged from \pm 1.0 to $\pm 2.5 \mathrm{~cm} / \mathrm{s}$ for both $u$ and $v$, which were in general smaller than those for the June 1989 survey (Figure 2.33, lower). Compared with the residual flow field on June 7-12, 1989, a relatively weaker northeastward flow was found during May 19-21 on the western flank of Georges Bank, even though the magnitudes of the coastal currents over the western flank of the GSC were similar in both 

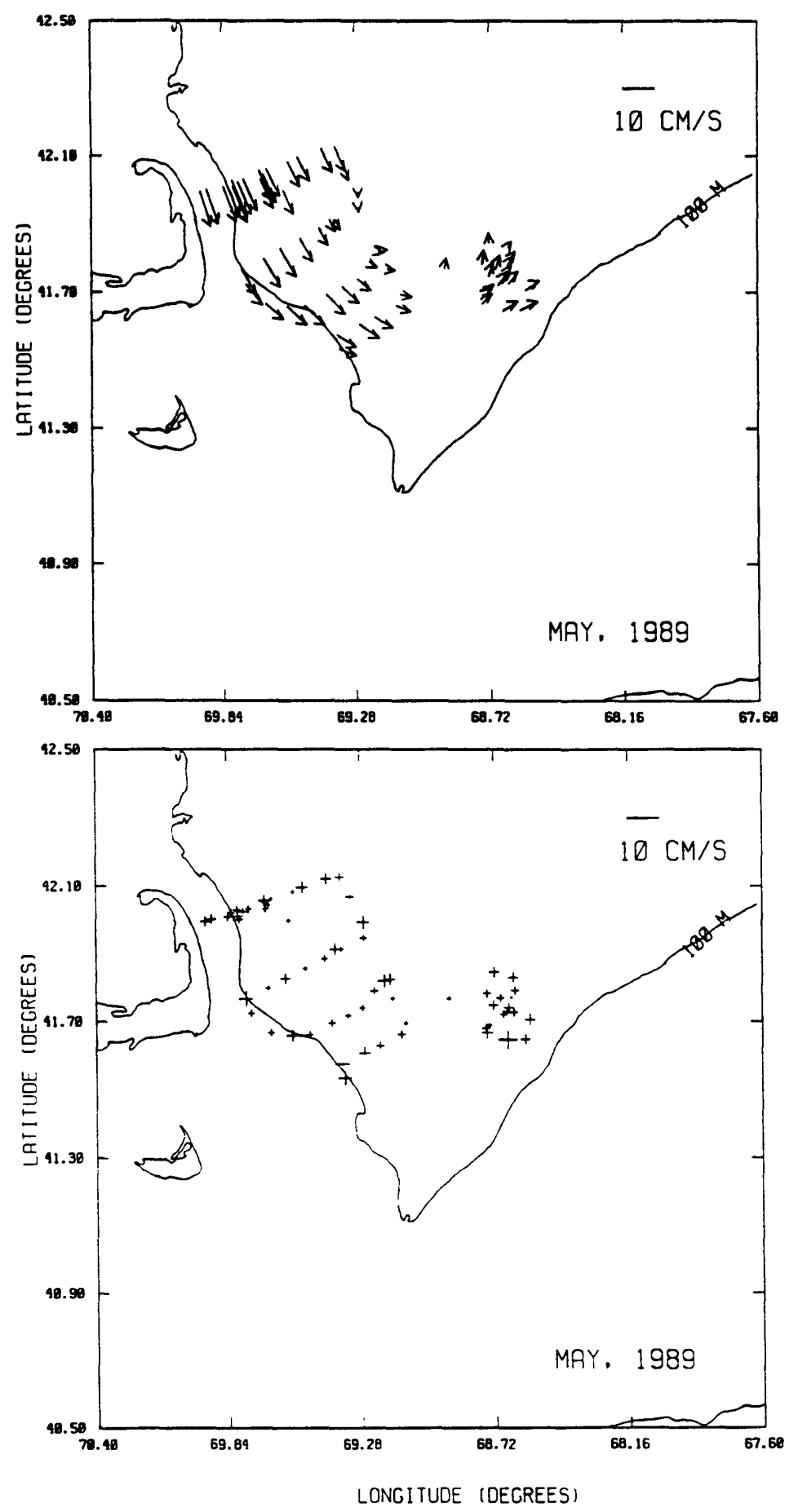

Figure 2.33: Distributions of vertically averaged residual flow (upper) and fitting uncertainty (lower) in the northern GSC during the May 1989 CTD/ADCP survey. The plotting scale for the current vector and fitting uncertainty is in centimeters per second. 

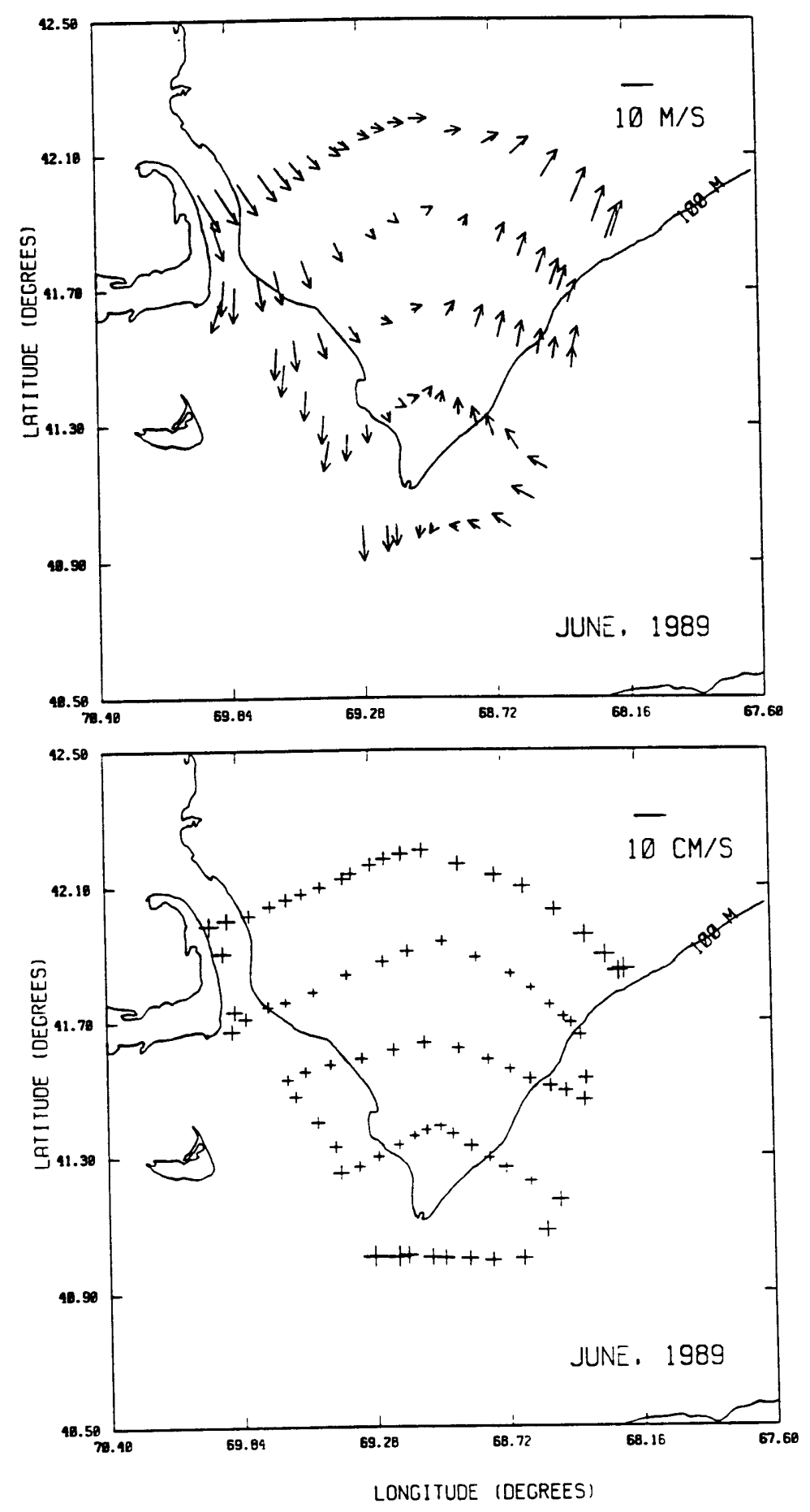

Figure 2.34: Distributions of vertically averaged residual flow (upper) and fitting uncertainty (lower) in the northern GSC during the June 1989 CTD/ADCP survey. The plotting scale for the current vector and fitting uncertainty is in centimeters per second. 


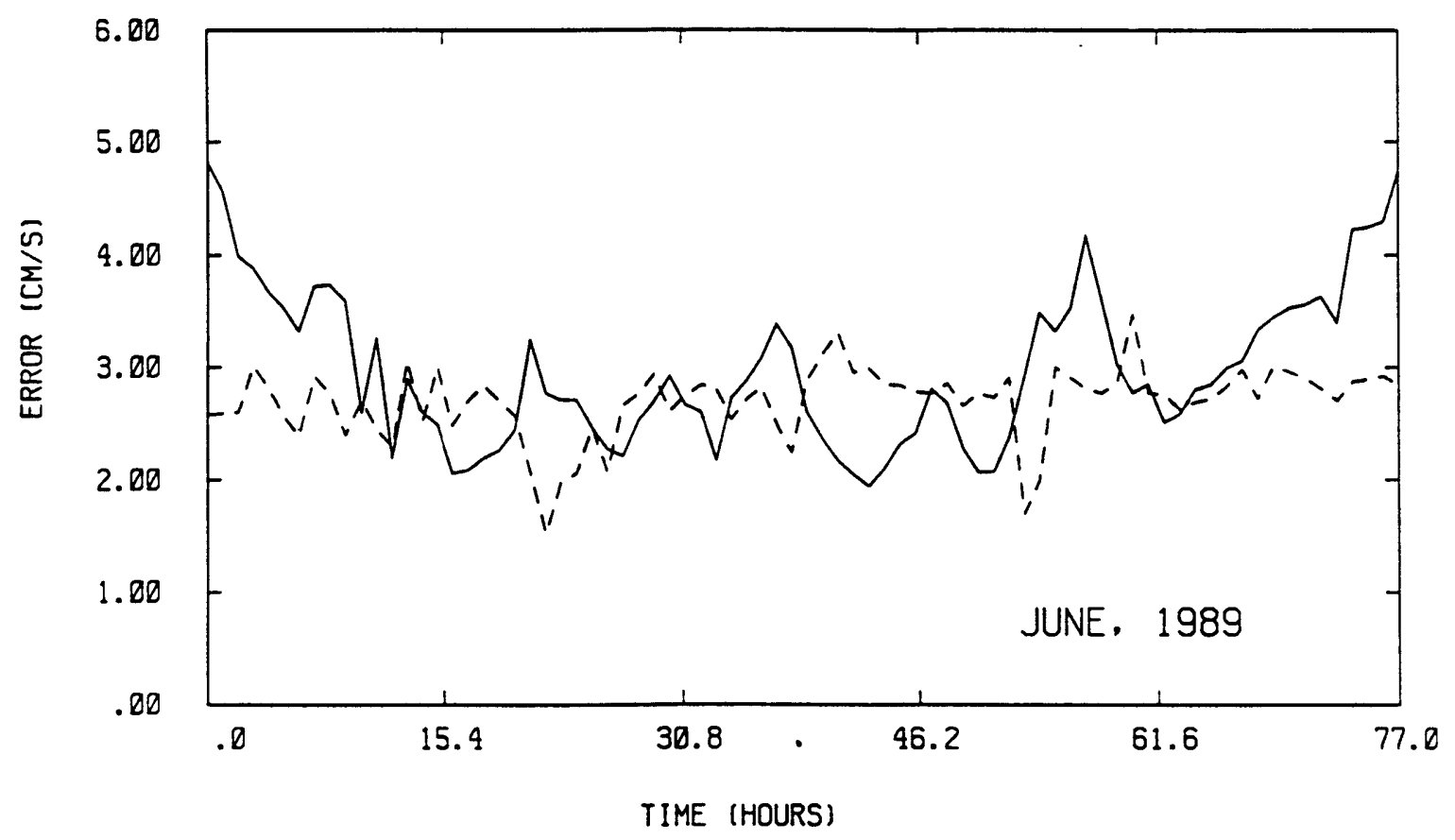

Figure 2.35: Comparison between the fitting (solid line) and measurement (dashed line) errors along the ship's track during June 7-12, 1989 CTD/ADCP surveys.

May and June, 1989. This result suggests a good picture of the seasonal spin-up of cyclonic circulation in the northern GSC that was intensifying in late spring with an increase of eastward net mass transport from May to June, 1989.

A similar approach was also used to fit the April, 1988 vertically averaged ADCP data. The field of fitted vertically averaged residual vectors and fitting uncertainty for $u$ and $v$ at a $95 \%$ confidence level are shown in Figure 2.37. The $F$-test shows a best fit for the "raw" residual currents with a second order polynomial, resulting in a correlation between the "raw" and fitted residual currents of 0.60 for $u$ and 0.89 for $v$ and a standard deviation of $4.5 \mathrm{~cm} / \mathrm{s}$ for $u$ and $6.5 \mathrm{~cm} / \mathrm{s}$ for $v$. The fitting errors estimated by the formula (2.14) were distributed as a function of space which ranged from \pm 2.5 to $\pm 5.0 \mathrm{~cm} / \mathrm{s}$ in $u$ and \pm 3.0 to $\pm 7.5 \mathrm{~cm} / \mathrm{s}$ in $v$. Since the measurement errors were unknown for the whole April, 1988 ADCP survey, we can not tell from our analysis whether or not these fitting errors were caused by the ADCP instrument. Anyway, the vertically averaged residual 

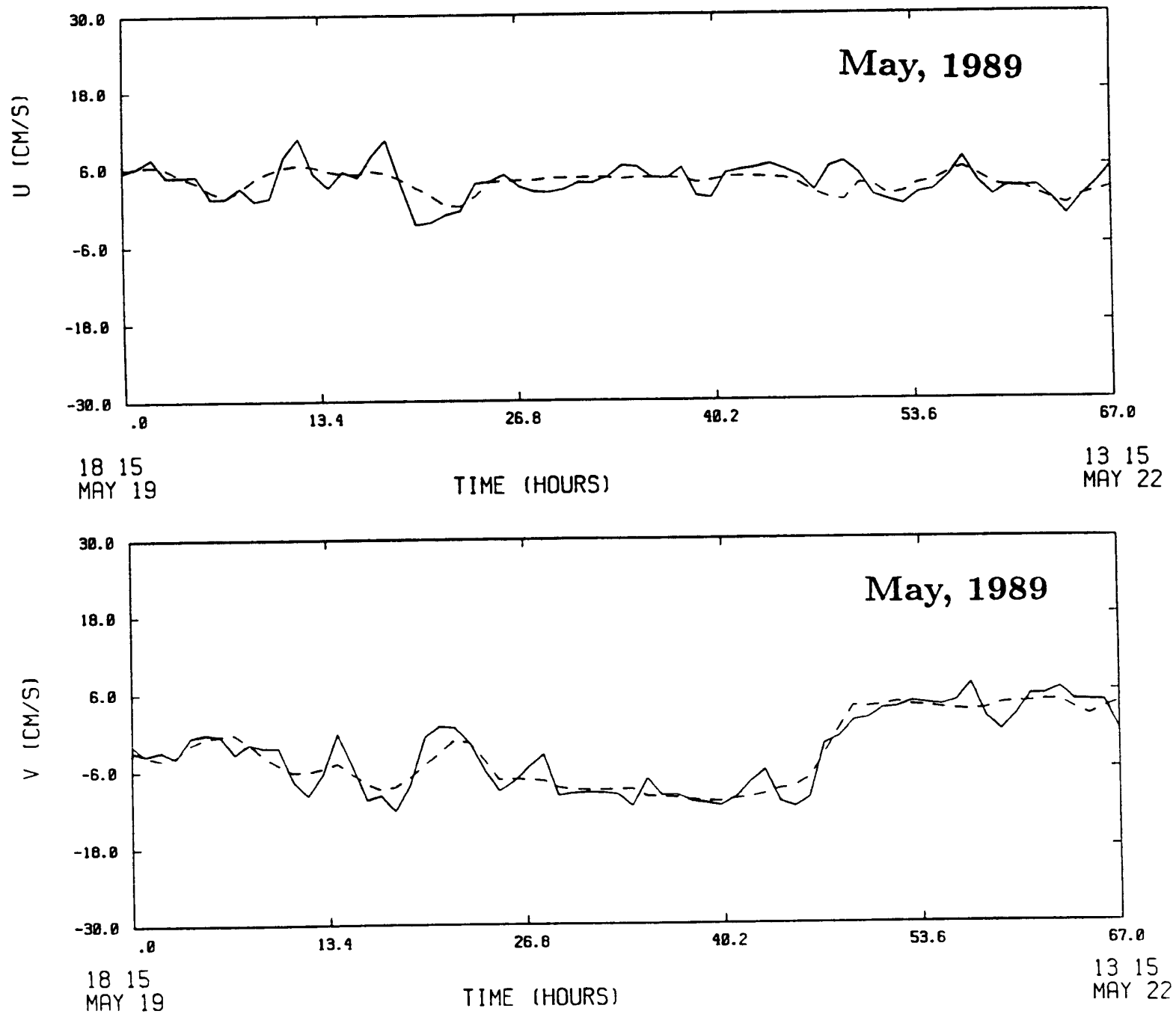

Figure 2.36: Comparison between the "raw" (solid line) and fitted (dashed line) vertically averaged residual current components $u$ (upper) and $v$ (lower) along the ship's track during May 19-22, 1989 CTD/ADCP survey. The time resolution is one-hour and velocities are in centimeters per second. 

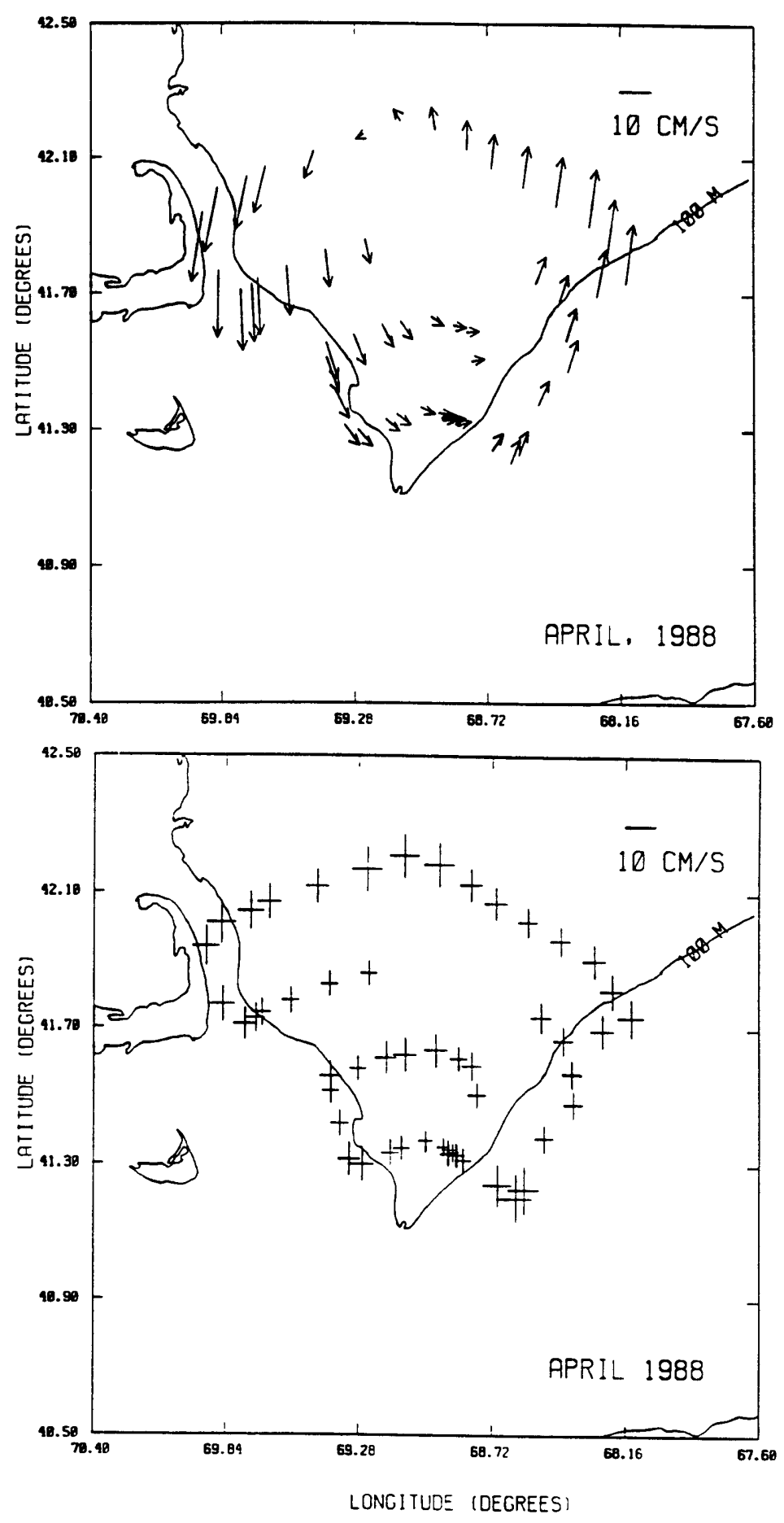

Figure 2.37: Distributions of vertically averaged residual flow (upper) and fitting uncertainty (lower) in the northern GSC during the April, 1988 CTD/ADCP surveys. The plotting scale for the current vector and fitting uncertainty is in centimeters per second. 
flow in the northern GSC in late April, 1988 was characterized by a cyclonic circulation pattern that was very similar to that found in May and June, 1989 except for differences in the amplitude and local current directions near Cape Cod. Unlike the May and June, 1989 surveys, in late April, 1988, the vertically averaged residual current had a significant onshore component over the western flank of the northern GSC. The exact cause of this is not known, although a period of strong $(>15 \mathrm{~m} / \mathrm{s})$ southward wind did occur just before the April, 1988 SCOPEX survey (see Figure 2.13).

Five small-scale biological ADCP surveys taken from May 22 to June 4, 1989 provided ADCP records of at least 20-hour duration within small areas about $5 \times 5 \mathrm{~km}^{2}$. Within these areas, we treated the ADCP data as a simple time series and used a least squares fit to separate the tidal and residual currents from the ADCP data. The resulting vertically averaged residual current vectors are shown here in Figure 2.38 to compare with those obtained from the mid-June, 1989 large-scale ADCP survey, approximately one half to one month later. For the sake of comparison, the small-scale ADCP survey stations are numbered here 1-5 from the east coast of Cape Cod to the western flank of Georges Bank (see Figure 2.26). Good agreement was found in current direction between these ADCP surveys, implying a relatively stable cyclonic circulation pattern in the northern GSC, at least in late spring, 1989. Good agreement was also found in magnitude of the vertically averaged residual current between the small-and large-scale surveys within the uncertainties of fitting errors except at station 5 where a weaker northeastward current of $1.85 \mathrm{~cm} / \mathrm{s}$ was observed during May 22-24, almost three weeks earlier than the large-scale survey. Subtracting this value from that for the mid-June, large-scale survey, we found a difference of about $5.9 \pm 2.5 \mathrm{~cm} / \mathrm{s}$ from May 22 to June 12, 1989 at that point, which may be due to the temporal variability of residual currents near the western flank of Georges Bank.

There had been few direct current measurements made in the northern GSC before SCOPEX except over Nantucket Shoals (see Figure 1.3 in Chapter 1). The subtidal current 


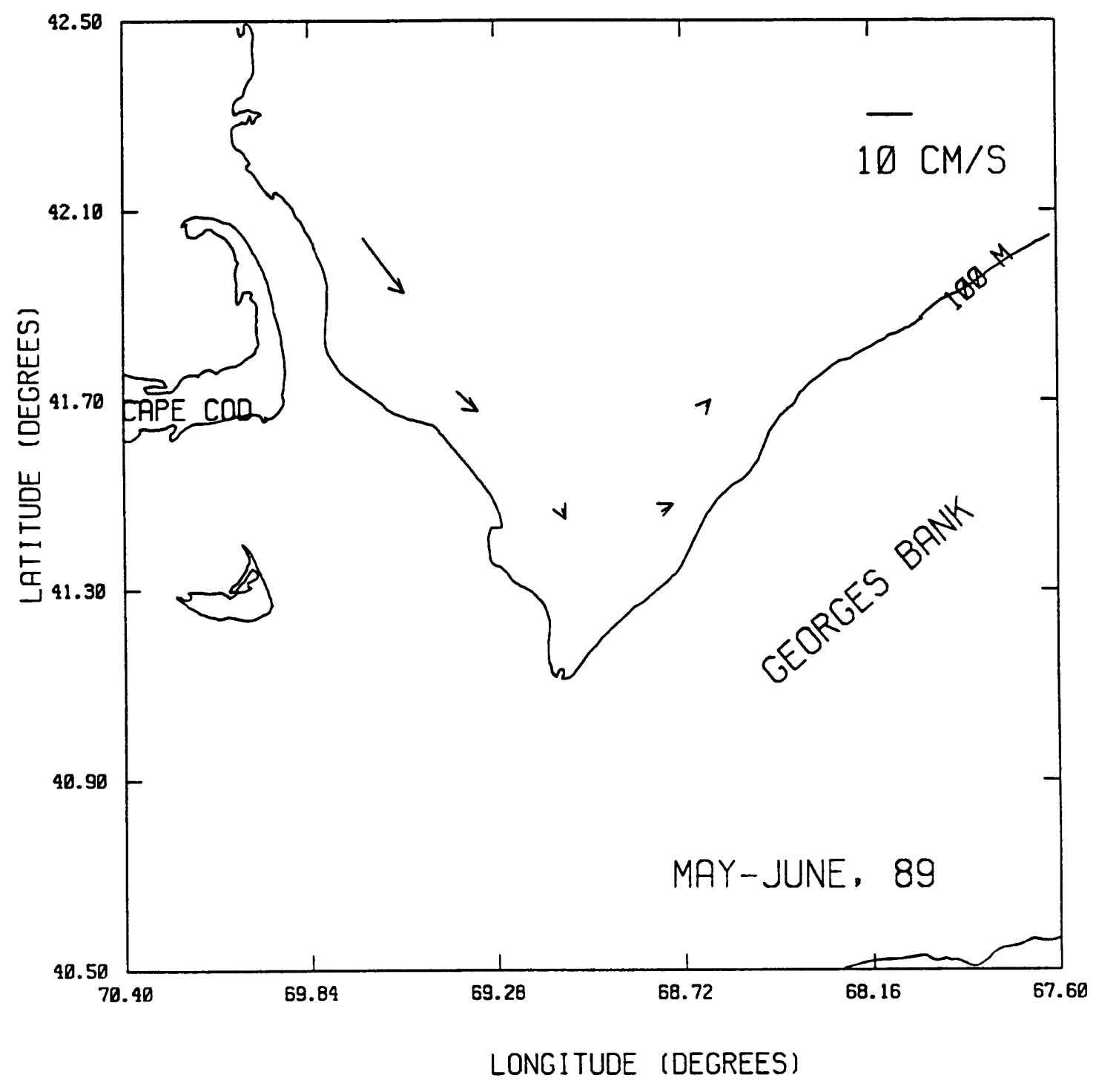

Figure 2.38: Distributions of vertically averaged residual flow in the northern GSC during the May-June, 1989 small-scale, 24-hour anchor CTD/ADCP surveys. The plotting scale for the current vector is in centimeters per second. 
over Nantucket Shoals tends to flow southward at all depths and varies from about $5 \mathrm{~cm} / \mathrm{s}$ in January-March to $10 \mathrm{~cm} / \mathrm{s}$ or more in June-July (Limeburner and Beardsley, 1982). This result agrees well with the results of the ADCP analysis during the June, 1989 survey where a southward flow of about $10 \mathrm{~cm} / \mathrm{s}$ was observed. However, the circulation pattern deduced from the small- and large-scale ADCP data for both 1988 and 1989 in the northern GSC is very different from the $M_{2}$ tidally driven residual current pattern predicted numerically by Greenberg (1983). He showed a closed eddy in the northern GSC with a speed of order 1-3 cm/s (see Figure 1.4 in Chapter 1). This difference implies that the semidiurnal tidal current is not a dominant process driving the residual current in the northern GSC, especially at the northern boundary of our survey region where the water depth is greater than $150 \mathrm{~m}$. What is the most important forcing in that region? We will discuss this next.

\section{Vertical Structure of Residual Flow in the Northern GSC}

Equations (2.1)-(2.4) were also used to fit one-hour-averaged ADCP data recorded every $4 \mathrm{~m}$ in the vertical during the April, 1988 and May-June, 1989 SCOPEX surveys. $F$-tests showed a best fit for the residual flows with a second order polynomial for the April, 1988 and June, 1989 surveys, but a third-order polynomial was required in the upper $100 \mathrm{~m}$ for the May, 1989 survey. The standard deviations were inversely proportional to the degrees of freedom for fitting, and the correlations between raw and fitted residual flows were generally larger near the surface and bottom and smaller in mid-depths. For the June, 1989 survey, the standard deviation was $2.0-3.0 \mathrm{~cm} / \mathrm{s}$ in $u$ and $v$ at depths of $20-100 \mathrm{~m}$ where the correlation ranged from 0.85 to 0.95 . However, the standard deviation increased to $5.0-7.0 \mathrm{~cm} / \mathrm{s}$ near the surface due to low correlation of about 0.50 in $u$ and 0.75 in $v$ and below $100 \mathrm{~m}$ due to small numbers of degrees of freedom. Similarly, for the May, 1989 survey, the standard deviation was about $1.5-3.5 \mathrm{~cm} / \mathrm{s}$ in $u$ and $v$ in the upper $120 \mathrm{~m}$ where the correlation was about $0.70-0.80$ in $u$ and $0.9-0.95$ in $v$, while it became about $4.0-5.0 \mathrm{~cm} / \mathrm{s}$ below $120 \mathrm{~m}$ where the numbers of quality ADCP data values were reduced and correlations became bad. For the April, 1988 survey, however, large measurement noise 
caused relatively low correlations and large deviations, which were up to $7.0-8.0 \mathrm{~cm} / \mathrm{s}$ in deviation at each measurement depth and down to 0.45 in correlation, especially in the $u$ component. Fitting uncertainty for the residual flow at a $95 \%$ confidence level was about the same order as the standard deviation, even though there indeed existed a spatial distribution in fitting errors at each measurement depth. Compared with the magnitude of the fitted residual flow, the fitting errors were relatively smaller for the 1989 surveys, especially in the region of strong current on both sides of the GSC, but they were rather larger for the April, 1988 survey, so that the vertical structure of residual flow obtained from the April, 1988 ADCP data is meaningful only qualitatively rather than quantitatively.

In Figure 2.39 are shown vertical sections of temperature and residual flow normal to the northernmost cross-channel transect (section E, corresponding to the temperature and salinity sections shown in Figures 2.8 and 2.10) for both the April, 1988 and June, 1989 SCOPEX surveys. In both surveys, the horizontal circulation was cyclonic at all levels throughout at least the upper $120 \mathrm{~m}$, with maximum inflow and outflow occurring at the western and eastern ends of the transect, respectively. In April, 1988, one maximum inflow occurred at a depth of $20 \mathrm{~m}$, corresponding to the coastal-trapped low-salinity water near the surface, the other maximum inflow and outflow were centered near $80 \mathrm{~m}$, at the core of MIW whose temperature minimum was about $4.4^{\circ} \mathrm{C}$. Similarly, in June, 1989, one maximum inflow occurred in a broad band centered between 40 and $70 \mathrm{~m}$, which was also located in the center of MIW, and a second maximum was observed in the upper $20 \mathrm{~m}$, associated with the strong thermocline. A narrow core of temperature minimum in a range of $3.2^{\circ}$ to $4.4^{\circ} \mathrm{C}$ was observed in July, 1989 near the western coast over a horizontal distance of $40 \mathrm{~km}$ between 40 and $120 \mathrm{~m}$ at section $\mathrm{E}$. This core, in contrast to the observations of April, 1988, seemed to be coastal water separated from the interior MIW. This colder and relatively fresh (hence lighter) water spread offshore to mix with the interior MIW as it flowed southward from section $\mathrm{E}$ to section A (Figure 2.11). Correspondingly, a strong coastal jet-like flow was found over the western flank of the GSC at section E, with a maximum velocity of about $18.0 \mathrm{~cm} / \mathrm{s}$ at mid-depth and a strong horizontal shear of $10 \mathrm{~cm} / \mathrm{s}$ over $40 \mathrm{~km}$ away from the 

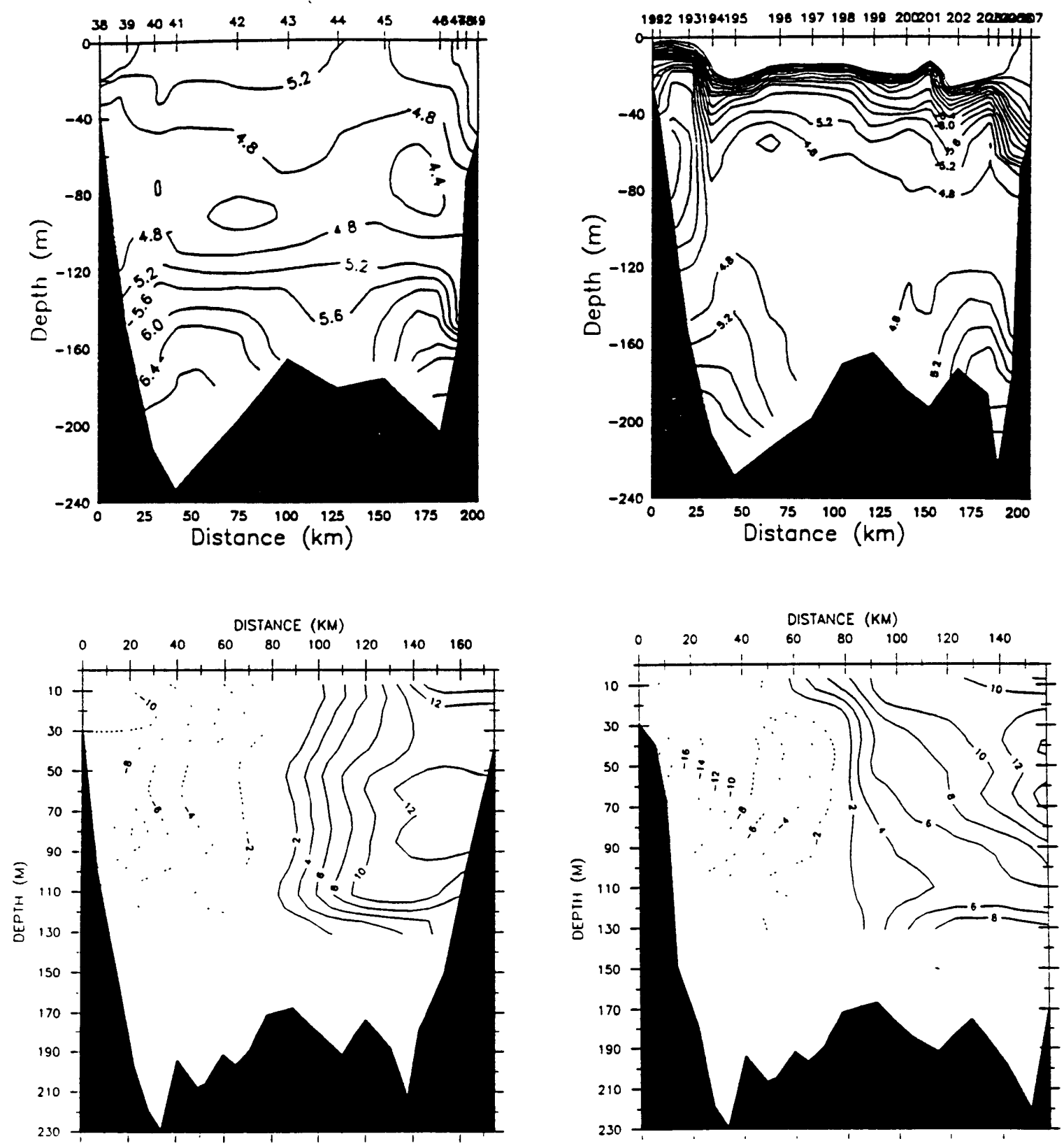

Figure 2.39: Vertical sections of temperature (upper) and residual flow normal to temperature section (lower) on the northernmost section E for April, 1988 and June, 1989 surveys. The velocity scale is in centimeters per second. A negative sign means flow into the northern GSC (southward) and a positive sign means flow out of the northern GSC (northward). 
coast. A continuous path of this deep inflow can be traced in subsequent sections $D$ to $B$ in Figure 2.40, where the lighter and colder coastal water was continuously carried into the northern GSC, flowing southward first along the $100-\mathrm{m}$ isobath to mix with interior MIW and warm, saline Maine Bottom Water, and then turning northward to flow out of the northern GSC along the western flank of Georges Bank as MIW with the relatively weak temperature minimum. The strength of this deep current weakened southward. It decreased from $18.0 \mathrm{~cm} / \mathrm{s}$ at section $\mathrm{E}$ to $8.0 \mathrm{~cm} / \mathrm{s}$ at section $\mathrm{B}$ as it flowed along the western flank and then increased again to $12.0 \mathrm{~cm} / \mathrm{s}$ as it left the northern GSC along the western flank of Georges Bank, indicating that a large part of the deep MIW turned eastward as it flowed into the northern GSC and only a small portion of this water was carried southward to section B. There did exist an outflow directly through the GSC from the western GOM on the western side of the southernmost section $A$ in the upper $50 \mathrm{~m}$, consistent with the $\mathrm{T} / \mathrm{S}$ described in section 2.2. This implies that the outflow through the GSC in the upper $50 \mathrm{~m}$ is a mixture water of low-salinity plume and MSW.

Vertical sections of temperature and residual flow normal to the temperature sections for the May 1989 survey are plotted in Figure 2.41 to investigate the seasonal spin-up of cyclonic circulation in the northern GSC, especially for the deep current in association with the MIW. Strong stratification was just beginning to form in the upper $20 \mathrm{~m}$ in late May, 1989. The narrow coastal core of temperature minimum water, which was found in June 7-12, 1989, can be traced back to May 19-21, 1989 at the northernmost section F but it was about $0.8^{\circ} \mathrm{C}$ warmer. This fresher and colder coastal water was still continuously detected southward on subsequent temperature sections $\mathrm{E}$ to $\mathrm{C}$, implying that the cold water was flowing into the northern GSC along the western coast of GOM in late May, 1989. Correspondingly, on the northernmost section $\mathrm{F}$, one maximum southward current in excess of $14.0 \mathrm{~cm} / \mathrm{s}$ was found at the strong thermocline at surface, and a second maximum southward flow of order $12.0 \mathrm{~cm} / \mathrm{s}$ was observed at the center of the temperature 

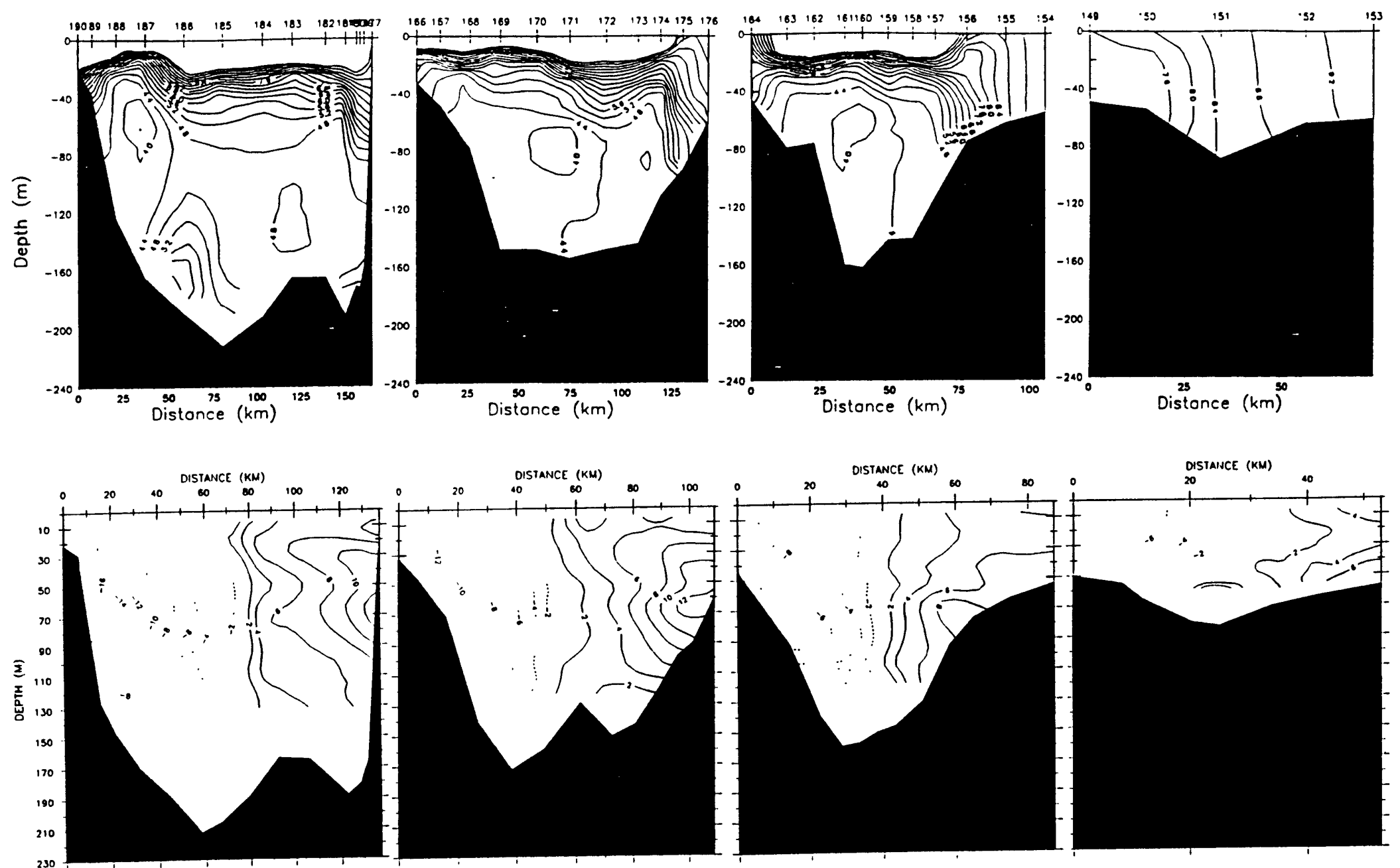

Figure 2.40: Vertical sections of temperature (upper) and residual flow normal to temperature section (lower) southward from section $\mathrm{D}$ to section A during the June, 1989 survey. The velocity scale is in centimeters per second. A negative sign means flow into the northern GSC (southward) and a positive sign means flow out of the northern GSC (northward). 


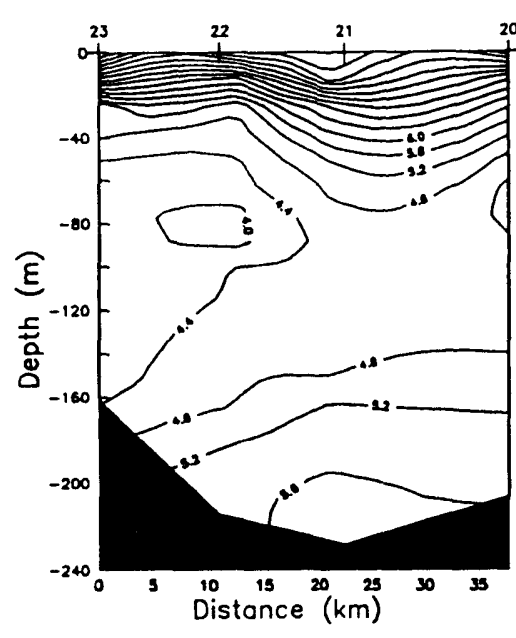

DISTANCE (KM)

e

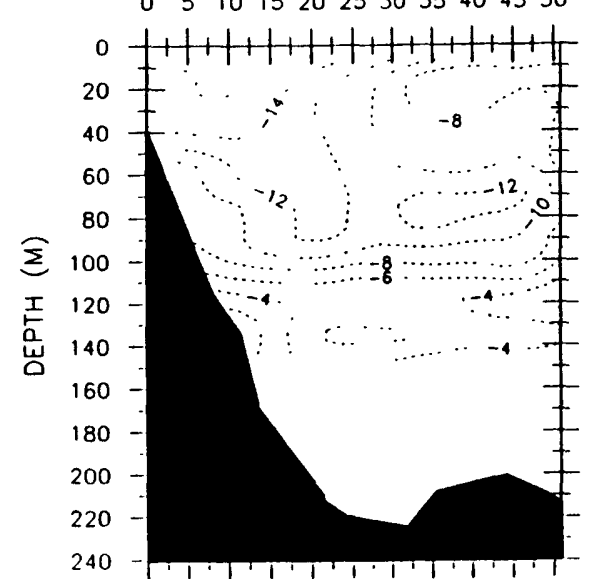

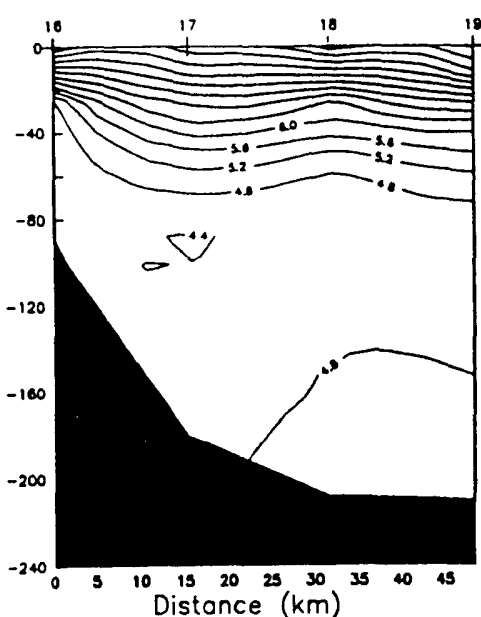

CIISTANCE (KM) $\begin{array}{llllllll}0 & 5 & 10 & 15 & 20 & 25 & 30 \quad 35 \quad 40 \quad 45\end{array}$

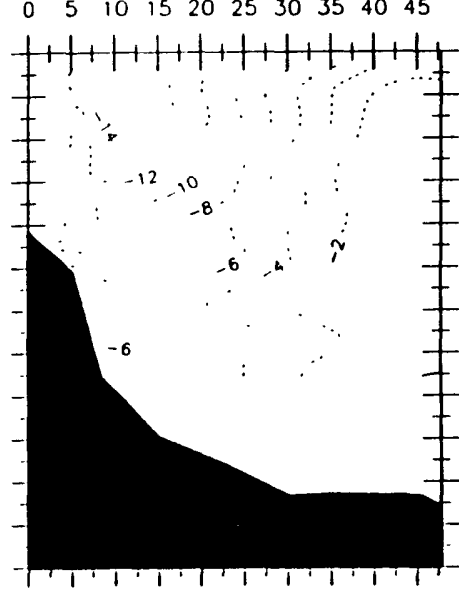

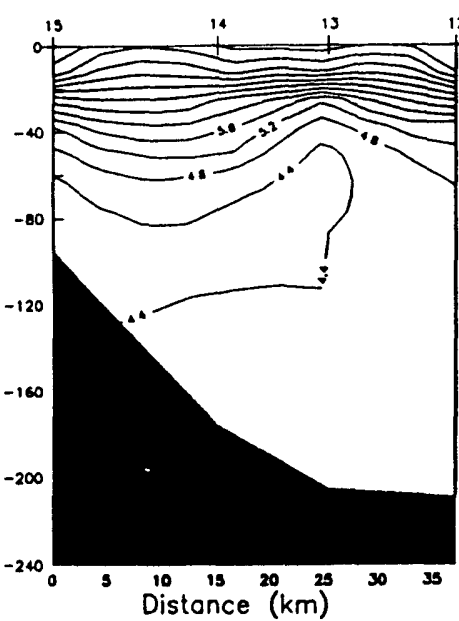

DISIANCE (KM)

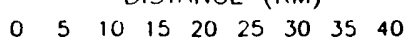

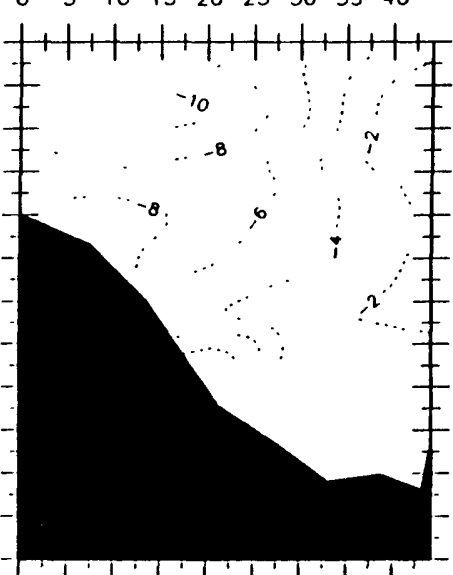

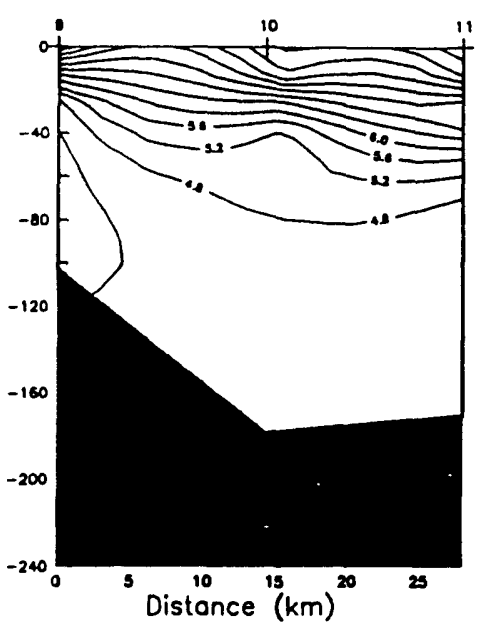

DISIANCE (KM)

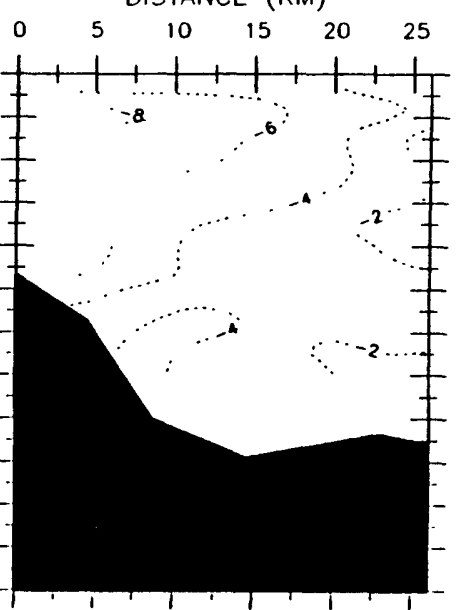

Figure 2.41: Vertical sections of temperature (upper) and residual flow normal to temperature section (lower) from section $\mathrm{F}$ to section $\mathrm{C}$ for the May 19-22, 1989 CTD/ADCP survey. The velocity scale is in centimeters per second. A negative sign means flow into the northern GSC (southward). 
minimum ${ }^{5}$. The deep current tended to weaken southward as it flowed into the northern GSC. It decreased to $6.0 \mathrm{~cm} / \mathrm{s}$ at section $\mathrm{C}$ at about $41.0^{\circ} \mathrm{N}$. Comparison with the vertical distributions of residual flow and temperature on June 7-12, 1989 provides us with a good view of the seasonal evolution of this coastal jet-like deep current. As the temperature minimum decreased from $4.0^{\circ} \mathrm{C}$ on May $19-21,1989$ to $3.2^{\circ} \mathrm{C}$ on June $7-12,1989$, the deep current became $6.0 \mathrm{~cm} / \mathrm{s}$ stronger (a $50 \%$ increase), which clearly showed a seasonal spin-up of this deep current at mid-depth in late spring. Since we did not see a coastal trapped temperature minimum in April, 1988, we can not tell if this coastal current is a permanent feature of the deep circulation in the western GOM in spring or whether it starts to flow into the GSC during springtime. A series of CTD/ADCP surveys should be conducted in the western GOM to resolve the complete seasonal evolution of this deep current.

During each regional survey, satellite-tracked drifters were deployed at $5 \mathrm{~m}$ to measure Lagrangian movement of the near-surface water and at $50 \mathrm{~m}$ to observe sub-thermocline water movement (Limeburner and Beardsley, 1990). The resulting low-passed trajectories of the 5 and $50 \mathrm{~m}$ drifters from April 28 or May 1 to May 31, 1988 and from June 11 to June 30, 1989 are re-plotted here in the upper panels of Figures 2.42-2.45, respectively, to show the spatial structure of water movement near the surface and at $50 \mathrm{~m}$. In April-May, 1988, the near-surface Lagrangian flow looked very complex, especially in the central and northern GSC. In spite of that, the daily path of near-surface water over the western flank west of $69.0^{\circ} \mathrm{W}$ was southward, with some water flowing out of the GOM and onto the New England shelf. The near-surface water in the GSC, especially near and east of $69.0^{\circ} \mathrm{W}$ between $41.1^{\circ} \mathrm{N}$ and $41.6^{\circ} \mathrm{N}$, tended to drift eastward and northeastward along the western and northern flank of Georges Bank. Above $41.7^{\circ} \mathrm{N}$, the near-surface water flowed northward and then turned northeastward to join an anticyclonic gyre along Georges Bank. The daily averaged drifter speed varied from 0.1 to $16.0 \mathrm{~cm} / \mathrm{s}$, stronger over the shallow region

\footnotetext{
${ }^{5}$ Note: the May, 1989 ADCP survey was basically conducted along the CTD stations but it extended westward about $15 \mathrm{~km}$ on the northernmost section $\mathrm{F}$, so that comparison between temperature and residual flow should start at the point where the water depth was the same for both maps rather than at the origin of the horizontal coordinate.
} 

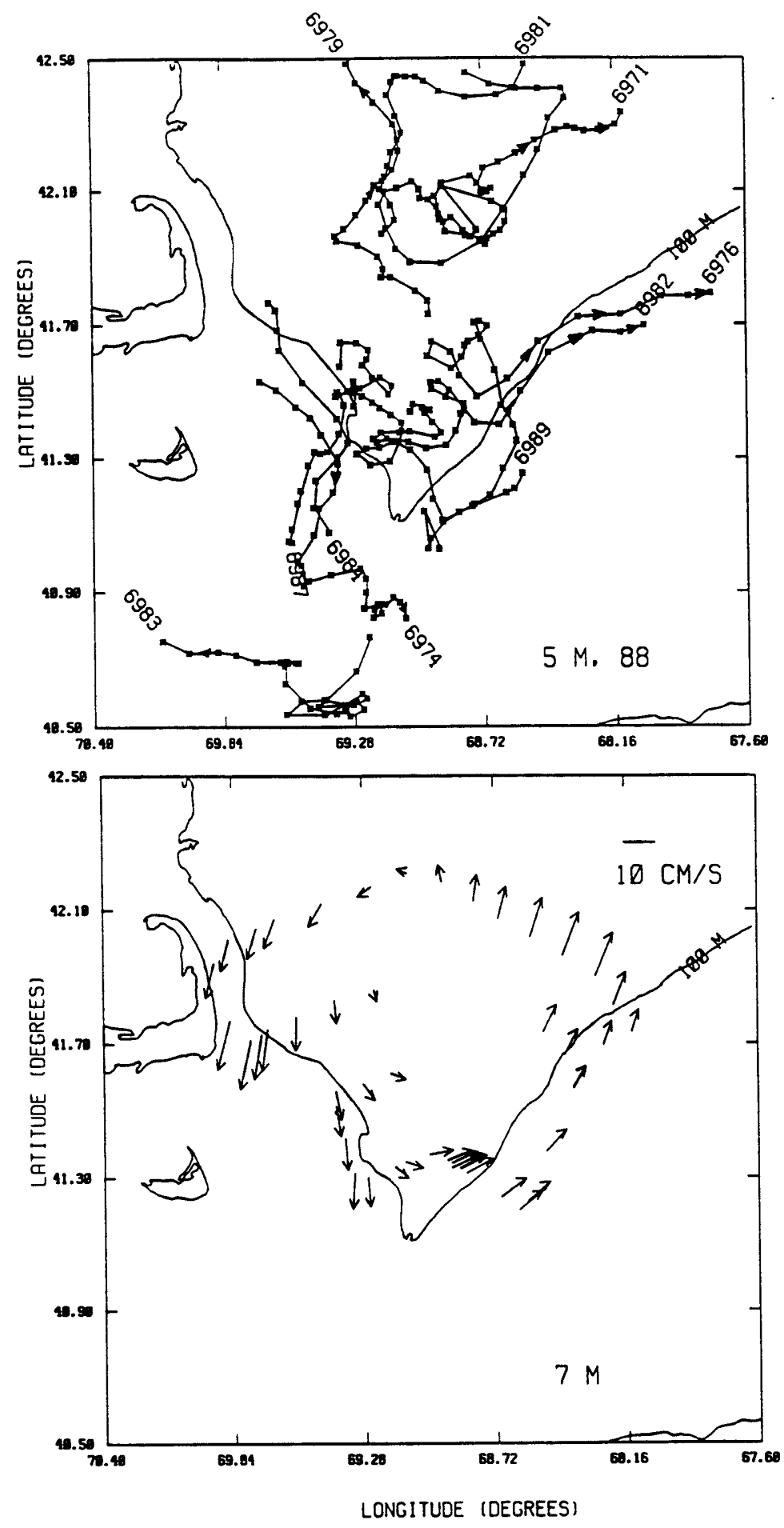

Figure 2.42: Low-passed trajectories of 5-m drifters (upper) from April 28 or May 1 to May 31, 1988, and maps of residual flow at $7 \mathrm{~m}$ (lower) in the northern GSC obtained during the April, $1988 \mathrm{CTD} / \mathrm{ADCP}$ survey . 


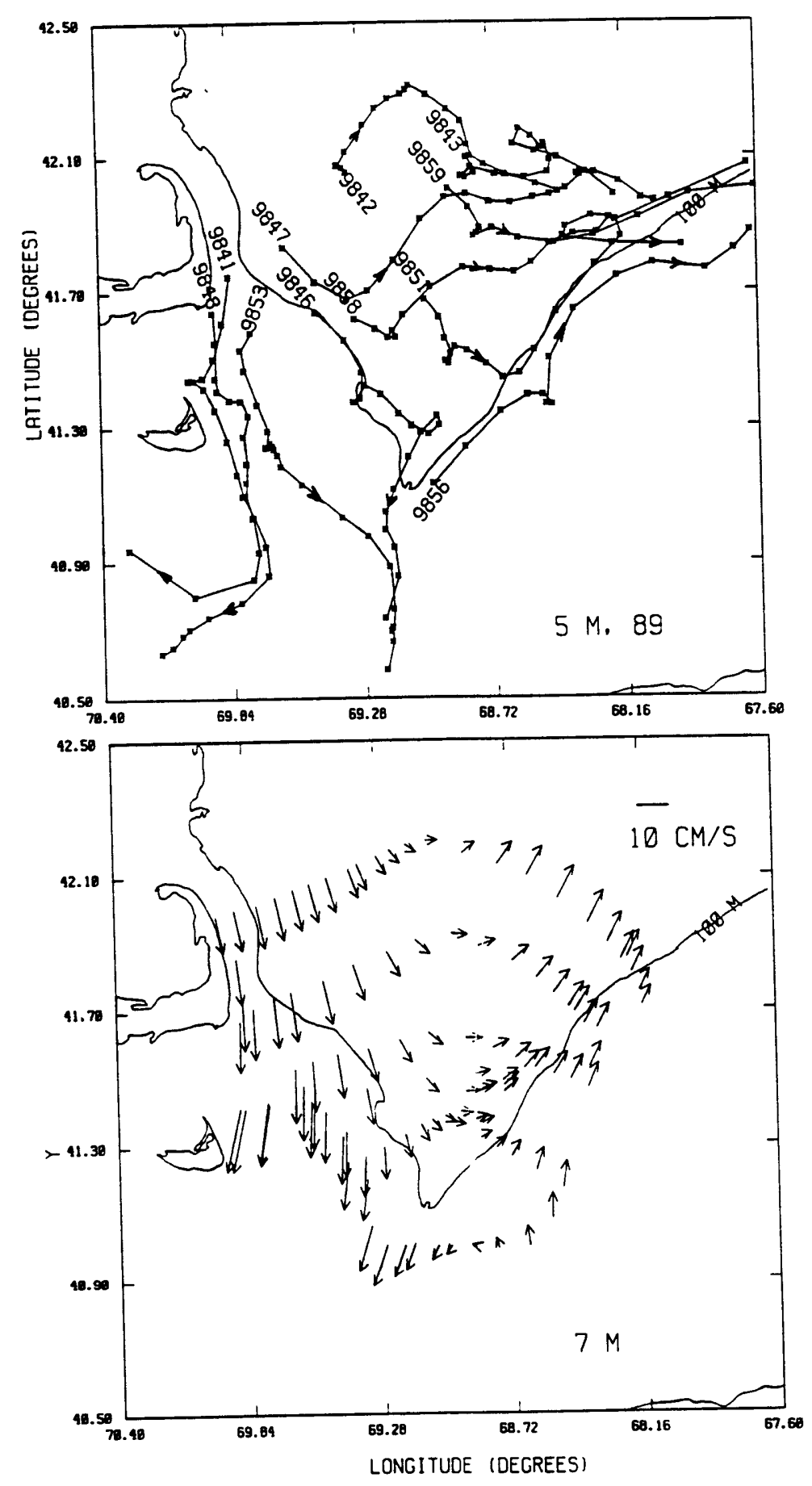

Figure 2.43: Low-passed trajectories of 5-m drifters (upper) from June 11 to June 30, 1989, and maps of residual flow at $7 \mathrm{~m}$ (lower) in the northern GSC obtained during the June, 1989 (right) CTD/ADCP survey . 

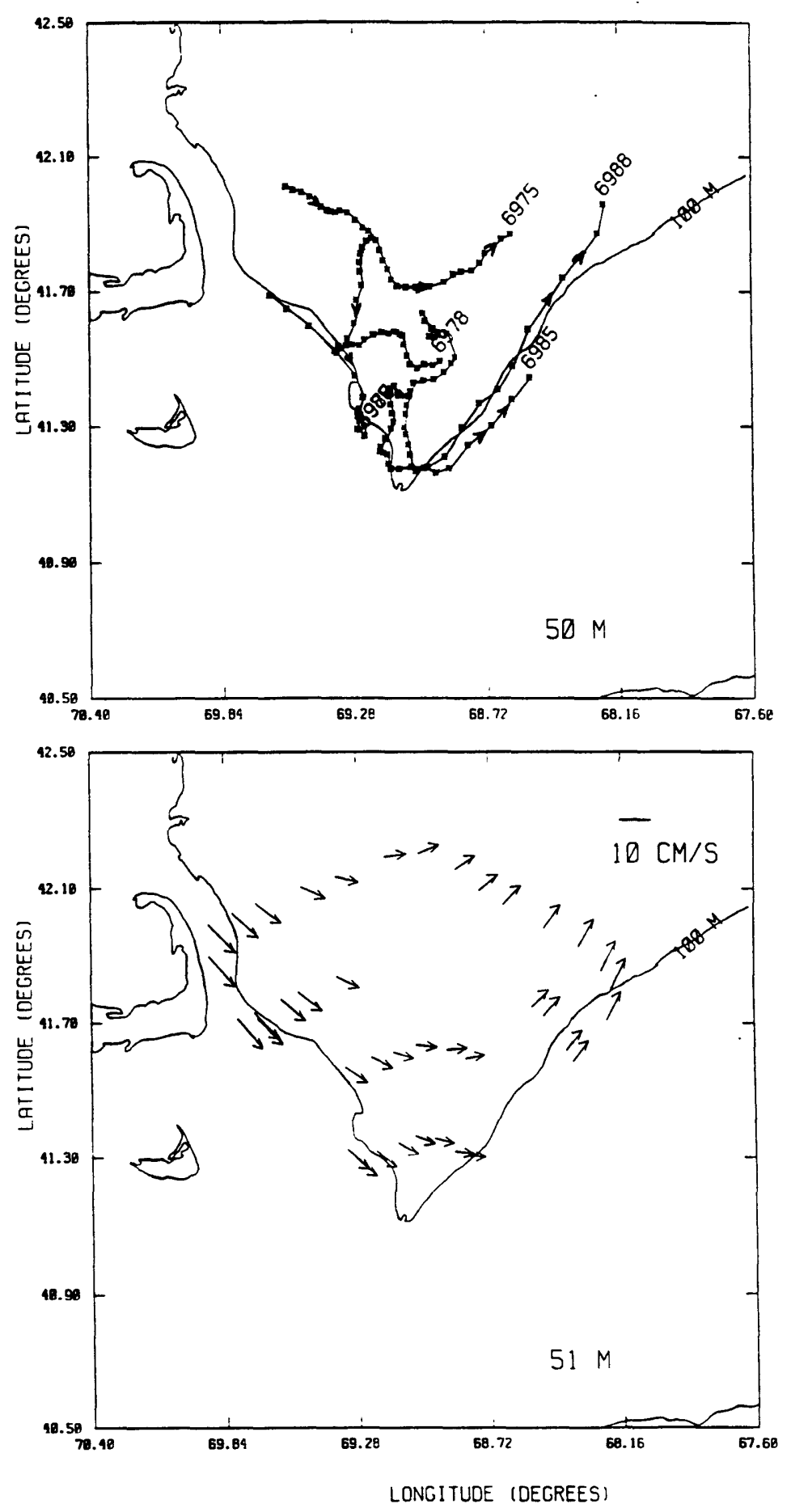

Figure 2.44: Low-passed trajectories of 50-m drifters (upper) from April 28 or May 1 to May 31, 1988, and maps of residual flow at $51 \mathrm{~m}$ (lower) in the northern GSC obtained during the April $1988 \mathrm{CTD} / \mathrm{ADCP}$ survey . 

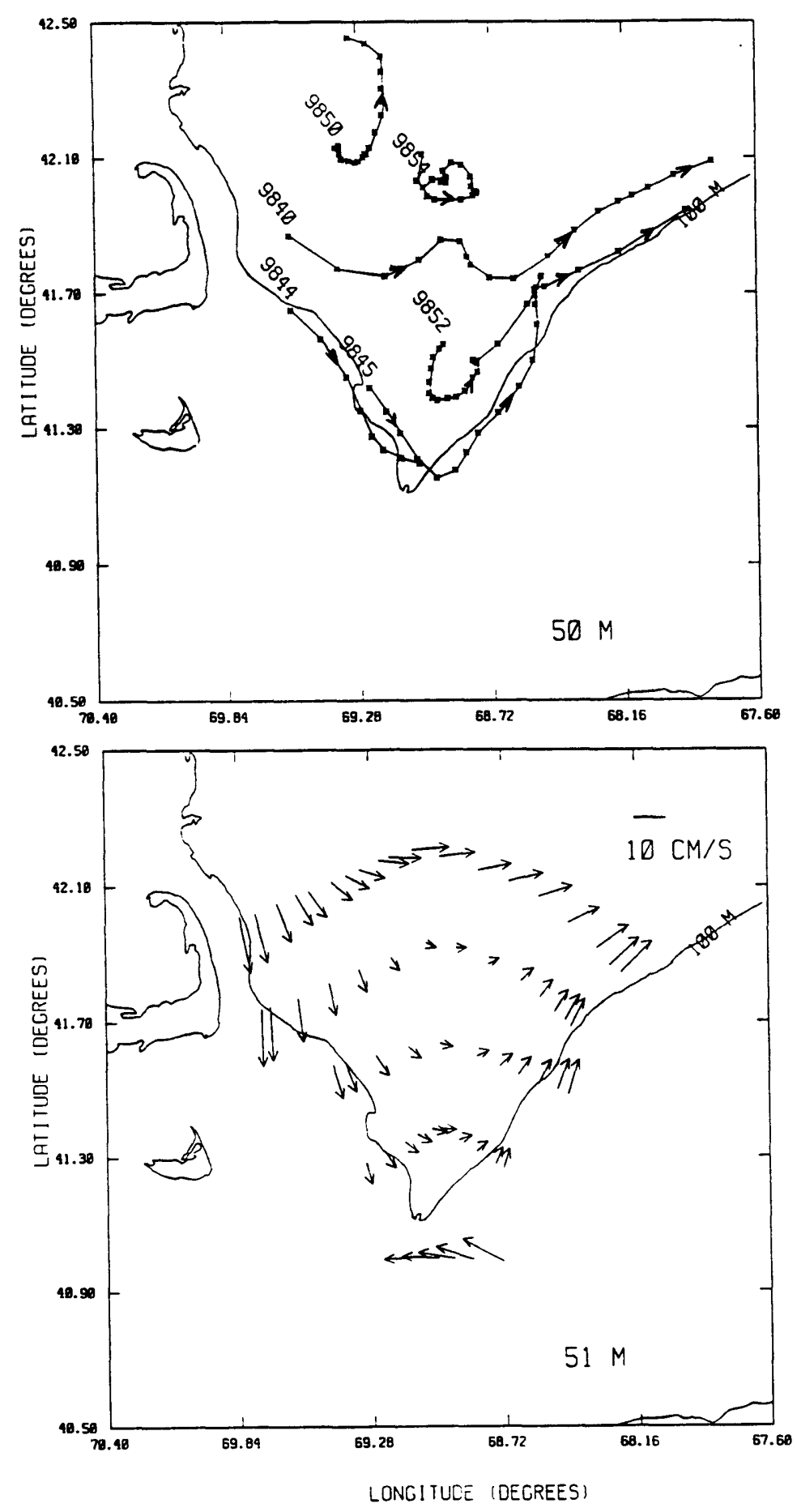

Figure 2.45: Low-passed trajectories of 50-m drifters (upper) from June 11 to June 30, 1989, and maps of residual flow at $51 \mathrm{~m}$ (lower) in the northern GSC obtained during the June, 1989 CTD/ADCP surveys. 
and weaker in the northern GSC between $41.3^{\circ} \mathrm{N}$ and $41.5^{\circ} \mathrm{N}$. Similar daily mean trajectories of near-surface water were found in June, 1989 where the low-passed Lagrangian flows were clearly identified as having three main paths: (1) flow southward out of the GOM along the western flank west of $69.0^{\circ} \mathrm{W},(2)$ flow eastward and then northeastward from the central and northern GSC to the northern flank of Georges Bank, and (3) the anticyclonic circulation round Georges Bank. The daily-averaged drifter speed in June, 1989 varied from 0.1 to $15.0 \mathrm{~cm} / \mathrm{s}$, with relatively weak flow in the center of the GSC between $41.3^{\circ} \mathrm{N}$ to $41.5^{\circ} \mathrm{N}$. The $50-\mathrm{m}$ Lagrangian flow in the northern GSC during April-May, 1988 and June, 1989 was generally cyclonic from east of Cape Cod to the northern flank of Georges Bank. In April-May, 1988, the daily averaged $50-\mathrm{m}$ drifter speeds varied from 0.5 to $5.0 \mathrm{~cm} / \mathrm{s}$ in the western GSC where the local water depth is deeper than $100 \mathrm{~m}$, but the drifter speeds increased to $15.0-20.0 \mathrm{~cm} / \mathrm{s}$ as they drifted into the eastern GSC. In June, 1989, the daily averaged $50-\mathrm{m}$ drifter speeds varied from 5 to $25 \mathrm{~cm} / \mathrm{s}$, except in the center of the GSC and north of $42.0^{\circ} \mathrm{N}$ where the water speed was found to be $0.5-2.0 \mathrm{~cm} / \mathrm{s}$.

The fields of horizontal residual flow obtained at $7 \mathrm{~m}$ and $51 \mathrm{~m}$ from the ADCP surveys in late April, 1988 and mid-June, 1989 are shown in the lower panels of Figures 2.422.45 , respectively, to compare with the field of Lagrangian flow at 5 and $50 \mathrm{~m}$. By definition, the Lagrangian velocity refers to the velocity of a fluid element which is dependent on position and time, while the Eulerian velocity is the velocity at a fixed point. If the flow field is steady, or, if the local derivatives of the flow are equal to zero, then the trajectory of a fluid element is equal to the streamline so that the Lagrangian velocity is equivalent to the Eulerian velocity at a fixed point. In April-May, 1988 and June, 1989, however, the Lagrangian velocity data at 5 and $50 \mathrm{~m}$ started one or two days after the regional ADCP surveys were completed, so that it is difficult to compare quantitatively the Lagrangian velocity deduced from the drifters with the regional Eulerian velocity obtained from the ADCP data. In spite of this time mismatch, the Eulerian flow field agreed well in direction and spatial structure with the mean paths of the drifters in April-May, 1988 and June, 1989 , especially at mid-depth near $50 \mathrm{~m}$. This structure can be also seen in the horizontal 

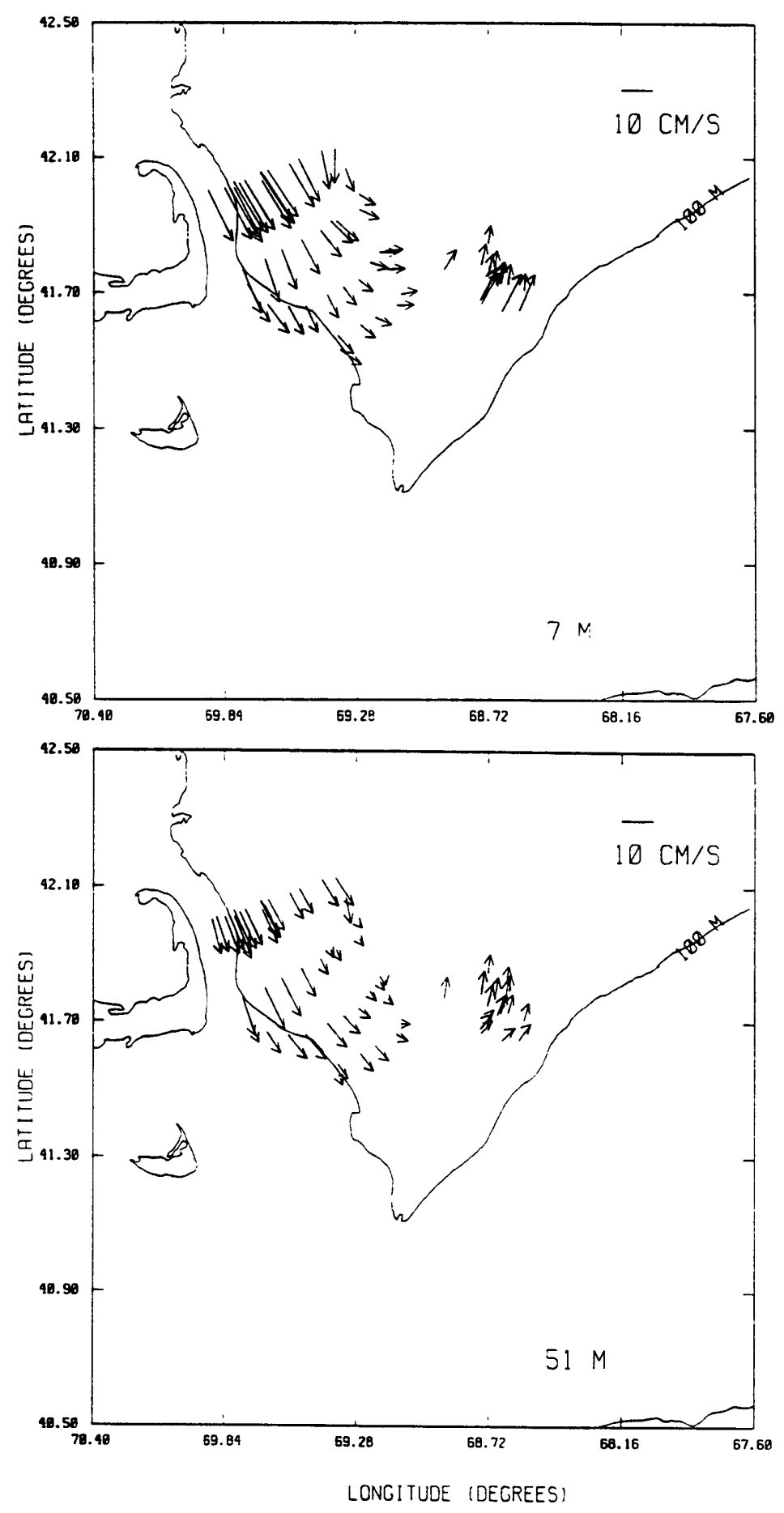

Figure 2.46: Distributions of $7 \mathrm{~m}$ (upper) and $51 \mathrm{~m}$ (lower) residual flows in the northern GSC during the May 19-22, 1989 CTD/ADCP survey. The plotting scale for the current vector is in centimeters per second. 
field of the 7 and 51-m residual flow on May 19-21, 1989 in Figure 2.46. This implies a relatively stable flow pattern in the northern GSC during late spring, at least at mid-depth in the MIW.

\section{Comparison Between the Vertical Shears of the ADCP Residual and Geostrophic Flows}

As an example, Figure 2.47 shows the geostrophic (right panel) and residual (left panel) shears relative to a depth of $50 \mathrm{~m}$ on transect $\mathrm{E}$ for the June, 1989 survey. In general, the maximum vertical velocity shear should occur where the vertical gradient of relative velocity is strongest. For the geostrophic calculation, a multiple cell pattern of relative velocity was shown in the upper $30 \mathrm{~m}$ across the whole transect, with maximum vertical shears of about $2.0 \mathrm{~cm} / \mathrm{s}$ over a vertical depth of $5 \mathrm{~m}$, about $10-20 \mathrm{~km}$ from the western end of the transect and about $30-40 \mathrm{~km}$ from the eastern end of the transect. Between 50 and $130 \mathrm{~m}$, a strong vertical shear of geostrophic velocity was found on both sides of the transect, with a maximum value of $2.0 \mathrm{~cm} / \mathrm{s}$ over a vertical distance of $3-4 \mathrm{~m}$ between the depths of $70 \mathrm{~m}$ and $90 \mathrm{~m}$ in a narrow band of $30 \mathrm{~km}$ from the western end of the transect but with a second maximum of about $2.0 \mathrm{~cm} / \mathrm{s}$ over a vertical distance of $10 \mathrm{~m}$ at a depth of about $120 \mathrm{~m}$ near the eastern end of the transect. In the middle of the transect below $40 \mathrm{~m}$, the vertical and horizontal density gradients were so small that the vertical geostrophic shear is not visible in Figure 2.47 where the contour interval of $2 \mathrm{~cm} / \mathrm{s}$ was chosen.

In general, comparison between geostrophic and ADCP residual vertical shears for section $E$ showed good agreement near both ends of the transect between 80 and $100 \mathrm{~m}$, but poor agreement over the deep center of the section. The broad horizontal structure of ADCP residual vertical shear may be due to both the low degree of the "best fit" polynomial used and the polynomial fitting errors since the uncertainty in the fitted residual flow was about $3.0 \mathrm{~cm} / \mathrm{s}$ in the vertical in the ADCP data. This implies that the baroclinic motion in the northernmost GSC is controlled more by density forcing in late spring than tidal 
rectification, and that the strong inflow of cold MIW water occurred as a narrow jet along the flank through section $\mathrm{E}$, rather than the broad inflow indicated by the ADCP residual flow. Comparing the ADCP residual currents with tidally rectified flow predicted numerically by Greenberg (1983) and Lynch and Naimie (1992), we can also estimate quantitatively the relative contribution of tidal and buoyancy driving to the mean flow in the GSC. Over the western flank east of Cape Cod where the water depth is shallower than $60 \mathrm{~m}$, the numerical models predict a southward tidally rectified current of $7-10 \mathrm{~cm} / \mathrm{s}$, about the same order as the ADCP mean current. In deeper water around the GSC and in the central region of the GSC where the water depth is about $100-200 \mathrm{~m}$, however, the predicted tidally rectified current is about $0.1-1.0 \mathrm{~cm} / \mathrm{s}$, only about $1-10 \%$ of the ADCP mean flow. Therefore, we conclude here that buoyancy driving is dominant in the central region of the GSC and tidal rectification more important over the shallower sides of the GSC in late spring.

More calculations are made here to estimate the absolute geostrophic velocity using the ADCP residual flow at $50 \mathrm{~m}$ as reference velocity (Figure 2.48). Regardless of the upper $30 \mathrm{~m}$ where the surface mixed layer was located, the resulting absolute geostrophic flow showed a cyclonic pattern at all levels throughout the upper $100 \mathrm{~m}$, which is in good agreement with the circulation pattern obtained directly from the ADCP residual flow. Below $100 \mathrm{~m}$ in the region of Maine Bottom Water (MBW), the structure of absolute geostrophic velocity seems much more complicated. A strong northward current of order $8.0 \mathrm{~cm} / \mathrm{s}$ was found at depths of $100-150 \mathrm{~m}$ in a narrow $(5-\mathrm{km})$ region over the western flank due to a strong baroclinic effect relative to $50 \mathrm{~m}$ (see Figure 2.47), which was twice as large as the fitting uncertainty in the ADCP analysis. This means that if the currents were geostrophic there would be a northward jet flow at depths of 100-150 m over the western flank. Since it is hard to imagine where this jet flow would originate in such a semi-enclosed deep region, we question here the validity of geostrophic calculations for the movement of MBW, especially near the flanks where topography, tidal mixing and lateral friction may be important. The geostrophic velocity relative to the bottom is also shown in Figure 2.48. 

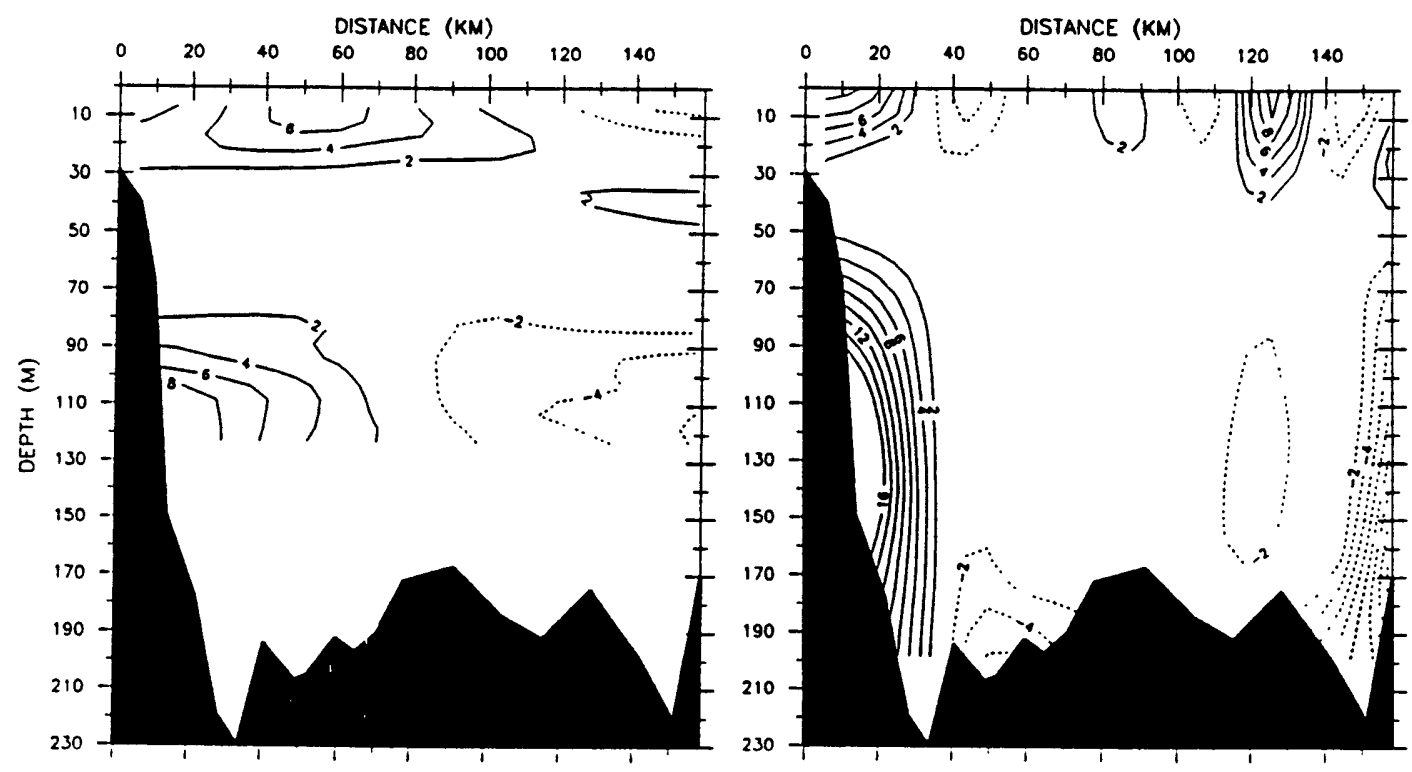

Figure 2.47: Residual (left) and geostrophic (right) velocity shears relative to a depth of $50 \mathrm{~m}$ in centimeters per second on the northernmost cross-channel transect for the June, 1989 SCOPEX CTD/ADCP survey.
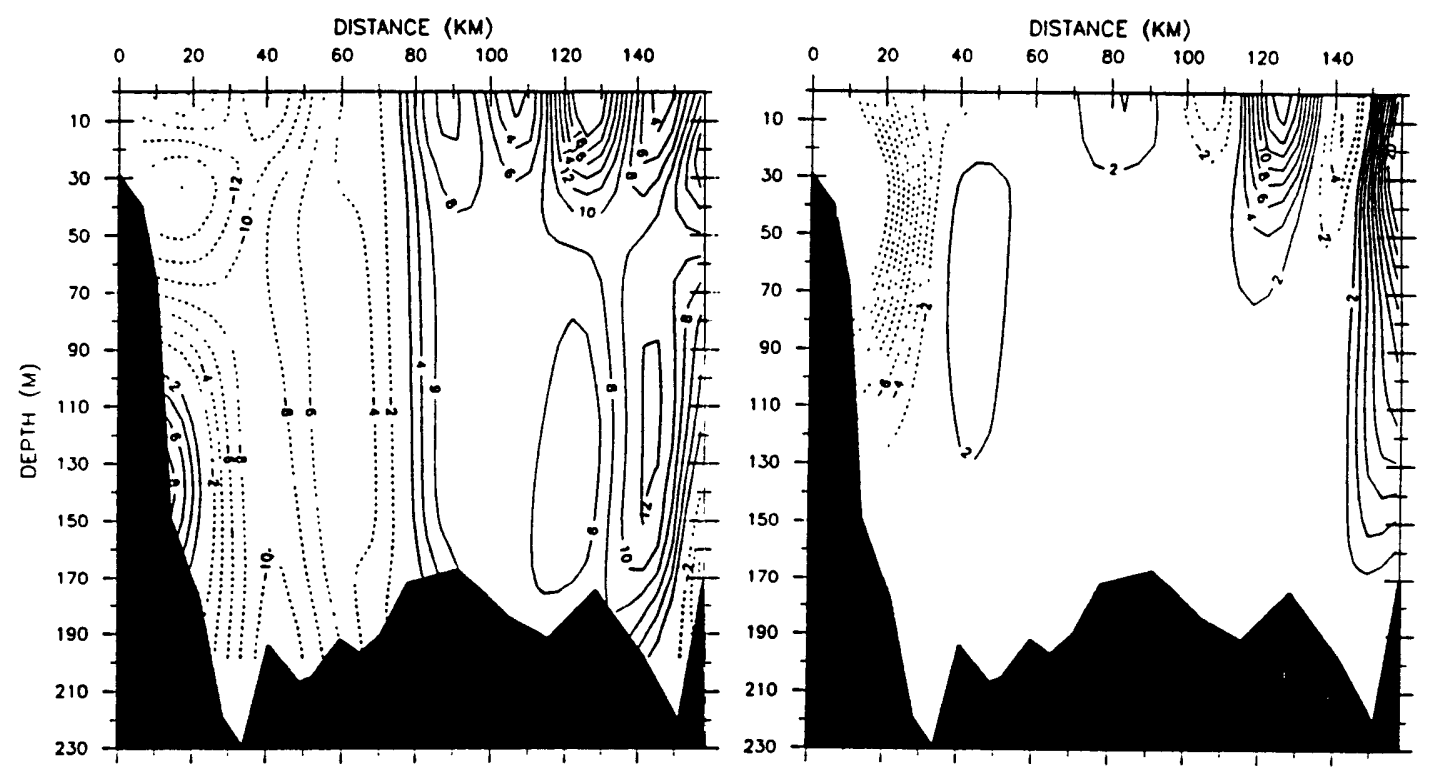

Figure 2.48: Vertical sections of absolute geostrophic velocity with an ADCP residual flow at $50 \mathrm{~m}$ as reference velocity (left) and geostrophic velocity relative to the bottom (right) on the northernmost cross-channel transect for the June, 1989 SCOPEX CTD/ADCP survey. Velocity is in centimeters per second. 
Although this calculation misses a large barotropic flow component, it did clearly show a cyclonic baroclinic circulation at all levels, with maximum inflow and outflow occurring at the western and eastern ends of the transect, respectively.

In summary, the vertical shear of the ADCP residual velocity is in good agreement with the geostrophic shear in the northern GSC within the uncertainty of the ADCP fitting using only a second degree polynomial. The buoyancy-driven flow is dominant in the central area of the GSC while tidally rectified flow is dominant over the shallow western flank of the GSC.

\section{Southward Transports of the Low-Salinity Plume and MIW}

It is difficult to estimate the total mass transport across different sections using the SCOPEX CTD/ADCP measurements because the ADCP profiler could not resolve currents below $140 \mathrm{~m}$. Brooks (1990) deployed three current meters in the deep region at Lindenkohl Sill between Georges Basin and Wilkinson Basin in June, 1986. The resulting low-frequency

\section{Table 2.4: Southward Transports of the Low Salinity Plume (LSP) and MIW.}

\begin{tabular}{|c|c|c|}
\hline \multirow{2}{*}{ Survey } & \multicolumn{2}{|c|}{ Transport } \\
\cline { 2 - 3 } time & LSP (Sv) & MIW (Sv) \\
\hline April, 1988 & $-0.07 \pm 0.03$ & $-0.31 \pm 0.38$ \\
\hline June, 1989 & $-0.12 \pm 0.06$ & $-0.66 \pm 0.14$ \\
\hline
\end{tabular}

current near the bottom showed a good correlation with the surface wind when the density stratification was relatively weak but they were decoupled when density stratification became stronger. A strong current of order $100 \mathrm{~cm} / \mathrm{s}$ was found near the bottom during wind-coherent intervals, while currents were intensified at mid-depth and upper level during wind-current decoupled intervals. This suggests that MBW may contribute a large part of 
the total mass transport across the northern GSC. For this reason, we focus our study only on transports of the near-surface, low-salinity plume and MIW.

Table 2.4 shows transports of the low-salinity plume and MIW across the northernmost section E for the April, 1988 and June, 1989 surveys. In April, 1988, the low-salinity plume was identified by a strong salinity front of $32.5 \%$ on the western flank of the northern GSC, with a maximum depth of about $40 \mathrm{~m}$ and a cross-shelf scale of about $40 \mathrm{~km}$. The southward volume transport of this plume was about $-0.07 \pm 0.03 \mathrm{~Sv}$ with a mean salinity of $32.2 \%$. In June, 1989 , the low-salinity plume as defined by the $32.5 \%$ isohaline was much fresher and occupied a large area of the northern GSC. The southward volume transport of the plume was increased to $-0.12 \pm 0.06 \mathrm{~Sv}$ with a mean salinity of $31.4^{\circ} \%$ o almost twice as large as that in April, 1988. As we mentioned before, the difference in the transport of the low-salinity plume between these two years is mainly due to the increased freshwater river discharge in late spring, 1989. The transport of MIW was estimated from the flow of water with $\mathrm{T} \leq 4.8^{\circ}$ located between 40 and $120 \mathrm{~m}$ depths. There is a big difference in the southward transport between the April, 1988 and June, 1989 surveys. The southward transport of MIW was about $-0.31 \pm 0.38 \mathrm{~Sv}$ in April, 1988, while it increased to $-0.66 \pm 0.14 \mathrm{~Sv}$ in June, 1989. The larger southward transport of MIW in June, 1989 is due to the existence of the strong coastal jet flow over the western flank of the northern GSC. There is also a big difference in the net flux of MIW across the northern GSC between these two surveys. In April, 1988, the net flux across section E was northward, with a transport of $0.73 \pm 0.47 \mathrm{~Sv}$, while in June, 1989, it was southward, with a transport of $-0.015 \pm 0.056 \mathrm{~Sv}$. The large northward transport found in the upper $120 \mathrm{~m}$ in April, 1988 suggests an intrusion of the anticyclonic Georges Bank circulation into the northern GSC during the measurement time since part of the water in the eastern side of the GSC was different from that in the western side of the GSCA and can be traced back to Georges Bank. A strong southward wind higher than $15 \mathrm{~m} / \mathrm{s}$, recorded just before the April, 1988 survey, may have caused the westward shift of the anticyclonic gyre around Georges Bank, and hence increased the northward transport on the eastern side of the northern GSC. The 
small net southward transport found in June, 1989 suggests that most of the MIW was recirculated cyclonically along the local isobaths and only a small amount of MIW flowed southward through the GSC onto the outer shelf. This result is consistent with the $\mathrm{T} / \mathrm{S}$ analysis mentioned in section 2.2 . 


\subsection{Possible Relationship Between the High Concentration of Zooplankton and the Physical Environment in the Northern GSC}

Beardsley and Limeburner suggested that the unusually dense aggregations of the zooplankton Calanus finmarchicus, which occur in springtime in the GSC, may be directly or indirectly related to some unique aspect of the physical environment, such as tidal mixing, advection of the low-salinity plume, and lateral mixing between the low-salinity plume and Georges Bank water. In order to examine possible mechanisms for physical-biological interaction in the northern GSC during late spring, a variety of multiple measurements were made on the R/V Endeavor in SCOPEX; these include three regional CTD/ADCP surveys, several small-scale CTD tow-yo/ADCP surveys, and zooplankton sampling with both a MOCNESS (Multiple Opening and Closing Environmental Sampling System) and a towed $200-\mathrm{kHz}$ acoustic profiler. As an example, the small-scale spatial variability in stratification and zooplankton distribution near a feeding right whale in the GSC during June 2-3, 1989 was discussed by Beardsley et al. (1990). They found that at that time the zooplankton was concentrated in the main thermocline and halocline where a strong vertical shear of horizontal velocity occurred. Wishner et al. (1990) observed daily vertical migration of Calanus in the GSC in late spring of 1986-1989. Since these small animals can swim vertically at speeds of about $50 \mathrm{~m} /$ hour $(\sim 2 \mathrm{~cm} / \mathrm{s})$, they should be free from upwelling or downwelling. However, since the cause of such vertical migration is so poorly understood, we will focus our discussion here on the possible relationship between the large-scale horizontal distribution of zooplankton and the physical environment.

Zooplankton aggregations were found in different regions of the GSC in late spring for 1988 and 1989. The zooplankton was concentrated on the western flank of the GSC in April, 1988 but moved onto the western flank of Georges Bank in June, 1989 (Figure 1.6). This shift in location is consistent with the movement of the near-surface, low-salinity plume, which was limited to the western flank of the GSC during April, 1988 but extended eastward 

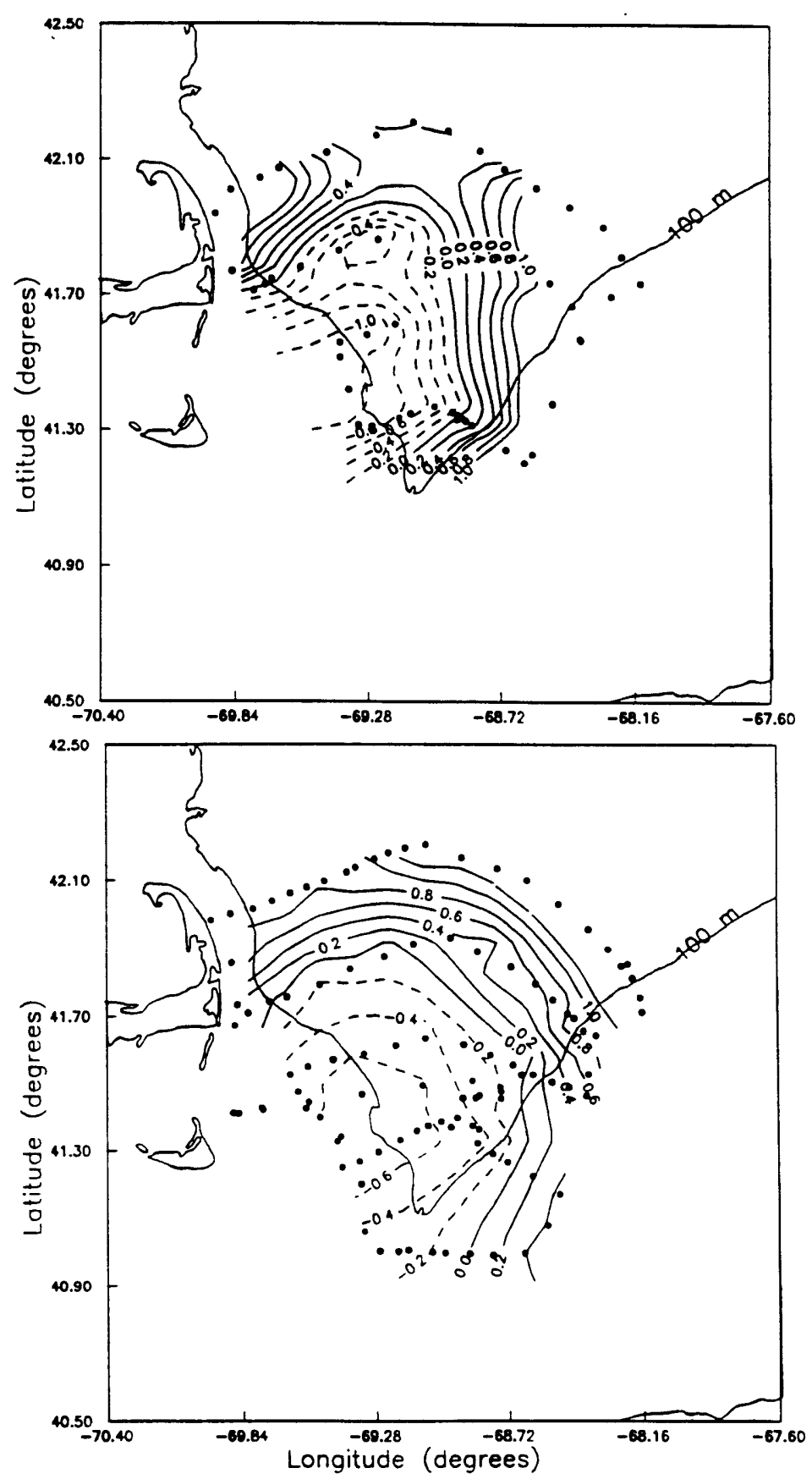

Figure 2.49: Distributions of fluid convergence (dashed lines) and divergence (solid lines) at $7 \mathrm{~m}$ during April, 1988 (left) and June, 1989 (right) CTD/ADCP surveys. The contours in the maps are plotted in a scale of $10^{-6} s^{-1}$. 
to the interior GSC during May-June, 1989 (Figure 2.4). These coherent pictures support Beardsley and Limeburner's second hypothesis that the advection of the low-salinity plume from the northwestern GOM may lead in part to the concentration of zooplankton near the salinity front in the GSC. The 1988 and 1989 SCOPEX drifter trajectories and ADCP currents clearly showed three different currents near the surface: (1) southward low-salinity current along the western flank of the GSC, (2) the cyclonic circulation along the local 100$m$ isobath across the GSC, and (3) the northeastward current around Georges Bank. This surface circulation pattern supports Beardsley and Limeburner's third hypothesis that the unusual concentration of zooplankton in the GSC may be in part caused by lateral mixing of the southward flowing GOM low-salinity plume and vertically well-mixed shelf water flowing northward through the GSC around Georges Bank. However, since this surface circulation pattern is a stable feature in the GSC, it does not contribute directly to the shift in the zooplankton concentration observed in 1988 and 1989. A V-shaped tidal front is observed in the shallow region less than $100 \mathrm{~m}$ across the Nantucket Shoals, GSC and Georges Bank (Figure 2.14). Although the oscillatory advection and fortnightly variation in the strength of tidal mixing may cause a $10-\mathrm{km}$ deviation of the location of the tidal front in the north-south direction, this front seems to be relatively stable in the GSC during late spring. This suggests that the tidal mixing does not contribute to the horizontal shift in the zooplankton concentration across the GSC from 1988 to 1989 , even though it may contribute to zooplankton concentration near the tidal front.

Olson and Backus (1985) developed a simple advection/diffusion model for the study of fish concentration in the frontal zone at the eastern edge of a warm-core Gulf Stream ring in April and June, 1982. They found that the abundance of fish in the ring can be accounted for by fluid convergence. The fluid convergence in the GSC can be calculated using the fitted 1988 and 1989 SCOPEX ADCP residual current fields. Since the uncertainty of these calculations in some areas of the GSC is comparable in magnitude to the estimated values, we will focus our discussion only on the qualitative pattern. A large convergence area was found near the surface in the GSC in April, 1988 and June, 1989 (Figure 2.49). 
In April, 1988, the convergence area was confined to the western flank of the GSC, while in June, 1989, it spread eastward to the western flank of Georges Bank as the low-salinity plume extended offshore in late spring. Compared with the distribution of zooplankton, the maximum convergence was found at the highest zooplankton concentration on the western flank of the GSC in April, 1988 but in June, 1989, the highest concentration of zooplankton was found in the convergence region but not at the maximum convergence. This suggests that the near-surface fluid convergence may be a necessary but insufficient condition for the development of zooplankton aggregations in the GSC during late spring. 


\subsection{Conclusion and Discussion}

As components of the main field experiment of SCOPEX, three regional CTD/ADCP surveys coupled with satellite-tracked drifter deployments were conducted in the northern GSC during April 26-27, 1988, May 18-21 and June 6-12, 1989, to investigate the variability of water properties and local circulation in the region of enhanced biological productivity. The main results are summarized here.

The surface salinity patterns observed in late April, 1988 and May-June, 1989 differ significantly in the extent of the freshwater plume which occurs east of Cape Cod in spring. In April, 1988, the surface plume was just beginning to form along the outer coast of Cape Cod while three to six weeks later in 1989, the minimum salinity was about $1.5^{\circ} \%$ less and a large pool of water fresher than $31.6^{\circ} \%$ ood pushed east over much of the northern GSC region. The difference in the amount of freshening observed between the two surveys is due primarily to the six-week difference in the seasonal cycle and the increased river discharge in 1989. The seasonal offshore extrusion of the low-salinity plume may be driven by either an upwelling-favorable wind stress or a deep cyclonic circulation. A significant difference was also found at mid-depth in the MIW in late spring during the 1988 and 1989 surveys. In April, 1988, the seasonal pycnocline was just beginning to form, and the spatial structure of MIW was relatively uniform. In May-June, 1989, a narrow core of temperature minimum water (with $T_{\min }$ in a range of $3.2^{\circ}$ to $4.4^{\circ} \mathrm{C}$ ) was found along the western flank of the northern GSC between 40 and $120 \mathrm{~m}$. This colder, fresher, and lighter water spread to mix with the interior MIW as the core flowed southward into the northern GSC.

During each regional hydrographic or small-scale biological survey, continuous acoustic Doppler current profiler data were collected using the R/V Endeavor $150-\mathrm{kHz}$ ADCP. The relatively shallow depth of the SCOPEX region allowed bottom tracking during the entire survey (except in a small region of the April, 1988 survey) so that high-quality 
absolute current profile data over the upper $140 \mathrm{~m}$ were obtained. Instantaneous absolute currents in the GSC were dominated by a strong tidal signal so that an empirical least squares fit method was used to separate the absolute ADCP current into tidal and residual current components for each survey and anchor stations. The resulting field of vertically averaged residual flow shows a cyclonic circulation pattern in the northern GSC, with strong currents in excess of $10 \mathrm{~cm} / \mathrm{s}$ flowing southward and southeastward along the western flank of the northern GSC, and turning northeastward to flow along the western flank of Georges Bank. This cyclonic circulation was found at all levels throughout at least the upper $140 \mathrm{~m}$, with maximum inflow and outflow occurring at the western and eastern ends of the crosschannel transects, respectively. There were two velocity maxima in the vertical: one was near the surface to drive the surface water, the other was at mid-depth to carry MIW into the northern GSC region. The residual ADCP current patterns are consistent with the vertical distributions of water masses and the trajectories of the satellite-tracked drifters drogued at 5 and $50 \mathrm{~m}$ in the GSC.

Significant differences were found in the southward transport of the low-salinity plume and MIW between April, 1988 and June, 1989. In April, 1988, the southward transports of low-salinity plume water and MIW were about $-0.07 \pm 0.03 \mathrm{~Sv}$ and $-0.31 \pm$ $0.38 \mathrm{~Sv}$, respectively, while in June, 1989 , they increased to about $-0.12 \pm 0.06 \mathrm{~Sv}$ and $-0.66 \pm 0.14 \mathrm{~Sv}$, respectively. The larger transport of low-salinity plume water and MIW found in June, 1989 are due to the increased freshwater river discharge and occurrence of the deep coastal jet current along the western flank of the GSC.

Comparison between the vertical shears of the geostrophic and residual currents on the northernmost CTD transect for the June, 1989 survey shows good agreement between geostrophic and residual shears in the range of MIW on both ends of the transect. Direct comparison between the ADCP mean flow and tidally rectified flow predicted numerically by Greenberg (1983) suggests that the residual current is mainly driven by tidal rectification 
over the shallower sides of the GSC and by buoyancy forcing over the deep flanks and in the central region of the GSC.

A relatively permanent $\mathrm{V}$-shaped thermal front was located in the shallow region of the GSC (less than $100 \mathrm{~m}$ ) across Nantucket Shoals, GSC, and Georges Bank during late spring of 1988 and 1989. The location of this front moved roughly $10 \mathrm{~km}$ in the north-south direction, most likely due to oscillatory tidal advection and monthly/fortnightly variation in the strength of tidal mixing in the GSC.

The dense aggregations of the zooplankton Calanus finmarchicus in the northern GSC during late spring appears to be related to the regional circulation. In particular, a large scale near-surface convergence appears to be a necessary condition for the high concentration of zooplankton, while the horizontal shift of the region of high zooplankton concentration between 1988 and 1989 is associated with the movement of the near-surface, low-salinity plume.

Finally, it should be pointed out that the formation of the coastal jet-like current found at mid-depth of the northern GSC has not been clearly described in this work because of limited observations. We do not know yet why the narrow core of temperature minimum water occurred in late spring 1989 but not in 1988. Is it related to the increase of freshwater river discharge in the western GOM that occurred in 1989, or to the difference in the surface heat fluxes during the proceeding winters, or to the seasonal evolution of a coastal-trapped jet which forms upstream in May? To answer these questions, we need to make more CTD and ADCP observations (over at least two years) in the western GOM, particularly in the upstream region of the northern GSC. 


\section{Chapter 3. Numerical Study of Stratified Tidal Rectification Over Georges Bank}

\subsection{Introduction}

Long-term direct Eulerian and Lagrangian current measurements summarized by Butman et al. (1982) clearly demonstrate a clockwise circulation around Georges Bank that varies seasonally with a maximum in the along-bank flow in summer and fall and a minimum in winter. Trajectories of the 5-m SCOPEX 1989 drifters taken by Limeburner and Beardsley (1989) nicely illustrate the partially closed nature of the along-bank circulation in summer (hence the term gyre) caused by re-circulation of water, flowing southwestward along the southern flank and turning northward through the GSC to feed the narrow northeastward jet on the northern flank. This re-circulation appears most pronounced at the surface in late summer and fall when the vertical density stratification is strongest. Seasonal intensification of the along-bank mean circulation is associated with the seasonal variability of the stratification over the Bank. Hydrographic observations show tidal mixing fronts between the vertically well-mixed and stratified waters over Georges Bank and a shelf/slope front between the relatively fresh shelf water and the more saline upper slope water at the southern edge of the Bank. The location of tidally induced fronts also varies seasonally over Georges Bank (Flagg, 1987). During late spring and summer, the fronts are located along the 40-m isobath on the northern flank and along the 50-60-m isobath on the southern flank. During winter, however, the tidal front disappears on the southern flank and is much weaker on the northern flank, even though the position of this front remains almost stationary on the northern edge of the Bank. The shelf/slope front occurs at the shelf break near the 100 -m isobath on the southern flank, and moves steadily onshore from winter to summer and then suddenly adjusts offshore as fall turns to winter (Flagg, 1987). As a result, the along-bank residual flow intensifies during summer and fall on the northern 
edge of the Bank, over the top of the Bank, and near the shelf break on the southern flank where the stronger tidal and shelf/slope fronts are located.

Theoretical studies predict that an along-bank residual flow can be generated by tidal rectification, which transfers tidal vorticity and momentum into the mean current field as a long barotropic tidal wave propagates from the deep ocean onto a variable bottom topography such as Georges Bank (Huthnance, 1973, 1981; Loder, 1980; Wright and Loder, 1985; Zimmerman, 1978, 1980). The seasonal intensification of the mean aroundbank flow over Georges Bank is believed mainly due to seasonal variation in stratification associated with surface heating and strong tidally induced vertical mixing over the Bank (Loder and Wright, 1985; Maas and Zimmerman, $1989 a$ and $b$ ). ${ }^{1}$ However, analytical models published to date do not provide a complete picture of tidally rectified flow over Georges Bank, especially the detailed structure of residual currents influenced by finite topography, stratification, and tidal mixing. The large and asymmetrical bottom slope on the sides of Georges Bank may cause a horizontally asymmetrical structure of residual flow over Georges Bank through a large cross-bank divergence of the tidal momentum flux and strong turbulence over the Bank. Effects of stratification on the vertical structure and strength of tidally rectified flow can be produced by either internal tidal generation and rectification or a tide-induced mixing front. As a barotropic tidal wave propagates onto the slope, the topography-induced vertical velocity will displace the isopycnals over the slope, producing an oscillating buoyancy force to generate internal waves at the tidal frequency, i.e, the internal tides (Wunsch, 1975; Baines, 1982). In a nonlinear system, the nonlinear interaction between barotropic and internal tidal currents as well as between internal tidal currents can transfer momentum from tidal frequency to mean field, generating the residual currents over the topographic feature. On the other hand, the strong tidal current will mix the water over the shelf and then create density fronts on both sides of the bank to separate the vertically well-mixed water over the shelf from the stratified water on the slope. The existence of

\footnotetext{
${ }^{1}$ Note: The intrusion of the slope water through the buoyancy-driven shelf/slope front may also contribute to the intensification of along-bank mean current on the southern flank. We have not included it here because we want to focus our study on the influence of stratification on tidal rectification.
} 
tide-induced fronts on the shelf can either produce an along-isobath buoyancy-driven mean current by the horizontal density gradient or act like a sponge to absorb and dissipate all energies of internal tidal waves propagating onto the shelf. Since all of these dynamical processes are coupled in a strongly nonlinear way, the relative importance of the effects of local bottom topography, stratification and tidal mixing on the tidal rectification are still unclear. For this reason, we have used the primitive equation model developed by Blumberg and Mellor (1987) to study the influence of stratification on tidal rectification over Georges Bank.

To simplify our scientific problem, we have developed a two-dimensional version of the ECOM3D-SI by introducing periodic boundary conditions in the along-bank direction and considering only a thin slice of the three-dimensional model in the cross-bank direction. A barotropic tidal forcing is imposed at the seaward open boundary by specifying a free surface elevation as a cosine function of time. Also, a gravity wave radiation boundary condition plus a sponge layer is added at both seaward and coastal open boundaries to allow barotropic and internal waves to propagate continually out of the study domain without reflection (Chapman, 1985). In order to understand the simple dynamics, we first conduct a sequence of initial value experiments for the two-dimensional unstratified tidalrectification problem (associated with the propagation of a single semidiurnal tidal wave over a finite-amplitude symmetrical bank), and then add stratification to investigate the effects of stratified tidal rectification and tidal mixing on the structure of the residual flow. Finally, we apply this model to the asymmetric bottom topography of Georges Bank and then compare the model results with observations.

This chapter consists of seven sections. In section 3.2 , we will review previous analytical and semi-analytical theories of tidal rectification over variable bottom topography. In section 3.3, we will describe the Blumberg and Mellor numerical model in detail and discuss the turbulent mixing mechanism involved in the Mellor and Yamada (1974 and 1982) turbulent boundary layer model. The barotropic tidal-rectification process over a 
symmetrical topographic bank will be discussed in section 3.4 , in which the influence of the size and shape of the bank on the residual flow will be studied, and the parameterization of vertical eddy viscosity in the Mellor and Yamada turbulent closure model will be checked using tidal observations in the limit of no stratification. The sequence of initial value experiments conducted in section 3.4 will be repeated in section 3.5 for given initial stratification in which stratified tidal rectification and tidal mixing will be discussed. In section 3.6, we will apply the model to study stratified tidal rectification over Georges Bank using realistic topography and winter and summer stratification. Conclusions and discussion will be given in section 3.7, in which some limits of our model will be discussed and some future works will be described. 


\subsection{Review and Discussion of Previous Theoretical Works}

The basic mechanism of tidal rectification over variable bottom topography was first studied by Huthnance (1973), who showed that an along-isobath residual current can be generated against bottom friction in a two dimensional (i.e., no along-isobath variation) homogeneous fluid by a nonlinear transfer of the momentum of oscillating tidal currents to mean flow. Loder (1980) extended Huthnance's model to consider the depth-independent tidal-rectification process over Georges Bank. Using simple scaling analysis for the momentum balance, he showed that the tidally rectified flow can be produced only when the tidal wave length is much longer than the scale of bottom topography. As a barotropic semidiurnal tidal current oscillates across Georges Bank, rotation, mass continuity and friction can cause the cross-isobath momentum flux of along-bank tidal current to diverge on the side of positive gradient bottom slope and to converge on the side of negative gradient bottom slope, thus driving a clockwise mean circulation around the Bank against friction. The time-averaged, along-bank momentum balance in this depth-independent model is simply given by

$$
\frac{\partial}{\partial x}(h \overline{U V})=-k \bar{v}
$$

where $x$ is the cross-isobath coordinate, $U$ and $V$ are the $x$ and $y$ components of tidal currents, $\bar{v}$ is the along-isobath residual flow, $h$ is the wave depth, $k$ is a linear bottom friction coefficient, and the overbar indicates the time average over a tidal cycle.

This mechanism can be alternatively interpreted using the vorticity argument. Since the vertical integral volume transport remains unchanged across the Bank when the topographic scale is much shorter than the tidal wave length, the time-averaged along-bank momentum balance can be rewritten by

$$
h \overline{U \omega}=-k \bar{v}
$$


where $\omega=\partial V / \partial x$ is relative vorticity. This momentum balance suggests that the alongbank mean current can be generated by the transfer of relative vorticity from oscillating tidal currents to mean flow if there is a net cross-bank flux of tidal vorticity over a tidal cycle (Robinson, 1981; Zimmerman, 1978, 1980). For a constant $k$, the relative vorticity of the mean current is generated by a net cross-bank advection of relative tidal vorticity against friction over a tidal cycle since

$$
\overline{h U \frac{\partial \omega}{\partial x}}=-k \bar{\omega}
$$

where $\bar{\omega}$ is mean vorticity.

The energy equation in the mean field for oscillating tidal rectification is rather simple, which is characterized by the balance between the cross-bank advection of total potential and kinetic energies and the dissipation of kinetic energy, i.e.,

$$
\overline{h u \frac{\partial}{\partial x}\left(q^{2}+g \zeta\right)}=-k \overline{q^{2}}
$$

where $q^{2}=\frac{1}{2}\left(u^{2}+v^{2}\right)$ is the kinetic energy and $u$ and $v$ are $x$ and $y$ components of both tidal and mean velocities. This simple energy balance also suggests that the kinetic energy of mean flow is supported by the cross-bank advection of total energy against dissipation. Based on these simple dynamic balances, Loder (1980) used the harmonic truncation method to solve this problem analytically and predicted the tidally rectified current peak of order $8 \mathrm{~cm} / \mathrm{s}$ and $16 \mathrm{~cm} / \mathrm{s}$ on the southern and northern flanks of Georges Bank, respectively (Figure 3.1). Loder also applied this method to study analytically the tidal rectification on a step topography and found that the along-isobath residual flow reaches its maximum at the step and exponentially decays to both sides. This result provides a good estimate for the along-bank transport of residual current but it gives a wrong impression of the width of residual flow over a step (Young, 1983). In fact, the topographic tidal-rectification process 

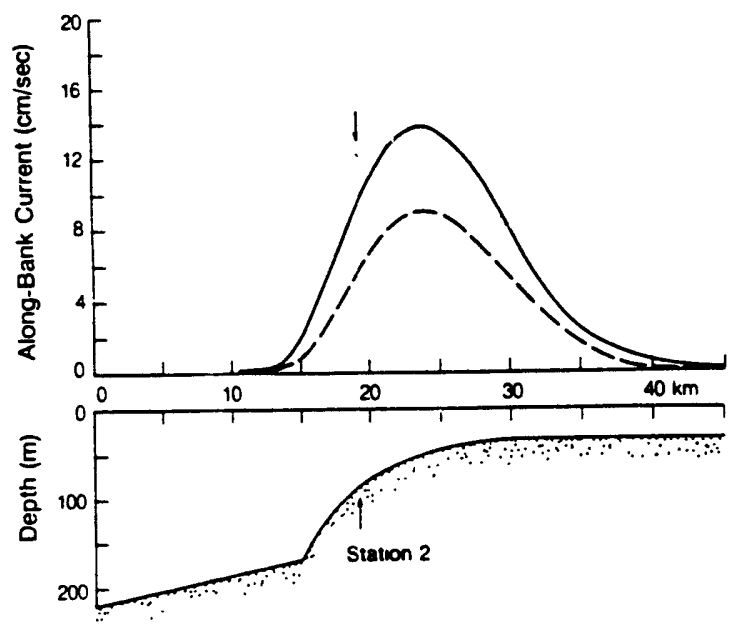

(a)
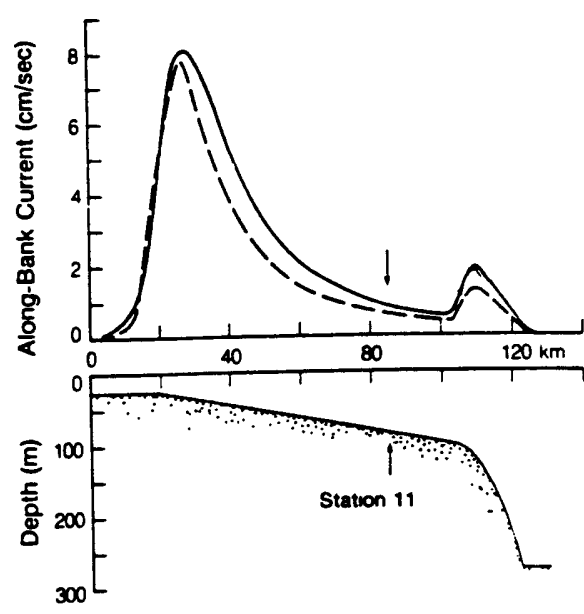

(b)

Figure 3.1: Along bank mean Eulerian (solid line) and mean Lagrangian (dashed line) currents $(\mathrm{cm} / \mathrm{s})$ on the northern (a) and southern (b) flanks of Georges Bank as predicted by Loder's (1980) idealized depth independent model of tidal rectification with the interaction of mean and tidal currents included. The deep water forcing parameters (in Loder's notation) are $H_{d}=220 \mathrm{~m}, U_{d}=13 \mathrm{~cm} / \mathrm{s}, R=0.5$, and $\phi_{r}=-\pi / 9$ for the northern flank and $H_{d}=276 \mathrm{~m}, U_{d}=10 \mathrm{~cm} / \mathrm{s}, R=0.75$, and $\phi_{r}=-\pi / 2$ for the southern flank (Butman et al., 1987)

over a step topography only involves water parcels within one tidal excursion length from the step. There should be no change of relative vorticity observed beyond the distance of one tidal excursion from the step, and hence no residual flow should be generated there (Maas and Zimmerman, 1987). The cross-step exponential decay structure of residual current found by Loder (1980) depends critically upon the harmonic truncation method. Scaling analysis argues that this method is not appropriate for a step topography because it is valid only when the length scale of the slope is much greater than the length of a tidal excursion (i.e., $L_{e} / 2 L_{t} \ll 1$, where the cross-isobath tidal excursion length $L_{e}=2 U / \sigma$ and the length scale of the slope $\left.L_{t}=h(d h / d x)^{-1}\right)$.

The vertical structure of tidally rectified flow is mainly characterized by friction and stratification. Wright and Loder (1985) examined the frictionally induced vertical structure of residual flow due to tidal rectification in a two-dimensional homogeneous fluid using a simplified depth-dependent model. The model neglects the interaction of tidal and mean flows on an assumption of weak nonlinearity and is closed using an artificial 
assumption of zero depth averaged mean flow across the isobath. Tidal motion in this model is linear for a uniform and time-independent vertical eddy viscosity which ignores feedback effects from mean flow to tidal currents. Applying this model to Georges Bank, Wright and Loder found that the predicted along-bank flow is in a clockwise sense at all depths with a maximum at the surface and it generally decays monotonically with depth. However, the vertical structure of the cross-isobath mean current predicted in their model is very sensitive to the parameterization of vertical eddy viscosity, which is dominated by a high modal pattern for small Ekman number but tends to vanish as the Ekman number become larger. This fact poses a difficult problem in the numerical modeling of tidal rectification without a turbulent closure model, especially in predicting the cross-bank mean flow, because we do not really know which appropriate form of vertical eddy viscosity is most relevant for the model.

As stratification is added, the vertical structure of the residual current can be modified by a tide-induced front due to turbulent mixing (Loder and Wright, 1985), internal tidal generation over the slope (Maas and Zimmerman,1989a and $b$ ), and modified internal and bottom friction due to stratification (Loder and Wright, 1985; Tee, 1985). Garrett and Loder (1981) developed a steady analytically tractable diagnostic model for the quasi-geostrophic mean circulation associated with a density front on the slope in a twodimensional continuously stratified fluid. This linear model predicts the geostrophic flow along the front driven by the horizontal gradient of density and a double cell circulation pattern across the front caused by the pressure gradient force against friction. Coupling linearly the diagnostic frontal model into the depth-dependent model with a zero across-bank flux of vertical integral transport, Loder and Wright (1985) studied effects of the density front on the tidally rectified currents. They found that the along-bank mean flow intensifies at the surface due to the density front and a relatively strong cross-bank double cell circulation occurs on either side of the Bank (Figures 3.2 and 3.3). However, linear superposition of frictionally controlled, tidally-rectified, and density front-induced currents results in two surface-intensified maximum cores of along-bank mean current on the northern flank of the 

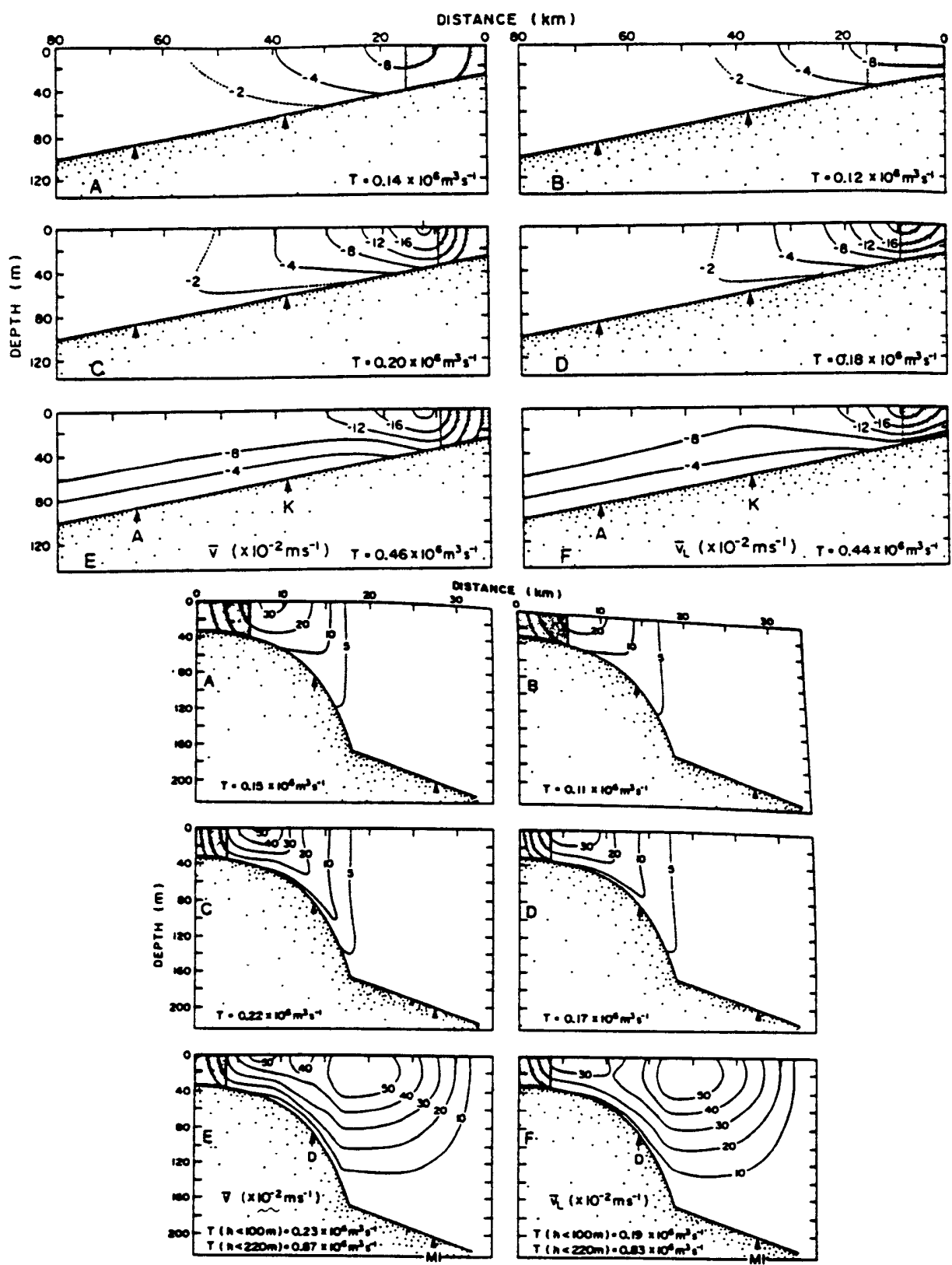

Figure 3.2: Model predictions of along-isobath mean currents and transports, $\mathrm{T}$, on the open ocean side of Georges Bank (upper) and on the northwestern side of the Bank (lower). Eulerian (A, C, E) and Lagrangian $(B, D, F)$ results are shown for the three cases: $(A, B)$ wintertime currents associated with tidal rectification - no stratification influence and $u_{\star_{w}}=1.8 \mathrm{~cm} / \mathrm{s} ;(C, D)$ summertime currents associated with tidal rectification $-K_{m}$ reduced and $u_{\star_{w}}=1.1 \mathrm{~cm} / \mathrm{s} ;$ and $(\mathrm{E}, \mathrm{F})$ superposition of the summertime currents associated with tidal rectification and density-driven frontal circulation with friction parameters as in (C, D). The shaded areas indicate the region in which the strength of vertical mixing may be over-estimated (Loder and Wright, 1985). 

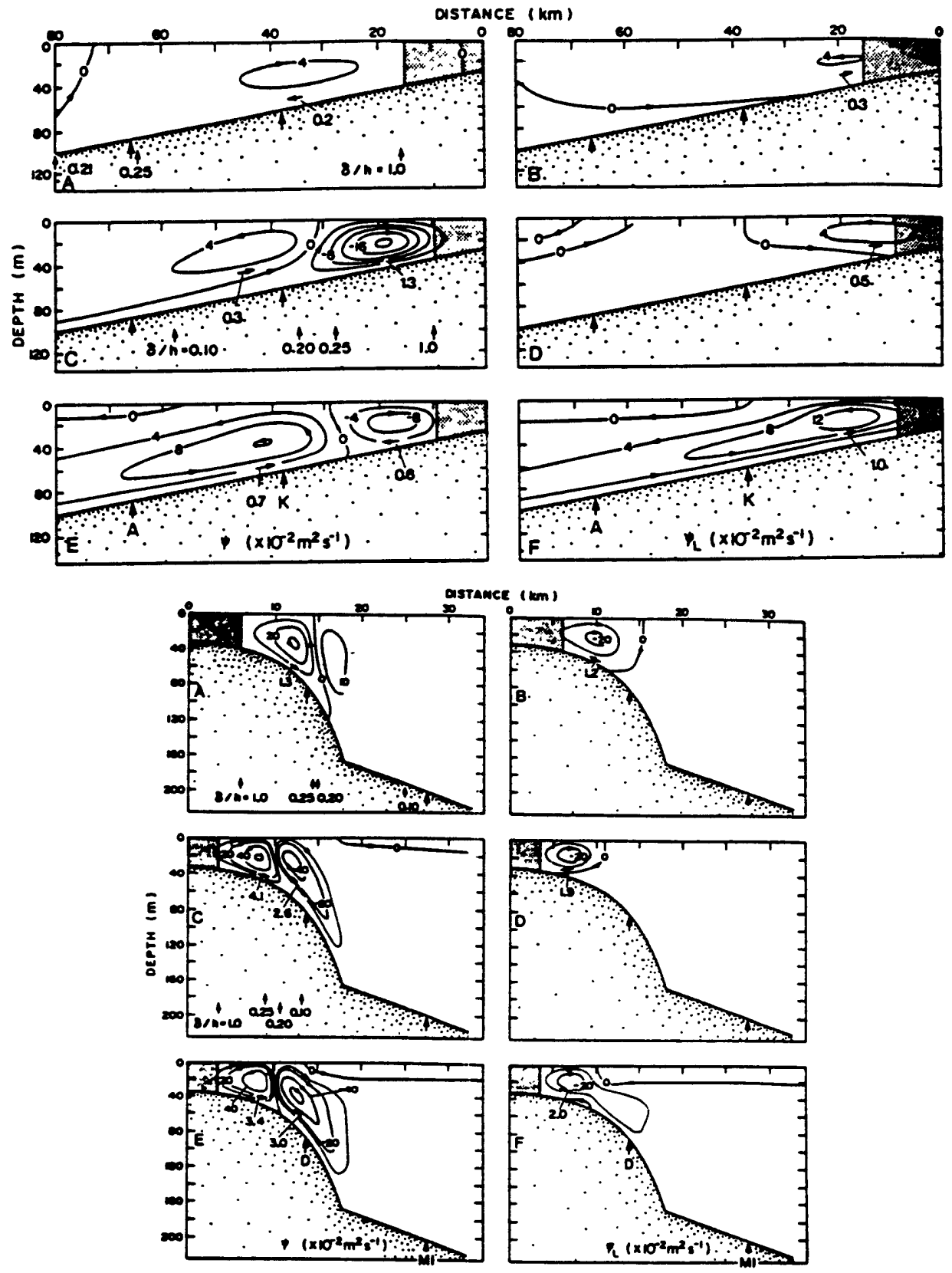

Figure 3.3: Stream functions for cross-isobath Eulerian (A, C, E) and Lagrangian (B, D, F) currents on the open ocean (upper) and northwestern (lower) sides of Georges Bank for the three cases in Figure 3.2. The shaded areas indicate the regions in which the strength of vertical mixing may be over-estimated. The bold numbers by the current vectors indicate the speed in units of $10^{-2} \mathrm{~m} \mathrm{~s}^{-1}$ (Loder and Wright, 1985). 
Bank. One is located at the $40-\mathrm{m}$ isobath due to frictionally controlled tidal rectification and the other is near the shelf break at the $200-\mathrm{m}$ isobath due to the density front. This structure of an along-bank mean current is poorly supported by the observations on the northern side of Georges Bank where the along-bank mean current flows northeastward as a single along-bank jet (Loder et al., 1992). In fact, the stratified tidal-rectification process is a nonlinear problem so that friction and density-front-induced currents may not be reproduced in detail with such a linear superposition.

Maas and Zimmerman (1989a and $b$ ) recently investigated internal tidal rectification using multiple scale analysis. Based on two different length scales of barotropic and internal tidal waves, they considered the oscillation of a barotropic tidal current in a linearly stratified fluid over small-amplitude bottom topography. This problem is analytically tractable provided that friction is weak and the feedback influence of residual flow to tidal current is ignored. Interaction of barotropic tidal currents over topography in a linearly stratified fluid produces a damped propagating internal tidal wave across the variable bottom topography and then the nonlinear interaction between either the barotropic and internal tidal currents or internal tidal currents themselves causes a non-propagating transient which generates the along- and cross-isobath tidally rectified flow over the topography. Unlike the homogeneous case, the structure of the harmonic and residual currents critically depends on the strength of stratification which causes the internal tidal and residual currents to be bottom intensified as the internal Rossby deformation radius $\left(\ell_{i}=\mathrm{NH} / \mathrm{f}\right)$ approaches and exceeds the excursion amplitude of the barotropic tidal current $\left(\ell_{0}=U / \sigma\right)$ or the topographic length scale $\left(\ell_{t}\right)$ (for example, see Figure 3.4). These features may be applicable for Georges Bank, especially on the northern flank where $\ell_{i}$ is estimated to be equal or larger than $\ell_{o}$. However, direct application of Maas and Zimmerman's model to study stratified tidal rectification over Georges Bank is impossible both because of the limitations of small-amplitude topography and weak nonlinearity and the absence of tidal mixing. 

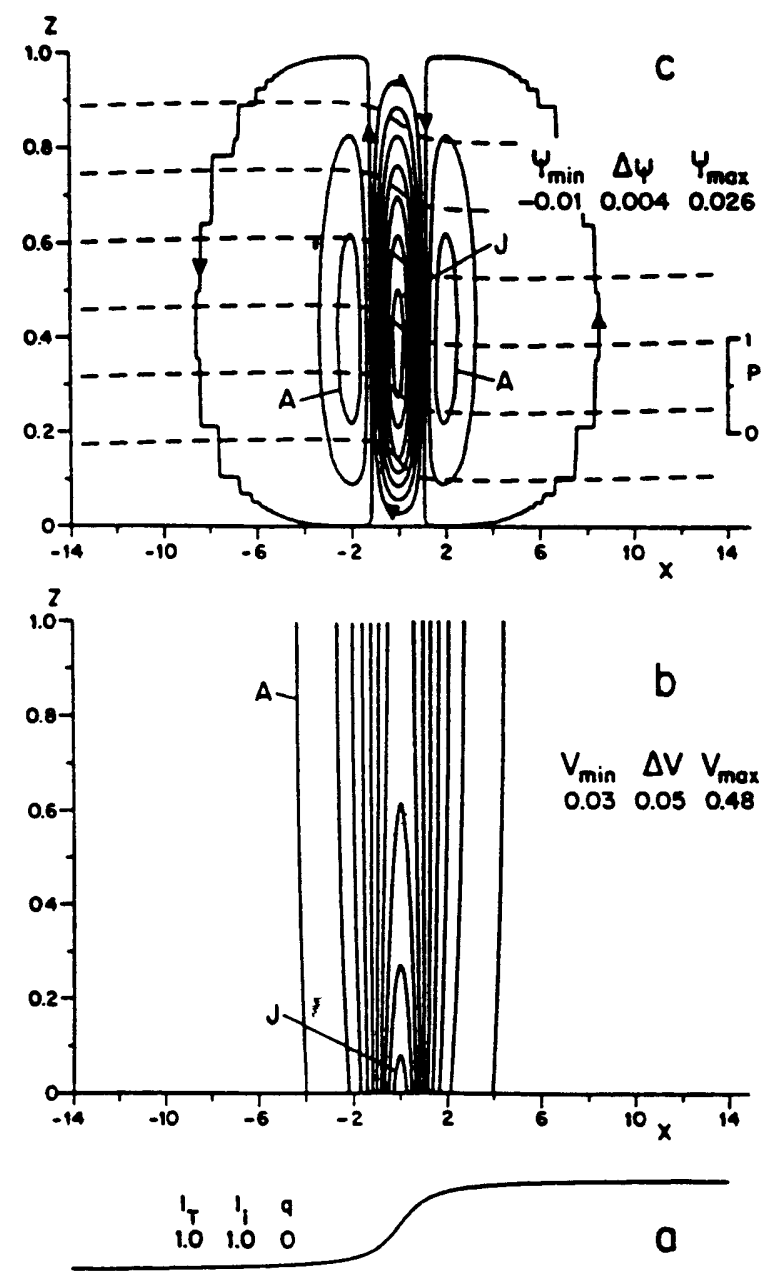

Figure 3.4: (a) Topography; (b) along-isobath residual velocity $V$; and (c) streamfunction $\Psi$ (solid line) and pressure field (dashed lines) as a function of $\mathrm{x}=x_{\star} / \ell_{0}$ and $\mathrm{z}=z_{\star} / H_{0}$ for the case of $\ell_{t}=\ell_{0}=\ell_{2}$, where $\ell_{t}, \ell_{0}$ and $\ell_{1}$ are the topographic length scale, the barotropic tidal excursion, and the internal Rossby radius deformation, respectively (Haas and Zimmerman, 1989b). 
The effects of the strength of friction in a stratified fluid were examined by Loder and Wright (1985), who showed an increase in the along-and cross-isobath residual currents when friction is reduced due to stratification (see Figures 3.2 and 3.3). A similar result was also found by Tee (1985) in a study of the sensitivity of residual flow to different values and forms of vertical eddy viscosity. Over Georges Bank, in addition to increasing the magnitude of the residual flow, the reduction of friction due to stratification may modify the structure of the cross-isobath residual current, especially over the southern flank of the Bank where the bottom slope is small (see Figures 3.2 and 3.3).

It should be mentioned here that some efforts have also been made to study the structure of mean currents flowing along Georges Bank due to local (Hopkins and Garfield, 1981; Brink, 1983) or regional wind stress (Csanady, 1974; Beardsley and Haidvogel, 1981; Greenberg, 1983) and intrusion of shelf break water (Flagg, 1977; Flagg et al., 1982; Gawarkiewicz and Chapman, 1992; Wright et al., 1986). Since our interest here is the tidal-rectification process, we will not give a detailed discussion of these different mechanisms in this section.

Although much work has been done on the subject of tidal rectification over variable bottom topography, there are still gaps in our understanding, especially about the combined influence of internal tidal interaction and tidal mixing on the vertical structure and strength of residual flow over bottom topography. Analytical theory is good to study the basic mechanisms of residual flow generation, however, it is not tractable for more realistic cases with strong nonlinear interaction. For this reason, a three-dimensional numerical model developed by Blumberg and Mellor (1987) is used here to study the stratified tidal rectification over Georges Bank. 


\subsection{The Numerical Model}

The Blumberg and Mellor (1991) model ECOM3D-SI is a three-dimensional Boussinesq, hydrostatic nonlinear coastal ocean circulation model. It incorporates Mellor and Yamada's (1982) level $2 \frac{1}{2}$ turbulent closure model to provide a realistic parameterization of vertical mixing and a free surface to simulate surface wave propagation such as tides and long gravity waves. A $\sigma$-coordinate transformation is used in the vertical and a curvilinear coordinate system in the horizontal, which allow smooth representation of finite-amplitude irregular bottom topography and real coastal geometry. The model formulation is described in detail by Blumberg and Mellor (1987) and a new version of the model with a semi-implicit numerical scheme (called ECOM3D-SI) is described by Blumberg (1991).

Garrett (1972) and Greenberg (1979) showed that the GOM is nearly resonant at the $\left(M_{2}\right)$ semidiurnal tidal period, resulting in a large oscillating inflow and outflow from the adjacent North Atlantic across Georges Bank into the rest of the GOM. The cotidal and phase lines of the $M_{2}$ tidal component are almost parallel to local isobaths around Georges Bank, varying from about $10 \mathrm{~cm} / \mathrm{s}$ outside of the southern and northern flanks to about $100 \mathrm{~cm} / \mathrm{s}$ on top of the Bank. Brown (1984) made a diagnostic analysis of tidally driven forces using current and bottom pressure data. He found that the $M_{2}$ tide behaves as a progressive inertial gravity wave propagating directly across Georges Bank, with the momentum balance among the tidal acceleration, Coriolis, and pressure gradient terms in the cross-bank direction and between the inertial and Coriolis terms in the along-bank direction. These results encourage us to simplify our study of stratified tidal rectification to a two-dimensional problem in which the along-isobath variation for all independent variables is ignored. To do this mathematically, we simply consider a thin slice of the model domain in the cross-bank direction and impose periodic boundary conditions in the along-bank direction. 
The governing equations for the two-dimensional version of the ECOM3D-SI consist of three momentum, an incompressible continuity, and conservative potential temperature and salt equations. The Boussinesq approximation is used to simplify the momentum equations in which the density variation is neglected for momentum balance except its contribution to the buoyancy body force in the vertical. Hydrostatic balance is assumed in the model, which implies the hydrostatic stability in the vertical motion where the free convection due to gravitational buoyancy force is balanced by the vertical pressure gradient. Potential density is evaluated as a function of potential temperature and salinity and is calculated using an equation of state developed by Fofonoff (1962). In a system of orthogonal Cartesian coordinates with $x$ increasing northward (in the cross-isobath direction), $y$ increasing westward (in the along-isobath direction), and $z$ increasing upward, the primitive equations in a two-dimensional form $(\partial / \partial y=0)$ can be written as

$$
\begin{aligned}
\frac{\partial u}{\partial t}+u \frac{\partial u}{\partial x}+w \frac{\partial u}{\partial z}-f v & =-\frac{1}{\rho_{o}} \frac{\partial P}{\partial x}+\frac{\partial}{\partial z} K_{m} \frac{\partial u}{\partial z}+\mathbf{F}_{\mathbf{u}} \\
\frac{\partial v}{\partial t}+u \frac{\partial v}{\partial x}+w \frac{\partial v}{\partial z}+f u & =\frac{\partial}{\partial z} K_{m} \frac{\partial v}{\partial z}+\mathbf{F}_{\mathbf{v}} \\
\frac{\partial P}{\partial z} & =-\rho g \\
\frac{\partial u}{\partial x}+\frac{\partial w}{\partial z} & =0 \\
\frac{\partial \theta}{\partial t}+u \frac{\partial \theta}{\partial x}+w \frac{\partial \theta}{\partial z} & =\frac{\partial}{\partial z} K_{H} \frac{\partial \theta}{\partial z}+\mathbf{F}_{\theta} \\
\frac{\partial s}{\partial t}+\frac{\partial s}{\partial x}+w \frac{\partial s}{\partial z} & =\frac{\partial}{\partial z} K_{H} \frac{\partial s}{\partial z}+\mathbf{F}_{\mathbf{S}} \\
\rho & =\rho(\theta, s)
\end{aligned}
$$

where $u, v$ and $w$ are the $x, y$, and $z$ velocity components, $\theta$ the potential temperature, $s$ the salinity, $p$ the pressure, $f$ the Coriolis parameter, $g$ the gravitational acceleration, $K_{m}$ the vertical eddy viscosity coefficient, $K_{H}$ the thermal vertical eddy friction coefficient. $\mathbf{F}_{\mathbf{u}}, \mathbf{F}_{\mathbf{v}}, \mathbf{F}_{\theta}$ and $\mathbf{F}_{\mathbf{s}}$ represent the horizontal momentum, thermal and salt diffusion terms. $\rho$ and $\rho_{o}$ are the perturbation and reference densities, which satisfy

$$
\rho_{\text {total }}=\rho_{o}+\rho
$$


The equations are not mathematically closed because there are nine unknown variables in seven equations. A second order turbulent closure scheme (level $2 \frac{1}{2}$ ) developed by Mellor and Yamada (1974 and 1982) is introduced into the model in which the vertical eddy viscosity and diffusion coefficients are parameterized based on turbulent kinetic energy and turbulent macroscale equations. Unlike the level 2 model where the turbulent production term is balanced by the turbulent dissipation term, the level $2 \frac{1}{2}$ model describes a timedependent turbulent mixing process in which the local variation of turbulent kinetic energy depends not only on the turbulent production against dissipation but also on the horizontal and vertical advections of turbulent kinetic energy. Under the boundary layer approximation where the shear production of turbulent energy can be neglected except in the vertical, the two-dimensional turbulent kinetic energy and turbulent macroscale equations can be simplified as

$$
\begin{gathered}
\frac{\partial q^{2}}{\partial t}+u \frac{\partial q^{2}}{\partial x}+w \frac{\partial q^{2}}{\partial z}=2\left(P_{s}+P_{b}-\varepsilon\right)+\frac{\partial}{\partial z}\left(K_{q} \frac{\partial q^{2}}{\partial z}\right)+F_{q} \\
\frac{\partial q^{2} \ell}{\partial t}+u \frac{\partial q^{2} \ell}{\partial x}+w \frac{\partial q^{2} \ell}{\partial z}=\ell E_{1}\left(P_{s}+P_{b}-\varepsilon \widetilde{W}\right)+\frac{\partial}{\partial z}\left(K_{q} \frac{\partial q^{2} \ell}{\partial z}\right)+F_{\ell},
\end{gathered}
$$

where $q^{2}=\frac{1}{2}\left(u^{\prime 2}+v^{2}\right)$ is the turbulent kinetic energy, $\ell$ is the turbulent macroscale, $K_{q}$ is the eddy diffusion coefficient of the turbulent kinetic energy, $F_{q}$ and $F_{\ell}$ represent horizontal diffusion of the turbulent kinetic energy, $P_{s}$ is the shear production of turbulent energy defined as

$$
P_{s}=K_{M}\left[\left(\frac{\partial u}{\partial z}\right)^{2}+\left(\frac{\partial v}{\partial z}\right)^{2}\right]
$$

$P_{b}$ is buoyancy production of turbulent energy defined as

$$
P_{b}=\frac{g}{\rho} K_{H} \frac{\partial \rho}{\partial z}
$$


$\varepsilon$ is the turbulent energy dissipation given by

$$
\varepsilon=\frac{q^{3}}{B_{1} \ell}
$$

$\widetilde{W}$ is a wall proximity function defined as

$$
\widetilde{W}=1+E_{2}\left(\frac{\ell}{\kappa L}\right)
$$

and

$$
(L)^{-1}=(\eta-z)^{-1}+(H+z)^{-1}
$$

$\kappa$ is the von Karman constant, $\mathrm{H}$ is the water depth, $\eta$ the free surface elevation, and $E_{1}, E_{2}$ and $B_{1}$ are empirical constants. Parameterization has been made for vertical turbulent momentum and heat fluxes in the model where the vertical fluxes of horizontal turbulent momentum $-\left\langle w^{\prime} u^{\prime}\right\rangle$ and $-\left\langle w^{\prime} v^{\prime}\right\rangle$ have been expressed in terms of the product of vertical eddy viscosity and vertical shear of the horizontal velocity and the vertical heat flux $-\left\langle\theta^{\prime} w^{\prime}\right\rangle$ has been given in terms of the product of the vertical thermal diffusion coefficient and vertical density gradient.

The turbulent kinetic energy and macroscale equations are closed by defining

$$
K_{M}=\ell q S_{M}, K_{H}=\ell q S_{H}, K_{q}=\ell q S_{q}
$$

where $S_{M}, S_{H}$, and $S_{q}$ are stability functions which satisfy

$$
\begin{aligned}
S_{M}\left[6 A_{1} A_{2} G_{M}\right]+S_{H}\left[1-2 A_{2} B_{2} G_{H}-12 A_{1} A 2 G_{H}\right] & =A_{2} \\
S_{M}\left[1+6 A_{1}^{2} G_{M}-9 A_{1} A_{2} G_{H}\right]-S_{H}\left[12 A_{1}^{2} G_{H}+9 A_{1} A_{2} G_{H}\right] & =A_{1}\left(1-3 C_{1}\right) \\
S_{q} & =0.20
\end{aligned}
$$


and where

$$
\begin{aligned}
G_{M} & =\frac{\ell^{2}}{q^{2}}\left(\left(\frac{\partial u}{\partial z}\right)^{2}+\left(\frac{\partial v}{\partial z}\right)^{2}\right) \\
G_{H} & =\frac{\ell^{2}}{q^{2}}\left(\frac{g}{\rho_{0}} \frac{\partial \rho}{\partial z}\right) .
\end{aligned}
$$

With the empirical constants given, the stability functions can be analytically determined by solving a linear algebraic equation, and the solutions depend on the vertical shear of mean flow and stratification. All empirical constants mentioned above are assigned values based on laboratory experiments (Mellor and Yamada, 1974; 1982) as follows:

$$
\left(A_{1}, A_{2}, B_{1}, B_{2}, C_{1}, E_{1}, E_{2}\right)=(0.92,0.74,16.6,10.1,0.08,1.8,1.33)
$$

In the absence of wind stress and surface and bottom heat fluxes, the surface and bottom boundary conditions can be expressed as

$$
\left.\begin{array}{l}
\frac{\partial u}{\partial z}=\frac{\partial v}{\partial z}=0, \\
\frac{\partial \theta}{\partial z}=\frac{\partial s}{\partial z}=0, \\
q^{2}=q^{2} \ell=0, \\
w=\frac{\partial \eta}{\partial t}+u \frac{\partial \eta}{\partial x}
\end{array}\right\} \quad \text { at } z=\eta(x, y, t)
$$

$$
\begin{aligned}
& K_{M}\left(\frac{\partial u}{\partial z}, \frac{\partial v}{\partial z}\right)=\left(\frac{\tau_{b x}}{\rho_{o}}, \frac{\tau_{b y}}{\rho_{o}}\right), \\
& q^{2}=B_{1}^{\frac{2}{3}} u_{\tau b}^{2} \\
& q^{2} \ell=0 \\
& w=-u \frac{\partial H}{\partial x}
\end{aligned}
$$

where $\tau_{b x}$ and $\tau_{b y}$ are the $x$ and $y$ components of the bottom stress, and $u_{\tau b}$ the friction velocity associated with the bottom stress. The bottom stress is determined by 
matching the velocity with the logarithmic layer such that

$$
\left(\tau_{b x}, \tau_{b y}\right)=C_{d} \sqrt{u^{2}+v^{2}}(u, v)
$$

where

$$
C_{d}=\operatorname{Max}\left(\frac{\kappa^{2}}{\ln \left(\frac{z}{z_{o}}\right)^{2}}, 0.0025\right)
$$

and $z_{o}$ is the roughness parameter of the bottom topography.

A $\sigma$-coordinate transformation is used to map the irregular bottom topography into a flat bottom domain in which it is much easier to design a numerical grid. The $\sigma$-coordinate transformation used in the model is given by

$$
\sigma=\frac{z-\eta}{H+\eta}
$$

Instead of varying from $-\mathrm{H}$ to $\eta$, the vertical coordinate in this $\sigma$ system varies from -1 at the bottom to 0 at the surface. The expression of the governing equations in the $\sigma$-coordinate are given in detail by Blumberg and Mellor (1987), so we will not repeat it here.

In all our numerical experiments, the $M_{2}$ tidal elevation will be input (as a wave maker) at the southern boundary of the numerical domain. Also, a gravity wave radiation boundary condition with a propagation speed of $\sqrt{g H}$ is specified at the northern boundary to allow the tidal wave to propagate out of the computational domain with minimum reflection (for a comprehensive discussion, see Chapman, 1985). However, the reflection of internal waves propagating to boundaries at each vertical level may occur at open boundaries since the radiation condition in the finite-difference numerical scheme only allows one internal wave to travel out of the computational domain. In other words, the radiation condition works only for the case where the energy spectrum of internal waves is 
very narrow in the frequency or wave number domain. In order to overcome this problem, we added a sponge layer into the radiation condition to absorb the rest of the "noise" waves in the region near the boundary (see Appendix B). Some initial numerical experiments for surface gravity waves in different cases were conducted to check if the radiation condition plus a sponge layer works in our model; they showed good agreement between the model and analytical solutions.

Non-uniform horizontal resolution is used in all our numerical experiments. A fine grid is taken across and near the bank or Georges Bank and then a linear increasing function is used to connect it to a coarse grid near the open boundary. The advantage of this non-uniform grid is to filter the short internal waves propagating toward the open boundaries without losing the horizontal resolution in our domain of interest and hence to reduce the strength of the artificial sponge layer at the boundaries.

Vertical resolution is chosen based on resolving the bottom boundary layer while minimizing the computational error over steep bottom topography due to the $\sigma$-coordinate transformation. Some numerical experiments for the barotropic tidally driven Ekman layer over a flat bottom were conducted to study the effects of vertical resolution on the numerical solutions. Comparison with the analytical solution shows that the vertical resolution may seriously influence the Ekman transport even though it may not distort the vertical structure of the flow. These experiments indicate that 31 grid points in the vertical are required to resolve the bottom boundary layer in the case where the water depth is $100 \mathrm{~m}$ and the thickness of the Ekman layer is $40 \mathrm{~m}$ for a given constant vertical eddy viscosity of $K_{m}=0.01 \mathrm{~m}^{2} / \mathrm{s}$ (see Figure A.2 in the Appendix). This relation implies that a rough estimate can be made for the vertical resolution if the thickness of the boundary layer is known. Weatherly and Martin (1978) compared field observations with results obtained with the Mellor and Yamada level 2 turbulent closure model and found that the thickness 
of the bottom boundary layer in stable stratifications can be estimated by

$$
h=\frac{A u_{*}}{f\left[1+\left(N_{o}^{2} / f^{2}\right)\right]^{\frac{1}{4}}}
$$

where $u_{*}$ is the friction velocity, $N_{o}$ the Brunt-Väisälä frequency, and $A$ an empirical constant determined as 1.3 from observations. Although this formula may not provide us with an accurate estimate of the thickness of the bottom boundary layer in our case where time-dependent turbulence over a sloping bottom is considered, we can still use it as a guide to make an initial guess for the thickness of the boundary layer.

Haney (1991) recently discussed the numerical errors in computing the baroclinic pressure gradient due to the $\sigma$-coordinate transformation, and pointed out that the computational errors can result in an along-isobath circulation over a steep bottom slope if the vertical resolution is low and the finite-difference scheme is not hydrostatically consistent. Using a similar analytical method, we found that the maximum pressure gradient error due to the $\sigma$-coordinate transformation in the ECOM3D-SI can be estimated by

$$
\Delta P_{x(\max )} \sim N^{\prime 2} \alpha D \Delta \sigma / 2
$$

where $N^{\prime}$ is the vertical perturbation buoyancy frequency, $\alpha$ the bottom slope, and $D$ the water depth. The error becomes big when the gradient of perturbation density or the topographic slope is large, while the error becomes smaller when the vertical resolution is increased. The along-isobath error current can be estimated from the geostrophic balance as

$$
\Delta V_{\max } \sim \frac{1}{f} \Delta P_{x(\max )} .
$$


If we want the maximum error of the current to be less than $1.0 \mathrm{~cm} / \mathrm{s}$ in our model results, the vertical resolution must be constrained by

$$
\Delta \sigma<2 f / N^{\prime 2} \alpha D
$$

This condition and formula (3.30) are used as criteria to choose the vertical resolution in our numerical experiments. A detailed discussion of this issue may be found in Appendix A of this thesis.

The equations with boundary condition are solved by finite-difference techniques. A staggered Arakawa C grid (Arakawa and Lamb, 1977) is used for the numerical computation. An implicit numerical scheme is used to calculate the vertical friction terms (Roache, 1972). Unlike the original Blumberg and Mellor time-splitting model, a semiimplicit scheme is introduced in the horizontal for the barotropic model; this scheme treats the barotropic pressure gradient in the momentum equation and the velocity convergence in the continuity equation implicitly. Instead of using a smaller time-step with an explicit scheme or computing a large set of linear algebraic equations by a fully implicit scheme, this method leads to a linear symmetrical diagonal system at each time-step which can be solved efficiently by a preconditioned conjugate gradient method with no sacrifice in computational time (Casulli, 1990). Although the horizontal advection terms are still treated explicitly in the semi-implicit scheme, the time-step criterion for the advection computation is inversely proportional to the magnitude of velocity rather than the speed of the gravity wave, i.e.,

$$
\Delta t \leq\left[\frac{|u|}{\Delta x}+\frac{|v|}{\Delta y}\right]^{-1}
$$

Two other issues need to be considered before using the numerical model to study stratified tidal rectification over Georges Bank. One is the hydrostatic approximation and the other is the horizontal diffusion coefficient. Hydrostatic balance is strictly valid only 
for low-frequency long waves where the horizontal wave length is much larger than the local water depth. Internal tides on the northern flank of Georges Bank were recently observed with horizontal wave lengths of order $20-50 \mathrm{~km}$ (Loder and Horne, 1989), roughly a factor of $10^{3}$ larger than the water depth, and the wave frequency is much smaller than the Brunt-Väisälä frequency $N$ over the slope $\left(N \sim 10^{-2} \mathrm{~s}^{-1}\right.$, Flagg, 1987). The hydrostatic approximation should be adequate to study such low-frequency internal tides over Georges Bank. Since the horizontal diffusion terms are in general opposite to the nonlinear advection over the sloping bottom topography, an arbitrary choice for a large horizontal diffusion coefficient in the model may lead to meaningless numerical results in the problem of tidal rectification where nonlinear advection is a key driving force. An implicit numerical scheme used for the computation of vertical friction terms in the Blumberg and Mellor model is effective in reducing the need for horizontal diffusion because horizontal advection followed by vertical mixing acts like horizontal diffusion in a real physical sense (Blumberg and Mellor, 1987). We have used a horizontal diffusion coefficient of $1 \mathrm{~m}^{2} / \mathrm{s}$ in barotropic cases and of about $20 \mathrm{~m}^{2} / \mathrm{s}$ in baroclinic cases. In both sets of cases, the effective horizontal diffusion is several orders of magnitude smaller than nonlinear advection. 


\subsection{Barotropic Tidal Rectification Over a Symmetrical Bank}

There have been many theoretical papers written on the subject of unstratified tidal rectification over variable bottom topography (Huthnance, 1973; Loder, 1980; Zimmerman, 1978, 1980, 1981; Tee, 1979, 1980, 1985, 1987; Robinson, 1981); these papers provide a good view on the dynamical mechanism of the generation of residual flow. However, the analytical models cannot be applied to the quantitative prediction of the detailed spatial structure of residual flow over the real bottom topography of Georges Bank because of theoretical limitations of either small-amplitude bottom topography (Zimmerman, 1978, 1980,1981 ) or vertically averaged approximation (Loder, 1980) and artificial assumptions of mixing coefficients. Although Greenberg's (1983) numerical model directly gives us insight into the vertically averaged pattern of residual flow over Georges Bank, it misses the vertical structure of residual flow, especially near the maximum axis of along-isobath flow where the second order cross-isobath circulation may be generated due to friction (Wright and Loder, 1985). In a depth-independent model, the time-averaged along-isobath momentum balance between the horizontal advection and friction terms suggests that the structure of residual currents depends on how friction is parameterized. In a depth-dependent model, however, turbulent friction in the bottom boundary layer causes vertical shear in tidal currents, which may lead to vertical non-uniform momentum transports for both the tidal and mean flows (Tee, 1979 and 1980; Wright and Loder, 1985). In addition, the transport model driven by oscillating tidal currents (under the assumption of a rigid lid) is a good approximation for small-amplitude bottom topography where the tidal wave length is much larger than the topographic scale, but it may either over-estimate residual currents in cases where the slope of bottom topography is small or significantly influence the cross-isobath residual currents due to missing of the on-bank Stokes drift (Tee, 1985). In this section, we will try to answer the following key questions. What does the vertical structure of residual flow look like over finite-amplitude bottom topography? How is the numerical solution for residual currents 


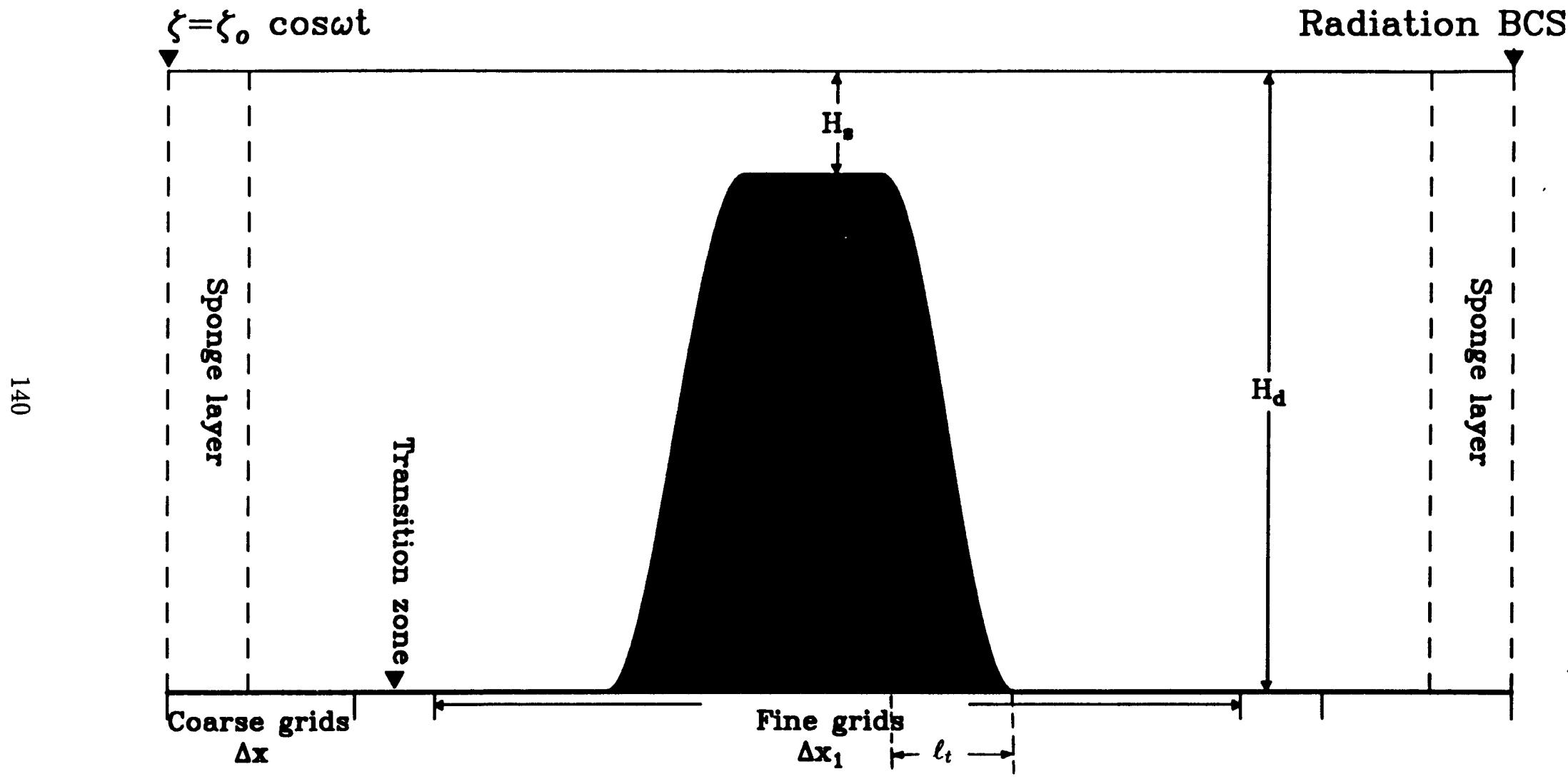

Figure 3.5: Numerical model domain. The $\zeta$ is the free surface, $\zeta_{0}$ the amplitude of the free surface $M_{2}$ tidal wave at the upstream boundary, $\omega$ is the $M_{2}$ tidal frequency, $H_{s}$ and $H_{d}$ the water depths on and off the bank, and $\Delta x$ and $\Delta x_{1}$ the coarse and fine grids, respectively. The bank is symmetric about its center. $\ell_{t}$ is the topography scale. 


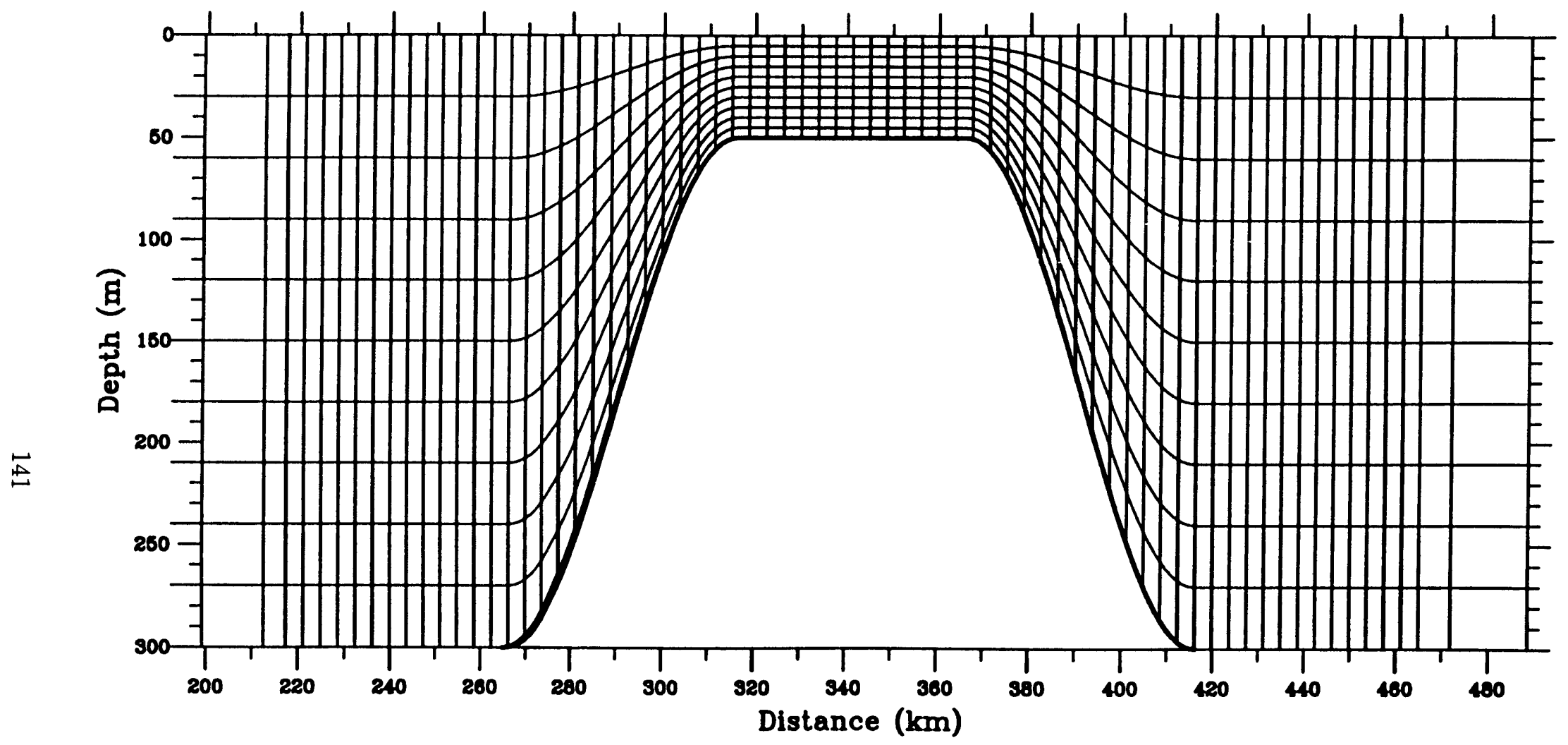

Figure 3.6: Numerical grid for the case of $H_{s}=50 \mathrm{~m}$ and $\ell_{t}=50 \mathrm{~km}$. The grid lines are plotted every three points in both horizontal and vertical directions. The horizontal resolution is $2.5 \mathrm{~km}$ over and near the bank and $11.96 \mathrm{~km}$ out of the domain of interest. A transition zone of 10 grid points with linearly-increasing spacing is used to connect the fine and coarse horizontal grids. The vertical resolution for $\sigma$ is -0.033 (31 points), which corresponds to a $\Delta z$ of $10 \mathrm{~m}$ in the deep region (for $H_{d}=300 \mathrm{~m}$ ) and $1.7 \mathrm{~m}$ (for $H_{s}=50 \mathrm{~m}$ ) over the bank. 
sensitive to the parameterization of vertical turbulent mixing coefficients in a homogeneous fluid?

By requiring no variation in the along-isobath direction, the numerical model for unstratified tidal rectification over variable bottom topography can be simplified into a twodimensional problem on a constant $f$-plane as sketched in Figure 3.5, where $x, y, z$ are a right-handed Cartesian coordinate system with $x$ and $y$ normal and parallel to the local isobath and $z$ increasing upward. To generalize this problem in a systematic way, we start our numerical experiments for a finite-amplitude symmetrical topographic feature or bank in this section and then extend our study to the real asymmetrical bottom topography of Georges Bank in section 3.6 where the tidal wave reflection due to the steep sloping bottom will be discussed. The depth distribution of the symmetrical bank used in this section is expressed analytically by

$$
h(x)= \begin{cases}H_{d} & x \leq x_{c_{1}} \\ \frac{1}{2}\left(H_{d}+H_{s}\right)+\frac{1}{2}\left(H_{d}-H_{s}\right) \cos \left(\frac{\pi\left(x-x_{c_{1}}\right)}{x_{s_{1}}-x_{c_{1}}}\right) & x_{c_{1}} \leq x \leq x_{s_{1}} \\ H_{s} & x_{c_{1}} \leq x \leq x_{s_{1}} \\ \frac{1}{2}\left(H_{d}+H_{s}\right)+\frac{1}{2}\left(H_{d}-H_{s}\right) \cos \left(\frac{\pi\left(x-x_{c_{2}}\right)}{x_{c_{2}}-x_{s_{2}}}\right) & x_{s_{2}} \leq x \leq x_{c_{2}} \\ H_{d} & x_{c_{2}} \leq x\end{cases}
$$

where $h(x)$ is the height of the bank and $H_{s}$ and $H_{d}$ are the water depths over the top of the bank and in the deep region away from the bank, respectively (see Figure 3.5). This formula was first used by Loder (1980) in his barotropic model, which provides smooth bottom topography with continuous first derivatives $h^{\prime}(x)$ across the bank. The numerical experiments are conducted in such a domain that the water depth is $300 \mathrm{~m}$ in the deep region away from the bank and $100 \mathrm{~m}$ or $50 \mathrm{~m}$ over the top of the bank. A barotropic semidiurnal tidal forcing is imposed at the left open boundary by specifying a free surface wave with an amplitude of $0.5 \mathrm{~m}$. A gravity wave radiation boundary condition plus a sponge layer is added at the right open boundary to allow the tidal wave to propagate continually out of the model domain without reflection (Chapman, 1985). To avoid transients due to sharp 
initial conditions, we ramp up the model forcing from zero to its full value over one tidal cycle. The numerical grid for $H_{s}=50 \mathrm{~m}$ and $\ell_{t}=50 \mathrm{~km}$ is shown in Figure 3.6. The horizontal resolution is $2.5 \mathrm{~km}$ over and near the bank and $11.96 \mathrm{~km}$ away from the domain of interest. A transition zone of 10 grid points with linearly increasing spacing is used to connect the fine and coarse horizontal grids. The vertical resolution for the $\sigma$ is -0.033 (31 points), which corresponds to a $\Delta z$ of $10 \mathrm{~m}$ in the deep region (for $H_{d}=300 \mathrm{~m}$ ) and $1.7 \mathrm{~m}$ (for $H_{s}=50 \mathrm{~m}$ ) over the bank.

A five-day time series of cross- and along-isobath currents and surface elevation at $\sigma=-0.033$ at five reference points is shown in Figure 3.7 for the case where $H_{s}=100 \mathrm{~m}$ and $\ell_{t}$ is $50 \mathrm{~km}$. The reference points (labeled A-E in Figure 3.8) are located across the bank with $A$ and $E$ in deep water, $B$ and $D$ on the upper slope and $C$ in the center of the bank. It takes about $2 \frac{1}{2}$ days for the model tidal elevation and currents to become stable and then the residual flow to be established. The stable tidal currents are symmetric across the bank, with an amplitude of about $10 \mathrm{~cm} / \mathrm{s}$ in the deep regions and about $45 \mathrm{~cm} / \mathrm{s}$ in $u$ and $35 \mathrm{~cm} / \mathrm{s}$ in $v$ over the top of the bank. The along-isobath residual currents are found over the slopes where the amplitude of the tidal current shifts upward on the left side and downward on the right side. The cross-isobath distribution of along-isobath residual flow is plotted in Figure 3.8a, which shows a symmetrical clockwise jet-like circulation along the bank with a maximum value of $2.4 \mathrm{~cm} / \mathrm{s}$ at the surface near the shelf break.

In addition, the model has been run for several different slopes $\left(\ell_{t}=25 \mathrm{~km}\right.$ and $12.5 \mathrm{~km}$ ) and height $\left(H_{s}=50 \mathrm{~m}\right)$ of the bank (Figures 3.8 and 3.9). The strength and vertical distribution of along-isobath residual flow strongly depend on the height of the bank and topographic slope. The maximum velocity increases to $7.0 \mathrm{~cm} / \mathrm{s}$ as the water depth over the top of the bank decreases to $50 \mathrm{~m}$. When the topographic scale $\ell_{t}$ reduces to $25 \mathrm{~km}$, the maximum current intensifies about $1.3 \mathrm{~cm} / \mathrm{s}$ in the case of $H_{s}=100 \mathrm{~m}$ but about $4.2 \mathrm{~cm} / \mathrm{s}$ in the case of $H_{s}=50 \mathrm{~m}$. The same order of increase is also found when the topographic scale is decreased to $12.5 \mathrm{~km}$ for both depth cases. The horizontal scale 

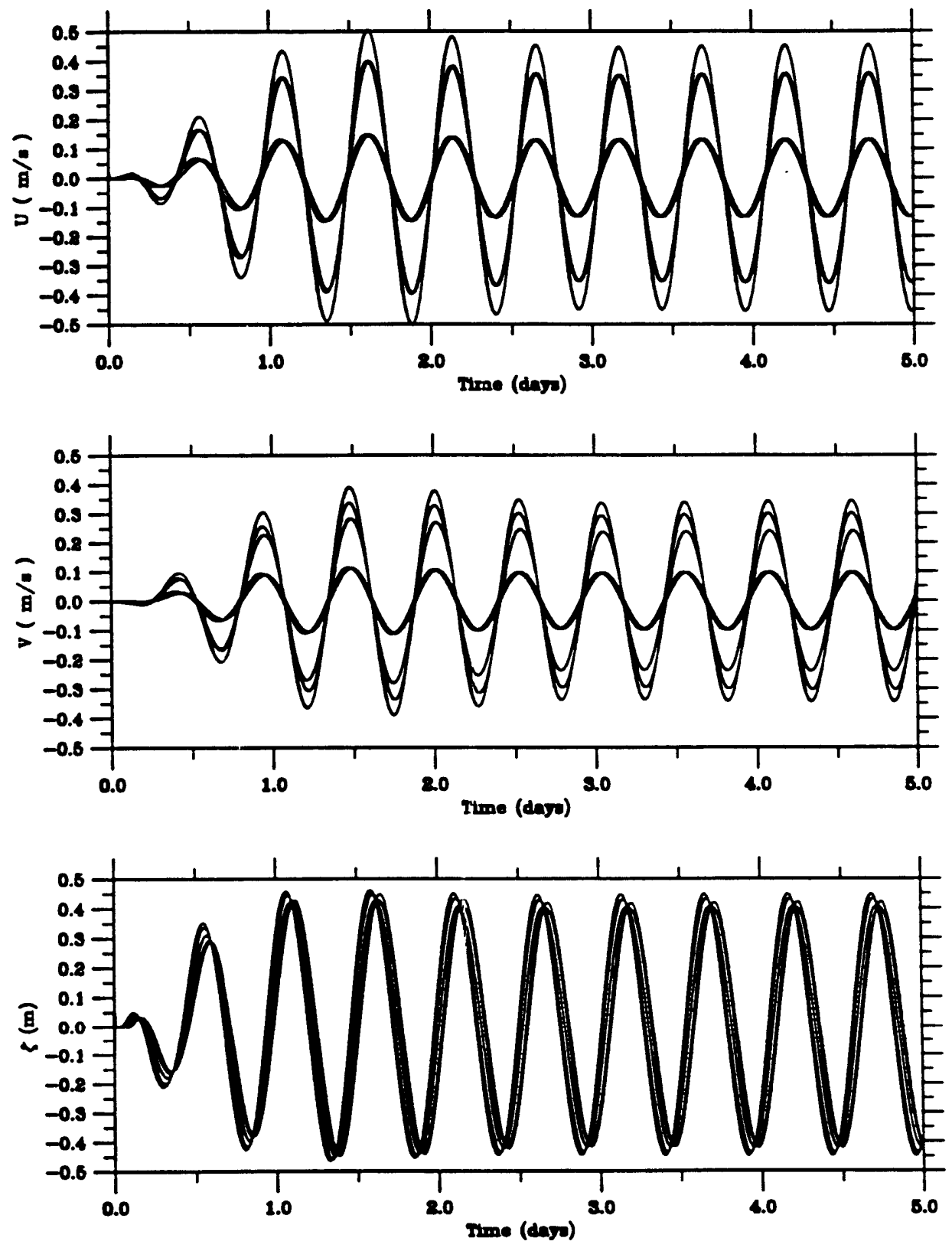

Figure 3.7: Time series of cross- and along-isobath tidal currents $(u$ and $v)$ and surface elevation $(\zeta)$ at $\sigma=-0.033$ at the five reference points (A-E) shown at the top in Figure 3.8a. 
of along-isobath residual flow is controlled by the topographic scale, which reduces as the topographic slope becomes steep for a given height.

The maximum cross-isobath and vertical residual currents are of order $0.1-1.0 \mathrm{~cm} / \mathrm{s}$ in $u$ and $10^{-3} \mathrm{~cm} / \mathrm{s}$ or less in $w$ for the six different topographic shapes. The distributions of mean surface elevation and cross-isobath and vertical residual current for the case of $H_{s}=100 \mathrm{~m}$ and $\ell_{t}=25 \mathrm{~km}$ are shown in Figure 3.10 as examples. There are the spatial decrease in amplitude and phase shift in tidal elevation observed across the bank as the tidal wave propagates toward the right away from its source region (see Figure 3.7 for an example). After averaging over one tidal cycle, two minima in mean sea elevation are found near the points of maximum along-isobath residual flow, which correspond to a maximum horizontal divergence in the cross-isobath residual current (see Figures $3.10 \mathbf{a}$ and b). Similar to tidal elevation, a spatial decrease and phase shift in mean surface elevation are always observed in these model results, even though the elevation gradients are too weak to drive a significant residual flow. A double-cell second-order Eulerian circulation pattern is found centered near the shelf break on both sides of the bank, where water is upwelled along the slopes and recirculated on both sides of the shelf break (Figure $3.10 \mathrm{c}$ ). ${ }^{2}$

The turbulent closure model provides a parabolic structure for the vertical mean turbulent mixing coefficient $K_{m}$. An example for the case of $H_{s}=100 \mathrm{~m}$ and $\ell_{t}=50 \mathrm{~km}$ is shown in Figure 3.11 where the mean $K_{m}$ has a maximum near mid water depth over the bank and decreases as the water becomes deep or away from the bank. The distribution of $K_{m}$ is very similar to that used by Brown (1984) to find a best fit for observed semidiurnal tidal currents over Georges Bank. The maximum value is about $0.06 \mathrm{~m}^{2} / \mathrm{s}$ on the top of the bank, which is close to an empirical value of $0.075 \mathrm{~m}^{2} / \mathrm{s}$ estimated for a transport of $\mathrm{H} \bar{U}=30 \mathrm{~m}^{2} / \mathrm{s}$ using the empirical formula $K_{m}=C_{d} \bar{U} H$ (where $C_{d}$ is the friction coefficient given as $2.5 \times 10^{-3}, \bar{U}$ is the vertically averaged tidal current, and $H$ is total depth; see Garrett, 1975). Time sequences of $K_{m}$ and tidal currents at three reference

\footnotetext{
${ }^{2}$ Note: all decriptions of cross-bank residual flow given here and later are based on the Eulerian currents only. The Eulerian currents may be very different from the Lagrangian currents because of Stokes drift.
} 

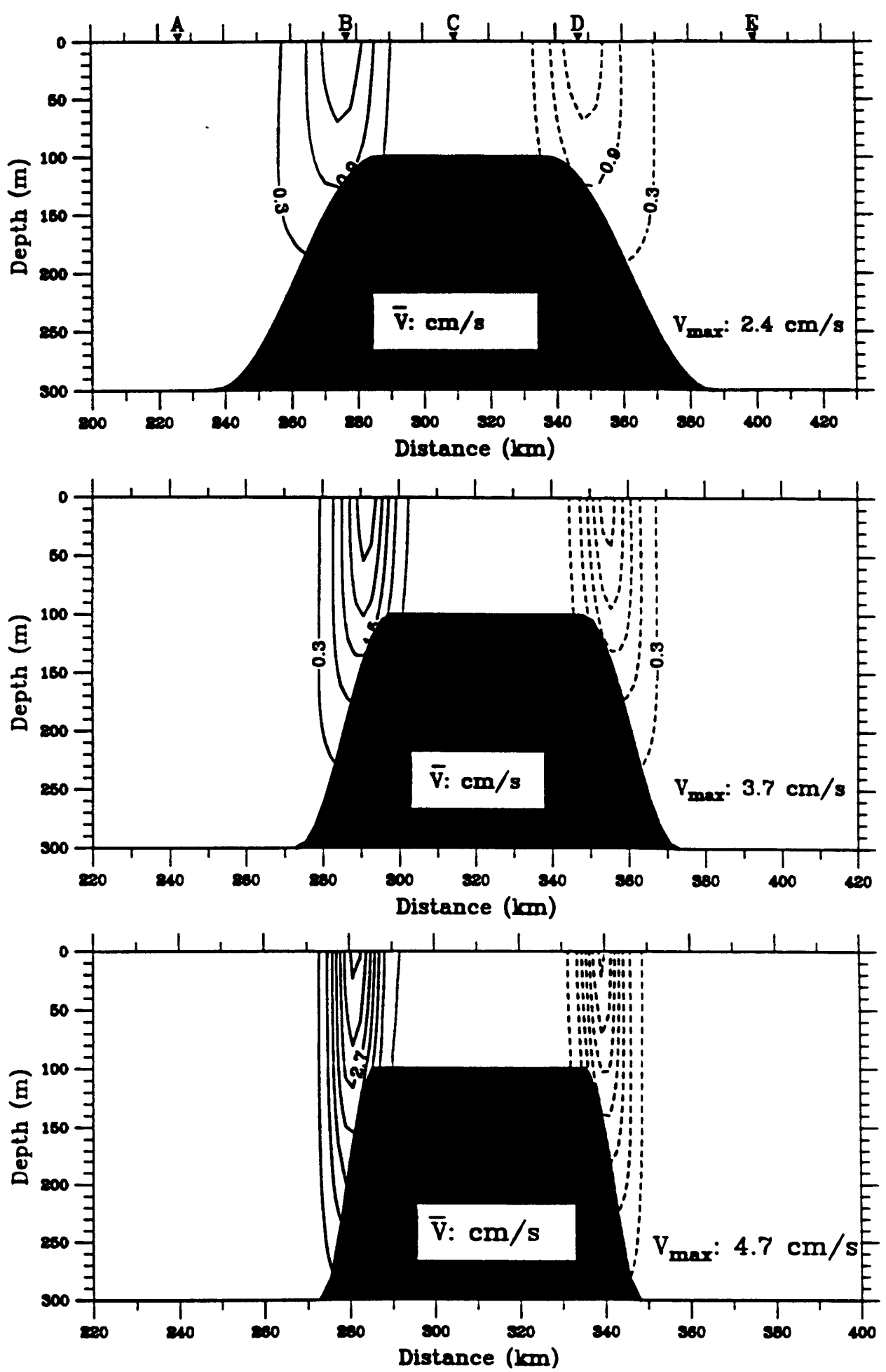

Figure 3.8: Structure of along-isobath residual current for the topographic scale $\ell_{t}=50 \mathrm{~km}(\mathrm{a}), 25 \mathrm{~km}$ (b) and $12.5 \mathrm{~km}$ (c). The contour interval is $0.6 \mathrm{~cm} / \mathrm{s}$ and the water depth is $100 \mathrm{~m}$ over the bank and $300 \mathrm{~m}$ away from the bank. The maximum velocity is shown in the lower right corner in each case. 

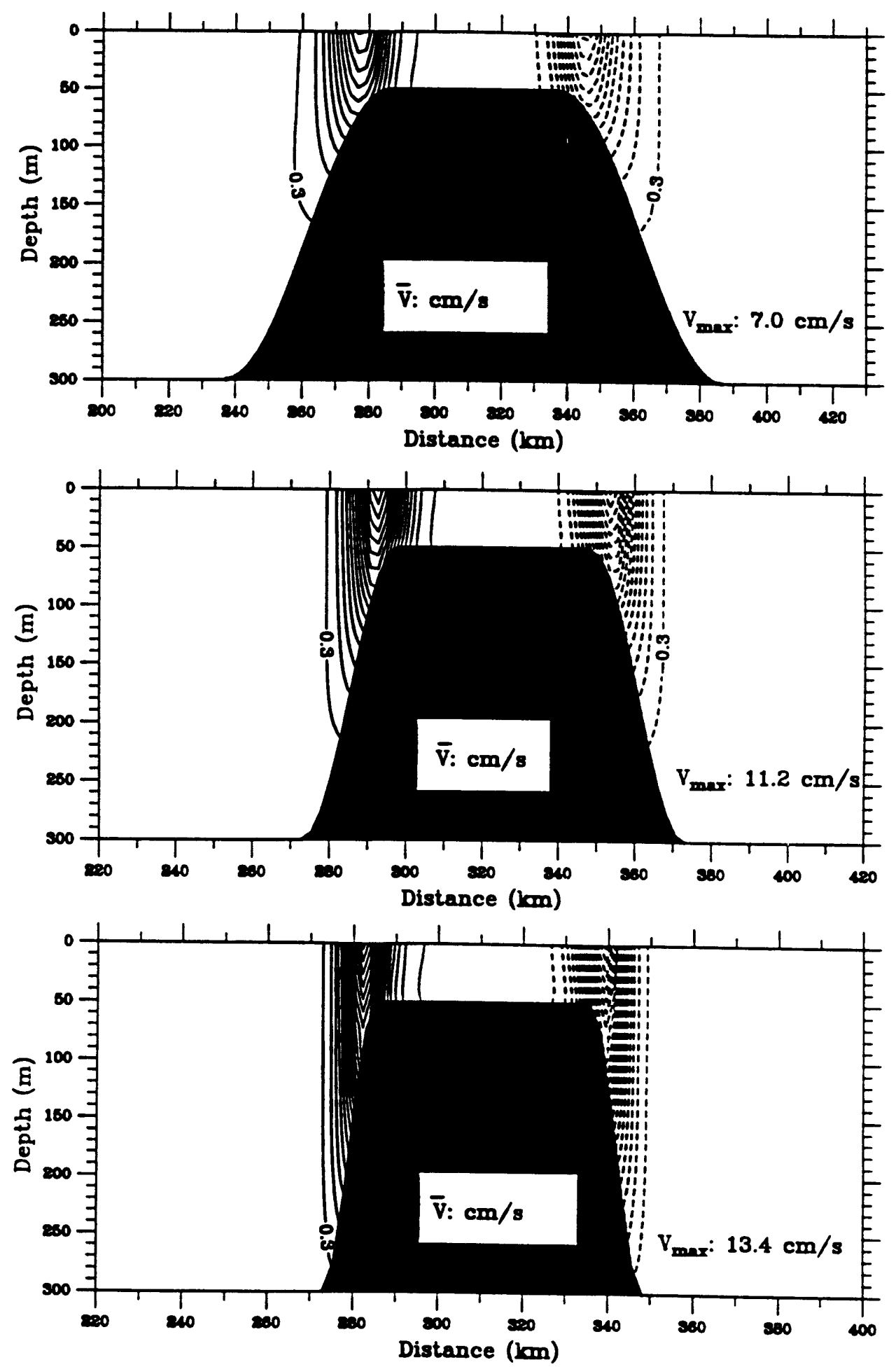

Figure 3.9: Structure of along-isobath residual current for the topographic scale $\ell_{t}=50 \mathrm{~km}(\mathrm{a}), 25 \mathrm{~km}$ (b) and $12.5 \mathrm{~km}$ (c). The contour interval is $0.6 \mathrm{~cm} / \mathrm{s}$ and the water depth is $50 \mathrm{~m}$ over the bank and $300 \mathrm{~m}$ away from the bank. The maximum velocity is shown in the lower right corner in each case. 

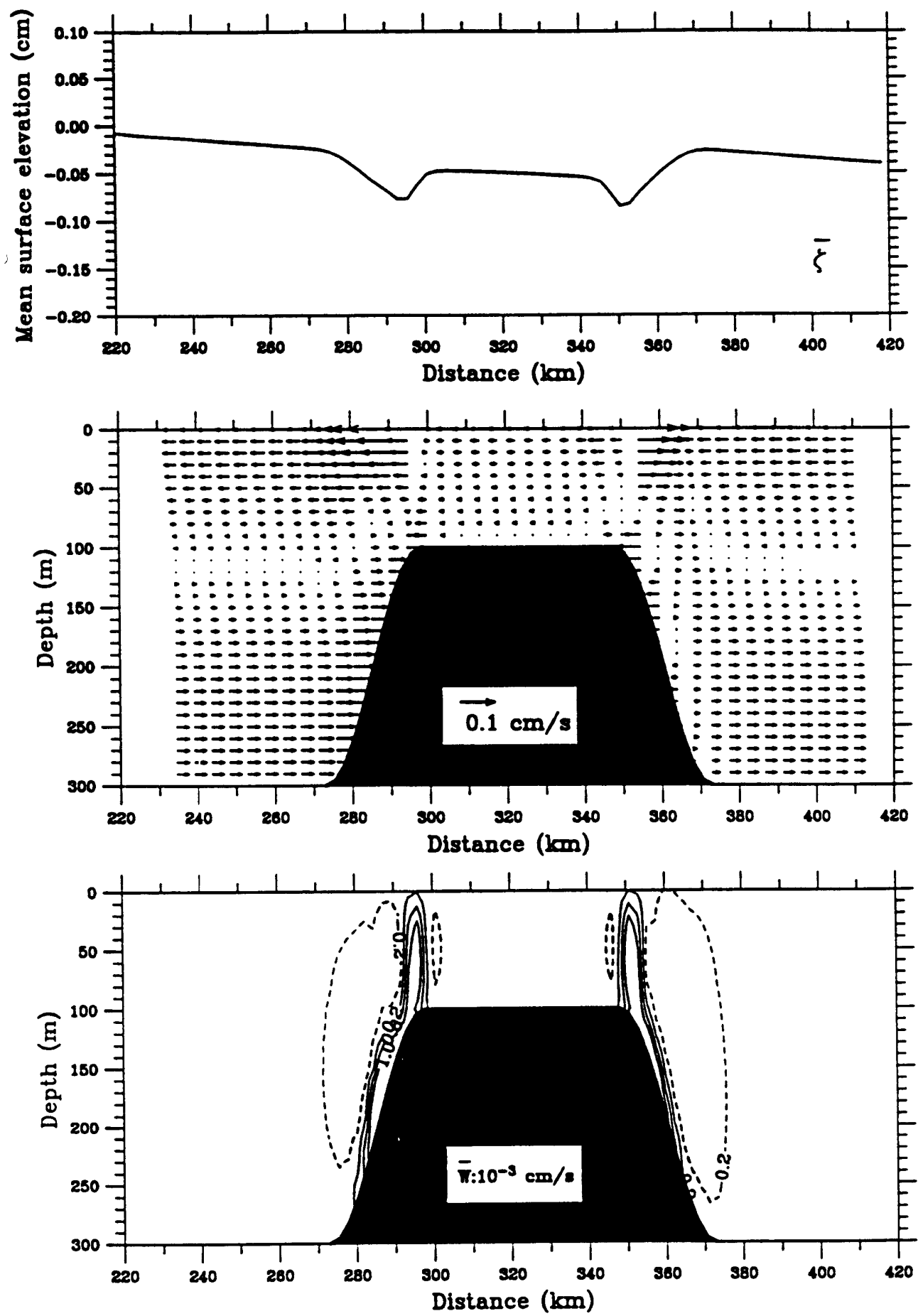

Figure 3.10: Cross-bank distribution of the mean surface elevation (a), cross-isobath horizontal residual current (b) and vertical residual current (c) for the case of $H s=100 \mathrm{~m}$ and $\ell_{t}=25 \mathrm{~km}$. The contour interval is $0.4 \times 10^{-3} \mathrm{~cm} / \mathrm{s}$ for the vertical velocity. In $\mathrm{c}$, the dashed line is the negative value and solid line is the positive value. 


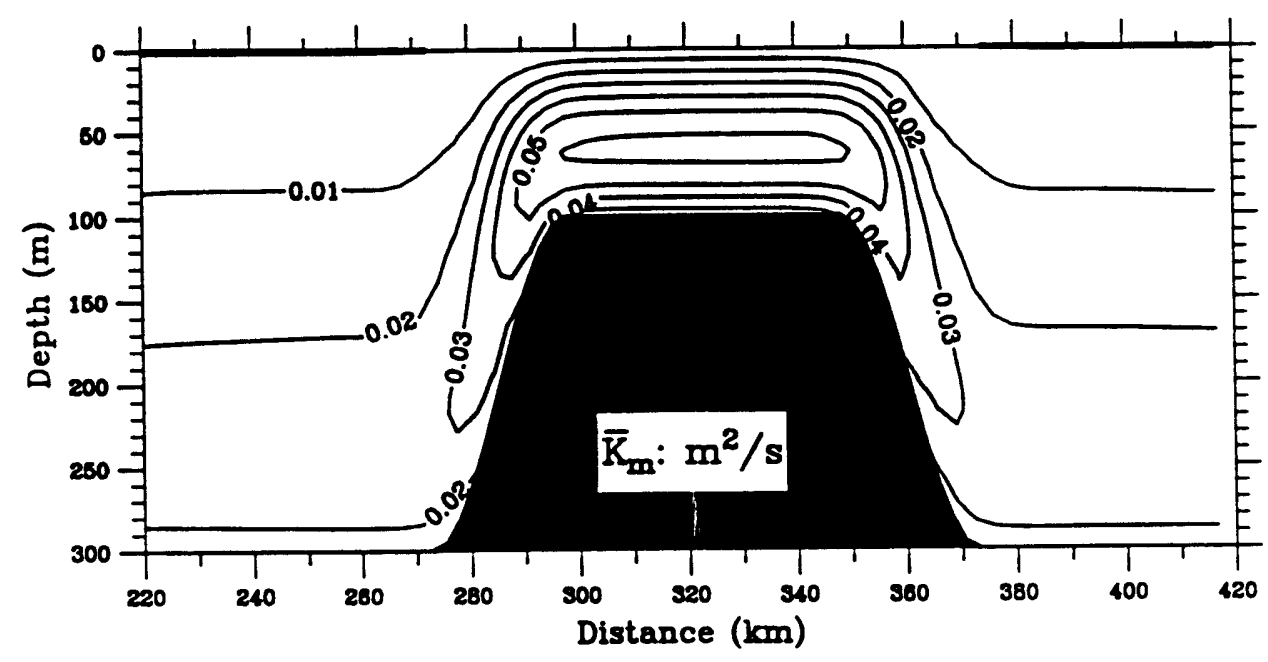

Figure 3.11: Cross-bank distribution of mean vertical eddy viscosity for the case of $H_{0}=100 \mathrm{~m}$ and $\ell_{t}=25 \mathrm{~km}$. The contour interval is $0.01 \mathrm{~m}^{2} / \mathrm{s}$.

points are presented every 0.83 hour over half of one tidal cycle in Figure 3.12 to examine the time-dependent variation of $K_{m}$ during the tidal period. Although there is some variation in the maximum value of $K_{m}$ over it tidal cycle, its vertical structure remains stable with time. The time-dependent variation of maximum $K_{m}$ during the tidal period is about $0.01 \mathrm{~m}^{2} / \mathrm{s}$ and $0.02 \mathrm{~m}^{2} / \mathrm{s}$ on the top of the bank and near the shelf break, respectively, while there is little variation in the deep water. In order to investigate the sensitivity of the residual flow to the specific form of $K_{m}$, we have run the model with a constant vertical eddy viscosity $K_{m}=0.01 \mathrm{~m}^{2} / \mathrm{s}$ and $0.075 \mathrm{~m}^{2} / \mathrm{s}$ (chosen by the empirical estimate mentioned above). A comparison of the results from the constant eddy viscosity model and the turbulent closure model is shown in Figure 3.13 where the vertical distributions of $K_{m}$, along-and cross-isobath residual flows $\bar{u}$ and $\bar{v}$ at three reference points are presented. Reasonable agreement is found in the vertical shear of velocities between the turbulent closure model and the constant $K_{m}$ model over the bank for $K_{m}=0.075 \mathrm{~m}^{2} / \mathrm{s}$ and away from the bank for $K_{m}=0.01 \mathrm{~m}^{2} / \mathrm{s}$, suggesting that the horizontal distribution of $K_{m}$ is 

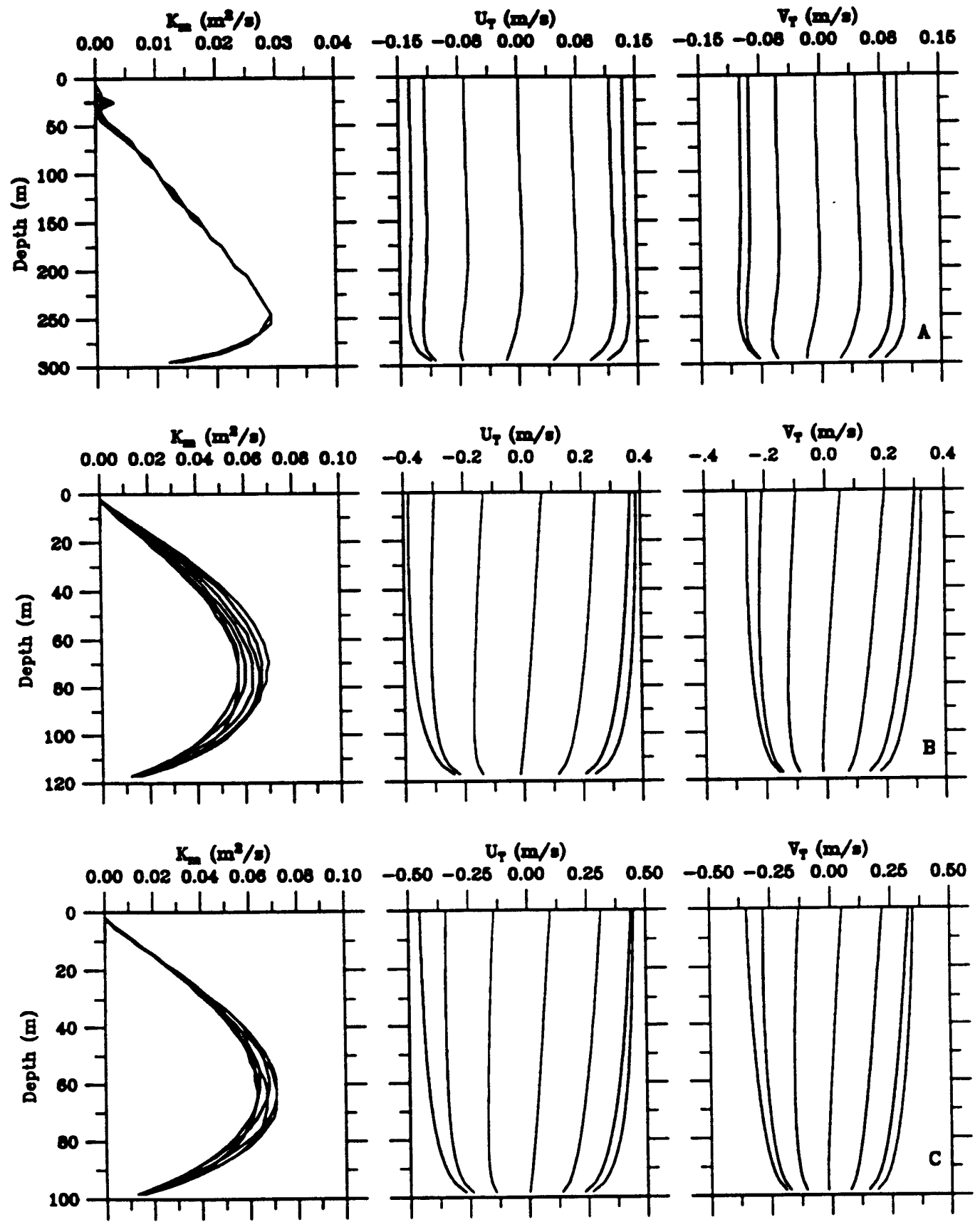

Figure 3.12: Time sequences of vertical eddy viscosity (left), cross- (middle) and along- (right) isobath tidal currents during half tidal cycle at three reference points (A-C) shown in Figure 3.8. The time interval is 0.83 hour. $H_{s}=100 \mathrm{~m}$ and $\ell_{t}=50 \mathrm{~km}$. 

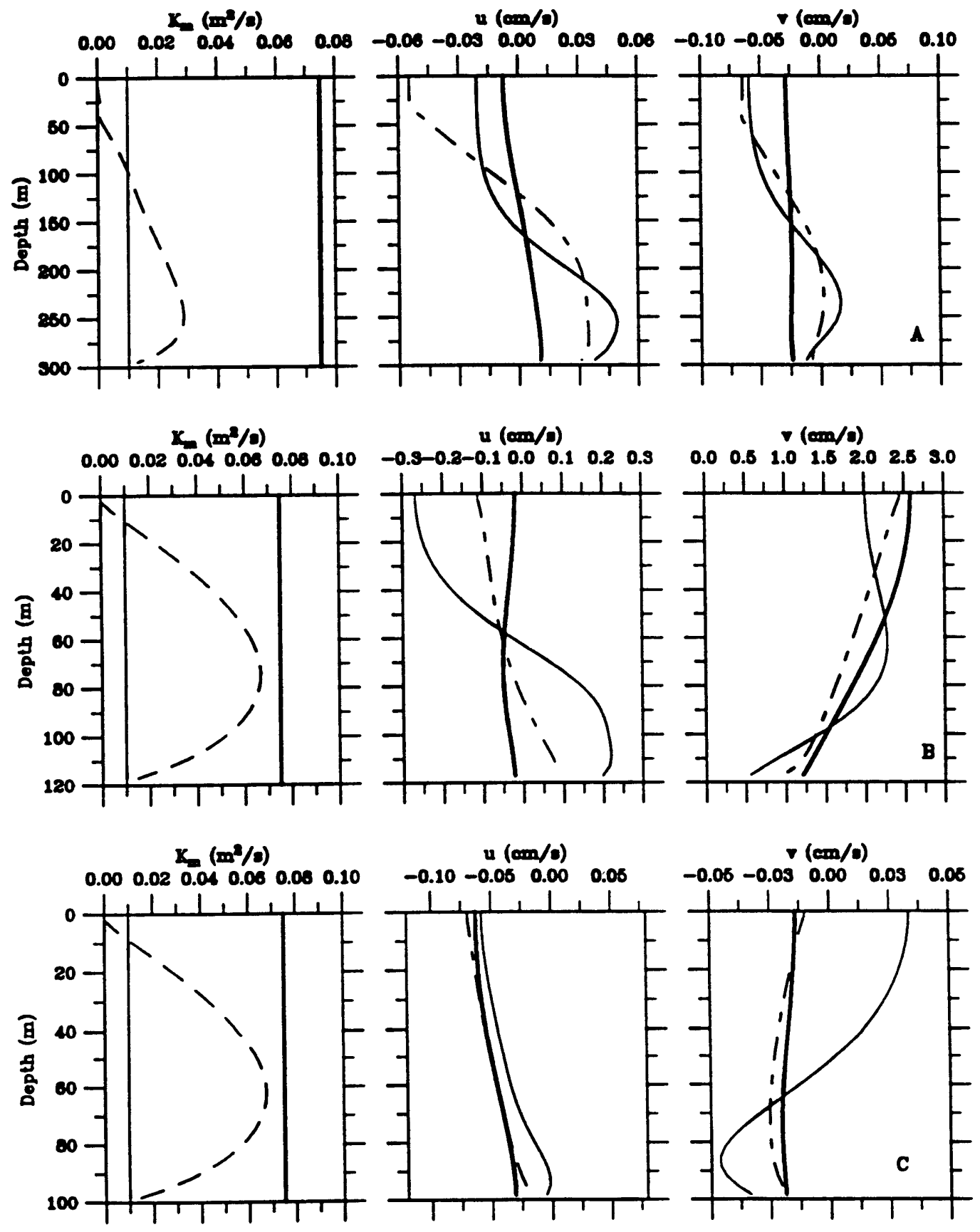

Figure 3.13: Vertical distribution of vertical viscosity $K_{m}$ (left), cross- (middle) and along- (right) isobath residual currents for $K_{\mathrm{m}}=0.01 \mathrm{~m}^{2} / \mathrm{s}$ (solid line), $0.075 \mathrm{~m}^{2} / \mathrm{s}$ (heavy solid line) and time/spatial dependent $K_{m}$ (dashed line) from the turbulent closure model at three reference points (A-C). All these three experiments have been done for the case of $H_{s}=100 \mathrm{~m}$ and $e l l_{t}=50 \mathrm{~km}$. 
more important than the vertical distribution in determining the structure of the residual flow in the unstratified fluid.

Model results obtained from these numerical experiments are qualitatively consistent with analytical solutions from either simplified depth-averaged models (Loder, 1980; Zimmerman, 1978, 1980) or depth-dependent models (Wright and Loder, 1985; Tee, 1985, 1987). Based on the harmonic truncation method, Loder (1980) analytically predicted a clockwise residual circulation around Georges Bank with a maximum near the shelf break and decaying exponentially away from the Bank. Young (1983) and Maas et al. (1987) separately used second-order momentum and spectral theories to argue that the residual flow should be a jet-like circulation limited over the Bank. Our numerical results do show a clockwise jet-like residual circulation along a finite-amplitude symmetrical bank, which is in good agreement with their theories. Loder and Wright (1985) considered a depthdependent model with a constant vertical eddy viscosity and linear stress law at the bottom boundary and predicted a double cell cross-isobath mean circulation over Georges Bank due to friction. Similar structures were found numerically by Tee (1987) with a no-slip bottom boundary condition. This is also true in our model results where a double cell cross-isobath circulation pattern is centered at the shelf break on both sides of the bank.

Unlike most of the previous theoretical work, however, our model is forced by a surface tidal wave rather than a transport (oscillating tidal current) and also includes the contribution of nonlinear interaction between tidal and mean currents to tidal motion. A coherent picture of minimum mean surface elevation associated with cross-isobath current divergence suggests a different dynamical mechanism for the existence of a horizontal gradient of mean surface elevation because no mass convergence or divergence is allowed in the transport model. The spatial decrease and phase shift of the tidal and mean surface elevation downstream across the bank always exist in our numerical results, which are independent of open boundary conditions, since tidal currents in the upper layer of the deep water are symmetric on both sides of the bank. In addition, the weak time-dependent 
variation of $K_{m}$ in the deep region away from the bank during the tidal cycle suggests a difference between turbulent mixing in rotating and non-rotating fluids. In order to answer these questions, we have run the model in some diagnostic ways to examine the basic balance in the field of residual flow and effects of rotation on the time variation of turbulent kinetic energy.

As an example, a diagnostic analysis has been made for the case of $H_{s}=50 \mathrm{~m}$ and $\ell_{t}=50 \mathrm{~km}$. Time series of each dynamic term at $\sigma=-0.033$ (near the surface) and -0.9 (near the bottom) in the momentum equations at three reference stations $\mathrm{A}-\mathrm{C}$ are shown in Figures 3.14-3.16. At station A, $40 \mathrm{~km}$ away from the bank, the nonlinear advection terms are at least two orders of magnitude smaller than the Coriolis force term so that the momentum balance is characterized by linear inertial-gravity wave equations in which the local acceleration term is balanced principally by the sum of the Coriolis and pressure gradient terms in the cross-isobath direction and by the Coriolis force term in the along-isobath direction. At station B on the slope of the bank where the mean water depth is $127 \mathrm{~m}$, nonlinear terms start to affect the tidal current as a first-order modification for the inertial-gravity wave, and increase as the water depth becomes shallower, and reach the maximum near the shelf break where the strongest along-isobath residual current is located. At station $\mathrm{C}$ in the center of the bank, the nonlinear effects almost disappear because of the flat bottom, so that the tidal momentum balance is characterized again by linear inertial-gravity wave equations plus the first-order correction due to the vertical friction. Similar results were found by Brown (1984), who estimated terms in vertically averaged momentum equations using observations of currents and bottom pressures over Georges Bank and concluded that the $M_{2}$ tide is a progressive inertial-gravity wave over Georges Bank.

Cross-bank distributions of each dynamic term averaged over a tidal cycle in the momentum equations are plotted in Figures 3.17 and 3.18. Unlike the vertically averaged model where the cross-isobath mean current is assumed to be zero, the Coriolis force in 

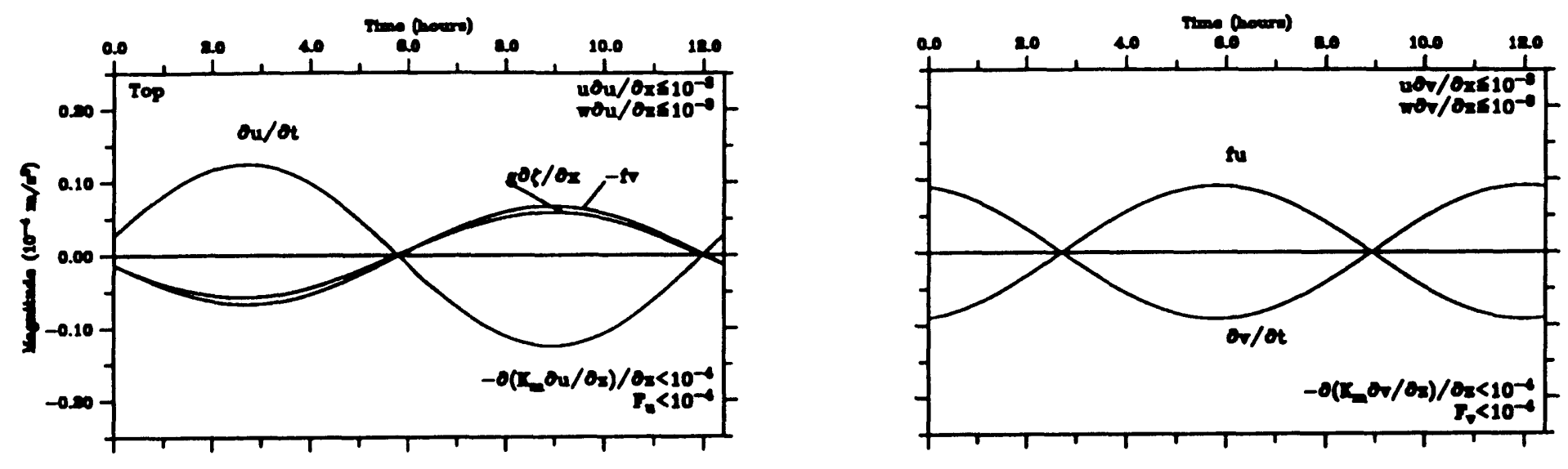

矛
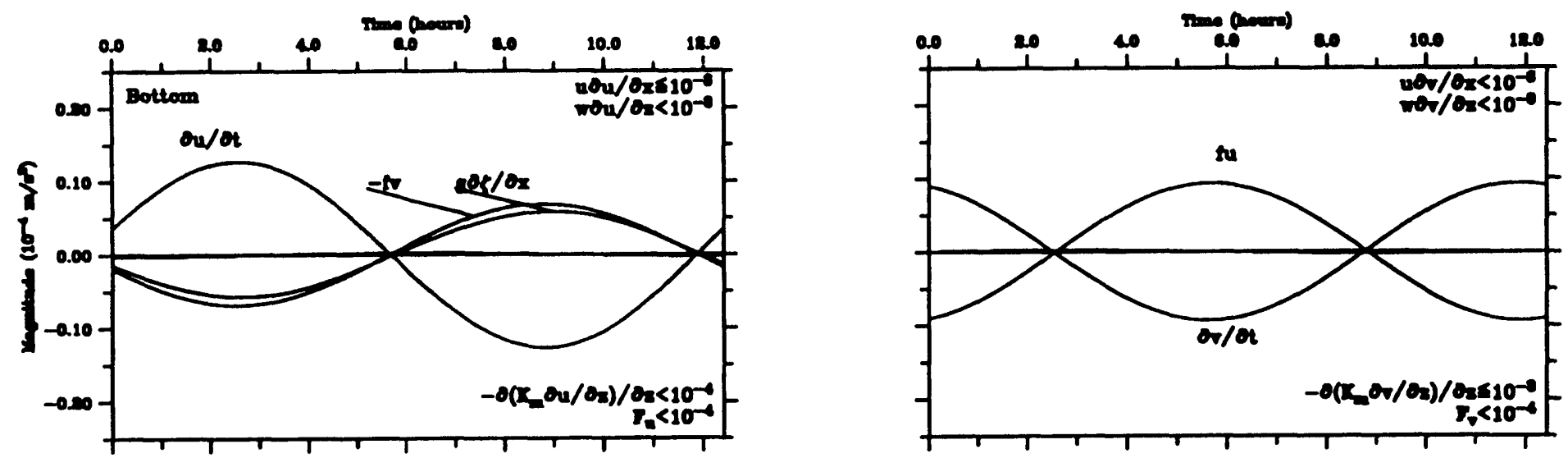

Figure 3.14: Time series of each dynamic term in the tidal momentum equations at $\sigma=-0.033$ near the surface (upper) and $\sigma=-0.9$ near the bottom (lower) at station A shown in Figure 3.17 for the barotropic case. 

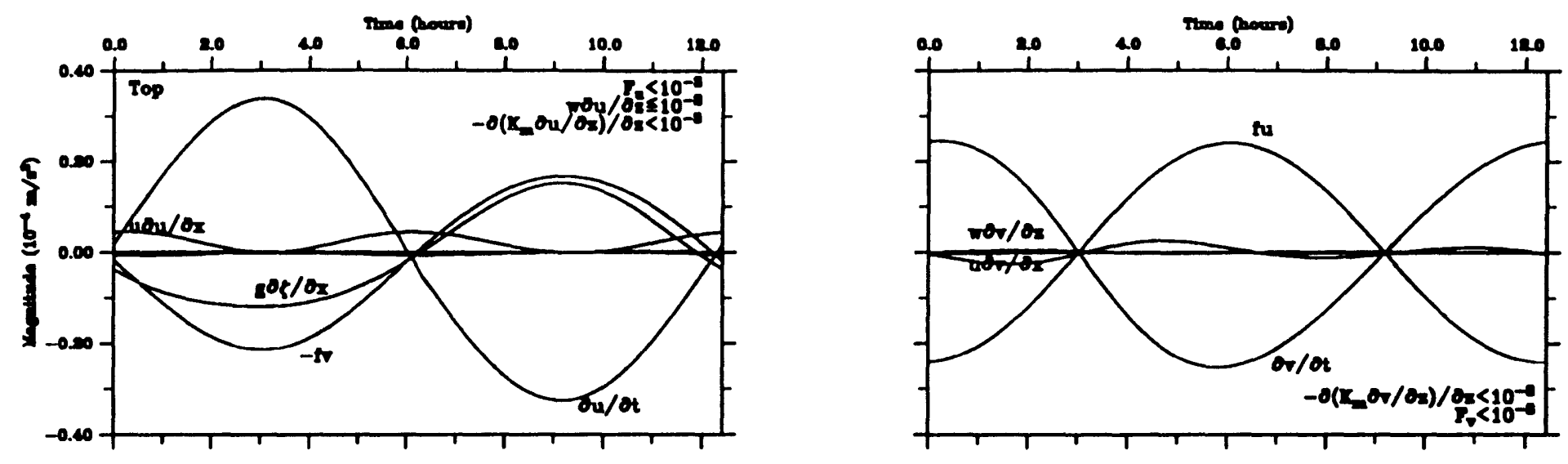

방
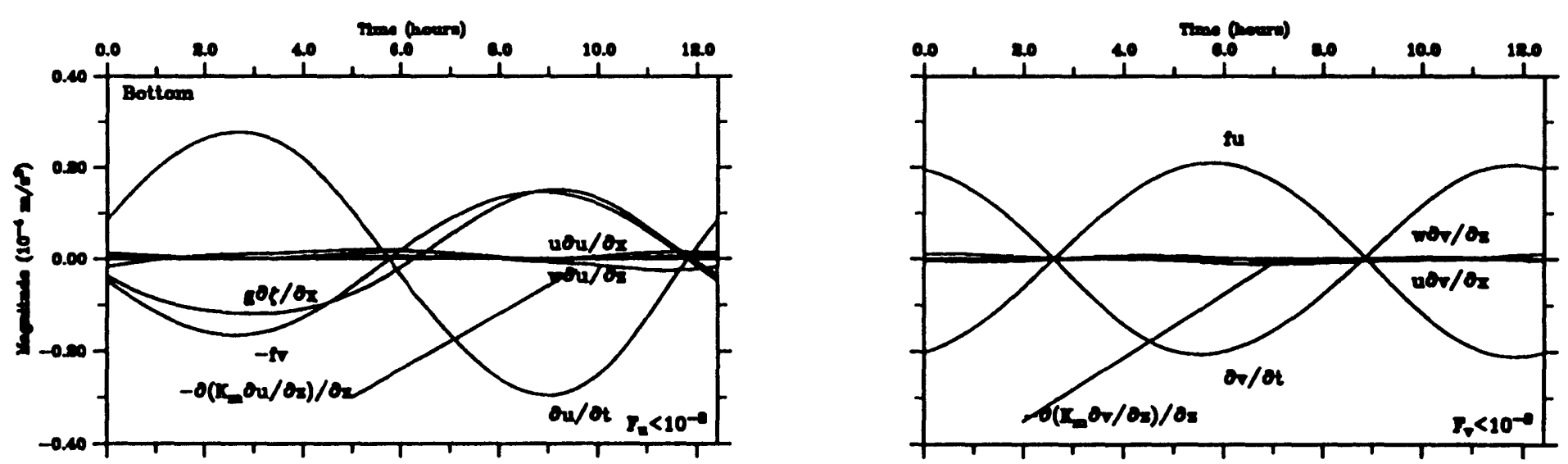

Figure 3.15: Time series of each dynamic term in the tidal momentum equations at $\sigma=-0.033$ near the surface (upper) and $\sigma=-0.9$ near the bottom (lower) at station B shown in Figure 3.17 for the barotropic case. 

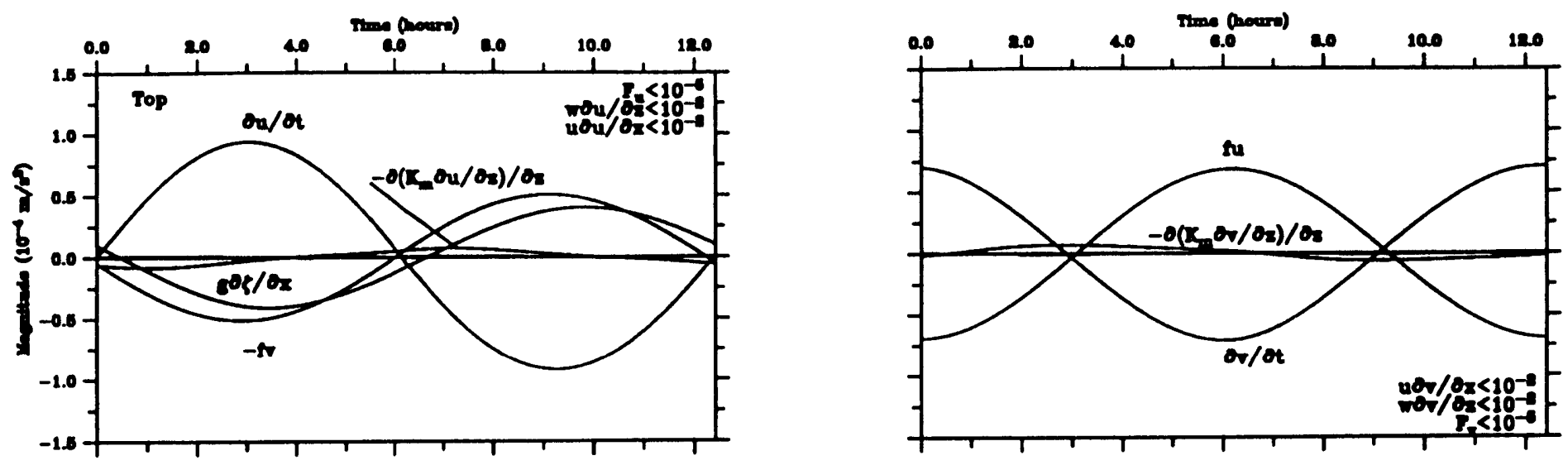

矛
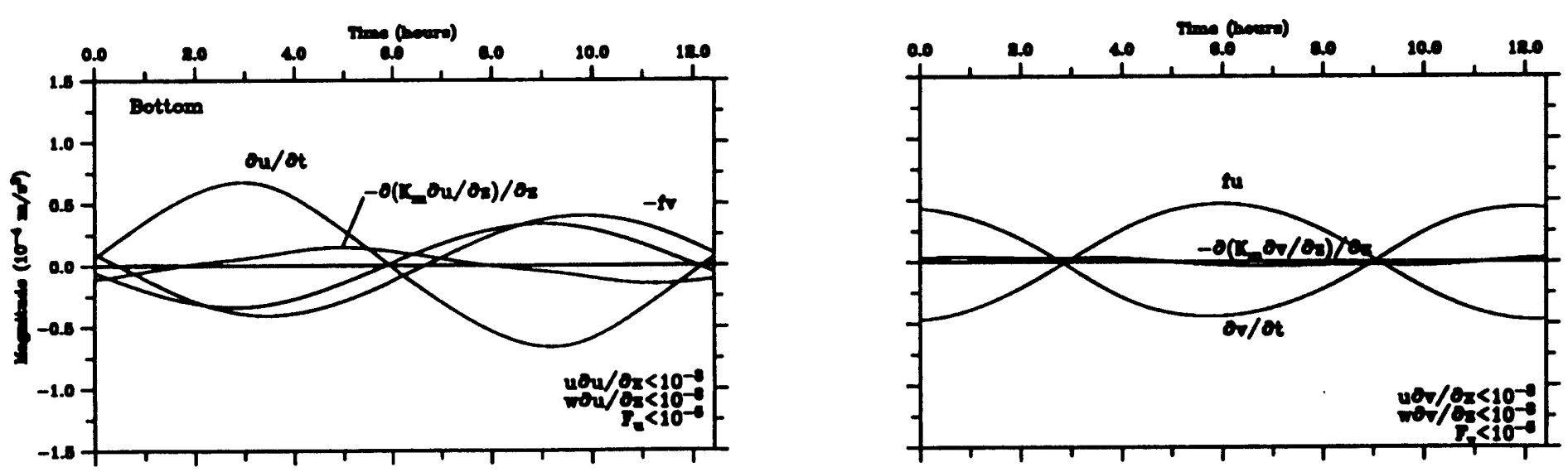

Figure 3.16: Time series of each dynamic term in the tidal momentum equations at $\sigma=-0.033$ near the surface (upper) and $\sigma=-0.9$ near the bottom (lower) at station $\mathrm{C}$ shown in Figure 3.17 for the barotropic case. 
the along-isobath direction becomes relatively important in the along-isobath momentum balance, and adds together with the horizontal advection to balance the vertical friction. The basic balance in the cross-isobath direction is between the horizontal advective and Coriolis terms. The cross-isobath gradient of mean surface elevation is in general one order smaller than the horizontal advective term except at the edge of the bank, $7 \mathrm{~km}$ away from the maximum core of along-isobath residual current where the residual current is relatively small. In summary, the mean momentum balance is given by

$$
\begin{aligned}
& \overline{u \frac{\partial u}{\partial x}}-f \bar{v}=\left(-\overline{g \frac{\partial \zeta}{\partial x}}\right), \\
& \overline{u \frac{\partial v}{\partial x}}+f \bar{u}=\overline{\frac{\partial}{\partial z}\left(K_{m} \frac{\partial v}{\partial z}\right)},
\end{aligned}
$$

where the curved brackets in (3.35) indicate that the term they enclose is, in general, one order smaller than the other terms in the equation. These balance relations indicate a simple driving mechanism by which the along-isobath mean flow is generated through the momentum transfer from tidal currents to mean flow against friction. The flow reaches its steady state when the Coriolis force term is balanced by the nonlinear advection term in the cross-isobath direction and by the nonlinear advection and vertical friction terms in the along-isobath direction.

Vertical distributions of each term in the mean momentum equations at the shelf break on the 52-m isobath are shown in Figure 3.19 to study the driving mechanism for the cross-bank double cell mean circulation pattern. Loder (1980) suggested a driving mechanism for cross-bank circulation based on the balance between the surface pressure gradient and the bottom friction in the bottom boundary layer and pointed out that a bottom flow can be driven in the $-\mathrm{x}$ direction by the positive unbalanced cross-isobath surface pressure gradient against the bottom friction, and in turn the bottom current flows toward $+\mathrm{x}$ when the surface pressure gradient is negative. Wright and Loder (1985) examined this 

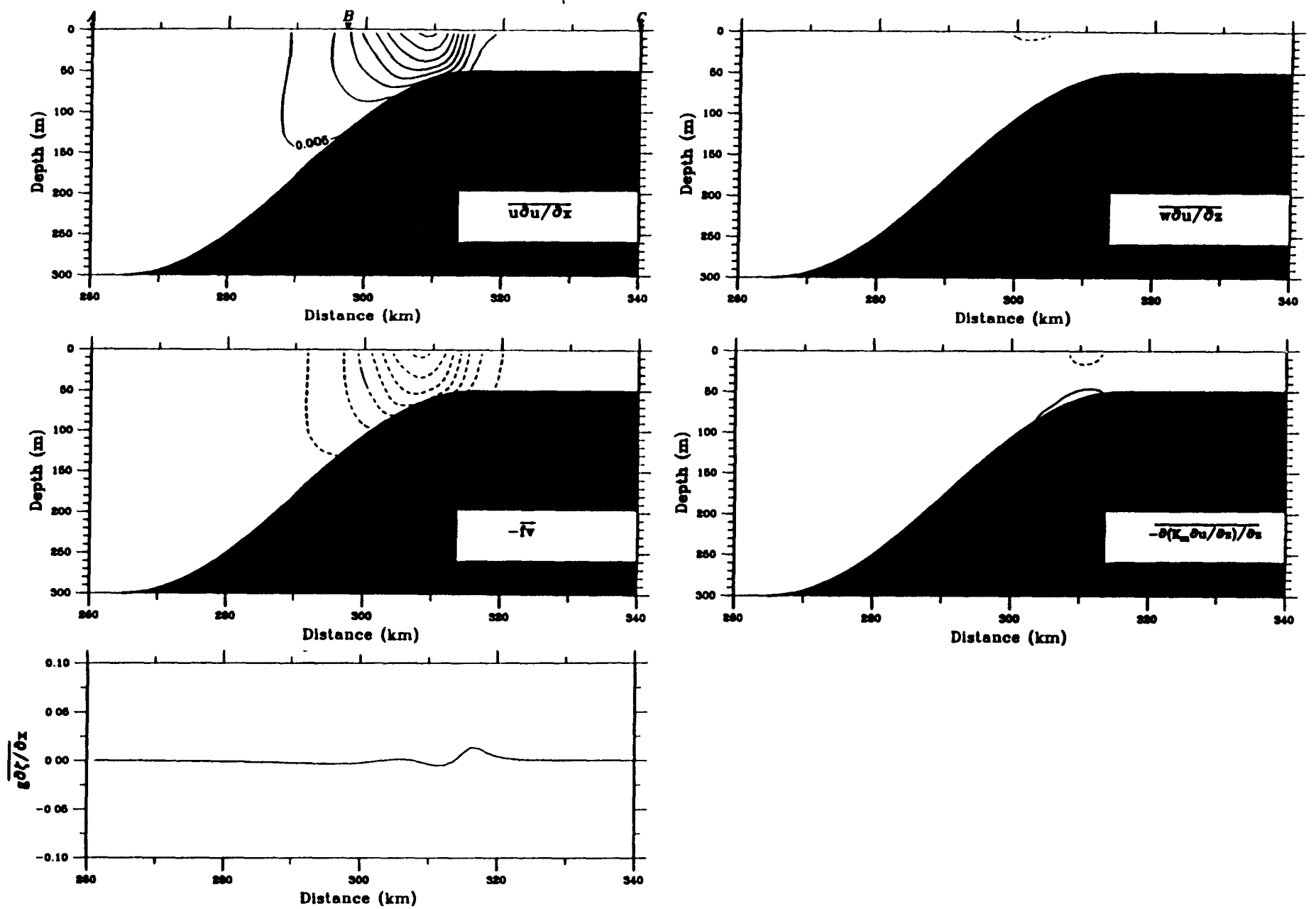

Figure 3.17: Cross-bank distribution of each dynamic term averaged over a tidal cycle in the cross-bank momentum equation for the barotropic case. The unit is $10^{-4} \mathrm{~m} / \mathrm{s}^{2}$ and the contour interval is $0.01 \times 10^{-4} \mathrm{~m} / \mathrm{s}^{2}$. 

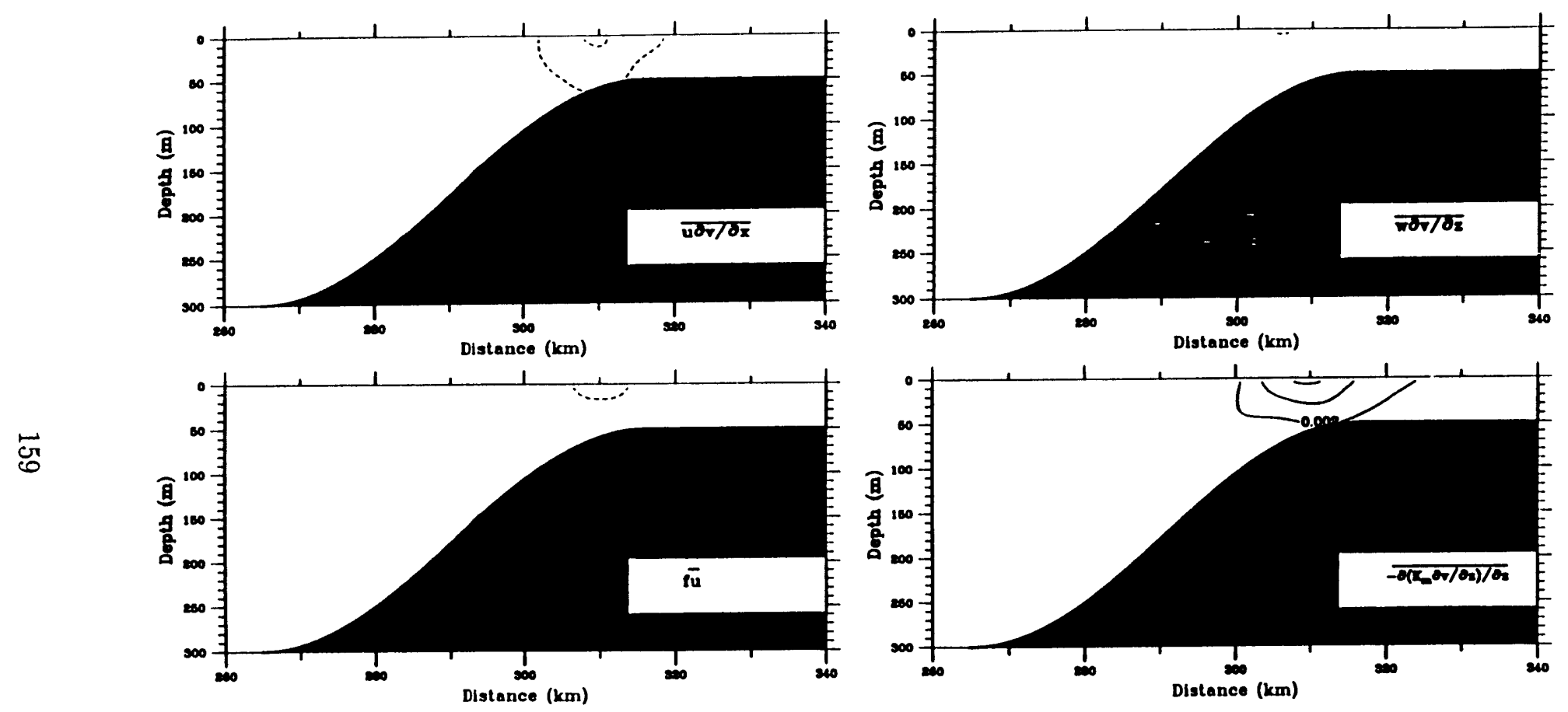

Figure 3.18: Cross-bank distribution of each dynamic term averaged over a tidal cycle in the along-bank momentum equation for the barotropic case. The unit is $10^{-4} \mathrm{~m} / \mathrm{s}^{2}$ and the contour interval is $0.005 \times 10^{-4} \mathrm{~m} / \mathrm{s}^{2}$. 

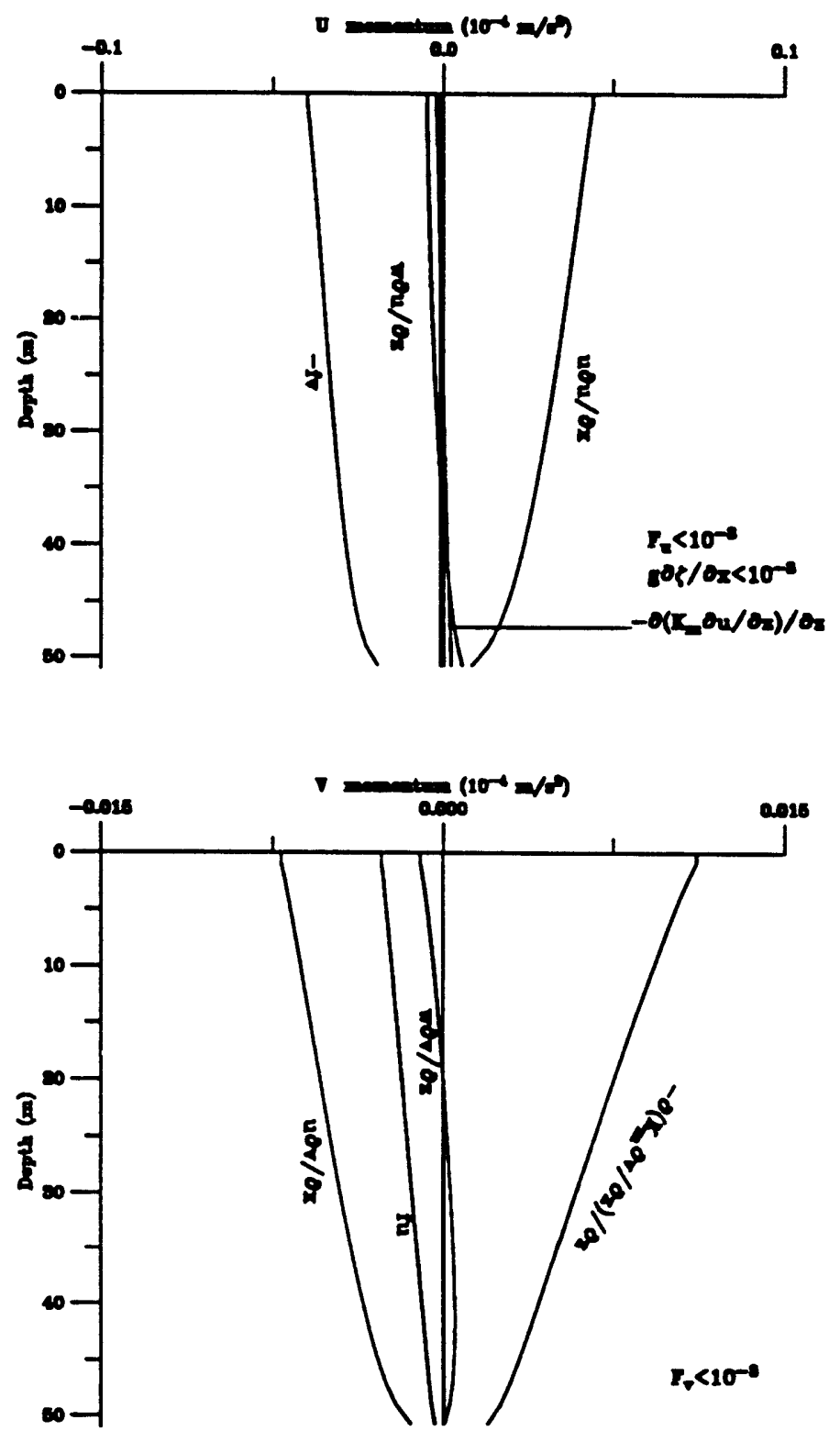

Figure 3.19: Vertical distribution of individual terms in the cross- (upper) and along- (lower) bank mean momentum equations at the $52-\mathrm{m}$ isobath near the shelf break for the barotropic case. The unit is $10^{-4} \mathrm{~m} / \mathrm{s}^{2}$. Note: two different scales are used in these two figures. It varies from -0.1 to 0.1 in the upper one while it varies from -0.015 to 0.015 in the lower one. 
mechanism using a depth-dependent model and found that it is not valid in the bottom boundary layer. In the strong tidal rectification process over finite-amplitude bottom topography, the surface pressure gradient is in general one or two orders of magnitude smaller than the horizontal advection in the cross-isobath direction, so that the bottom boundary layer is characterized by the momentum balance among the Coriolis force, the horizontal advection, and vertical friction in both along- and cross-isobath directions, i.e., the nonlinear Ekman layer (Figure 3.19). The existence of an along-isobath clockwise residual flow would produce a momentum transport toward the top of the bank by the Coriolis force. This transport overcomes the bottom friction to force upwelling near the bottom along the slope and then causes recirculation cells on both sides of the shelf break for mass conservation.

The minimum surface elevation near the shelf break may be a direct result of horizontal velocity divergence. With tidal cycle averaging, the continuity equation for the steady state becomes

$$
\overline{\frac{\partial}{\partial x}(H+\zeta) u_{a}}=0, \text { or }, \overline{(H+\zeta) u_{a}}=\text { constant }
$$

where $u_{a}$ is the vertically averaged cross-isobath velocity defined as $\frac{1}{H+\zeta} \int_{-H}^{\zeta} u d z$. Since no cross-isobath divergence or convergence of total mass transport is allowed in the steady state, the sea surface elevation must decrease when $u_{a}$ becomes large or the water depth $H$ becomes deep. This argument is supported by our numerical results where such a coherent picture exists between the mean surface elevation and cross-isobath velocity.

The tendency for the tidal and mean surface elevation to decrease downstream in our model results is probably due to wave dissipation in a nonlinear system. As we know, the gradient of free surface elevation is a depth-independent forcing associated with vertically averaged properties of the motion rather than its detailed vertical structure. When a tidal wave propagates away from its source in a viscous system, it must lose energy and momentum through friction. Although the vertically averaged model with a simple linear 
bottom stress reveals a decrease in amplitude and the phase shift for a propagating tidal wave, such a linear system could not generate a steady non-zero mean surface elevation. Therefore, the spatial decay of the mean surface elevation must be related to a nonlinear process. Batchelor (1967) pointed out that when the amplitude and phase of an oscillatory wave current vary with distance due to bottom friction, the nonlinear interaction between the wave currents can cause a net vertical transport of horizontal momentum across the bottom boundary layer to the interior, thus generating a weak mean or "steady streaming" current. This idea can be directly extended to interpret the steady non-zero mean surface elevation observed in our model since both of them are caused by the combined mechanism of friction and nonlinear advection. The vertically averaged streaming velocities due to the decrease in mean surface elevation can be estimated based on mass conservation and the geostrophic relation. Let $\bar{u}_{a 1}$ and $\bar{u}_{a 2}$ be the vertically averaged cross-isobath residual flow at points of $x=220$ and $420 \mathrm{~km}$ and $\bar{v}$ be the mean along-isobath residual current between these two points; then mass conservation and the geostrophic relation yield

$$
\left(\frac{\bar{u}_{a 1}}{\bar{u}_{a 2}}-1\right)=\frac{\eta_{2}-\eta_{1}}{H}=O\left(10^{-6}\right)
$$

and

$$
\bar{v}=\frac{g}{f} \frac{\overline{\partial \zeta}}{\partial x}=O\left(10^{-2}\right) \mathrm{cm} / \mathrm{s}
$$

where the horizontal decrease of mean surface elevation is $0.03 \mathrm{~cm}$ over a distance of $200 \mathrm{~km}$ from $x=220$ to $420 \mathrm{~km}$. Since the streaming velocities are much weaker than the tidal residual flow, we can safely neglect them in our model results.

Bowden (1960) studied oscillatory tidal flow and turbulent mixing in the Mersey estuary and found the time-dependent structure of vertical eddy viscosity to vary by a factor of 3-5 over the tidal cycle. Similar results were reported by Jonsson and Carlsen (1976), who found experimentally and theoretically that high-frequency flows have strongly timedependent eddy viscosity. Lavelle and Mofjeld (1983) investigated effects of time-varying 
viscosity on oscillatory turbulent channel flow using a semi-analytical model for the timedependent bottom boundary layer. They found that neglecting time variations in viscosity may result in underestimates of maximum bottom stress and distortion of the flow profile near times of flow reversal. All of these results, however, are limited to a non-rotational case where the kinetic energy of the flow varies significantly with time during the flow period. When the Coriolis force is included, the motion becomes rotary and the time variation of kinetic energy may decrease such that the vertical eddy viscosity exhibits only weak timedependence. Numerical experiments for both non-rotating and rotating tidal flow over a flat bottom have been conducted using the ECOM3D-SI model. In these experiments, the water depth is $300 \mathrm{~m}$ and the amplitude of the imposed semidiurnal $M_{2}$ tidal wave at the boundary is $0.5 \mathrm{~m}$. Model results are shown in Figures 3.20 and 3.21 where time series of turbulent kinetic energy and vertical eddy viscosity are plotted for non-rotating and rotating cases, respectively. A coherent structure is found between the turbulent kinetic energy $\left(q^{2}\right)$ and vertical eddy viscosity $\left(K_{m}\right) . \quad K_{m}$ varies periodically with time as a result of periodic variation of $q^{2}$ in the non-rotating case, while it remains almost constant in the rotating case since the time-dependent variation of $q^{2}$ is weak over a tidal cycle. In fact, the vertical eddy viscosity in the Mellor and Yamada level $2 \frac{1}{2}$ model is defined as a function of turbulent kinetic energy and turbulent macroscale. Since the turbulent macroscale is proportional to the turbulent kinetic energy, the time variation of vertical eddy viscosity is determined by the time-dependent structure of the turbulent kinetic energy. In these two experiments, the time variation of turbulent kinetic energy is equal to the net contribution of turbulent shear production against dissipation and vertical turbulent energy diffusion. With the parameterization of vertical turbulent momentum flux using the vertical shear of horizontal tidal currents, the turbulent shear production becomes directly proportional to the sum of the squared shears of horizontal tidal velocities. In the non-rotating case, the vertical shear of horizontal velocity varies periodically with time as the tidal current oscillates in one direction during the tidal cycle, resulting in a large periodic change in the turbulent kinetic energy. In the rotating case, however, the two velocity components of 

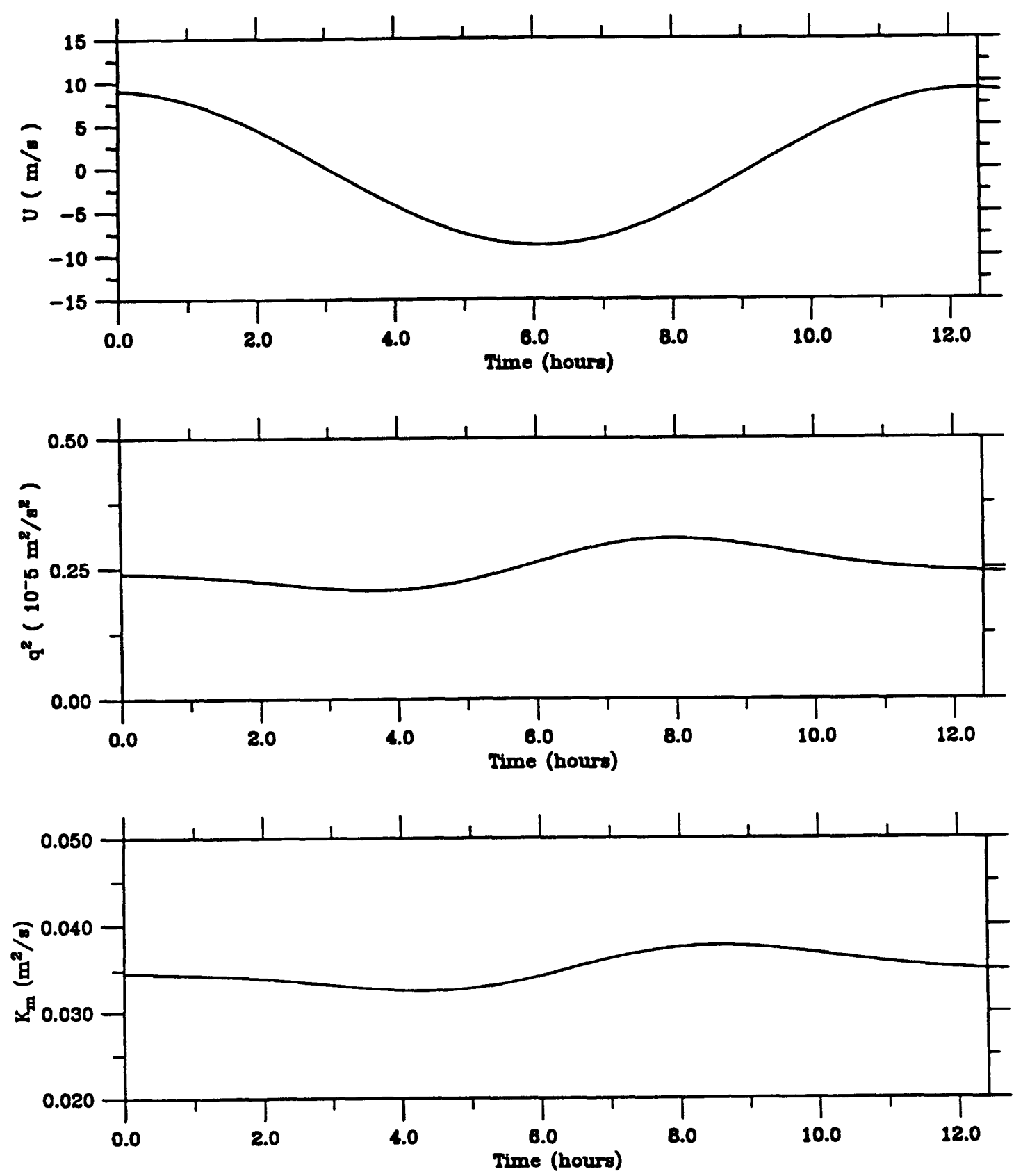

Figure 3.20: Time series of the cross-isobath tidal current, turbulent kinetic energy and vertical eddy viscosity at $\sigma=-0.94$ over a tidal cycle in the non-rotating case. The 52 vertical grid points are chosen to resolve the bottom boundary layer. 

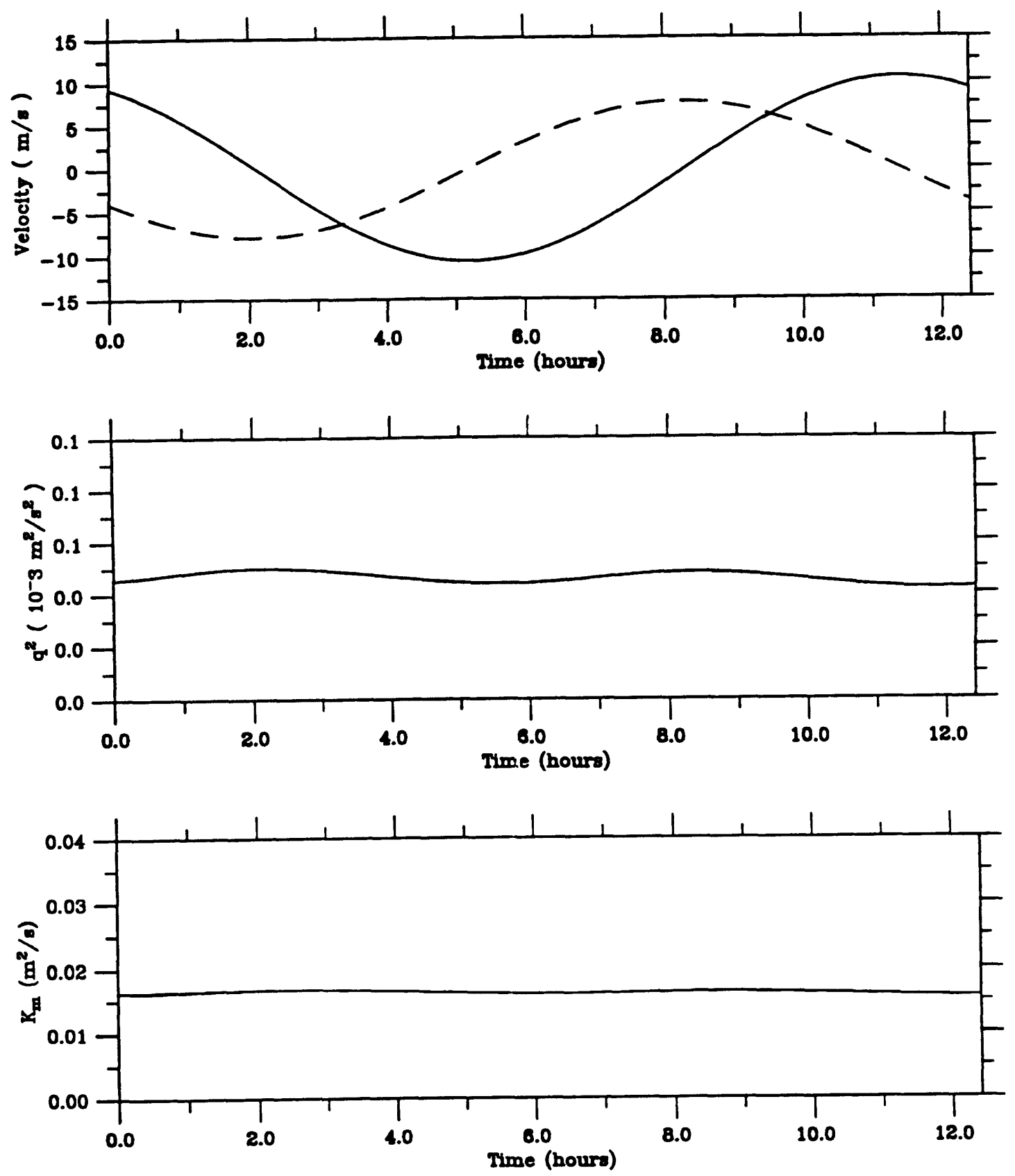

Figure 3.21: Time series of the cross-and along-isobath tidal currents, turbulent kinetic energy and vertical eddy viscosity at sigma $=-0.94$ over a tidal cycle in the rotating case. The 52 vertical grid points are chosen to resolve the bottom boundary layer. 
the tide oscillate with a phase difference of $90^{\circ}$, so that the sum of the squared shears of horizontal tidal velocities is relatively constant over a tidal cycle, leading to a weak timedependent structure of turbulent kinetic energy and hence of vertical eddy energy. The relatively large time variation of vertical eddy viscosity found over the slope is due to the difference in amplitude of the two components of tidal velocity. In the deep region, the tidal velocity amplitude is almost constant because the tidal ellipse is close to a circle, while in the shallow region, the tidal velocity amplitude varies with time during the tidal cycle because the tidal ellipse is rather elongated.

The results of our numerical study of barotropic tidal rectification over a finiteamplitude symmetric bank are summarized as follows. The model predicts a clockwise jetlike residual circulation along the bank and a friction-induced, double-cell, cross-isobath, mean circulation centered near the shelf break on both flanks of the bank, consistent with the results of previous theoretical studies. Over a finite-amplitude bank where nonlinear interaction is strong, the along-isobath residual flow is generated through the momentum transfer from tidal currents to mean flow against friction and reaches its steady state when the Coriolis force term is balanced by the nonlinear advection term in the cross-isobath direction. Unlike the vertically averaged model, the Coriolis force term in the along-isobath momentum equation becomes relatively important in the momentum balance, while the cross-isobath gradient of mean surface elevation is in general much smaller than horizontal advection so the cross-isobath momentum balance is not geostrophic. Mass conservation suggests that the minimum mean surface elevation found near the shelf break on both sides of the bank is due to a divergence of the vertically averaged cross-isobath mean flow. The bottom cross-isobath upwelling flow (hence the double cell circulation pattern) is mainly driven by the nonlinear Ekman layer mechanism. Since the $M_{2}$ tide propagates as a progressive inertial gravity wave and tidal currents are rotary with a phase difference of $90^{\circ}$, the sum of the squared shears of horizontal tidal velocity does not vary much over the tidal cycle; therefore the turbulent kinetic energy and hence vertical eddy viscosity exhibit weak time dependence. Finally, the vertical structure of the residual current is more 
sensitive to the horizontal rather than the vertical structure of the vertical eddy viscosity, suggesting that the constant eddy viscosity model is adequate to provide a qualitative picture of barotropic tidal rectification over finite-amplitude topography. 


\subsection{Stratified Tidal Rectification Over a Symmetrical Bank}

Numerical experiments with the barotropic model provide us with a good view of the basic mechanism for the generation of residual currents over a finite-amplitude bottom topography. However, the barotropic model cannot quantitatively predict the magnitude of residual flow and its seasonal variability because it ignores tidal mixing and tidal rectification associated with stratification. The tidal mixing mechanism in a stratified fluid is different from that in a homogeneous fluid. Unlike a homogeneous fluid where turbulent kinetic energy for tidal mixing is mainly generated by turbulent shear production against turbulent dissipation and vertical friction, both shear and buoyancy turbulent production are important in generating the turbulent kinetic energy in a stratified fluid so that tidal mixing occurs only when tidal energy dissipation is stronger than buoyancy input. For given tidal currents, the thickness of the bottom boundary layer depends on the ratio of turbulent energy dissipation to stratification (Simpson and Hunter, 1974) rather than the ratio of vertical eddy viscosity to the Coriolis parameter as a classic Ekman layer in a homogeneous fluid.

Since the friction is not only a time-dependent function of tidal currents but also of stratification, parameterizing the vertical eddy viscosity $\left(K_{m}\right)$ becomes a critical problem for the study of stratified tidal rectification. Loder and Wright (1985) considered the influence of stratification on the magnitude of $K_{m}$ in the buoyancy-driven flow at density fronts. To find an analytical solution, they simplified $K_{m}$ to be a function of time-independent gradient Richardson number, which led to a vertically uniform $K_{m}$ for a given stratification. A similar approach was also taken by Maas and Zimmerman (1989a and $b$ ), who assumed $K_{m}$ to be a function of time-independent Brunt-Väisälä frequency for the problem of baroclinic tidal rectification. It is questionable, however, whether or not their results can be quantitatively applied to the real ocean because of the uncertainty in the vertically uniform eddy viscosity. In fact, $K_{m}$ is a function of Richardson number only in a case where the turbulent shear and buoyancy production are balanced by turbulent dissipation (Mellor and Yamada, 1982). 
Since Richardson number changes during the process of tidal mixing, $K_{m}$ is in general flow-dependent rather than constant. In addition to the net contribution of turbulent shear and buoyancy production against turbulent dissipation and vertical friction to the time variation of the turbulent energy, in a strongly nonlinear system, the horizontal and vertical advection of turbulent energy may redistribute the tidal mixing energy and then produce a non-uniform spatial distribution of the tidal mixing coefficient. Therefore, the simplified vertically uniform $K_{m}$ may no longer be valid in such a system.

We therefore have chosen to use the Mellor and Yamada level $2 \frac{1}{2}$ turbulent closure model to calculate the time- and space-dependent turbulent kinetic energy and turbulent macroscale by which $K_{m}$ can be directly estimated at each time step. In order to understand the basic mechanisms of the stratified tidal-rectification process, we first consider numerical experiments with a finite-amplitude symmetric bank in this section, and then extend our work to the real asymmetrical bottom topography over Georges Bank in section 3.6 where stratified tidal rectification during summer and winter will be discussed.

Numerical experiments for stratified tidal rectification over a symmetrical finiteamplitude bank have been conducted with the same initial and boundary conditions as the unstratified problem except that the initial temperature (density) field has been chosen to be a non-zero linear function of $z$. In order to reduce errors due to stratification in the $\sigma$-coordinate transformation over the sloping bottom, we chose a finer horizontal grid of $\Delta x_{1}=1.25 \mathrm{~km}$ near and over the bank and a coarse grid of $\Delta x_{2}=11.96 \mathrm{~km}$ outside of our domain of interest. A linearly increasing function of the horizontal grid is also used to connect these two different grids over 20-25 grid points. The vertical grid has a uniform resolution of $\Delta \sigma=-0.033$, which corresponds to a $\Delta z$ of $10 \mathrm{~m}$ in the deep region for $H_{s}=300 \mathrm{~m}$ and $1.7 \mathrm{~m}$ for $H_{s}=50 \mathrm{~m}$ or $3.3 \mathrm{~m}$ for $H_{s}=100 \mathrm{~m}$.

The model has been run for both strong and weak initial stratification over the different topographic shapes considered in section 3.4. The vertical distribution of initial 
temperature is given by

$$
T=T_{o}+\frac{T_{b}-T_{o}}{H_{d}} z
$$

where $T_{o}$ and $T_{b}$ are the initial surface and bottom (at the depth of $H_{d}=300 \mathrm{~m}$ ) temperatures, respectively. The values of $T_{0}$ and $T_{b}$ are chosen based on summer and winter monthly averaged distributions of temperature across Georges Bank (Flagg, 1987). For the case of strong stratification, $T_{o}$ and $T_{b}$ are taken as $20^{\circ} \mathrm{C}$ and $11^{\circ} \mathrm{C}$. For the case of weak stratification, $T_{o}$ and $T_{b}$ are taken as $13^{\circ} \mathrm{C}$ and $11^{\circ} \mathrm{C}$ (Figure 3.22).

To organize our discussion, we will first concentrate on the case of $H_{s}=50 \mathrm{~m}$ and $\ell_{t}=50 \mathrm{~km}$ with strong stratification. Secondly, we will extend our discussion to include effects of different topographic slopes and heights of the bank, and then discuss the effects of weak stratification. A diagnostic analysis for momentum and heat balances will be made to investigate the dynamic mechanism for stratified tidal rectification and the energy argument used to derive a simple formula for tidal mixing.

\section{Model Results}

Strong Stratification. Fifteen-day time series of cross-and along-isobath tidal currents at $\sigma=-0.033$ at five reference points $\mathrm{A}-\mathrm{E}$ (shown in Figure 3.22) are presented in Figure 3.23 for the case of $H_{s}=50 \mathrm{~m}$ and $\ell_{t}=50 \mathrm{~m}$, and the associated time series of temperature at $\sigma=-0.033$ at the three reference points on the left side of the bank from $\mathrm{A}-\mathrm{C}$ are shown in Figure 3.24. Although it took about five days for the model surface tidal currents to become stable and the steady residual flow to be established, it took much longer for temperature to become stable in the deep regions and over the slope, implying a long thermal diffusion time scale. We stopped the computation after fifteen days when the mean temperature distribution became relatively constant for the last two tidal cycles. With a tidal wave forcing of $50 \mathrm{~cm}$ in amplitude at the left open boundary, the surface tidal currents are symmetric across the bank, with a maximum speed of about $10 \mathrm{~cm} / \mathrm{s}$ in the 

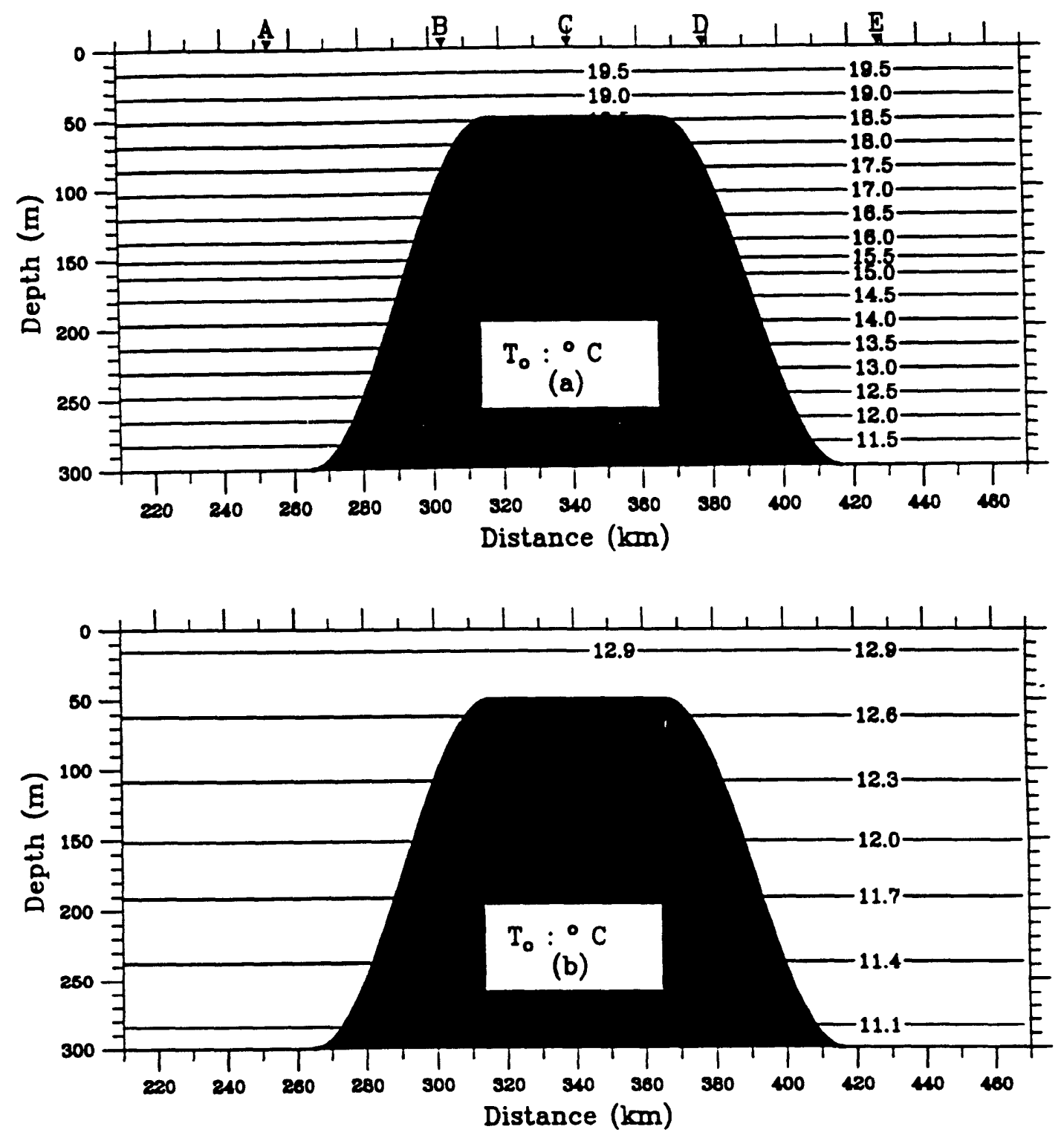

Figure 3.22: Initial temperature distribution for the cases of strong (a) and weak (b) stratification. The surface and bottom (at a depth of $300 \mathrm{~m}$ ) temperatures are $20^{\circ} \mathrm{C}$ and $11^{\circ} \mathrm{C}$ for the case of strong stratification but $13^{\circ} \mathrm{C}$ and $11^{\circ} \mathrm{C}$ for the case of weak stratification. The contour interval is $0.5^{\circ}$ in a and $0.3^{\circ} \mathrm{C}$ in $\mathrm{b}$. 

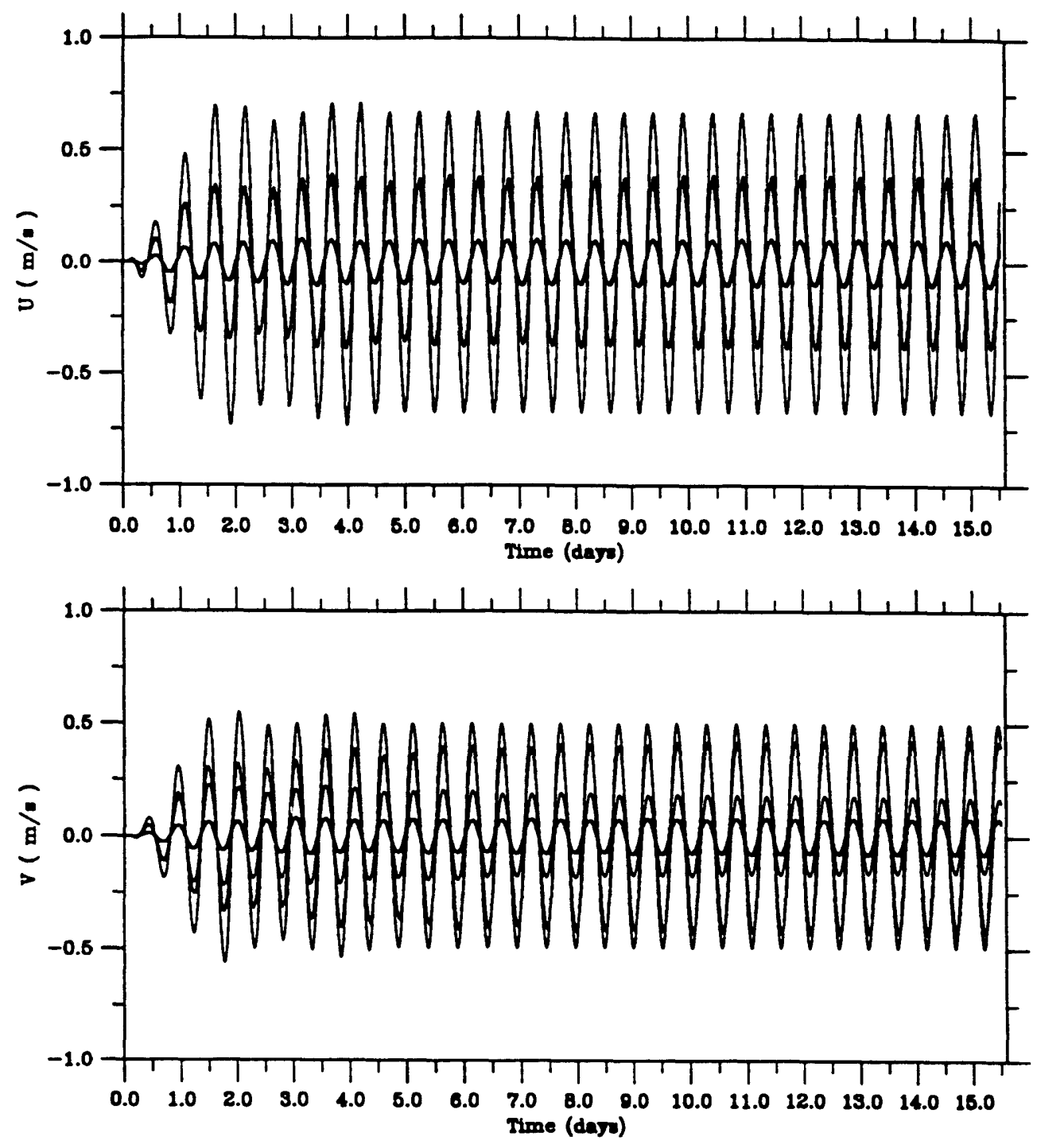

Figure 3.23: Time series of cross- and along-isobath tidal currents $(u$ and $v)$ at $\sigma=-0.033$ at five reference points shown in Figure 3.22. The topographic scale $\ell_{t}=50 \mathrm{~km}$, and the water depth $H_{s}=50 \mathrm{~m}$ over the bank and $H_{d}=300 \mathrm{~m}$ away from the bank. 
deep region and about $70 \mathrm{~cm} / \mathrm{s}$ in $u$ and $50 \mathrm{~cm} / \mathrm{s}$ in $v$ on the top of the bank. Turbulent mixing associated with strong tidal currents makes the water temperature well mixed on a time scale of about two days (Figure 3.24c) on the top of the bank and then creates well defined thermal fronts at the shelf break on both sides of the bank, separating the wellmixed water on the top of the bank from the stratified water on the slope (Figure 3.25a). Next to the front over the slope, tidal mixing plus the thermal diffusion also generates a bottom mixed layer parallel to the slope, thinner in the deep region and thicker as the water becomes shallower. As a result, the along-isobath clockwise residual current intensifies with a maximum of about $16 \mathrm{~cm} / \mathrm{s}$ at a depth of $25 \mathrm{~m}$ at the front and the axis of the strongest currents coincides with the top of the bottom boundary layer along the slope (Figure 3.25b). The cross-isobath scale of residual current is about $70 \mathrm{~km}$ on either side of the bank, which is about twice as wide as that in the homogeneous case but much larger than the internal Rossby deformation radius $\left(\ell_{i}=N H / f=15-30 \mathrm{~km}\right.$ for $H=150-300 \mathrm{~m}, N=10^{-2} \mathrm{~s}^{-1}$, and $f=10^{-4} \mathrm{~s}^{-1}$ ).

A relatively strong cross-isobath double cell circulation pattern is found on both sides of the bank where the water is upwelled to the surface along the bottom slope and the axis of maximum horizontal gradient of temperature and then downwelled on both sides of the front, causing convergence near the bottom and divergence near the surface (Figures 3.26 and 3.27). The maximum vertical velocity is about $3 \times 10^{-2} \mathrm{~cm} / \mathrm{s}$ in the upwelling region and about $5 \times 10^{-3} \mathrm{~cm} / \mathrm{s}$ in the downwelling region, corresponding to a cross-isobath current of about $2.0 \mathrm{~cm} / \mathrm{s}$ near the surface and bottom at the front. Unlike the homogeneous case where the contribution of surface pressure gradient to the along-isobath residual flow is negligible, a very smooth and large gradient of mean surface elevation is observed across the bank in the strongly stratified case, which can result in an along-isobath geostrophic mean current of about $4.3 \mathrm{~cm} / \mathrm{s}$ at a place where the upwelling is strongest (Figure 3.28). This barotropic current accounts for almost one-fourth of the total along-isobath residual current, implying that the surface pressure gradient becomes important in the momentum balance as stratification and tidal mixing are included. 

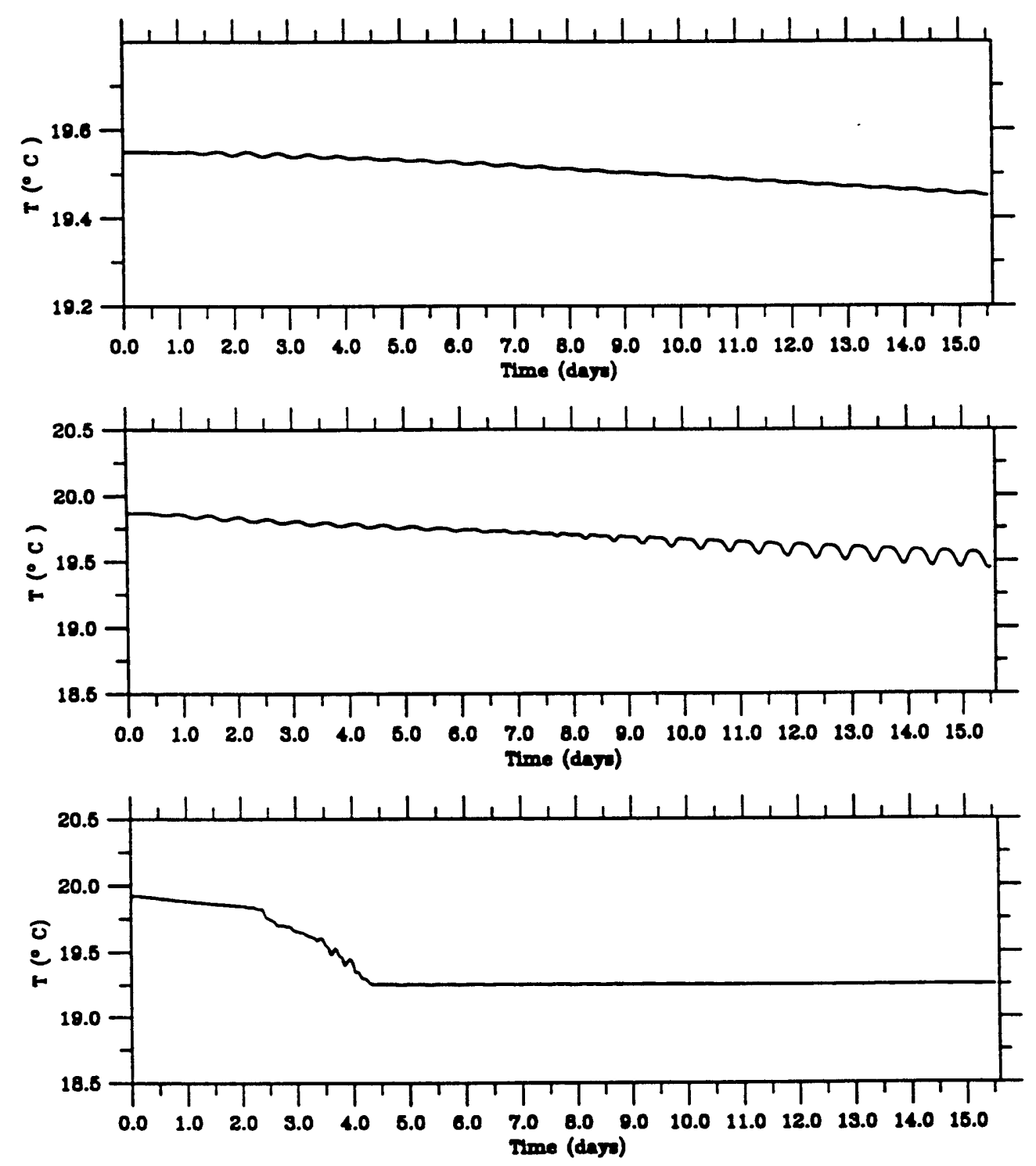

Figure 3.24: Time series of temperature at $\sigma=-0.033$ at three reference points $(\mathrm{A}-\mathrm{C})$ shown in Figure 3.22. The topographic scale $\ell_{\mathrm{t}}=50 \mathrm{~km}$, and the water depth $H_{s}=50 \mathrm{~m}$ over the bank and $H_{d}=300 \mathrm{~m}$ away from the bank. 

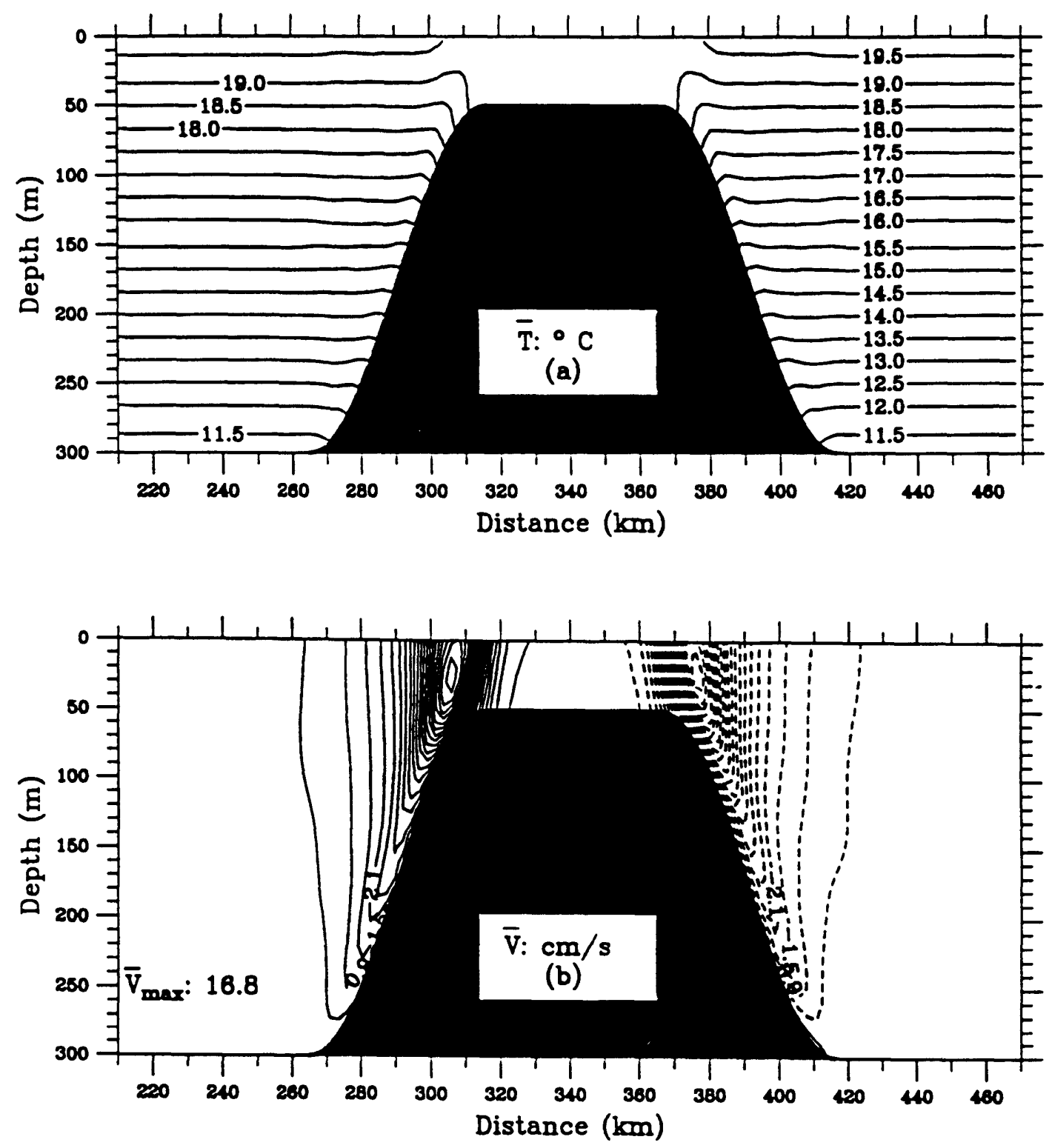

Figure 3.25: The structure of mean temperature averaged over a tidal period (a) and along-isobath residual current (b) for the topographic scale $\ell_{t}=50 \mathrm{~km}$. The amplitude of input tidal elevation at the left open boundary is $0.5 \mathrm{~m}$, the water depth $H_{s}=50 \mathrm{~m}$ over the bank and $H_{d}=300 \mathrm{~m}$ away from the bank. The contour interval is $0.5^{\circ} \mathrm{C}$ for temperature and $0.6 \mathrm{~cm} / \mathrm{s}$ for residual currents. 

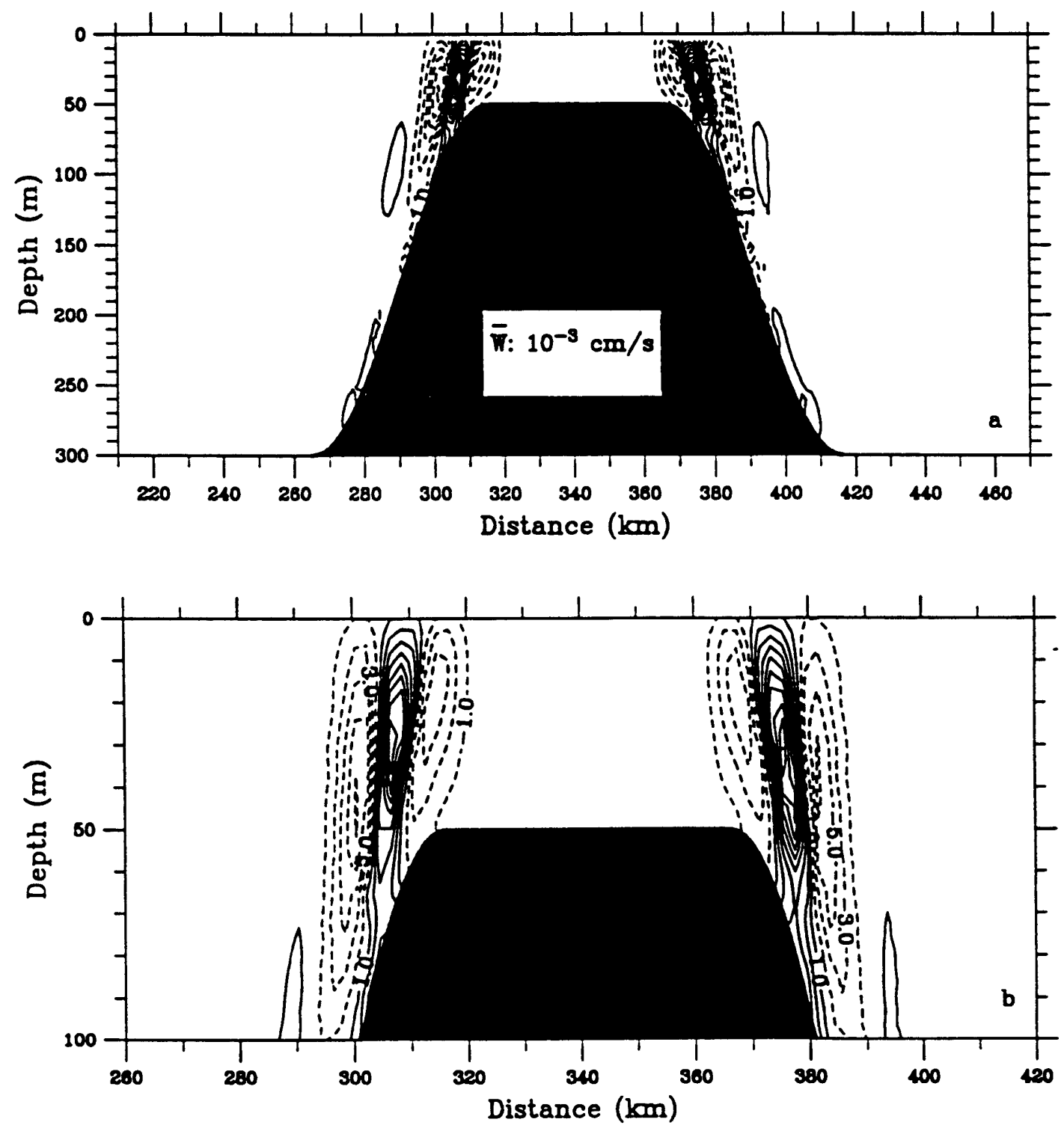

Figure 3.26: The structure of residual vertical velocity (a) for the topographic scale $\ell_{t}=50 \mathrm{~km}$. The amplitude of input tidal elevation at the left open boundary is $0.5 \mathrm{~m}$, the water depth is $50 \mathrm{~m}$ over the bank and $300 \mathrm{~m}$ away from the bank. The contour interval is $1 \times 10^{-} 3 \mathrm{~cm} / \mathrm{s}$. The contours in the upper $100 \mathrm{~m}$ are shown in $\mathbf{b}$. 

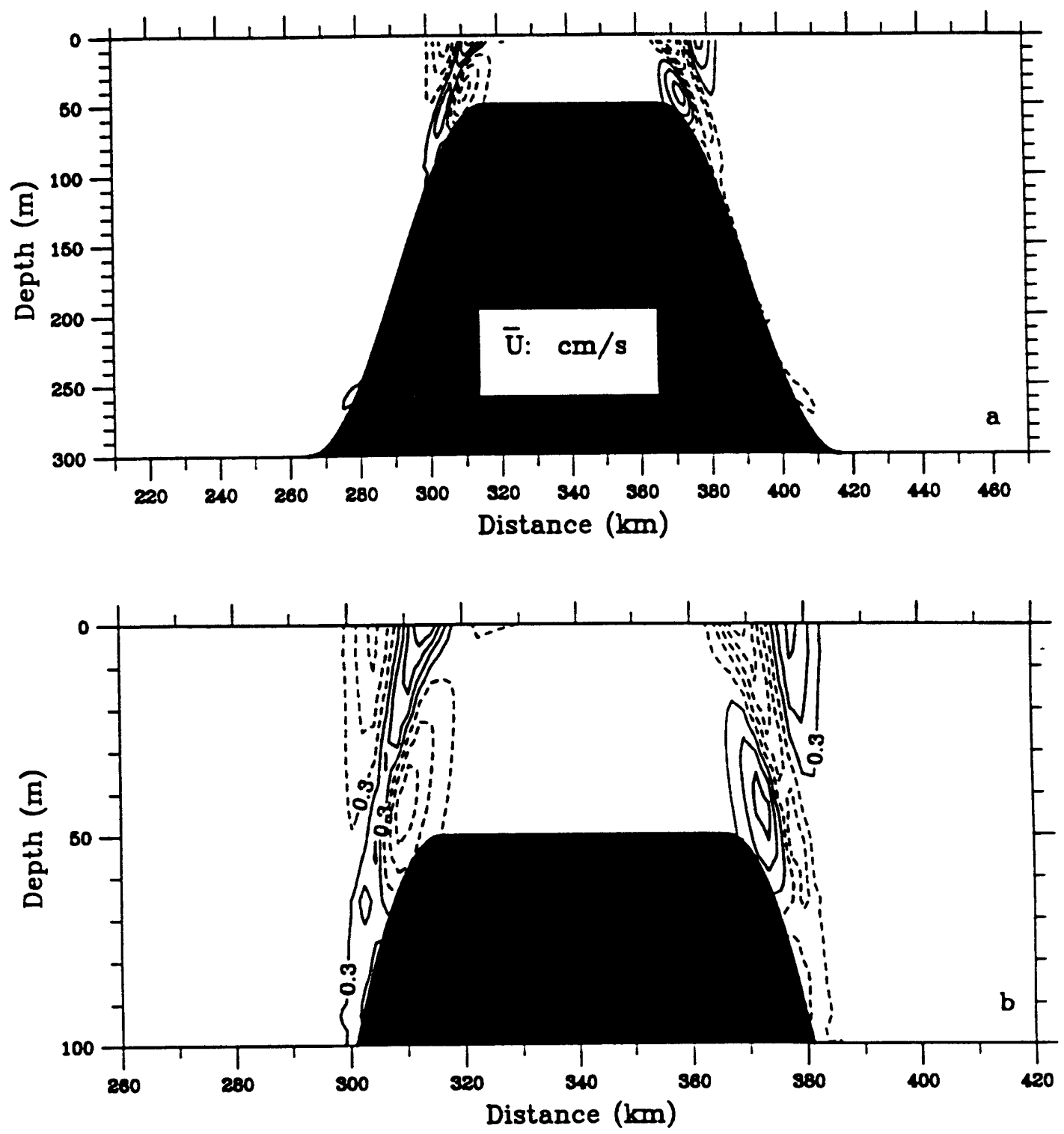

Figure 3.27: The structure of cross-isobath residual velocity (a) for the topographic scale $\ell_{t}=50 \mathrm{~km}$. The amplitude of input tidal elevation at the left open boundary is $0.5 \mathrm{~m}$, the water depth $H_{s}=50 \mathrm{~m}$ over the bank and $H_{d}=300 \mathrm{~m}$ away from the bank. The contour interval is $0.6 \mathrm{~cm} / \mathrm{s}$. The contours in the upper $100 \mathrm{~m}$ are amplified in $\mathrm{b}$. 
The model was next run with the different topographic slopes and heights to investigate the dependence of tidal mixing and residual currents on bottom topography with strong stratification. For fixed tidal forcing with an amplitude of $\zeta_{0}=50 \mathrm{~cm}$ at the left open boundary and topographic scale of $\ell_{t}=50 \mathrm{~km}$, the decrease in height of the bank tends to reduce the tidal currents over the bank so as to weaken the tidal mixing there. As the height of the bank is reduced to $200 \mathrm{~m}\left(H_{s}=100 \mathrm{~m}\right)$, the tidal currents decrease to $40 \mathrm{~cm} / \mathrm{s}$ in $u$ and $30 \mathrm{~cm} / \mathrm{s}$ in $v$ on the top of the bank, and hence tide-induced turbulence only mixed the water temperature about $40 \mathrm{~m}$ above the bottom on the top of the bank (Figure $3.29 \mathrm{a}$ ). As a result, the along-isobath residual current intensifies near the bottom at the front with a maximum of about $9.4 \mathrm{~cm} / \mathrm{s}$ at a height of $20 \mathrm{~m}$ above the bottom near the shelf break on both sides of the bank (Figure 3.29b). As the amplitude of tidal forcing is increased to $100 \mathrm{~cm}$, the tidal mixed layer is extended up to $70 \mathrm{~m}$ above the bottom on the top of the bank (Figure 3.30a), and hence the core of maximum along-isobath residual current is stretched upward along the front with a maximum of about $22.6 \mathrm{~cm} / \mathrm{s}$ at a height of $50 \mathrm{~m}$ above the bottom where the horizontal density gradient is strongest (Figure $3.30 \mathrm{~b}$ ). On the other hand, as the topographic scale of the bank $\ell_{t}$ is reduced to $25 \mathrm{~km}$, the thickness of the tidal mixed layer remains constant on the top of the bank (Figure 3.31a) but the structure of along-isobath residual flow becomes more complicated (Figure 3.31b). In addition to the front-intensified mean along-isobath current near the bottom, increasing bottom slope causes a second maximum of along-isobath residual current near the surface a few kilometers away from the shelf break on both sides of the bank and anti-clockwise currents either inside the fronts on the top of the bank or at a height of $80 \mathrm{~m}$ above the bottom outside of the slope on both sides of the bank. The horizontal length scales of these currents are about $10 \mathrm{~km}$ on the top of the bank and $20 \mathrm{~km}$ in the deep region away from the bank. The occurrence of a second maximum residual current over such a steep bottom is mainly due to the barotropic topographic response which shows a consistent intensification of the mean current near the shelf break as the topography becomes steep (see Figure 3.8). Since they do not exist in homogeneous cases, stratification is believed to be responsible for 

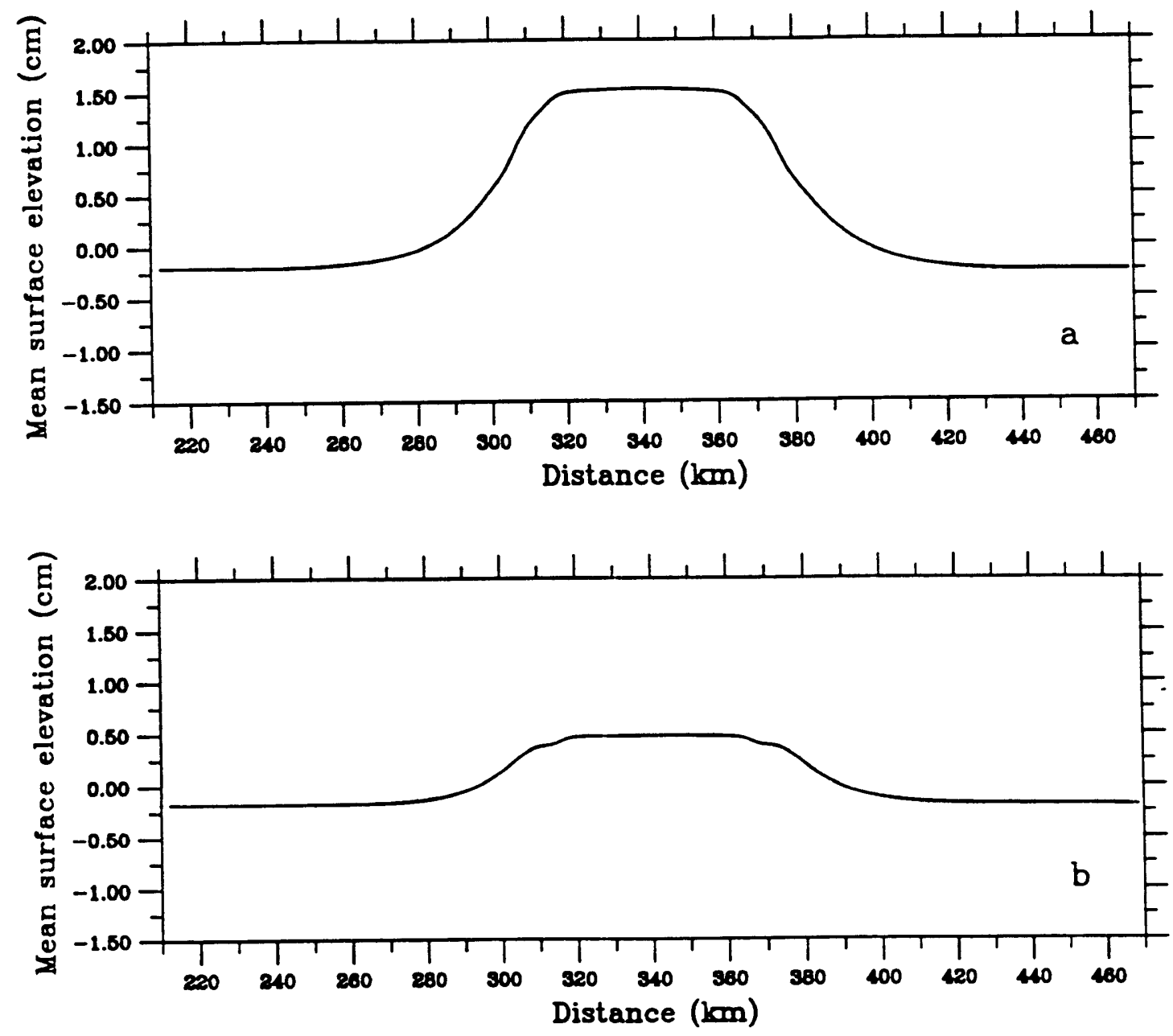

Figure 3.28: The cross-isobath distribution of mean surface elevation for the cases of strong (a) and weak (b) stratification. The topographic scale $\ell_{t}=50 \mathrm{~km}$ and the amplitude of input tidal elevation at the left open boundary is $0.5 \mathrm{~m}$, the water depth $H_{s}=50 \mathrm{~m}$ over the bank and $H_{d}=300 \mathrm{~m}$ away from the bank. 

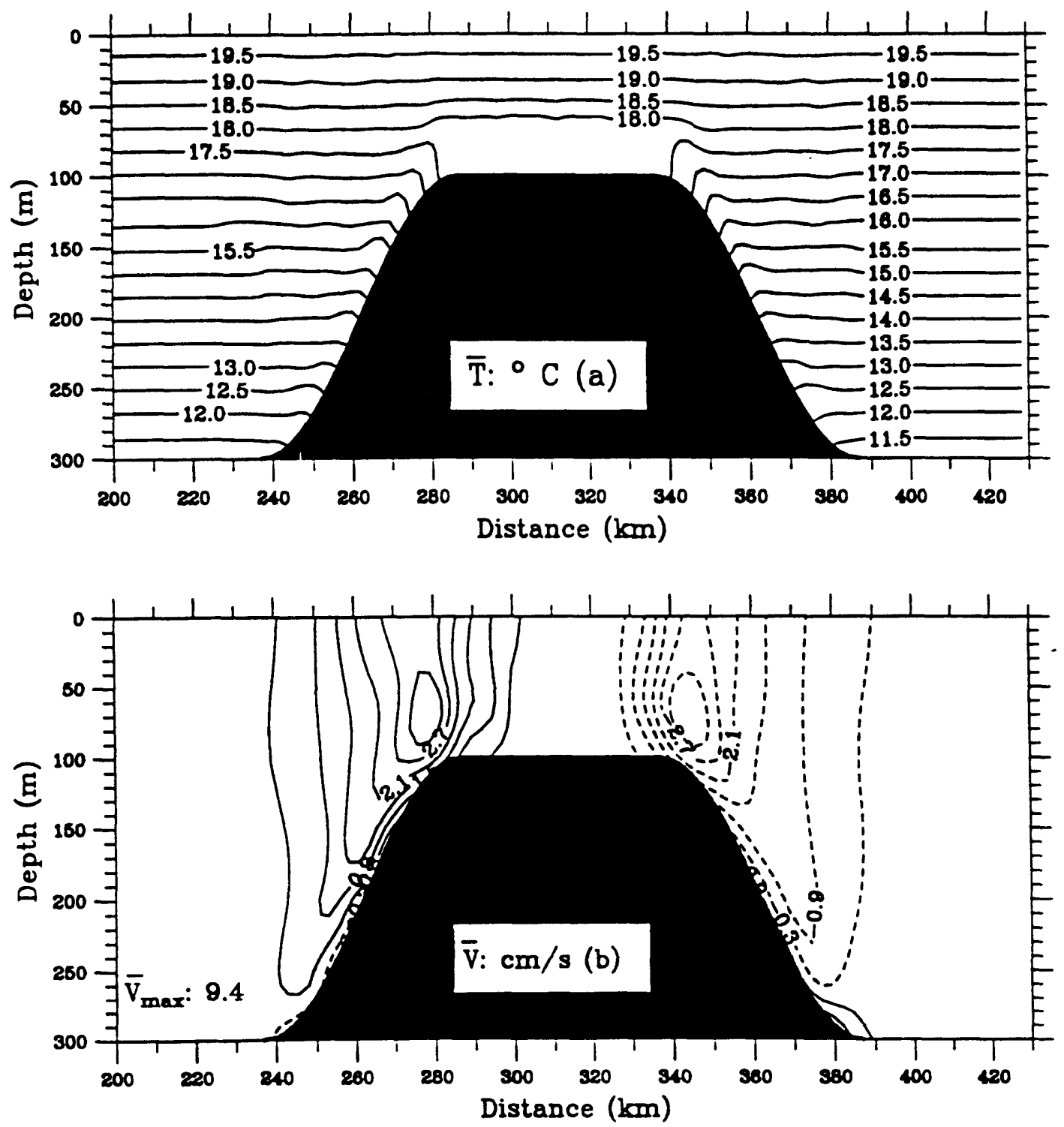

Figure 3.29: The structure of mean temperature averaged over a tidal period (a) and along-isobath residual current (b) for the topographic scale $\ell_{t}=50 \mathrm{~km}$. The amplitude of input tidal elevation at the left open boundary is $0.5 \mathrm{~m}$, the water depth $H_{s}=100 \mathrm{~m}$ over the bank and $H_{d}=300 \mathrm{~m}$ away from the bank. The contour interval is $0.5^{\circ} \mathrm{C}$ for temperature and $0.6 \mathrm{~cm} / \mathrm{s}$ for the residual current. Note: the contour plotting program PPlus is unable to resolve contours higher than $3.3 \mathrm{~cm} / \mathrm{s}$ in this case. 

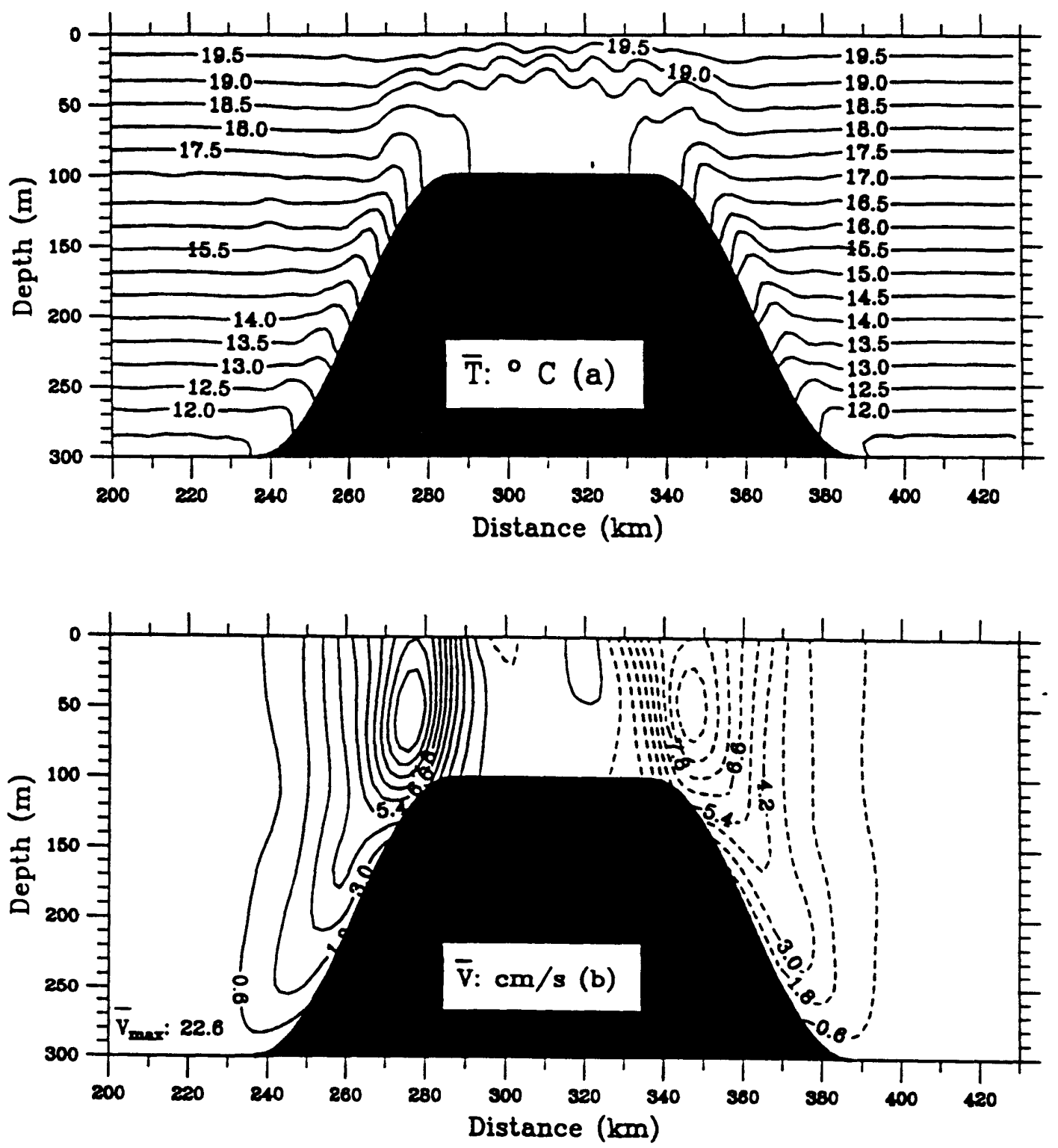

Figure 3.30: The structure of mean temperature averaged over a tidal period (a) and along-isobath residual current (b) for the topographic scale $\ell_{t}=50 \mathrm{~km}$. The amplitude of input tidal elevation at the left open boundary is $1.0 \mathrm{~m}$, the water depth $H_{s}=100 \mathrm{~m}$ over the bank and $H_{d}=300 \mathrm{~m}$ away from the bank. The contour interval is $0.5^{\circ} \mathrm{C}$ for temperature and $0.6 \mathrm{~cm} / \mathrm{s}$ for the residual current. Note: the contour plotting program PPlus is unable to resolve contours higher than $8.4 \mathrm{~cm} / \mathrm{s}$ in this case. 
the formation of these jets. On the top of the bank inside the fronts, tidal mixing produces a mixed layer of about $40 \mathrm{~m}$ above the bottom which separates the well-mixed water in the bottom boundary layer from the stratified water in the upper $60 \mathrm{~m}$. The vertical turbulent eddy viscosity is large in the bottom mixed layer but small in the upper stratified layer, suggesting that both turbulent tidal mixing and stratification may be important for the existence of these jets inside the fronts. In the deep region, however, the tidal mixing plus thermal-diffusion bottom boundary layer is only a few meters thicker above the sloping bottom than for $\ell_{t}=50 \mathrm{~km}$ while the anti-clockwise jet is located about 10-20 km away from the slope where stratification is strong and tidal mixing is weaker. Therefore, boundary mixing theories (Wunsch, 1970; Phillips, 1970; Garrett, 1990, 1992) cannot be applied to explain this jet. We will propose and discuss later one possible mechanism associated with the nonlinear interaction of internal tidal waves with the bottom slope.

In addition, the turbulent closure model makes the vertical mixing coefficient large in the tidally mixed region and very small in the stratified region. As an example, the crossisobath distribution of mean $K_{m}$ is shown in Figure 3.32 for two cases where $\ell_{t}=50 \mathrm{~km}$ is fixed and $H_{s}=100 \mathrm{~m}$ and $50 \mathrm{~m}$, respectively. In the case of $H_{s}=50 \mathrm{~m}$, the model predicts a parabolic structure of $K_{m}$ with a maximum of about $0.06 \mathrm{~m}^{2} / \mathrm{s}$ at a height of $25 \mathrm{~m}$ above the bottom inside the fronts where the water temperature is well mixed throughout the whole water column but a negligible value in the stratified region away from the bank. As the height of the bank is decreased to $200 \mathrm{~m}\left(H_{s}=100 \mathrm{~m}\right)$, however, the maximum of $K_{m}$ is at a height of $10 \mathrm{~m}$ above the bottom inside the tidal mixing front and $K_{m}$ decays quickly upward in a distance of $10 \mathrm{~m}$ since the water in the upper $60 \mathrm{~m}$ remains stratified. We also find a relatively large value of $K_{m}$ near the bottom over the slope. The spatial structure of mean $K_{m}$ in the mixed layer is similar to that found in the homogeneous case, implying a time-dependent adjustment of the vertical mixing coefficient as the turbulent shear production increases and the turbulent buoyancy production decreases during tidal mixing. 

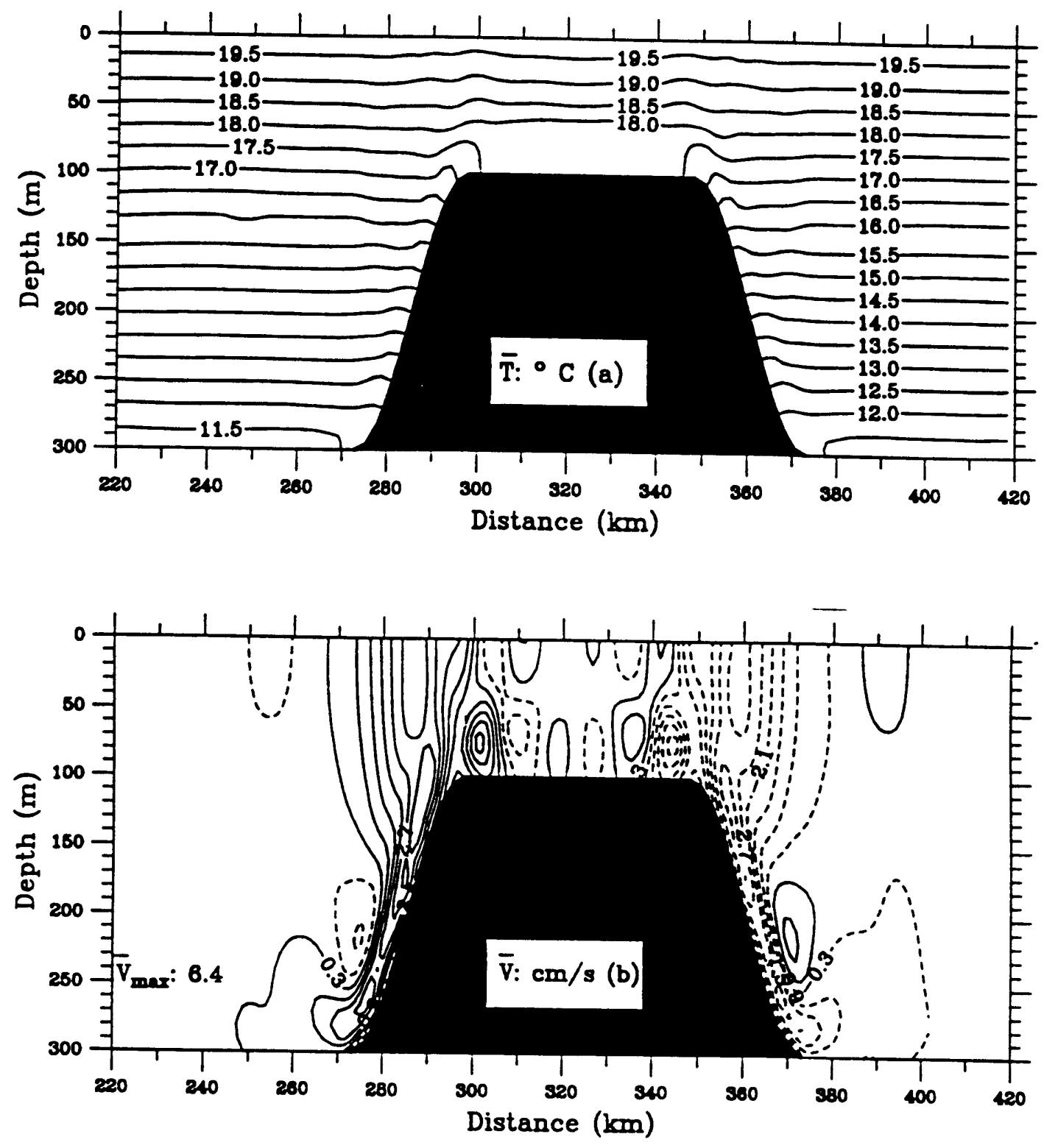

Figure 3.31: The structure of mean temperature averaged over a tidal period (a) and along-isobath residual current (b) for the topographic scale $\ell_{t}=25 \mathrm{~km}$. The amplitude of input tidal elevation at the left open boundary is $0.5 \mathrm{~m}$, the water depth $H_{s}=100 \mathrm{~m}$ over the bank and $H_{d}=300 \mathrm{~m}$ away from the bank. The contour interval is $0.5^{\circ} \mathrm{C}$ for temperature and $0.6 \mathrm{~cm} / \mathrm{s}$ for the residual current. 

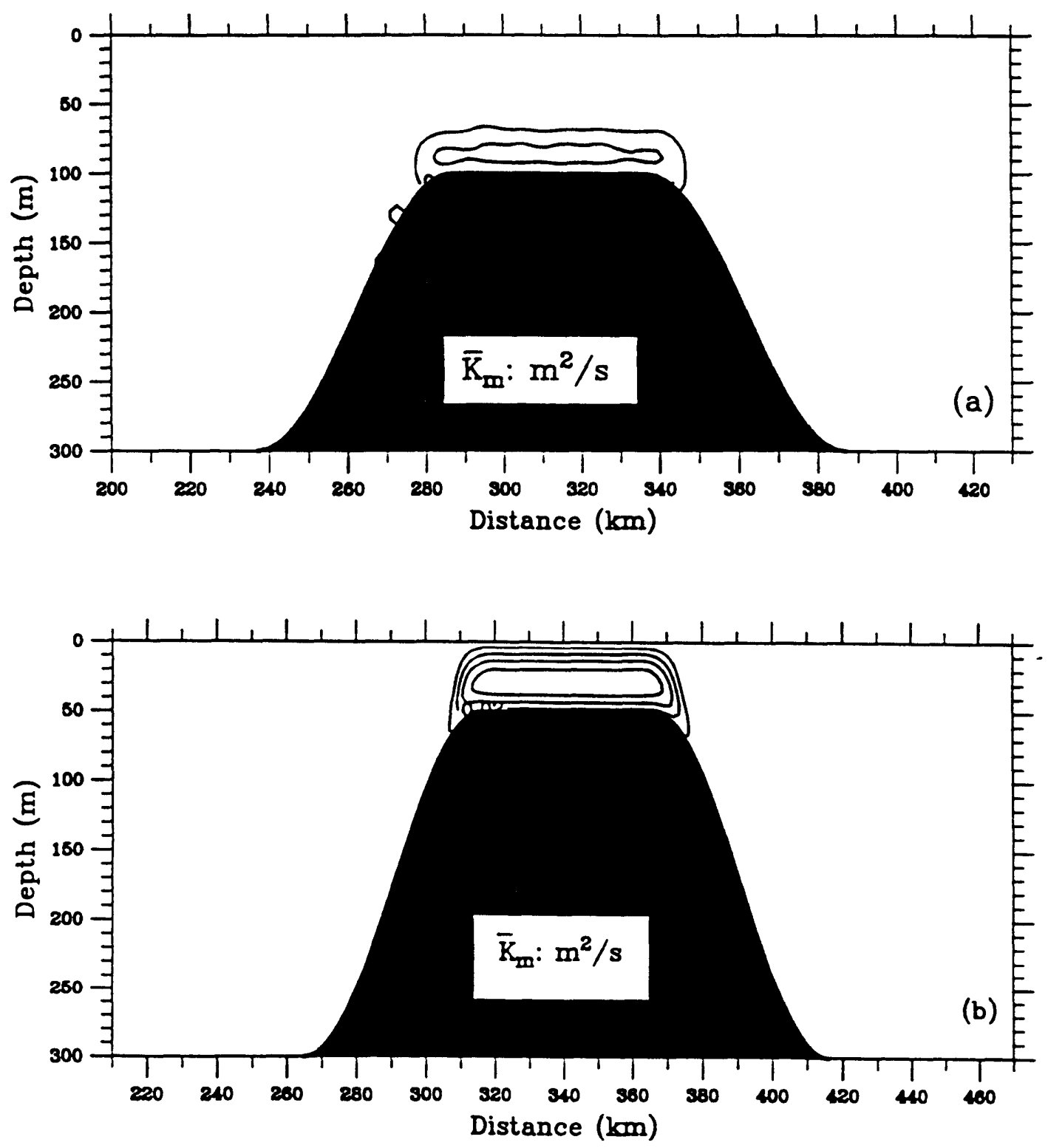

Figure 3.32: The structure of mean vertical eddy viscosity for strong stratification with a fixed topographic scale $\ell_{t}=50 \mathrm{~km}$ and $H_{s}=100 \mathrm{~m}(\mathrm{a})$ and $50 \mathrm{~m}(\mathrm{~b})$. The contour interval is $0.01 \mathrm{~m}^{2} / \mathrm{s}$ for both plots. 
Weak Stratification. Figure 3.33 shows the cross-isobath vertical distribution of mean temperature and along-isobath residual current for the case of $H_{s}=50 \mathrm{~m}, \ell_{t}=50 \mathrm{~km}$, and initial $N=3 \times 10^{-3} \mathrm{~s}^{-1}$. The structures of the tidally mixed front and bottom tidally mixed/thermal-diffusion boundary layer are very similar to those found in the case of strong stratification except for the strength of the horizontal temperature gradient. Unlike the case of strong stratification where the current is intensified at depth in the front, weak stratification leads to the surface intensification of along-isobath residual current at the front, even though the axis of strongest current remains fixed at the maximum temperature gradient along the front and the top of the bottom boundary layer over the slope. The maximum velocity is about $9.4 \mathrm{~cm} / \mathrm{s}$ at the surface at the tidal front, about $6 \mathrm{~km}$ away from the shelf break. The cross-bank scale of the mean flow is about $50 \mathrm{~km}, 10 \mathrm{~km}$ wider than the barotropic case but $20 \mathrm{~km}$ narrower than the case of strong stratification, implying that stratification tends to widen the horizontal scale of residual current in stratified tidal rectification. The fact that the internal Rossby deformation radius is much smaller than the scales of the residual current and the topography suggests that stratified tidal rectification is controlled not only by stratification and rotation but also by other dynamic factors such as topography and friction.

Similar to the case of strong stratification, the cross-isobath residual current is dominated by a double cell circulation pattern in which the water is upwelled along the slope bottom to the surface at the center of the front and then downwelled on both sides of the front (Figures 3.34 and 3.35). The maximum vertical velocity is about $5.0 \times 10^{-2} \mathrm{~cm} / \mathrm{s}$ in the upwelling region and $1.0-2.0 \times 10^{-3} \mathrm{~cm} / \mathrm{s}$ in the downwelling region, much weaker than those found in the case of strong stratification. The maximum cross-isobath horizontal velocity is about $1.0 \mathrm{~cm} / \mathrm{s}$ at the surface and $0.5 \mathrm{~cm} / \mathrm{s}$ near the bottom, almost one order smaller than in the case of strong stratification. A relatively smooth curve of mean surface elevation is observed in the case of weak stratification (Figure 3.28b). The maximum horizontal surface pressure gradient is about $1.9 \times 10^{-7}$ over the slope, resulting in an 

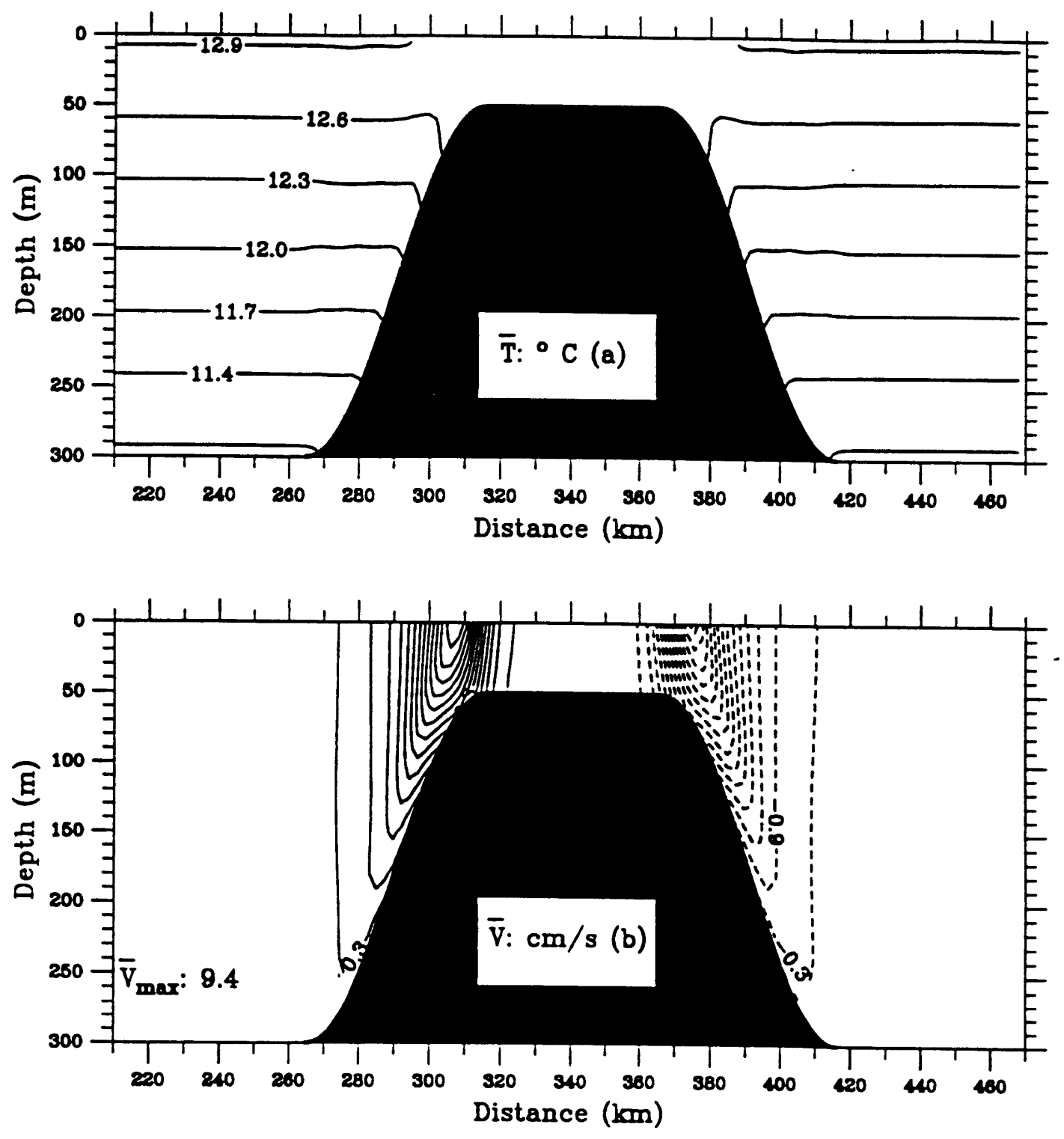

Figure 3.33: The structure of mean temperature averaged over a tidal period (a) and along-isobath residual current (b) for the case of weak stratification. The topographic scale $\ell_{\mathfrak{t}}=50 \mathrm{~km}$ and the amplitude of input tidal elevation at the left open boundary is $0.5 \mathrm{~m}$, the water depth $H_{s}=50 \mathrm{~m}$ over the bank and $H_{d}=300 \mathrm{~m}$ away from the bank. The contour interval is $0.3^{\circ} \mathrm{C}$ for temperature and $0.6 \mathrm{~cm} / \mathrm{s}$ for residual currents. 

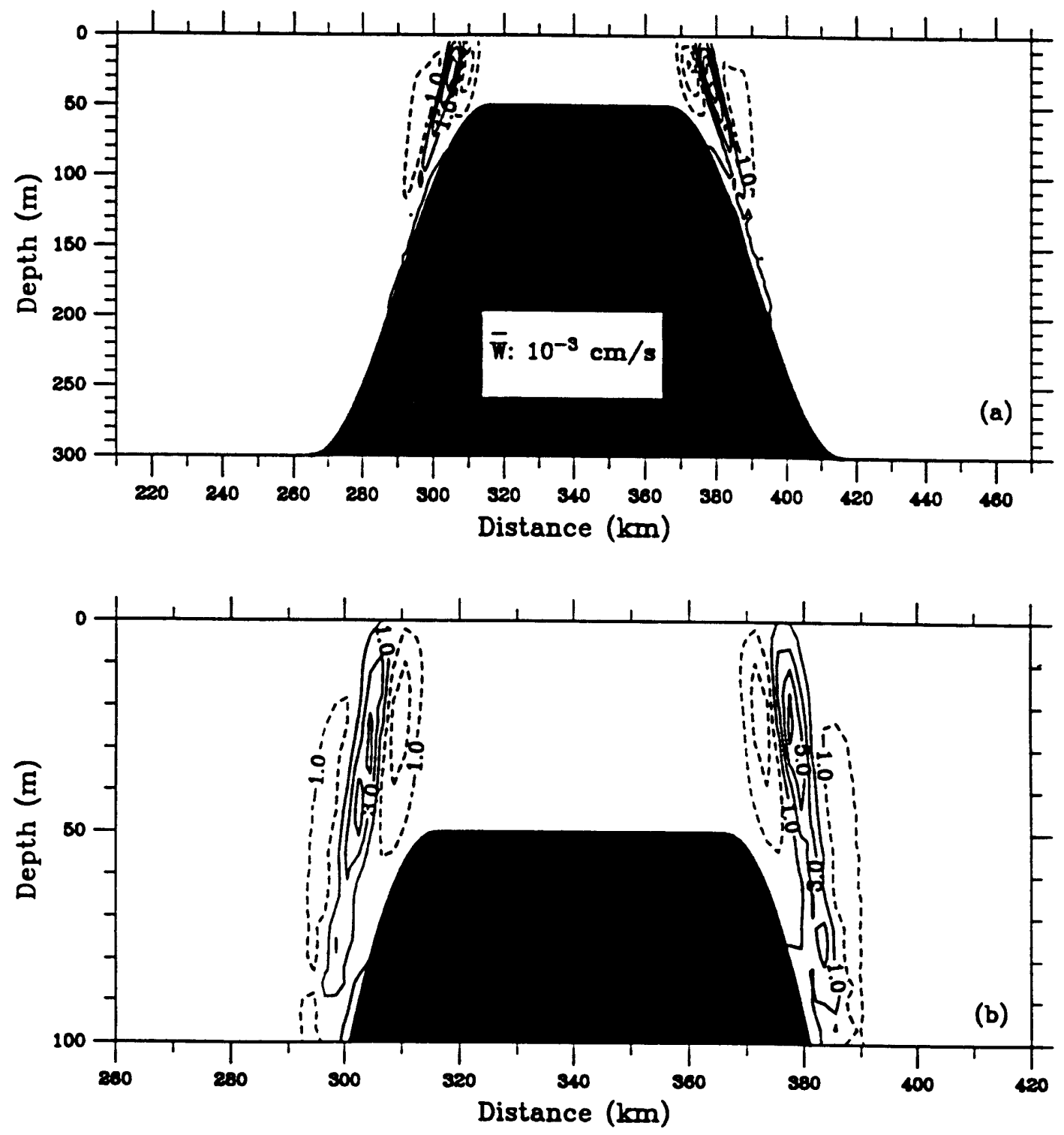

Figure 3.34: The structure of residual vertical velocity (a) for the case of weak stratification. The topographic scale $\ell_{t}=50 \mathrm{~km}$ and the amplitude of input tidal elevation at the left open boundary is $0.5 \mathrm{~m}$. The water depth $H_{s}=50 \mathrm{~m}$ over the bank and $H_{d}=300 \mathrm{~m}$ away from the bank. The contour interval is $1 \times 10^{-} 3 \mathrm{~cm} / \mathrm{s}$. The contours in the upper $100 \mathrm{~m}$ are amplified in $\mathrm{b}$. 

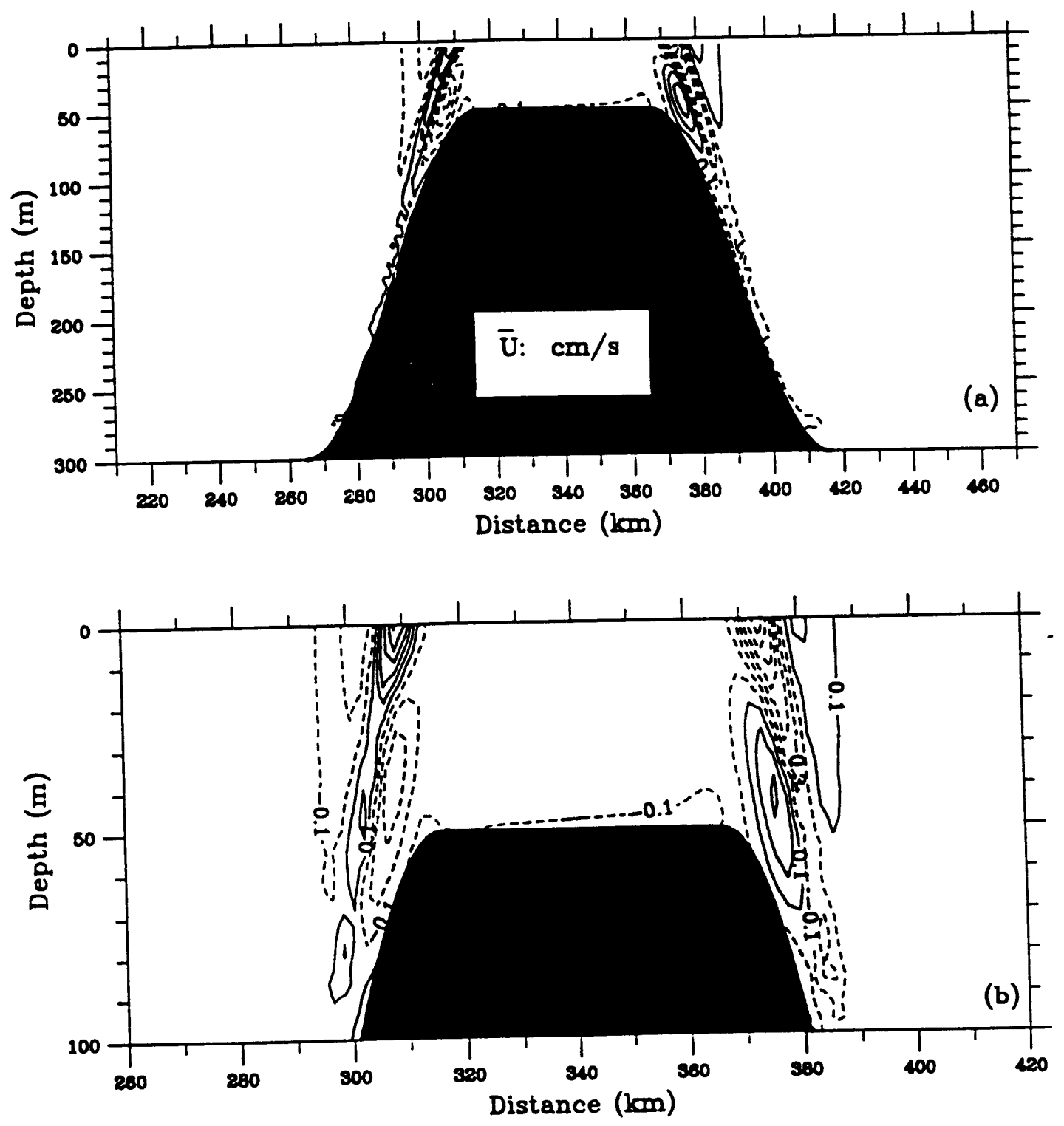

Figure 3.35: The structure of cross-isobath residual velocity (a) for the case of weak stratification. The topographic scale $\ell_{t}=50 \mathrm{~km}$ and the amplitude of input tidal elevation at the left open boundary is $0.5 \mathrm{~m}$. The water depth $H_{s}=50 \mathrm{~m}$ over the bank and $H_{d}=300 \mathrm{~m}$ away from the bank. The contour interval is $0.6 \mathrm{~cm} / \mathrm{s}$. The contours in the upper $100 \mathrm{~m}$ are amplified in $\mathrm{b}$. 
along-isobath barotropic current of about $1.8 \mathrm{~cm} / \mathrm{s}, 2.5 \mathrm{~cm} / \mathrm{s}$ smaller than that in the case of strong stratification.

As the height of the bank is decreased to $200 \mathrm{~m}\left(H_{s}=100 \mathrm{~m}\right)$, turbulent tidal mixing is not strong enough to mix the whole water column, even though the initial stratification is weak (Figure 3.36a). However, a decrease in stratification does increase the thickness of the bottom mixed layer to $60 \mathrm{~m}$ above the bottom. As a result, the alongisobath residual current is trapped near the bottom at the front, although the maximum current strength is weaker and cross-isobath motion scale is narrower compared with those in the case of strong stratification (Figure 3.37b).

\section{Discussion}

Numerical experiments with weak and strong stratification over the different topographic slopes and heights of the symmetrical bank provide us with a quantitative view of the effects of stratification and bottom topography on tidal mixing and tidal rectification. As stratification is added, stratified tidal rectification and tidal mixing intensify the alongand cross-isobath residual currents, and create tidal fronts which hence modify the vertical structure of the residual current field. The thickness of the tidally mixed bottom boundary layer depends strongly on the strength of stratification and height of the bank; it increases as stratification becomes weak or the bank becomes higher. No correlation has been found between the thickness of the tidal mixed layer and bottom slope, suggesting that the tidal mixing is directly associated with the turbulent kinetic energy dissipation characterized by the tidal currents rather than the horizontal shear of tidal velocity. As a result of tidal mixing, the along-isobath residual current becomes larger at the front as stratification is increased. Unlike the homogeneous case, increasing the bottom slope in the stratified fluid not only increases the magnitude of along-isobath residual current but also generates anticlockwise jets inside the front on the top of the bank and in the deep region beyond the bottom slope. The existence of these jets over the bank implies a complex vertical structure of dynamical balances in the momentum equations and heat equation during stratified tidal 

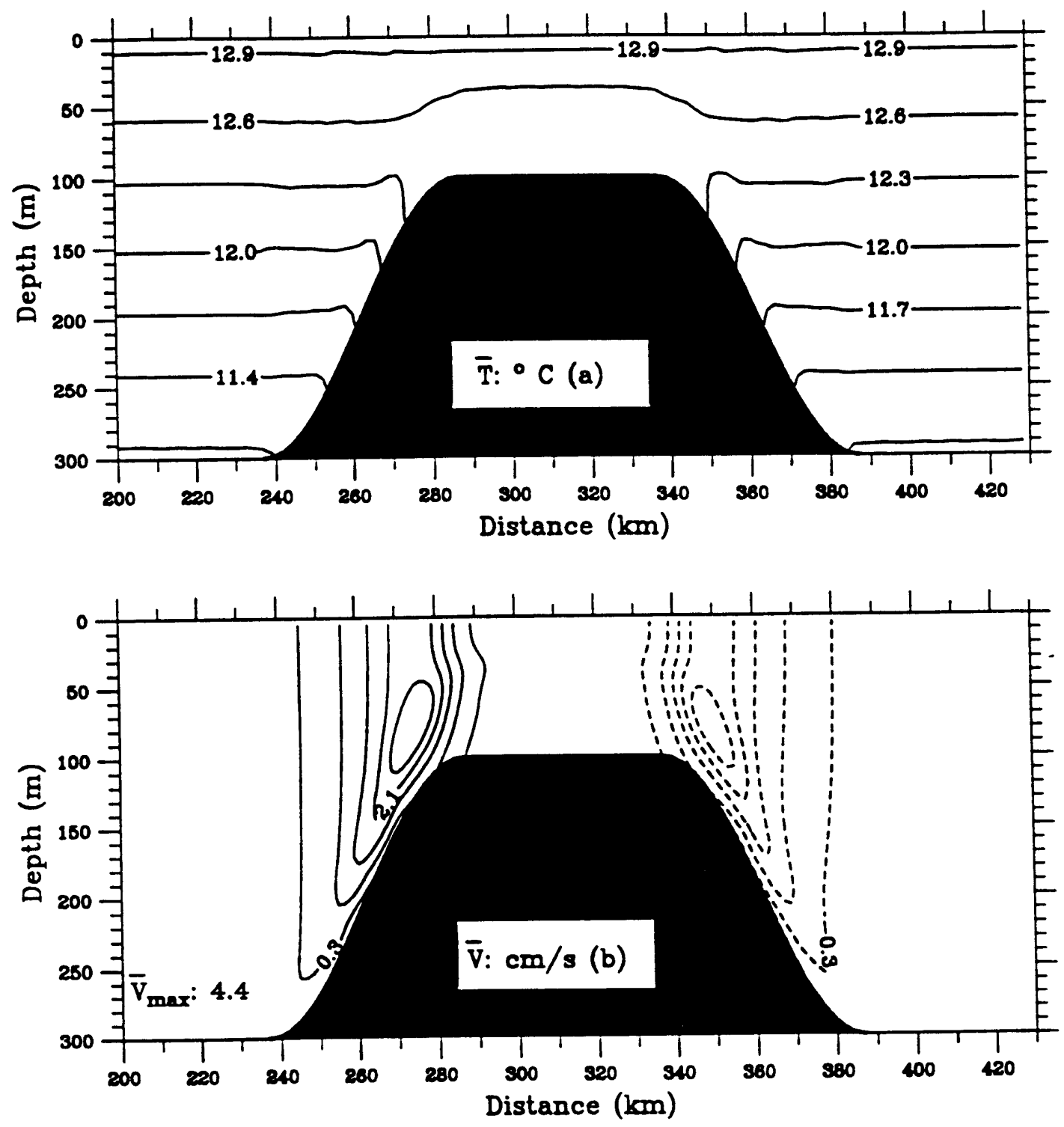

Figure 3.36: The structure of mean temperature averaged over a tidal period (a) and along-isobath residual current (b) for the case of weak stratification. The topographic scale $\ell_{t}=50 \mathrm{~km}$ and the amplitude of input tidal elevation at the left open boundary is $0.5 \mathrm{~m}$, the water depth $H_{s}=100 \mathrm{~m}$ over the bank and $H_{d}=300 \mathrm{~m}$ away from the bank. The contour interval is $0.3^{\circ} \mathrm{C}$ for temperature and $0.6 \mathrm{~cm} / \mathrm{s}$ for the residual current. 
rectification. What are the basic balances in the momentum and heat equations? Since the cross-isobath horizontal and vertical velocities may be opposite over a variable topographic bottom in some cases, the simple scaling method may not be applied to study the mechanism of tidally or topographically driven flow where the current direction is important in the dynamic balance. In addition, because the relatively strong eddies only occur as the bottom slope becomes steeper and are located away from the slope where stratification is strong and vertical friction is weak, the boundary layer mixing theories cannot be used to explain the mechanism of formation of these eddies. What causes the mean anti-clockwise currents in the deep region beyond the slope? The prediction for the thickness of tidal mixed layer by our model is limited to some special case studies. If the model result is right, it should be consistent with those results predicted by empirical energy arguments (Simpson and Hunter, 1974; Garrett et al., 1978). We will try to answer these questions next.

Diagnostic Analysis of the Tidal Motion. A diagnostic analysis has been made for the case of $H_{s}=50 \mathrm{~m}$ and $\ell_{t}=50 \mathrm{~km}$ with strong stratification. Time series of each dynamic or thermodynamic term in the momentum and heat equations at two reference points (A and B) (see Figure 3.40) are shown in Figures 3.37-3.38. Similar to the homogeneous case, at station A, $40 \mathrm{~km}$ away from the bank, the nonlinear terms are at least two orders of magnitude smaller than the Coriolis force term so that the momentum balance is characterized by linear inertial-gravity wave equations except near the bottom where the baroclinic pressure gradient makes a first-order contribution to the $x$ momentum balance. Over the slope of the bank at station B where the mean water depth is $127 \mathrm{~m}$, nonlinear terms and the baroclinic pressure gradient start to affect the tidal current as a first-order modification to the inertial-gravity wave; these terms increase as the water depth become shallower and reach their maximum near the shelf break where the tidal mixing front is located. Although the momentum equations in the upper layer far away from the bottom are mainly characterized by the inertial-gravity wave equations modified with first-order horizontal and vertical advection, the dynamic balance is very complex near the bottom 

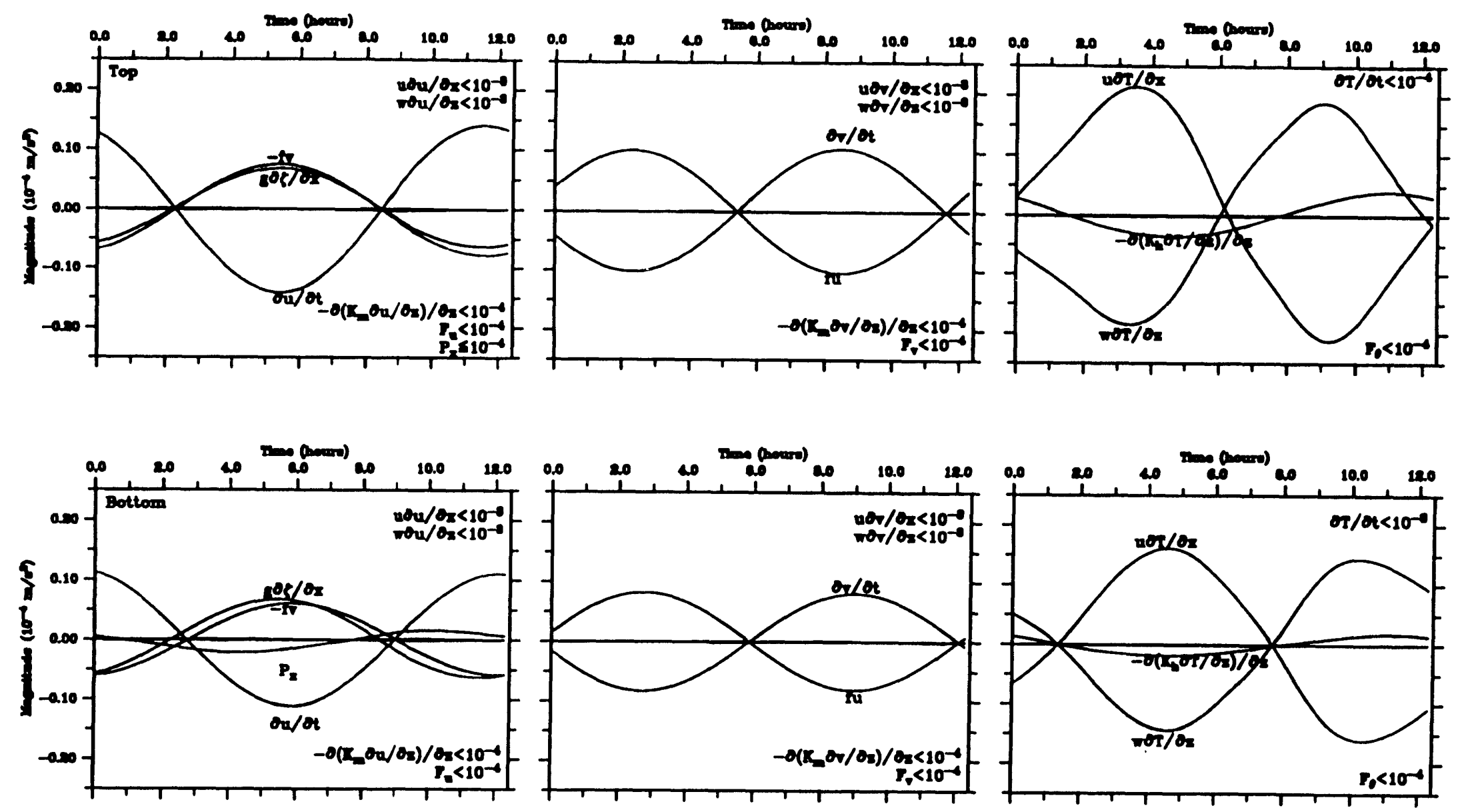

Figure 3.37: Time series of each dynamic or thermodynamic term in the tidal momentum and heat equations at $\sigma=-0.033$ near the surface (upper) and $\sigma=-0.9$ near the bottom (lower) at station A shown in Figure 3.40 for the case of strong stratification. 

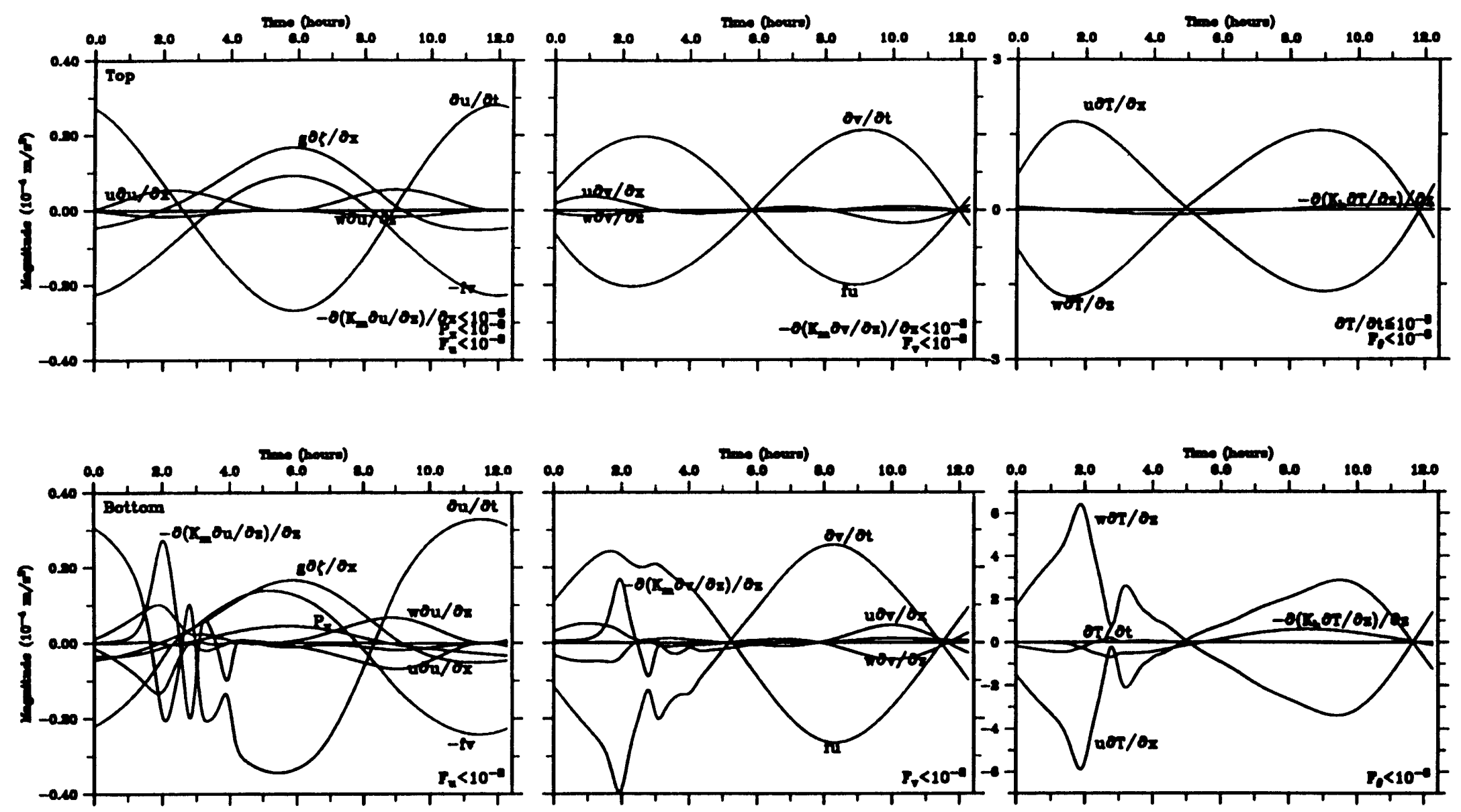

Figure 3.38: Time series of each dynamic or thermodynamic term in the tidal momentum and heat equations at $\sigma=-0.033$ near the surface (upper) and $\sigma=-0.9$ near the bottom (lower) at station B shown in Figure 3.40 for the case of strong stratification. 


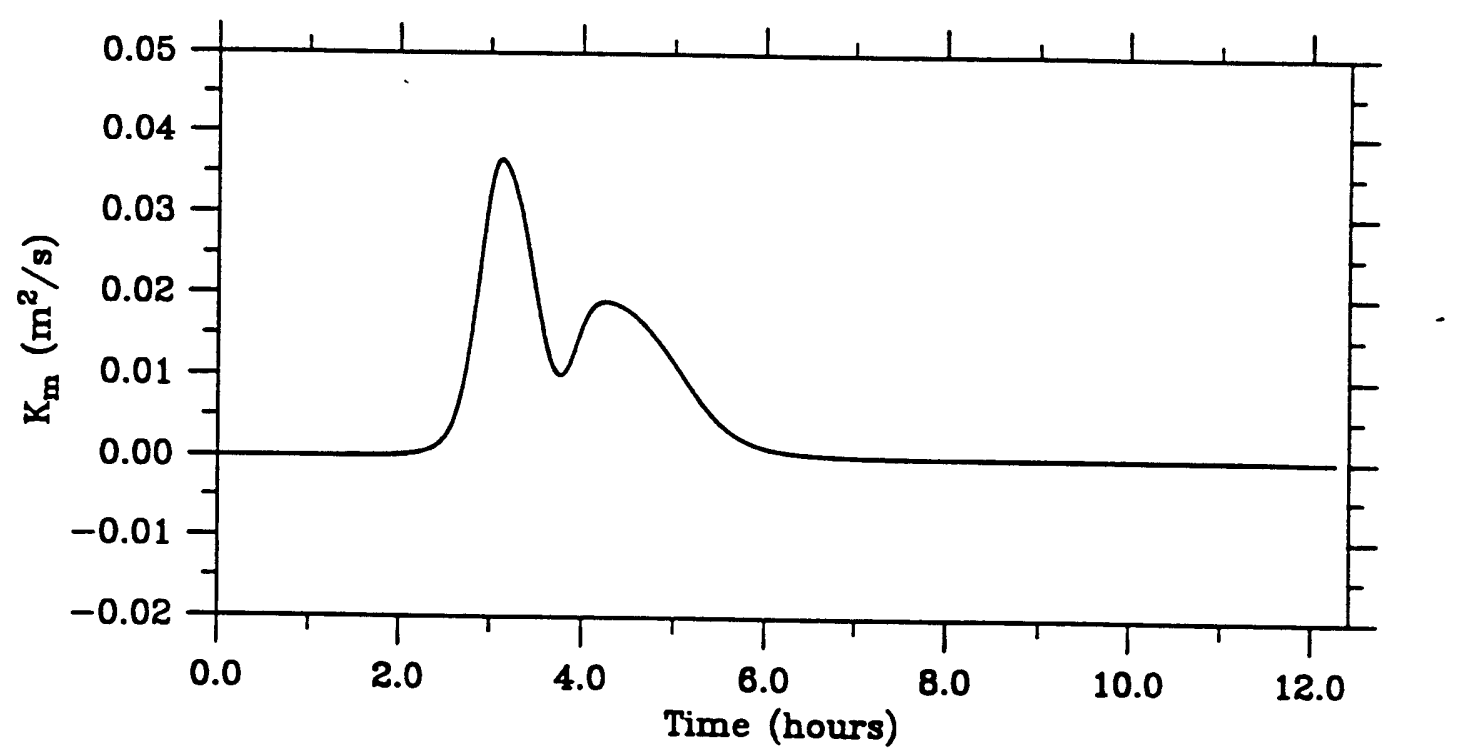

Figure 3.39: Time series of vertical eddy viscosity $K_{m}$ at $\sigma=-0.9$ near the bottom during a tidal cycle at the bottom at station B shown in Figure 3.40 for the case of strong stratification.

and exhibits significant differences between periods of flood (on-bank current) and ebb (offbank current) tides. The vertical friction term gradually increases as the tide floods onto the bank and reaches a maximum after the maximum tide occurs, causing a high-frequency internal wave bore-like structure in $u$ and a small peak in $T$ (see the time series of $u$ and $T$ ). The period of this fluctuation is about three hours; it damps very rapidly as the flood tide weakens and then returns to the modified inertial-gravity wave equations similar to the upper layer during the ebb tide. Since the horizontal and vertical momentum fluxes are of the same order but opposite sign, they tend to cancel each other during the tidal period over the slope. Thus the vertical viscosity term may be responsible for the generation of the high-frequency internal wave bore. The vertical eddy viscosity $K_{m}$ is significantly larger during the flood tide period but rapidly decays with a damped oscillation as the tidal current ebbs (Figure 3.39), suggesting that the turbulent induced friction plays an important role in dissipating the energy of the internal wave bore. On the top of the bank where the water is vertically well mixed and nonlinear terms are smaller, the tidal momentum balance is characterized again by linear inertial-gravity wave equations plus the first order correction 
due to vertical friction, the same as that found in the barotropic case. The horizontal temperature advection term is always opposite to the vertical temperature advection term in the deep region and over the slope. These two terms thus tend to cancel each other and the residue is generally balanced by the vertical thermal diffusion, resulting in a small local time variation of temperature in the deep region and over the slope. Vertical thermal diffusion becomes important near the tidal front where the vertical temperature advection is weak and the time variation of temperature is balanced by the horizontal temperature advection and vertical temperature diffusion.

Diagnostic Analysis of the Residual Flow. Cross-bank distributions of each dynamic or thermodynamic term averaged over a tidal cycle in the momentum and heat equations are shown in Figures 3.40-3.41. Unlike the homogeneous case where the momentum balance is between Coriolis force and nonlinear advection terms in the cross-isobath direction and among the horizontal advection, Coriolis force and vertical friction terms in the along-isobath direction, all the other dynamic terms except the cross-isobath vertical friction become important in the cross- and along-isobath momentum balances over bottom topography as strong stratification is added. In the cross-isobath direction, the horizontal advection term, which is significant only in the upper $150 \mathrm{~m}$ near the shelf break and intensifies at the surface in the barotropic case, extends downward along the slope to the edge of the bottom topography and has a maximum at a depth of about $20 \mathrm{~m}$ near the tidal front. Associated with increasing stratification, the vertical advection term becomes larger, with a negative extreme near the surface but positive maximum near the bottom, just like a first baroclinic mode. The tidal mixing front creates a core of maximum cross-isobath baroclinic pressure gradient centered at a depth of about $70 \mathrm{~m}$ and $100 \mathrm{~km}$ away from the shelf break. This pressure gradient and the horizontal and vertical advection intensify the along-isobath residual current at the sub-surface and over the slope. Good coherence is found in positions of maxima of the horizontal advection term and along-isobath residual flow, suggesting that the stratified tidal rectification may be equivalent to the tidal-mixing- 

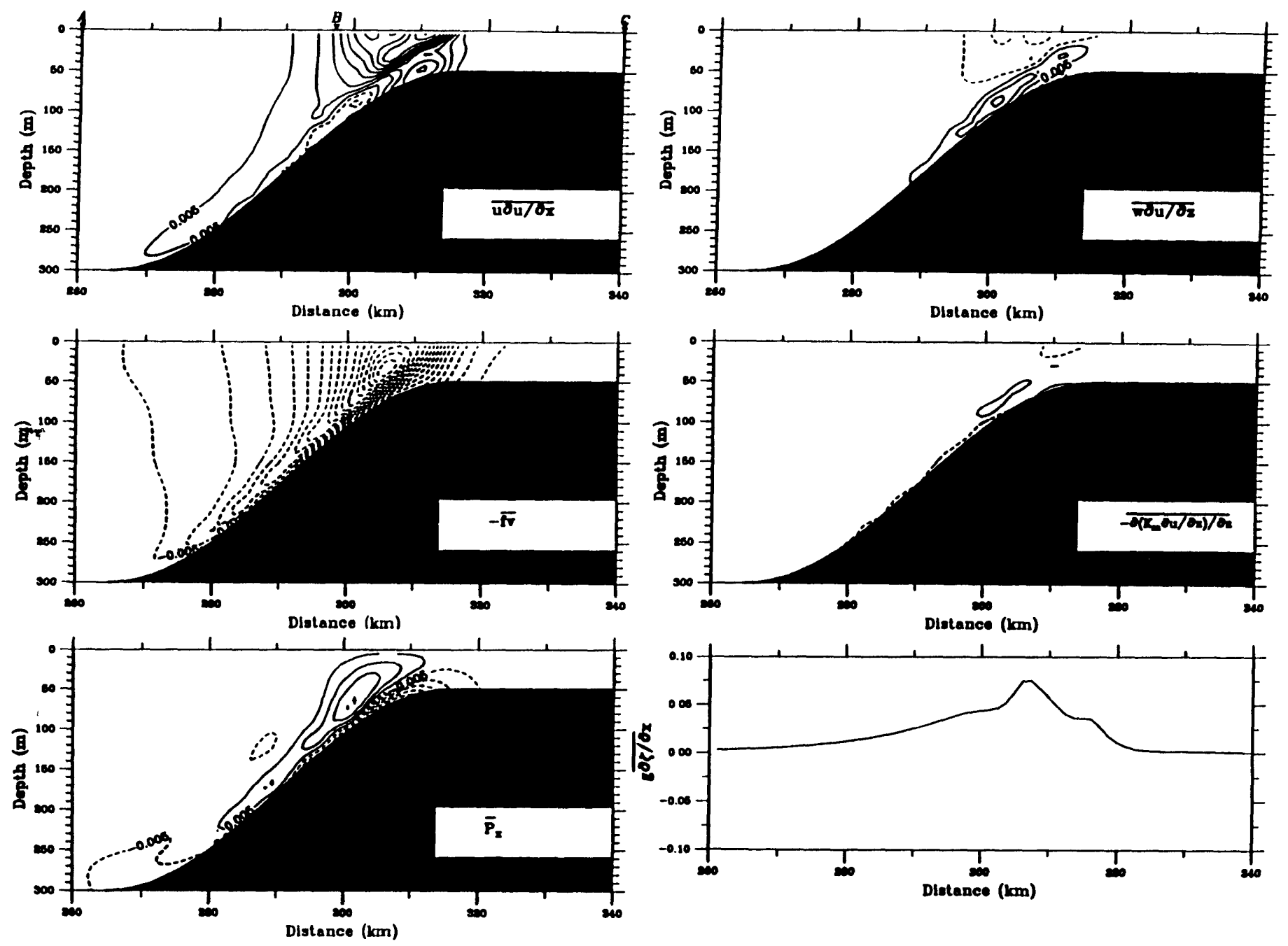

Figure 3.40: Distribution of each dynamic term averaged over a tidal cycle in the cross-bank momentum equation for the case of stron stratification. The unit is $10^{-4} \mathrm{~m} / \mathrm{s}^{2}$ and the contour interval is $0.01 \times 10^{-4} \mathrm{~m} / \mathrm{s}^{2}$. 

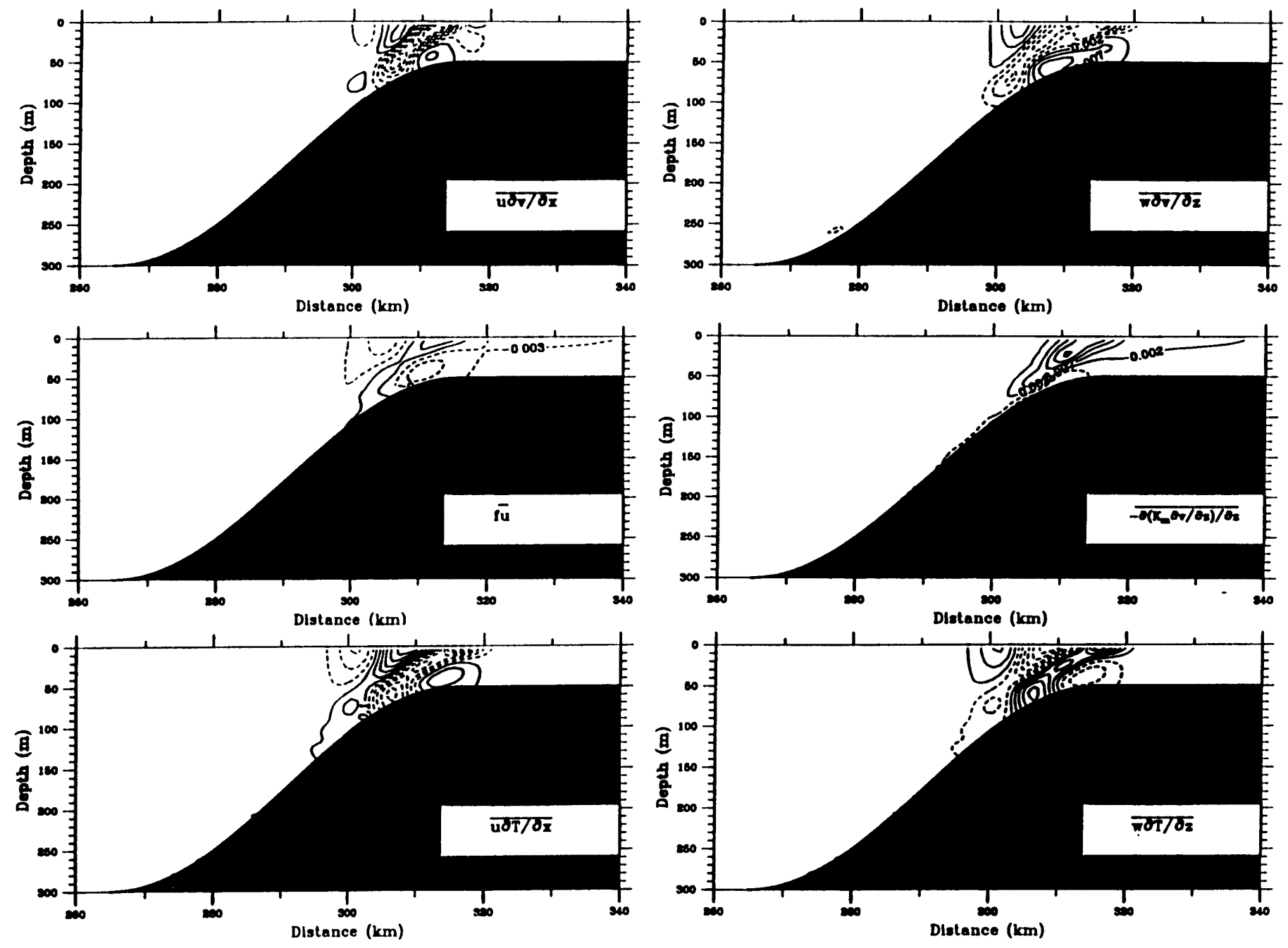

Figure 3.41: Distribution of each dynamic or thermodynamic term averaged over a tidal cycle in the along-bank momentum and hr equations for the case of strong stratification. The unit is $10^{-4} \mathrm{~m} / \mathrm{s}^{2}$ and the contour interval is $0.005 \times 10^{-4} \mathrm{~m} / \mathrm{s}^{2}$ for the dynamic terms a $0.2 \times 10^{-40} \mathrm{C} / \mathrm{s}$ for heat fluxes. 
induced horizontal density gradient in the intensification of along-isobath residual current over the slope.

In the along-isobath direction, the horizontal and vertical advection terms, which are relatively weak and surface-intensified in the barotropic case, are considerably increased as strong stratification is included and are mainly characterized by a first baroclinic mode in the vertical. Associated with such vertical distributions of nonlinear terms, the crossisobath current diverges at the front and converges on both sides of the front near the surface and reverses near the bottom, resulting in a cross-isobath double cell circulation pattern centered at the front near the shelf break. The mean temperature balance is relatively simple around tidal mixing fronts. Since vertical thermal diffusion is in general one or two orders of magnitude weaker than the advection terms in the heat equation, the mean temperature field is maintained through a basic balance between horizontal and vertical temperature advection (Figure 3.41).

The momentum balance in the bottom boundary layer is complex compared with that in the barotropic case. Stratification reduces the vertical friction and hence weakens vertical mixing. Tidal mixing on the top of the bank results in a larger horizontal density gradient at the tidal front and strengthens the horizontal and vertical advection, so that classic Ekman theory is no longer valid in the bottom boundary layer over the slope where the water is stratified. As an example, the vertical distributions of the terms in the momentum and heat equations at the shelf break at the 51-m isobath are plotted in Figure 3.42. Although the Ekman balance is approximately correct in the along-isobath direction near the bottom where the horizontal and vertical advections tend to cancel each other, the cross-isobath momentum equation near the bottom exhibits a strong nonlinear feature, representing a balance among the Coriolis force, baroclinic and surface pressure gradients, and horizontal and vertical advection. To give a better view of the basic mean momentum and temperature balances for stratified tidal rectification over a finite-amplitude 

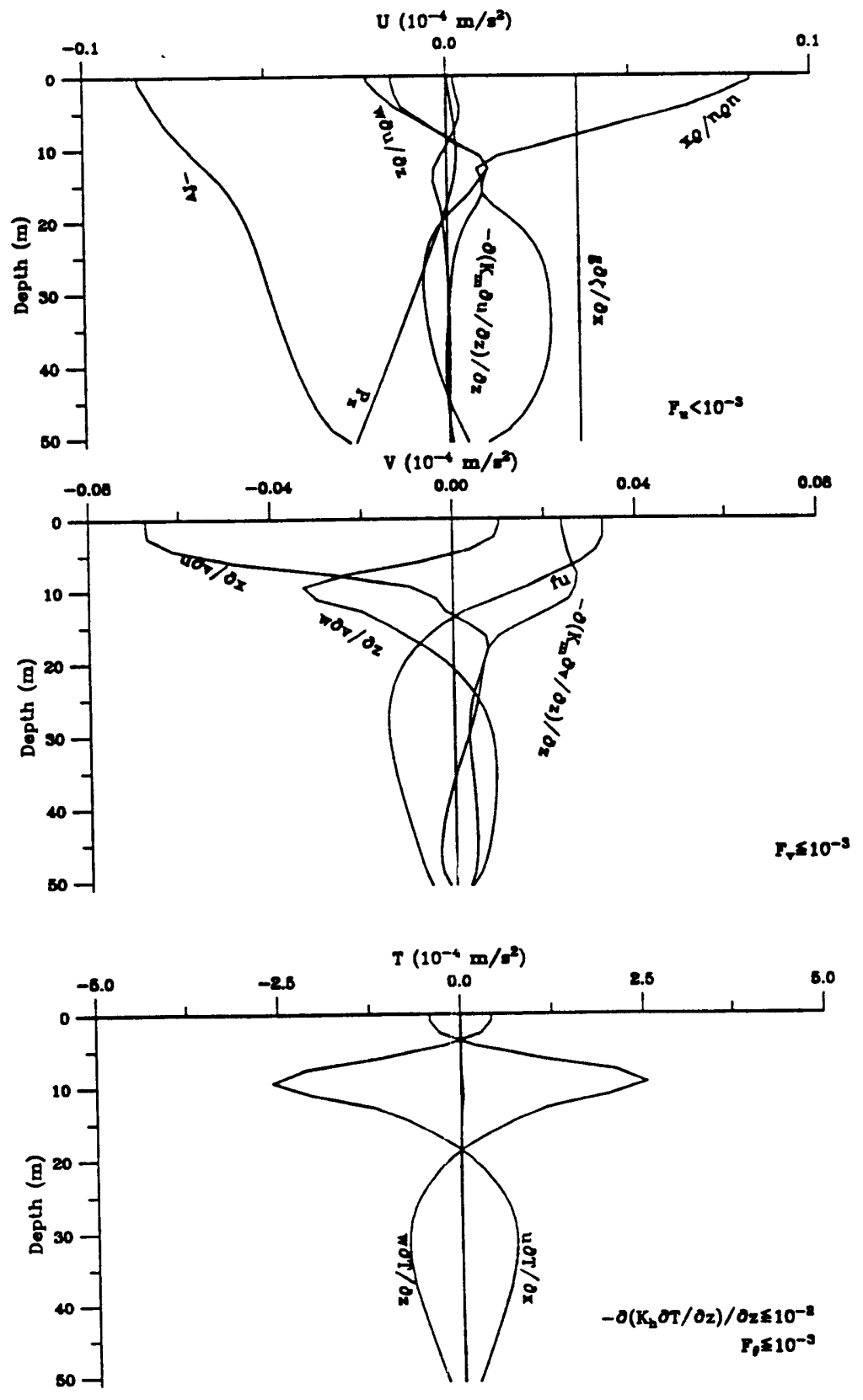

Figure 3.42: Vertical distributions of individual terms in the mean momentum and heat equations at the 51-m isobath near the shelf break for the case of strong stratification. 
bank, we summarize our diagnostic results using the forms of mean equations as follows,

$$
\begin{aligned}
\overline{u \frac{\partial u}{\partial x}}+\overline{w \frac{\partial u}{\partial z}}-f \bar{v} & \sim-g \frac{\partial \zeta}{\partial x}-\overline{P_{x}} \\
\overline{u \frac{\partial u}{\partial x}}+\overline{w \frac{\partial v}{\partial z}}+f \bar{u} & \sim \overline{\frac{\partial}{\partial z}\left(K_{m} \frac{\partial v}{\partial z}\right)} \\
\left(\overline{u \frac{\partial T}{\partial x}}+\overline{w \frac{\partial T}{\partial z}}\right) & \sim 0 .
\end{aligned}
$$

As an example, a simple analysis is made next for the case of $H_{s}=50 \mathrm{~m}$ and $\ell_{t}=50 \mathrm{~km}$ to estimate the contributions of the tidal mixing front and stratified tidal rectification to the intensification of along-isobath residual current. Near the shelf break where the maximum along-isobath residual current is located, the mean baroclinic pressure gradient associated with the tidal mixing front is equal to

$$
\bar{P}_{x}=0.04 \times 10^{-4} \mathrm{~m} / \mathrm{s}^{2}
$$

while the maximum horizontal advection in the cross-isobath direction is given by

$$
\left(\overline{u \frac{\partial u}{\partial x}}\right)_{h}=0.07 \times 10^{-4} \mathrm{~m} / \mathrm{s}^{2} \quad \text { for the homogenous case }
$$

and

$$
\left(\overline{u \frac{\partial u}{\partial x}}\right)_{s}=0.1 \times 10^{-4} \mathrm{~m} / \mathrm{s}^{2} \quad \text { for the strong stratification case }
$$

Thus, the mean baroclinic horizontal advection associated with stratified tidal rectification can be estimated as

$$
\left(\overline{u \frac{\partial u}{\partial x}}\right)_{b}=\left(\overline{u \frac{\partial u}{\partial x}}\right)_{s}-\left(\overline{u \frac{\partial u}{\partial x}}\right)_{h}=0.03 \times 10^{-4} \mathrm{~m} / \mathrm{s}^{2}
$$

which is the same order of magnitude as the mean baroclinic pressure gradient. Therefore, both the tidal mixing front and stratified tidal rectification contribute equally to the 
intensification of along-isobath residual flow over the sloping bottom. It is because the nonlinear interaction due to the internal tide is so important near the tidal mixing front that the semi-geostrophic balance, which holds in a linear system, is no longer valid over finite-amplitude sloping bottom topography.

A Simple Formula for Tidal Mixing. The mechanism of tidally induced vertical mixing in a shallow sea was first studied by Simpson and Hunter (1974). Ignoring the effects of wind mixing, horizontal friction and advection, and freshwater input from rainfall and river discharge, vertical mixing is predominantly controlled by the surface buoyancy flux and tidal energy dissipation. When tidal energy dissipation is strong enough to overcome the buoyancy input, the water will be vertically well mixed. Otherwise, the water will remain stratified. The transition zone between well-mixed and stratified regions is located at a place where these two processes are balanced. We extend this energy argument here to discuss the effects of stratification and tidal currents on vertical tidal mixing in our model.

Suppose that the surface heat input leads to a linearly vertical distribution of density in the water column of height $H$, i.e.,

$$
\rho=\rho_{s}+\frac{\rho_{b}-\rho_{s}}{H} z=\rho_{s}-\frac{\rho_{o}}{g} N^{2} z
$$

where $\rho_{s}$ and $\rho_{b}$ are the surface and bottom densities, and $N$ is the Brunt-Väisälä frequency. After a time interval $\Delta T$, the tidal current becomes stable and the water density is vertically mixed in a layer of depth $h_{m}$ above the bottom while remaining stratified in the rest of the water column. The relation of the thickness of the tide-induced mixed layer to the initial stratification and tidal currents can be simply derived based on an energy argument introduced by Simpson and Hunter (1974). The potential energies before and 
after tidal mixing are given by

$$
\begin{aligned}
\Phi_{t}=0 & =g h^{2}\left(\frac{1}{2} \rho_{s}+\frac{1}{3 g} \rho_{o} N^{2} h\right) \\
\Phi_{t=\Delta T} & =\frac{1}{2} \rho_{s} g h^{2}+\frac{\rho_{o}}{3} N^{2}\left(h-h_{m}\right)^{3}+\frac{\rho_{o}}{4} N^{2}\left(2 h-h_{m}\right)^{2} h_{m} .
\end{aligned}
$$

Then the potential energy required for the vertical mixing is equal to

$$
\Delta \Phi=\Phi_{t=0}-\Phi_{t}=\Delta T=\frac{\rho_{o} N^{2}}{12} h_{m}^{3}
$$

Let $\gamma$ be the bottom friction coefficient and $U$ be a typical tidal current, then the average rate of dissipation of tidal kinetic energy can be given approximately by

$$
\epsilon=\overline{u_{b} \tau_{b}}=\frac{4}{3} \pi \gamma \rho_{o} U^{3}
$$

where $\tau_{b}$ is the bottom stress and $u_{b}$ a bottom velocity related to $U$ (Simpson and Hunter, 1974). Part of this tidal kinetic energy dissipation $(\delta)$ is used over the period $\Delta T$ to produce the potential energy needed for vertical mixing, so

$$
\frac{\rho_{o} N^{2}}{12} h_{m}^{3}=\left(\frac{4}{3} \pi \gamma \rho_{o} U^{3} \Delta T\right) \delta
$$

Thus

$$
h_{m}=\left(16 \pi \gamma \delta \Delta T U^{3} / N^{2}\right)^{\frac{1}{3}} .
$$

This criterion implies that the thickness of the tidal mixed layer depends directly on the magnitude of the tidal current and inversely on the initial stratification rather than either horizontal or vertical shear of the tidal current. Approximate values of $\delta$ and $\gamma$ can be determined from laboratory experiments and field observations. Simpson and Hunter (1974) suggested a value of $\delta=0.0037$ for the Irish Sea, which later was derived theoretically by Hearn (1984). In many theoretical and numerical works, $\gamma$ is usually taken as 
$2.5 \times 10^{-3}$. Provided that these two empirical constants are applicable to our numerical experiments, this criterion should correctly predict the thickness of tidal mixed layer in our model experiments.

The time scale of tidal mixing over the top of the bank is about two days for the case of strong stratification with an initial $N=10^{-2} \mathrm{~s}^{-1}$ and $\sim 0.5$ day for weak stratification with an initial $N=3 \times 10^{-3} \mathrm{~s}^{-1}$, so that the thicknesses of the tidal mixed layer over the top of the bank in both weak and strong stratification can be estimated using equation (3.47) for given $\delta=0.0037$ and $\gamma=2.5 \times 10^{-3}$.

Table 3.1: Comparison Between the Thicknesses of Tidal Mixing Layer Predicted by the Energy Argument and the Numerical Model

\begin{tabular}{|c|c|c|c|c|c|}
\hline $\begin{array}{c}N \\
\left(\mathrm{~s}^{-1}\right)\end{array}$ & $\begin{array}{c}U \\
(\mathrm{~cm} / \mathrm{s})\end{array}$ & $\begin{array}{c}H_{s} \\
(\mathrm{~m})\end{array}$ & $\begin{array}{c}\Delta T \\
(\text { day })\end{array}$ & $\begin{array}{c}h_{m} \\
(\mathrm{~m})\end{array}$ & $\begin{array}{c}\hat{h}_{m} \\
(\mathrm{~m})\end{array}$ \\
\hline $10^{-2}$ & 60 & 50 & 2 & 56 & 57 \\
$10^{-2}$ & 45 & 100 & 2 & 41 & 40 \\
\hline $3 \times 10^{-3}$ & 60 & 50 & 0.5 & 78 & 80 \\
$3 \times 10^{-3}$ & 45 & 100 & 0.5 & 59 & 60 \\
\hline
\end{tabular}

Note: $N$ is the Brunt-Väisälä frequency; $H_{s}$ is the depth of the bank; $U$ is the typical tidal current; $\Delta T$ is the mixing time scale, and $h_{m}$ and $\widehat{h}_{m}$ are the thicknesses of the tidal mixing layer predicted by the energy argument and the numerical model.

Table 3.1 shows good agreement between the thickness of the tidal mixing layer predicted by the numerical model and by the energy argument (equation 3.47) for both weak and strong stratification, implying that the Mellor and Yamada (1982) level $2 \frac{1}{2}$ turbulent closure model is correctly simulating tidal mixing.

It should be pointed out that the good agreement found above is based on the choice of the mixing efficiency $\delta$. In general, $\delta$ is a function of the bottom drag coefficient $\gamma$, the surface heat flux $Q$, the local water depth $H$, and the typical tidal current $U$. 
Garrett et. al (1978) estimated a value of $\delta=0.0026$ for the summertime tidal mixing in the GOM based on $\gamma=0.0024, Q=170 \mathrm{~W} \mathrm{~m}{ }^{-2}$ and the ratio of $H / U^{3}=70 \mathrm{~m}^{-2} \mathrm{~s}^{3}$. In our numerical experiments, $\gamma$ varies in the range of 0.0025 (for $H_{s}=100 \mathrm{~m}$ ) to 0.0035 (for $H_{s}=50 \mathrm{~m}$ ) over the top of the bank. Taking this variation into account, we can estimate the change of $\delta$ due to the bottom drag coefficient in both weak and strong stratification using the model predicted thickness of the tidal mixing layer, which varies in the range of 0.0025-0.0028 for $\gamma=0.0035$ and $0.0035-0.0040$ for $\gamma=0.0025$. Therefore, the mixing efficiency suggested by Simpson and Hunter (1974) represents an averaged value predicted by our model for $\gamma=0.0025$.

Formation of Anti-clockwise Current. Occurrence of a relatively strong anticlockwise jet flow in the deep region away from the steep sloping side of the bank is believed due to internal wave reflection over the bottom topography. Consider reflection of an internal gravity wave (or an internal tidal wave) from a sloping bottom which is inclined an angle of $\alpha$ to the horizontal. The boundary condition of zero normal velocity and Snell's law lead to the simple relationship between wave numbers of incident and reflected waves:

$$
\begin{aligned}
& k_{r}=k_{i}\left(\frac{1+\alpha / R}{1-\alpha / R}\right), \\
& m_{\tau}=-m_{i}\left(\frac{1+\alpha / R}{1-\alpha / R}\right),
\end{aligned}
$$

where the subscripts $r$ and $i$ refer to reflected and incident waves, respectively, $k$ and $m$ are horizontal and vertical wave numbers, and $R$ is the slope of the ray (or the slope of the constant phase plane along which the wave energy propagates). Assuming hydrostatic balance, $R$ is given by

$$
R^{2}=\frac{\omega^{2}-f^{2}}{N^{2}} .
$$

The direction of energy flux depends on the critical value of $\alpha / R$. When the slope of the ray is greater than that of the bottom topography $(\alpha / R<1)$, the energy reflects about 
the vertical axis and the horizontal component of the energy flux keeps the same direction after reflection as it had before. On the other hand, when the bottom slope is steeper than the ray slope, the energy reflects about the horizontal axis and the horizontal energy flux is reversed. Applying these ideas to the internal tidal waves considered in our numerical experiments over the bank of $H_{s}=100 \mathrm{~m}$, we find that $\alpha / R$ increases from 40 to 80 as the topographic scale decreases from $\ell_{t}=50 \mathrm{~km}$ to $\ell_{t}=25 \mathrm{~km}$, implying that the internal tidal energy will be reflected back into the deep region if the friction near the slope is weak. The existence of vertical eddy viscosity may dissipate the internal tidal wave energy and thus weaken the wave reflection. A thermally diffusive boundary layer is considered over the slope in the stratified fluid, where the diffusion tends to mix the water in the vertical and make no density flux into the boundary (Wunsch, 1970). With a vertically linear initial distribution of density, the thickness of this thermal boundary layer depends on the bottom slope. Since the bending of the density contours to satisfy the no normal flux condition decreases as the bottom slope increases, the vertical eddy viscosity tends to become smaller as the bottom slope becomes steeper. For this reason, we expect that strong reflection will occur for the steep bottom slope case of $\ell_{t}=25 \mathrm{~km}$ where the critical value of $\alpha / R$ is big and friction over the deeper part of the slope is weak. Since $(1+\alpha / R) /(1-\alpha / R)$ is very close to -1 as the bottom slope becomes steep, the wavelength of the reflected wave remains almost the same as the incident wave as the topographic scale is reduced to $25 \mathrm{~km}$. In addition, since the energy of the internal tide is always dominant in the first mode (see Figures 3.43 and 3.44), we can estimate the length of the internal tide over the slope using the temperature structure, which shows a horizontal wavelength of $15-20 \mathrm{~km}$ in our model (Figure 3.45). This wavelength is similar to that of the anti-clockwise jet flow found in the residual current field, suggesting that the formation of this jet flow may be due to the wave reflection. As internal tidal waves reflect off the slope, nonlinear interaction may transfer the tidal energy to the mean field, and then an anti-clockwise jet flow can be formed in the deep water where friction is weak and barotropic topographic tidal rectification is small. 
Structure of the Internal Tide. It is interesting to compare the structure of the model-predicted internal tide with observations and theories. There has been much observational work done on the internal tidal waves over Georges Bank, the Nova Scotian, Australian, and California shelves (Trites, 1978; Moody et al., 1984; Marsden, 1986; Loder et al., 1992; Petrie, 1974; Holloway, 1983, 1984, 1985; Winant and Olson, 1976; Winant, 1979; Rosenfeld, 1987). Marsden (1986) examined current meter records at three locations on the northern edge of Georges Bank during October and early November, 1977, and observed the $M_{2}$ internal tide with a horizontal wavelength of about $20-30 \mathrm{~km}$ on the northern flank of Georges Bank. Similar results were also found early by Trites (1978) and Moody et al. (1984) and later by Loder et al. (1992). Holloway (1983 and 1984) conducted a relatively long term current meter measurement on the northwestern Australian shelf and found that the vertical structure of the internal tide was primarily dominated by the first baroclinic mode, which decreased in amplitude as stratification was reduced. Applying a critical value of $\alpha / R$ to observed tidal data, he reported that the baroclinic tidal currents were bottom intensified as $\alpha / R>1$. Similar features were also reported by Rosenfeld (1987) in the moored current meter data over the northern California continental shelf where the kinetic energy of the observed semidiurnal internal tide was concentrated in the first baroclinic mode and increased as stratification became strong.

The generation of an internal tide over the slope and shelf is believed due to the interaction of the barotropic tidal wave with topography in a stratified fluid (Wunsch, 1969, 1975; Baines, 1982), while the predominance of the first mode of the internal tide on the shelf is probably due to dissipation by bottom friction (Brink, 1988). Brink studied the energy dissipation of the internal tide over the shelf for a relatively weakly damped wave and found that the internal wave energies tend to be dissipated rapidly in modes higher than the first as the waves propagate onto the shelf where bottom friction is larger and stratification is weaker. The decay distance of the internal tidal energy propagation in his model is directly proportional to the water depth, stratification, and vertical internal wave modes and inversely proportional to bottom friction. 
Time sequences of temperature, cross-isobath and vertical velocities during a tidal cycle at three reference points on the left side of the bank (shown in Figure 3.22, A-C) are presented in Figures 3.43 and 3.44 for the case of $H_{s}=50 \mathrm{~m}$ and $\ell_{t}=50 \mathrm{~km}$. In the deep region, the vertical structure of temperature remains linear and time-independent during the tidal cycle, and the cross-isobath tidal current exhibits little vertical shear in the interior except in the bottom boundary layer where tidal mixing is strong. The vertical velocity is dominated by the first mode with a maximum at mid-depth and decay toward the surface and the bottom. Significant time variations of temperature and currents are found in a lower layer about $40 \mathrm{~m}$ above the bottom over the slope at station $\mathrm{B}$. The vertical gradient of temperature above the bottom boundary layer increases as the vertical shear of cross-isobath current becomes larger during the tidal cycle. As a result, the vertical velocity intensifies at the top of the bottom boundary layer where the thermocline is the strongest. At station $\mathbf{C}$ on top of the bank, however, the water temperature is well-mixed throughout the water column, so the vertical structure of currents reverts to the homogeneous case where the vertical velocity profile is linear.

We do find a near-bottom intensification of tidal currents on the slope where $\alpha / R>1$ (see Figures 3.43 and 3.44), consistent with observation (Holloway, 1984) and theory (Wunsch, 1975). It shall be noted here that the time-dependent profiles of model output temperature and tidal currents mentioned above cannot be used directly to describe the internal tidal propagation because of the nonlinear coupling between bottom friction and stratification. The existence of the nonlinear bottom friction can induce vertical tidal motion even in a homogeneous fluid, so that vertical modes predicted from the model should include both the friction-induced barotropic and stratification-induced baroclinic modes. Since these two factors are nonlinearly coupled, they cannot be directly separated from our model results. For this reason, we calculated the eigenmodes of the vertical velocity from temperature predicted from the model and the associated phase speeds for the first three modes using a fourth order Runge-Kutta shooting method. Distributions of temperature, Brunt-Väisälä frequency, and the first three vertical velocity modes at two reference points 

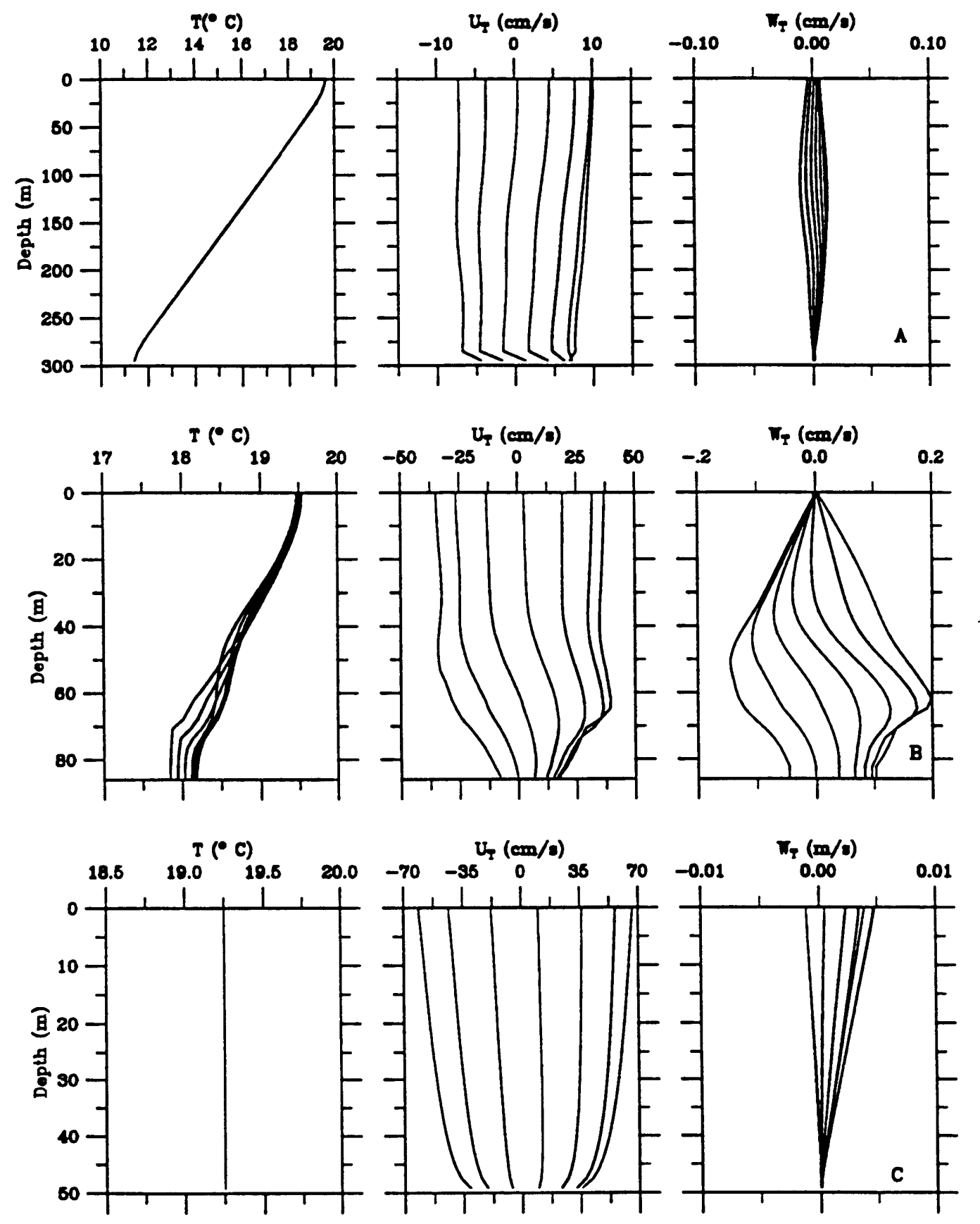

Figure 3.43: Time sequences of temperature (left), cross isobath (middle) and vertical (right) tidal currents during the period of positive cross-isobath acceleration $(\partial u / \partial t>0)$ at three reference points $(A-C)$ in Figure 3.22. The time interval is 0.83 hour. The water depth over the bank $H_{s}=50 \mathrm{~m}$ and topographic scale $\ell_{t}=50 \mathrm{~km}$. 

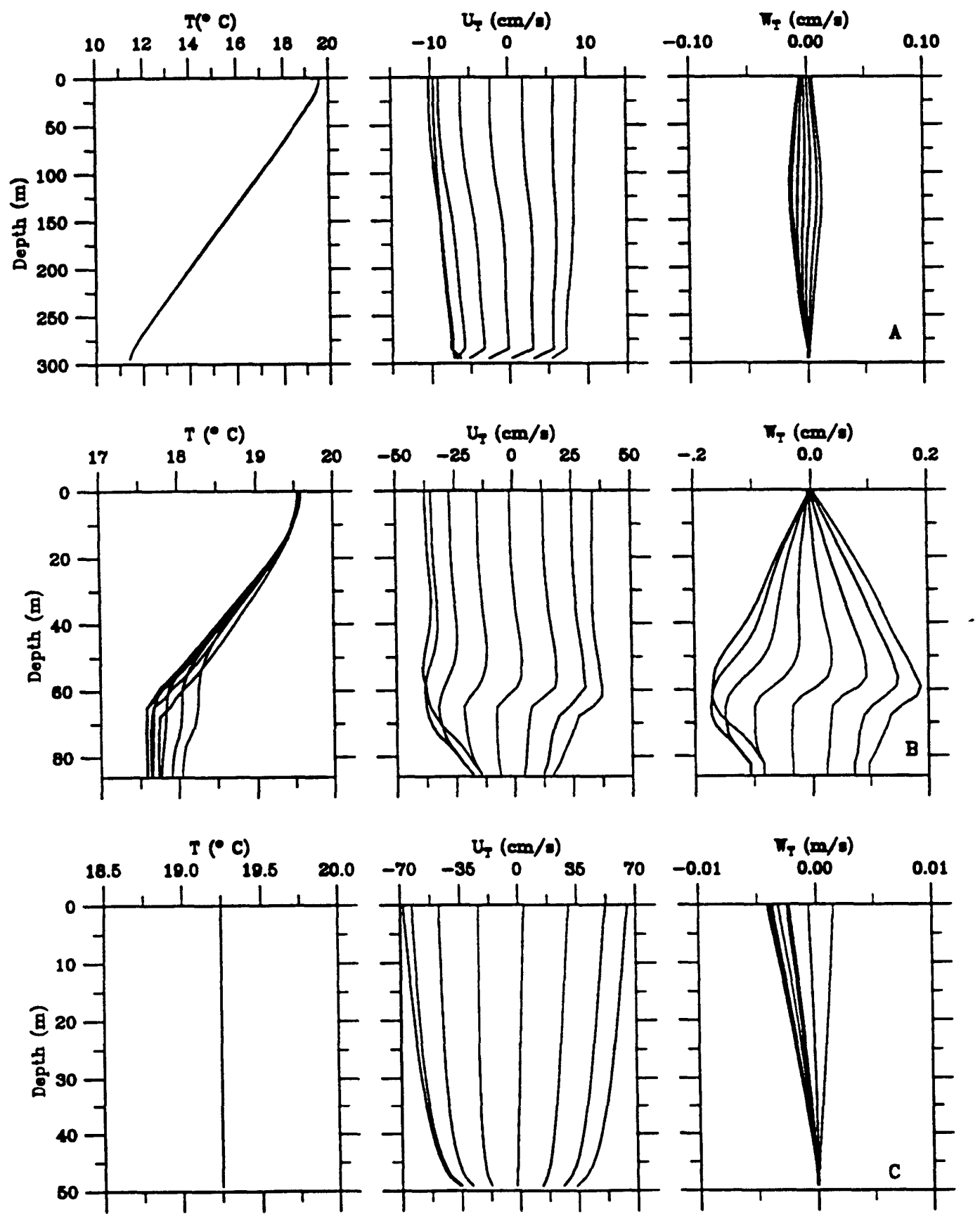

Figure 3.44: Time sequences of temperature (left), cross-isobath (middle) and vertical (right) tidal currents during the period of negative cross-isobath acceleration $(\partial u / \partial t<0)$ at three reference points $(\mathrm{A}-\mathrm{C})$ in Figure 3.22. The time interval is 0.83 hour. The water depth over the bank $H_{s}=50 \mathrm{~m}$ and topographic scale $\ell_{t}=50 \mathrm{~km}$. 

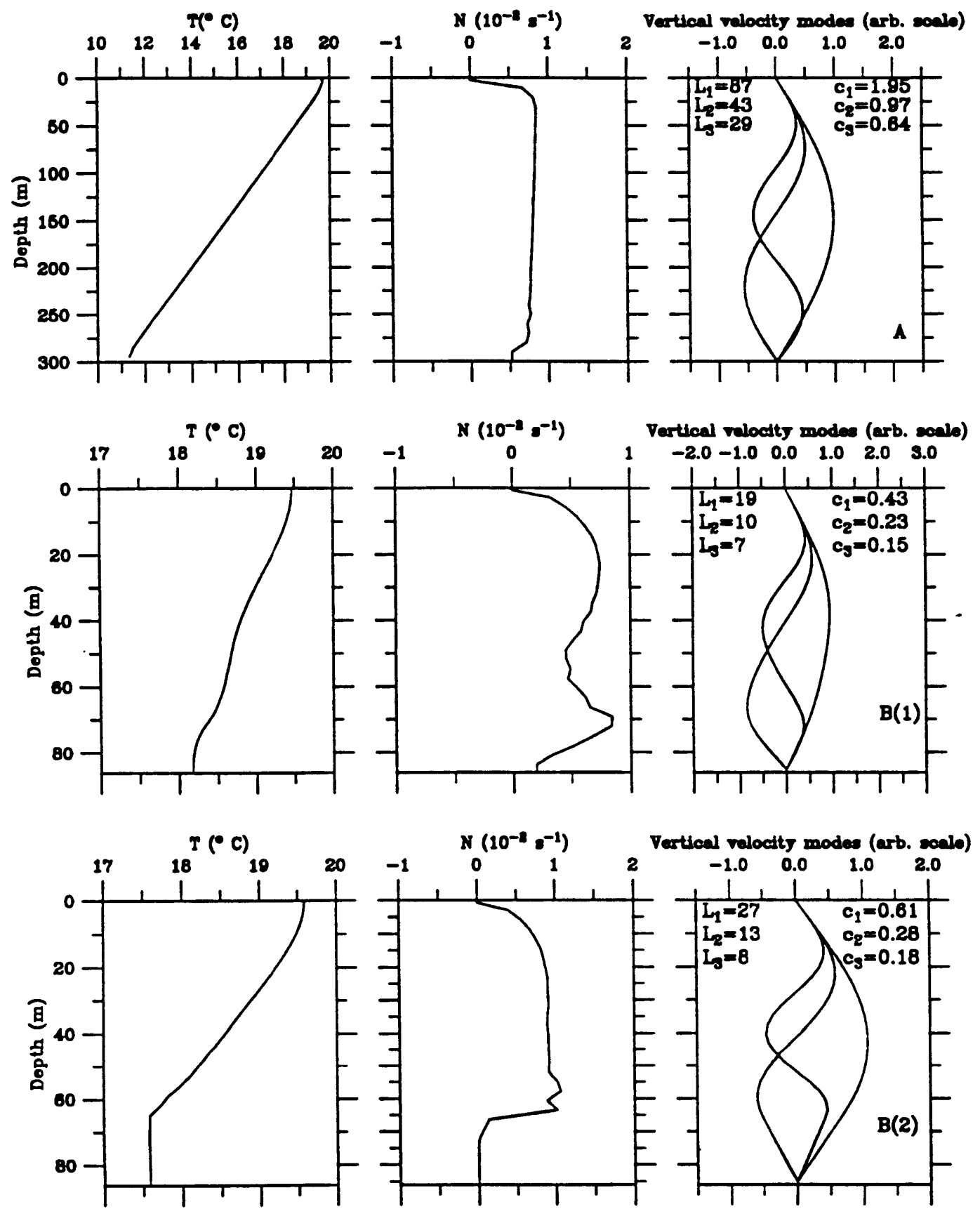

Figure 3.45: Distribution of temperature (left), $\mathrm{N}$ (middle) and vertical velocity modes (right) at two reference points (A and B) in Figure 3.22. The units are $\mathrm{km}$ for wavelength and $\mathrm{m} / \mathrm{s}$ for phase speed $\left(c_{\mathrm{t}}\right)$. $B(1)$ and $B(2)$ are two different cases during a tidal cycle at station $B$. 
(A-B) on the left side of the bank are shown in Figure 3.45. These pictures show that the internal tide tends to decrease its wavelength and reduce its phase speed as it propagates from the deep region onto the slope. The first mode of the internal tide propagates with a phase speed of $1.95 \mathrm{~m} / \mathrm{s}$ in the deep region but slows down to $0.25 \mathrm{~m} / \mathrm{s}$ (weak vertical temperature gradient) or $0.48 \mathrm{~m} / \mathrm{s}$ (strong vertical temperature gradient) as it climbs onto the slope. Correspondingly, the wavelength of the first internal mode is $87 \mathrm{~km}$ in the deep region, while it is only $11 \mathrm{~km}$ (weak vertical temperature gradient) or $20 \mathrm{~km}$ (strong vertical temperature gradient) over the slope. It is also true for the second and third modes, implying a strong internal wave dissipation over the slope where tidal mixing is significant.

Based on the Froude number $F=U / c_{i}$ where $U$ is vertically averaged tidal current and $c_{i}$ is the phase speed for mode $\mathbf{i}$, we found that the internal tidal motion is always subcritical in the deep region $(U=10 \mathrm{~cm} / \mathrm{s})$, while it is super-critical over the slope when the tidal current is strong and subcritical near the transition times between flood and ebb tides. The existence of two critical states over the slope implies that the hydraulic jump or lee wave may occur at a time during the tidal cycle on the lee side of the bank as the internal wave propagates across the bank (Farmer and Smith, 1980; Pratt, 1991; Loder et al., 1992). In other words, as the barotropic tidal current propagates onto and across the bank, the internal waves can be generated by the interaction of the barotropic tide with the topography (Wunsch, 1969; Baines, 1984) and interaction of internal tidal waves (Hibiya, 1986). On the lee side of the bank, the tidal advection prevents the internal waves from propagating upstream onto the shelf, causing an accumulation of wave energy there (hydraulic jump). When the tidal current relaxes or reverses, these accumulated waves will propagate and advect onto the bank (Lee and Beardsley, 1974; Maxworthy,1979; Loder et $a l ., 1991)$. However, we have not seen such a hydraulic jump or lee wave in any time series of temperature in our model.

A big gap between our knowledge of hydraulic jump phenomena and the real ocean exists since most of the theories in this field consider simple layered stratification in a 
channel (Pratt, 1986, 1991; Hogg, 1983; Maxworthy, 1979; Whitehead et al., 1974; Lee and Beardsley, 1974). To our knowledge, it is still unknown how the hydraulic models can be critically influenced by time-dependent flow, continuous stratification and strong friction. In other words, we do not know yet how the amplitude of a hydraulic jump varies with stratification, friction and time variation of tidal flow over a finite-amplitude bank or sill, and how it will be influenced by nonlinear interaction among these factors. In principle, the strong and continuous vertical stratification may reduce the amplitude of a hydraulic jump since the tidal current and phase speed of internal waves are depth-dependent. Strong friction is sufficient to dissipate the high-frequency short internal waves over the slope or shelf (Brink, 1988), so that the hydraulic jump-induced transitional wave may be dissipated completely before it gets onto the shelf. Rotation can also redistribute the accumulated internal wave energy to the along-bank direction and hence reduce the amplitude of the hydraulic jump. In addition, the tidal fronts at the shelf break act like a sponge to absorb and dissipate the internal wave energy so that no high-frequency wave can be measured near the front. Moreover, the amplitude of the hydraulic jump may be too small to be resolved in our model grid where the vertical and horizontal resolutions are about 2-10 m and $1 \mathrm{~km}$, respectively. Anyway, since these factors are nonlinearly coupled in our model, it is difficult to say here which is the most important for preventing or reducing the hydraulic jump. We would like to leave this question open since we do not really know the answer.

\section{Conclusion}

Numerical experiments with weak and strong stratification over different symmetrical finite-amplitude banks provide a detailed picture of stratified tidal rectification. As stratification is included, strong turbulent tidal mixing creates tidal fronts near the shelf break on both sides of the bank, which in turn produce large horizontal density gradients and strengthen the horizontal and vertical advection. A tidal mixing front and stratified tidal rectification result in the subsurface intensification of the along-isobath residual current at the front and at the top of the bottom mixed layer over the slope. The structure of 
the cross-isobath residual current is dominated by a double cell circulation pattern centered at the front near the shelf break on either side of the bank. Modeling results for tidal mixing are consistent with the energy argument in which the thickness of the tidal mixed layer is proportional directly to the tidal current and inversely to stratification. Tidal currents are mainly characterized by the inertial-gravity wave equations that are modified by the baroclinic pressure gradient and nonlinear advection over the slope and vertical friction on top of the bank. The vertical eddy viscosity near the bottom over the slope is significantly larger during the flood-tide period but weaker as the tidal current ebbs, resulting in a rapidly damped internal wave bore in the flood-tide period. All dynamic terms except the cross-isobath vertical friction term are important in the cross- and along-isobath momentum balances for the residual currents, and thus simple Ekman layer theory is no longer valid in the bottom boundary layer. Since the vertical thermal friction is in general one or two orders of magnitude weaker than nonlinear terms in the heat equation, the steady state temperature field is maintained throughout the basic balance between horizontal and vertical heat advection. The existence of the anti-clockwise jet flow in the deep region outside of the steep bank is probably due to the nonlinear interaction of internal tidal currents reflected from the bottom slope. 


\subsection{Tidal Rectification Over Georges Bank}

Successful work on unstratified/stratified tidal rectification over a finite-amplitude symmetrical bank encourages us to apply the model to the asymmetric bottom topography over Georges Bank where the $M_{2}$ tidally driven flow is dominant. Since the cotidal and phase lines of the $M_{2}$ tidal component are almost parallel to the local isobaths over Georges Bank, we can simplify our initial study on tidal rectification over the Bank to a two-dimensional problem in which the along-isobath variation for all independent variables is ignored. A cross-bank section cut from southeast to northwest was chosen for our numerical experiment, and the bottom topography across this section was taken from the NOAA National Ocean Survey bathymetry map No. 1-451 (Uchupi and Austin, 1987, see Figure 3.46).

The grid for the numerical computation is shown in Figure 3.47. The horizontal resolution is $1.0 \mathrm{~km}$ near and on the Bank and linearly increases to $11.96 \mathrm{~km}$ over an interval of 15 grid points outside the domain of interest. The vertical resolution for $\sigma$ is -0.033 (31 points in the vertical) which corresponds to a vertical $\Delta z$ of $10 \mathrm{~m}$ in the deep region $\left(H_{d}=300 \mathrm{~m}\right)$ and $4-1.3 \mathrm{~m}$ over the Bank where $H_{s}$ varies from 120 to $40 \mathrm{~m}$. The initial temperature distribution for summer and winter stratification is simply given by a linear function of $z$ based on observational data during summer and winter, 1979 (Figure 3.48). The initial surface and bottom (at depth of $300 \mathrm{~m}$ ) temperatures are taken as $20^{\circ} \mathrm{C}$ and $11^{\circ} \mathrm{C}$ for the summer case and $13^{\circ} \mathrm{C}$ and $11^{\circ} \mathrm{C}$ for the winter case (Figure 3.49). To simplify the modelling problem and focus on the effects of tidal mixing in producing thermal fronts, we ignore the spatial structure of the background salinity and make salinity constant $\left(35^{\circ} \%\right.$ o $)$ everywhere in the computational domain.

Initial experiments with a homogeneous fluid show a relatively strong surface wave reflection on the southern side of the Bank, which reduced by about half the amplitude of the incident tidal current in the deep region and thus reduced the mass transport across the 


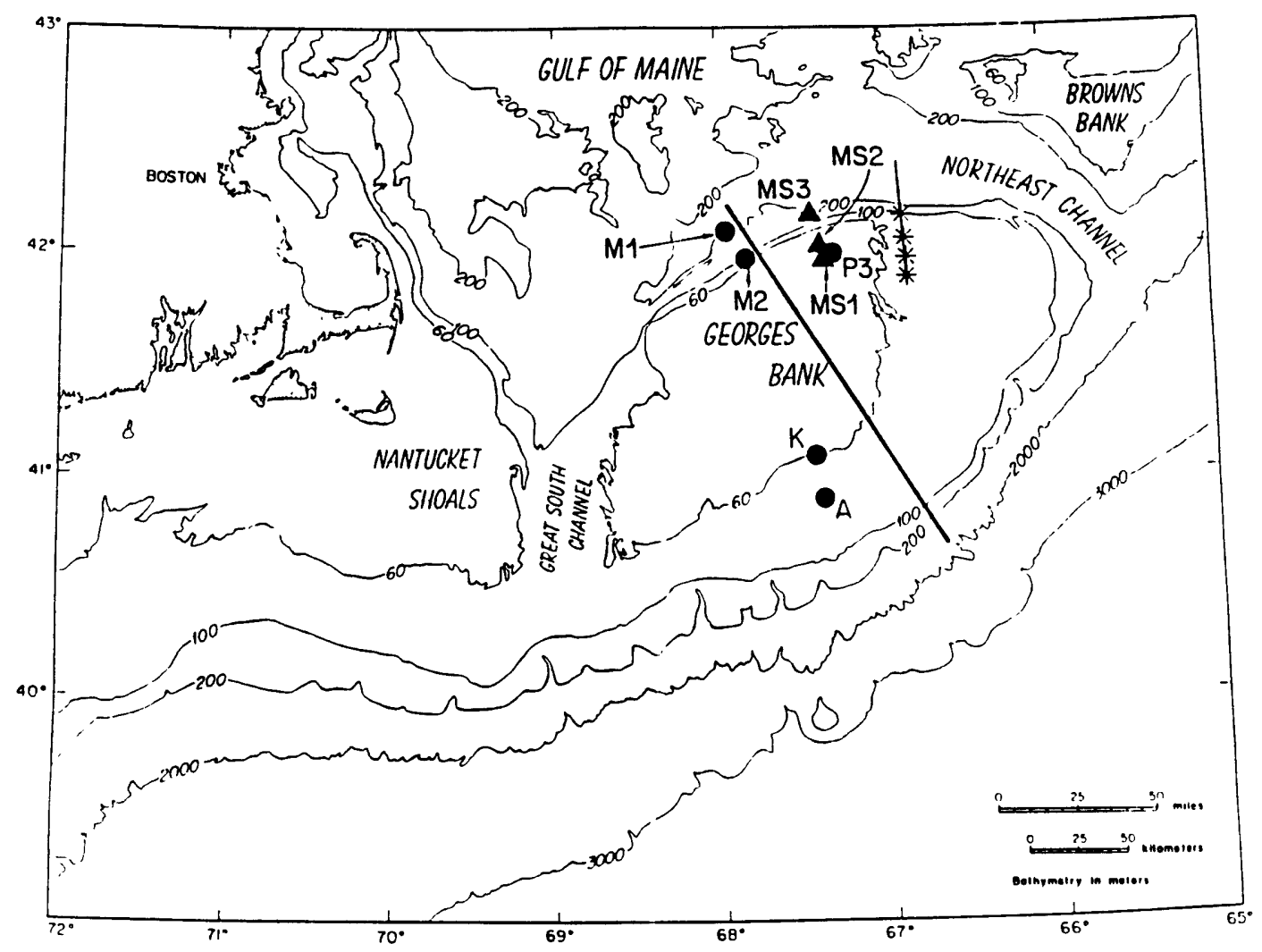

Figure 3.46: Bathymetry (in meters) of the southern New England continental margin (Uchupi and Austin, 1987). The heavy solid line is the section for our numerical experiment and light solid line is the $1988 \mathrm{CTD}$, ADCP and moored current meter measurement section conducted by Loder et al. (1992). Solid circles with capital letters and numbers are moored current meter stations summarized by Butman et al. (1982). Solid triangles and asterisks are moored current meter stations deployed by Marsden (1986) and Loder et al. (1992), respectively. 


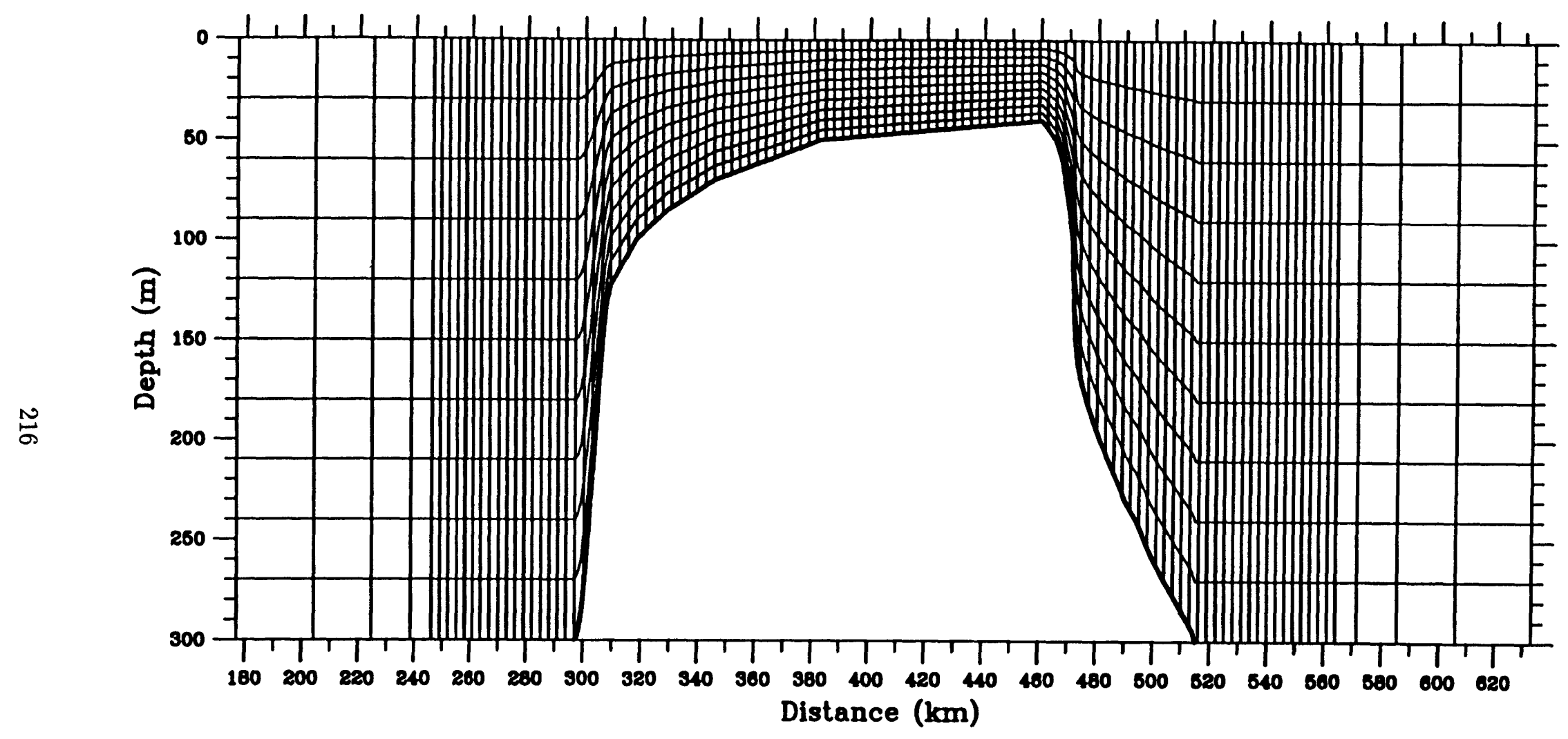

Figure 3.47: Numerical grid for Georges Bank model plotted every three points in both the horizontal and the vertical. The horizontal resolution is $1.0 \mathrm{~km}$ near and across the Bank from 249 to $561 \mathrm{~km}$, and then linearly increases to $11.96 \mathrm{~km}$ over an interval of $15 \mathrm{grid}$ points outside of the domain of interest. The vertical resolution for $\sigma$ is $-\mathbf{0 . 0 3 3}$. The shallowest water depth is $40 \mathrm{~m}$ at $460 \mathrm{~km}$ away from the southern open boundary. 
Bank. Occurrence of the reflected wave is believed due to the very steep bottom topography of the Bank, a process which can be interpreted using a simple theory of long gravity wave reflection over step topography (Leblond and Mysak, 1978). Since our focus is on simulating flow conditions over the Bank, we have adjusted the surface forcing at the southern open boundary so that the combined incident and reflected waves produce the desired mass transport across the Bank. In this geometry, a forcing amplitude of $\zeta_{0}=90 \mathrm{~cm}$ produces a cross-bank barotropic tidal surface current of about $12 \mathrm{~cm} / \mathrm{s}$ in the deep region and $90 \mathrm{~cm} / \mathrm{s}$ over the top of the Bank, consistent with tidal current measurements over Georges Bank reported by Moody et al. (1984).

This section consists of four parts. First, the model will be run for unstratified and summer and winter stratification to investigate effects of an asymmetric bottom topography, stratified tidal rectification and tide-induced mixing on the strength and spatial structure of the residual current. Second, a diagnostic analysis will be made to study the driving mechanism for tidal residual currents during winter and summer. Third, the model results will be compared with observations and previous analytical results, and finally, the model results and discussion will be summarized and some suggestions for future work are given.

\section{Model Results}

Homogeneous Case. It took about five days for the model tidal elevation and currents to become stable and the residual flow to be established. The distribution of stable tidal currents is proportional to the water depth, with an amplitude of about $12 \mathrm{~cm} / \mathrm{s}$ at the surface in the deep region and about $90 \mathrm{~cm} / \mathrm{s}$ over the top of the Bank. After averaging over a tidal cycle, the model predicts a topographically controlled clockwise residual circulation around the Bank, flowing northeastward as a strong jet with a maximum speed of about $16 \mathrm{~cm} / \mathrm{s}$ along the northern flank and then recirculating from the top of the Bank to the southern flank as a relatively weak and broader southwestward flow with a maximum of about $3 \mathrm{~cm} / \mathrm{s}$ at the $50-\mathrm{m}$ isobath (Figure 3.50 ). The predicted cross-bank residual current is characterized by a single circulation cell on either side of the Bank where the water 

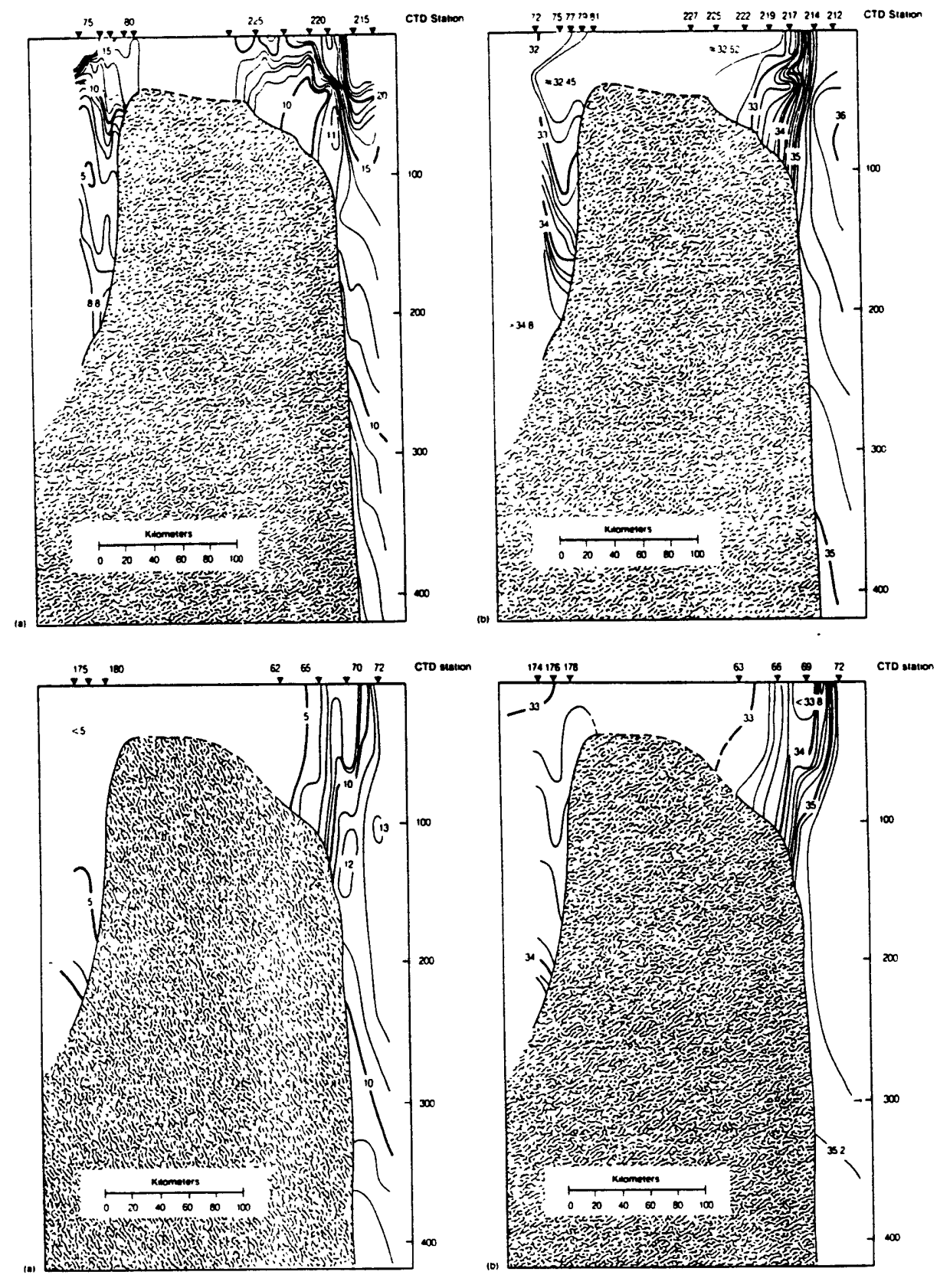

Figure 3.48: Cross-bank transects of temperature $\left({ }^{\circ} \mathrm{C}\right)$ and salinity $(\%$ during August 14-23, 1979 (a) and during March 17-29, 1979 (b). Combined CTD and XBT data were used in these plots (Flagg, 1987). 

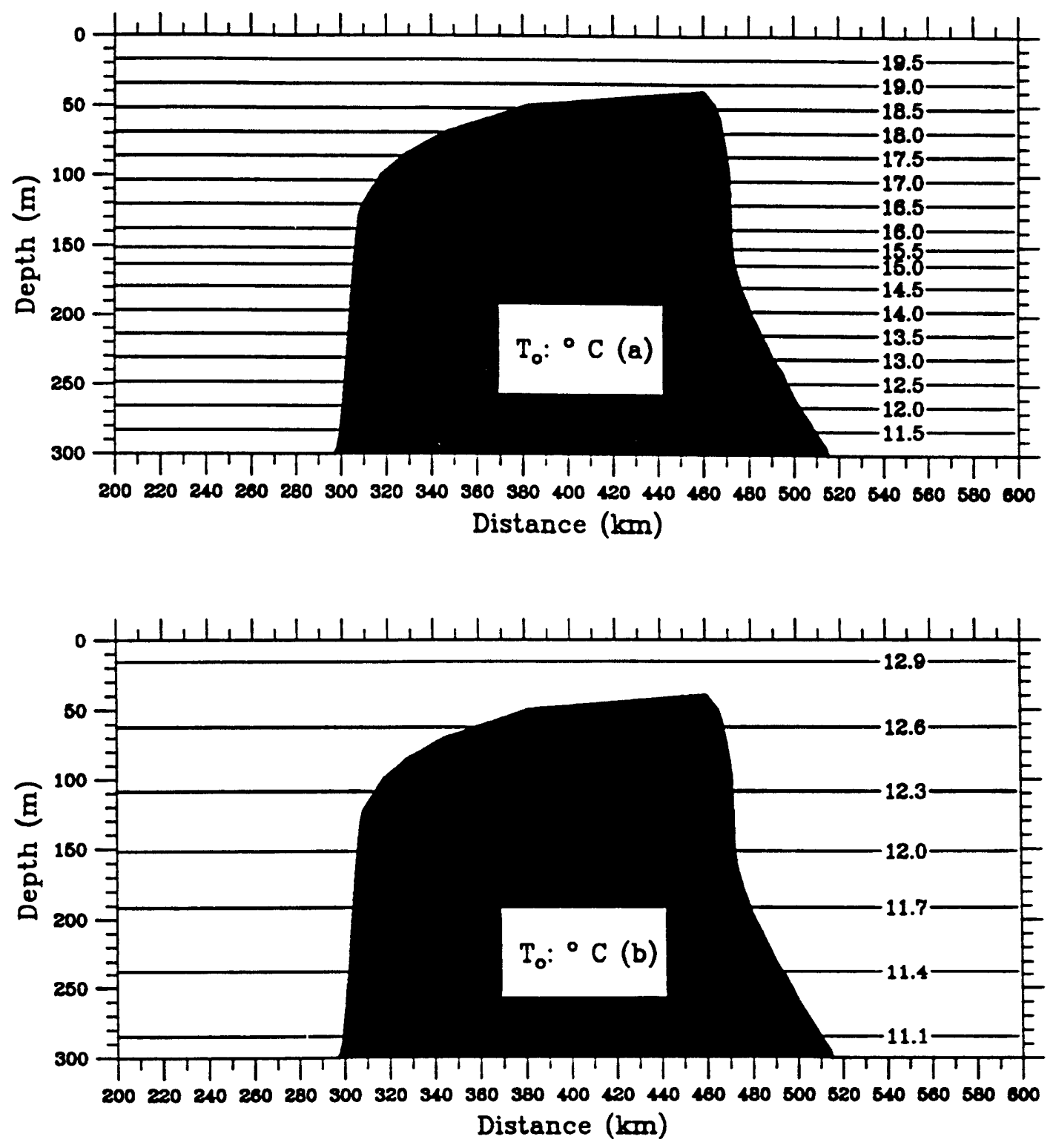

Figure 3.49: Initial temperature distribution for the summer (strong stratification) case (a) and winter (weak stratification) case (b). The temperature contours are plotted with an interval of $0.5^{\circ}$ for summer and of $0.3^{\circ}$ for winter. 


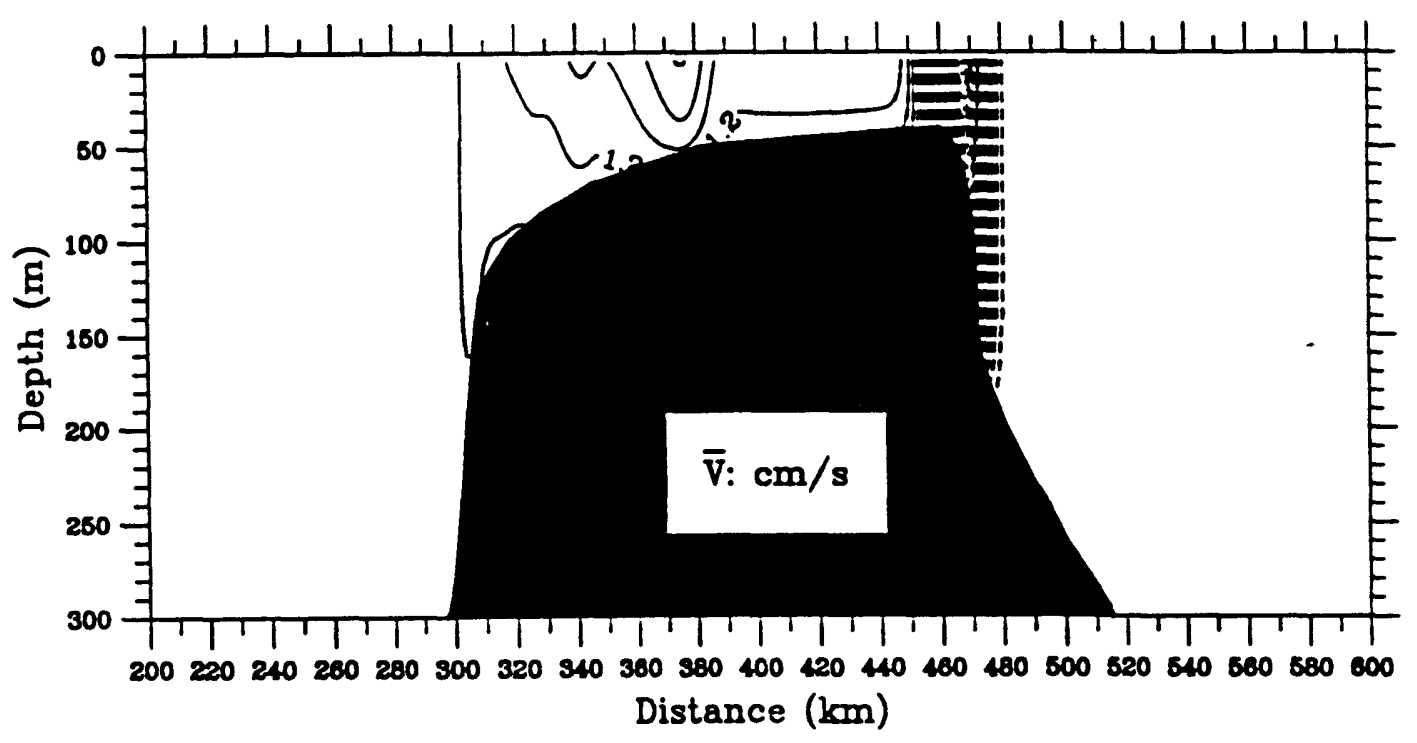

Figure 3.50: The cross-bank structure of the along-bank barotropic residual current. The contour interval is $0.6 \mathrm{~cm} / \mathrm{s}$. The maximum velocity is $3.2 \mathrm{~cm} / \mathrm{s}$ on the southern flank and $16.4 \mathrm{~cm} / \mathrm{s}$ on the northern flank.

upwells along the sloping bottom and then downwells to the outer flank. The maximum upwelling velocity is about $2.8 \times 10^{-2} \mathrm{~cm} / \mathrm{s}$ on the northern flank and $4 \times 10^{-3} \mathrm{~cm} / \mathrm{s}$ on the southern flank, while the maximum downwelling velocity is about $2.2 \times 10^{-2} \mathrm{~cm} / \mathrm{s}$ to the northern side of the shelf break on the northern flank and $3 \times 10^{-3} \mathrm{~cm} / \mathrm{s}$ to the southern side of the shelf break (Figure 3.51a) on the southern flank. The water over the top of the Bank tends to flow southward off the Bank at all depths with a maximum velocity of $0.6 \mathrm{~cm} / \mathrm{s}$ at the surface near the northern flank and decreasing as the water becomes deeper (Figure 3.51b). A tendency for the mean surface elevation to increase northward is found over the top of the Bank, and a relatively strong reversed gradient of mean surface elevation occurs over the northern flank (Figure 3.52a). In addition, an asymmetric distribution of vertical eddy viscosity is observed across the Bank, strongest over the northern flank where the water depth is shallowest and reduced as the water becomes deeper (Figure 3.52b). The cross-bank Eulerian circulation pattern is very similar to that predicted by Tee (1985).

Summer (Strong Stratification) Case. As strong stratification is added, the strong tidal currents mix the water over the top of the Bank and then well-defined tide- 

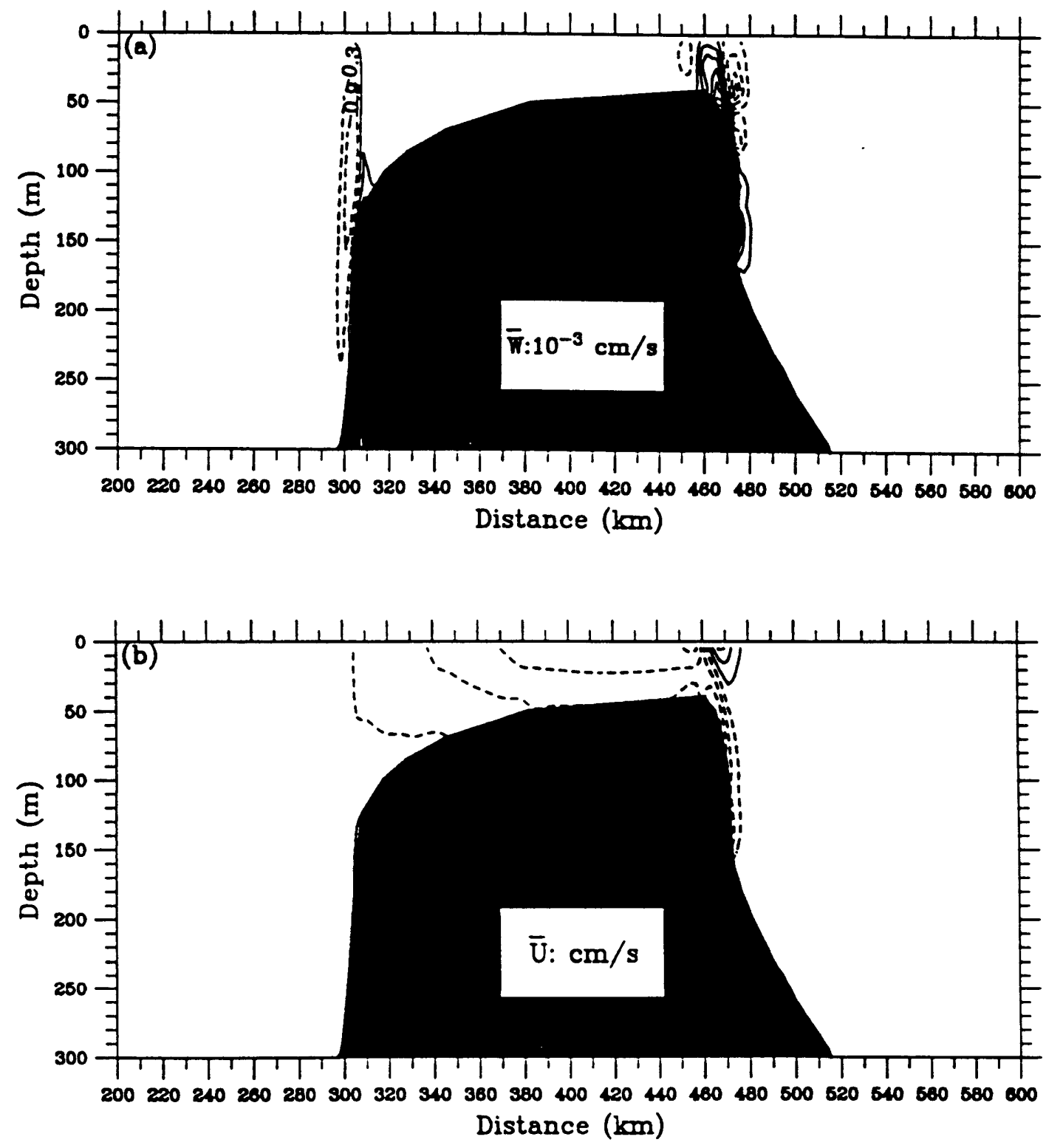

Figure 3.51: The structure of the cross-bank vertical (a) and horizontal (b) residual currents for the homogeneous case. The contour interval is $0.6 \times 10^{-3} \mathrm{~cm} / \mathrm{s}$ for $\bar{w}$ and $0.2 \mathrm{~cm} / \mathrm{s}$ for $\bar{u}$. 

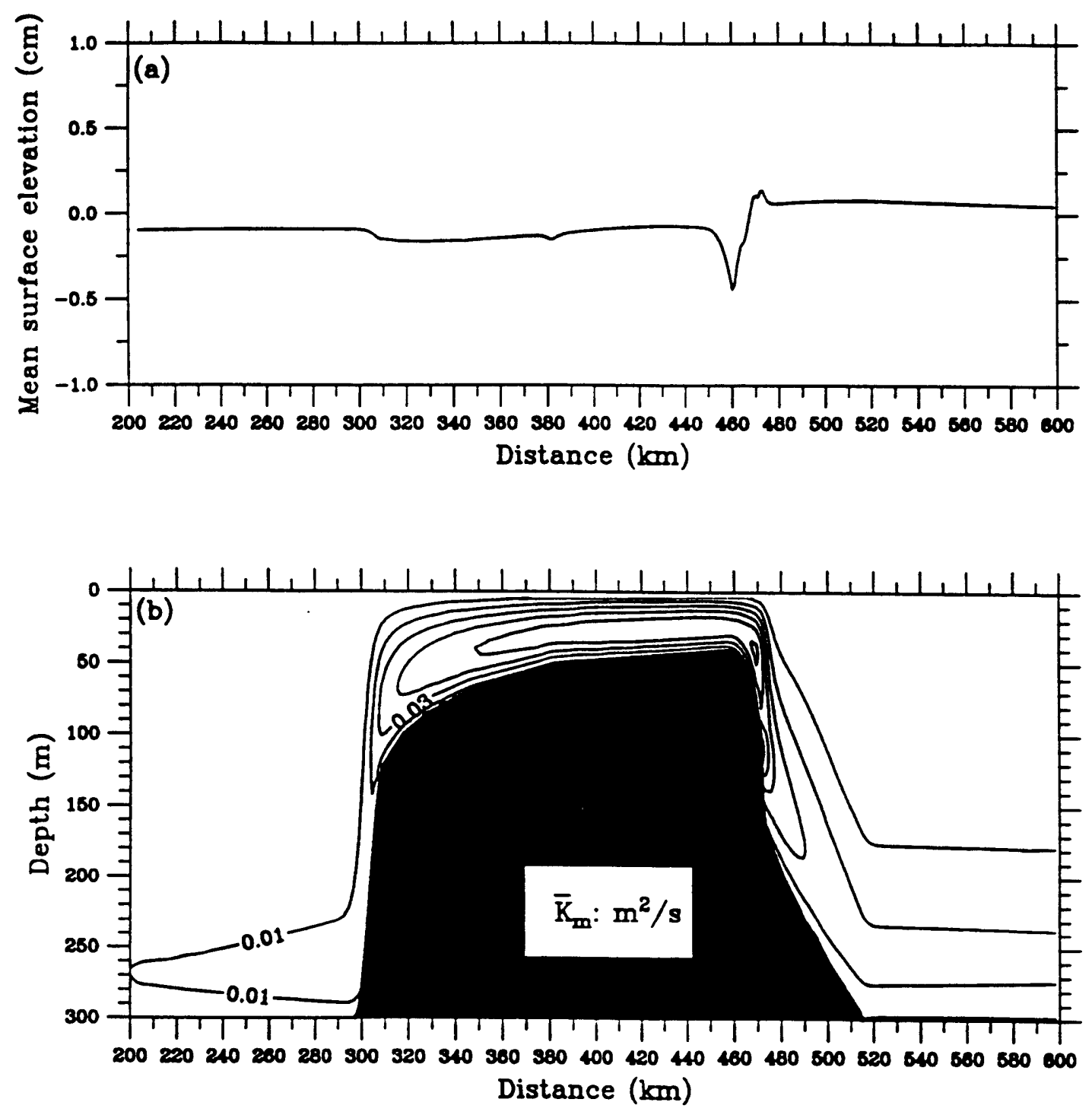

Figure 3.52: The cross-bank structure of the mean surface elevation (a) and the vertical eddy viscosity (b) for the barotropic case. The contour interval is $0.01 \mathrm{~m}^{2} / \mathrm{s}$ for $\bar{K}_{m}$. 
induced thermal fronts are generated at about the $40-\mathrm{m}$ isobath on the northern flank and at the $60-\mathrm{m}$ isobath on the southern flank, separating the vertically well mixed water over the Bank from the stratified water over the outer flanks (Figure 3.53a). The tidally mixed bottom boundary layer is clearly identified over the southern shelf to the southern side of the tidal front. The thickness of this bottom boundary layer decreases seaward as the water depth increases and finally disappears at the shelf break. Corresponding to the tide-induced thermal front and the bottom mixed layer, the along-bank clockwise residual current intensifies along the axis of maximum baroclinic temperature gradient (the top of the bottom mixed layer) on the southern flank and near the shelf break on the northern flank (Figure 3.53b). The maximum along-bank residual current increases to $32 \mathrm{~cm} / \mathrm{s}$ at a depth of about $20 \mathrm{~m}$, about $7 \mathrm{~km}$ to the north of the shelf break on the northern flank, and to $8 \mathrm{~cm} / \mathrm{s}$ at a height of $27 \mathrm{~m}$ above the bottom, about $10 \mathrm{~km}$ inside of the shelf break on the southern flank. A second core of maximum residual current is found at a depth of $190 \mathrm{~m}$ on the northern side of the Bank, which corresponds to the tidal mixing and thermally diffused mixed layer there (Figure 3.53a,b). In contrast to the upper mean circulation pattern, the along-bank residual current below $200 \mathrm{~m}$ near the bottom flows in a counter-clockwise sense around the Bank with recirculation farther offbank, thus forming weak anticyclonic eddies in the deep water both north and south of the Bank. Since no strong vertical mixing is found beyond the slope, the existence of the deep eddy-like mean circulation is due in part to the internal wave reflection as discussed in the previous section.

The structure of the cross-bank and vertical residual currents is shown in Figure 3.54 and enlarged pictures of the contours in the upper $130 \mathrm{~m}$ are replotted in Figures 3.55 and 3.56 for the southern and northern flanks of the Bank, respectively. The cross-bank circulation exhibits a strong asymmetry with respect to the two sides of the Bank. On the southern side, the cross-bank current is characterized by multiple circulation cells, which are strongest near the shelf break where the bottom slope is steep and mixing is weak (Figure $3.55 \mathrm{a}, \mathbf{b}$ ) and become weaker as the water becomes shallower and vertical mixing increases. The magnitudes of the cross-bank and vertical residual currents are about 

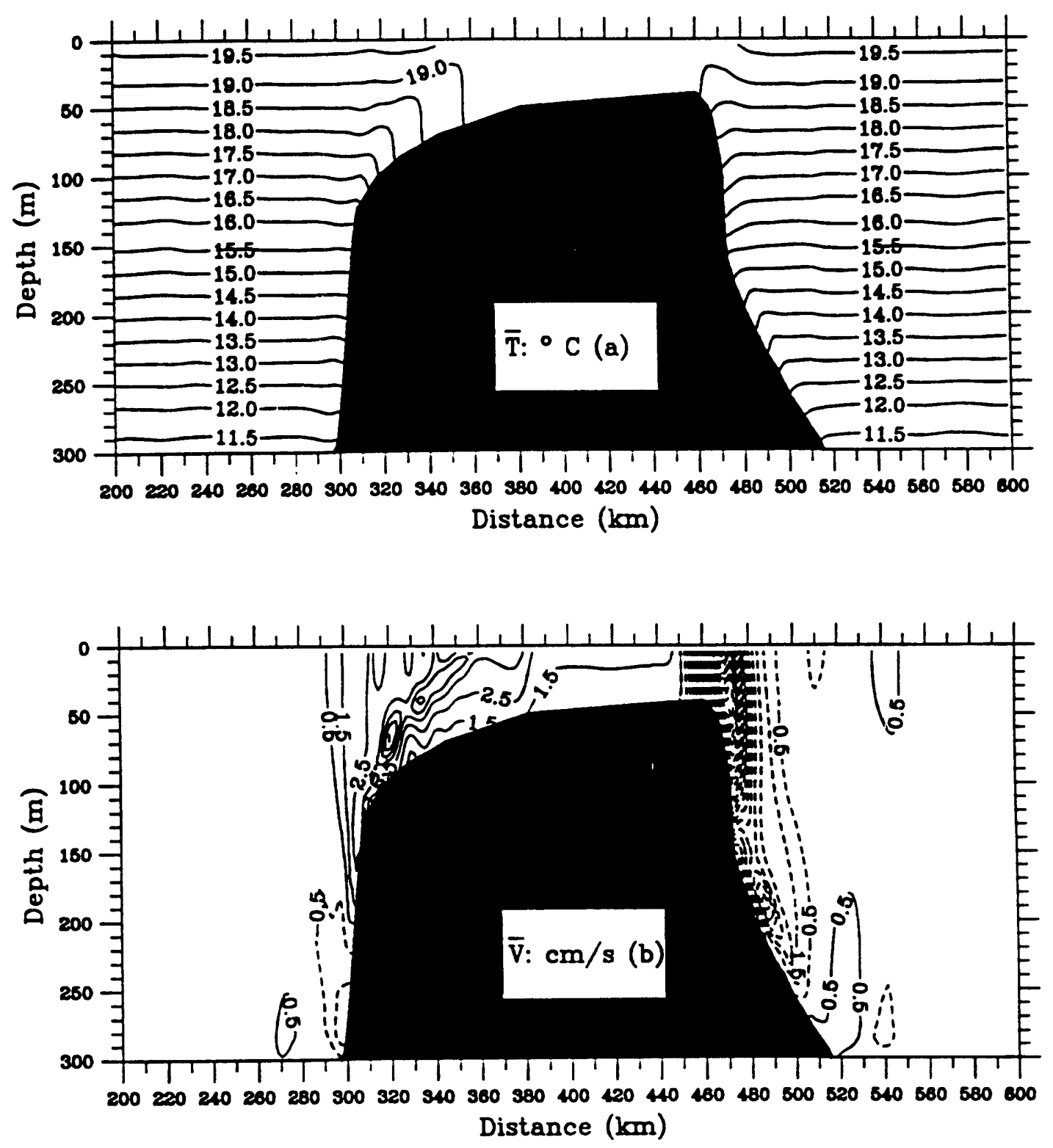

Figure 3.53: The cross-bank structure of the mean temperature averaged over a tidal period (a) and along-bank residual current (b) for the summer (strong stratification) case. The contour interval is $0.5^{\circ} \mathrm{C}$ for $\bar{T}$ and $1.0 \mathrm{~cm} / \mathrm{s}$ for $\bar{v}$. 

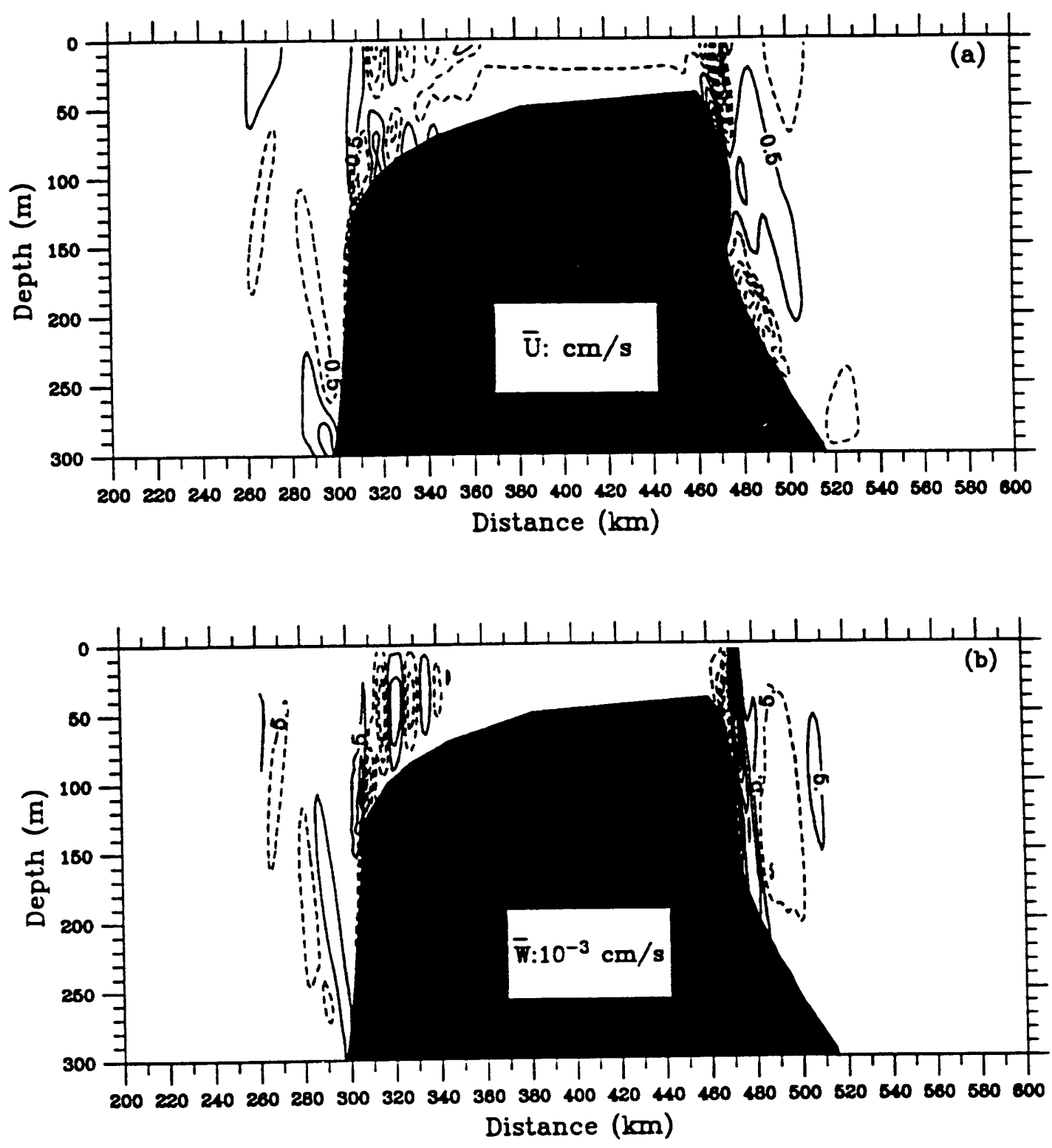

Figure 3.54: The structure of the cross-bank horizontal (a) and vertical (b) residual currents for the summer (strong stratification) case. The contour interval is $10 \times 10^{-3} \mathrm{~cm} / \mathrm{s}$ for $\bar{w}$ and $1.0 \mathrm{~cm} / \mathrm{s}$ for $\bar{u}$. 

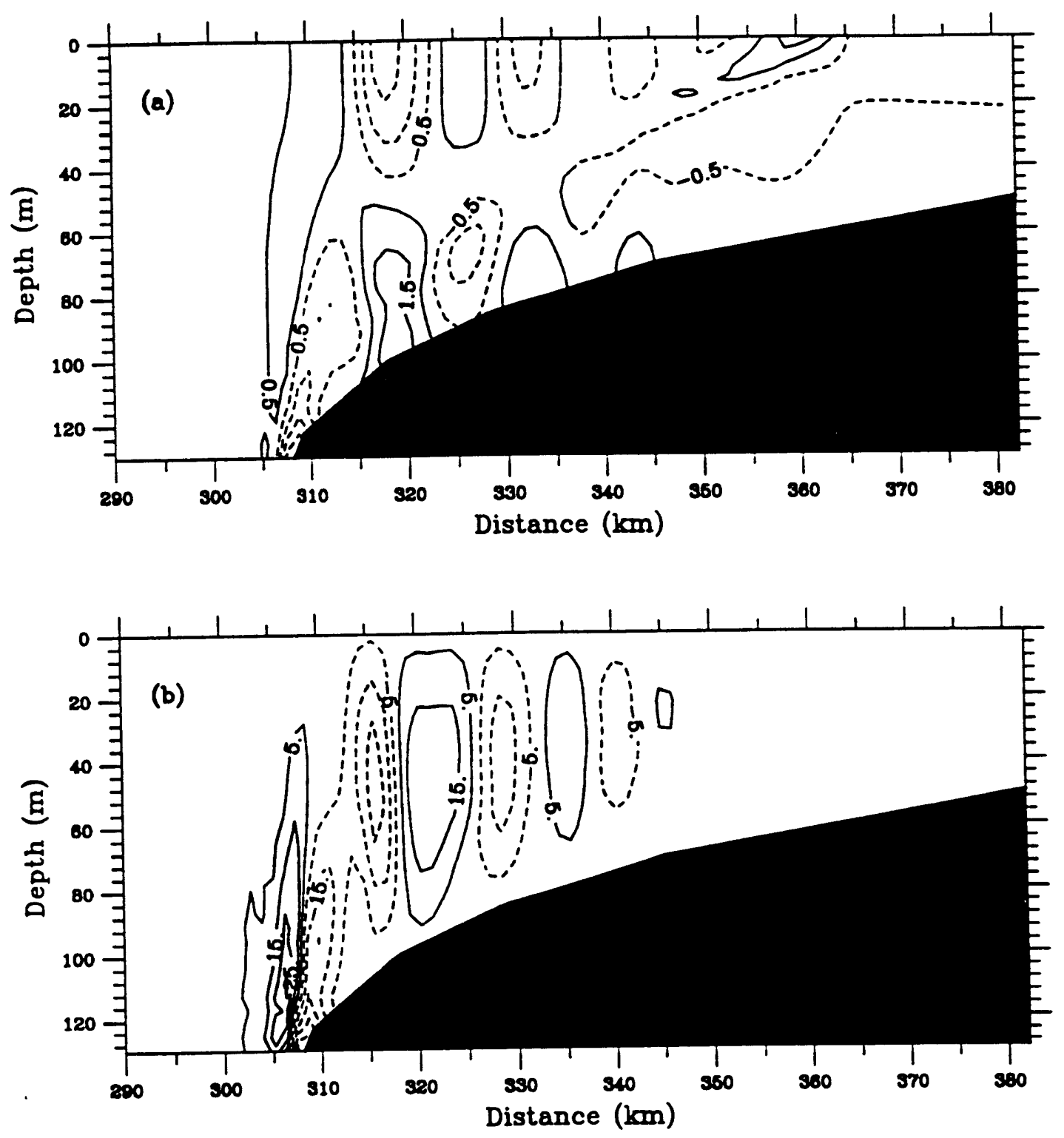

Figure 3.55: The structure of the cross-bank horizontal (a) and vertical (b) residual currents in the upper $130 \mathrm{~m}$ on the southern flank for the summer case. The contour interval is $10 \times 10^{-3} \mathrm{~cm} / \mathrm{s}$ for $\bar{w}$ and $1.0 \mathrm{~cm} / \mathrm{s}$ for $\bar{u}$. 

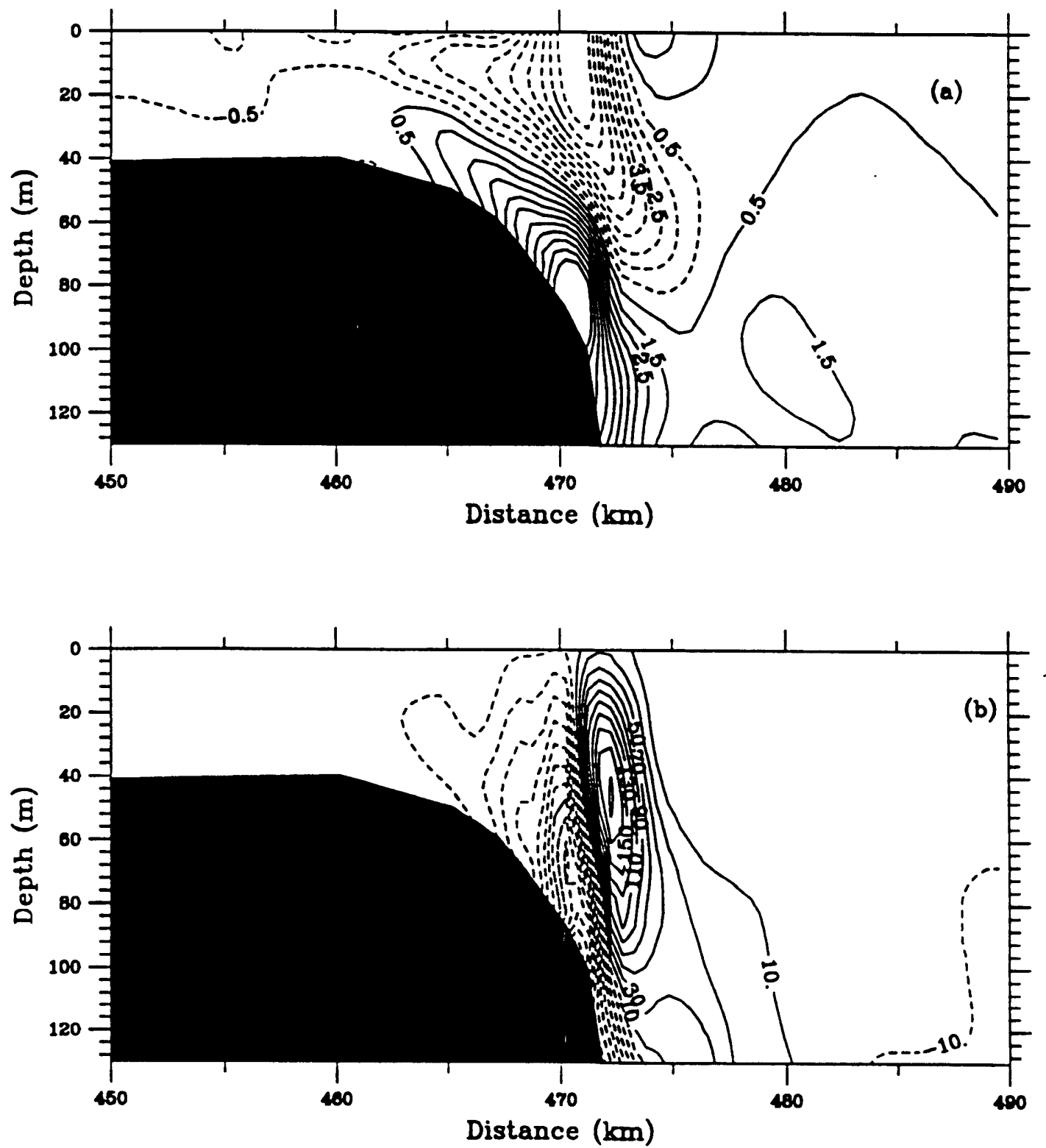

Figure 3.56: The structure of the cross-bank horizontal (a) and vertical (b) residual currents in the upper $130 \mathrm{~m}$ on the northern flank for the summer case. The contour interval is $10 \times 10^{-3} \mathrm{~cm} / \mathrm{s}$ for $\bar{w}$ and $1.0 \mathrm{~cm} / \mathrm{s}$ for $\bar{u}$. 
$5 \mathrm{~cm} / \mathrm{s}$ in $\bar{u}$ and $0.1 \mathrm{~cm} / \mathrm{s}$ in $\bar{w}$ at a depth of $120 \mathrm{~m}$ near the shelf break and decrease to $0.5 \mathrm{~cm} / \mathrm{s}$ in $\bar{u}$ and $0.01 \mathrm{~cm} / \mathrm{s}$ in $\bar{w}$ at the tide-induced thermal front. On the northern side, however, the cross-bank current is dominated by a single strong circulation cell centered at the tidal front where the water is carried down along the sloping bottom to about $150 \mathrm{~m}$ deep and then upwelled again to the northern side of the front. The maximum velocity is about $10 \mathrm{~cm} / \mathrm{s}$ in $\bar{u}$ and $0.3 \mathrm{~cm} / \mathrm{s}$ in $\bar{w}$, occurring at a depth of $50 \mathrm{~m}$ near the shelf break and near the surface on the northern side of the front. Over the top of the Bank inside the fronts, the water tends to flow southward off the Bank at all depths at a speed of about $0.6 \mathrm{~cm} / \mathrm{s}$ at the surface and $0.1 \mathrm{~cm} / \mathrm{s}$ near the bottom. Associated with the along- and cross-bank residual currents, the cross-bank mean surface elevation increases up to $1.25 \mathrm{~cm}$ over a distance of about $140 \mathrm{~km}$ from the southern flank to the northern flank as the water depth decreases and then sharply falls to $-0.75 \mathrm{~cm}$ over a distance of about $30 \mathrm{~km}$ on the northern shelf break (Figure 3.57a).

Winter (Weak Stratification) Case: To study change in residual flow due to the seasonal variation of stratification over Georges Bank, we have also run the model for the winter case with an initial stratification of $\mathrm{N}=3 \times 10^{-3} \mathrm{~s}^{-1}$. With this weak initial stratification, the tide can mix more of the water column, thus causing a shift in the thermal front on the southern flank near the shelf break southward to the $90-\mathrm{m}$ isobath and the front at the northern edge of the Bank to about $5 \mathrm{~km}$ north from the shelf break (Figure 3.58a). Although the position of maximum temperature gradient remains approximately fixed on the northern side, it moves to the shelf break on the southern flank. Corresponding to this seasonal shift in the location of the tidal mixing front, the winter maximum alongbank residual current occurs at the surface on both sides of the Bank, with a maximum of about $6 \mathrm{~cm} / \mathrm{s}$ at the southern shelf break and of about $26 \mathrm{~cm} / \mathrm{s}$ on the northern flank (Figure $3.58 \mathrm{~b}$ ). In addition, when stratification is weak, the width of the northern jet flow becomes smaller and the anti-clockwise deep current below $200 \mathrm{~m}$ near the bottom disappears. The circulation over the top of the Bank is dominated by a barotropic clockwise 

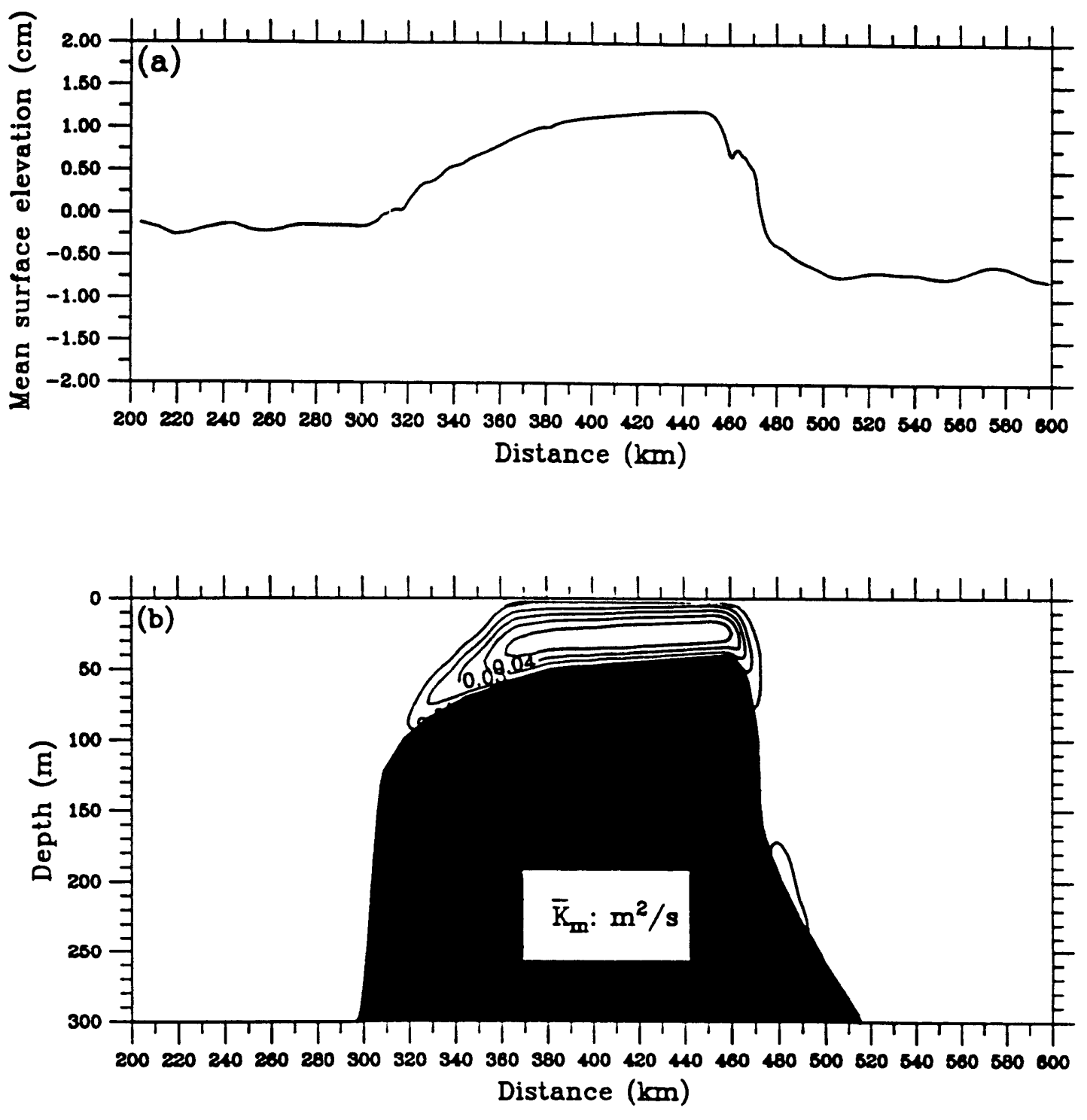

Figure 3.57: The cross-bank structure of the mean surface elevation (a) and the vertical eddy viscosity (b) for the summer case. The contour interval is $0.01 \mathrm{~m}^{2} / \mathrm{s}$ for $\bar{K}_{m}$. 

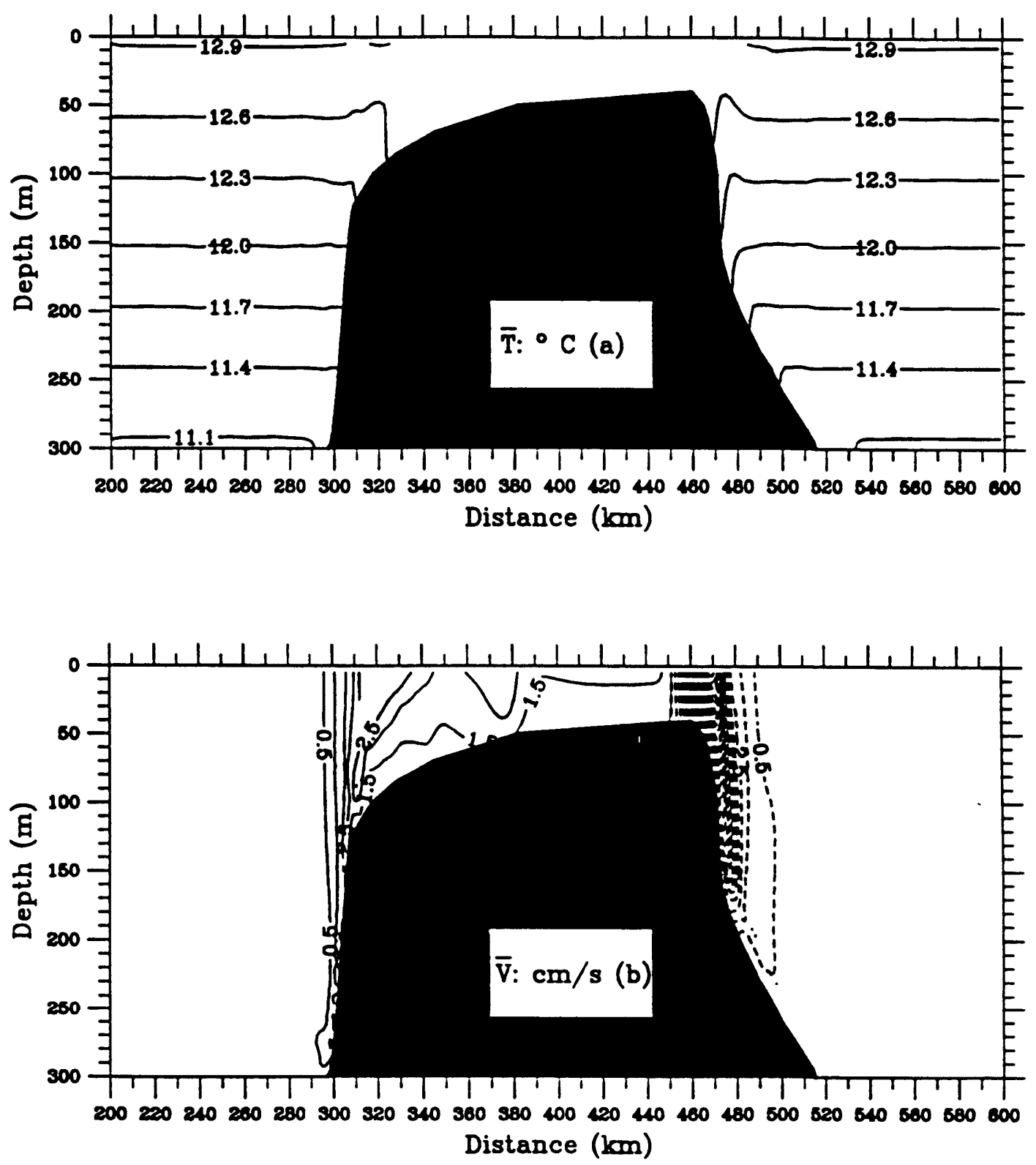

Figure 3.58: The cross-bank structure of the mean temperature averaged over a tidal period (a) and along-bank residual current (b) for the winter (weak stratification) case. The contour interval is $0.3^{\circ} \mathrm{C}$ for $\bar{T}$ and $1.0 \mathrm{~cm} / \mathrm{s}$ for $\bar{v}$. 
current with a second maximum at the $50-\mathrm{m}$ isobath about $70 \mathrm{~km}$ inward of the southern shelf break (Figure 3.58b).

The structures of the cross-bank and vertical residual currents are shown in Figures 3.59-3.61. Along with the shift of the tidal front to deeper water, the cross-bank circulation is dominated by a double circulation cell pattern on both sides of the Bank. The maximum of cross-bank and vertical residual currents is about $1.8 \mathrm{~cm} / \mathrm{s}$ in $\bar{u}$ and either $0.08 \mathrm{~cm} / \mathrm{s}$ in $\bar{w}$ at a depth of $60 \mathrm{~m}$ in the downwelling region or $0.05 \mathrm{~cm} / \mathrm{s}$ in $\bar{w}$ at a depth of $56 \mathrm{~m}$ in the upwelling region on the southern flank, while it is about $3.5 \mathrm{~cm} / \mathrm{s}$ in $\bar{u}$ and either $0.12 \mathrm{~cm} / \mathrm{s}$ in $\bar{w}$ at a depth of $70 \mathrm{~m}$ in the downwelling region along the slope or $0.18 \mathrm{~cm} / \mathrm{s}$ in $\bar{w}$ at a depth of $83 \mathrm{~m}$ in the upwelling region on the northern flank. Compared with the summer case, the cross-bank gradient of mean surface elevation decreases as stratification becomes weaker and a reversed structure of pressure gradient occurs at the northern flank (Figure 3.62a), suggesting that the cross-bank, double-circulation cell may be driven by the surface pressure gradient against bottom friction (Loder, 1980). However, a rather weak reversed structure of mean surface elevation on the southern side is not sufficient to drive such a double cell circulation near the southern shelf break, implying that other baroclinic forcing is important for that. We will discuss this problem later.

\section{Diagnostic Analysis for Dynamic Balances}

In order to understand the driving mechanisms of stratified tidal rectification over Georges Bank, we have made diagnostic computations for each dynamic term in the momentum equations for both the barotropic and strong stratification cases and show the resulting cross-bank distributions of these terms in Figures 3.63-3.66. For the barotropic case, the cross-bank momentum is still balanced among the horizontal advection, Coriolis force and the surface pressure gradient on both the southern and northern flanks where the alongbank residual currents are strongest. The along-bank momentum balances on the southern and northern flanks are very different. On the southern flank, the along-bank momentum is balanced among horizontal advection, Coriolis force and the vertical friction terms 

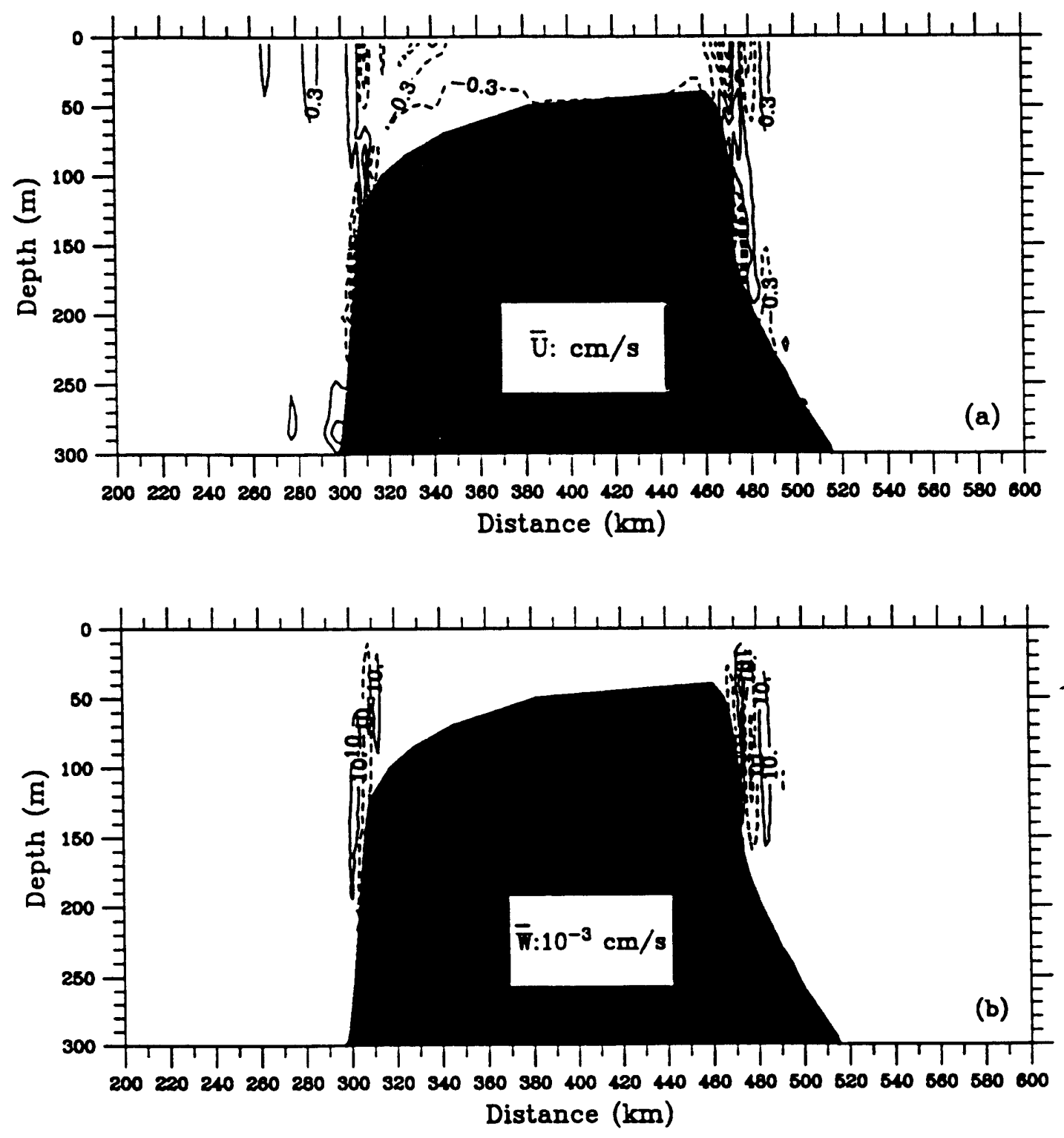

Figure 3.59: The cross-bank structure of the cross-bank horizontal (a) and vertical (b) residual currents for the winter (weak stratification) case. The contour interval is $10 \times 10^{-3} \mathrm{~cm} / \mathrm{s}$ for $\bar{w}$ and $1.0 \mathrm{~cm} / \mathrm{s}$ for $\bar{u}$. 

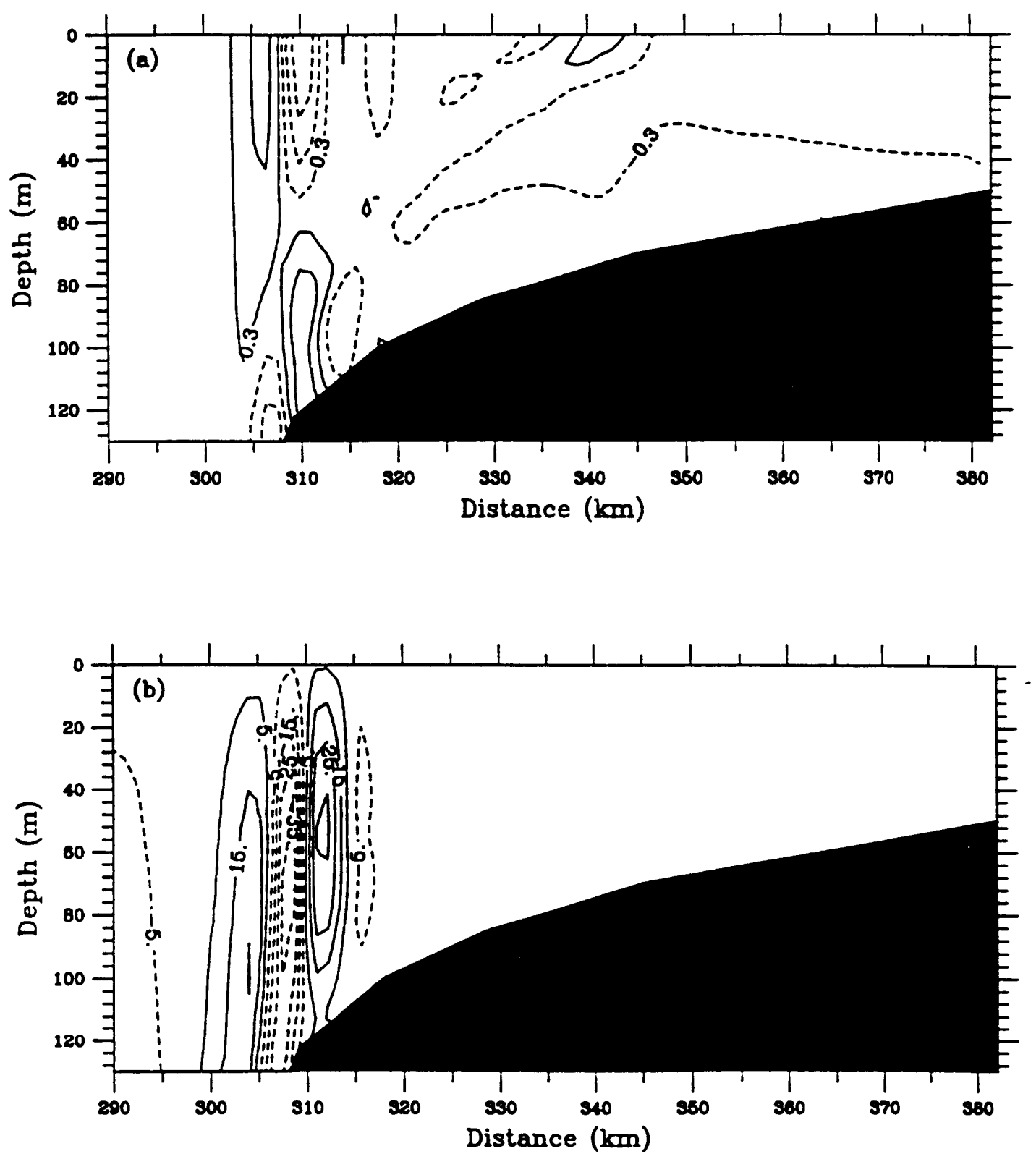

Figure 3.60: The structure of the cross-bank horizontal (a) and vertical (b) residual currents in the upper $140 \mathrm{~m}$ on the southern flank for the winter (weak stratification) case. The contour interval is $10 \times 10^{-3} \mathrm{~cm} / \mathrm{s}$ for $\bar{w}$ and $1.0 \mathrm{~cm} / \mathrm{s}$ for $\bar{u}$. 

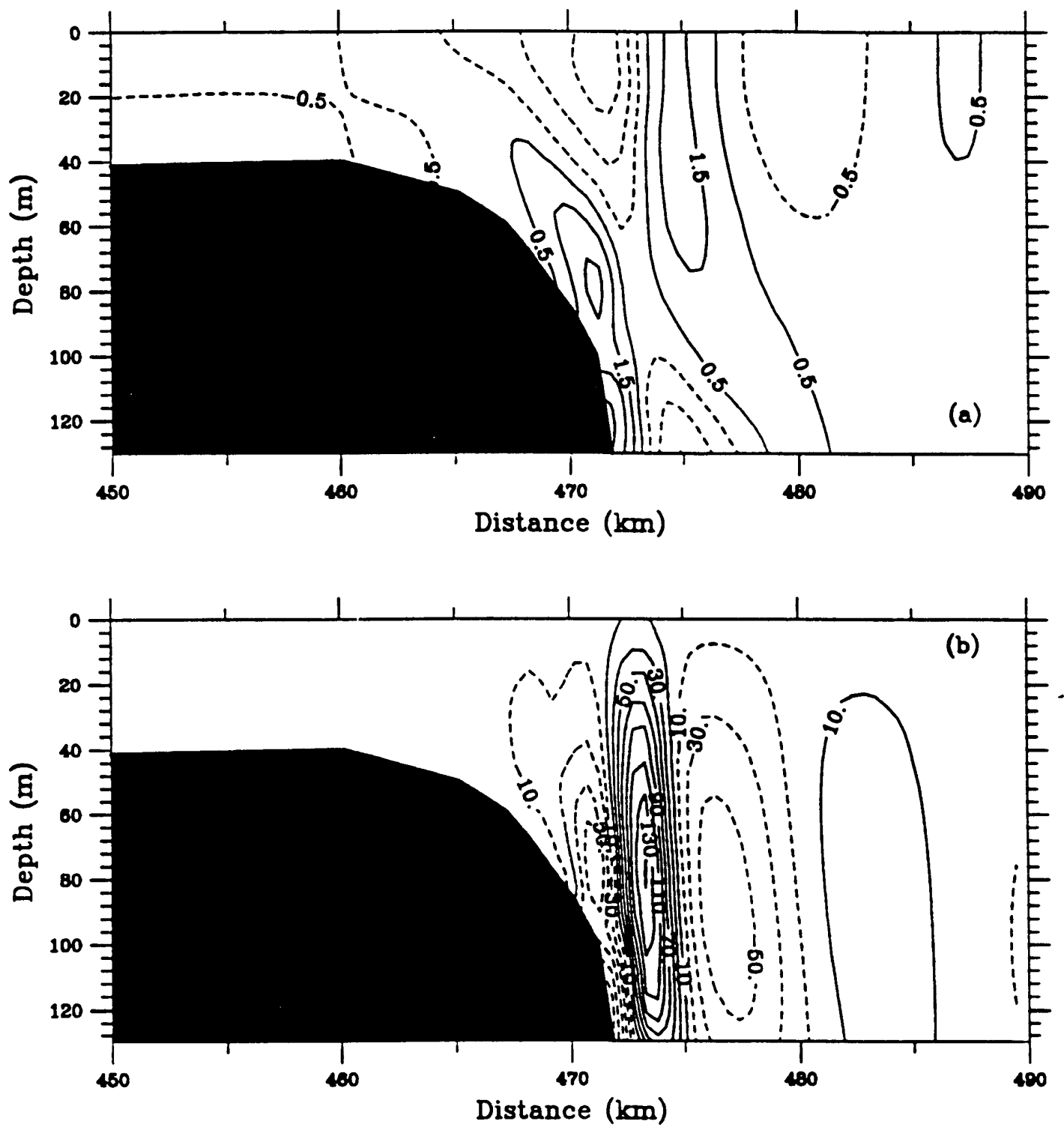

Figure 3.61: The structure of the cross-bank horizontal (a) and vertical (b) residual currents in the upper $140 \mathrm{~m}$ on the northern flank for the winter (weak stratification) case. The contour interval is $10 \times 10^{-3} \mathrm{~cm} / \mathrm{s}$ for $\bar{w}$ and $1.0 \mathrm{~cm} / \mathrm{s}$ for $\bar{u}$. 

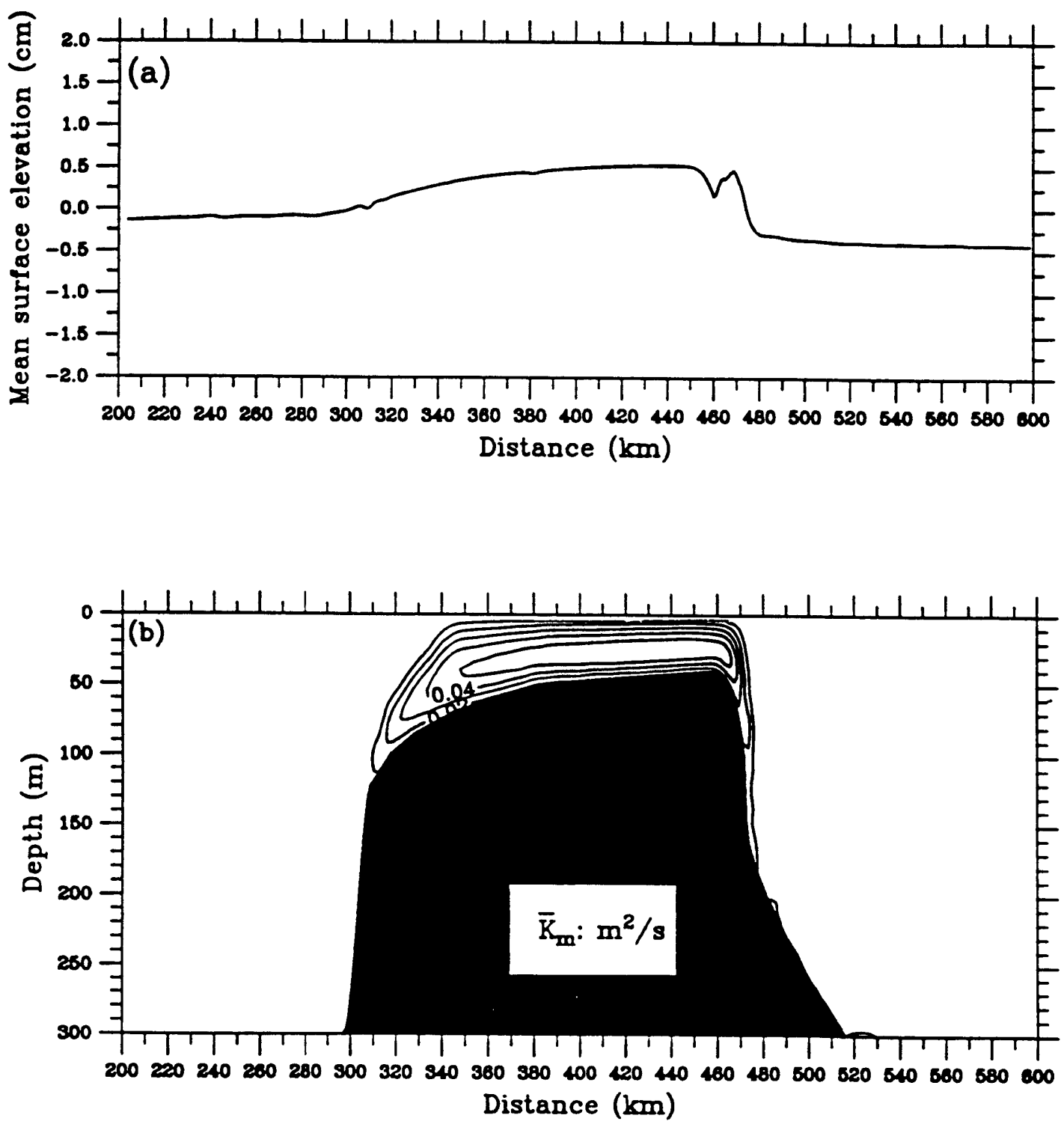

Figure 3.62: The cross-bank structure of the mean surface elevation (a) and the vertical eddy viscosity (b) for the winter (weak stratification) case. The contour interval is $0.01 \mathrm{~m}^{2} / \mathrm{s}$ for $\bar{K}_{m}$. 

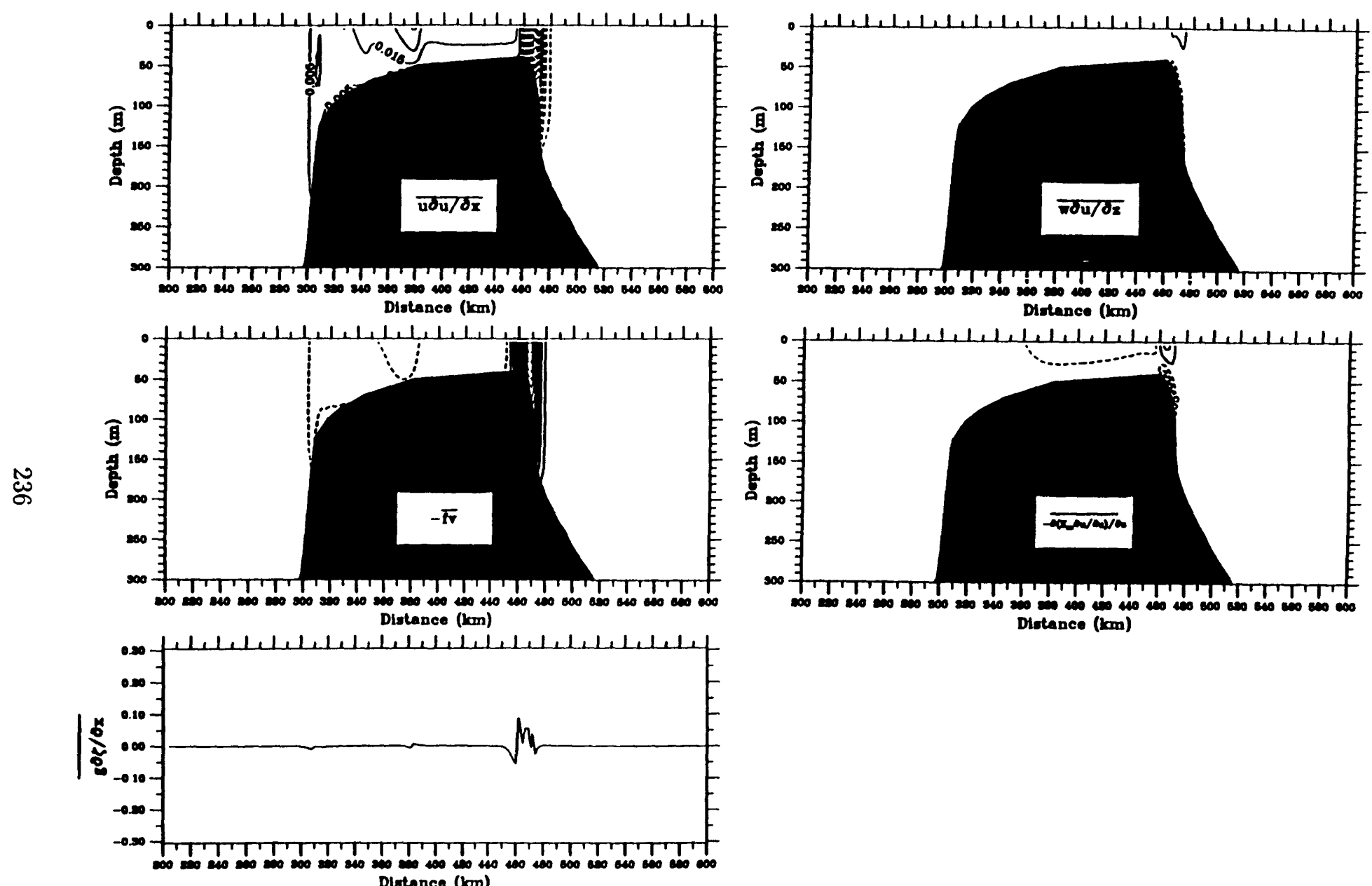

Distance ( $(\mathbf{k m})$

Figure 3.63: Cross-bank distribution of each dynamic term in the cross-bank mean momentum equation for the barotropic case. The contour interval is $0.01 \times 10^{-4} \mathrm{~m} / \mathrm{s}^{2}$ for all plots. 


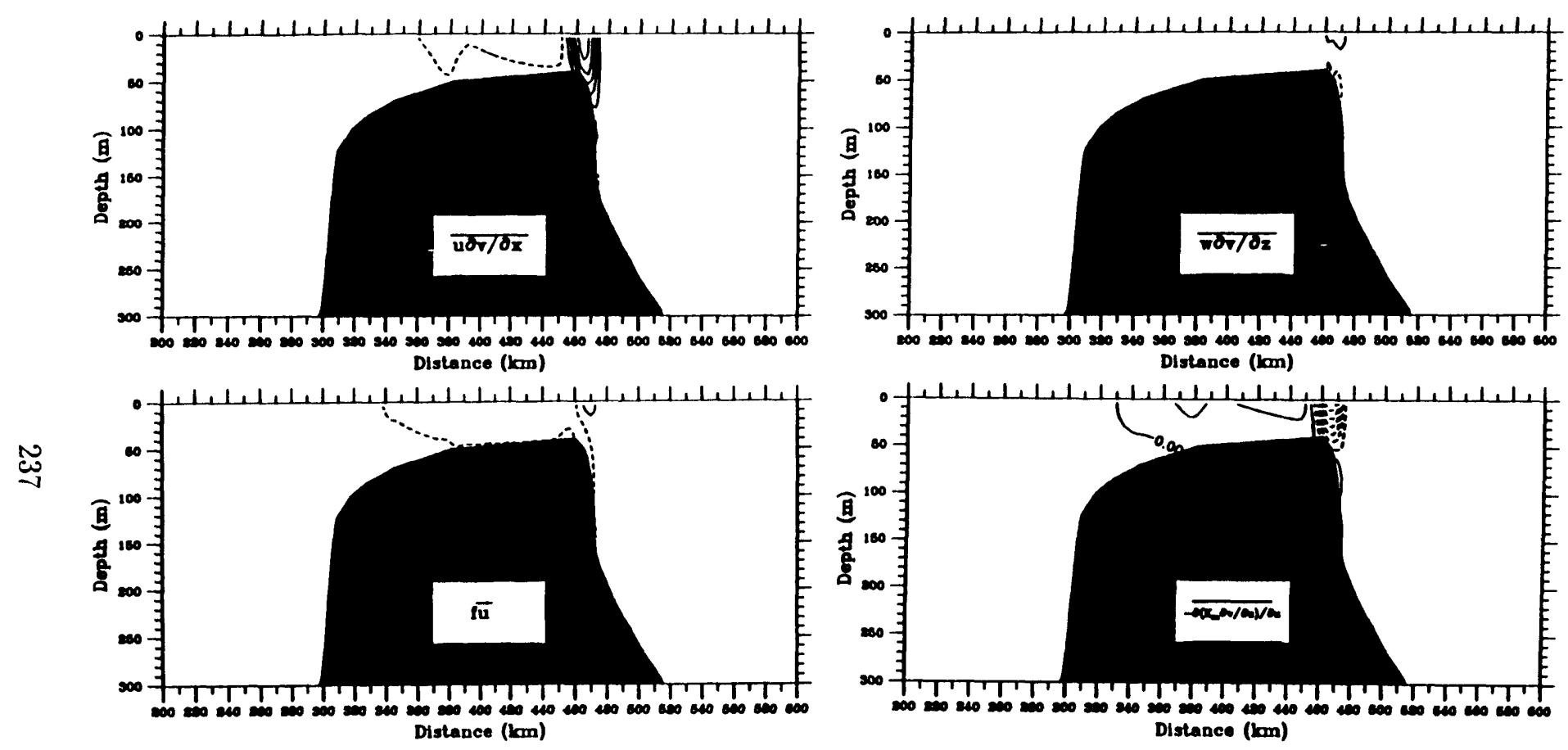

Figure 3.64: Cross-bank distribution of each dynamic term in the along-bank mean momentum equation for the barotropic case. The contour interval is $0.005 \times 10^{-4} \mathrm{~m} / \mathrm{s}^{2}$ for all plots. 
where the vertical advection is too small to be important. On the northern flank, however, steep bottom topography leads to much larger along-bank horizontal advection and vertical friction terms than Coriolis force, so that the along-bank momentum is mainly balanced between horizontal advection and vertical friction terms. This dynamic balance is consistent with the scale analysis for vertically averaged tidal rectification (Loder, 1980; Zimmerman, 1978) and diagnostic results for a symmetrical bank discussed in section 3.3. Except on the southern flank where the bottom slope is smaller, the along-bank horizontal advection is weaker so that the Coriolis force becomes important in the along-bank momentum balance. As stratification is added, all terms increase across the Bank except the vertical friction term in the cross-bank momentum equation. On both flanks of the Bank, the momentum is mainly balanced among horizontal and vertical advection, Coriolis force, and surface and baroclinic pressure gradients in the cross-bank direction, and among horizontal and vertical advection, Coriolis force, and vertical friction in the along-bank direction.

The driving mechanism for homogeneous and stratified tidal rectification is discussed next. In the homogeneous case, the momentum balance over the Bank suggests that the along-bank residual current is mainly generated by nonlinear transfer of momentum from the fluctuating tidal currents to the mean flow. This mechanism is consistent with previous analytical and numerical results for vertically averaged or depth-dependent models (Loder, 1980; Greenberg, 1983; Loder and Wright, 1985). Two reference points in the cross-bank circulation cell on either side of the Bank are chosen as examples to study the mean momentum balance in the bottom boundary layer (see Figures 3.67 and 3.68). On the southern flank at the $175-$ and $120-\mathrm{m}$ isobaths, the two biggest terms in the momentum balance near the bottom are surface pressure gradient and horizontal advection in the cross-bank direction and the Coriolis force and vertical friction in the along-bank direction (Figure 3.67). Magnitudes of vertical friction, Coriolis force and vertical advection in the cross-bank direction are relatively small near the bottom at the $175-\mathrm{m}$ isobath but they increase up to the same order as the surface pressure gradient at the $120-\mathrm{m}$ isobath. A similar tendency is also found in the along-bank direction where horizontal and vertical 


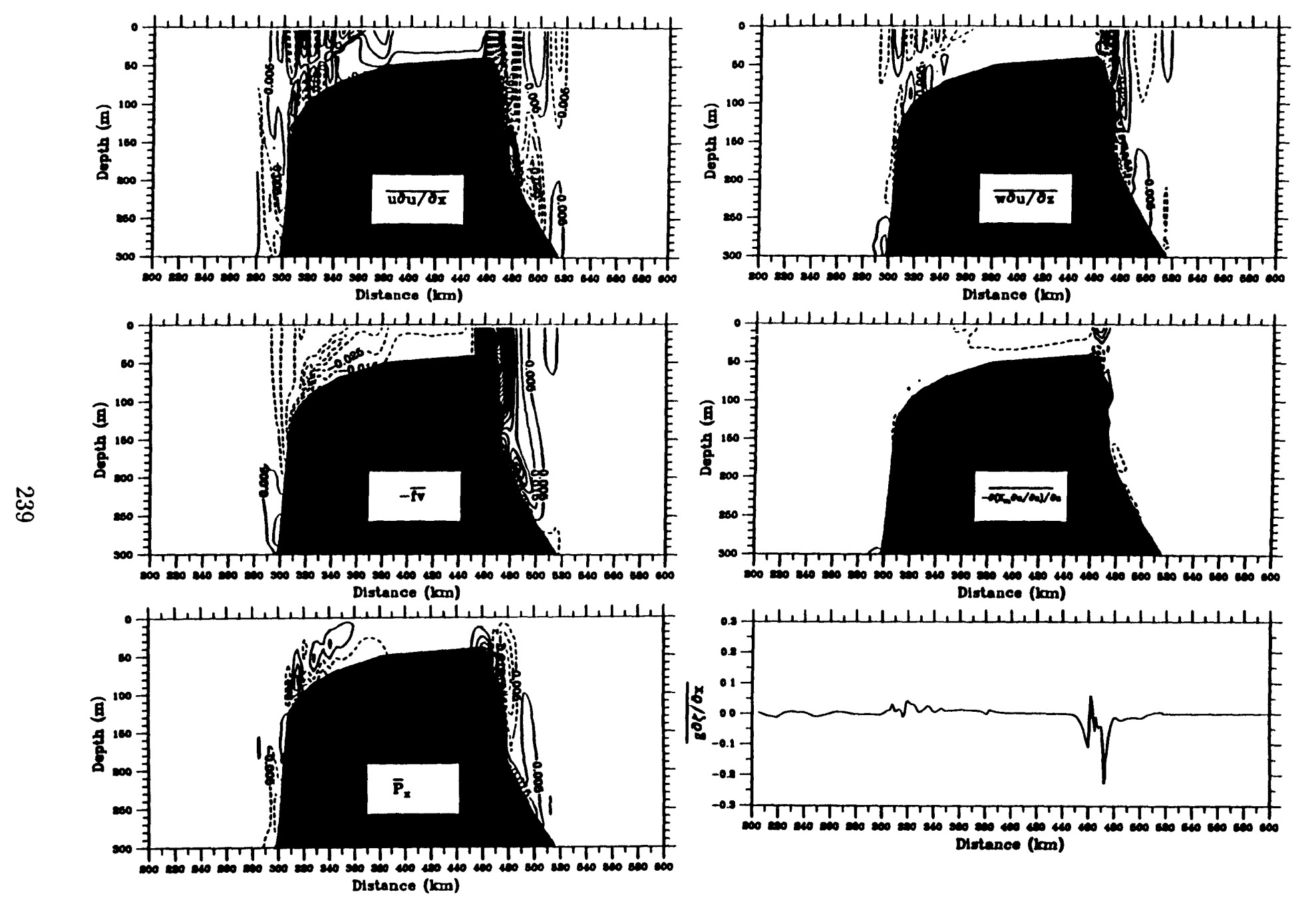

Figure 3.65: Cross-bank distribution of each dy namic term in the cross-bank mean momentum equation for the summer (strong stratification) case. The contour interval is $0.01 \times 10^{-4} \mathrm{~m} / \mathrm{s}^{2}$ for all plots. 

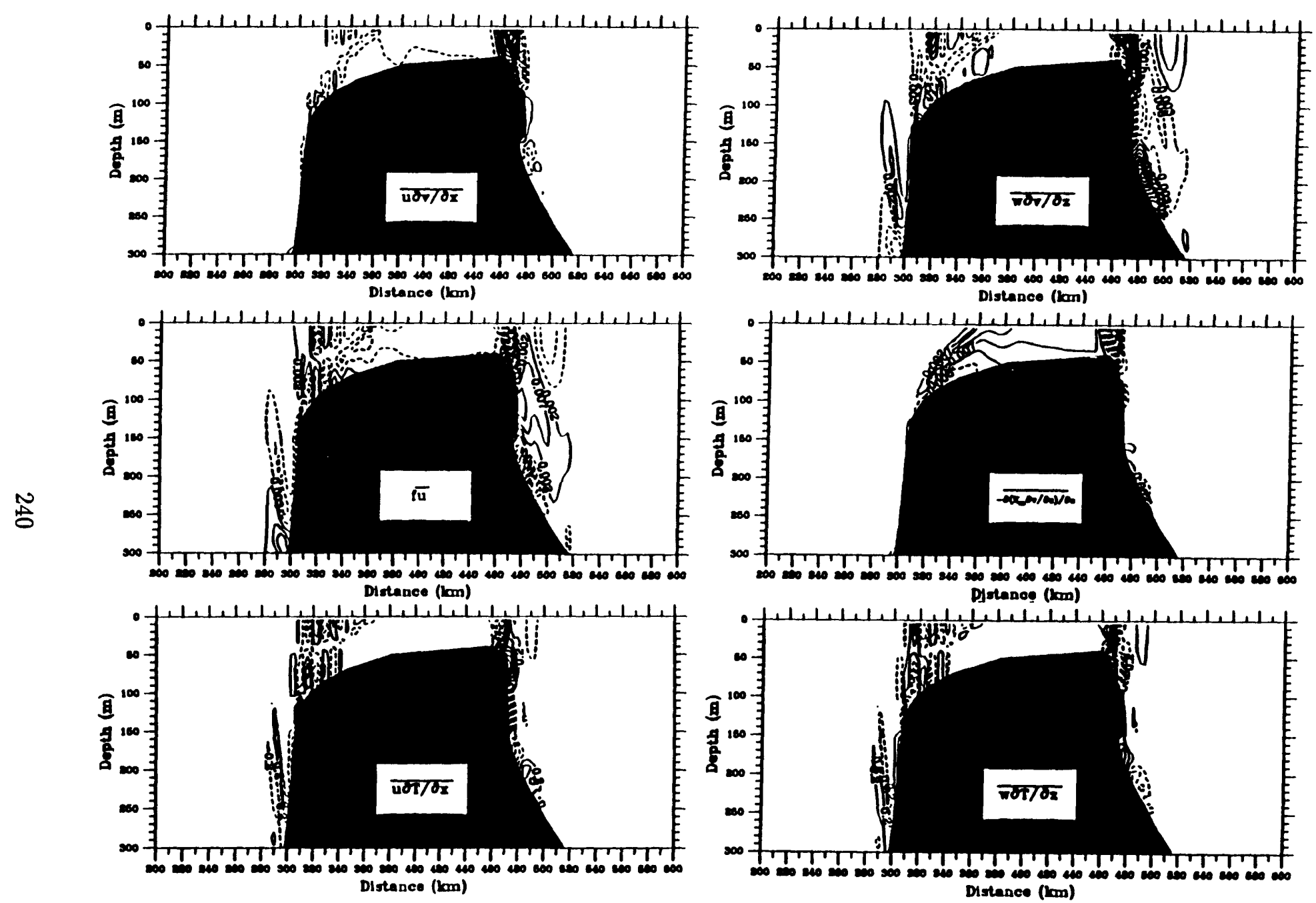

Distance (kom)

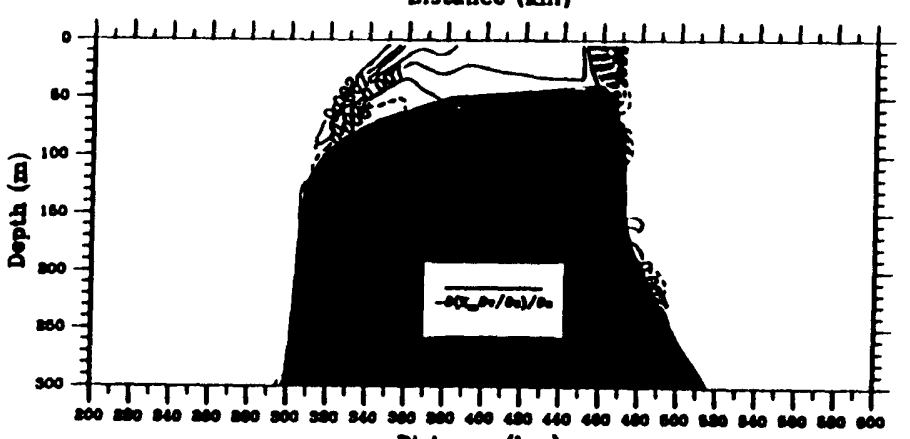

Djatanog $(\mathrm{km})$

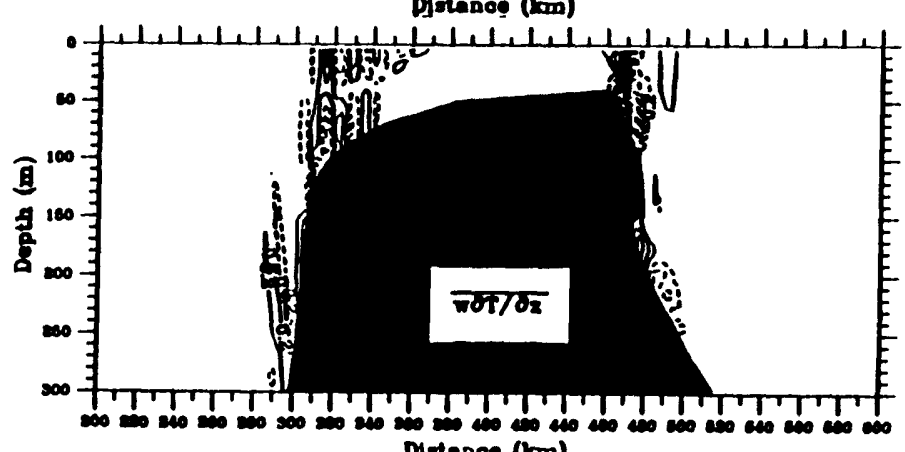

Figure 3.66: Cross-bank distribution of each dynamic term in the along-bank mean momentum and heat equations for the summer (strong stratification) case. The contour interval is $0.005 \times 10^{-4} \mathrm{~m} / \mathrm{s}^{2}$ for dynamic terms and $0.2 \times 10^{-4}{ }^{\circ} \mathrm{C} / \mathrm{s}$ for heat fluxes. 

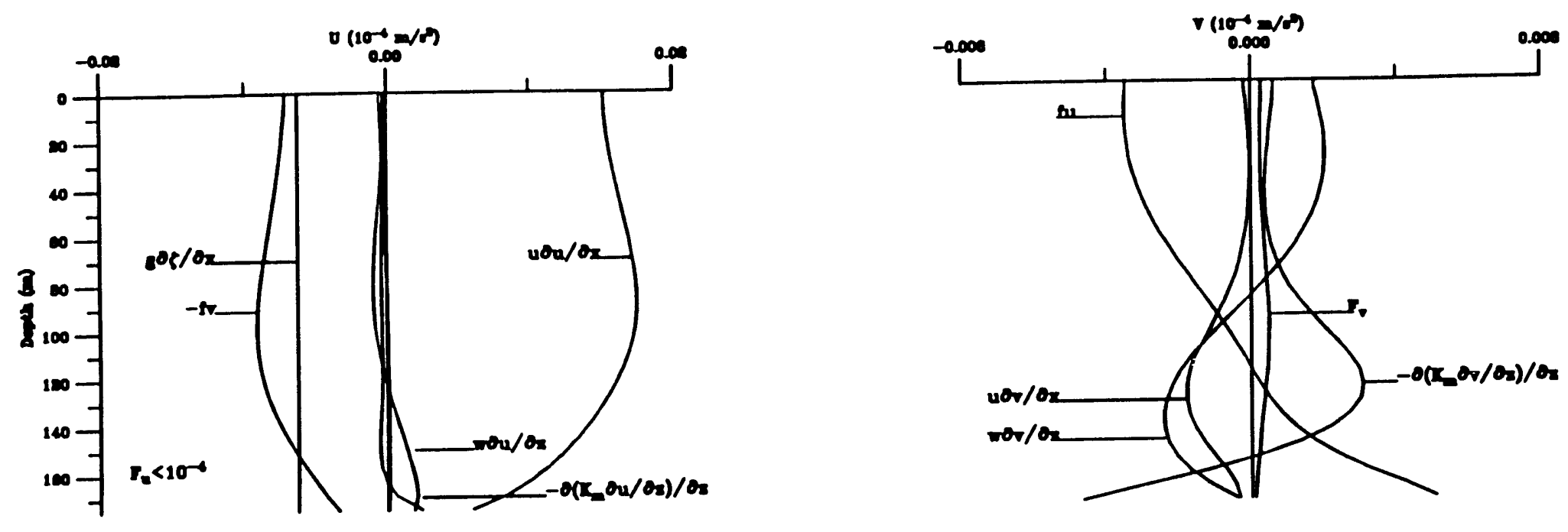

$\tilde{\underline{H}}$
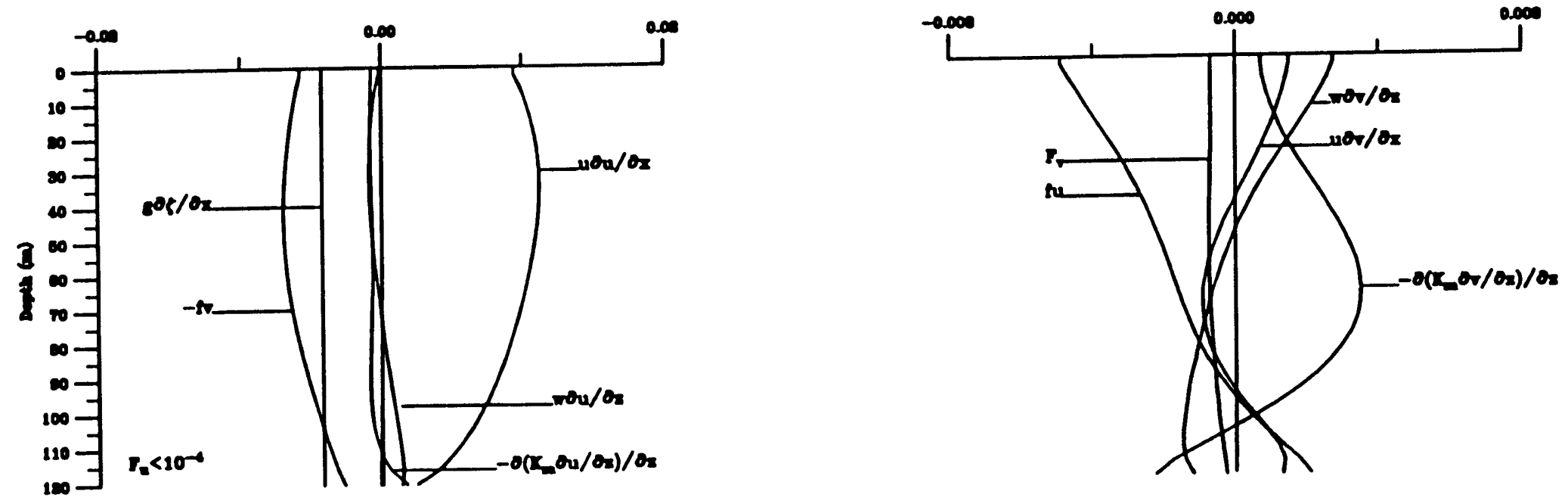

Figure 3.67: The vertical profile of each dynamic term in the cross-and along-bank momentum mean equations at the 175 and 120 -m isobath on the southern flank for the homogeneous case. 
advection are small near the bottom at the $175-\mathrm{m}$ isobath but increase up to the same order as the vertical friction term at the $120-\mathrm{m}$ isobath. Since the nonlinear terms in the along-bank direction are relatively small at the $175-\mathrm{m}$ isobath and are opposite at the $120-\mathrm{m}$ isobath, the along-bank momentum is primitively balanced by Coriolis force and vertical friction terms just like an Ekman layer. However, the cross-bank momentum balance is very complex near the bottom where all nonlinear terms, Coriolis force, surface pressure gradient, and vertical friction are important in the bottom boundary layer. Similar results can be found on the northern flank at the $41-$ and $85-\mathrm{m}$ isobaths except that the steep bottom topography causes both horizontal and vertical advection to be important in the bottom boundary layer in the along-bank direction (Figure 3.68). Regardless of the complex cross-bank momentum balance, the cross-bank circulation cell can be interpreted here by Ekman theory in which the positive along-bank vertical friction drives the bottom water onto the Bank on the southern flank and off the Bank on the northern flank.

In the case of strong stratification, both stratified tidal rectification and tidal mixing fronts modify the vertical structure and strength of along-and cross-bank residual currents. Evidence of stratified tidal rectification can be seen in the intensification of nonlinear interaction due to stratification. The cross-bank horizontal advection, which has a single maximum of about $0.3 \times 10^{-5} \mathrm{~m} / \mathrm{s}^{2}$ on the southern flank or about $0.2 \times 10^{-4} \mathrm{~m} / \mathrm{s}^{2}$ on the northern flank in the homogeneous case, is characterized by multiple cells on both flanks of the Bank in the summer case and its magnitude increases to $0.8 \times 10^{-5} \mathrm{~m} / \mathrm{s}^{2}$ on the southern flank and $0.4 \times 10^{-4} \mathrm{~m} / \mathrm{s}^{2}$ on the northern flank. Similar results can be seen in the other nonlinear terms in both cross-and along-bank momentum equations. The relative contribution of stratified tidal rectification and tidal mixing fronts to the intensification of along-bank residual flow is very different across the Bank. On the southern flank, good correlation is found between the axes of maximum baroclinic pressure gradient and along-bank residual current, while horizontal and vertical advection are the same order of magnitude but cancel each other, suggesting that the tide-induced front plays a key role in the vertical intensification of along-bank flow there. On the northern flank, at the place where 

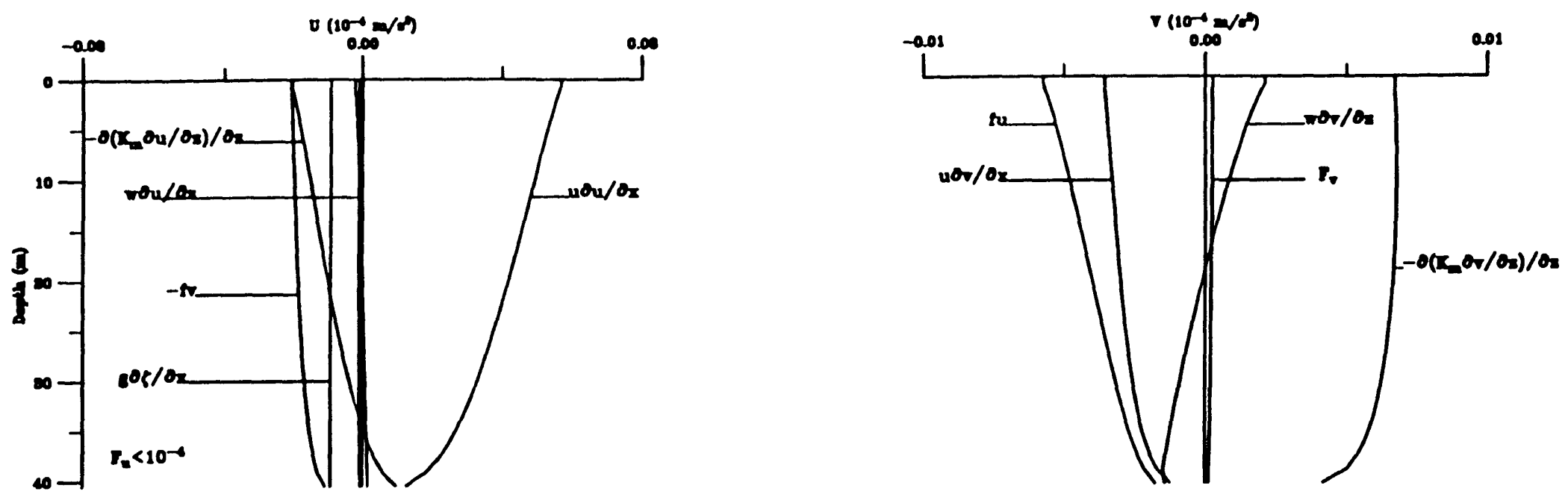

荄
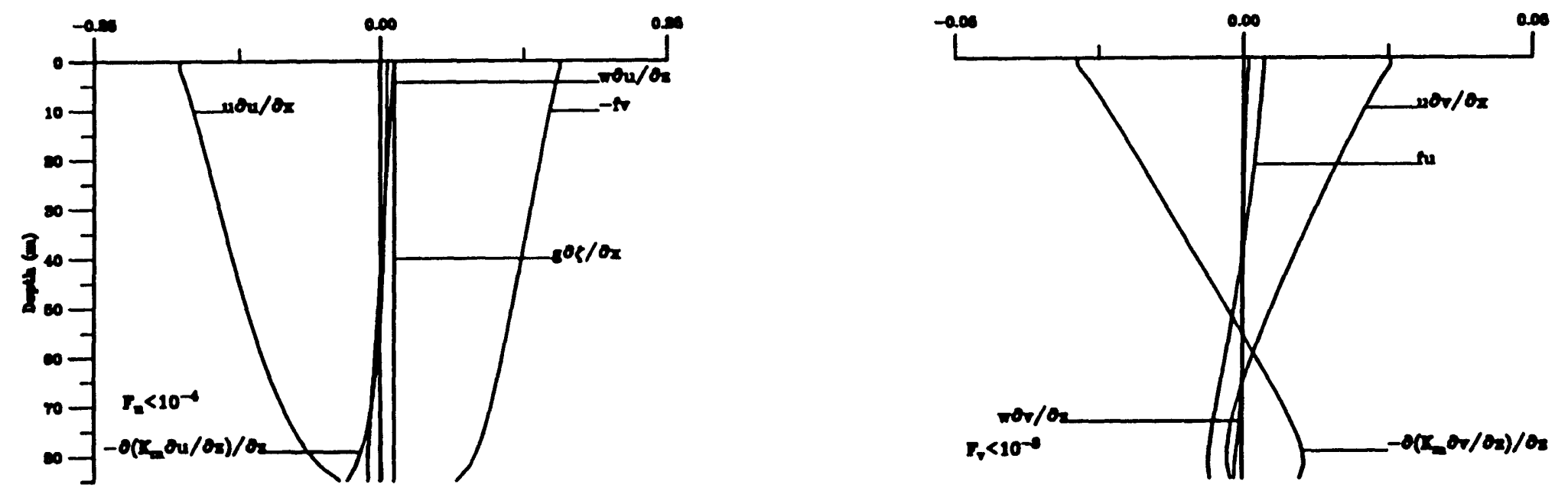

Figure 3.68: The vertical profile of each dynamic term in the cross- and along-bank momentum equations at the 41- and 85-m isobaths on the northern flank for the homogeneous case. 
the maximum along-bank residual flow is located, the baroclinic horizontal advection term (the difference between the horizontal advection terms for the summer and homogeneous cases) in the cross-bank direction is about $-0.13 \times 10^{-4} \mathrm{~m}^{2} / \mathrm{s}$ while the baroclinic pressure gradient is only about $-0.003 \times 10^{-4} \mathrm{~m}^{2} / \mathrm{s}$, implying that stratified tidal rectification is more important for the summertime intensification of along-bank residual current there.

The cross-bank residual current exhibits a pronounced asymmetry across the Bank. On the southern flank, the cross-bank residual flow is characterized by a multiple cell circulation pattern. However, there is just a single cell on the northern flank. Good correlation between the cross-bank horizontal advection and residual flow suggests a driving mechanism of cross-bank mean flow due to stratified tidal rectification. Maas and Zimmerman (1989a and $b$ ) analytically studied stratified tidal rectification over small-amplitude bottom topography and found that the cross-bank residual current pattern over the slope consists of three cells, whose horizontal scale is $2 \ell_{o}-4 \ell_{o}\left(\ell_{o}=\mathrm{U} / \omega\right.$, the barotropic tidal current excursion). Although no tidal mixing mechanism is included in Maas and Zimmerman's spectral model, we can still apply their theory to study stratified tidal rectification over the shelf region on the southern flank since the bottom slope is relatively small there. The barotropic tidal current excursion is about $3.5 \mathrm{~km}$ for a tidal current of about $50 \mathrm{~cm} / \mathrm{s}$ over the shelf, so that the horizontal scale of the cross-bank circulation cell caused by the $M_{2}$ internal tides is about $7-14 \mathrm{~km}$. The numerical model-predicted, cross-bank circulation cell on the southern flank has a horizontal scale of about $10 \mathrm{~km}$ in the case of strong stratification, which is in good agreement with the analytical solution. Using a fourth order Runge-Kutta shooting method, we can also estimate the wavelength of the $M_{2}$ internal tide from the inferred vertical temperature structure. The wavelength of the first baroclinic mode is about $15 \mathrm{~km}$ near the southern flank and decreases as the wave propagates onto the Bank, thus supporting our argument on the driving mechanism of stratified tidal rectification for the cross-bank circulation on the southern flank. The spatial distribution and strength of multiple circulation cells found on the southern flank are associated with the spatial distribution of temperature in which the tidal mixed layer is dominant near the bot- 
tom and increases as the water becomes shallower. Tidal mixing and bottom stress tend to dissipate the internal tidal energy as the wave propagates onto the shelf, and hence reduces the strength and vertical scale of the circulation cell as the water becomes shallower.

The driving mechanism for a single cross-bank circulation cell on the northern flank looks more complex since all dynamical terms are relatively strong there. To study this problem, we have taken two reference points in the cross-bank circulation cell on the northern flank and then compared the dynamical terms in the mean momentum equations (see Figure 3.69). Although the circulation pattern is consistent with the cross-bank surface pressure gradient (Loder, 1980), the momentum balance in the bottom boundary layer is very complicated at these two stations. At the $85-\mathrm{m}$ isobath, both Coriolis force and vertical friction terms in the cross-bank direction are positive near the bottom; they are balanced by surface and baroclinic pressure gradients and horizontal advection. In the alongbank direction, the vertical friction term is only half of the Coriolis force so that nonlinear interaction becomes more important in the along-bank momentum balance in the bottom boundary layer. At the $140-\mathrm{m}$ isobath, however, relatively strong stratification results in weak vertical friction in both along-and cross-bank directions throughout the water column. The momentum balance is then mainly between surface and baroclinic pressure gradients plus corrections due to the net contribution of horizontal and vertical advection and Coriolis force in the cross-bank direction, and between Coriolis force and the difference of horizontal and vertical advection in the along-bank direction. Relatively large nonlinear terms and baroclinic pressure gradient suggest that tidal mixing and stratified tidal rectification are both important for the formation of the cross-bank circulation cells during summer on the northern flank, where neither Ekman nor Loder's theory is valid as stratification is added.

\section{Comparison with Tidal Current Observations}

It is difficult to make simple comparisons between the predicted and observed tidal currents over Georges Bank because of the varying influence of the seasonal stratification cycle. Most of the measurements of the $M_{2}$ tidal currents over Georges Bank were made 

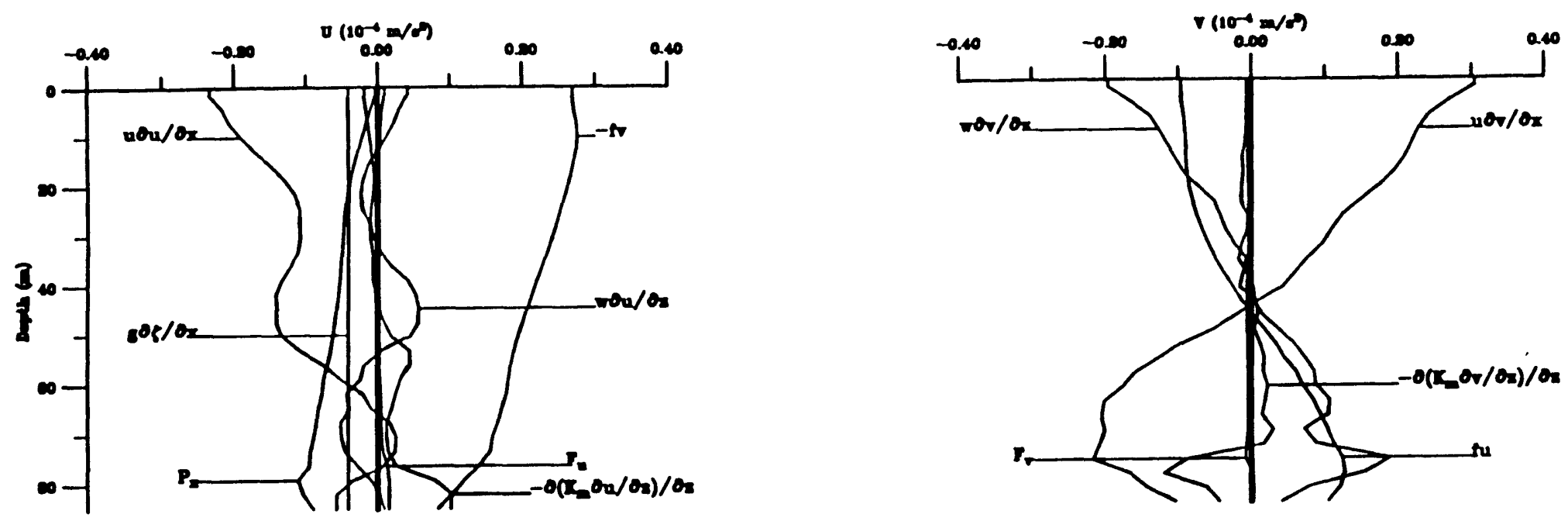

舍
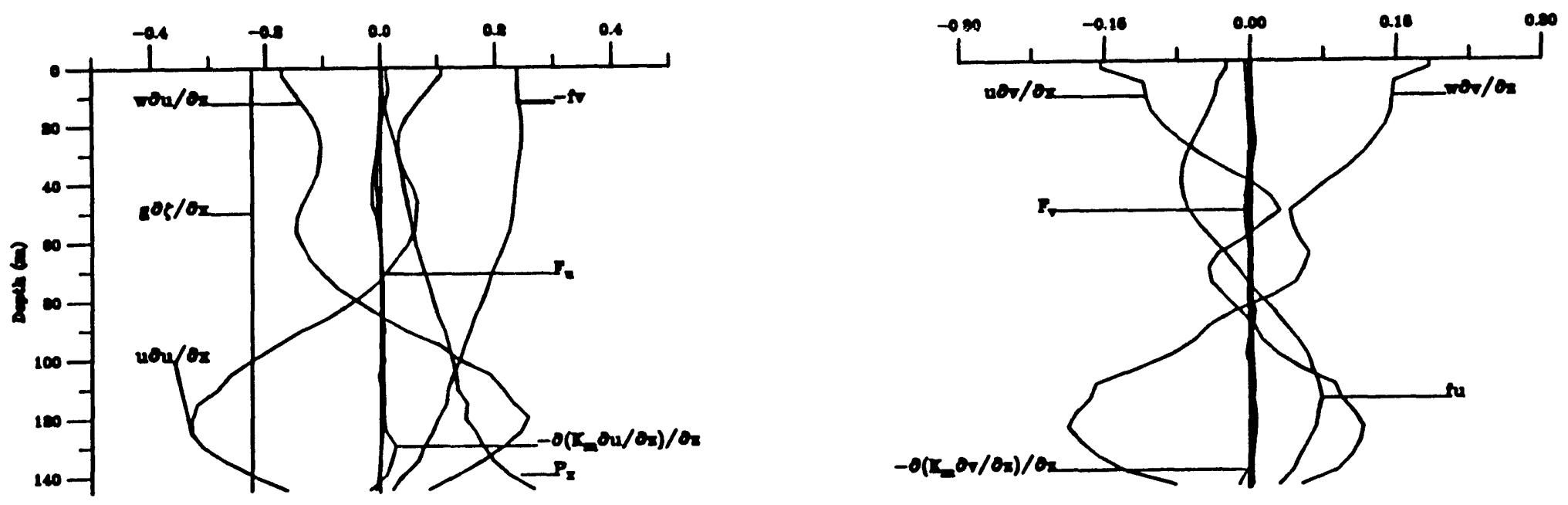

Figure 3.69: The vertical profile of individual terms in the cross- and along-bank momentum equations at the 85- and 145-m isobaths on the northern flank for the summer (strong stratification) case. 
during time periods of about 2-60 days (Moody et al., 1984; Marsden, 1986; Loder et al., 1992) where the temporal variability of the internal tide was included. Therefore, it might not make sense to compare predicted and observed tidal currents without considering the degree of stratification which existed during the measurement period. For this reason, our comparison with tidal observations will be limited to the barotropic cases where the observed tidal currents were taken either by averaging over all seasons or in the vertically well mixed region. Long-term current meter data were recorded for periods of $\sim 261-957$ days at station $\mathrm{A}$ at the $85-\mathrm{m}$ isobath, hence, the average amplitude of the $M_{2}$ tidal current there should be a good approximation to the barotropic tide. Although the record length of the current measurement was in the range of 58 to 232 days at station $\mathrm{K}$ at the $61-\mathrm{m}$ isobath and only about 15 days at station P3 at the 45-m isobath, the water was almost vertically well mixed there, so amplitudes of the $M_{2}$ tidal current should be primarily barotropic (Moody et al., 1984). We will now compare these observed $M_{2}$ tidal currents with the predicted barotropic tidal currents taken at the same isobath in our numerical model.

Figure 3.70 shows the vertical structure of the predicted and observed $M_{2}$ tidal currents at four stations. The amplitudes of predicted along-and cross-bank tidal currents are found to be in reasonable agreement with observations at stations $A$ and $K$ on the southern flank of the Bank within the measurement uncertainty, but about $6-8 \mathrm{~cm} / \mathrm{s}$ less at station P3 on the northeast side of the Bank. It may not be appropriate to compare predicted tidal currents with observed tidal data at station P3 because of the relatively large distance between our model section and that station. A maximum core of about 90$110 \mathrm{~cm} / \mathrm{s}$ was always observed in the cross-bank direction near station P3, $60 \mathrm{~km}$ east of our model section (Moody et al., 1984; Loder et al., 1992), suggesting that the relatively large difference between the model and observed results on the northern side of Georges Bank is probably due to the spatial variation of tidal currents there. Good agreement can be found between predicted and observed tidal currents if the observed tidal current at station P3 are interpolated to our model section. Considering the above uncertainty in our comparisons, 

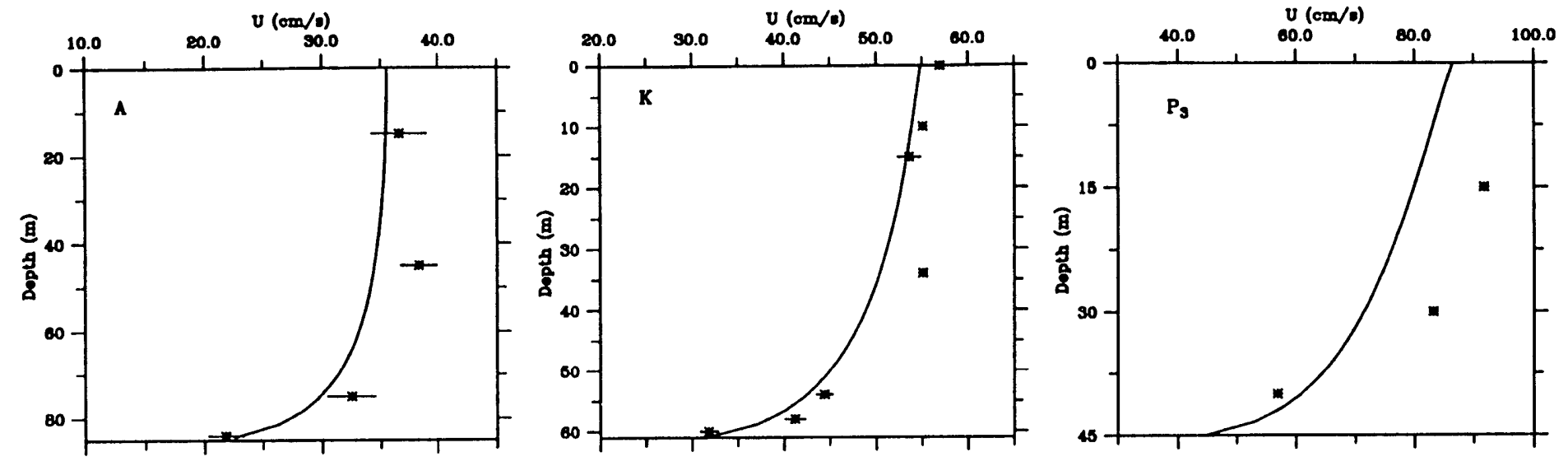

$\stackrel{\tilde{\infty}}{\infty}$
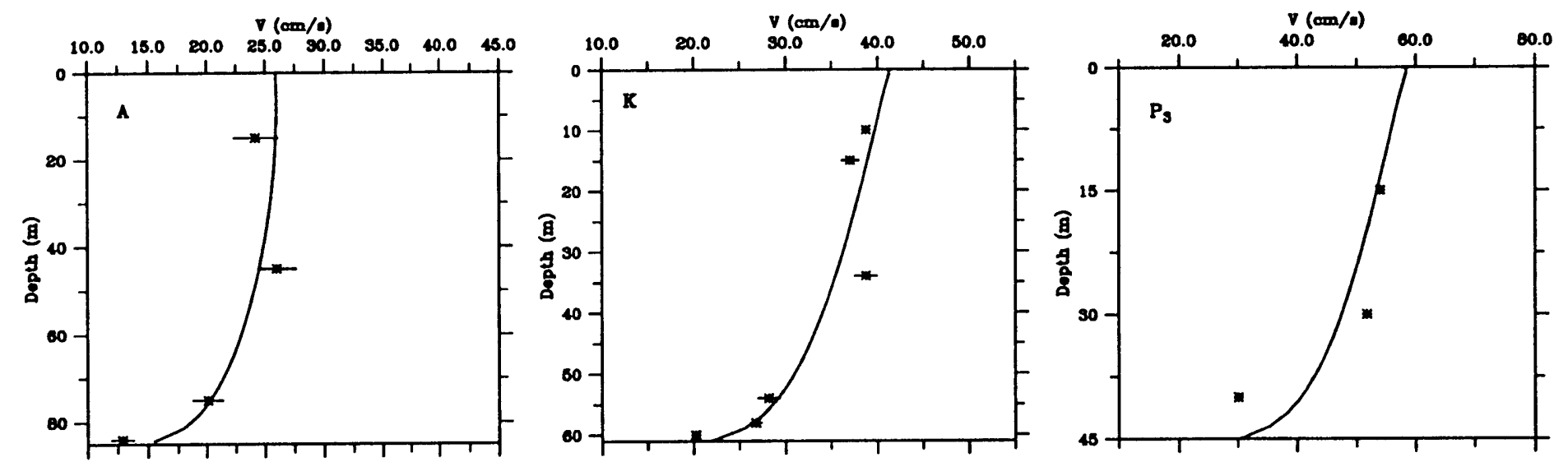

Figure 3.70: Comparison between amplitudes of the predicted and observed tidal currents at stations A, K, P3. The solid line is the components of predicted tidal currents. The symbol * is an individual tidal current datum at stations $\mathrm{A}, \mathrm{K}$, and $\mathrm{P} 3$. 
we conclude here that the model-predicted tidal currents coincide well within $10 \%$ with the $M_{2}$ tidal current components observed over Georges Bank.

\section{Comparison with Observations of the Tidal Mixing Fronts}

Georges Bank exhibits two well-known types of fronts: (1) the shelf/slope front and (2) tidal mixing fronts. The shelf/slope front (sometimes called the shelf break front) occurs at the shelf break near the $100-\mathrm{m}$ isobath on the southern flank, and moves steadily onshore from winter to summer and then suddenly adjusts offshore as fall turns to winter (Flagg, 1987). The tidal mixing front over Georges Bank also exhibits a seasonal variation (Flagg, 1987). During summer and fall, the tidal mixing fronts are located at the $40-\mathrm{m}$ isobath at the northern edge of the Bank and near the $50-60-\mathrm{m}$ isobath on the southern flank, about $70-80 \mathrm{~km}$ on-bank from the shelf break. During winter, however, the tidal mixing front disappears on the southern flank and is much weaker on the northern flank, even though the position of this front remains almost stationary on the northern edge of the Bank.

The observed seasonal variability in the strength and location of tidal mixing fronts is also seen in our model results for strong and weak stratification. For the summer case, the model shows the well-defined tidal-induced thermal fronts at both the $40-\mathrm{m}$ isobath on the northern flank and the $60-\mathrm{m}$ isobath on the southern flank. As stratification lessens in winter, the tidal mixing front on the southern flank moves toward the shelf break, while the tidal front on the northern flank weakens in intensity but remains almost fixed in position. These model results agree well with existing observations of the tidal mixing fronts. In addition to increased atmospheric cooling and storms in winter, the disappearance of the tidal mixing front on the southern flank during winter can also be interpreted as the offshore migration of the tidal front predicted on the southern flank for weak stratification. As the tidal mixing front moves to the shelf break, it merges with the shelf/slope front there, resulting in a single frontal structure on the southern flank. This result suggests an 
intensification of the shelf/slope front due to interaction with the tidal mixing front during winter since both fronts have similar density structures.

\section{Comparison with Observations of the Along-bank Mean Current}

Winter. Table 3.2 shows the comparison between the observed and predicted barotropic and weakly stratified mean currents during winter at station $\mathrm{A}$ on the southern flank and at stations M2 and M1 on the northern flank. The wintertime mean Eulerian current measurements summarized by Butman et al. (1982) and Flagg et al. (1982) clearly

\section{Table 3.2. Comparison Between the Observed and Predicted}

\section{Along-Bank Mean Currents During Winter}

\begin{tabular}{|c|c|c|c|c|c|}
\hline \multirow[b]{2}{*}{ Station } & \multirow[b]{2}{*}{$\begin{array}{c}\mathrm{H} \\
(\mathrm{m})\end{array}$} & \multirow[b]{2}{*}{$\begin{array}{l}Z_{m} \\
(\mathrm{~m})\end{array}$} & \multirow{2}{*}{$\begin{array}{c}\text { Observation } \\
\bar{v}_{o} \\
(\mathrm{~cm} / \mathrm{s})\end{array}$} & $\begin{array}{c}\text { Homogeneous } \\
\text { Case }\end{array}$ & Winter Case \\
\hline & & & & $\begin{array}{c}\bar{v}_{b} \\
(\mathrm{~cm} / \mathrm{s})\end{array}$ & $\begin{array}{c}\bar{v}_{w} \\
(\mathrm{~cm} / \mathrm{s})\end{array}$ \\
\hline \multirow{3}{*}{ A } & \multirow{3}{*}{85} & 45 & $2 \pm 3-8 \pm 8$ & 1.1 & 2.1 \\
\hline & & 75 & $1 \pm 3-7 \pm 7$ & 0.8 & 1.1 \\
\hline & & 84 & $1 \pm 3-5 \pm 5$ & 0.6 & 0.7 \\
\hline \multirow{3}{*}{ M2 } & \multirow{3}{*}{85} & 10 & $-19 \pm 10$ & -17.4 & -26.8 \\
\hline & & 44 & none & -14.6 & -18.1 \\
\hline & & 77 & $-8 \pm 7$ & -8.4 & -9.6 \\
\hline \multirow{3}{*}{ M1 } & \multirow{3}{*}{200} & 10 & $-15 \pm 13$ & -0.2 & -1.7 \\
\hline & & 77 & $-5 \pm 10$ & -0.2 & -2.9 \\
\hline & & 192 & $-2 \pm 8$ & -0.1 & -0.8 \\
\hline
\end{tabular}

Note: $H$ is the water depth; $Z_{m}$ is the instrument depth; $\bar{v}_{o}$ is the observed mean current plus the standard deviation; $\bar{v}_{b}$ and $\bar{v}_{w}$ are the model-predicted mean currents for the homogeneous and weak stratification cases.

showed a clockwise circulation around Georges Bank, flowing southwestward at a mean speed of about $5 \mathrm{~cm} / \mathrm{s}$ at a depth of $45 \mathrm{~m}$ at station $\mathrm{A}$ on the southern flank, and northeastward at a speed of about $-19 \mathrm{~cm} / \mathrm{s}$ near the surface at station $\mathrm{M} 1$ on the northern 
flank. Butman et al. (1982) estimated that only about $40 \%$ of the along-bank mean flow (about $2 \mathrm{~cm} / \mathrm{s}$ ) at a depth of $45 \mathrm{~m}$ at station $\mathrm{A}$ was driven by tidal rectification during winter. A similar circulation pattern is also predicted by our numerical model for the homogeneous and weakly stratified models. In the homogeneous case, the model predicts a southwest mean flow of about $1.1 \mathrm{~cm} / \mathrm{s}$ at a depth of $45 \mathrm{~m}$ at station $\mathrm{A}$ on the southern flank and a northwest mean flow of about $-17.4 \mathrm{~cm} / \mathrm{s}$ near the surface at station $\mathrm{M} 2$ on the northern flank. As weak stratification is included, the along-bank mean flow increases to about $2.1 \mathrm{~cm} / \mathrm{s}$ at station $\mathrm{A}$ and about $-26.9 \mathrm{~cm} / \mathrm{s}$ at station $\mathrm{M} 2$. Since stratification was different for the years during which the wintertime observations were made, the real currents should vary within the homogeneous and weakly stratified cases during winter. Considering the relatively large deviation of the measurements, we conclude here that the model results agree reasonably well with observations.

Summer. Table 3.3 shows the comparison between the observed and predicted mean currents during summer at station $A$ on the southern flank and at stations M2, M1, and MS2 on the northern flank. The summertime mean Eulerian current measurements reported by Butman et al. (1982) and Flagg et al. (1982) showed an intensified clockwise circulation around the Bank, flowing southwestward at a speed of about 8-17 cm/s at a depth of $15 \mathrm{~m}$ at station $\mathrm{A}$ on the southern flank, and northeastward at a speed of -30 to $-33 \mathrm{~cm} / \mathrm{s}$ and -3 to $-27 \mathrm{~cm} / \mathrm{s}$ at a depth of $10 \mathrm{~m}$ at stations $\mathrm{M} 2$ and $\mathrm{M} 1$ on the northern flank, respectively. A relatively long-term current measurement conducted by Marsden (1986) also reported northeastward flow of $-13.4 \pm 0.7 \mathrm{~cm} / \mathrm{s}$ at a depth of $15 \mathrm{~m}$ at station MS2. Such an intensified clockwise circulation was predicted at the same depth by our model for the strongly stratified case; it shows a southwest flow of about $3.8 \mathrm{~cm} / \mathrm{s}$ at station $A$ on the southern flank and a northeast flow of about $-14 \mathrm{~cm} / \mathrm{s},-27 \mathrm{~cm} / \mathrm{s}$, and $-3 \mathrm{~cm} / \mathrm{s}$ at stations MS2, M2, and M1, respectively, on northern flank.

Extensive CTD, ADCP and moored current meter measurements were made under light wind conditions by Loder et al. (1992) during July 2-3, 1988. They observed a strong 
tidal current on the northern flank with a maximum amplitude of $110 \mathrm{~cm} / \mathrm{s}$ in the crossbank direction and $70 \mathrm{~cm} / \mathrm{s}$ in the along-bank direction. A tidal mixing front was located on the $50-\mathrm{m}$ isobath near the shelf break, which separated the vertically well mixed water

\section{Table 3.3. Comparison Between the Observed and Predicted} Along-Bank Mean Currents During Summer

\begin{tabular}{|c|c|c|c|c|}
\hline \multirow[b]{2}{*}{ Station } & \multirow[b]{2}{*}{$\begin{array}{c}\mathrm{H} \\
(\mathrm{m})\end{array}$} & \multirow[b]{2}{*}{$\begin{array}{l}Z_{m} \\
(\mathrm{~m})\end{array}$} & \multirow{2}{*}{$\begin{array}{c}\text { Observation } \\
\bar{v}_{o} \\
(\mathrm{~cm} / \mathrm{s})\end{array}$} & \multirow{2}{*}{ 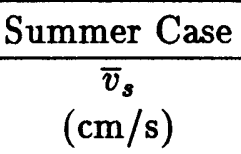 } \\
\hline & & & & \\
\hline \multirow{4}{*}{$\mathrm{A}$} & \multirow{4}{*}{85} & 15 & $8 \pm 5-17 \pm 9$ & 3.8 \\
\hline & & 45 & $10 \pm 6-13 \pm 7$ & 3.3 \\
\hline & & 75 & $2 \pm 2-4 \pm 5$ & 0.5 \\
\hline & & 84 & $1 \pm 2-3 \pm 2$ & 0.2 \\
\hline \multirow{3}{*}{ M2 } & \multirow{3}{*}{85} & 10 & $-30 \pm 9--33 \pm 8$ & -27.5 \\
\hline & & 44 & $-19 \pm 5--29 \pm 8$ & -21.0 \\
\hline & & 77 & $-6 \pm 6--9 \pm 5$ & -11.5 \\
\hline \multirow{3}{*}{ M1 } & \multirow{3}{*}{200} & 10 & $-3 \pm 7--27 \pm 11$ & -3.4 \\
\hline & & 77 & $-5 \pm 5--7 \pm 4$ & -3.8 \\
\hline & & 192 & $-2 \pm 3--2 \pm 6$ & -0.3 \\
\hline \multirow[b]{2}{*}{ MS2 } & \multirow[b]{2}{*}{45} & 15 & $-13.4 \pm 0.7$ & -14.1 \\
\hline & & 30 & $-7.9 \pm 0.5$ & -11.7 \\
\hline
\end{tabular}

Note: $H$ is the water depth; $Z_{m}$ is the instrument depth plus the standard deviation; $\bar{v}_{o}$ and $\bar{v}_{s}$ are the observed and predicted currents during summer.

on top of the Bank from the stratified water $\left(N \sim 1.3 \times 10^{-2} \mathrm{~s}^{-1}\right)$ over the slope. Associated with this tidal mixing front, both the ADCP and moored current meter data consistently showed a strong northeast along-bank mean current on the northern flank with a maximum of about $50 \mathrm{~cm} / \mathrm{s}$ at a depth of $20 \mathrm{~m}$ near the shelf break where the front-induced horizontal density gradient was strongest (see Figure 3.71, upper panel). Although this observed section is about $60 \mathrm{~km}$ east of our model section where the maximum cross-bank tidal 

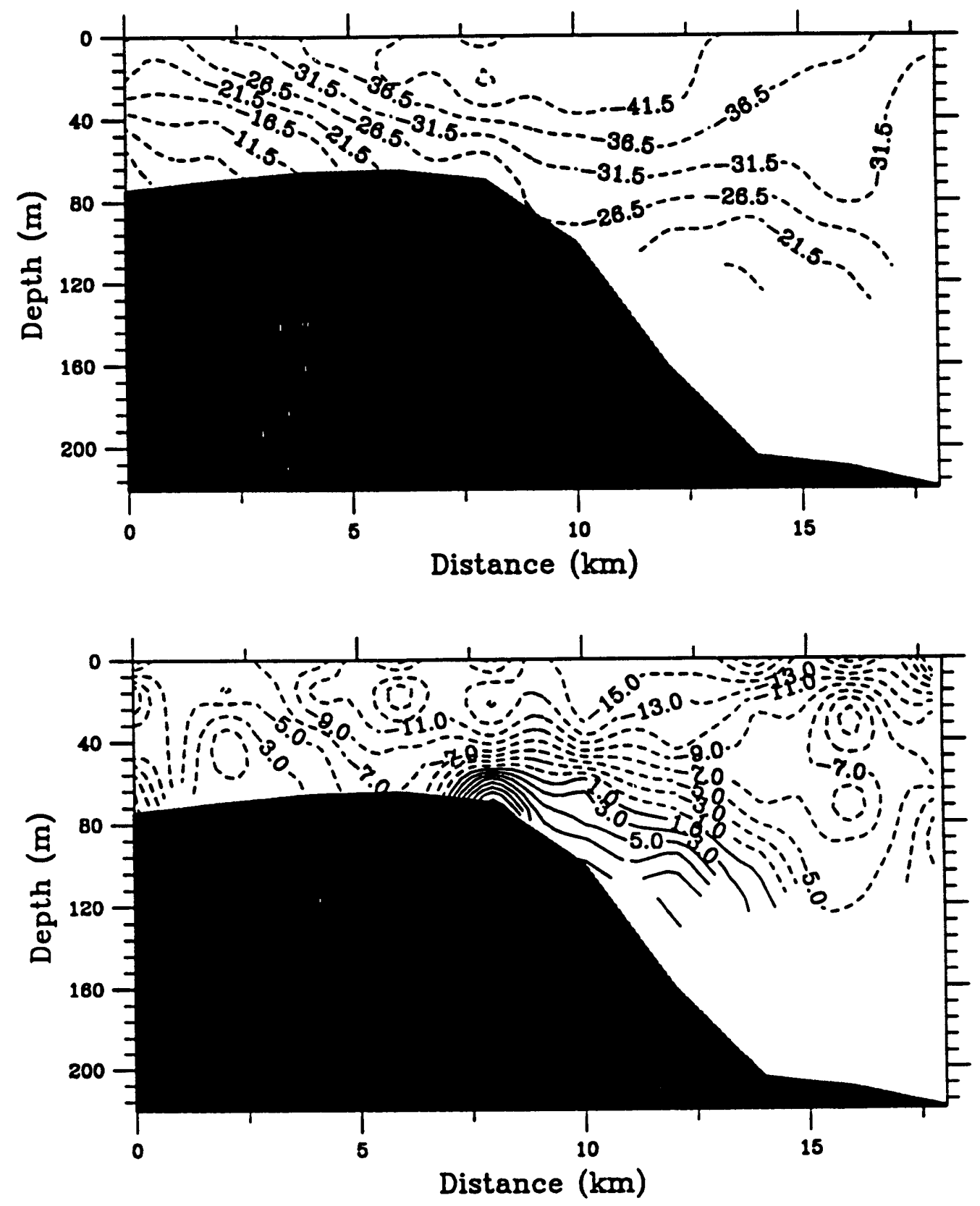

Figure 3.71: Distribution of along- (upper) and cross- (lower) bank residual currents across Loder et al.'s CTD/ADCP section in 1988 on the northern edge of Georges Bank. The negative along-bank residual current is northeastward, and negative cross-bank residual current is toward the bank. 
current is only about $90 \mathrm{~cm} / \mathrm{s}$, the structure of the observed along-bank flow is quite similar to our model results.

Lagrangian current measurements made with satellite-tracked drifters with drogues at 5 and $50 \mathrm{~m}$ during the 1989 SCOPEX survey (Limeburner and Beardsley, 1989) exhibited continuous trajectories of the drifters around Georges Bank, with a mean velocity of about $1-20 \mathrm{~cm} / \mathrm{s}$ on the southern flank and about $20-45 \mathrm{~cm} / \mathrm{s}$ on the northern flank. Theoretical study suggests that the tidally rectified Lagrangian current is generally less than the Eulerian current because of a Stokes velocity over the variable bottom slope (Loder, 1980; Zimmerman, 1978, 1980). With a barotropic harmonic truncation model, Loder (1980) estimated that the mean Lagrangian current is only about two-thirds of the mean Eulerian current. However, if the density front is included, the Lagrangian current may be the magnitude as the Eulerian current (Loder and Wright, 1985; see Figures 3.2 and 3.3). If this is true in our case, the model-predicted current is in reasonable agreement with the Lagrangian current on the northern flank of the Bank. The big difference between the observed and predicted along-bank residual currents on the southern flank is probably due to processes associated with the shelf break front, and surface and lateral buoyancy forcing.

\section{Comparison with Observations of the Cross-bank Mean Current}

Winter. Table 3.4 shows the comparison between the observed and predicted homogeneous and weak stratified cross-bank mean currents during winter at station $\mathrm{A}$ on

the southern flank and at stations M2 and M1 on the northern flank. The observations showed the opposite cross-bank mean circulation pattern on the sides of Georges Bank. On the southern flank, the water at station A tended to flow off-bank at a speed of -0.5 to $-1.0 \mathrm{~cm} / \mathrm{s}$ above $75 \mathrm{~m}$ and on-bank at the same speed near the bottom. On

the northern flank, however, the water at stations M2 and M1 tended to flow on-bank at a speed of -0.5 to $-1.3 \mathrm{~cm} / \mathrm{s}$ near the surface and off-bank at a speed of $2.0-4.8 \mathrm{~cm} / \mathrm{s}$ near the bottom. This circulation pattern is consistent with our model results for the weakly 
stratified case, which shows an off-bank flow of about $-0.29 \mathrm{~cm} / \mathrm{s}$ near the surface and an on-bank flow of $0.1-0.2 \mathrm{~cm} / \mathrm{s}$ near the bottom at station $A$, and an on-bank flow of about -0.1 to $-2.5 \mathrm{~cm} / \mathrm{s}$ near the surface and an off-bank flow of about $0.5-1.5 \mathrm{~cm} / \mathrm{s}$ near the bottom at stations M2 and M1.

\section{Table 3.4. Comparison Between the Observed and Predicted Cross-Bank Mean Currents During Winter}

\begin{tabular}{|c|c|c|c|c|c|}
\hline \multirow[b]{2}{*}{ Station } & \multirow[b]{2}{*}{$\begin{array}{c}\mathrm{H} \\
(\mathrm{m})\end{array}$} & \multirow[b]{2}{*}{$\begin{array}{c}Z_{m} \\
(\mathrm{~m})\end{array}$} & \multirow{2}{*}{$\begin{array}{c}\text { Observation } \\
\begin{array}{c}\bar{u}_{o} \\
(\mathrm{~cm} / \mathrm{s})\end{array}\end{array}$} & \multirow{2}{*}{$\begin{array}{c}\text { Homogeneous } \\
\text { Case }\end{array}$} & \multirow{2}{*}{$\begin{array}{c}\text { Winter Case } \\
\begin{array}{c}\bar{u}_{w} \\
(\mathrm{~cm} / \mathrm{s})\end{array}\end{array}$} \\
\hline & & & & & \\
\hline \multirow{3}{*}{ A } & \multirow{3}{*}{85} & 45 & $-0.5 \pm 3.0$ & -0.22 & -0.29 \\
\hline & & 75 & $-1.0 \pm 3.0$ & -0.07 & 0.05 \\
\hline & & 84 & $1.0 \pm 2.0$ & -0.02 & 0.15 \\
\hline \multirow{3}{*}{ M2 } & \multirow{3}{*}{85} & 10 & $-1.3 \pm 4.2$ & -0.36 & -2.48 \\
\hline & & 44 & none & -0.09 & 0.11 \\
\hline & & 77 & $4.8 \pm 3.2$ & -0.66 & 1.50 \\
\hline \multirow{3}{*}{ M1 } & \multirow{3}{*}{200} & 10 & $-0.5 \pm 6.5$ & 0.04 & -0.79 \\
\hline & & 77 & $-2.1 \pm 3.8$ & 0.01 & -0.14 \\
\hline & & 192 & $2.0 \pm 3.0$ & -0.07 & 0.55 \\
\hline
\end{tabular}

Note: $H$ is the water depth; $Z_{m}$ is the instrument depth; $\bar{u}_{o}$ is the observed cross-bank mean current plus the standard deviation; $\bar{u}_{b}$ and $\bar{u}_{w}$ are the model-predicted barotropic and weakly stratified mean currents.

Summer. Table 3.5 shows the comparison between the observed and predicted cross-bank mean currents during summer at station $A$ on the southern flank and at stations M2, M1, and MS2 on the northern flank. On the southern flank, the model predicts an onbank flow of $0.53 \mathrm{~cm} / \mathrm{s}$ near the surface and an off-bank flow of -0.03 to $-0.43 \mathrm{~cm} / \mathrm{s}$ near the bottom at station $\mathrm{A}$, in good agreement with observations considering the uncertainty of the measurements. On the northern flank, at station M2, the model shows an on-bank 
flow of $-9.0 \mathrm{~cm} / \mathrm{s}$ at a depth of $10 \mathrm{~m}$ near the surface and an off-bank flow of $13 \mathrm{~cm} / \mathrm{s}$ at a depth of $77 \mathrm{~m}$ near the bottom, in good comparison with observation in both magnitude

\section{Table 3.5. Comparison Between the Observed and Predicted Cross-Bank Mean Currents During Summer}

\begin{tabular}{|c|c|c|c|c|}
\hline \multirow[b]{2}{*}{ Station } & \multirow[b]{2}{*}{$\begin{array}{c}\mathrm{H} \\
(\mathrm{m})\end{array}$} & \multirow[b]{2}{*}{$\begin{array}{l}Z_{m} \\
(\mathrm{~m})\end{array}$} & Observation & \multirow{2}{*}{$\begin{array}{c}\text { Summer Case } \\
\bar{u}_{\mathrm{s}} \\
(\mathrm{cm} / \mathrm{s})\end{array}$} \\
\hline & & & $\begin{array}{c}\bar{u}_{o} \\
(\mathrm{~cm} / \mathrm{s})\end{array}$ & \\
\hline \multirow{4}{*}{ A } & \multirow{4}{*}{85} & 15 & $1 \pm 3-4 \pm 5$ & 0.53 \\
\hline & & 45 & $1 \pm 3-5 \pm 8$ & 0.16 \\
\hline & & 75 & $-1 \pm 1--2 \pm 3$ & -0.43 \\
\hline & & 84 & $1 \pm 1-2 \pm 2$ & -0.03 \\
\hline \multirow{3}{*}{ M2 } & \multirow{3}{*}{85} & 10 & $-3 \pm 5--5 \pm 6$ & -9.25 \\
\hline & & 44 & $-2 \pm 6--6 \pm 4$ & -1.44 \\
\hline & & 77 & $5 \pm 3--11 \pm 4$ & 12.85 \\
\hline \multirow{3}{*}{ M1 } & \multirow{3}{*}{200} & 10 & $-1 \pm 8--2 \pm 6$ & 0.34 \\
\hline & & 77 & $-1 \pm 3-2 \pm 3$ & 0.99 \\
\hline & & 192 & $-1 \pm 3-1 \pm 5$ & -4.88 \\
\hline \multirow{3}{*}{ MS2 } & \multirow{3}{*}{45} & 15 & $-1.4 \pm 1.1$ & -1.3 \\
\hline & & 30 & $2.2 \pm 0.8$ & 0.7 \\
\hline & & 40 & -1.3 & -0.1 \\
\hline
\end{tabular}

Note: $H$ is the water depth; $Z_{m}$ is the instrument depth; $\bar{u}_{0}$ is the observed current plus the standard deviation; and $\bar{u}_{s}$ is predicted current during summer.

and direction. A similar vertical structure was found by Loder et al. (1992) at the northern edge of Georges Bank, $60 \mathrm{~km}$ east to our numerical section. Both the moored current meter and ADCP data showed an on-bank flow in the upper $70 \mathrm{~m}$ and an off-bank return flow in the lower $80 \mathrm{~m}$ above the slope bottom with a maximum velocity of about $10 \mathrm{~cm} / \mathrm{s}$ near the surface and bottom (see Figure 3.71, lower panel). In addition, on the top of the Bank where the water is vertically well mixed, the model shows that the water tends to flow 
southward at all depths at a speed of about -0.1 to $-0.6 \mathrm{~cm} / \mathrm{s}$, consistent with the current meter data taken by Marsden (1986), who reported a southward flow at all depths of about -0.7 to $-3.2 \mathrm{~cm} / \mathrm{s}$ at the $40-\mathrm{m}$ isobath.

\section{Conclusion}

Unstratified and stratified tidal rectification over Georges Bank have been studied using a new two-dimensional version of the Blumberg and Mellor numerical circulation model. The model physics includes primitive equations in the horizontal direction, the hydrostatic approximation in the vertical, and Mellor and Yamada level $2 \frac{1}{2}$ turbulent closure model to simulate turbulent friction and mixing. The model domain consists of a vertical slice running roughly northwest across the center of Georges Bank, and the flow is forced by a simple elevation variation imposed at the southeast open boundary of the model with the period of the dominant $M_{2}$ tide $(12.42 \mathrm{hrs})$. In the unstratified case, the model predicts a topographically controlled clockwise residual circulation around the Bank, flowing northeastward as a strong surface intensified jet with a maximum speed of about $16 \mathrm{~cm} / \mathrm{s}$ along the northern flank, and then re-circulating as a relatively weak and broader southwestward flow with a maximum of about $3 \mathrm{~cm} / \mathrm{s}$ from the top of the Bank to the southern flank. The cross-bank circulation is characterized by a single circulation cell on the southern flank and by a double cell on the northern flank. As stratification is added, stratified tidal rectification and tidal mixing intensify along-and cross-bank residual currents and hence modify the vertical structure of the residual flow. During strong stratification in summer, the tidal front is located at about the 40-m isobath on the northern edge of the Bank and at the 50-60- $\mathrm{m}$ isobath on the southern flank, about 70-80 km on-bank from the shelf break. This results in an intensification of the clockwise along-bank residual current with a maximum of about $32 \mathrm{~cm} / \mathrm{s}$ on the northern flank and about $8 \mathrm{~cm} / \mathrm{s}$ on the southern flank. During weak stratification in winter, the position of the tidal front remains relatively fixed on the northern flank, however, it moves to the shelf break on the southern flank. The winter maximum of clockwise along-bank residual flow is about $26 \mathrm{~cm} / \mathrm{s}$ on the northern flank and 
about $6 \mathrm{~cm} / \mathrm{s}$ at the shelf break on the southern flank. The numerical model results are consistent with existing theories for stratified tidally rectified flow and observations of mean flow. The predicted along-bank residual current is relatively weaker than observed on the southern flank, suggesting that buoyancy driving associated with the shelf break front and the surface heat flux is also important in generating the residual flow on the southern flank.

Additional dynamic mechanisms become active in stratified tidal rectification. In the homogeneous case, the along-bank residual current is generated by the nonlinear transfer of momentum fluctuations from tidal currents to the mean flow, while the cross-bank mean current satisfies Ekman layer theory. When stratification is included, both stratified tidal rectification and tidal mixing play important roles in modifying the strength and distribution of residual currents over the Bank. On the southern flank, tidal mixing intensifies the along-bank residual flow along the axis of maximum baroclinic pressure gradient at the front and the top of the bottom boundary layer, and stratified tidal rectification plus tidal mixing cause the cross-bank multiple cell circulation pattern over the southern shelf. On the northern flank, both the stratified tidal rectification and tidal mixing are responsible for the intensification of along-bank residual current. Scaling analysis suggests that the stratified tidal rectification plays a more important role in increasing the maximum alongbank mean flow than the baroclinic density gradient due to the tidal mixing front on the northern flank. Ekman theory is no longer valid in the bottom boundary layer where the off-bank residual current is mainly driven by nonlinear advection and surface and baroclinic pressure gradients.

It should be pointed out here that our numerical experiments do not include the buoyancy-and wind-driven circulations caused by wintertime surface cooling and wind mixing, nor do they include the thermohaline circulations generated by either tidal mixing of the salinity field on either flank of the Bank or that associated with the shelf/slope front. Wintertime surface cooling can also mix water vertically over the top of the Bank, generating density fronts on both sides of the Bank. The study of the nonlinear interaction 
between tidal mixing and overturning on residual currents seems very interesting. Wind mixing can create a mixed layer near the surface over the slope and in the deep region, which may modify the spatial distribution and transport of along-bank mean flow. The seasonal variation of freshwater input around the Bank may cause a corresponding variation in the strength of the salinity fronts and thus influence the strength and distribution of residual currents over the Bank. In addition, since the Lagrangian currents are not described in the present work, we cannot provide a detailed time-dependent picture of mixing processes over Georges Bank. All these questions will be addressed with our numerical model in the future. 


\section{REFERENCES}

Arakawa, A., and V. R. Lamb, 1977. Computational design of the basic dynamical processes of the UCLA general circulation model. Methods in Computational Physics, Academic Press, 17, 174-265.

Baines, P. G., 1982. On internal tide generation models. Deep-Sea Research, 29, 307-338.

Batchelor, G. K., 1967. An Introduction to Fluid Dynamics. Cambridge University Press, $615 \mathrm{pp}$.

Beardsley, R. C., C. S. Chen, K. Wishner, and M. Macaulay, 1990. Spatial variability in stratification and zooplankton distribution near a feeding right whale in the Great South Channel of Gulf of Maine. Eos, Transactions of the American Geophysical Union, 71, 1990, abstract.

Beardsley, R. C., and D. B. Haidvogel, 1981. Model studies of the wind-driven transient circulation in the Middle Atlantic Bight: part 1, adiabatic boundary conditions. Journal of Physical Oceanography, 11, 355-375.

Bigelow, H. B., 1927. Physical oceanography of the Gulf of Maine. Bulletin of the U.S. Commerce Bureau, 40, 511-1027.

Blumberg, A. F., 1991. A primer for ECOM-SI. Technique Report of HydroQual, Inc., 66 pp.

Blumberg, A. F., and G. L. Mellor, 1987. A description of a three-dimensional coastal ocean circulation model. In: Three-Dimensional Coastal Ocean Model, N. S. Heaps, editor, Coastal and Estuarine Science, 4, 1-16.

Bowden, K. F., 1960. Circulation and mixing in the Mersey estuary. Commission of Surface Waters, Publication 51, International Association of Science and Hydrology, Gentbrugge, Belgium, 46-56. 
Brink, K. H., 1988. On the effect of bottom friction on internal waves. Continental Shelf Research, 8, 397-403.

Brooks, D. A., 1985. Vernal circulation in the Gulf of Maine. Journal of Geophysical Research, 90, 4687-4705.

Brooks, D. A., 1990. Currents at Lindenkohl Sill in the southern Gulf of Maine. Journal of Geophysical Research, 95, 22,173-22,192.

Brown, W. S., 1984. A comparison of Georges Bank, Gulf of Maine and New England Shelf tidal dynamics. Journal of Physical Oceanography, 14, 145-167.

Brown, W. S., and R. C. Beardsley, 1978. Winter circulation in the western Gulf of Maine. Part I: Cooling and water mass formation. Journal of Physical Oceanography, 8, 265-277.

Brown, W. S., and J. D. Irish, 1992. The annual evolution of geostrophic flow in the Gulf of Maine. Journal of Physical Oceanography, submitted.

Brownlee, K. A., 1965. Statistical theory and methodology in science and engineering. J. Wiley \& Sons, New York, 590 pp.

Butman, B., R. C. Beardsley, B. Magnell, D. Frye, J. A. Vermersch, R. Schlitz, R. Limeburner, W. R. Wright, and M. A Noble, 1982. Recent observations of the mean circulation on Georges Bank. Journal of Physical Oceanography, 12, 569-591.

Butman, B., J. W. Loder, and R. C. Beardsley, 1987. The seasonal mean circulation: observation and theory. In: Georges Bank, R. H. Backus, editor, The MIT Press, pp. 125-135.

Candela, J., R. C. Beardsley, and R. Limeburner, 1992. Separation of tidal and subtidal currents in ship-mounted Acoustic Doppler Current Profiler observations. Journal of Geophysical Research, 97, 769-788. 
Casulli, V., 1990. Semi-implicit finite-difference methods for the two-dimensional shallow water equations. Journal of Computational Physics, 86, 56-74.

Chao, S. Y., 1988. Wind-driven motion of estuarine plumes. Journal of Physical Oceanography, 18, 1144-1166.

Chapman, D. C., 1985. Numerical treatment of cross-shelf open boundaries in a barographic coastal model. Journal of Physical Oceanography, 15, 1060-1075.

Chelton, D. B., 1982. Statistical reliability and the seasonal cycle: comments on "Bottom pressure measurements across the Antarctic Circumpolar Current and their relation to the wind." Deep-Sea Research, 29, 1381-1388.

Chen, C., 1989. The structure of the Kuroshio west of Kyushu. M.S. Thesis, MIT/WHOI Joint Program, Woods Hole, Massachusetts, 137 pp.

Csanady, G. T., 1974. Barotropic currents over the continental shelf. Journal of Physical Oceanography, 4, 357-371.

Draper, N. R., and H. Smith, 1966. Applied Regression Analysis. J. Wiley \& Sons, New York, $407 \mathrm{pp}$.

Farmer, D. M., and J. D. Smith, 1980. Tidal interaction of stratified fluid with a sill in Knight Inlet. Deep-Sea Research, 27A, 239-254.

Flagg, C. N., 1977. The kinematics and dynamics of the New England continental shelf and shelf/slope front. Ph.D. Thesis, MIT/WHOI Joint Program. WHOI-77-67, 207 pp.

Flagg, C. N., 1987. Hydrographic structure and variability. In: Georges Bank, R. H. Backus, editor, The MIT Press, pp. 108-124.

Flagg, C. N., B. A. Magnell, D. Frye, J. J. Cura, S. E. McDowell, and R. I. Scaler, 1982. Interpretation of the physical oceanography of Georges Bank. Final report prepared for the New York OCS office, Bureau of Land Management, by EG\&G Environmental Consultants, Waltham, Massachusetts, 901 pp. 
Fofonoff, N. P., 1962. Physical properties of seawater. In: The Sea, 1, edited by N. M. Hill, Interscience, New York, pp. 3-30.

Fofonoff, N. P., and H. Bryden, 1975. Specific gravity and density of seawater at atmospheric pressure. Journal of Marine Research, 33 Supplement, 69-82.

Freeland, H. J., and M.G.G. Foreman, 1990. Tidal removal from ship-mounted Doppler measurements. Eos, Transactions of the American Geophysical Union, 71, 1990, abstract.

Garrett, C.J.R., 1972. Tidal resonance in the Bay of Fundy and Gulf of Maine. Nature, 238, 441-443.

Garrett, C.J.R., 1975. Tidal water movement. In: Effects of Energy-Related Activities on the Atlantic Continental Shelf, Bernard Manowitz, editor. Brookhaven National Laboratory, Upton, New York, pp. 48-58.

Garrett, C.J.R., 1990. The role of secondary circulation in boundary mixing. Journal of Geophysical Research, 95, 3181-3188.

Garrett, C.J.R., 1992. Marginal mixing theories. Atmosphere-Ocean, (R. W. Stewart Symposium Issue), submitted.

Garrett, C.J.R., J. R. Keeley, and D. A. Greenberg, 1978. Tidal mixing versus thermal stratification in the Bay of Fundy and Gulf of Maine. Atmosphere-Ocean, 16, 403-423.

Garrett, C.J.R, and J. W. Loder, 1981. Dynamical aspects of shallow sea fronts. Philosophical Transactions of the Royal Society, London, A 302,563-581.

Garrison, K., and W. S. Brown, 1989. Hydrographic survey in the Gulf of Maine (I: April 1987, II: July-August 1987). University of New Hampshire Technical Report UNHP-T/DR-SG-89-5.

Gawarkiewicz, G., and D. C. Chapman, 1992. The role of stratification in the formation and maintenance of shelfbreak fronts. Journal of Physical Oceanography, submitted. 
Geyer, R., and R. Signell, 1990. Tidal flow measurements around a headland with a shipboard Acoustic Doppler Current Profiler. Journal of Geophysical Research, 95, 3189-3197.

Greenberg, D. A., 1979. A numerical model investigation of tidal phenomena in the Bay of Fundy and Gulf of Maine. Marine Geodesy, 2, 161-187.

Greenberg, D. A., 1983. Modeling the mean barotropic circulation in the Bay of Fundy and Gulf of Maine. Journal of Physical Oceanography, 13, 886-904.

Haidvogel, D. B., J. H. Wilkin, and R. Young, 1991. A semi-spectral primitive equation ocean circulation model using vertical sigma and orthogonal curvilinear horizontal coordinates. Journal of Computational Physics, 94, 151-185.

Haney, R. L., 1991. On the pressure gradient force over steep topography in sigmacoordinate ocean models. Journal of Physical Oceanography, 21, 610-619.

Hearn, C. J., 1984. On the value of the mixing efficiency in the Simpson-Hunter $h / u^{3}$ criterion. Deutsche Hydrographisches Zeitschrift, 38, 133-145.

Hibiya, T., 1986. Generation mechanism of internal waves by tidal flow over a sill. Journal of Geophysical Research, 91, 7697-7708.

Hogg, N. G., 1983. Hydraulic control and flow separation in a multi-layered fluid with application to the Vema Channel. Journal of Physical Oceanography, 13, 695-708.

Holloway, P. E., 1983. Internal tides on the Australian North West Shelf: A preliminary investigation. Journal of Physical Oceanography, 13, 1357-1370.

Holloway, P. E., 1984. On the semi-diurnal internal tide at a shelfbreak region on the Australian North West Shelf. Journal of Physical Oceanography, 14, 1787-1799.

Holloway, P. E., 1985. A comparison of semi-diurnal internal tides from different bathymetric locations on the Australian North West Shelf. Journal of Physical Oceanography, $15,240-251$. 
Hopkins, T. S., and N. Garfield III, 1979. Gulf of Maine Intermediate Water. Journal of Marine Research, 37, 103-139.

Hopkins, T. S., and N. Garfield III, 1981. Physical origins of Georges Bank water. Journal of Marine Research, 39, 465-500.

Huthnance, J. M., 1973. Tidal current asymmetries over the Norfolk Sandbank. Estuarine and Coastal Marine Science, 1, 89-99.

Huthnance, J. M., 1981. On mass transports generated by tides and long waves. Journal of Fluid Mechanics, 102, 367-387.

Jonsson, I. G., and N. A. Carlsen, 1976. Experimental and theoretical investigations in an oscillatory turbulent boundary. Journal of Hydrological Research, 14, 45-60.

Joyce, T. M. 1989. On in-situ "calibration of shipboard ADCP's." Journal of Atmospheric and Oceanic Technology, 6, 169-172.

Large, W. S., and S. Pond, 1981. Open ocean momentum flux measurements in moderate to strong winds. Journal of Physical Oceanography, 11, 324-336.

Lavelle, J. W., and H. O. Mofjeld, 1983. Effects of time varying viscosity on oscillatory turbulent channel flow. Journal of Geophysical Research, 88, 7607-7616.

Leblond, P. H., and L. A. Mysak, 1978. Waves in the Ocean. Elsevier Scientific Publishing Company, New York, 602 pp.

Lee, C. Y., and R. C. Beardsley, 1974. The generation of long nonlinear internal waves in a weekly stratified shear flow. Journal of Geophysical Research, 79, 453-462.

Limeburner, R., and R. C. Beardsley, 1982. The seasonal hydrographic and circulation over Nantucket Shoals. Journal of Marine Research, 40 Supplement, 371-406. 
Limeburner, R., and R. C. Beardsley, 1989. CTD observations off the New England coast during the South Channel Ocean Productivity EXperiment, SCOPEX, June 1989. Woods Hole Oceanographic Institution Technical Report WHOI-89-52, 252 pp.

Limeburner, R., and R. C. Beardsley, 1989. Lagrangian circulation in the Great South Channel and on Georges Bank during summer. In: Proceedings of Third Georges Bank Research Workshop, Bedford Institute of Oceanography, $29 \mathrm{pp}$.

Loder, J. W., 1980. Topographic rectification of tidal currents on the sides of Georges Bank. Journal of Physical Oceanography, 10, 1399-1416.

Loder, J. W., D., Brickman, and P. W. Horne, 1992. Detailed structure of currents and hydrography on the northern side of Georges Bank. Journal of Geophysical Research, submitted.

Loder, J. W., and D. A. Greenberg, 1986. Predicted positions of tidal fronts in the Gulf of Maine region. Continental Shelf Research, 6, 397-414.

Loder, J. W., and E. P. W. Horne, 1989. Internal waves on the northern side of Georges Bank. In: Proceedings of Third Georges Bank Research Workshop at Bedford Institute of Oceanography. $29 \mathrm{pp}$.

Loder, J. W., and D. G. Wright, 1985. Tidal rectification and front circulation on the sides of Georges Bank. Journal of Marine Research, 43, 581-604.

Lwiza, K. M. M., and D. G. Bowers, 1990. Measurements of tidal and residual currents at a front in the North Sea using an acoustic Doppler current profiler. Eos, Transactions of the American Geophysical Union, 71, 1990, abstract.

Maas, L.R.M., J.T.F. Zimmerman, and N. M. Temme, 1987. On the exact shape of horizontal profile of a topographically rectified tidal flow. Geophysical and Astrophysical Fluid Dynamics, 38, 105-129. 
Maas L.R.M., and J.T.F. Zimmerman, 1989a. Tide-topography interaction in a stratified shelf sea, I: Basic equations for quasi-nonlinear internal tides. Geophysical and Astrophysical Fluid Dynamics, 45, 1-35.

Maas, L.R.M., and J.T.F. Zimmerman, 1989b. Tide-topography interaction in a stratified shelf sea, II: Bottom trapped internal tides and baroclinic residual currents. Geophysical and Astrophysical Fluid Dynamics, 45, 37-69.

Marsden, R. F., 1986. The internal tide on Georges Bank. Journal of Marine Research, $44,35-50$.

Maxworthy, T., 1979. A note on the internal solitary waves produced by tidal flow over a three-dimensional ridge. Journal of Geophysical Research, 84, 338-346.

Mellor, G. L., and T. Yamada, 1974. A hierarchy of turbulence closure models for planetary boundary layers. Journal of the Atmospheric Sciences, 33, 1791-1896.

Mellor G. L., and T. Yamada, 1982. Development of a turbulence closure model for geophysical fluid problem. Reviews of Geophysical and Space Physics, 20, 851-875.

Moody, J. A., B. Butman, R.C. Beardsley, W. S. Brown, P. Daifuku, J. D. Irish, D. A. Mayer, H. O. Mofjeld, B. Petrie, S. Ramp, P. Smith, and W. R. Wright, 1984. Atlas of tidal elevation and current observations on the northeast American continental shelf and slope. U.S. Geological Survey Bulletin 1611, 122 pp.

Mountain, D. G., and P. F. Jessen, 1987. Bottom waters of the Gulf of Maine. Journal of Marine Research, 45, 319-345.

Mountain, D. G., and J. P. Manning, 1991. Seasonal and interannual variability in the properties of the surface water of the Gulf of Maine, in preparation.

Olson, D. B., and R. H. Backus, 1985. The concentrating of organisms at fronts; A cold-water fish and a warm-core Gulf Stream ring. Journal of Marine Research, 43, 113-137. 
Petrie, B., 1974. $M_{2}$ surface and internal tides on the Scotian shelf and slope. Journal of Marine Research, 33, 303-323.

Phillips, O. M., 1970. On flows induced by diffusion in a stably stratified fluid. Deep-Sea Research, 17, 435-443.

Pratt, L. J., 1986. Hydraulic control of sill flow with bottom friction. Journal of Physical Oceanography, 16, 1970-1980.

Pratt, L. J., 1991. Hydraulics of rotating stratified and sill flow. Annual Reviews of Fluid Mechanics, 23, 81-106.

Ramp, S., R. Schlitz, and W. R. Wright, 1985. The deep flow through Northeast Channel, Gulf of Maine. Journal of Physical Oceanography, 15, 1790-1808.

Roache, D. J., 1972. Computational Fluid Dynamics, Hermosa Publishers, Albuquerque, New Mexico, pp. 345-349.

Robinson, I. S., 1981. Tidal vorticity and residual circulation. Deep-Sea Research, 28A, 195-212.

Rosenfeld, L. K., 1987. Tidal band current variability over the Northern California Continental Shelf. Ph.D Thesis, MIT/WHOI Joint Program, WHOI-87-11, 237 pp.

Saunders, P. M., 1977. Wind stress on the ocean over the eastern continental shelf of North America. Journal of Physical Oceanography, 7, 555-566.

Simpson, J. H., and J. R. Hunter, 1974. Fronts in the Irish Sea. Nature, 250, 404-406.

Smith, P. C., 1983. The mean seasonal circulation off southwest Nova Scotia. Journal of Physical Oceanography, 13, 1034-1054.

Stern, M., J. A. Whitehead, and B. L. Hua, 1982. The intrusion of a density current along the coast of a rotating fluid. Journal of Fluid Mechanics, 123, 237-265. 
Tee, K. T., 1979. The structure of three-dimensional tide-generating currents. Part I: Oscillating currents. Journal of Physical Oceanography, 9, 930-944.

Tee, K. T., 1980. The structure of three dimensional tide-generating currents. Part II: Residual currents. Journal of Physical Oceanography, 10, 2035-2057.

Tee, K. T., 1985. Depth-dependent studies of tidally induced residual current on the sides of Georges Bank. Journal of Physical Oceanography, 15, 1818-1846.

Tee, K. T., 1987. Simple models to simulate three-dimensional tidal and residual currents. In: Three-Dimensional Coastal Ocean Model, N. S. Heaps, editor, Coastal and Estuarine Science, 4, 125-147.

Trites, R., 1978. Marine Ecology Laboratory, Bedford Institute of Oceanography, Cruise Report 78-031, 12 pp.

Uchupi, E., and J. A. Austin, 1987. Morphology. In: Georges Bank, R. H. Backus, editor, The MIT Press, pp. 25-29.

Vermersch, J. A., R. C. Beardsley, and W. S. Brown, 1979. Winter circulation in the western Gulf of Maine, Part 2: Current and pressure observation. Journal of Physical Oceanography, 9, 768-784.

Weatherly, G. L., and P. J. Martin, 1978. On the structure and dynamics of the oceanic bottom boundary layer. Journal of Physical Oceanography, 8, 557-570.

Whitehead, J. A., A. Leetmaa, and R. A. Knox, 1974. Rotating hydraulics of strait and sill flows. Geophysical Fluid Dynamics, 6, 101-125.

Winant, C. D., 1979. Coastal current observations. Reviews of Geophysics and Space Physics, 17, 89-98.

Winant, C. D., and J. R. Olson, 1976. The vertical structure of coastal currents. Deep-Sea Research, 23, 925-936. 
Wishner, K., J. R. Schoenherr, and C. Gelfman, 1990. The spatial and temporal variability of zooplankton distributions and vertical migration in a right whale feeding area. Eos, Transactions of the American Geophysical Union, 71, 1990, abstract.

Wright, D. G., D. A. Greenberg, and J. W. Loder, 1986. The steady-state barotropic response of the Gulf of Maine and adjacent regions to surface wind stress. Journal of Physical Oceanography, 16, 949-966.

Wright, D. G., and J. W. Loder, 1985. A depth-dependent study of the topographic rectification of tidal currents. Geophysical and Astrophysical Fluid Dynamics, 31, 169-220.

Wunsch, C., 1969. Progressive internal waves on slopes. Journal of Fluid Mechanics, 35, 131-144.

Wunsch, C., 1970. On oceanic boundary mixing. Deep-Sea Research, 17, 293-301.

Wunsch, C., 1975. Internal tides in the ocean. Reviews of Geophysics and Space Physics, 13, $167-182$.

Young, W. R., 1983. Topographic rectification of tidal currents. Journal of Physical Oceanography, 13, 716-721.

Zimmerman, J.T.F., 1978. Topographic generation of residual circulation by oscillatory tidal currents. Geophysical and Astrophysical Fluid Dynamics, 11, 35-47.

Zimmerman, J.T.F., 1980. Vorticity transfer by tidal currents over an irregular topography. Journal of Marine Research, 38, 601-630.

Zimmerman, J.T.F., 1981. Topographical rectification: a comment on spectral representation. Journal of Physical Oceanography, 11, 1037-1039. 


\section{Appendix A: Discussion on Vertical Resolution and $\sigma$-Coordinate Errors}

Two main issues need to be considered in choosing the vertical resolution for the numerical computation: (1) resolving the bottom boundary layer and (2) minimizing the computational error over steep bottom topography due to the $\sigma$-coordinate transformation. To answer the first question, we analytically solved a linear Ekman layer model and compared it with the numerical results for different vertical resolutions. To estimate the $\sigma$-coordinate error, we used a simple scaling method introduced by Haney (1991) in which the relationship of pressure gradient error to stratification, the slope of bottom topography, and vertical resolution can be derived.

Consider the linear, barotropic, propagating $M_{2}$ tidal wave in the two-dimensional $(x-z$ plane $)$ interior ocean given by

$$
\begin{aligned}
u_{i} & =A_{\circ} \sqrt{\frac{g}{D}} \frac{\omega}{\sqrt{\omega^{2}-f^{2}}} \cos (\alpha x-\omega t), \\
v_{i} & =A_{\circ} \sqrt{\frac{g}{D}} \frac{f}{\sqrt{\omega^{2}-f^{2}}} \sin (\alpha x-\omega t), \\
\zeta_{i} & =A_{o} \cos (\alpha x-\omega t),
\end{aligned}
$$

where $\alpha=\sqrt{\left(\omega^{2}-f^{2}\right) / g D}$. The no-slip bottom boundary condition can induce a timedependent Ekman layer above the flat bottom which satisfies

$$
\begin{aligned}
& \frac{\partial u_{e}}{\partial t}-f v_{e}=\frac{\partial}{\partial z}\left(K_{m} \frac{\partial u_{e}}{\partial z}\right) \\
& \frac{\partial v_{e}}{\partial t}+f u_{e}=\frac{\partial}{\partial z}\left(K_{m} \frac{\partial v_{e}}{\partial z}\right)
\end{aligned}
$$

where

$$
\begin{gathered}
\frac{\partial u_{e}}{\partial z}=\frac{\partial v_{e}}{\partial z}=0, \quad \text { at } z=0 \\
u_{e}=-u_{i}, v_{e}=-v_{i}, \quad \text { at } z=-D .
\end{gathered}
$$


The solutions for the Ekman velocities are given by

$$
\begin{aligned}
& u_{e}=-\frac{1}{2}\left\{\left(\gamma_{1} \frac{G_{1}}{C_{1}}+\gamma_{2} \frac{G_{3}}{C_{2}}\right) \cos (\alpha x-\omega t)+\left(\gamma_{1} \frac{G_{2}}{C_{1}}+\gamma_{2} \frac{G_{4}}{C_{2}}\right) \sin (\alpha x-\omega t)\right\} \\
& v_{e}=\frac{1}{2}\left\{\left(\gamma_{1} \frac{G_{1}}{C_{1}}-\gamma_{2} \frac{G_{3}}{C_{2}}\right) \sin (\alpha x-\omega t)-\left(\gamma_{1} \frac{G_{2}}{C_{1}}-\gamma_{2} \frac{G_{4}}{C_{2}}\right) \cos (\alpha x-\omega t)\right\}
\end{aligned}
$$

where

$$
\begin{aligned}
& C_{1}=\cosh \frac{2 D}{\delta E_{1}}+\cos \frac{2 D}{\delta E_{1}} \\
& C_{2}=\cosh \frac{2 D}{\delta E_{2}}+\cos \frac{2 D}{\delta E_{2}} \\
& G_{1}=\cosh \frac{z+D}{\delta E_{1}} \cos \frac{z-D}{\delta E_{1}}+\cosh \frac{z-D}{\delta E_{1}} \cos \frac{z+D}{\delta E_{1}} \\
& G_{2}=\sinh \frac{z+D}{\delta E_{1}} \sin \frac{z-D}{\delta E_{1}}+\sinh \frac{z-D}{\delta E_{1}} \sin \frac{z+D}{\delta E_{1}} \\
& G_{3}=\cosh \frac{z+D}{\delta E_{2}} \cos \frac{z-D}{\delta E_{2}}+\cosh \frac{z-D}{\delta E_{2}} \cos \frac{z+D}{\delta E_{2}} \\
& G_{4}=\sinh \frac{z+D}{\delta E_{2}} \sin \frac{z-D}{\delta E_{2}}+\sinh \frac{z-D}{\delta E_{2}} \sin \frac{z+D}{\delta E_{2}}
\end{aligned}
$$

and

$$
\begin{aligned}
\gamma_{1} & =A_{o} \sqrt{\frac{g}{D}} \frac{\omega-f}{\sqrt{\omega^{2}-f^{2}}}, \\
\gamma_{2} & =A_{o} \sqrt{\frac{g}{G}} \frac{\omega+f}{\sqrt{\omega^{2}-f^{2}}}, \\
\alpha & =\sqrt{\frac{\omega^{2}-f^{2}}{g D}} .
\end{aligned}
$$

The Ekman transports are equal to

$$
\begin{aligned}
& E_{u_{e}}=-\frac{1}{4}\left\{\left(A_{1}+A_{2}\right) \cos (\alpha x-\omega t)+\left(B_{1}+B_{2}\right) \sin (\alpha x-\omega t)\right\}, \\
& E_{v_{e}}=\frac{1}{4}\left\{\left(A_{1}-A_{2}\right) \sin (\alpha x-\omega t)-\left(B_{1}-B_{2}\right) \cos (\alpha x-\omega t)\right\},
\end{aligned}
$$


where

$$
\begin{aligned}
& A_{1}=\frac{\gamma_{1}}{C_{1}} \delta E_{1}\left(\sin \frac{2 D}{\delta E_{1}}+\sinh \frac{2 D}{\delta E_{1}}\right), \\
& A_{2}=\frac{\gamma_{2}}{C_{2}} \delta E_{2}\left(\sin \frac{2 D}{\delta E_{2}}+\sinh \frac{2 D}{\delta E_{2}}\right), \\
& B_{1}=\frac{\gamma_{1}}{C_{1}} \delta E_{1}\left(\sin \frac{2 D}{\delta E_{1}}-\sinh \frac{2 D}{\delta E_{1}}\right), \\
& B_{2}=\frac{\gamma_{2}}{C_{2}} \delta E_{2}\left(\sin \frac{2 D}{\delta E_{2}}-\sinh \frac{2 D}{\delta E_{2}}\right) .
\end{aligned}
$$

Comparisons between analytical and numerical solutions for Ekman velocities and transports are shown in Figures A.1 and A.2 for the case of $K_{m}=0.01 \mathrm{~m}^{2} / \mathrm{s}, \mathrm{D}=100 \mathrm{~m}$ and $A_{o}=0.5 \mathrm{~m}$. Numerical results for Ekman transport tend to match analytical solutions as the vertical resolution increases from $\Delta \sigma=0.1(\Delta z=10 \mathrm{~m})$ to $\Delta \sigma=0.033(\Delta z=$ $3.1 \mathrm{~m}$ ), even though there is no big change in the vertical profiles of Ekman velocities. The fact that the vertical resolution seriously influences the Ekman transport rather than the vertical structure of the flow implies that a relatively high vertical resolution is required to resolve numerically the bottom boundary layer. The 31 vertical points needed to resolve the Ekman layer with a thickness of $40 \mathrm{~m}$ in our test case suggests that a rough estimate can be made for vertical resolution if the thickness of the boundary layer is known.

In the $\sigma$-coordinate system, the baroclinic pressure gradient over the slope in the cross-isobath direction is written by

$$
P_{x}=\frac{g}{\rho}\left(\frac{\partial D}{\partial x} \int_{\sigma}^{0} \sigma \frac{\partial \rho^{\prime}}{\partial \sigma} d \sigma-D \frac{\partial}{\partial x} \int_{\sigma}^{0} \rho^{\prime} d \sigma\right)
$$

where $P_{x}$ is defined as $-\frac{1}{\rho_{o}} \frac{\partial P}{\partial x}, \rho^{\prime}=\rho-\bar{\rho}(x, \sigma)$, and $\bar{\rho}(x, \sigma)$ is the horizontally uniform reference state density in the $\sigma$-coordinate, which is transformed from $\bar{\rho}(z)=$ $1 / L \int_{0}^{L} \rho\left(x, z, t_{0}\right) d x, L$ is the length of computational domain, the total depth $D=H(x)+$ $\zeta$, and $\rho_{o}$ the background density. The difference scheme for calculating the baroclinic 

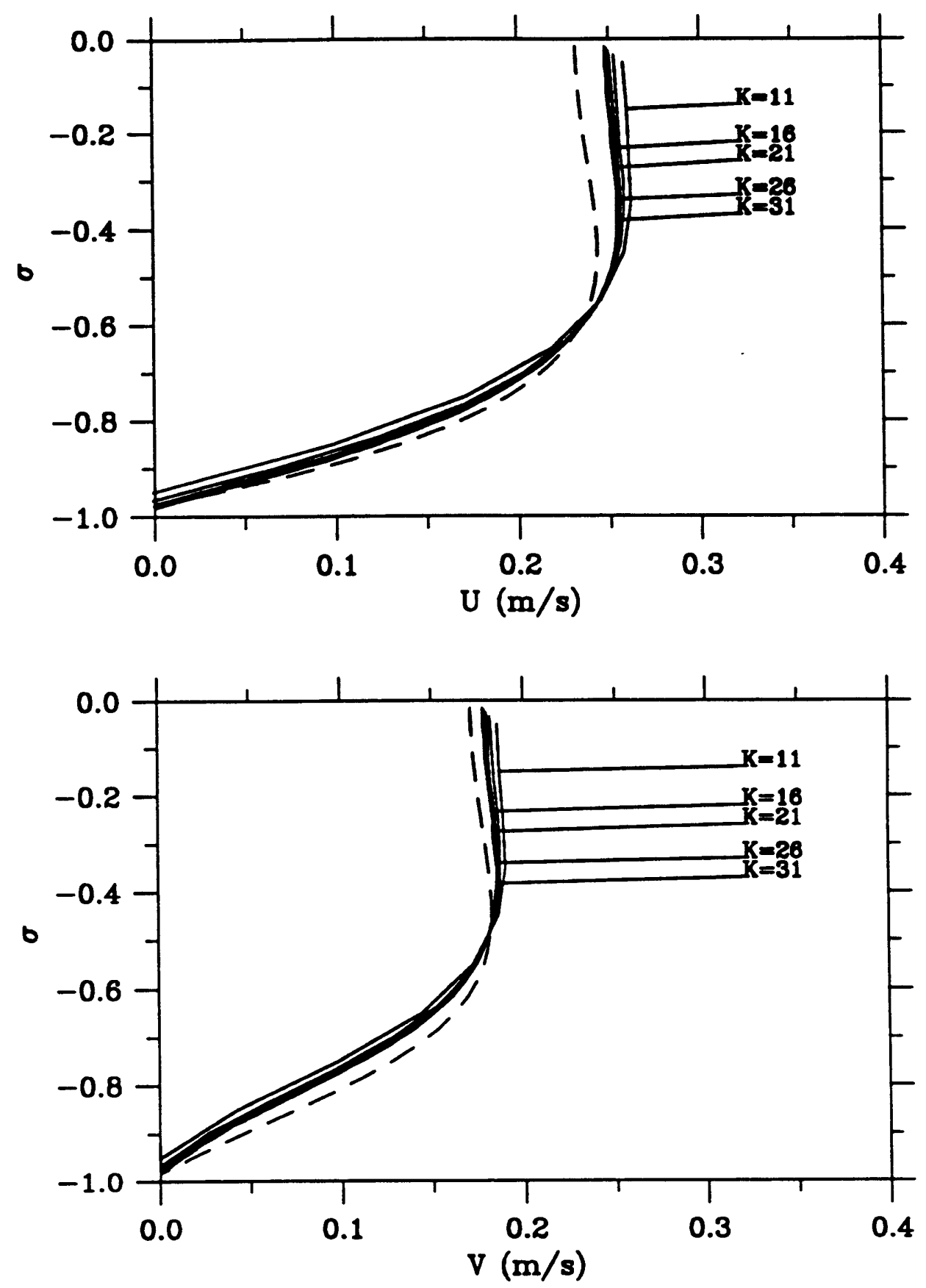

Figure A.1: Comparison between the analytical (dashed line) and numerical (solid line) Ekman velocities $u_{e}$ (upper) and $v_{e}$ (lower) for the case of $K_{m}=0.01 \mathrm{~m}^{2} / \mathrm{s}, \mathrm{D}=100 \mathrm{~m}$ and $A_{o}=0.5 \mathrm{~m}$. Symbol $K$ is the number of total vertical points in the $\sigma$ coordinate. 

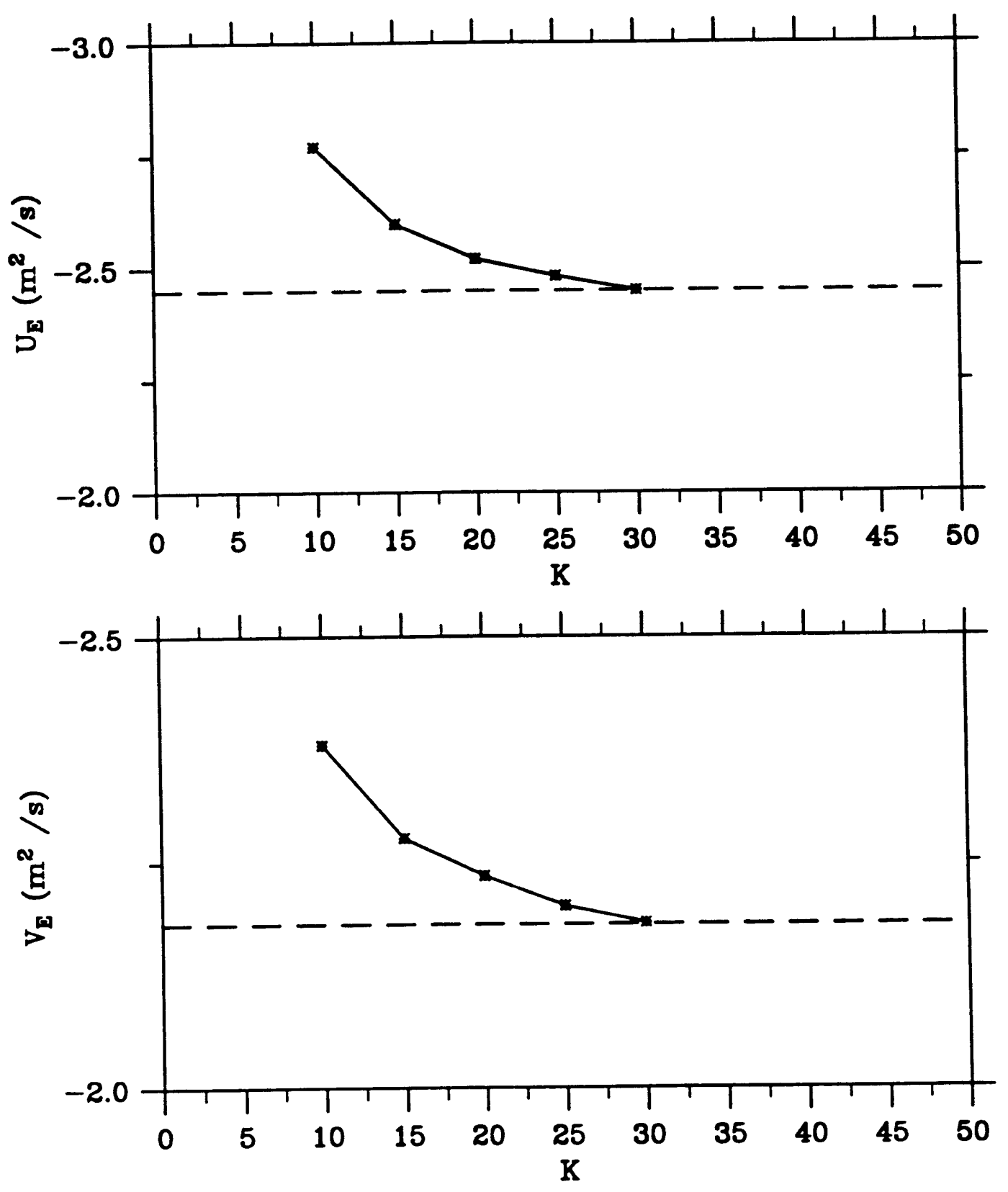

Figure A.2: Comparison between the analytical (dashed line) and numerical (solid line) Ekman transports in the $x$ (upper) and $y$ (lower) directions for the case of $K_{m}=0.01 \mathrm{~m}^{2} / \mathrm{s}, \mathrm{D}=100 \mathrm{~m}$ and $A_{o}=0.5 \mathrm{~m}$. Symbol $K$ is the number of total vertical points in the $\sigma$ coordinate. 
pressure gradient in the Blumberg and Mellor model is given by

$$
P_{x}=-\frac{g}{\rho_{o} \Delta x}\left(\sum \sigma \Delta_{x} D \Delta_{\sigma} \rho+D \Delta_{x} \rho \Delta \sigma\right)
$$

where $\Delta_{x}$ and $\Delta_{\sigma}$ are difference expressions in $x$ and $\sigma$ directions, respectively. The detailed expression of the difference equation in the Blumberg and Mellor model is

$$
\begin{aligned}
P_{x}(k)= & P_{x}(k-1)+\frac{g}{\rho_{o} \Delta x}\left\{\frac{1}{8}(\Delta \sigma(k)+\Delta \sigma(k-1))\right. \\
& \left(D_{i}+D_{i-1}\right)\left(\rho_{i}^{\prime}(k)-\rho_{i-1}^{\prime}(k)+\rho_{i}^{\prime}(k-1)-\rho_{i-1}^{\prime}(k-1)\right) \\
+ & \left.\frac{\sigma(k)}{2}\left(D_{i}-D_{i-1}\right)\left(\rho_{i}^{\prime}(k)+\rho_{i-1}^{\prime}(k)-\rho_{i}^{\prime}(k-1)-\rho_{i-1}^{\prime}(k-1)\right)\right\}
\end{aligned}
$$

where $i$ and $k$ are grid point indexes in $x$ and $\sigma$ directions, respectively.

For uniform spacing in $\sigma$,

$$
\Delta \sigma(k)=\Delta \sigma(k-1)=\cdots=\Delta \sigma(1)=\Delta \sigma
$$

so

$$
\begin{aligned}
P_{x}(k)= & P_{x}(k-1)+\frac{g}{\rho_{o} \Delta x} \\
& \left\{\frac{\Delta \sigma}{4}\left(D_{i}+D_{i-1}\right)\left(\rho_{i}^{\prime}(k)-\rho_{i-1}^{\prime}(k)+\rho_{i}^{\prime}(k-1)-\rho_{i-1}^{\prime}(k-1)\right)\right. \\
& \left.+\frac{\sigma(k)}{2}\left(D_{i}-D_{i-1}\right)\left(\rho_{i}^{\prime}(k)+\rho_{i-1}^{\prime}(k)-\rho_{i}^{\prime}(k-1)-\rho_{i-1}^{\prime}(k-1)\right)\right\}
\end{aligned}
$$

For simplification, we study next a case in which the density is linearly distributed in the vertical at $t=0$ and the bottom slope is constant (see Figure A.3), i.e.,

$$
\rho_{t=0}=\rho_{o}-\frac{\rho_{b}-\rho_{o}}{H_{o}} \text { and } D=H_{o}-\alpha_{1} x
$$

where $\alpha=\frac{H_{0}-H_{s}}{L_{s}}$. If no forcing is imposed on the system and no diffusion exists, numerical results for $P_{x}$ vanish at all times since the perturbation density is equal to zero. 
This suggests that the $\sigma$-coordinate transformation will not numerically generate extra motion over steep topography in an inviscous ocean at rest if only perturbation density relative to horizontal uniform state density is used to calculate the baroclinic pressure gradient. However, once diffusion occurs or an initial force is imposed on the system,

$$
\rho^{\prime} \neq 0
$$

which makes the fluid start to move by a non-zero pressure gradient (Chapman, personal communication). In general, the perturbation density is a function of $x, z$ and $t$ in our two-dimensional model that we can express as

$$
\rho^{\prime}=\vec{\rho}^{\prime}(z)+\rho^{\prime \prime}(x, z, t)
$$

where the average of $\rho^{\prime \prime}(x, z, t)$ in the $x$ direction is equal to zero. By the definition of pressure gradient, $\bar{\rho}^{\prime}(z)$ should not produce a horizontal pressure gradient since it is independent of $x$. However, the $\sigma$-coordinate transformation may numerically generate an extra horizontal pressure gradient error due to non-zero $\bar{\rho}^{\prime}(z)$ because of the finite-difference scheme. Using Haney's analysis method, we assume that

$$
\bar{\rho}^{\prime}(z)=\rho_{o}^{\prime}+\widehat{\beta} z \text { and } z=H(x) \sigma
$$

where $\hat{\beta}=-\partial \rho^{\prime} / \partial z$ is the vertical gradient of perturbation density. Substituting this into the analytical formula for the baroclinic pressure gradient, we get

$$
\begin{aligned}
\bar{P}_{x}^{\prime} & =\frac{g}{\rho_{o}}\left\{\frac{\partial H(x)}{\partial x} \int_{\sigma}^{0} \sigma \hat{\beta} H(x) d \sigma-H(x) \frac{\partial}{\partial x} \int_{\sigma}^{0}\left(\rho_{o}^{\prime}+\hat{\beta} \sigma H(x)\right) d \sigma\right\} \\
& =\frac{g}{\rho_{o}}\left(\frac{\partial H(x)}{\partial x} \hat{\beta} H(x)-\frac{\partial H(x)}{\partial x} \hat{\beta} H(x)\right) \int_{\sigma}^{0} \sigma d \sigma=0,
\end{aligned}
$$

but the finite-difference scheme results in

$$
\bar{P}_{x}^{\prime}(k)=-\frac{\hat{\beta} g \alpha_{1} k}{\rho_{o}}\left(D_{i}+D_{i-1}\right) \Delta \sigma^{2} / 4
$$


This indicates that the error in the baroclinic pressure gradient $\bar{P}_{x}^{\prime}(k)$ always exists in the Efficient Coastal Ocean Model 3-D Semi-Implicit (ECOM3D-SI) model if it is used for a stratified fluid over a sloping bottom. The error is directly proportional to $\alpha_{1}, \Delta \sigma$, $D_{i}, \mathrm{k}$ and $\hat{\beta}$. For given $\alpha_{1}, \Delta \sigma, D_{i}$ and $\hat{\beta}$, maximum error occurs at the bottom where $k=K B-1$ and $(K B-1) \Delta \sigma=1$ ( $K B$ is the total number of vertical grid points), that is

$$
\left(\bar{P}_{x}^{\prime}\right)_{\max }=-N_{o}^{\prime 2} \alpha_{1}\left(D_{i}+D_{i-1}\right) \Delta \sigma / 4
$$

where $N_{o}^{\prime 2}=g \hat{\beta} / \rho$. This pressure gradient error would result in an along-isobath error current over the slope, the magnitude of which can be estimated by the geostrophic balance as

$$
\Delta V_{\max } \sim-\frac{1}{f}\left(P_{x}^{\prime}\right)_{\max }
$$

In our numerical experiment for the case of strong stratification, $N_{o}^{\prime 2} \sim 10^{-5} \mathrm{~s}^{-2}, D_{i}+$ $D_{i-1} \sim 600 \mathrm{~m}$ (a maximum depth), and $f=1.03 \times 10^{-4}$ at $45^{\circ} \mathrm{N}$. The topographic scale $\ell_{t}$ varies in the range $50-12.5-\mathrm{km}$, which corresponds to a bottom slope $\alpha_{1}=0.004-0.016$ for $H_{s}=100 \mathrm{~m}$ and $0.005-0.02$ for $H_{s}=50 \mathrm{~m}$. For example, the computational errors of pressure gradient and along-isobath geostrophic velocity due to vertical resolution are estimated in Table A.1 for the slope $\alpha_{1}=0.016$, showing the decrease in computational error as the vertical resolution is increased.

Table A.1

\begin{tabular}{|c|c|c|c|c|c|}
\hline $\mathrm{KB}$ & 11 & 16 & 21 & 26 & 31 \\
\hline$\Delta \sigma$ & 0.10 & 0.07 & 0.05 & 0.04 & 0.03 \\
\hline$\left({\overline{P^{\prime}}}_{x}\right)_{\max }$ & $2.4 \times 10^{-4}$ & $1.6 \times 10^{-4}$ & $1.2 \times 10^{-4}$ & $1.0 \times 10^{-4}$ & $0.8 \times 10^{-4}$ \\
\hline$\Delta V_{\max }$ & 2.3 & 1.5 & 1.2 & 0.9 & 0.8 \\
\hline
\end{tabular}



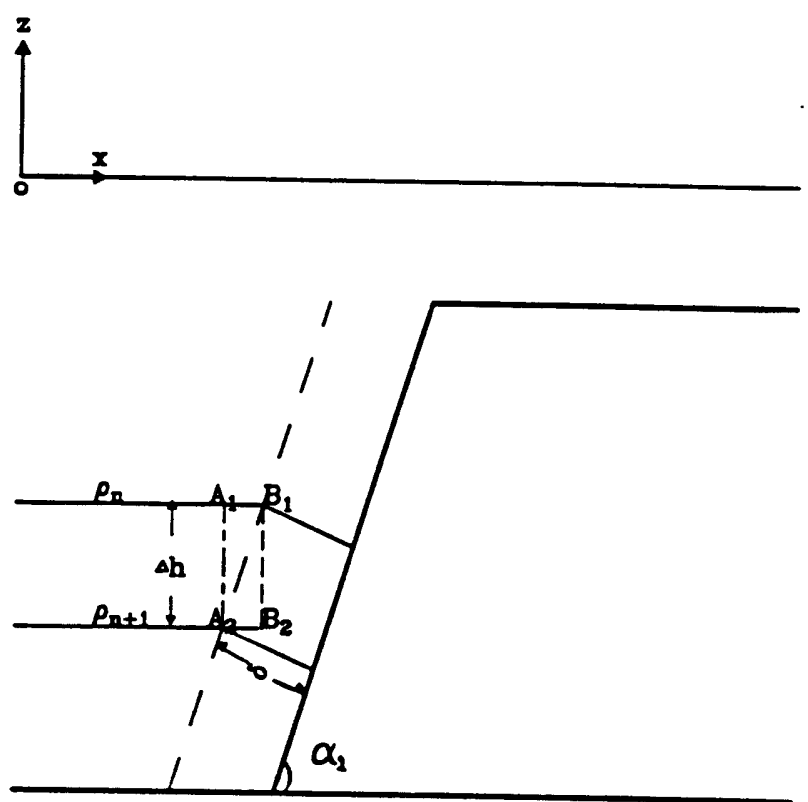

Figure A.3: Illustrative map for the density distribution in and out of the thermal boundary layer. Symbol $\delta$ is the thickness of the thermal boundary layer over the slope, $\alpha_{1}$ is the slope of bottom topography, and $\Delta \mathrm{h}$ is the vertical interval between the density surfaces $\rho_{n}$ and $\rho_{n+1}$.

In the real ocean, on the other hand, diffusion near the slope tends to mix the water in the vertical and the condition of no density flux into the sloping bottom requires that the density contours encounter the slope at a right angle (Wunsch, 1970; Phillips, 1970). Using a simple thermal boundary layer model, Wunsch (1970) estimated the thickness of the bottom diffusive layer, which is proportional to

$$
\delta \sim\left(K_{m} K_{H}\right)^{\frac{1}{4}} N^{\frac{1}{2}}
$$

When the fluid moves horizontally into the boundary, thermal diffusion will reduce its density and hence cause it to upwell along the slope. The vertical velocity based on Wunsch's theory is about

$$
w \sim\left(K_{m} K_{H}\right)^{\frac{1}{4}} N^{\frac{1}{2}}
$$

Whenever rotation is included, an along-isobath flow can be generated in such a thermal boundary layer. Facing downstream in the direction of the current, the lighter water is 
always on the observer's left. The fact that the maximum computational error in the baroclinic pressure gradient in the $\sigma$ coordinate system in the Blumberg and Mellor model occurs at the bottom and, in general, in the opposite direction to the diffusive current (true current) predicted by Wunsch's theory, provides us with a simple way to estimate the $\sigma$-coordinate error by checking if there is a consistent diffusive flow in our model results.

An example for comparison of our numerical results with Wunsch's theory is given next. In all our numerical computations, we assumed the turbulent Prandtl number to be 1 and specified $K_{m}=K_{H}$. The predicted thickness of the thermal boundary layer and vertical velocity can be simplified as

$$
\delta \sim \sqrt{\frac{K_{m}}{N}} \text { and } w \sim \sqrt{K_{m} N} .
$$

For the case of $H_{s}=50 \mathrm{~m}$ and $\ell_{t}=50-\mathrm{km}$, the numerically predicted $K_{m}=0.001 \mathrm{~m}^{2} / \mathrm{s}$ and $\mathrm{N}^{2}=10^{-5} \mathrm{~s}^{-2}$ at a depth of $281 \mathrm{~m}, \delta \sim 3 \mathrm{~m}$, and $w \sim 0.2 \times 10^{-3} \mathrm{~cm} / \mathrm{s}$ there. Substituting the numerical values of $K_{m}$ and $N$ into Wunsch's formula gives

$$
\delta \sim 57 \mathrm{~cm}, \text { and } w \sim 0.2 \mathrm{~cm} / \mathrm{s}
$$

which are the same order of magnitude as the numerical result. The along-isobath geostrophic velocity can be easily estimated based on Wunsch's theory. Let density outside and inside the thermal boundary layer be given by

$$
\rho= \begin{cases}\rho_{o}-\frac{\rho_{b}-\rho_{o}}{H} z & \text { outside the thermal boundary layer } \\ \rho_{o}-\frac{\rho_{b}-\rho_{o}}{H}\left(z+x^{\prime} \cot \alpha_{1}\right) & \text { inside the thermal boundary layer }\end{cases}
$$


so that the pressure at points $A_{2}$ and $B_{2}$ (see Figure A.3) is equal to

$$
\begin{aligned}
& P_{A_{2}}=P_{o}+g \rho_{o} \Delta h+g \frac{\rho_{b}-\rho_{o}}{2 H} \Delta h^{2}, \\
& P_{B_{2}}=P_{o}+g \rho_{o} \Delta h+g \frac{\rho_{b}-\rho_{o}}{2 H} \Delta h^{2}-g \frac{\rho_{b}-\rho_{o}}{H} \Delta x \cot \alpha_{1} \Delta h .
\end{aligned}
$$

Then an along-isobath geostrophic flow can be estimated as

$$
V=\frac{1}{f \rho_{o}} \frac{\partial P}{\partial x}=-\frac{N^{2}}{f} \cot \alpha_{1} \Delta h .
$$

Since $\Delta x=\delta \sin \alpha_{1}$, and $\Delta h=\Delta x \tan \alpha_{1}$, the above equation can be simplified to

$$
V=-\frac{N^{2}}{f} \delta \sin \alpha_{1}
$$

Taking $N^{2} \sim 10^{-5} \mathrm{~s}^{-2}, \delta=3 \mathrm{~m}$ and $\sin \alpha_{1}=0.005$, so

$$
V \sim-0.15 \mathrm{~cm} / \mathrm{s}
$$

In our numerical experiment, we found that

$$
V=-0.1 \sim 0.2 \mathrm{~cm} / \mathrm{s}
$$

This is in good agreement with the theoretical prediction, suggesting that the $\sigma$-coordinate transformation error should be at least one order of magnitude smaller than the true thermal current in this case. Based on similar analysis made for other numerical experiments, the numerical errors for along-isobath velocity $(\bar{v})$ over the steep bottom topography of Georges Bank are believed to be of order $1 \mathrm{~cm} / \mathrm{s}$ or less, about $10 \%$ of the maximum residual current on the southern flank while only $3 \%$ on the northern flank. 


\section{Appendix B: A Numerical Treatment of Open Boundary Conditions in the ECOM3D-SI}

\section{I: Radiation Boundary Condition}

A staggered Arakawa $\mathrm{C}$ grid is used for the numerical computation in the ECOM3DSI. For the barotropic case, unknown variables at either southern or northern open boundary are the surface elevation $\zeta$ and along-bank velocity $v$, while the cross-bank velocity $u$ can be directly calculated using the elevation and a linearized set of momentum equations. In the numerical experiment for the $M_{2}$ tidal rectification, we force the model with the $M_{2}$ tidal elevation at the southern boundary of the numerical domain. Also, an implicit gravity wave radiation boundary condition with a phase speed of $\sqrt{g H}$ is specified for $\zeta$ and $\bar{v}$ at the northern boundary and for $\bar{v}$ alone at the southern boundary ( $\bar{v}$ is the vertically averaged $v)$.

The numerical calculation for $\zeta$ at the northern boundary is given by

$$
\zeta_{b}^{n+1}=\left(\zeta_{b}^{n}+\mu \zeta_{b-1}^{n+1}\right) /(1+\mu)
$$

where $\mu=\sqrt{g H} \Delta t / \Delta x, n$ is the time step, and $\mathrm{b}$ is boundary point. In the numerical computation, the time step $\Delta t$ and the horizontal grid $\Delta x$ are chosen based on the condition of the minimum reflection $\mu=\sqrt{g H} \Delta t / \Delta x=0.5$ (Chapman, 1985).

Two steps are used to calculate $v$ at the open boundary. First, we separate $v$ as a sum of $v^{\prime}$ and $\bar{v}$, i.e,

$$
v=v^{\prime}+\bar{v}
$$

where $v^{\prime}$ is the difference between $v(x, y, z)$ and the vertically averaged velocity $\bar{v}(x, y)$. Then the radiation boundary condition for $\bar{v}$ is applied along with the gradient boundary 
condition for $v^{\prime}$ (i.e, $\left.v_{b}^{\prime}=v_{b-1}^{\prime}\right)$. So, $v$ at boundary is numerically given as

$$
v_{b}^{n+1}=v_{b-1}^{n+1}+\left(\bar{v}_{b}^{n}+\mu \bar{v}_{b-1}^{n+1}\right) /(1+\mu)
$$

\section{II: Sponge Layers}

The sponge layer is constructed by introducing linearly damping functions of $-\gamma_{1} \zeta$ in the continuity equation and $-\gamma_{2} v$ in the $v$ momentum equation over the $n$ grid points close to or at the open boundary. The damping coefficient $\gamma_{i}\left(i\right.$ is the index for $\gamma_{1}$ and $\left.\gamma_{2}\right)$ in the numerical form is given by

$$
\gamma_{i}=\frac{\gamma_{\text {imax }}}{n}(b-j), \quad j=b \pm 1,2,3, \ldots, n
$$

where $n$ is the number of grid points in the sponge, $\gamma_{i m a x}$ is the maximum $\gamma_{i}$ at the outer edge of the sponge, and $b$ is the boundary point. In the southern sponge layer, $\gamma_{1}$ is taken as zero because the model is forced by the surface elevation at the southern open boundary. The values of $\gamma_{1}$ in the northern sponge layer and $\gamma_{2}$ in both southern and northern sponge layers are chosen based on numerical experiments for linear propagating waves. In the stratified tidal rectification problem described in Chapter $3, \gamma_{2 \max }=0.019$ in both southern and northern sponge layers, and $\gamma_{1 \max }=0.01$ in the northern sponge layer. Five grid points were used for the symmetric bank case $(n=5)$ and ten for the asymmetric bank case $(n=10)$. 